\title{
Analysis of room transfer function and reverberant signal statistics Abstract of paper
}

Georganti, Eleftheria; Mourjopoulos, John; Jacobsen, Finn

Published in:

Acoustical Society of America. Journal

Link to article, DOI:

$10.1121 / 1.2935346$

Publication date:

2008

Document Version

Publisher's PDF, also known as Version of record

Link back to DTU Orbit

Citation (APA):

Georganti, E., Mourjopoulos, J., \& Jacobsen, F. (2008). Analysis of room transfer function and reverberant signal statistics: Abstract of paper. Acoustical Society of America. Journal, 123(5), 3761-3761.

https://doi.org/10.1121/1.2935346

\section{General rights}

Copyright and moral rights for the publications made accessible in the public portal are retained by the authors and/or other copyright owners and it is a condition of accessing publications that users recognise and abide by the legal requirements associated with these rights.

- Users may download and print one copy of any publication from the public portal for the purpose of private study or research.

- You may not further distribute the material or use it for any profit-making activity or commercial gain

- You may freely distribute the URL identifying the publication in the public portal

If you believe that this document breaches copyright please contact us providing details, and we will remove access to the work immediately and investigate your claim 


\title{
Session 5aAAa
}

\section{Architectural Acoustics: New Frontiers in Room Acoustical Modeling I}

\author{
Murray Hodgson, Cochair \\ The University of British Columbia, Department of Electrical and Computer Engineering, 2332 Main Mall, Vancouver, \\ BC V6T 1Z4, Canada \\ Vincent Valeau, Cochair \\ Laboratoire d'Etudes Aérodynamiques (LEA), Université de Poitiers - ENSMA - CNRS, Bâtiment K, \\ 40 Avenue du Recteur Pineau, Poitiers, F-86022, France
}

\section{Contributed Papers}

\section{8:00}

5aAAa1. Multiresolution geometrical-acoustics modeling. Benjamin Markham (Rensselaer Polytechnic Institute, Greene Bldg., 110 8th St., Troy, NY 12180, USA, markhb@ @pi.edu), Paul Calamia (Rensselaer Polytechnic Institute, Greene Bldg., 110 8th St., Troy, NY 12180, USA, calamp @ rpi.edu)

Geometrical-acoustics (GA) modeling techniques assume that surfaces are large relative to the wavelengths of interest. For a given scenario, practitioners typically create a single 3D model with large, flat surfaces that satisfy the assumption over a broad range of frequencies. Such geometric approximations lead to errors in the spatial distribution of the simulated sound field because geometric details that influence reflection and scattering behavior are omitted. To compensate for the approximations, modelers typically estimate scattering coefficients for the surfaces to account stochastically for the actual, wavelength-dependent variations in reflection directionality. A more deterministic approach could consider a series of models with increasing geometric detail, each to be analyzed at a corresponding frequency band for which the requirement of large surface dimensions is satisfied. Thus, to improve broadband spatial accuracy for GA simulations, we propose a multiresolution modeling approach. Using scale model measurements of a corrugated wall, comparisons of our method with non-GA techniques, and some simple listening tests, we will demonstrate that multiresolution geometry provides more spatially accurate results than single-resolution approximations when using GA techniques, and that this improved accuracy is aurally significant.

\section{$8: 20$}

5aAAa2. On the analysis of the time spreading of sound diffusers. Javier Redondo (IGIC - Universitat Politècnica de València, Cra. NazaretOliva S/N, E-46730 Gandia, Spain, fredondo@fis.upv.es), Rubén Picó (EPSG - Univ. Politécnica de Valencia, c/ Nazaret-Oliva s/n, 46780 Grau de Gandia, Spain, rpico@fis.upv.es), Mark R. Avis (University of Salford, Acoustics Research Centre, Newton Building, M5 4WT Salford, UK, m.r.avis@salford.ac.uk)

Since the invention of sound diffusers three decades ago a substantial effort has been made to predict the acoustic behaviour of these structures. BEM methods are well established for this purpose after a systematic comparison between simulations and experimental data. Volumetric methods such as finite element methods (FEM) or the finite difference time domain method (FDTD) are not often used, due to their large computational cost. However, near to far field transformations (NFFT) can overcome that problem. Recently some of the authors have shown that the FDTD method is a useful technique to analyse the time domain signature of sound diffusers. In this paper a careful analysis of the performance of diffusers in the time domain ("time spreading") are reported, opening a new field of research.

\section{Invited Papers}

\section{$8: 40$}

5aAAa3. Diffraction modeling in acoustic radiance transfer method. Samuel Siltanen (Helsinki University of Technology, P.O. Box 5400, 02015 TKK, Finland, Samuel.Siltanen@tml.hut.fi), Tapio Lokki (Helsinki University of Technology, P.O. Box 5400, 02015 TKK, Finland, Tapio.Lokki@tkk.fi)

The room acoustic radiance transfer method is a solution to recently presented room acoustic rendering equation which formulates the mathematical basis for all the ray-based (geometrical) room acoustic modeling algorithms. The basic acoustic transfer method gives as accurate results as the state-of-the-art commercial room acoustic modeling software. However, the basic method still lacks, e.g., diffraction modeling and modeling of complex reflections from surfaces. In this paper we discuss different diffraction modeling methods in the light of the acoustic radiance transfer method. The problems as well as benefits of each diffraction modeling method are summarized to understand which one of them can be implemented together with acoustic radiance transfer. Finally, some implementation examples are given.

\section{9:00}

5aAAa4. Can also diffracted sound be handled as flow of particles? Some new results of a beam tracing approach based on the uncertainty principle. Uwe M. Stephenson (Hafen City University Hamburg, Nelkenweg 10, 23843 Bad Oldesloe, Germany, post @umstephenson.de)

In computational room acoustics as well as noise immission prognosis efficient ray or beam tracing methods are well approved - but the problem of the neglected diffraction is still unsolved in general. The author's successful approach of 1986 based on Heisenbergs uncertainty principle was extended to the more efficient beam tracing technique and presented at the ICA 2007. The algorithm has now been generalized to recursive higher order diffraction. Now, not only single edge, but also multiple edge diffraction could be simulated 
(slit and "wide obstacle" with two edges). The results have again been compared with Svensson's exact wave-theoretical secondary edge source model. With some restrictions, they seem to indicate, that indeed even diffraction of sound - like light - may be handled as flow of particles. To avoid the feared explosion of computation time with higher order diffraction, a beam reunification may now be achieved by Quantized Pyramidal Beam Tracing.

\title{
Contributed Paper
}

9:20

5aAAa5. General impedance boundary conditions in pseudospectral time-domain methods for room acoustics. Carlos Spa (Universitat Pompeu Fabra - Fundació Barcelona Media, Ocata, 1, 08003 Barcelona, Spain, carlos.spa@upf.edu), Toni Mateos (Universitat Pompeu Fabra Fundació Barcelona Media, Ocata, 1, 08003 Barcelona, Spain, toni.mateos@barcelonamedia.org), Adan Garriga (Universitat Pompeu Fabra - Fundació Barcelona Media, Ocata, 1, 08003 Barcelona, Spain, adan .garriga@barcelonamedia.org)

Finite-differences in the time domain (FDTD) are among the most accurate numerical techniques to simulate wave phenomena. The main drawback of FDTD numerical schemes is their computational cost in large scale simulations. The recently developed Fourier pseudospectral time-domain (PSTD) techniques, by approximating the spatial derivatives more efficiently, have improved significantly the accuracy and time costs of the simulations of electromagnetic fields. As a step towards applying PSTD techniques to room acoustic problems, we present here a framework to properly deal with material modeling in terms of generic impedance boundary conditions, beyond the common perfectly matched layer absorbing boundaries. We apply our results to a few representative cases (simple but reverberant rooms) and analyse its performance in terms of accuracy and computational resources. In particular we analyse whether the mild constraints that PSTD imposes on space-time discretization lead to good enough results in room acoustic simulations.

9:40-11:00 Posters

Lecture sessions will recess for presentation of poster papers on various topics in acoustics. See poster sessions for topics and abstracts.

\section{Invited Papers}

\author{
11:00
}

5aAAa6. Ray-tracing prediction of sound-pressure and sound-intensity fields in empty and fitted rooms. Murray Hodgson (The University of British Columbia, Department of Electrical and Computer Engineering, 2332 Main Mall, Vancouver, BC V6T 1Z4, Canada, mhodgson@interchange.ubc.ca), Owen Cousins (The University of British Columbia, Department of Electrical and Computer Engineering, 2332 Main Mall, Vancouver, BC V6T 1Z4, Canada, owencousins@hotmail.com), Gary Chan (The University of British Columbia, Department of Electrical and Computer Engineering, 2332 Main Mall, Vancouver, BC V6T 1Z4, Canada, gakachan@interchg.ubc.ca), Vincent Valeau (Laboratoire d'Etudes Aérodynamiques (LEA), Université de Poitiers - ENSMA - CNRS, Bâtiment K, 40 Avenue du Recteur Pineau, F-86022 Poitiers, France, vincent.valeau@lea.univ-poitiers.fr)

A Monte-Carlo ray-tracing model has been adapted to the prediction of sound-pressure and sound-intensity fields in rooms with surfaces of arbitrary surface impedance, and containing parallelepiped obstacles. Phase changes due to propagation distance and wall reflection were accounted for. Diffraction around obstacle edges was modeled by the Unified Theory of Diffraction. The model was also extended to include the out-of-phase secondary sound source of a single-channel, global active-noise-control (ANC) system. The new model was validated in comparison with predictions by reference models (e.g. FEM) and with the results of experiments in an anechoic chamber and in real rooms. It was used to predict the effectiveness of ANC. This paper discusses the development of the new models, the results of the validation tests and ANC predictions.

\section{$11: 20$}

5aAAa7. The intensity in a reverberant field as an acoustic energy-density gradient. Vincent Valeau (Laboratoire d'Etudes Aérodynamiques (LEA), Université de Poitiers - ENSMA - CNRS, Bâtiment K, 40 Avenue du Recteur Pineau, F-86022 Poitiers, France, vincent.valeau@lea.univ-poitiers.fr), Matthieu Boirlaud (ESIP, Av. du Recteur Pineau, 86022 Poitiers Cedex, France, matthieu.boirlaud@etu.univ-poitiers.fr), Judicaël Picaut (Lab. Central des Ponts et Chaussées, Division Entretien, Sécurité et Acoustique des Routes, Route de Bouaye - BP 4129, 44341 Bouguenais Cedex, France, Judicael.Picaut@lcpc.fr), Murray Hodgson (The University of British Columbia, Department of Electrical and Computer Engineering, 2332 Main Mall, Vancouver, BC V6T 1Z4, Canada, mhodgson@interchange.ubc.ca)

The classical assumption for diffuse reverberant sound fields is that the acoustic energy flow at any location in a room -- i.e., the acoustic intensity -- is null. For rooms with disproportionate dimensions and/or uneven absorption distributions, this assumption no longer holds. Over the past few years, a room-acoustic diffusion model has been developed that can be seen as an extension of the statistical theory to nondiffuse sound fields. This work investigates the basic gradient-equation underlying this diffusion theory: it states that the energy-flow vector is proportional to both the gradient of the acoustic energy density, and to the room diffusion constant. The gradient equation for the acoustic intensity is confirmed numerically by comparison with calculations of the intensity vector using a particle-tracing model and a ray-tracing model. Examples are presented for a room with a diffuse sound field, and for elongated rooms where the sound field is strongly non-diffuse, in the case of both diffuse and specular reflections. 
5aAAa8. Prediction and tailoring of steady-state broadband sound fields in enclosures using absorption scaling and energy-intensity boundary elements. Donald B. Bliss (Duke University, Mechanical Engineering and Materials Science, 148B Hudson Hall, Durham, NC 27708, USA, dbb@duke.edu), Krista A. Michalis (Duke University, Mechanical Engineering and Materials Science, 148B Hudson Hall, Durham, NC 27708, USA, kam49@duke.edu), Linda P. Franzoni (Duke University, Mechanical Engineering and Materials Science, 148B Hudson Hall, Durham, NC 27708, USA, franzoni@duke.edu)

Enclosures with diffuse reflection boundaries are modeled with an energy-intensity boundary element method using uncorrelated broadband directional sources. An absorption-based perturbation analysis shows the spatial variation of the acoustic field obeys certain scaling laws. A series expansion in terms of average absorption gives separate boundary integral problems at each order. The lowestorder solution has a uniform level proportional to the reciprocal of the average absorption. The next-order solution is independent of average absorption and primarily responsible for spatial variation of the acoustic field. This solution depends on the spatial distribution of absorption and input power sources, but not their overall level. For the primary spatial variation, the effects of the relative distributions of absorption and input power are linear and uncoupled. These distributions can be expressed in terms of constituent spatial modes corresponding to the ways absorption and input power can be distributed. Solved numerically once for each mode, the acoustic field can be expressed in terms of the modal amplitudes in closed form. These amplitudes can be adjusted to tailor the spatial variation. Examples include how to distribute absorption to minimize sound levels in one location, or how to achieve a uniform interior field. (Sponsor: NSF)

\section{Contributed Papers}

\section{2:00}

5aAAa9. Analysis of room transfer function and reverberant signal statistics. Eleftheria Georganti (Acoustic Technology Department, Technical University of Denmark, Ørsted Plads, B352, DK-2800 Lyngby, Denmark, ege@oersted.dtu.dk), John Mourjopoulos (Audio and Acoustic Technology Group, Wire Communications Laboratory, Electrical Engineering \& Computer Technology Department, University of Patras, 26500 Patras, Greece, mourjop@wcl.ee.upatras.gr), Finn Jacobsen (Acoustic Technology Department, Technical University of Denmark, Ørsted Plads, B352, DK2800 Lyngby, Denmark, fja@ oersted.dtu.dk)

For some time now, statistical analysis has been a valuable tool in analyzing room transfer functions (RTFs). This work examines existing statistical time-frequency models and techniques for RTF analysis (e.g., Schroeder's stochastic model and the standard deviation over frequency bands for the RTF magnitude and phase). RTF fractional octave smoothing, as with $1 / 3$ octave analysis, may lead to RTF simplifications that can be useful for several audio applications, like room compensation, room modeling, auralisation purposes. The aim of this work is to identify the relationship of optimal response smoothing (e.g., as in complex smoothing) with respect to the original RTF statistics. More specifically, the RTF statistics, derived after the complex smoothing calculation, are compared to the original statistics across space inside typical rooms, by varying the source, the receiver position and the corresponding ratio of the direct and reverberant signal. In addition, this work examines the statistical quantities for speech and audio signals prior to their reproduction within rooms and when recorded in rooms. Histograms and other statistical distributions are used to compare RTF minima of typical "anechoic" and "reverberant" audio speech signals, in or- der to model the alterations due to room acoustics. The above results are obtained from both in-situ room response measurements and controlled acoustical response simulations.

\section{2:20}

5aAAa10. On the implementation of room acoustics modeling software using Digital Waveguide Mesh. José J. Lopez (Tech. Univ. of Valencia, Camino de Vera S/N, 46021 Valencia, Spain, jjlopez@dcom.upv.es), José Escolano (University of Jaén, Alfonso X, 28, E-23700 Linares, Spain, escolano@ujaen.es), Basilio Pueo (University of Alicante, Signals, Systems and Telecommunications, Cta San Vicente del Raspeig s/n, E-03690 Alicante, Spain, basilio@ua.es)

The Digital Waveguide Mesh (DWM) method for room acoustic simulation has been introduced in the last years to solve sound propagation problems numerically. However, the huge computer power needed in the modeling of large rooms and the complexity to incorporate realistic boundary conditions has delayed their general use, being restricted to the validation of theoretical concepts using simple and small rooms. This paper presents a complete DWM implementation where all the stages needed to analyze a room are discussed. The software starts the analysis from the architectural model of the room importing its geometry directly from a CAD file. After that, it generates the rectangular mesh of individual cells that conforms the volume to be simulated. Next, the time domain recursion is carried out using parallel computer techniques. Also the software includes a serious treatment of boundary conditions using different material with frequency dependence characteristics. Finally, the software can export the results. Additionally, some room simulation examples are presented and analyzed in detail. The work carried out demonstrate how it is possible, with the current power of the personal computer, to start to simulate real rooms with high amount of geometric details and frequency dependent boundary.

\section{2:40-2:00 Lunch Break}

\section{2:00}

5aAAa11. Defining perceptual requirements of dynamic (real-time) acoustic modeling and auralization. Linda Gedemer (5930 Penfield Ave., Woodland Hills, CA 91367, USA, gedeml@rpi.edu)

Acoustic modeling is evolving from static sources and receivers to dynamic sources and receivers as computational speeds allow for faster renderings. Current research has focused on creating highly accurate dynamic models and auralizations but still struggle with the trade-off between accuracy and available processing speed. The question remains as to how accurate do these models need to be if the receiver (listener) is moving dynamically through the acoustic model. The focus of this paper is to address the accuracy required of dynamic models and/or auralizations from a perceptual standpoint. From this, the necessity for creating models that are perhaps more accurate than required will be considered.

\section{2:20}

5aAAa12. The scanning and voxellisation of complex 3D objects for incorporation within finite difference time domain based acoustic prediction. Ian Drumm (The University of Salford, C.S.E., Salford University, The Crescent, M5 4WT Manchester, UK, i.drumm@salford.ac.uk)

This paper presents techniques developed to incorporate complex 3D objects within the author's own finite difference time domain based acoustic prediction software application. Using a combination of 3D laser scanning, ray based voxellisation and a least pth norm based filter design approach to emulate the absorption profiles of non-rigid boundaries; complex objects can be incorporated into a variety of FDTD based acoustic prediction scenarios. The paper will evaluate the success of the approach and explore its application. Of particular interest is the emulation of furniture, people, acoustic modifiers and other complex non planer structures within room acoustics predictions. 


\title{
Invited Paper
}

2:40

5aAAa13. Localization and mesoscopics in structures and rooms II. Richard Weaver (Dept. of Physics, University of Illinois, Urbana, IL 61801, USA, r-weaver@uiuc.edu)

A new theory is developed which makes quantitative predictions for energy transport that are consistent with localization. The theory promises ultimately to apply to a wide variety of systems. It is based on an assumption of S-matrices that are incoherent - as conventional diffuse field theories would have it - but then corrected to assure exact energy conservation. It is shown that the resulting system responses (they are concatenations of S matrices) have coherence, are consistent with localization, and predict Greens function retrieval. Localization emerges naturally from competition between rates of transport and rate of eigen-mode resolution. Examples are presented corresponding to coupled rooms and to diffusion in a multiply scattering medium. Future generalizations are discussed and the mathematical challenges are outlined. This is the second talk in a pair begun in a different session. Work supported by NSF CMS 05-28096.

FRIDAY MORNING, 4 JULY 2008

ROOM 243, 8:00 A.M. TO 3:20 P.M.

\section{Session 5aAAb}

\section{Architectural Acoustics and Noise: Airborne and Impact Sound Insulation I}

\author{
Berndt Zeitler, Cochair \\ NRC - Institute for Research in Construction, 1200 Montreal Road, Building M-27, Ottawa, ON K1A OR6, Canada \\ Patrizio Fausti, Cochair \\ Engineering Dept. - Univ. of Ferrara, Via Saragat, 1, Ferrara, 44100, Italy
}

\section{Contributed Paper}

8:00

5aAAb1. Maximizing field performance of thin, light-weight partition and floor assemblies. Ronald Eligator (Acoustic Dimensions, 145 Huguenot Street, New Rochelle, NY 10801, USA, religator@acousticdimensions .com)

Laboratory test reports are an important source of information regarding anticipated performance of a sound barrier construction. However, differences in construction conditions between the laboratory and a real building can greatly affect, usually negatively, the performance of a sound barrier assembly in the field. This reality, combined with the typical client's focus on reducing construction costs, requires that for a sound barrier construction to be successful, the effect of abutting constructions must be well understood. This paper reports on our firm's experience in translating lab-tested lightweight sound barrier assemblies to the field, relating design conditions to field results in terms of physical construction and noise reduction results.

\section{Invited Paper}

5aAAb2. Monitoring field and laboratory performance for airborne and impact sound insulation for 110,000 homes per annum. Sean Smith (BPC, Napier University, 42 Colinton Road, EH10 5BT Edinburgh, UK, se.smith@napier.ac.uk), Dave Baker (Robust Details Ltd, Davy Avenue, Knowlhill, MK5 8NB Milton Keynes, UK, dbaker@robustdetails.com), David Panter (Robust Details Ltd, Davy Avenue, Knowlhill, MK5 8NB Milton Keynes, UK, dpanter@robustdetails.com)

This paper provides an overview of a unique monitoring system used in England and Wales for tracking the sound insulation performance for 110000 new build homes per annum. The regulatory approach, known as Robust Details was established within the building regulations and passed by parliament in 2004 after an 18 month initial research project. Annually over 5000 construction sites now use this system approach for separating walls and floors for attached houses and apartments. Acoustic site inspections and sound insulation field testing reports provide systematic feedback to the design and performance review. Since the RD systems inception on-site sound insulation compliance rates have risen from $40 \%$ to $97 \%$. This paper will discuss the role of laboratory benchmark testing for products and also the role of acoustic "field" inspectors in tracking the airborne and impact performance. In addition the role of intervention and statistical analysis in upgrading or withdrawing specific wall and floor constructions will be presented. 


\title{
Contributed Paper
}

\section{8:40}

5aAAb3. Changed sound properties due to minor construction changes in a lightweight building. Fredrik Ljunggren (Luleå Univ. of Technology, Div. of Sound and Vibration, S-97187 Luleå, Sweden, fredrik.ljunggren@ltu .se)

This paper relates to building acoustic measurement inside a two-story office house. The construction, which is known as lightweight, is prefabricated in volumes at a factory and is then transported to the building yard for assembling. It is build up of a wooden frame with particle boards and plaster boards attached. The building consists of a number of nominally, or almost nominally, identical rooms with assumed identical sound properties. In the projection stage, the construction was slightly modified in some of the rooms in order to see in what way the sound properties would be affected. In total eight impact sound pressure level measurements and eight sound reduction index measurements were performed and analysed for the different setups.

\section{Invited Papers}

\begin{abstract}
9:00
5aAAb4. The drying process influence on the brick walls sound reduction index: laboratory evaluations and theoretical analysis. Chiara Scrosati (Construction Technologies Institute of Italian National Research Council, Viale Lombardia, 49, 20098 San Giuliano Milanese (MI), Italy, labacustica@itc.cnr.it), Fabio Scamoni (Construction Technologies Institute of Italian National Research Council, Viale Lombardia, 49, 20098 San Giuliano Milanese (MI), Italy, Fabio.Scamoni@itc.cnr.it), Fabrizio Valentini (Construction Technologies Institute of Italian National Research Council, Viale Lombardia, 49, 20098 San Giuliano Milanese (MI), Italy, Fabrizio.Valentini@itc.cnr.it)
\end{abstract}

This paper presents experimental results showing the sound reduction index of different types of brick walls; the analysis was in particular carried out on double walls, single walls, and single walls with external lining. Its main purpose is to find the minimum drying time of the structure necessary to obtain the sound reduction indexes of brick walls as close as possible to laboratory real values. Moreover, it aims at making results of different laboratories as comparable as possible in terms of repeatability and reproducibility. All the fundamental quantities, such as the sound reduction index $(\mathrm{R})$, the weighted sound reduction index $\left(\mathrm{R} \_\{\mathrm{w}\}\right)$, the spectrum adaptation terms $\mathrm{C}_{\text {and }} \mathrm{C}_{-}\{\mathrm{tr}\}$, and temperature $(\mathrm{T})$ and relative moisture, have been analyzed. A qualitative analysis of the curves slope of the sound reduction index versus frequency has been conducted for each drying time for each type of evaluated wall. The measurements were carried out in the acoustic laboratory of ITC-CNR (the Construction Technologies Institute of the Italian National Research Council) in Milan, according to the procedures of ISO 140 part 3.

$$
\text { 9:20 }
$$

5aAAb5. Acoustical proprieties of light brick walls and its effects on flanking transmission. Giovanni Semprini (University, DIENCA Dept. Facoltà di Ingegneria, Viale Risorgimento 2, 40136 Bologna, Italy, giovanni.semprini@mail.ing.unibo.it), Luca Barbaresi (DIENCA - Univ. of Bologna, Viale Risorgimento 2, 40136 Bologna, Italy, luca.barbaresi@mail.ing.unibo.it)

Light brick walls 8-10 $\mathrm{cm}$ thick are typical structures frequently used in Italian building constructions as a internal partitions between dwellings and as internal layer of double façade walls. Due to low surface mass and rigid connection to other horizontal and vertical structures, light brick walls are often responsible of high flanking transmission. The simplest acoustic modeling of this structure for the evaluation of sound transmission in buildings, is the approach proposed by the EN 12354 standards, where the brick wall can be considered as a homogeneous structure and main acoustic parameters required are the sound reduction index and the vibration reduction index Kij. The aim of this paper is to analyse results of physical and acoustical properties (sound reduction index, structural reverberation time, radiation efficiency) of a typical $8 \mathrm{~cm}$ thick brick wall measured in laboratory testing facility, used as a partition in different test configuration: single layer wall, double brick layer wall with internal thermal layer and as a component of lateral wall, where the Kij index flanking transmission were also evaluated. Experimental results are then compared with theoretical values in order to analyse difference with homogeneous structures.

9:40-11:00 Posters

Lecture sessions will recess for presentation of poster papers on various topics in acoustics. See poster sessions for topics and abstracts.

\section{Contributed Paper}

11:00

5aAAb6. Lightweight system Technostar specified in the CCTV tower Beijing. Andrew Koster (Saint-Gobain Insulation, Parallelweg 20, 4878AH Etten-Leur, Netherlands, andrew.koster@saint-gobain.com)

The role and possibilities of lightweight mineral wool is known for quite some years, acting as the optimum spring in a mass spring mass system one can reach high acoustic values. Compared to the massive constructions (e.g., concrete) the use of a mass spring mass construction has also other advantages (e.g., logistic, dimension of foundation and labour costs). SaintGobain Isover developed a wall system called Technostar, originally defined for cinema walls. In this construction an optimal mass spring mass construction is created. The theory of mass spring mass systems will shortly come up. All elements of the system are described. The acoustical performance of the Technostar construction is calculated with acoustic software (Stiff) and compared with laboratory measurements; the influence of different parameters on the measured acoustical performance will be shown. The practical use of the Technostar wall will be shown on the basis of the project CCTV tower in Beijing. For this project the acoustic requirements, the translation in terms of the wall construction with the specific details will be shown. The system will be build in March 2008 . 


\section{Invited Paper}

\section{1:20}

5aAAb7. Influence of static-load on airflow resistivity determination. Alessandro Schiavi (Istituto Nazionale di Ricerca Metrologica, str. delle Cacce, 91, 10135 Turin, Italy, a.schiavi@inrim.it), Claudio Guglielmone (Istituto Nazionale di Ricerca Metrologica, str. delle Cacce, 91, 10135 Turin, Italy, c.guglielmone@inrim.it), Piercarlo Miglietta (FIAT Group Automobiles S.p.A., Corso G. Agnelli, 220, 10135 Turin, Italy, piercarlo.miglietta@ fiat.com), Franco Alasia (Istituto Nazionale di Ricerca Metrologica, str. delle Cacce, 91, 10135 Turin, Italy, f.alasia@inrim.it)

Dynamic stiffness of resilient materials used as underlayer in floating floors give useful knowledge on the acoustical behaviour of a floor in impact sound insulation. In previous works the influence of the static load and the compressive behaviour of the resilient layers on dynamic stiffness evaluation has been shown. A new measurement technique of the airflow resistance (according to ISO 9053) in terms of static load applied on the resilient materials with open cells is proposed. Usually the airflow resistance of porous materials used as underlayers is evaluated on unloaded samples of material. In dynamic stiffness measurements the material is subject to a static load of $2 \mathrm{kPa}$. Because the dynamic stiffness value depends on airflow resistivity $\mathrm{r}\left[\mathrm{Pa} \times \mathrm{s} / \mathrm{m}^{2}\right]$ (in particular when $10 \mathrm{kPas} / \mathrm{m}^{2}<\mathrm{r}<100$ $\mathrm{kPas} / \mathrm{m}^{2}$ ) through the material, it is important to evaluate the airflow resistivity behaviour in the same condition of the dynamic stiffness measurement, i.e., under a static load of $2 \mathrm{kPa}$. A new apparatus to evaluate the airflow resistivity in terms of different static loads has been designed and realized at I.N.RI.M. of Turin. The first experimental results show evidence of the dependence of the airflow resistivity of the resilient materials on the applied load.

\section{Contributed Papers}

$11: 40$

5aAAb8. Compression of felt-type thermal insulation layer for underfloor heating system and floor impact sound. Tongjun Cho (SKEC, Jung-Gu, Soonhwa-Dong 66, SK Soonhwa B/D, R\&D Center, 100130 Seoul, Republic of Korea, tjc@skec.co.kr), Hyun-Min Kim (SKEC, Jung-Gu, Soonhwa-Dong 66, SK Soonhwa B/D, R\&D Center, 100130 Seoul, Republic of Korea, thek80@skec.co.kr)

In Korea, almost every house uses underfloor heating, which has advantages of thermal comfort and energy efficiency. However, when it is constructed for high-rise apartment houses, it yields a problem in floor impact sound insulation. It accounts for the fact that a foam-type thermal insulator sandwiched between structural slab and heating floor functions as a spring and easily transmits impacts on the floor to the slab. In that case, the system's transmissibility is determined by dynamic stiffness of the thermal insulation layer and the lower the dynamic stiffness is, the more the floor impact is isolated. For that reason, apartments construction companies are attempting to lower the dynamic stiffness of the thermal insulation layer for impact sound reduction. As part of the attempt, felt-type materials with relatively low dynamic stiffness such as glass wool or polyester felt are considered as a substitution for the foam-type thermal insulator. However, there is a possibility that compression of the felt-type materials would increase the dynamic stiffness and the impact sound insulation effect at early stage might be weakened in the long term. This paper investigates the correlation between gradual compression of the felt-type thermal insulation layer and the impact sound variation.

\section{2:00}

5aAAb9. Acoustic and vibration characteristics of floated floors concrete structures. Seungyup Y. Yoo (Hanyang University, 133-791 Seoul, Republic of Korea, syrus81@hanyang.ac.kr), Jin Yong Jeon (Hanyang University, Department of Architectural Engineering, 133791 Seoul, Republic of Korea, jyjeon@ hanyang.ac.kr)

The floating floor with multilayers of resilient materials was investigated for the isolation of floor impact sound. The floor structures were evaluated in the reduction of the heavy-weight impact sound, which is mainly affected by the boundary conditions of the tested floors. The values of single-number ratings for floor impact sounds decrease dramatically for floated floors. Insertion loss is determined by the vibration characteristics of a bare slab, such as transmissibility. The logarithmic decrement $(\xi)$ of the vibration responses was 4 times higher than that of the bare slab. Above $125 \mathrm{~Hz}$, the vibration between the slab structure and floating floor is isolated effectively. It was revealed that the resonant frequency of the slab structure and the damping factor of the floating floor are important aspects for impact sound isolation.

\section{Invited Paper}

5aAAb10. Interactions between floor and ceiling panels in presence of a fibrous material in the cavity. Thomas Scelo (Marshall Day Acoustics LTD, P. O. Box 5811, Wellesley St., 1000 Auckland, New Zealand, thomas.scelo@marshallday.co.nz)

The mechanisms governing the acoustic transmission through building elements such as floor/ceiling assemblies are complex. The vibroacoustic interactions between the finite size structure and the enclosed volume are, in particular, rendered more complex at low frequencies when the finite dimensions of the building are taken into consideration. A model developed to predict the low frequencies vibroacoustics response of mutliplates systems offers the opportunity to explore, among other geometrical and material parameters, the effects of a fibrous material in the ceiling plenum on the acoustics behaviour of the whole structure. This paper presents the theoretical approach employed to predict the interactions between the floor, the ceiling panel and the enclosed volume. It also explores the effects of varying the material characteristics and overall thickness on these interactions.

\section{2:40-1:40 Lunch Break}




\section{Invited Paper}

\section{$1: 40$}

5aAAb11. Methods to control low frequency impact noise in wood-frame construction. Berndt Zeitler (NRC - Institute for Research in Construction, 1200 Montreal Road, Building M-27, Ottawa, ON K1A 0R6, Canada, berndt.zeitler@nrc-cnrc.gc.ca), Trevor Nightingale (NRC - Institute for Research in Construction, 1200 Montreal Road, Building M-27, Ottawa, ON K1A 0R6, Canada, Trevor.Nightingale@nrc-cnrc.gc.ca), Frances King (NRC - Institute for Research in Construction, 1200 Montreal Road, Building M-27, Ottawa, ON K1A 0R6, Canada, Frances.King @nrc-cnrc.gc.ca)

The control of low frequency impact noise is of great importance in Japan and Korea. For impact noise rating, both countries use standardised heavy and soft impactors that inject high levels of low frequency power into a wood-frame floor due to the high force applied and "good" impedance match between the source and the floor. This paper reports on a parametric study designed to confirm the important parameters for controlling low frequency impact noise and how these parameters are affected by changes to construction details. The paper shows that increasing the drive point impedance floor surface while minimising the structural coupling between the gypsum board ceiling and the structural framing (joists) are important elements. Additionally, a well-designed floor topping can be very effective when applied to a suitable floor. The paper presents data to show the relative importance of direct and flanking transmission paths. Furthermore, it is shown that repeated impacts by the Bang Machine physically change the floor assembly and as a consequence resulting impact sound pressure levels in the receive room also change. The paper concludes with a general discussion and recommendations.

\section{Contributed Papers}

2:00

5aAAb12. Field airborne and impact sound insulation of wood truss floor systems. Chad N. Himmel (JEAcoustics, 1705 West Koenig Lane, Austin, TX 78756, USA, Himmel@JEAcoustics.com), Daniel J. Kupersztoch (JEAcoustics, 1705 West Koenig Lane, Austin, TX 78756, USA, Kupersztoch@JEAcoustics.com)

A series of field measurements on wood truss floors of various types was recently completed at four residential apartment complexes. About 40 floors with different floor finishes, gypsum concrete underlayment, noise control underlayment systems, truss span lengths, ceiling types, and resilient metal channel types were constructed and measured. Trusses were all similar engineered prefabricated parallel chord wood trusses. Room volumes and room absorptive characteristics are varied and nonstandard. Measurements are normalized according to ASTM E 1007 and proposed normalized impact sound rating (NISR) procedures to provide a reasonably consistent set for analysis. Low frequency measurements were conducted to $12.5 \mathrm{~Hz}$ one-third octave band. For both transmission loss and impact sound, many of the results compare well with predictions using simple regression analysis developed by others using variables such as the mass of the layers, truss depth and spacing, insulation thickness and density, and resilient metal channel spacing. The measurements and results will be presented in the paper.
2:20

5aAAb13. Impact sound insulating performance of access floors. Francesco Asdrubali (Università degli studi di Perugia, Via G. Duranti 67, 06125 Perugia, Italy, fasdruba@unipg.it), Francesco D'Alessandro (Università degli studi di Perugia, Via G. Duranti 67, 06125 Perugia, Italy, dalessandro.unipg@ciriaf.it)

Access floor are raised floor systems consisting of modular panels supported by posts at a certain height to create a gap below the floor surface. Electrical wires and pipes can be placed inside the gap in order to avoid exposed installations and to simplify operations involving the inspection, repair, changing, or adding of system elements. This paper presents the results of the measurement campaign carried out on seven different access floor configurations, obtained by changing different floor elements: panels, surface finishes, damping materials under the posts base. Impact sound insulating properties of access floors combined with false ceilings were also tested and are reported in the paper. The tests were executed in two overlapping reverberating rooms available at the Acoustics Laboratory of the University of Perugia using the procedures given by ISO 140-6 Standard. The results are expressed in terms of normalized sound impact pressure level Ln and of the corresponding single number index Ln,W.

\section{Invited Papers}

\section{2:40}

5aAAb14. Measurement of room-to-room airborne sound insulation with an access floor in a dwelling unit of condominium. Takashi Koga (Kajima Technical Research Institute, 2-19-1 Tobitakyu, Chofu, 182-0036 Tokyo, Japan, tkoga@kajima.com)

It is preferred covering a wooden access floor on the concrete floor for condominium apartments in Japan. The access floor usually installed after every partition in each dwelling unit. If access floor and ceiling were done before partition, one can obtain accuracy of construction and reduce waste material. Therefore, one can provide building more green. But, there is concern that may deteriorate sound insulation because of flanking path through under the access floor. On the other hand, there is also another dominant flanking path irrespectively to these construction methods: via doors leading into hallway. A number of field measurements of sound insulation were done with opening/closing doors to evaluate the flanking transmission via the doors. These results show that the doors affect especially in high frequency, but not significant in low-mid frequency. Next, the sound transmission performances of access floors and ceilings measured at laboratory are compared with direct sound transmission through wall itself. As a conclusion, field measurement results show that this construction method difference less affect to airborne sound insulation performance.

5aAAb15. The acoustical effect of reveal blocks, from measuring method to prediction. Frigyes Reis (Budapest University of Technology and Economics, Lab. of Building Acoustics, Mûegyetem rakpart 3., 1111 Budapest, Hungary, reis@mail.bme.hu), Balázs Tóth (Wienerberger Co., Bártfay str. 34, 1119 Budapest, Hungary, balazs.toth@wienerberger.hu)

The analysis of the efforts to create high quality building from the point of view of energy-efficiency, and the resulting variety of new products, building constructions and systems leads to the conclusion: there are characteristic deficiencies in the field of measuring and prediction methods related to sound insulation, and there is a need to stop the gap. The example on which the problem is shown is 
the acoustical effects of reveal blocks, used around window openings, to reduce heat propagation and increase energy efficiency. The reveal blocks include more sound propagation paths, the sound insulation of which can not be determined using the existing measuring standards. The paper describes new measuring concept and method to characterise the sound insulation characteristics of reveal blocks in their built-in form. The methods are fitted to the standardised measuring methods of sound insulation. Proposal is presented for the modification in the prediction procedure of sound insulation of façade constructions, utilising the results of the new measuring method.

FRIDAY MORNING, 4 JULY 2008

P2-B, LEVEL 2, 9:40 TO 11:20 A.M.

Session 5aAAc

\title{
Architectural Acoustics and Noise: Airborne and Impact Sound Insulation II (Poster Session)
}

\author{
Berndt Zeitler, Cochair \\ NRC - Institute for Research in Construction \\ Patrizio Fausti, Cochair \\ Engineering Dept. - Univ. of Ferrara
}

\begin{abstract}
All posters will be on display from 9:40 a.m. to 11:20 a.m. To allow contributors an opportunity to see other posters, contributors of odd-numbered papers will be at their posters from 9:40 a.m. to 10:30 a.m. and contributors of even-numbered papers will be at their posters from 10:30 a.m. to 11:20 a.m.
\end{abstract}

\section{Contributed Papers}

5aAAc1. Enhancement of impact sound insulation for "Gründerzeit-Häuser" build around 1900 during refurbishment using concrete-wood composite floors or suspended ceilings. Thomas Bednar (University of Technology, Karlsplatz 13/206, A-1040 Vienna, Austria, thomas.bednar@tuwien.ac.at)

During refurbishment of Viennese buidlings that have been erected around 1900 an important aspect is to enhance the impact sound insulation of the old wooden floors. Usually the floors are wood beam floors and the uppermost floor is a massive wooden floor called Dippelbaumdecke. Two important measures are taken to increase the impact sound insulation to achieve the minimum requirement of $\operatorname{LnTw}<=48 \leqslant \mathrm{~dB}$. The paper describes the design of an optimal suspended ceiling to avoid additional measures and as an alternative the impact of an additional thin concrete floor on top of the Dippelbaum-floor to achive minimum floor heights. As the thin concrete floor has a much larger area than the rooms below the measured results showed a big influence of concrete floor area on the impact sound level.

5aAAc2. Sound insulation properties of building elements, considering the frequency range below $100 \mathrm{~Hz}$. Herbert Muellner (TGM Federal Institute of Technology, Wexstrasse 19-23, 1200 Vienna, Austria, herbert.muellner@tgm.ac.at), Anna Frey (University of Applied Sciences, FH Campus Vienna, Daumegasse 3, 1100 Vienna, Austria, anna.frey@chello.at), Catrin Humer (TGM Federal Institute of Technology, Wexstrasse 19-23, 1200 Vienna, Austria, catrin.humer@tgm.ac.at)

The constantly increasing standard of comfort in general and the rising number of apartment buildings in lightweight mode of construction directed the focus of research to the sound insulation properties of timber frame and massive wood element buildings. The complaints of the residents of terraced houses and multiple dwellings in lightweight buildings mainly concern noise events characterised by dominant low frequency. This problem regards airborne sound insulation as well as impact sound insulation. Based on empirical data the comparison of the sound insulation properties of the building elements investigated shows the specific problem of each category of construction mode in the frequency range below $100 \mathrm{~Hz}$. The typical sound insulation characteristics regarding the low frequency range are discussed in the light of the current applied sound insulation requirements as well as in relation to the problem to comply with the resident's apperception of sound insulation quality.

5aAAc3. Effect of the frequency spectrum trend on the determination of the weighted normalized impact sound pressure level for floor structures. Federica Bettarello (Engineering Dept. - Univ. of Ferrara, Via Saragat, 1, 44100 Ferrara, Italy, federica.bettarello@unife.it), Patrizio Fausti (Engineering Dept. - Univ. of Ferrara, Via Saragat, 1, 44100 Ferrara, Italy, patrizio.fausti@unife.it)

The most common floor constructions in Italian buildings are composed of two parts: the structural one, made of concrete beams and perforated bricks, and the floating floor. The results of the in situ measurements of the impact sound pressure level, realized on the structural part of the floors, show a frequency spectrum trend that increases with the frequency. The spectrum is also characterized by many peaks and dips at the high frequency range that strongly influence the determination of the weighted normalized impact sound pressure level, also for structures with similar mass and geometry. The frequency trend of the beam and brick structures is completely different from the characteristic spectrum of homogeneous concrete slabs or lightweight structures. Considerations on how those differences can influence the obtainable results in terms of the reduction of impact sound pressure level referred to the same floating floor typology, the spectrum adaptation terms and the other acoustic quantities described in the standards are reported.

5aAAc4. Influence of flanking transmission in typical Italian constructions. Simone Secchi (Univ. of Florence, Via San Niccolò 89/a, 50125 Florence, Italy, simone.secchi@taed.unifi.it), Gianfranco Cellai (Univ. of Florence, Via San Niccolò 89/a, 50125 Florence, Italy, gianfranco.cellai@unifi.it), Elisa Nannipieri (Univ. of Florence, Via San Niccolò 89/a, 50125 Florence, Italy, elisa.nannipieri@ taed.unifi.it)

Actually acoustic performance of building components are analysed with reference to standardised conditions, in laboratories with suppressed flanking transmission. Laboratory results often show values of the performances quite different from those measured in real buildings, as a consequence of flanking transmission and of different conditions of realisation in 
situ. Moreover, in spite of all efforts aimed to standardise laboratory test conditions, often we find different certified performances of same building components obtained in different laboratory. In some cases, these differences may be very relevant. Besides, the Italian decree on acoustic requirement of buildings prescribes limiting values to be measured in situ. For these reasons, many producers of building materials and components more frequently presents in their depliants both laboratory values and in situ values of acoustic performances of their products. The paper shows the preliminary results of an research aimed to verify acoustic performances of partition walls and floors in a laboratory which respect conditions of ISO 140-1, but with the presence of relevant flanking transmission, similar to real buildings. The relevance of the flanking transmission has also been modified by means of a lining applied to the ceiling of the receiving room of the laboratory.

5aAAc5. Survey on the insulation performance of acoustic louvres. Lieven De Geetere (Belgian Building Research Institute, Lombardstraat 42, B-1000 Brussel, Belgium, lieven.de.geetere@bbri.be), Bart Ingelaere (BBRI, rue du Lombard, 42, B-1000 Brussel, Belgium, bart .ingelaere@bbri.be)

Machinery such as compressors, boilers and generators require adequate ventilation and extract. To avoid excessive noise breakout, acoustic louvres are frequently used. These are usually made of tilted hollow metallic blades filled with absorptive materials and perforated at the inside face. This study investigates the important characteristics of common shaped acoustic louvres with regard to sound insulation. First, a market survey on 109 existing acoustic louvres of 24 producers worldwide is made. Second, laboratory measurements of sound insulation on 38 prototypes of $1 \mathrm{~m}^{2}$ acoustic louvres are compared. The influence of several parameters are studied such as louvre depth, blade angle, blade shape, blade thickness, blade spacing, kind of absorptive material, perforation degree, and edge filling.

5aAAc6. Sound insulation characteristics of shipboard windows. Sangryul R. Kim (Acoustics Team, Korea Institute of Machinery and Materials, 171 Jang-dong, Yuseong-gu, 305-343 Daejeon, Republic of Korea, srkim@kimm.re.kr), Hyun-Sil Kim (Acoustics Team, Korea Institute of Machinery and Materials, 171 Jang-dong, Yuseong-gu, 305-343 Daejeon,
Republic of Korea, hskim@kimm.re.kr),Hyun-Ju Kang(Acoustics Team, Korea Institute of Machinery and Materials, 171 Jang-dong, Yuseong-gu, 305-343 Daejeon, Republic of Korea, kanghj@kimm.re.kr)

Although small-size windows are used in a ship, shipboard windows are a multilayered structure with glasses and air-gaps for high sound insulation. This paper discusses how to improve the sound insulation performance of shipboard windows. First, the sound transmission loss (STL) obtained from various experiments with shipboard windows are introduced and studied. The results show the layer arrangement as well as the material property of each layer makes an effect on the window's STL. It is also found that the higher the sound insulation performance of the window is, the more important the effect of the window frame is. Next, theoretical investigations are carried out and discussed in comparison with the experimental results. The comparison provides possible clues to increase the STL of the window.

5aAAc7. Acoustic analysis of the framework and walls stage in the construction of a housing block. Maria Jesus Ballesteros (Universidad de Castilla-La Mancha, Campus Universitario, 16071 Cuenca, Spain, MJesus.Ballesteros1@alu.uclm.es), Samuel Quintana (Universidad de Castilla-La Mancha, Campus Universitario, 16071 Cuenca, Spain, Samuel.Quintana@uclm.es), Marcos D. Fernandez (Universidad de Castilla-La Mancha, Campus Universitario, 16071 Cuenca, Spain, Marcos.Fernandez@uclm.es), Jose A. Ballesteros (Universidad de Castilla-La Mancha, Campus Universitario, 16071 Cuenca, Spain, Josea.Ballesteros@uclm.es), Laura Rodriguez (Universidad de Castilla-La Mancha, Campus Universitario, 16071 Cuenca, Spain, Laura.Rodriguez @uclm.es)

The noise in the construction process is one of the main environmental and industrial noise sources. There are no specific regulations in several European countries for assessing such kind of noise, neither reference indexes for its evaluation. Therefore, the limits adopted are those for environmental and industrial noise, although they do not take into account the intrinsic characteristics of this noise. A measurement procedure has been stated for assessing the noise in building sites in which, the most appropriate indexes for this noise have been analyzed and used to derive the acoustic characteristics of the framework and walls stage. This stage is considered as one of the most annoying in the construction process of a housing block. 


\title{
Session 5aAAd
}

\section{Architectural Acoustics: Coupled Volume Acoustics I (Poster Session)}

\author{
Jason Summers, Cochair \\ U.S. Naval Res. Lab. \\ Alexis Billon, Cochair \\ Universite de Liege
}

\begin{abstract}
All posters will be on display from 9:40 a.m. to 11:20 a.m. To allow contributors an opportunity to see other posters, contributors of odd-numbered papers will be at their posters from 9:40 a.m. to 10:30 a.m. and contributors of even-numbered papers will be at their posters from 10:30 a.m. to 11:20 a.m.
\end{abstract}

\section{Contributed Paper}

5aAAd1. Investigations on real-size coupled rooms based on Bayesian estimation method. Zuhre Su (Russ Berger Design Group, 4006 Belt Line Road Suite 160, Addison, TX 75001, USA, tayasu7@yahoo.com), Dave Woolworth (Oxford Acoustics, Inc., 356 CR 102, Oxford, MS 38655, USA dave@oxfordacoustics.com), Ning Xiang (Rensselaer Polytechnic Institute, Greene Building, School of Architecture, 110 8th Street, Troy, NY 12180, USA, xiangn@rpi.edu)

As a case study, this work aims to experiment coupled-volume systems with scientifically grounded quantifiers using Bayesian analysis, a reliable analysis method in evaluating effects of different parameters on sound energy decays of coupled rooms [Xiang \& Goggans, J. Acoust. Soc. Am. 110,
1415 - 1424 (2001)]. This research uses the well-defined indicators developed by Xiang and Goggans to assess sound energy decays in a real-size hall. Derived from the model-based Bayesian inference, level differences $(\Delta \mathrm{L})$, diverse decay times, and decay time ratios are the quantitative descriptors of sound energy decays in acoustically coupled spaces. The room impulse responses of Howorth Theater in Oxford, Mississippi coupled to the adjacent lobby are experimentally measured in the case study. The two rooms are connected with two separate apertures and have distinct natural reverberation times that allow experimenting different setups for probable acoustical coupling at certain locations. In this paper, frequency, and location dependence of double-slope characteristics of sound energy decays are discussed. 


\title{
Session 5aAAe
}

\section{Architectural Acoustics: New Frontiers in Room Acoustical Modeling II (Poster Session)}

\author{
Murray Hodgson, Cochair \\ University of British Columbia \\ Vincent Valeau, Cochair \\ Laboratoire d'Etudes Aérodynamiques (LEA)
}

\begin{abstract}
All posters will be on display from 9:40 a.m. to 11:20 a.m. To allow contributors an opportunity to see other posters, contributors of odd-numbered papers will be at their posters from 9:40 a.m. to 10:30 a.m. and contributors of even-numbered papers will be at their posters from 10:30 a.m. to 11:20 a.m.
\end{abstract}

\section{Contributed Papers}

5aAAe1. Predicting the acoustics of historic Istanbul Tunnel: Simulation, calculation methods and geometrical details. Filiz Bal Kocyigit (Karabuk University, 232. Str. No: 5/5 Ilkbahar Mah., Cankaya, 06550 Ankara, Turkey, filizbkocyigit@yahoo.com)

Istanbul Tunnel was designed and constructed by Eugene Henry Gavand in 1875 is the third Metro and second underground railway system in the world after Washington Metro (1868). In this paper, the acoustics of Istanbul Tunnel is investigated. This is a special case which sets up a challenge to these prediction methods. The shape of the tunnel and therefore focusing the sound, reverberant wall, and therefore fluctuation effect demands high accuracy in predicting the early reflections. The energy dissipates quickly in this type of enclosures and there is little masking effect of the reverberation. Another aspect that has been shown to give very different results in this case study is the geometrical detailing of the models. When Istanbul Tunnel compared with modern metrosystems; nowadays railway systems are controlled by modern electronic and mechanical system, but in the 19th century this electronic systems could not used. Solutions could be with the shape of the building. The aim of this paper is to clarify some of the problems that can arise in this type of constructions, and give guidelines for how they can be overcome/avoided. Another objective is to emphasize that room acoustic computer simulations, although very useful, need careful consideration about the underlying calculation methods.

5aAAe2. Size-adaptive spherical receptor acceleration method for acoustical ray tracing. Stéphane Lesoinne (University of Liège - INTELSIG, Institut Montéfiore, Sart-Tilman, Batiment 28, 4000 Liège, Belgium, s.lesoinne@ulg.ac.be), Jean-Jacques Embrechts (University of Liège - INTELSIG, Institut Montéfiore, Sart-Tilman, Batiment 28, 4000 Liège, Belgium,jjembrechts@ulg.ac.be)

Randomized ray tracing in room acoustics can be used to compute echograms, but the results computed at the receptor are affected by statistical errors [1]. To decrease these statistical errors, the usual solution is to increase the number of rays, but this also increases the computation time. Another solution is to increase the receptor size, but this lowers the spatial resolution of the computed sound field, which is particularly important for the early part of the echogram. In order to decrease the computation time and keep a sufficient resolution, the method proposed in this paper is based on a progressive modification of the receptor size (spherical type) as long as the ray's travel grows. At the same time, the number of rays into the room can be decreased as the sound ray lengths increase while keeping the statistical errors more or less constant. The consequence is that the sound field spatial resolution evolves from "precise" at the beginning of the echogram to "rough" at its end. The first applications of this new method show a significant gain of CPU time. [Broad spectrum diffusion model for room acous- tics ray-tracing algorithms, J. J. Embrechts, Jnl. Acoust. Soc. Am. 107(4) 2068-2081 (2000)].

5aAAe3. Variation of mean free path length over time and its effect on room impulse response. Miomir Mijic (Faculty of Electrical Engineering, Bulevar Kralja Aleksandra 73, 11000 Belgrade, Serby, emijic@etf.bg.ac.yu), Dragana Sumarac Pavlovic (Faculty of Electrical Engineering, Bulevar Kralja Aleksandra 73, 11000 Belgrade, Serby, dsumarac@etf.bg.ac.yu)

In their previous paper (Forum acusticum 2005, Proceedings, 23992404) the authors presented the local effect found in a large sports hall, described as significantly shorter reverberation time in the first $400-500 \mathrm{~ms}$ than that in the rest of the hall's long impulse response. To analyse this phenomenon, a new measure - mean free path length (MFPL) variation in time over the impulse response - was introduced. It is identified as the function of time obtained as an ensemble average in some fixed interval traced along the impulse response duration. The ray tracing simulation was used to calculate ray paths approaching the receiver in time. Analyzing MFPL variations over time is a new insight into the structure of room impulse response, and this paper presents its main characteristics, its dependence on geometrical properties of rooms, and also its relation to statistically defined mean free path length $(4 \mathrm{~V} / \mathrm{S})$. The paper also presents a correlation between MFPL variation over time and the sound decay curve. The MFPL variation over time reveals the causes of the local effect introduced in the previous paper, but it is generally useful in explaining the sound decay monotony deviations.

5aAAe4. Towards a realistic ray tracing for room acoustics. Jaume Durany (Universitat Pompeu Fabra - Fundació Barcelona Media, Ocata, 1, $08003 \quad$ Barcelona, Spain, jaume.durany@upf.edu), Adan Garriga (Universitat Pompeu Fabra - Fundació Barcelona Media, Ocata, 1, 08003 Barcelona, Spain, adan.garriga@barcelonamedia.org), Toni Mateos (Universitat Pompeu Fabra - Fundació Barcelona Media, Ocata, 1, 08003 Barcelona, Spain, toni.mateos@barcelonamedia.org)

We present a recently developed ray tracing engine for room acoustics computations whose improved physics provide more realistic results than present state of the art methods. Ray tracing has been widely applied within the graphics community to obtain realistic shadings, but the computational cost reduction has oriented efforts towards fast computations of apparently realistic images rather than physically correct results. In the case of audio ray tracers, a similar tendency has led to relatively fast shading algorithms that often miss its physical basis, preventing the resulting impulse responses from converging to physically correct solutions. Our engine improves this situation by focusing on two main issues. First, it ensures the mathematical convergence of the algorithms by maintaining the coherence between the 
computation of the direction of the reflected rays and the computation of the contribution of the sources to a given point in the environment. Second, the engine works directly in the frequency domain. This allows for the implementation of frequency-dependent phenomena, from surface impedance and reflections to frequency-dependent source directivity patterns. We present a comparative study of the proposed ray tracing engine with the state of the art, emphasizing properties like realism, coherence and convergence.

5aAAe5. A geometric acoustics simulation proposal for curved geometry. Arthur Van Der Harten (Rensselaer Polytechnic Institute, Greene Bldg., 110 8th St., Troy, NY 12180, USA, Arthur .vanderharten@gmail.com), Paul Calamia (Rensselaer Polytechnic Institute, Greene Bldg., 110 8th St., Troy, NY 12180, USA, calamp@rpi.edu)
Current methods for acoustical simulations based on geometrical acoustics are designed to ascertain the properties of rooms using models comprising large flat polygons. Typically these same methods are used on models of spaces with curved surfaces in which the curves are approximated using planar facets. In such cases, errors are introduced in the simulation when the infinitely varying normal of a curve is replaced with a finite number of piecewise constant normals, one for each facet. NURBS - nonuniform rational B-splines - offer an alternative geometric representation that allows curves to be represented with precision. Using Rhinoceros, a commonly used NURBS-based CAD program, as a platform for an acoustic simulation tool for models of both NURBS and polygon geometry, we can begin to discover whether it is possible to conceptualize a geometrical acoustics method that is more accurate for curved surfaces. This talk will cover the implementation and early testing of an acoustic-simulation plug-in for Rhinoceros

FRIDAY MORNING, 4 JULY 2008

P2-B, LEVEL 2, 9:40 TO 11:20 A.M.

\title{
Session 5aAAf
}

\section{Architectural Acoustics and Musical Acoustics: New Measurement Parameters in Performing Arts Spaces I (Poster Session)}

\author{
Lily Wang, Cochair \\ University of Nebraska - Lincoln \\ Brian Katz, Cochair \\ LIMSI-CNRS
}

\begin{abstract}
All posters will be on display from 9:40 a.m. to 11:20 a.m. To allow contributors an opportunity to see other posters, contributors of odd-numbered papers will be at their posters from 9:40 a.m. to 10:30 a.m. and contributors of even-numbered papers will be at their posters from 10:30 a.m. to 11:20 a.m.
\end{abstract}

\section{Contributed Papers}

5aAAf1. Converging evidence in evaluating acoustical intimacy. Bobby E. Gibbs (Rensselaer Polytechnic Institute, Greene Bldg., 110 8th St., Troy, NY 12180, USA, gibbsb@rpi.edu), Jonas Braasch (Rensselaer Polytechnic Institute, Greene Bldg., 110 8th St., Troy, NY 12180, USA, braasj@rpi.edu), Ted Krueger (Rensselaer Polytechnic Institute, Greene Bldg., 110 8th St., Troy, NY 12180, USA, krueger@rpi.edu)

Previously, we presented a novel interactive pilot experiment in which participants modified parameters in a virtual acoustical environment so that they corresponded to three intimacy settings: low, medium, and high. Additionally, participants were asked to rank the -- yet unknown -- parameters (volume, direct-to-reverberant energy ratio, frequency attenuation, and room size) in terms of the importance in making their judgments. Based on a larger body of data, the direct-to-reverberant energy ratio emerges as the strongest acoustical correlate of intimacy. A repeated-measures test revealed that the "preservation" of this parameter (the degree to which a participant changed the value from an optimum setting) varies the least across intimacy levels and across levels of previous training. Additionally, this parameter is consistently ranked as being the most important in the ranking portion of the test. We also found that salience of some of the other parameters varied significantly across intimacy levels, possibly suggesting a weighting system is warranted. These results are compared with binaural measurements of the virtual system. In particular, we will address disparities from the pilot experiment and recent data that resulted from differences in measuring the direct-to-reverberant energy ratio. [Work supported by RPI HAAS Fellowship and ASA Minority Fellowship.]
5aAAf2. Effects of multiple directional sources on quantitative and qualitative analyses of concert hall acoustics. Youngmin Kwon (Acoustic Dimensions, 15505 Wright Brothers Drive, Addison, TX 75001, USA, ykwon@acousticdimensions.com), Gary W. Siebein (Univ. of Florida, 231 Arch, PO Box 115702, Gainesville, FL 32611, USA, gsiebein@siebeinacoustic.com), Martin A. Gold (Univ. of Florida, 231 Arch, P.O. Box 115702, Gainesville, FL 32611, USA, mgold@ufl.edu)

The "orchestral impulse reponse" measurement technique using multiple directional sources and its effects on quantitative analysis of concert hall acoustics were discussed earlier [Kwon and Siebein, J. Acoust. Soc. Am. 120, 3263(A) (2006)]. An array with 16 directional loudspeakers approximating overall sound radiation patterns of each instrumental group of an orchestra was incorporated for room acoustical measurements and analyses. As a continuous study, this paper discusses qualitative assessments of concert hall acoustics over room subjective parameters including reverberance, clarity, warmth, spaciousness, envelopment, etc. The music signals binaurally recorded in a performance hall with the same source array were evaluated by means of subjective listening tests in comparison to those recorded with the single omni-directional source (dodec). The results showed that some perceived differences are present between the array with multiple directional sources and the one with a single omni-directional source. In the main orchestra seating, in particular, reverberance and clarity are perceived more but warmth is perceived less when compared to the recordings through the single omni-source. The results will be further discussed in conjunction with their quantitative measures resulted from the above measurement technique. 
5aAAf3. Room acoustics measurements with an approximately spherical source of 120 drivers. Roger Schwenke (Meyer Sound Laboratories, 2832 San Pablo Ave, Berkeley, CA 94702, USA, rogers@meyersound.com), Franz Zotter (Institute of Electronic Music and Acoustics, Inffeldgasse 10/3, 8010 Graz, Austria, zotter@iem.at), David Wessel (Center for New Music and Audio Technologies, 1750 Arch Street, Berkeley, CA 94720, USA, wessel@cnmat.berkeley.edu.), Andrew Schmeder (Center for New Music and Audio Technologies, 1750 Arch Street, Berkeley, CA 94720, USA, andy@cnmat.berkeley.edu)

An approximately spherical source of 120 individually controlled drivers is used to perform impulse response measurements in a room with a $1.4 \mathrm{~s}$ reverberation time and a distinct echo. The signal to the drivers is processed to produce both omni-directional and unidirectional patterns. The omnidirectional pattern is compared with measurements made with a traditional 12 sided source. The unidirectional patterns are measured both pointed towards and away from the listener position. Intelligibility metrics for the different directionalities and orientations are measured. The unidirectional pattern is aimed in different directions to minimally and maximally excite the distinct echo in the room, and locate it's origin.

5aAAf4. Measurement-based fuzzy interpolation of room impulse responses. Csaba Huszty (Budapest University of Technology and Economics, BME Dept. of Telecommunications, Magyar tudósok körútja 2, H-1117 Budapest, Hungary, huszty@hit.bme.hu), Bottyán Németh (Budapest University of Technology and Economics, BME Dept. of Telecommunications, Magyar tudósok körútja 2, H-1117 Budapest, Hungary, bottyan@tmit.bme.hu), Péter Baranyi (Budapest University of Technology and Economics, BME Dept. of Telecommunications, Magyar tudósok körútja 2, H-1117 Budapest, Hungary, baranyi@tmit.bme.hu), Fülöp Augusztinovicz (Budapest University of Technology and Economics, BME Dept. of Telecommunications, Magyar tudósok körútja 2, H-1117 Budapest, Hungary, fulop@ hit.bme.hu)

Application of room impulse responses (RIRs) to acoustic evaluation and auralization often requires many measurements to get enough information about the hall, or to provide enough flexibility for virtual sound source placements in convolution reverberation. In this paper we propose a measurement-based fuzzy modeling method to approximate the RIR function at an arbitrary location between available measured points, without a priori information on the hall geometry or wall reflection parameters. For the fuzzy model identification we define an accuracy indicator of the spatial density of the source positions and predict the required number of them in a selected hall. This indicator quantifies the relationship of the early reflections, determined for various measured positions. This paper also proposes a method that treats nonuniform spatial sampling of the measurement positions, and its implementation for $2 \mathrm{D}$ cases is shown. Nonuniform spatial sampling can be useful when RIRs at some source positions -- e.g. positions of musicians on a stage of a concert hall -- are known or have to be measured precisely, but RIRs at locations in between require an approximation only. The proposed fuzzy model of RIRs actually transforms the measured information into a uniform and tensor product form, enabling the analyst to use further matrix and tensor algebra based numerical methods.

FRIDAY MORNING, 4 JULY 2008

AMPHI BLEU, 8:00 A.M. TO 5:40 P.M.

\title{
Session 5aABa
}

\section{Animal Bioacoustics: General Topics in Animal Bioacoustics II}

\author{
Richard R. Fay, Cochair \\ Loyola University Chicago, Parmly Hearing Institute, 6525 N. Sheridan Rd., Chicago, IL 60626, USA \\ Michel Andre, Cochair \\ Laboratori d'Aplicacions Bioacústiques (Universitat Politècnica de Catalunya), avda. Rambla Exposició s/n, \\ Vilanova i la Geltrú, 08800, Spain
}

\section{Contributed Papers}

\section{8:00}

5aABa1. Acoustic production mechanisms in pinnipeds. Marija Spasikova (Centre for Social Learning and Cognitive Evolution, University of St. Andrews, School of Psychology, KY16 9JP St. Andrews, Fife, UK, marija.spasikova@gmail.com), William T. Fitch (Centre for Social Learning and Cognitive Evolution, University of St. Andrews, School of Psychology, KY16 9JP St. Andrews, Fife, UK, wtsf@st-andrews.ac.uk), Colleen Reichmuth (UCSC Institute of Marine Sciences, Long Marine Lab - University of California, 100 Shaffer Road, Santa Cruz, CA 95060, USA, coll@ucsc.edu), Ronald J. Schusterman (UCSC Institute of Marine Sciences, Long Marine Lab - University of California, 100 Shaffer Road, Santa Cruz, CA 95060, USA, rjschust@ucsc.edu)

The ability to develop articulate speech relies on capabilities that became available to our ancestors through changes in both peripheral mechanisms (vocal acoustics and anatomy) and neural mechanisms (vocal control and imitation). Few distantly related species (cetaceans, birds and pinnipeds) have shown the ability of vocal imitation, but of the three, only the pinnipeds use the same vocal tract as us humans. For this reason we choose to study two closely related pinniped species: the harbour seal (Phoca Vitulina), a good vocal imitator (capable of complex vocal learning), and the
Californian sea lion (Zalophus californicus), a close relative with very limited vocal imitation capabilities (no evidence of complex vocal learning) to look at the acoustical production mechanisms of the species as well as the neural mechanisms underlying the same. Using previously acquired data gathered in California at the Long Marine Lab, we performed video and acoustic analysis to show correlation between jaw opening and formants frequencies in the harbour seal (Phoca Vitulina), in speech like vocalisations. We are examining the involvement of tongue movement during these vocalisations, hypothesising that it will account for the remaining residuals in the correlation between the formants excursions and the jaw movements.

\section{$8: 20$}

5aABa2. Habitat occupation time-series of St. Lawrence belugas from passive acoustic monitoring. Nathalie Roy (Fisheries and Oceans Canada \& ISMER-UQAR, 850 route de la Mer, P.O. Box 1000, Mont-Joli, QC G5H-3Z4, Canada, royn@dfo-mpo.gc.ca), Yvan Simard (Fisheries and Oceans Canada \& ISMER-UQAR, 850 route de la Mer, P.O. Box 1000, Mont-Joli, QC G5H-3Z4, Canada, simardy@dfo-mpo.gc.ca), Catherine 
Bédard(Marine Science Institute, University of Québec, 310 Allée des Ursulines, P.O. Box 3300, Rimouski, QC G5L-3A1, Canada, catherine.becard01@uqar.qc.ca), Elisabeth Leblanc (Marine Science Institute, University of Québec, 310 Allée des Ursulines, P.O. Box 3300, Rimouski, QC G5L-3A1, Canada, elisabeth.leblanc@uqar.qc.ca)

Passive acoustic monitoring (PAM) was used to generate a six-week continuous time-series of occupation of a segment of the St. Lawrence Estuary by belugas. The PAM data were acquired from a cabled hydrophone deployed at mid-depth into the 300-m deep Laurentian channel off Cap-deBon-Desir during summer 2003. Beluga vocalisation activity time-series was obtained from the $[0.5-5.0 \mathrm{kHz}]$ bandpassed PAM data that were processed to filter out noise from the heavy shipping on the nearby St. Lawrence Seaway and from occasional whale watching boats, and lowfrequency traces of clicks, via adaptive spectral subtraction and image processing methods applied to the spectrogram. The remaining traces on the binary image of the spectrogram were summed up to generate a vocalisation index, which was essentially due to belugas in this region. The vocalisation index time-series was then analysed for presence of circadian rhythms and correlations with the semi-diurnal tidal cycle, currents from an acoustic Doppler current profiler and wind. Results are interpreted from the knowledge of the biological and physical oceanographic processes occurring in the monitored area.

\section{8:40}

5aABa3. Comparison of automatic classification methods for beluga whales vocalizations. Elisabeth Leblanc (Marine Science Institute, University of Québec, 310 Allée des Ursulines, P.O. Box 3300, Rimouski, QC G5L-3A1, Canada, elisabeth.leblanc@uqar.qc.ca), Mohammed Bahoura (Eng. Dept., Université du Québec, 300 Allée des Ursulines, P.O. Box 3300, Rimouski, QC G5L-3A1, Canada, mohammed_bahoura@uqar.qc.ca), Yvan Simard (Fisheries and Oceans Canada \& ISMER-UQAR, 850 route de la Mer, P.O. Box 1000, Mont-Joli, QC G5H-3Z4, Canada, simardy@dfo-mpo.gc.ca)

The beluga whale is a loquacious marine mammal with a complex vocal repertory. It produces a wide variety of whistles and pulsed tones as well as high-frequency echolocation clicks. Automatic detection of the longdistance propagating communication calls are desirable for implementing passive acoustic monitoring (PAM) systems in their environment, for habitat utilisation studies and real-time early warning devices in anthropogenic impact mitigation systems. Signal processing methods were developed to classify beluga vocalizations from a PAM data set recorded in Cap-de-BonDésir in the Saguenay-St. Lawrence Marine Park in summer 2003 using a 6-hydrophones array with a 10-100 kHz sampling rate. The performance of an MFCC-based HMM system and a polynomial model applied to extracted time-frequency contours of vocalizations will be evaluated. The feasibility of real-time implementation will then be analysed for both methods.

\section{9:00}

5aABa4. One plus one equals one: The beluga whale (Delphinapterus leucas) produces two pulses to form its echolocation click. Marc $\mathrm{O}$. Lammers (Hawaii Institute of Marine Biology, P. O. Box 1106, Kailua, HI 96734, USA, lammers@hawaii.edu), Manuel Castellote (L’Oceanografic, C/. Junta de Murs i Valls, s/n, 46013 Valencia, Spain, mcastellote @oceanografic.org)

Empirical results have shown that two sound generators exist in the nasal system of delphinids. It had been previously assumed that these function independently, with one primarily involved in pulse production and the other in the generation of tonal signals. Here we report that in the beluga whale both generators appear to be simultaneously involved in the production of the echolocation click measured in front of the animal. A study was conducted that examined the propagation of the echolocation click of a captive 12 year-old female beluga whale at $15^{\circ}, 30^{\circ}, 45^{\circ}, 60^{\circ}, 75^{\circ}$, and $90^{\circ}$ off the center axis of both sides of the outgoing beam. The results reveal that at angles greater than $30^{\circ}$ two distinct pulses are measured that cannot be attributed to multipath reflections from the surface or any structure in the tank. The two pulses are maximally separated in time at $90^{\circ}$ off-axis and gradually converge with each $15^{\circ}$ measurement closer to the beam center. At $30^{\circ}$ the two pulses begin to form a single, albeit distorted pulse. The phenomenon is symmetrical on both sides of the animal. These findings shed new light on how beluga whales and perhaps other odontocetes form their echolocation pulse.

\section{9:20}

5aABa5. The nanomechanics of mechanosensory neurones in vivo. James F. Windmill (University of Bristol, D34 School of Biological Sciences, Woodland Road, BS8 1UG Bristol, UK, james.windmill@bristol.ac.uk), Victoria G. Pook (University of Bristol, School of Biological Sciences, Woodland Road, BS8 1UG Bristol, UK, vicky.pook@bristol.ac.uk), Daniel Robert (University of Bristol, D34 School of Biological Sciences, Woodland Road, BS8 1UG Bristol, UK, d.robert@bris.ac.uk)

The ability to detect and process sound is a sense particularly important in many animals, including insects, playing a key role in predator, prey and mate detection. Acute hearing, both in the sense of extreme sensitivity to sound and sharp frequency selectivity, relies on the active participation of auditory mechanoreceptors. In insects, active auditory mechanics was first demonstrated in mosquitoes, whereby auditory sensitivity is enhanced by the action and reaction of mechanosensory neurones to sound-induced vibrations. The mosquito's auditory neurones can generate motions that mechanically drive the antenna and tune it to biologically relevant sounds. The mechanosensory neurones are capable of detecting exquisitely small mechanical displacements, down to 100 picometres. In the mosquito's Johnston's organ ( $300 \mu \mathrm{m}$ in diameter) there is a high density of these neurones (16 000 units). The mechanical response of the mechanoreceptors was measured in vivo using an atomic force microscope, in response to stimulation of the external antenna. The work establishes the link between the previously measured non-linearities of the mosquito's antennal vibrations and the nanoscale mechanics of the mechanosensory neurones.

\section{9:40-11:00 Posters}

Lecture sessions will recess for the presentation of poster papers on various topics in acoustics. See poster sessions for topics and abstracts

\section{Contributed Paper}

\section{1:00}

5aABa6. Computer aided design of audio signal classifier systems. Sebastian V. Huebner (Pestalozzistrasse 5, 14482 Potsdam, Germany, sebastian@sejona.de)

The problem of programming classifier systems for deterministic events in bioacoustic signals is seen as a knowledge engineering problem. Advantages and disadvantages of traditional approaches are discussed. A knowledge engineering approach for interactive visual classifier system design is described and advantages of the approach are highlighted. The basic archi- tecture of a general purpose knowledge engineering framework for the acoustics domain is outlined. Emphasis is placed on both expert knowledge and on the role of data mining within the classifier system design process. Several examples illustrate how the approach can be used to create classifier systems for bioacoustic patterns in the time-frequency domain. Examples include multiscale spectrographic visualization, techniques to create and interactively modify subsymbolic and symbolic classifier systems for timefrequency patterns as well as the usage of three-dimensional visualizations to display hierarchical dependencies within complex classifier systems. 


\section{Invited Paper}

11:20

5aABa7. Sounds from a neonate harbour porpoise and their potential use in acoustic monitoring. Magnus Wahlberg (Fjord\&Bælt / University of Southern Denmark, Margrethes Plads 1, 5300 Kerteminde, Denmark, magnus@ @jord-baelt.dk)

Harbour porpoises (Phocoena phocoena) emit ultrasonic $(130 \mathrm{kHz})$ clicks with a stereotyped source parameters for echolocation and communication. Recently it was observed that the first days of living, a neonate harbour porpoise emitted contact calls of much a much lower frequency emphasis, around a few $\mathrm{kHz}$. The echolocation clicks produced by the calf significantly differed in duration and band width during the first four months postpartum. The acoustic signals from harbour porpoise calves are ideal for automated detection and therefore as a tool in population studies of this species. Both hydrophone array systems towed from boats, and automated click detectors (such as T-PODs) may incorporate routines for automated detection of young calves. These findings may have a large potential for facilitating biologists in gathering important biological data from this otherwise very difficult-to-study species of marine mammals.

\section{Contributed Papers}

\section{1:40}

5aABa8. Two-dimensional sonar beam characteristics of single harbour porpoise (Phocoena phocoena) echolocation clicks measured with a 16 hydrophone array. Jens C. Koblitz (Eberhard-Karls-Universität Tübingen, Zool. Institut, Abt. Tierphysiologie, Auf der Morgenstelle 28, 72076 Tübingen, Germany, Jens.Koblitz@uni-tuebingen.de), Magnus

Wahlberg (Fjord\&Bælt/University of Southern Denmark, Margrethes Plads 1, 5300 Kerteminde, Denmark, magnus@fjord-baelt.dk), Peter T. Madsen (University of Aarhus, Biological Sciences, Zoophysiology, C. F. Møllers Allé, Building 1131, DK-8000 Aarhus, Denmark, peter.madsen@biology.au.dk), Peter Stilz (Eberhard-Karls-Universität Tübingen, Zool. Institut, Abt. Tierphysiologie, Auf der Morgenstelle 28, 72076 Tübingen, Germany, peter.stilz@uni-tuebingen.de), Hans-Ulrich Schnitzler (Eberhard-Karls-Universität Tübingen, Zool. Institut, Abt. Tierphysiologie, Auf der Morgenstelle 28, 72076 Tübingen, Germany, hansulrich.schnitzler@uni-tuebingen.de)

For the first time, the beam structure of single harbour porpoise echolocation clicks was simultaneously measured in both the vertical and horizontal plane. Echolocation clicks of a stationed animal and free-swimming animals catching fish were recorded with a "plus-shaped" array consisting of 16 hydrophones. The $-3 \mathrm{~dB}$ beam width in the horizontal and vertical planes is in most cases similar to what has been previously measured by Au et al. (1999). However, individual clicks are sometimes broader or narrower than what has been previously reported. This may be caused by either voluntary control of the animal's beam width, or it may be a secondary effect of variations in intensity and air content within the sound production system.
12:00

5aABa9. Dolphin modulation rate transfer functions resulting from exposure to frequency modulated signals. Dorian $S$. Houser (Biomimetica, 7951 Shantung Dr., Santee, CA 92071, USA, dhouser@spawar.navy.mil), James J. Finneran (US Navy Marine Mammal Program, Space and Naval Warfare Systems Center, 53560 Hull St., Code 71510, San Diego, CA 92152, USA, james.finneran@navy.mil)

Frequency modulated (FM) signals are useful in audiometric studies where the test environment contributes to multipath interference and produces spatial variations in the sound pressure field. Rhythmic FM stimuli are useful in evoked potential audiometry because of the auditory steady state response (ASSR) that is created within the auditory system. Although some research on dolphin ASSRs resulting from rhythmic FM signals has been performed, relationships between modulation depth, modulation rate, and ASSR amplitude have not been fully explored. To address this, two dolphins were exposed to rhythmic FM signals (120 dB SPL and center frequency $=40 \mathrm{kHz}$ ) and the resulting ASSR recorded. The modulation depth (peak-to-peak) and modulation rate were varied from 0.1-10\% and 200-2500 $\mathrm{Hz}$, respectively. A total of 512 epochs were recorded for each stimulus presentation and the averaged waveform was analyzed in the frequency domain. The amplitude peak corresponding to the modulation rate was used to create the modulation rate transfer function (MRTF). Both dolphins showed a MRTF peak at a $1 \mathrm{kHz}$ modulation rate, while one dolphin showed a second peak at $500 \mathrm{~Hz}$. The use of rhythmic FM signals in ASSR studies may be a useful for testing in less than ideal acoustic environments.

12:20-1:40 Lunch Break

\section{Contributed Papers}

\section{1:40}

5aABa10. Correcting gray whale (Eschrichtius robustus) call rates in San Ignacio Lagoon, using sound exposure level measurements of ambient noise. Melania Guerra (Marine Physical Laboratory, Scripps Institution of Oceanography, 9500 Gilman Dr, MC 0238, La Jolla, CA $92093-$ 0238, USA, meguerra@ucsd.edu), Aaron Thode (Marine Physical Laboratory, Scripps Institution of Oceanography, 9500 Gilman Dr, MC 0238, La Jolla, CA 92093-0238, USA, athode@ucsd.edu), Jorge Urban (Laboratorio de Mamiferos Marinos, Universidad Autonoma de Baja California Sur, La Paz, Apartado Postal 19-B, 23080 La Paz, Mexico, jurban@uabcs .mx), Sergio Gonzalez (Laboratorio de Mamiferos Marinos, Universidad Autonoma de Baja California Sur, La Paz, Apartado Postal 19-B, 23080 La Paz, Mexico, sergiog@uabcs.mx)

Autonomous acoustic recordings of gray whales (Eschrichtius robustus) were made in San Ignacio Lagoon, in February 2005-2008, while animals were present to breed and raise calves. Counts were made of the gray whales' most common vocalization, type S1. A sequence of semi-automated procedures was implemented to assist with call detection. Hourly call rates were computed for all seasons and adjusted for expected changes in detection range, caused by variations in the ambient background noise level. In this environment, the underwater acoustic background combines biological, oceanographic and man-made sources and can present changes of $10 \mathrm{~dB}$ above the average base level of $96 \mathrm{~dB}$ re $1 \mathrm{uPa} 2$-s between 350 and $750 \mathrm{~Hz}$ over semidiurnal scales. The relative changes in call rates in 2006 and 2008 are compared with visual survey counts conducted over the same period. The definition of SNR in the present study develops from energy flux densities or sound exposure levels (SEL). SEL were calculated experimentally through even sampling in time and individual sampling for each call. By assuming that the background masks a proportion of the detected calls, corrections were applied to determine the vocal activity within a fixed detection range. 


\section{2:00}

5aABa11. The acoustic field on the melon of echolocating Atlantic bottlenose dolphin (Tursiops truncatus). Whitlow W. Au (Univ. of Hawaii, P.O. Box 1106, Kailua, HI 96734, USA, wau@hawaii.edu), Dorian S. Houser (Biomimetica, 7951 Shantung Dr., Santee, CA 92071, USA, dhouser@spawar.navy.mil), James J. Finneran (US Navy Marine Mammal Program, Space and Naval Warfare Systems Center, 53560 Hull St., Code 71510, San Diego, CA 92152, USA, james.finneran@navy.mil), Lois Dankiewicz (SAIC, 4065 Hancock Street, MS: Q1-A, San Diego, CA 92110, USA, lois.dankiewicz@saic.com), Wu-Jung Lee (MIT-WHOI Joint Program, 266 Woods Hole Road, Woods Hole, MA 02543, USA, wjlee@whoi.edu), Patrick W. Moore (US Space \& Naval Warfare Systems Center, 49620 Beluga Road, San Diego, CA 92151, USA, pmoore@spawar .navy.mil)

An array of five broadband suction cup hydrophones were placed on the melon of two bottlenose dolphins to determine where on the melon the echolocation beam emerges and to examine how signals in the acoustic near-field relate to signals in the far-field at $1 \mathrm{~m}$. Four different array geometries were used: a linear one with hydrophones arranged along the midline of the melon between 2.8 and $3.7 \mathrm{~cm}$ apart, and two around the front of the melon at 1.4 and $4.2 \mathrm{~cm}$ above the melon-rostrum crease and one across the melon in certain locations not measured by other configurations. The beam axis was found to be close to the mid-line of the melon, approximately 5.4 $\mathrm{cm}$ above the melon-rostrum crease for both animals. The signal path coincided with the low-density, low-velocity core of the melon supporting the melon hypothesis postulated many years ago by Kenneth Norris. Slight asymmetry in the signal was found with higher amplitudes on the starboard side of the melon. Although the signal waveform measured on the melon appeared distorted, when they were mathematically summed in the far-field while preserving the relative time of arrival, the resultant waveform matched that measured by the hydrophone located at $1 \mathrm{~m}$.

\section{2:20}

5aABa12. Detection of beaked whales using near surface towed hydrophones: prospects for survey and mitigation. Douglas Gillespie (Sea Mammal Research Unit, Gatty Marine Laboratory, University of St. Andrews, KY16 8LB St. Andrews, UK, dg50@st-andrews.ac.uk), Jonathan Gordon (Sea Mammal Research Unit, Gatty Marine Laboratory, University of St. Andrews, KY16 8LB St. Andrews, UK, jg20@st-andrews.ac.uk), Marjolaine Caillat (Sea Mammal Research Unit, Gatty Marine Laboratory, University of St. Andrews, KY16 8LB St Andrews, UK, marjolaine.caillat@club-internet.fr), Diane Claridge (Bahamas Marine Mammal Research Organisation, P.O. Box AB20714, Abaco, 0 Marsh Harbour, Bahamas, bmms@oii.net), David Moretti (NAVSEA, Newport Undersea Warfare Center, Newport, RI RI 02841, USA, MorettiDJ@npt.nuwc.navy.mil), Ian Boyd (Sea Mammal Research Unit, Gatty Marine Laboratory, University of St Andrews, KY16 8LB St Andrews, UK, ilb@st-andrews.ac.uk)

Beaked whales are extremely difficult to sight at sea and this hampers attempts to study them, and makes real time mitigation difficult. Passive acoustic monitoring could improve detection efficiency. Blainville's beaked whales, (Mesoplodon densirostris) are known to produce most of their vocalizations at depth. They are routinely detected on bottom mounted hydrophones arrays but the extent to which they can be detected using nearsurface hydrophones is not known. Continuous recordings were made at a sampling rate of $192 \mathrm{kHz}$ from towed hydrophone arrays during line transect surveys in the Bahamas in conjunction with teams monitoring bottommounted hydrophones at the AUTEC Tongue of the Ocean navy range. A beaked whale click detector and classifier was developed within Rainbow Click and PAMGUARD and this was both run in real time and used to analyze recordings to pick out beaked whale click trains. Detected click trains correlated well with detection of beaked whales on bottom-mounted hydrophones. Three species of beaked whale were encountered visually and detected acoustically: Mesoplodon densirostris, Ziphius cavirostris and Mesoplodon europaeus. Target motion analysis of bearings to sequences of clicks suggests a maximum detection range of approximately three kilometers and preliminary results indicate that clicks can be identified to species.
2:40

5aABa13. Acoustic localization of two distinct blue whale (Balaenoptera musculus) subspecies in the South-West Indian Ocean. Flore Samaran (CEBC-CNRS, Centre d'Etude Biologique de Chizé, 79360 Villiers en Bois, France, samaran@cebc.cnrs.fr), Olivier Adam (Université Paris Est, 61, avenue de Gaulle, 94000 Creteil, France, adam@univ-paris12.fr), Jean-François Motsch (Université Paris Est, 61, avenue de Gaulle, 94000 Creteil, France, motsch@univ-paris12.fr), Yves Cansi (Commissariat à l'Energie Atomique, Centre DAM - Ile de France, Bruyères-le-Châtel, 91297 Arpajon Cedex, France, yves.cansi@cea.fr), Gérard Ruzié (Commissariat à l'Energie Atomique, Centre DAM - Ile de France, Bruyères-le-Châtel, 91297 Arpajon Cedex, France, gerard.ruzie@cea.fr), Christophe Guinet (CEBC-CNRS, Centre d'Etude Biologique de Chizé, 79360 Villiers en Bois, France, guinet@cebc .cnrs.fr)

Analysis of one year of acoustic signal recordings from the five permanent autonomous hydrophones of the International Monitoring System in the South-West Indian Ocean reveals low frequency with high intensity calls produced by two blue whale subspecies. The "Antarctic" or "true" blue whale (B. m. intermedia) calls and the "Madagascar-type" Pygmy blue whale calls (B. m. brevicauda) were automatically detected through the matched filtering method. The potential movements were investigated by using the time difference of arrival (TDOA) of calls to assess the bearing of the sound source. The fully range dependent parabolic equation code (RAM - range-dependent acoustic model) and the PMCC code (progressive multichannel correlation) are applied to estimate the range between our system and the vocalising animals. Our results show that (1) the variation of call number revealed two distinct patterns of seasonal whale occurrences and (2) the distances from the hydrophones to the blue whales reached up to $50 \mathrm{~km}$. Tracking whales is possible when whales are concentrated of the hydrophone array.

\section{3:00}

5aABa14. Biosonar performance of a false killer whale (Pseudorca crassidens) improved with practice. Robert Gisiner (Marine Mammal Commission, 4340 East-West Highway, Room 700, Bethesda, CA 20814, USA, bgisiner@mmc.gov)

Cylinder wall thickness discrimination tasks have been used to assess the limits of dolphin biosonar. An attempt to replicate the benchmark data from a bottlenose dolphin (Tursiops truncatus) resulted in a surprising order of magnitude better performance by a false killer whale. The improvement came over multiple testing episodes, which suggests that initial limits to discrimination were overcome by learning; either through better understanding of the reinforcement contingencies, attention to novel sources of information within the returning echoes, or both. These results offer important insights into methodological considerations for testing animal psychophysical performance, especially for sensory performances like echolocation in which the subject exercises active control over the sensory input. The ability to improve sensory performance with practice also offers insights into the way the echo information is processed into an internal representation of external physical reality by the central nervous system, a process that is very likely open to improvement through experience, or learning, throughout the individual's life.

\section{3:20}

5aABa15. An analysis of Humpback whale songs for individual classification. Suleman Mazhar (URA Laboratory, Institute of Industrial Science, The University of Tokyo, 4-6-1, Komaba, Meguro, 153-8505 Tokyo, Japan, suleman@iis.u-tokyo.ac.jp), Tamaki Ura (URA Laboratory, Institute of Industrial Science, The University of Tokyo, 4-6-1, Komaba, Meguro, 153-8505 Tokyo, Japan, ura@iis.u-tokyo.ac.jp), Rajendar Bahl (Indian Institute of Technology, Delhi, Hauz Khas, 110016 New Delhi, India, rbahl@care.iitd.ernet.in)

Acoustics based tracking systems are in use for studying cetacean behaviour. Such noninvasive techniques can also prove efficient for population assessment of vocally active species. One problem in this regard is to distinguish already counted reappearing cetaceans, even if reappearance occurs after a brief interval. In this connection we propose development of in- 
dividual identification system for humpback whales (Megaptera novaeangliae) based on unique acoustic features underlying a song. Earlier we analyzed recognition performance of cepstrum based voice signature in seven humpback whales. The results were based on training and test data sampled from the same song. In this work, we test our hypothesis using data from different songs (i.e., recording timings of training and test data sets do not overlap) for two different individuals. Recognition rates for two individuals are above $80 \%$ and $95 \%$, respectively. However an eight years old song gives degraded recognition rate of around $58 \%$. The results give an insight into the effects of temporal song evolution on accuracy of our system. Our observations are followed by harmonic analysis of song units in two individuals. Cepstral coefficients and support vector machine (SVM) were used for classification and signal band-crossing rate was used for segmenting song units.

\section{$3: 40$}

5aABa16. Phonemic segment characterization of Norwegian killer whale call types. Ari D. Shapiro (Woods Hole Oceanographic Institution, Applied Ocean Physics \& Engineering Dept., Woods Hole, MA 02543, USA, ashapiro@whoi.edu), Stephanie Seneff (Massachusetts Institute of Technology, Computer Science and Aritifical Intelligence Laboratory, Spoken Language Systems Group, Cambridge, MA 02139, USA, seneff@csail.mit.edu), Peter Tyack (Woods Hole Oceanographic Institution, Applied Ocean Physics \& Engineering Dept., Woods Hole, MA 02543, USA, ptyack@whoi.edu)

An important issue in animal vocal communication concerns the fundamental unit used to build signals into a repertoire and the syntactic rules associated with that assembly. Killer whale vocal production has traditionally been categorized by human observers into a set of discrete call types. These call types often contain internal spectral shifts, silent gaps and synchronously produced low and high frequency components. Such features motivated the analysis here which tested whether call types could be represented by a set of flexibly arranged and smaller phonemic segments. We evaluated whether segmented characterizations of stereotyped Norwegian killer whale calls yielded automated classification results of contour traces that paralleled a classification scheme using whole call type designations. Representations of calls in their entirety or as sets of either distinct or shared syllables did achieve similar performance. Calls composed of shared segments may provide a more parsimonious approach to parsing the vocal stream since there were fewer segments than call types, nearly $75 \%$ of all call types contained at least one shared syllable, and some syntactic patterns were evident. Such a system could flexibly generate new call types and contain the killer whale vocal repertoire within a subset of the possible combinations of segments.

\section{4:00}

5aABa17. AFM and BSEM: Novel approaches to the Basilar Membrane. Darlene R. Ketten (NIH/NIDCD and WHOI, Section on Auditory Mechanics, Bldg 10-5D49, Bethesda, MD 20892, USA, dketten@whoi.edu), Emilios K. Dimitriadis (NIH/NIDCD and WHOI, Section on Auditory Mechanics, Bldg 10-5D49, Bethesda, MD 20892, USA, dimitria@helix.nih.gov), Richard S. Chadwick (NIH/NIDCD and WHOI, Section on Auditory Mechanics, Bldg 10-5D49, Bethesda, MD 20892, USA, chadwick@helix.nih.gov), David Mountain (Boston University, Biomedical Engineering Dept., 44 Cummington St., Boston, MA 02215, USA, dcm @bu.edu)

For over a half century, researchers have probed cochlear biophysics with increasingly sophisticated technologies. Recently, we brought two new approaches, atomic force microscopy (AFM) and backscatter electron microscopy (BSEM), to bear on the question of how basilar membrane structural variations affect mechanical responses. Hemi-cochleae and basilar membrane segments were obtained from rat, guinea pig, and gerbil ears by temporal bone microdissection. Hemi-cochleae in PBS or formalin were imaged in a hydrated, uncoated state (VP wet mode) in Petri dishes mounted on boutons using an Hitachi S3400 N-1 SEM. Membrane segments were excised, trimmed of laminae and ligament remnants, and mounted on polylysine/albumin-coated glass for AFM imaging and force measurements. Both BSEM and AFM data indicate two distinct regions consistent with softer ground substance separating relatively stiff, ordered fiber bundles ori- ented radially in pectinate regions. Fibers and bundles ranged 0.3 to $1 \mu \mathrm{m}$ in diameter with elasticity values, based on a Hertzian contact model, in the hundreds of $\mathrm{kPa}$ range, consistent with mixed elastin and collagen. Greatest variations occurred in matrix spacing, suggesting differences are attributable largely to packing density. Middle turn ground substance spacing averaged $1.5 \mu \mathrm{m}$, consistent with previously reported values (Naidu and Mountain, 2007; Fung, 1993).

\section{4:20}

5aABa18. Depth, orientation, and acoustics of sperm whales (Physeter macrocephalus) under natural and depredation foraging conditions in the Gulf of Alaska. Delphine Mathias (Marine Physical Laboratory, Scripps Institution of Oceanography, 9500 Gilman Dr, MC 0238, La Jolla, CA 92093-0238, USA, delphine.mathias@gmail.com), Aaron Thode (Marine Physical Laboratory, Scripps Institution of Oceanography, 9500 Gilman Dr, MC 0238, La Jolla, CA 92093-0238, USA, athode@ucsd.edu), Jan Straley (University of Alaska SE, 1332 Seward Ave, Sitka, AK 99835, USA, jan.straley@uas.alaska.edu), Kendall Folkert (PO Box 6497, Sitka, AK 99835, USA, kendallcobra@yahoo.com), John Calambokidis (Cascadia Research Collective, 218 1/2 W. 4th Ave., Olympia, WA 98501, USA, calambokidis@cascadiaresearch.org), Greg Schorr (Cascadia Research Collective, 218 1/2 W. 4th Ave., Olympia, WA 98501, USA, GSchorr@cascadiaresearch.org), William C. Burgess (Greeneridge Sciences Inc, 6060 Graham Hill Rd, suite f, Felton, CA 95018, USA, burgess@greeneridge.com), Chris Lunsford (National Marine Fisheries Service Auke Bay Laboratory, 11305 Glacier Hwy, Juneau, AK 99801-8626, USA, chris.lunsford@noaa.gov)

In July 2007 bioacoustic tags were attached to adult sperm whales in the Gulf of Alaska under both natural foraging conditions, and situations wherein the animals were depredating sablefish from commercial longlining vessels. A small Rigid-Hull Inflatable was used to approach and attach a suction-cup acoustic recording tag on 13 occasions and stayed on animals for a total of 168 hours, yielding $80 \mathrm{~h}$ of depth, orientation, and acoustic data. These results, combined with passive acoustic tracking and underwater video-camera data, indicate that sperm whales depredate at depths shallower than $50 \mathrm{~m}$, compared to natural foraging depths of 300-400 $\mathrm{m}$ in the area. During depredation the animals demonstrate changes in pitch and roll that are greater when compared with normal foraging behavior. Observations on the acoustic behavior of the animals are noted as well. The fact that these normally deep-diving animals depredate so close to the surface has interesting implications for both depredation-reduction strategies and biosonar research. [Work conducted under the SEASWAP program, supported by the North Pacific Research Board and the National Geographic Society.]

5aABa19. Fin whale (Balaenoptera physalus) movements along the Spanish mediterranean coast. Manuel Castellote (L'Oceanografic, C/. Junta de Murs i Valls, s/n, 46013 Valencia, Spain, mcastellote @oceanografic.org), José-Antonio Esteban (Research Department, Parques Reunidos Valencia S. A. L'Oceanogràfic, Ciudad de las Artes y las Ciencias, 46013 Valencia, Spain, investigacion@oceanografic.org), Christopher W. Clark (Cornell University Laboratory of Ornithology, Bioacoustics Research Program, 159 Sapsucker Woods Road, Ithaca, NY 14850, USA, cwc2 @ cornell.edu)

The Ligurian-Corsican-Provençal Basin is a fin whale summer feeding ground, but little is known about the seasonal movement patterns of this population. We acoustically monitored two regions off Spain (eastern and southern) during summer-fall-winter 2006 using seafloor autonomous recording units. Long patterned sequences of $20 \mathrm{~Hz}$ pulses and back-beats from Mediterranean fin whales were identified in both study regions using acoustic features as indicators of population identity. Eastern area: fin whale sounds were detected at very high rates (122 pulses/hour) and between 40$80 \%$ of time/day. The detection pattern can be explained by whales moving gradually through the acoustic recorder's detection area, suggesting that the area is probably used as a passage between summer and winter grounds. Southern area: detections from the same population were less abundant $(21$ pulses/hour) and between 10-35\% of time/day. The detection pattern shows a highly variable distribution with no clear trend, suggesting that Mediter- 
ranean fin whales continuously remained in and moved out of the detection area of the recorder from November to January. These results show that this southern area is potentially used as a winter ground. This work documents a new dispersion path towards the Alboran sea, a new potential winter ground.

\section{5:00}

5aABa20. Accurate estimation of the duration of tonal signals emitted by marine mammals. Nicolas Sentenac (Thales Underwater Systems, 525, route des dolines, BP 157, 06903 Sophia Antipolis, France, nicolas .sentenac@fr.thalesgroup.com)

Passive acoustic marine mammals detection and classification is of great importance nowadays. The goal of this article is to present a tool that can be used to accurately measure the duration of tonal emissions from marine mammals, helping to classify them. Marine mammals emissions can be split into two different kinds and one of them is tonal emission. It can be defined by its narrow band frequency pattern. Narrow band signals can be detected in the time/frequency space thanks to the use of the spectrogram and thanks to a quadratic contrast criterion. The expression of this criterion for signalonly and noise-only is straightforward. This article will introduce the expression of a lower bound of the expectation of the criterion when signal and noise are mixed. As this lower bound is a function of signal to noise ratio, it can be used to threshold the criterion with the desired signal to noise ratio value. When the criterion is computed along time on a sliding window, narrow band signals with a higher snr than the thresholding one are detected as soon as they appear and until they vanish, giving an accurate estimation of the duration of such signals.

\title{
Invited Paper
}

5aABa21. Ultrasonic production and reception in frogs: Lessons from Asia. Peter Narins (UCLA, Dept. of Physiological Science, 621 Charles E. Young Drive S., Los Angeles, CA 90095-1606, USA, pnarins@ucla.edu)

Among the vertebrates, only microchiropteran bats, cetaceans and some rodents are known to produce and detect ultrasonic (US) frequencies for the purpose of communication and/or echolocation, suggesting that this capacity may be restricted to mammals. We have recently provided the first evidence of ultrasonic communication in an amphibian - the concave-eared torrent frog, Amolops tormotus (Ranidae) from Huangshan Hot Springs, China. Males of A. tormotus produce diverse birdlike melodic calls with pronounced frequency modulations that often contain spectral energy in the US range. Acoustic playback experiments conducted in the animal's natural habitat confirmed that the audible as well as the US components of an A. tormotus call could effectively evoke males' vocal responses. Electrophysiological recordings from the auditory midbrain confirmed the US hearing capacity of these frogs and that of a sympatric species facing similar environmental constraints. This extraordinary upward extension into the ultrasonic range of both the harmonic content of the advertisement calls and the frog's hearing sensitivity is likely to have coevolved in response to the intense, predominately lowfrequency ambient noise from local streams. Supported by NIH grant DC-00222.

FRIDAY MORNING, 4 JULY 2008

P3-C, LEVEL 3, 9:40 TO 11:20 A.M.

\section{Session $5 \mathbf{a A B b}$}

\section{Animal Bioacoustics: General Topics in Animal Bioacoustics III (Poster Session)}

\author{
Richard Fay, Cochair \\ Loyola University Chicago \\ Michel Andre, Cochair \\ Laboratori d'Aplicacions Bioacústiques (Universitat Politècnica de Catalunya)
}

\begin{abstract}
All posters will be on display from 9:40 a.m. to 11:20 a.m. To allow contributors an opportunity to see other posters, contributors of odd-numbered papers will be at their posters from 9:40 a.m. to 10:30 a.m. and contributors of even-numbered papers will be at their posters from 10:30 a.m. to 11:20 a.m.
\end{abstract}

\section{Contributed Papers}

5aABb1. A brownian energy depot model of the basilar membrane oscillation. Chul Koo Kim (Yonsei University, Department of Physics, Shinchon-dong, 120-749 Seoul, Republic of Korea, ckkim@yonsei.ac.kr)

Response of a living basilar membrane (BM) is significantly different from that of the cadaver and is known to be essentially active. The best candidate for a generator of the active force in mammals is the outer hair cells. By employing the outer hair cells as the energy depot, we newly propose an interactive energy depot model for the basilar membrane. In this model, the nonlinear responses and the spontaneous basilar membrane oscillation are obtained. In the regime of small vibration, this model is reduced to the wellknown Hopf bifurcation model. Our model suggests two-fold roles of the outer hair cells in hearing: an amplification for a weak stimulation and a protection for a strong stimulation.
5aABb2. Slow chaotic changes in the excitability of the auditory units Nikolay G. Bibikov (N.N. Andreyev Acustical Institute, Shvernik st. 4, 117036 Moscow, Russian Federation, bibikov@akin.ru), Alexandre B. Dymov (N.N. Andreyev Acustical Institute, Shvernik st. 4, 117036 Moscow, Russian Federation, dymov@mail.ru)

We explored the statistical properties of the spontaneous and evoked firing activity in the brainstem auditory units of the grass frog (Rana $t$ temporaria). We estimated the properties of spontaneous firing using the following functions: interspike interval distribution, hazard function, autocorrelation functions for original and shifted sequence of interspike intervals, function of interdependence of neighboring intervals. The dependences of Fano and Allans factors upon the values of counting time (window size) were also obtained. We observed a considerable deviation of the spontane- 
ous activity from the renewal process for the majority of units located both in the dorsal medullar nucleus (homolog of cochlear nucleus in the mammals) and in torus semicircularis (homolog of inferior colliculus). The negative correlation between the neighboring short interspike intervals was typical for some (mainly toral) units. However, generally week but quite reliable positive correlation between neighboring intervals was typical for the great majority of neurons. The values of Fano and Allan factors were close to 1 at window size less than $100-500 \mathrm{~ms}$ and increased in proportion to some power for larger window sizes. The exponents of these power dependences were different for different units.

5aABb3. Synchrony and neuromechanical sensitivity in the mosquito hearing organ. Joseph C. Jackson (University of Bristol, D34 School of Biological Sciences, Woodland Road, BS8 1UG Bristol, UK, j.c.jackson@bris.ac.uk), Daniel Robert (University of Bristol, D34 School of Biological Sciences, Woodland Road, BS8 1UG Bristol, UK, d.robert @ bris.ac.uk)

Sound detection is a fundamental tool for many biological organisms to sense their environment. Sensing the particle velocity component of sound waves has led to the evolution of exquisitely sensitive auditory organs: small oscillators that are driven resonantly by the sound field. The sound detection threshold is often indistinguishable from Brownian motion. To achieve such low thresholds, the mammalian cochlea incorporates active mechanisms, adding energy to the sound oscillations, and so enhancing sensitivity and selectivity. Mosquito antennae are also endowed with active auditory mechanics. Males use these highly sensitive external antennae to detect the flight sound of females. Sound-induced oscillation of the antenna stimulates thousands of mechanosensory neurones. The antenna exhibits dynamic responses remarkably similar to vertebrate auditory systems: self-oscillation and amplitude-dependent bandwidth both occur. They also exhibit hitherto unseen nonlinear responses including mid-level amplification and hysteresis. The mechanisms for these effects are thought to derive from ciliary motility in the mechanosensory neurones. We show that synchronization in the motile neurones generates large stable coherent forces that provide the antenna with its nonlinear response characteristics. Synchronization ensures a coherent neuronal output, improving signal fidelity. Finally, coherent force modifies the dissipation of antennal energy, changing the bandwidth and enhancing sensitivity.

5aABb4. Changes in structure of Redwing's (Turdus iliacus) local dialect in time. Ludmila Osipova (St. Petersburg State University, Universitetskaya emb., 7/9, 199034 St. Petersburg, Russian Federation, osipoval @yandex.ru)

The question of the period of existance of a local dialect is yet to be answered. Were there a changes in time-and-frequency parameters of dialects with time? If there were, what parameters had changed? On the basis of original data for 2005 - 2007 period, and archives of Department of Vertebrate Zoology of Saint-Petersburg State University, the history of local dialect of Redwing (Turdus iliacus) from Saint-Petersburg's outskirt (Peterhof) was investigated.The archive contains recordings of local dialects of Peterhof for 1960-1991 period. Until now, the longest period of local dialect observation for this species did not exceed 10 years (Bjerke, 1981). In observed period of 48 years several changes were found. Number of elements in phrase, it's duration, frequency modulation of all elements, their configuration, duration and frequency range. The major changes occurred to the first element of song. It is gradual reduction with time is obvious.

5aABb5. Microacoustics: maintaining an ecologically relevant scale in insect bioacoustics. Erica L. Morley (University of Bristol, D34 School of Biological Sciences, Woodland Road, BS8 1UG Bristol, UK, Erica.Morley@bristol.ac.uk), Thorin Jonsson (University of Bristol, School of Biological Sciences, Woodland Road, BS8 1UG Bristol, UK, t.jonsson@bristol.ac.uk), Daniel Robert (University of Bristol, D34 School of Biological Sciences, Woodland Road, BS8 1UG Bristol, UK, d.robert @bris.ac.uk)

A major challenge when studying an organism is to maintain its environment and context as reliably as possible. When looking at sensory systems it is crucial not to assume the animal's perception the same as ours and instead measure and mimic more accurately the natural stimuli driving the sensors. In insect bioacoustics the usual practice in playback and recording techniques overlooks differences in scale and context in which the organism's sensory system evolved. Here we present an approach to emit and record low-amplitude near-field sound, while maintaining an ecologically relevant scale, in Drosophila melanogaster. To mimic the effect of male courtship song on the female sound receiver (antennae) a mechanical microwing was constructed simulating, in power and geometry, particle velocity signals emitted by singing males, with its efficacy tested by behavioural assay. Development of a miniature particle velocity microphone was also initiated for recording signals in the fly's immediate vicinity $(<2 \mathrm{~mm})$ to elucidate the magnitude, temporal and radiation characteristics of the produced sound-field.

5aABb6. The effect of courtship song components in Drosophila melanogaster. Yu-Po Chen (University of Bristol, D34 School of Biological Sciences, Woodland Road, BS8 1UG Bristol, UK, Yu-po.Chen@bristol.ac.uk), Joseph C. Jackson (University of Bristol, D34 School of Biological Sciences, Woodland Road, BS8 1UG Bristol, UK, j.c.jackson@bris.ac.uk), James F. Windmill (University of Bristol, D34 School of Biological Sciences, Woodland Road, BS8 1UG Bristol, UK, james.windmill@bristol.ac.uk), Daniel Robert (University of Bristol, D34 School of Biological Sciences, Woodland Road, BS8 1UG Bristol, UK, d.robert@bris.ac.uk)

Courtship between male and female Drosophila melanogaster involves a complex emission of sounds produced by the male. This song comprises two aspects: a low-frequency sine song followed by a series of intense pulses. These songs increase the chances of the female accepting the male. However, the reason for the complexity of the song, and the effect on the female of the song's components, is poorly understood. Using measurements of the male courtship song for a canonical stimulus, we investigate the importance of the sine song on both the mechanical and electrophysiological responses of the female antenna: the nonlinear auditory sensor. While stimulating the antenna with modified courtship songs, antennal motion was measured using laser Doppler vibrometry, and compound potentials were simultaneously measured from the auditory neurones. Results show that, even at the periphery, there exists a significant change in the way the antenna responds to the pulses as a function of the sine song intensity, most emphatically through the neurophysiological signals. Results indicate that the sine song is an advantageous trait used to increase the female perception of the pulses in both time and amplitude sensitivity, and therefore improve the male's chance of successfully courting a female.

5aABb7. The Locust's tympanal mechanics. James F. Windmill (University of Bristol, D34 School of Biological Sciences, Woodland Road, BS8 1UG Bristol, UK, james.windmill@bristol.ac.uk), Samuel D. Bockenhauer (Stanford University, 208 Rosse Ln, \#305, Stanford, 94305, USA, sbockenhauer@gmail.com), Thomas R. McDonagh (University of Bristol, School of Biological Sciences, Woodland Road, BS8 1UG Bristol, UK, tm3377@bristol.ac.uk), Daniel Robert (University of Bristol, D34 School of Biological Sciences, Woodland Road, BS8 1UG Bristol, UK, d.robert@bris.ac.uk)

In the ear of the desert locust frequency analysis arises from the mechanical properties of the tympanal membrane. Incident sound is spatially decomposed into discrete frequency components through a tympanal travelling wave that funnels mechanical energy to specific tympanal locations, where distinct groups of mechanoreceptor neurones project. Initial analysis of the travelling waves employs conventional, steady state FFT, allowing a detailed analysis of the spatial composition of different frequencies onto the membrane. To further understand the exact mechanics of the tympanal travelling wave, its motion was also measured in the time domain to characterise its response to single impulse and single frequency stimuli, with a resolution of $390 \mathrm{~ns}$. This allows the measurement of instantaneous wave velocity and the direct observation of wave compression across the tympanum. The locust tympanal membrane locust exploits tonotopic frequency analysis, in a similar sense to that of the travelling waves of von Békésy on the mammalian basilar membrane. However, von Békésy's wave is born from interactions between the anisotropic basilar membrane and surrounding incom- 
pressible fluids, whereas the locust's wave rides on an anisotropic membrane suspended in air. The locust's tympanum thus combines the functions of both sound reception and frequency analysis.

5aABb8. Automated acoustic identification of beetle larvae in imported goods using time domain analysis. James Schofield (University of York, Department of Electronics, Heslington, YO10 5DD York, UK, js517@york.ac.uk), David Chesmore (University of York, Department of Electronics, Heslington, YO10 5DD York, UK, edc1@ohm.york.ac.uk)

The detection of insect pests in imported goods is of considerable economic importance and the automation of this process is becoming more viable both technologically and financially. As a result, the Department for Environment, Food and Rural Affairs in the UK has funded a research project to develop instrumentation facilitating real-time acoustic detection of the feeding activity of insect larvae inside imported goods, such as timber. The instrumentation will also be capable of species-level identification. Previous work at York has shown that detection of beetle larvae in wood is possible using low cost piezoelectric sensors. The project described here extends this work by investigating a number of signal analysis methods for robust detection of biting events, including fractal dimension analysis. Identification is currently being carried out using time domain signal coding and artificial neural networks. This paper will concentrate on the results of various algorithms for the estimation of fractal dimension and their relative suitability for bite detection. The effects of varying sampling rates, threshold levels and signal-to-noise ratio on the detection rate will be demonstrated.

5aABb9. Sound insulation of artificial and natural sound signals in reeds habitats. Igor Ianovschi (Universitetskaja nab. 7/9, Dep. Vertebrate Zoology, 199034 St. Petersburg, Russian Federation, igorul@mail.ru), Ludmila Osipova (St. Petersburg State University, Universitetskaya emb., 7/9, 199034 St. Petersburg, Russian Federation, osipoval @ yandex.ru)

From the point of view of acoustical communication, dense fields of reeds are strong acoustical filtre which distorts original sound signal. In this research the rate of sound signals sound insulation in massives of reeds was estimated. As a models were used artificially synthesized, modulated by frequency signals of three frequency lines: $1-2 \mathrm{kHz}, 2-4 \mathrm{kHz}$, and $4-8 \mathrm{kHz}$ with duration of 0.1 and $0.25 \mathrm{msec}$. For comparison were also used elements of bird songs. The least damping was noted in signals with descending frequency modulation in $8-4 \mathrm{kHz}$ range in the upper level of reeds $(6 \mathrm{db}$ on $10 \mathrm{~m})$. The most damping were noted in signals with descending frequency modulation in $2-1 \mathrm{kHz}$ in the middle level of juncaceous massive $(18 \mathrm{db}$ to $10 \mathrm{~m})$. The signals with frequency modulation of $4-2 \mathrm{kHz}$ were extending better in the upper level of reeds $(7-8 \mathrm{db})$, whereas in the lower and upper levels were noted dependence of damping rate on duration of sound impulse - the signals with duration of 0.25 mec were damping less than signals with duration of $0.1 \mathrm{msec}$. The elements of bird songs during their emission in the middle level of juncaceous massive were damping by $15-20 \mathrm{db}$ on $10 \mathrm{~m}$.

5aABb10. Echolocation and flight strategies of Japanese house bats (Pipistrellus abramus) to attack a prey in the field revealed by a microphone array. Emyo Fujioka (Faculty of Engineering, Doshisha Univ., 1-3 Miyakodani Tatara, 610-0321 Kyotanabe, Japan, dth0902@mail4.doshisha.ac.jp), Shigeki Mantani (Faculty of Engineering, Doshisha Univ., 1-3 Miyakodani Tatara, 610-0321 Kyotanabe, Japan, bte2058@mail4.doshisha.ac.jp), Michihiro Fukuda (Faculty of Engineering, Doshisha Univ., 1-3 Miyakodani Tatara, 610-0321 Kyotanabe, Japan, dtg0108@mail4.doshisha.ac.jp), Shizuko Hiryu (Faculty of Engineering, Doshisha Univ., 1-3 Miyakodani Tatara, 610-0321 Kyotanabe, Japan, shiryu@mail.doshisha.ac.jp), Hiroshi Riquimaroux (Faculty of Engineering, Doshisha Univ., 1-3 Miyakodani Tatara, 610-0321 Kyotanabe, Japan, hrikimar@mail.doshisha.ac.jp), Yoshiaki Watanabe (Faculty of Engineering, Doshisha Univ., 1-3 Miyakodani Tatara, 610-0321 Kyotanabe, Japan, kwatanab@mail.doshisha.ac.jp)

Bats are supposed to have effective strategies for achieving a good balance between echolocation and flight behaviors while capturing small moving insects in the field. To reveal their strategies for catching insects, we successfully reconstructed 3-D flight trajectories for the bat to forage in the field by a four-microphone array system, and conducted both acoustical and behavioral analyses for capturing behavior. Data show that the flying bats changed their flight direction flexibly, and sometime repeated capturing insects every two to three seconds. During the search phase, the bat moved 0.5-0.8 $\mathrm{m}$ during an interval between successive pulses (IPI) and then decreased that the moving distance during an IPI up to $0.1 \mathrm{~m}$ just before capturing a prey. Interestingly, we found that the bat tended to descend toward a prey from above when the approach phase started. This suggests that foraging bats may effectively utilize gravity for an easy acceleration toward the prey to concentrate on the complex echolocation for capturing moving insects. [Supported by a grant to RCAST at Doshisha Univ. from MEXT of Japan: Special Research Grants for Development of Characteristic Education from the Promotion and Mutual Aid Corporation for Private Schools Japan, Innovative Cluster Creation Project.]

5aABb11. Optimization of the characteristics of receiving echoes by free-flying echolocating bats, revealed by onboard pulse-echo recordings. Shizuko Hiryu (Faculty of Engineering, Doshisha Univ., 1-3 Miyakodani Tatara, 610-0321 Kyotanabe, Japan, shiryu@mail.doshisha.ac.jp), Yu Shiori (Faculty of Engineering, Doshisha Univ., 1-3 Miyakodani Tatara, 610-0321 Kyotanabe, Japan, dtg0165@mail4.doshisha.ac.jp), Tatsuro Hosokawa (Faculty of Engineering, Doshisha Univ., 1-3 Miyakodani Tatara, 610-0321 Kyotanabe, Japan, dtg0119@mail4.doshisha.ac.jp), Hiroshi Riquimaroux (Faculty of Engineering, Doshisha Univ., 1-3 Miyakodani Tatara, 610-0321 Kyotanabe, Japan, hrikimar@mail.doshisha.ac.jp), Yoshiaki Watanabe (Faculty of Engineering, Doshisha Univ., 1-3 Miyakodani Tatara, 610-0321 Kyotanabe, Japan, kwatanab@mail.doshisha.ac.jp)

To understand complex sensory-motor behavior underlying echolocation by bats, we need to precisely measure acoustic characteristics of echoes that the bats actually listen to during flight. However, it has been technicallydifficult to develop a small and light enough microphone set at the position of the bat's ear, and information about the echoes is so far virtually lacked. Here, we have successfully monitored these returning echoes by means of an onboard telemetry microphone mounted on the bats, and we confirmed that the free-flying horseshoe bats compensated for Doppler-shifts by adjusting their call frequency, thus maintaining the echo frequency at constant. Furthermore, we found that the bats also adjusted pulse amplitude with the distance to maintain the echo intensity within the narrow range. Receiving echoes with stable amplitude could help the bats to sustain consistent analysis of successive echoes, and combined frequency and amplitude compensation may be for optimization of successive echoes for target range estimation to control approach and landing. [Supported by a grant to RCAST at Doshisha Univ. from MEXT of Japan: Special Research Grants for the Development of Characteristic Education from the Promotion and Mutual Aid Corporation for Private Schools of Japan and the Innovative Cluster Creation Project.]

5aABb12. Some problems of analyzing bio-sonar echolocation signals generated by echolocating animals living in the water and in the air. Tadeusz Gudra (Wroclaw University of Technology/Institute of Telecommunications, Teleinformatics and Acoustics, Wybrzeze Wyspianskiego 27, 50-370 Wroclaw, Poland, Tadeusz.Gudra@pwr.wroc.pl), Krzysztof Herman (Wroclaw University of Technology/Institute of Telecommunications, Teleinformatics and Acoustics, Wybrzeze Wyspianskiego 27, 50-370 Wroclaw, Poland, krzysztof.herman@pwr.wroc.pl)

In this paper some similarities and differences of bio-sonar echolocation signals generated in the water and in the air are presented. The echolocation cues of marine mammals and bats are usually frequency and amplitude signals modulated simultaneously. The main problems of the analysis of such type of signals are envelope detecting and time-frequency decomposition. In this paper some digital signal processing (DSP) algorithms that may be used to analyze this type of signals are presented. The basic linear spectrogram (STFS), the nonlinear Winger-Vill'e spectrogram and some time-scale representations of signals using wavelets methods of estimation of the fre- 
quency modulation function have been compared. An analysis of possibilities for acoustic identification of particular species of bats by means of echolocation is also presented.

5aABb13. Blue whale calls characterization using chirplet transform Mohammed Bahoura (Eng. Dept., Université du Québec, 300 Allée des Ursulines, P.O. Box 3300, Rimouski, QC G5L-3A1, Canada, mohammed_bahoura@uqar.qc.ca), Yvan Simard (Fisheries and Oceans Canada \& ISMER-UQAR, 850 route de la Mer, P.O. Box 1000, Mont-Joli, QC G5H-3Z4, Canada, simardy@dfo-mpo.gc.ca)

The blue whale, Balaenoptera musculus, frequently produce distinctive low-frequency $(<100 \mathrm{~Hz})$ signature calls that propagate over large distances in deep oceans. Their efficient detection and identification under variable noise conditions in long-term recordings is a basic requirement of passive acoustic monitoring systems used for studying distributional ecology and habitat selection of these large-scale migrant animals. In North Atlantic, blue whale signature calls are the A and B infrasounds $(15-20 \mathrm{~Hz})$, which often occur together in $\mathrm{AB}$ phrases, and the audible D-call $(35-120 \mathrm{~Hz})$, also known as arch sound. Given the distinction of these calls by their frequency band and rate of change in frequency, the chirplet transform appears well adapted to characterize such calls. We test here this new approach from subset of vocalizations of blue whale sounds recorded in St. Lawrence Estuary. The recordings are band-pass filtered and segmented to isolate individual calls. Then, a feature vector based on the chirplet transform is extracted from each call. Finally, vector quantization (VQ) is used to classify the calls into A, B, and D vocalizations. The performance of the method is compared for various VQ code book sizes. Small code book sizes using only 3 features produced classification rates exceeding $92 \%$.

5aABb14. Burst pulses produced by free-ranging bottlenose dolphins in Tampa Bay, Florida and Mississippi Sound, Mississippi. Natalija Lace (University of Southern Mississippi, 118 College Drive, Box 5025, Hattiesburg, MS 39406, USA, kodzaks@yahoo.com), Stan A. Kuczaj (University of Southern Mississippi, 118 College Drive, Box 5025, Hattiesburg, MS 39406, USA, s.kuczaj@usm.edu), Marc O. Lammers (Hawaii Institute of Marine Biology, P.O. Box 1106, Kailua, HI 96734, USA, lammers@ hawaii.edu)

Investigations of the bottlenose dolphin's acoustic repertoire have mainly focused on whistles and echolocation clicks. However, despite their widespread occurrence, burst pulses have not received much attention. The primary function of burst pulses remains unknown and a comparative analysis can be used to advance our knowledge of the function of burst pulses. We recorded the acoustic repertoires of free-ranging bottlenose dolphins in the Tampa Bay and Mississippi Sound areas using broadband recording equipment. The two habitats differ in their environmental features, including water temperature, ambient noise levels and water turbidity. Our preliminary data show that several acoustic parameters such as peak frequency and center frequency of burst pulses differ among habitats. We also report other acoustic parameters, such as the number of clicks, inter-click interval, 3-dB and rms bandwidth. In our ongoing study, we will examine relationships between environmental features of the habitats and the acoustic characteristics of burst pulses.

5aABb15. First report of burst-pulse vocalizations from white-beaked dolphins (Lagenorhynchus albirostris). Peter Simard (University of South Florida, College of Marine Science, 140 7th Ave. S., St. Petersburg, FL 33701, USA, psimard@marine.usf.edu), David Mann (University of South Florida, College of Marine Science, 140 7th Ave. S., St. Petersburg, FL 33701, USA, dmann@marine.usf.edu), Shannon Gowans (Eckerd College, Galbraith Marine Science Laboratory, 4200 54th Ave South, St. Petersburg, FL 33711, USA, gowanss@eckerd.edu)

Dolphin vocalizations are generally categorized as tonal whistles or pulsed clicks. Pulsed signals in dolphins are usually associated with echolocation, however an increasing number of species are found to produce burst pulse signals which may be used for communication. Groups of whitebeaked dolphins (Lagenorhynchus albirostris) were recorded from a $4.2 \mathrm{~m}$ rigid inflatable boat near Halifax, Canada with a hydrophone towed $25 \mathrm{~m}$ behind the boat at a depth of approximately 5m, and an M-Audio 24/96 digital recorder (16 bit, $96 \mathrm{kHz}$ ). During one 23-minute encounter, 11 burst pulse segments were recorded. Mean burst pulse duration was $0.83 \mathrm{~s}$ (SD 0.51 , range $0.22 \mathrm{~s}-1.74 \mathrm{~s})$. Seven segments with high signal to noise ratios were manually selected for analysis in Matlab. Mean pulse rate was $765 \mathrm{~Hz}$ (SD 182.2, range $552 \mathrm{~Hz}-941 \mathrm{~Hz}$ ). These durations and pulse rates are similar to those reported for other dolphin species. These vocalizations were made immediately before the group began actively swimming away from the boat at high speed, suggesting that these vocalizations are used in agonistic encounters as suggested for other species of dolphins. This is the first time burst-pulse vocalizations have been reported in this species.

5aABb16. Real-time acoustic monitoring of the deep-ocean environment. Michel Andre (Laboratori d'Aplicacions Bioacústiques (Universitat Politècnica de Catalunya), avda. Rambla Exposició s/n, 08800 Vilanova i la Geltrú, Spain, michel.andre@upc.edu), Mike Van Der Schaar (Laboratori d'Aplicacions Bioacústiques (Universitat Politècnica de Catalunya), avda. Rambla Exposició s/n, 08800 Vilanova i la Geltrú, Spain, mike.vanderschaar@upc.edu), A. Mas (Laboratori d'Aplicacions Bioacústiques (Universitat Politècnica de Catalunya), avda. Rambla Exposició s/n, 08800 Vilanova i la Geltrú, Spain, alex.mas@lab.upc.edu), A. Roma (Laboratori d'Aplicacions Bioacústiques (Universitat Politècnica de Catalunya), avda. Rambla Exposició s/n, 08800 Vilanova i la Geltrú, Spain, agnes.roma@lab.upc.edu), J.v. Castell (Laboratori d'Aplicacions Bioacústiques (Universitat Politècnica de Catalunya), avda. Rambla Exposició s/n, 08800 Vilanova i la Geltrú, Spain, joan.v.castell@lab.upc.edu), Maria Morell (Laboratori d'Aplicacions Bioacústiques (Universitat Politècnica de Catalunya), avda. Rambla Exposició s/n, 08800 Vilanova i la Geltrú, Spain, maria.morell@lab.upc.edu), M. Solè (Laboratori d'Aplicacions Bioacústiques (Universitat Politècnica de Catalunya), avda. Rambla Exposició s/n, 08800 Vilanova i la Geltrú, Spain, marta.sole@lab.upc.edu), J.f. Rolin (IFREMER, Centre de Brest, BP 70, 29280 Plouzane, France, jean.francois.rolin@ifremer.fr), Roland Person (IFREMER, Centre de Brest, BP 70, 29280 Plouzane, France, roland.person@ifremer.fr)

ESONET is a European Network of Excellence (NoE) associating 50 partners (research centres, universities, industrials and SMEs) from 14 countries: France, Germany, Italy, UK, Spain, Portugal, Greece, Belgium, Ireland, the Netherlands, Norway, Sweden, Bulgaria, and Turkey. More than 300 scientists and engineers will participate to its activities. The aim of the ESONET NoE is the lasting integration of European research on deep-sea multidisciplinary observatories. ESONET is particularly sensitive on the effects of noise on marine organisms. Because our knowledge is still quite limited, ESONET is developing a Demonstration Mission, called LIDO, Listening to the Deep-Ocean Environment, a research program that will help establishing a scientific base to allow (1) the real-time automatic identification and classification of nonbiological and biological sounds, (2) the monitoring of marine organisms and population dynamics, (3) the assessment and control of the long term effects of anthropogenic sources on marine organisms, (4) the education of the public, end-users and the administration, and (5) the approval of ethical guidelines and procedures. This latter point constitutes a major criterion to award the ESONET LABEL to the observatories. This paper presents the data management architecture and the RT analysis processes that will be carried out at the observatories.

5aABb17. The acoustic role of supralaryngeal air sacs. Bart De Boer (Spuistraat 210, 1012VT Amsterdam, Netherlands, b.g.deboer@uva.nl)

This paper investigates the acoustic effect of supralaryngeal air sacs on the range of sounds that a given vocal tract can generate. Humans do not have supralaryngeal air sacs, whereas apes (chimpanzees, orangutans and gorillas) do. It has also been found that the anatomy of the hyoid bone of Neanderthals (the Kebara hyoid) is consistent with the absence of air sacs, while that of Australopithecines (the Dikika baby) is consistent with their presence. As an important difference between humans and all other primates is that humans speak, it has been suggested that the loss of air sacs has something to do with the evolution of speech. Here an articulatory model is used to compare the acoustic properties of a humanlike vocal tract with and without a chimpanzeelike air sac. The articulatory model is based on the Mermelstein model, and the air sac is a simplified model of a side branch with the approximate size of a chimpanzee air sac. It is found that the air sac reduces the range of articulations, and decreases the average formant 
frequency. This would provide an explanation for the presence of air sacs in apes (size exaggeration) and their disappearance in humans (increased articulatory range).

5aABb18. Quantitative assessment of bone properties during defect healing in an animal defect model after augmentation with different bone graft materials using scanning acoustic microscopy. Mathias Schulz (ZHBO Universitätsklinikum Halle, Dept. of Traumatology and Reconstructive Surgery, Ernst-Grube-Str. 40, 06120 Halle, Germany, mathias.schulz@medizin.uni-halle.de), Kay Raum (Martin Luther University of Halle-Wittenberg, Dept. of Orthopedics, Q-BAM Group, Magdeburger Str. 22, 06097 Halle, Germany, kay.raum@medizin.uni-halle.de), Joerg Brandt (Martin Luther University of Halle-Wittenberg, Dept. of Orthopedics, Q-BAM Group, Magdeburger Str. 22, 06097 Halle, Germany, joerg.brandt@medizin.uni-halle.de), Kay Brehme (ZHBO Universitätsklinikum Halle, Dept. of Traumatology and Reconstructive Surgery, Ernst-Grube-Str. 40, 06120 Halle, Germany, kay.brehme@medizin.uni-halle de)

The aim of this study was to evaluate the dynamic process of bone healing and bone remodeling in an animal defect model. A nanocrystalline hydroxyapatite in an aqueous suspension paste and mixed with either autogenous or allogenic bone was implanted. The investigation was performed using quantitative acoustic microscopy at a frequency of $50 \mathrm{MHz}$. 4-mm diameter defects were prepared on each femur at distal metaphysis in 60 white New Zealand rabbits. The animals were sacrificed after 2, 4, 6, 8, or 12 weeks. The influences on the acoustic impedance values only in the newly formed bone were analyzed by two-factor analysis of variance and post-hoc multiple comparison tests. Moreover, the kinetics of bone stiffening was evaluated by fitting the impedance data to an exponential growth model. In all treatment groups the impedance increased with healing time. Significant differences between the treatment groups were observed 4,6 , and 8 weeks after treatment $(\mathrm{p}<0.05)$. The experimental results agreed with the exponential growth model with coefficients of correlation (R2) between 0.6 and 0.8 . Nanocrystalline hydroxyapatite paste in combination with autogenous bone was found to be superior to the other evaluated treatment strategies.

5aABb19. An opportunistic passive acoustics study of the spatial and temporal distribution and vocal behavior of Blainville's beaked whale ("Mesoplodon densirostris") in the presence of mid-frequency active sonar. David Moretti (NAVSEA, Newport Undersea Warfare Center, Newport, RI RI 02841, USA, MorettiDJ@npt.nuwc.navy.mil), Ronald P. Morrissey (Naval Undersea Warfare Center Division Newport, 1176 Howell Street, Bldg 1351, 2nd Floor, Newport, RI 02841, USA, morrisseyrp@npt.nuwc.navy.mil), Nancy A. Dimarzio (Naval Undersea Warfare Center Division Newport, 1176 Howell Street, Bldg 1351, 2nd Floor, Newport, RI 02841, USA, dimarziona@npt.nuwc.navy.mil), Jessica Ward (NAVSEA, Newport Undersea Warfare Center, Newport, RI RI
02841, USA, wardja@npt.nuwc.navy.mil),Susan Jarvis(Naval Undersea Warfare Center Division Newport, 1176 Howell Street, Bldg 1351, 2nd Floor, Newport, RI 02841, USA, Sjarvis@wpi.edu), Elena McCarthy (Naval Undersea Warfare Center Division Newport, 1176 Howell Street, Bldg 1351, 2nd Floor, Newport, RI 02841, USA, mccarthy@nurc .nato.int), Annamaria Izzi (Naval Undersea Warfare Center Division Newport, 1176 Howell Street, Bldg 1351, 2nd Floor, Newport, RI 02841, USA, izzia.ctrmccarthy@nurc.nato.int)

The effect of mid-frequency active sonar, has increasingly become an issue with navies worldwide. The U.S. navy ranges have been used to develop passive acoustic algorithms and tools to detect, classify, and localize marine mammal vocalizations which have been applied to an opportunistic passive acoustic study of Blainville's beaked whales. Based on Woods Hole Oceanographic Digital Tags (Dtags), these animals are known to produce echo-location clicks only during deep foraging dives. Using passive acoustics detection of vocalizations, foraging groups of animals were isolated and the duration of vocalizations was used as a measure of foraging behavior. The animals' vocal behavior and spatial and temporal distribution were characterized during periods with no active sonar on range. These results are compared to those derived from opportunistic data obtained during multiship active mid-frequency sonar operations.

$5 a A B b 20$. Testing the acoustic tolerance of harbour porpoise hearing for impulsive sounds. Klaus Lucke (FTZ Westkueste/University of Kiel, Hafentoern 1, 25761 Buesum, Germany, lucke@ftz-west.uni-kiel.de), Paul A. Lepper (Loughborough University, Electronic \& Electrical Engineering, LE113TU Leicestershire, UK, p.a.lepper@lboro.ac.uk), Marie-Anne Blanchet (Fjord\&Bælt / University of Southern Denmark, Margrethes Plads 1, 5300 Kerteminde, Denmark, marie@ @jord-baelt.dk), Ursula Siebert (FTZ Westkueste / University of Kiel, Hafentoern 1, 25761 Buesum, Germany, ursula.siebert@ftz-west.uni-kiel.de)

The planned construction of offshore wind turbines in the North and Baltic Seas involves the emission of high numbers of intense impulsive sounds when the foundations of the turbines are being driven into the ground by pile driving. Based on information from other odontocete cetaceans it can be assumed that the source levels which will on average exceed $225 \mathrm{~dB}$ re $1 \mu \mathrm{Pa}$ pose a risk at least for temporary threshold shift (TTS) on harbour porpoises which inhabit these waters. In order to base the definition of noise exposure criteria on information on the tolerance of the hearing of this species a TTS study was conducted on one of the harbour porpoises held at the Fjord and Baelt in Kerteminde, Denmark. The hearing data were collected by using the AEP method. An airgun was chosen as sound source for the fatiguing sound stimulus to simulate the impulsive sounds at sufficiently high levels. This study comprises the testing of the animals normal hearing sensitivity and subsequent repetitions of these tests after an exposure to single impulsive sounds from the airgun at increasing levels. The baseline hearing data, thresholds for behavioural reactions and the resulting TTS levels will be presented. 


\title{
Session 5aAO
}

\section{Acoustical Oceanography and ECUA: General Topics in Acoustical Oceanography II}

\author{
Andone Lavery, Cochair \\ Woods Hole Oceanographic Institution, Applied Ocean Physics \& Engineering Department, 98 Water Street, MS \#11, \\ Woods Hole, MA 02543, USA \\ James H. Miller, Cochair \\ University of Rhode Island, Department of Ocean Engineering, Narragansett Bay Campus, Narragansett, RI 02882, USA
}

\section{Contributed Papers}

11:00

5aA01. Analysis of Munk waveguide normal modes based on the Rayleigh-Schrödinger perturbation and adiabatic invariant theories. Luiz Guimarães (Dept. Física Nuclear, Instituto de Física, Universidade Federal do Rio de Janeiro, Cx Postal 68528, 21945-970 Rio de Janeiro, Brazil, LULA@IF.UFRJ.BR)

This work concerns to obtain accurate explicit formulas related to discrete spectrum of the Munk deepwater acoustic wave guide problem. To this end, based on the Rayleigh-Schrödinger perturbation theory, we developed analytic results related to the discrete values of the radial wave numbers as well as to the eigen pressure felds. We compare these previous results with JWKB ones. In addition, based on invariant adiabatic theory to wave equation $[1,2]$, we tried to improve the accuracy of the JWKB calculation applying Olver's uniform asymptotic expansion (UAE) theory for the solution of the second-order differential equation with two turning points [3-5]. Comparing these three above particular frameworks, we conclude that UAE theory is accurate and it well describes the Munk wave guide normal modes. [1] L. M. Brekhovskikh and Yu. P. Lysanov, Fundamentals of Ocean Acoustics, Spinger, NY, 2001. [2] B. G. Katsnelson and V. G. Petnikov, Shallow Water Acoustics. Spinger, UK, 2002. [3] F. W. J. Olver, Asymptotics and special functions, Academic Press, London, 1974. [4] L. G. Guimarães and H. M. Nussenzveig, J. Mod. Optic., 41, 625 (1994). [5] P. C. G. de Moraes and L. G. Guimarães, JQSRT, 74757 (2002).

\section{$11: 20$}

5aA02. High-resolution population density imaging of random scatterers through cross-spectral coherence in matched filter variance. Mark Andrews (Northeastern University, 302 Stearns Center, Rm 311, 360 Huntington Ave, Boston, MA 02115, USA, Andrews.mar@ neu.edu), Zheng Gong (Northeastern University, 302 Stearns Center, Rm 311, 360 Huntington Ave, Boston, MA 02115, USA, zgong@ece.neu.edu), Daniel Cocuzzo (Northeastern University, 302 Stearns Center, Rm 311, 360 Huntington Ave, Boston, MA 02115, USA, dcocuzzo@ece.neu.edu), Purnima Ratilal (Northeastern University, 302 Stearns Center, Rm 311, 360 Huntington Ave, Boston, MA 02115, USA, purnima@ece.neu.edu)

The matched filter enables imaging with high spatial resolution and high signal-to-noise ratio by coherent correlation with the expected field from what is assumed to be a discrete scatterer. In many imaging systems, however, returns from large numbers of scatterers are received together and the coherent or expected field vanishes. This is the case when imaging schools of fish, other groups of marine life, or other diffuse scatterers in sonar or ultrasound applications. Here we show that despite the absence of an expected field, cross spectral coherence in the matched filter variance retains a pulse compression property that enables high-resolution imaging of scatterer population density. Both analytic and numerical models are developed for active imaging systems. We show the conditions for when the coherent intensity can be neglected. The model is implemented for several scenarios where single scattering dominates and also for cases where multiple scattering is important. It can applied to imaging in both free space and waveguide environments.

\section{1:40}

5aA03. Using angular dependence of multibeam echo features in seabed classification. Zbigniew Lubniewski (Gdansk University of Technology, Department of Geoinformatics, Narutowicza 11/12, 80-952 Gdansk, Poland, lubniew@eti.pg.gda.pl), Andrzej Chybicki (Gdansk University of Technology, Department of Geoinformatics, Narutowicza 11/12, 80-952 Gdansk, Poland, andrzej.chybicki@eti.pg.gda.pl)

The approach to seabed classification based on processing multibeam sonar echoes is presented. The multibeam sonars, besides their well verified and widely used applications like high resolution bathymetry measurements or underwater object imaging, are also the promising tool in seafloor identification and classification, having several advantages over conventional single beam echosounders. The proposed seabed classification method assumes calculation of a set of parameters of an echo envelope, similarly as in single beam classification. These parameters include echo energy, echo length, statistical moments of echo energy and the set of echo shape descriptors. They are extracted for each consecutive beam allowing the estimation of their dependence on seafloor incident angle. The characteristic features of this dependence are described quantitatively and constitute the input information for an automatic supervised seabed classifier. The results of the simple classification procedure applied for multibeam data records acquired from several bottom types in Gdańsk Bay region are presented and discussed.

\section{2:00}

5aAO4. Applications of compression techniques for reducing the size of multibeam sonar records. Andrzej Chybicki (Gdansk University of Technology, Department of Geoinformatics, Narutowicza 11/12, 80-952 Gdansk, Poland, andrzej.chybicki@eti.pg.gda.pl), Marek Moszynski (Gdansk University of Technology, Department of Geoinformatics, Narutowicza 11/12, 80-952 Gdansk, Poland, marmo@eti.pg.gda.pl)

High efficiency of multibeam sonar system (MBS) hardware due to operational requirements (i.e., high frequency, high ping rate, and high resolution of collected data) results in very large volumes of datasets stored on local hard drives of operator's station. In this context, the process of archiving of such warehouse of data collected in previous surveys becomes crucial problem. The paper investigates various lossy and lossless compression methods that can be applied to multibeam sonar data to reduce the size of acquired files without loosing relevant information. The specific character of MBS data allows applying various signal, image, and video compression methods to achieve better results than when using standard ones. Various techniques of reordering the data were analysed to achieve best possible compression ratio. 


\title{
Session 5aBBa
}

\section{Biomedical Ultrasound/Bioresponse to Vibration and Engineering Acoustics: Transducers for Medical Imaging and Therapy I}

\author{
Jeffrey A. Ketterling, Cochair \\ Frederic L. Lizzi Center for Biomedical Engineering, Riverside Research Institute, 156 William St., New York, NY 10038, USA \\ Marc Lethiecq, Cochair \\ LUSSI, 10 Bd Tonellé, Tours, 37032, France
}

Invited Papers

8:00

\begin{abstract}
5aBBa1. Designing advanced piezoelectric ceramics for novel ultrasonic applications. Erling Ringgaard (Ferroperm Piezoceramics A/S, Hejreskovvej 18A, 3490 Kvistgaard, Denmark, er@ferroperm.net), Wanda W. Wolny (Ferroperm Piezoceramics A/S, Hejreskovvej 18A, 3490 Kvistgaard, Denmark, ww@ferroperm.net), Torsten Bove (Ferroperm Piezoceramics A/S, Hejreskovvej 18A, 3490 Kvistgaard, Denmark, tb@ferroperm.net)

As new applications of medical ultrasound continue to emerge and the development of electronics continues, there is an increased interest in specialised piezoelectric ceramics with optimised properties. With the present level of knowledge about piezoceramics it is possible to design materials combining selected characteristics - although of course the laws of physics prevent changing certain properties independently. The main parameters in the design process are composition modification and controlled porosity. One of the new types of materials developed is especially intended for high-intensity focused ultrasound (HIFU) applications, where the ability to continuously generate high acoustic power should be combined with specifications for electrical impedance matching. Since the first requirement calls for very low dielectric and mechanical losses (hard doping), whereas for typical applications the second one calls for a high permittivity (soft material), a new combination was needed. The new PZT-based materials Ferroperm Pz52 and Pz54 fulfil these requirements and are already being extensively used for HIFU applications. Another example of new materials is the commercial family of porous PZT. The introduction of porosity affects all properties, and by optimising composition and porosity level it is possible to design materials that can replace lead metaniobate-based ceramics for a number of applications.
\end{abstract}

$8: 20$

5aBBa2. Dual-mode ultrasound array (DMUA) systems for noninvasive surgery. John Ballard (University of Minnesota, 200 Union St SE, Rm 4-174 EECS Bldg, Minneapolis, MN 55455, USA, ball250@umn.edu), Yayun Wan (University of Minnesota, 200 Union St SE, Rm 4-174 EECS Bldg, Minneapolis, MN 55455, USA, wanx0028@umn.edu), Emad S. Ebbini (University of Minnesota, 200 Union St SE, Rm 4-174 EECS Bldg, Minneapolis, MN 55455, USA, emad@umn.edu)

Advances in microelectronics and piezocomposite transducer technology have made it possible to design, fabricate, and test therapeutic arrays with imaging capabilities suitable for image-guided noninvasive surgery. Prototype DMUAs have been recently tested and were shown to be capable of generating therapeutic HIFU beams suitable for tissue ablation while intermittently imaging the target volume before, during, and after lesion formation. An additional advantage of imaging with DMUAs is the potential for identifying critical regions in the treatment field for targeting or avoidance by the HIFU beam. We have developed an image-based refocusing algorithm that allows for maximizing the power deposition at the location of HIFU focus while minimizing the power deposition at critical targets to be avoided. Grayscale images obtained using the DMUA are used to define the coordinate of the target and the grid points in the treatment region. A major application of this capability is the targeting of liver tumors by large-aperture arrays in the presence of the rib cage. In this paper, we present experimental validation of this algorithm in vitro. Quantitative analysis of the improvements in the quality of the HIFU beam at the target will be presented and discussed.

\section{$8: 40$}

5aBBa3. High power sources for ultrasound thermal therapy and shock wave lithotripsy. Neil Owen (INSERM, U556, 151 Cours Albert Thomas, 69003 Lyon, France, Neil.Owen@lyon.inserm.fr), Dominique Cathignol (INSERM, U556, 151 Cours Albert Thomas, 69003 Lyon, France, cathignol@lyon.inserm.fr), David Melodelima (INSERM, U556, 151 Cours Albert Thomas, 69003 Lyon, France, melodelima@lyon.inserm.fr), Alain Birer (INSERM, U556, 151 Cours Albert Thomas, 69003 Lyon, France, birer@lyon .inserm.fr), Jean Yves Chapelon (INSERM, U556, 151 Cours Albert Thomas, 69003 Lyon, France, chapelon@lyon.inserm.fr), Cyril Lafon (INSERM, U556, 151 Cours Albert Thomas, 69003 Lyon, France, lafon@lyon.inserm.fr)

We present a summary of Inserm's experience with high power sources, which are necessary for ultrasound thermal therapy and lithotripsy. Moreover, generating high intensity pseudo-continuous waveforms or high pressure pulses imposes different constraints on the transducer materials, specifically heat and mechanical stress. For thermal therapy, miniature piezoceramic transducers were used for interstitial, intratumoral, and endoluminal applicators. These probes operated at surface intensities up to $50 \mathrm{~W} / \mathrm{cm}^{2}$ and generated elementary lesions in vivo within tens of seconds. Piezocomposite transducers were developed for large-aperture, highly-focused beams used in extracorporeal or intraoperative treatments. Focal intensities were $1000 \mathrm{~W} / \mathrm{cm}^{2}$ or higher and up to 256 elements were utilized. Miniaturized piezocomposite transducers are currently being developed for dual-mode imaging and therapy. For lithotripsy, piezoelec- 
tric shock wave generators were developed as alternatives to electrohydraulic or electromagnetic generators. Using piezocomposite materials and a novel prestraining method increased transducer surface pressure compared to a multielement piezoceramic design, and therefore halved the aperture diameter. In in vitro tests, plaster kidney stone models were comminuted with $\sim 200$ shock waves, a number comparable to values published for electrohydraulic generators, the current "gold" standard. This work contributes to the advancement of transducer performance in therapeutic ultrasound. [Supported by Inserm Post-doctoral Fellowship.]

\section{9:00}

5aBBa4. An eight-element annular array for image-guided high intensity focused ultrasound therapy. Peter Kaczkowski (Center for Industrial and Medical Ultrasound, Applied Physics Lab., University of Washington, 1013 NE 40th St., Seattle, WA 98105, USA, peter@apl.washington.edu), Bryan Cunitz (Center for Industrial and Medical Ultrasound, Applied Physics Lab., University of Washington, 1013 NE 40th St., Seattle, WA 98105, USA, bwc@apl.washington.edu), George Keilman (Sonic Concepts, 11807 North Creek Parkway South - Suite 111, Bothell, WA 98011, USA, gkeilman@sonicconcepts.com)

The investigation of high intensity focused ultrasound (HIFU) as a tool for noninvasive thermally ablative therapy has required deeper understanding of the relative roles of nonlinear mechanisms involved in heat deposition. Attempts at quantifying the dose response to particular exposure conditions in vitro are complicated by the interplay of several mechanisms. These include microbubble cavitation, nonlinear acoustic propagation and attenuation, dependence of tissue parameters on temperature and temperature history, and formation and evolution of vapor bubbles due to boiling. One immediately evident consequence of such effects is distortion of coagulative lesion shape and size, colloquially evolving from "cigars" to "tadpoles". Developing a quantitative understanding of the relative roles of relevant nonlinear mechanisms is not straightforward, yet is desirable for design of algorithms for therapy planning and real time monitoring using ultrasound. A historical perspective of research toward this end will be presented along with a recommendation for suitable terminology for the various physical acoustic regimes encountered in HIFU therapy. [Work supported by Army MRMC, NIH DK43881, NSBRI SMS00402, and RFBR.]

\section{9:20}

5aBBa5. Transducers for reduced aberration in HIFU by nonlinear harmonic focusing. Gregory Clement (Harvard Medical School, Focused Ultrasound Lab - BWH Radiology, 221 Longwood Ave RM 521, Boston, MA 02115, USA, gclement@hms.harvard.edu), Jason White (Harvard Medical School, Focused Ultrasound Lab - BWH Radiology, 221 Longwood Ave RM 521, Boston, MA 02115, USA, white@bwh.harvard.edu)

We have been investigating a low-frequency transducer design that promotes the divergence of the low frequency beam while promoting the focusing of nonlinear-induced higher harmonic frequencies. In this manner, a low frequency - and thus less absorbed and less distorted - beam is passed through the near field, peaking before the buildup of higher-frequency components. The high frequency components are then focused by means of the wavefront design. Using this approach, we expect reduced distortion of the ultrasound focus, while allowing a beam that uses mechanical or thermal effects for ablation, as opposed to cavitation. In our preliminary study we have been working to establish feasibility of the approach. Two source transducers $(0.272 \mathrm{MHz}$ and $0.272 \mathrm{MHz})$ with identical geometries were used to propagate through ex vivo human skull, representing strong nearfield aberration. Transmitted fields were scanned after transsskull propagation with a pvdf needle hydrophone. Reconstructions were performed at the fundamental frequencies for both scans, and for the $0.272 \mathrm{MHz}$ transducer, an additional reconstruction was performed at its second harmonic (by design $0.544 \mathrm{MHz}$ ). The harmonic signal was observed to be significantly less distorted than the same frequency directly propagated from the transducer. A numeric study for optimizing the method will also be presented.

9:40-11:00 Posters

Lecture sessions will recess for presentation of poster papers on various topics in acoustics. See poster sessions for topics and abstracts.

\section{Contributed Papers}

\section{1:00}

5aBBa6. Combined two frequency array for optoacoustics and acoustics. Kirsten Maass (Fraunhofer IBMT, Ensheimerstrasse 48, 66386 Sankt Ingbert, Germany, kirsten.maass@ibmt.fhg.de), Christian Degel (Fraunhofer IBMT, Ensheimer Strasse 48, 66386 Sankt Ingbert, Germany, christian.degel@ibmt.fraunhofer.de), Marc Fournelle (Fraunhofer IBMT, Ensheimer Strasse 48, 66386 Sankt Ingbert, Germany, marc.fournelle@ibmt.fhg.de), Heinrich Fonfara (Fraunhofer IBMT, Ensheimerstrasse 48, 66386 Sankt Ingbert, Germany, heinrich.fonfara@ibmt fhg.de), Robert M. Lemor (Fraunhofer IBMT, Ensheimer Strasse 48, 66386 Sankt Ingbert, Germany, robert.lemor@ibmt.fhg.de)

A combined transducer for optoacoustics and b-mode ultrasound has to be built according to the specific requirements of both modalities. In order to combine the two tasks in one transducer, we have designed a probe consisting of a PZT array and a PVDF array in a sandwich assembly. The $20 \mathrm{MHz}$ PZT array is a linear array with 128 elements bearing a $150 \mu \mathrm{m}$ pitch. It operates as a high resolution transmit and receive array for the visualization of geometrical structures in biological tissue. A thin piezoelectric polymer film (PVDF) that is structured as receiving array is superposed to the PZT array. The PVDF array allows to detect the small optoacoustic pressure transients resulting of laser irradiation of small biological structures. Its acoustic impedance of approximately 4-4,5 MRayl allows to use it in a double function as optoacoustic receiving array and matching layer for the PZT array. The combined array is driven by a custom made multichannel read-out system supporting sampling frequencies of $80 \mathrm{MHz}$ and combined imaging with b-mode and optoacoustics. This combined probe integrates the benefits of a high resolution PZT array and gives access to the specificity of optoacoustic signal generation.

\section{1:20}

5aBBa7. Dual-frequency driving transducer for ultrasonic echography. Iwaki Akiyama (Shonan Institute of Technology, 1-1-25 Tsujidonishikaigan, 251-8511 Fujisawa, Japan, akiyama@iwaki.org), Natsuki Yoshizumi (Shonan Institute of Technology, 1-1-25 Tsujido-nishikaigan, 251-8511 Fujisawa, Japan, yoshizumi@pari.go.jp), Shigemi Saito (Tokai University, 3-20-1 Orito, Shimizu-ku, 424-8610 Shizuoka, Japan, ssaito@scc.u-tokai.ac.jp), Daisuke Koyama (Tokyo Institute of Technology, 4259 Nagatsuda, 226-8503 Yokohama, Japan, dkoyama@sonic 
.pi.titech.ac.j),Kentarou Nakamura(Tokyo Institute of Technology, $4259 \mathrm{Na}$ gatsuda, 226-8503 Yokohama, Japan, knakamur@sonic.pi.titech.ac.jp)

We propose a novel ultrasonic imaging technique by transmitting ultrasonic pulse of dual-frequency and receiving multifrequency echoes from the biological tissues. When the ultrasonic pulse of two frequencies is transmitted from a transducer, the secondary waves are generated during the nonlinear propagation through the biological media. A choice of $\mathrm{f}_{0}$ and $4 \mathrm{f}_{0}$ as the dual-frequency results in the generation of $2 \mathrm{f}_{0}, 3 \mathrm{f}_{0}$, and $5 \mathrm{f}_{0}$ components as the secondary waves. Multifrequency echoes are capable of improving the image quality by reducing the speckle noise. We have developed the following annular type transducer. The PZT disk of $7 \mathrm{~mm}$ in diameter is coaxially arranged in the PZT ring of $9 \mathrm{~mm}$ in inner diameter and $17 \mathrm{~mm}$ in outer diameter. The ring and circular transducers transmit the pulses of 2 and 8 $\mathrm{MHz}$, respectively. It was confirmed that this transducer formed the ultrasonic beams of 4,6 , and $10 \mathrm{MHz}$ as well as the beams of 2 and $8 \mathrm{MHz}$ in degassed water. Then the experiments of imaging the agar-gel phantom and pork meat were carried out by mechanical scanning. As a result, we also confirmed the improvement of image quality by reducing the speckle noise.

\section{1:40}

5aBBa8. Progress towards transducers and arrays for real-time high frequency biomedical ultrasound imaging. Sandy Cochran (Institute for Medical Science and Technology, University of Dundee, Wilson House, 1 Wurzburg Loan, DD2 1FD Dundee, UK, s.cochran@dundee.ac.uk), Jeff Bamber (Institute of Cancer Research, 15 Cotswold Road, Belmont, Sutton, SM2 5NG Surrey, UK, jeff.bamber@icr.ac.uk), Anne L Bernassau (Institute for Medical Science and Technology, University of Dundee, Wilson House, 1 Wurzburg Loan, DD2 1FD Dundee, UK, a.1.bernassau@dundee.ac.uk), Nigel Bush (Piezo Composite Transducers Ltd, Aberdeen Science Park, Balgownie Drive, Bridge of Don, AB22 8GU Aberdeen, UK, nigel.bush@icr.ac.uk), Tim W. Button (Functional Materials Group, IRC in Materials Processing, The University of Birmingham, Edgbaston, B15 2TT Birmingham, UK, t.w.button@bham.ac.uk), Christine E. Demore (Institute for Medical Science and Technology, University of Dundee, Wilson House, 1 Wurzburg Loan, DD2 1FD Dundee, UK, c.demore@dundee.ac.uk), Luis Garcia-Gancedo (Tritech International Ltd, Peregrine Road, Westhill Business Park, Westhill, AB32 6LJ Aberdeen, UK, 1.garciagancedo@bham.ac.uk), David Hutson (Institute for Medical Science and Technology, University of Dundee, Wilson House, 1 Wurzburg Loan, DD2 1FD Dundee, UK, d.hutson@dundee.ac.uk), Duncan P. Maclennan (University of Strathclyde, DTC in Medical Devices, Bioengineering Department, Wolfson Building, 106 Rottenrow, G4 0NW Glasgow, UK, duncan.p.maclennan@strath.ac.uk), Paul M. Maher (Tritech International Ltd, Peregrine Road, Westhill Business Park, Westhill, AB32 6LJ Aberdeen, UK, paulmichaelmaher@ hotmail.com), Carl Meggs (Tritech International Ltd, Peregrine Road, Westhill Business Park, Westhill, AB32 6LJ Aberdeen, UK, c.meggs@bham.ac.uk), Rhiannon A. Webster (Tritech International Ltd, Peregrine Road, Westhill Business Park, Westhill, AB32 6LJ Aberdeen, UK, raw160@bham.ac.uk)

As research into transducers and arrays for real-time high frequency biomedical ultrasound imaging continues, it is becoming increasingly clear that major applications exist for systems with significantly higher spatial resolution than those already available for human clinical imaging. A key area of research remains the design and fabrication of the transducer or array. It is now accepted that piezocomposite is the material of choice and composite design and conventional dice-and-fill fabrication techniques have been optimised to allow $40 \mathrm{MHz}$ operation, corresponding to wavelengths around $40 \mathrm{um}$. This expands the range of applications of piezocomposites but is still limited in terms of resolution of fine structures, for example at cellular leve and to explore harmonic imaging. In this paper, we report continuing progress in work on new design techniques and fabrication processes with the potential for fabricating arrays that operate up to $100 \mathrm{MHz}$. The netshape micromoulding fabrication technique for the ceramic within our composites is outlined, new single element transducer designs and array fabrication based on advanced surface finishing and photolithographic processes are described, and results are presented illustrating key performance data such as the point spread function, insertion loss and imaging of post mortem human tissue.

\section{2:00}

5aBBa9. Micromachined linear array with $100 \mathrm{MHz}$ center frequency. Eike C. Weiss (Fraunhofer IBMT, Ensheimer Strasse 48, 66386 Sankt Ingbert, Germany, eike.weiss@ibmt.fraunhofer.de), Anette Jakob (Fraunhofer IBMT, Ensheimerstrasse 48, 66386 Sankt Ingbert, Germany, anette.jakob @ibmt.fraunhofer.de), Steffen H. Tretbar (Fraunhofer IBMT, Ensheimerstrasse 48, 66386 Sankt Ingbert, Germany, steffen.tretbar@ibmt.fraunhofer .de), Werner Haberer (Fraunhofer IBMT, Ensheimerstrasse 48, 66386 Sankt Ingbert, Germany, werner.haberer@ibmt.fraunhofer.de), Thorsten Knoll (Fraunhofer IBMT, Ensheimerstrasse 48, 66386 Sankt Ingbert, Germany, thorsten.knoll@ibmt.fraunhofer.de), Frank Bauerfeld (Fraunhofer IBMT, Ensheimerstrasse 48, 66386 Sankt Ingbert, Germany, frank.bauerfeld @ibmt.fraunhofer.de), Joerg Herrmann (Fraunhofer IBMT, Ensheimerstrasse 48, 66386 Sankt Ingbert, Germany, joerg.herrmann@ibmt.fraunhofer .de), Robert M. Lemor (Fraunhofer IBMT, Ensheimer Strasse 48, 66386 Sankt Ingbert, Germany, robert.lemor@ibmt.fhg.de)

High-frequency ultrasound systems based on single element transducers in the frequency range of $50-120 \mathrm{MHz}$ and mechanical scanning of the transducer. Linear arrays with electrical scanning can be used to increase the speed of scanning and reduce the size of the transducers. We present a linear array combined with multiplexer and single channel electronics. Working at $100 \mathrm{MHz}$, lateral resolution better than $100 \mu \mathrm{m}$ is possible with an aperture of $1 \mathrm{~mm}^{2}$. The $100 \mathrm{MHz}$ array is based on silicon micro machining with a $\mathrm{ZnO}$ membrane as active material. The most important steps are the deposition of a $26 \mu \mathrm{m}$-thick $\mathrm{ZnO}$ thin film by magnetron sputtering and the anisotropic etching of the backside of the silicon wafer to fabricate the $\mathrm{ZnO}$ membrane. The individual elements of the transducer are defined by patterning a gold electrode with the desired array structure via photolithography and subsequently wet etching. Results from 32 element arrays with an 8 element 500 by $500 \mu \mathrm{m}$ aperture agree well with numerical simulations and the shifting of the aperture works as well. Due to the cost-effectiveness of the process, a large number of applications have come now into reach for high-frequency ultrasound imaging.

\section{$12: 20$}

5aBBa10. Lead magnesium niobate-lead titanate single crystal thick films on silicon substrate for high-frequency micromachined ultrasonic transducers. Jue Peng (Department of Applied Physics and Materials Research Centre, The Hong Kong Polytechnic University, Hunghom, Kowloon Hong Kong, China, aperica@inet.polyu.edu.hk), Chen Chao (Department of Applied Physics and Materials Research Centre, The Hong Kong Polytechnic University, Hunghom, Kowloon Hong Kong, China, rikeynes@ @inet .polyu.edu.hk), Jiyan Dai (Department of Applied Physics and Materials Research Centre, The Hong Kong Polytechnic University, Hunghom, Kowloon Hong Kong, China, apdaijy@inet.polyu.edu.hk), Helen L. W. Chan (Department of Applied Physics and Materials Research Centre, The Hong Kong Polytechnic University, Hunghom, Kowloon Hong Kong, China, apahlcha@inet.polyu.edu.hk), Haosu Luo (The Shanghai Institute of Ceramics, Chinese Academy of Sciences, 201800 Shanghai, China, hsluo @ mail.sic.ac.cn)

Some new clinical applications requiring ultrasonic frequencies higher than $30 \mathrm{MHz}$ are emerging, such as ophthalmological and dermatological imaging and intravascular imaging with probes mounted on catheter tips. High frequency ultrasonic transducer (HFUT) has thus been a growing research area in recent years. Most of the HFUTs reported are using piezoelectric PZT ceramic thin plates, $\mathrm{ZnO}$ films or PVDF films as the transducer materials. The PMN-PT ((1-x) $\left.\mathrm{Pb}\left(\mathrm{Mg}_{1 / 3} \mathrm{Nb}_{2 / 3}\right) \mathrm{O}_{3-\mathrm{x}} \mathrm{PbTiO}_{3}\right)$ single crystal, although possessing the highest piezoelectric coefficients, has seldom been used for this purpose because it is difficult to lap down the brittle single crystals to thin plates and handle them in conventional way of transducer fabrication. In this work, a novel high-frequency ultrasonic transducer structure is realized by using PMN-PT-on-silicon technology and silicon micromachining. A hybrid processing method involving wafer bonding, mechanical lapping and wet chemical thinning is successfully developed. PMN-PT thick films with thickness ranging from several to tens of microns have been demonstrated and they have properties comparable to those of PMN-PT bulk samples. A prototype high frequency ultrasonic transducer is being fabricated and characterized. Acknowledgement Financial support from the Innovation and Technology Fund (K-ZP21) is acknowledged. 


\title{
Session 5aBBb
}

\section{Biomedical Ultrasound/Bioresponse to Vibration: Ultrasonic Characterization of Bone III}

\author{
Keith A. Wear, Cochair \\ U.S. Food and Drug Administration, Center for Devices and Radiological Health, 10903 New Hampshire Ave, Bldg 62, \\ Rm 3108, Silver Spring, MD 20993, USA \\ Emmanuel Bossy, Cochair \\ Laboratoire Photons et Matière, ESPCI/CNRS, 10 rue Vauquelin, Paris Cedex 05, 75231, France
}

\section{Invited Paper}

\author{
$8: 20$
}

\begin{abstract}
5aBBb1. Microelastic imaging of mineralized tissues - Principles and applications in musculoskeletal research. Kay Raum (Martin Luther University of Halle-Wittenberg, Dept. of Orthopedics, Q-BAM Group, Magdeburger Str. 22, 06097 Halle, Germany, kay.raum@medizin.uni-halle.de)
\end{abstract}

\begin{abstract}
High frequency ultrasound has become one of the most powerful tools for microelastic characterization of hard biological materials. A major advantage is the possibility to map not only the microstructure, but also the heterogeneous anisotropic elasticity of mineralized tissues. Quantitative concepts for deriving anisotropic elastic parameters with frequencies from $50 \mathrm{MHz}$ to the $\mathrm{GHz}$ range will be presented. Due to the scalability ultrasound can be applied for large animal studies, e.g., to predict the mechanical stability in sheep tibiae after callus distraction, as well as for small animal models, e.g., to study the effects of genetic differences in inbred strain mice. Moreover, SAM data are suitable for numerical deformation or sound propagation analyses on "real- life" models. Such models are crucial for the development and validation of new non-invasive diagnostic tools dedicated to the prediction of an individual fracture risk. For example, it has been shown that the velocity of the first arriving signal - measured with the axial transmission technique at the radius - can be predicted by a nonlinear combination of fracture determining parameters, i.e., porosity, cortical thickness and tissue elasticity, assessed by $50-\mathrm{MHz}$ SAM. These findings may lead to the establishment of pathology specific treatment and regeneration monitoring strategies.
\end{abstract}

\section{Contributed Papers}

\section{8:40}

5aBBb2. Assessment of cortical bone density and anisotropy in human femur using ultrasound and x-ray. Dorian Cretin (Furuno Electric Co., Ltd, 9-52 Ashihara-cho, 662-8580 Nishinomiya, Japan, dorian.cretin@furuno.co.jp), Ryouichi Suetoshi (Furuno Electric Co., Ltd, 9-52 Ashihara-cho, 662-8580 Nishinomiya, Japan, ryohichi.suetoshi@furuno.co.jp), Atsushi Uodome (Furuno Electric Co., Ltd, 9-52 Ashihara-cho, 662-8580 Nishinomiya, Japan, atsushi.uodome@furuno.co.jp), Shinji Ogawa (Furuno Electric Co., Ltd, 9-52 Ashihara-cho, 662-8580 Nishinomiya, Japan, shinji.ogawa@furuno.co.jp), Sakaya Miyabe (Graduate School of Engineering, Osaka Univ., Division of Materials and Manufacturing Science, 2-1 Yamadaoka, Suita, 565-0871 Osaka, Japan, miyabe@mat.eng.osaka-u.ac.jp), Takayoshi Nakano (Graduate School of Engineering, Osaka Univ., Division of Materials and Manufacturing Science, 2-1 Yamadaoka, Suita, 565-0871 Osaka, Japan, nakano@mat.eng .osaka-u.ac.jp)

The purpose of this research is to investigate the contribution of the intrinsic properties of human bone to the ultrasound velocities in the axial, radial, and tangential directions. Samples of cortical bone were cut from 19 human medial femurs and polished to a rectangular parallelepiped shape. The orientation of apatite crystallites was assessed by microbeam x-ray diffraction, the density with a densitometer using the Archimedes' principle. Bone mineral density (BMD) was also measured with peripheral quantitative computed tomography (pQCT) and dual-x-ray absorptiometry (DXA). The speed of sound (SOS) for each three directions was measured with 3 $\mathrm{MHz}$ ultrasonic broadband transducers. Radial and tangential SOS demonstrated a strong correlation with density $(\mathrm{R}=0.83, \mathrm{p}<0.0001$ and $\mathrm{R}=0$ $.85, \mathrm{p}<0.0001$, respectively). In the axial direction, the correlation between density and SOS was moderate $(\mathrm{R}=0.59)$. However, a significant correlation was found by using density and apatite orientation of c-axis in a multiple regression analysis $(\mathrm{R}=0.85, \mathrm{p}<0.001)$. Axial SOS can be explained by contributions of both density and crystal orientation.

\section{9:00}

5aBBb3. Experimental determination of Young modulus and Poisson ratio in cortical bone tissue using high resolution scanning acoustic microscopy and nanoindentation. Fabienne Rupin (Université Paris 6, Laboratoire d'Imagerie Paramétrique, 15, rue de l'Ecole de Médecine, 75006 Paris, France, fabienne.rupin@lip.bhdc.jussieu.fr), Amena Saied (Université Paris 6, Laboratoire d'Imagerie Paramétrique, 15, rue de l'Ecole de Médecine, 75006 Paris, France, saied@lip.bhdc.jussieu.fr), Davy Dalmas (CNRS/Saint-Gobain, SVI Saint-Gobain Recherche, 93303 Aubervilliers Cedex, France, Davy.Dalmas@saint-gobain.com), Françoise Peyrin (Creatis, UMR CNRS 5515, INSERM U630, 69621 Villeurbanne Cedex, France, peyrin@esrf.fr), Sylvain Haupert (Université Paris 6, Laboratoire d'Imagerie Paramétrique, 15, rue de l'Ecole de Médecine, 75006 Paris, $\quad$ France, sylvain.haupert@lip.bhdc.jussieu.fr), Etienne Barthel (CNRS/Saint-Gobain, SVI Saint-Gobain Recherche, 93303 Aubervilliers Cedex, France, Etienne.Barthel@saint-gobain.com), Georges Boivin (Unité INSERM U831, Faculté de Médecine R. Laennec, 69372 Lyon Cedex 08, France, Georges.boivin@sante.univ-lyon1.fr), Pascal Laugier (Université Paris 6, Laboratoire d'Imagerie Paramétrique, 15, rue de l'Ecole de Médecine, 75006 Paris, France, laugier@lip.bhdc.jussieu.fr)

Nanoindentation allows measurements of local mechanical properties of bone tissue. Scanning acoustic microscopy (SAM) provides images related to bone density and elasticity. In both techniques, the estimation of Young modulus (E) relies on the accuracy of Poisson's ratio value $(\sigma)$. In cortical bone, $\sigma$ varies between 0.15 and 0.45 but, is classically set to 0.3 , resulting in an approximate value of $\mathrm{E}$. This study describes a new method combining SAM and nanoindentation techniques to locally evaluate $\sigma$ in human femo- 
ral cortex. A $200 \mathrm{MHz}$ SAM-based acoustic impedance $(8 \mu \mathrm{m}$ lateral resolution) was combined with synchrotron microtomography (to provide local bone mineral density) to map the distribution of near surface elastic modulus. Whereas, nanoindentation modulus was calculated on several osseous regions. Assuming the equalization rule, the intersection of both modulus curves versus $\sigma$ permits to accurately derive $\sigma$. The method was tested on aluminium, PMMA and polycarbonate samples of known $\sigma$ and provided experimental $\sigma$ values with a precision better than 3\%. In bone, $\sigma$ was $0.42 \pm 0.01$ corresponding to $\mathrm{E}=20 \pm 1 \mathrm{GPa}$. Our preliminary results indicate that combination of high-resolution SAM and nanoindentation may be relevant to accurately determine both Poisson ratio and Young modulus of bone tissue.

\section{9:20}

5aBBb4. Vibration spectroscopy and guided wave propagation data as indicators of structural and mechanical degradation of human bones. Erick Ogam (Laboratoire de Mécanique et d'Acoustique CNRS UPR-7051, 31, Chemin Joseph Aiguier, 13402 Marseille Cedex 20, France, ogam@1ma.cnrs-mrs.fr), Armand Wirgin (CNRS-Laboratoire de Mécanique et d'Acoustique, 31 Chemin Joseph Aiguier, 13402 Marseille, France, wirgin@1ma.cnrs-mrs.fr), Zine Fellah (CNRS-Laboratoire de Mécanique et d'Acoustique, 31 Chemin Joseph Aiguier, 13402 Marseille, France, fellah@1ma.cnrs-mrs.fr), Catherine Masson (INRETS - Laboratoire de Biomécanique Appliquée- UMRT 24, Faculté de Medecine, Bd. Pierre Dramard, 13916 Marseille, France, catherine.masson@inrets.fr), Philippe
Guillemain(Laboratoire de Mécanique et d'Acoustique CNRS UPR-7051, 31, Chemin Joseph Aiguier, 13402 Marseille Cedex 20, France, guillemain@lma.cnrs-mrs.fr), François Gabrielli (INRETS - Laboratoire de Biomécanique Appliquée- UMRT 24, Faculté de Medecine, Bd. Pierre Dramard, 13916 Marseille, France, francois.gabrielli@inrets.fr), Jean-Philippe Groby (DRE/L2S - UMR8506 CNRS/Supelec/Univ. Paris Sud 11, Département de Recherche en Electromagnétisme/Laboratoire des signaux et systèmes, 3 rue Joliot-Curie, 91192 Gif-sur-Yvette cedex, France, groby@1ss.supelec.fr), Robert Gilbert (University of Delaware, Department of Mathematics, 317 Ewing Hall, Newark, DE 19716, USA, gilbert @math.udel.edu)

Vibration spectroscopy and guided wave modes analysis of long bones are investigated as tools to assess bone quality or integrity. Two different methods of vibroacoustic experiments are undertaken to determine the health of dry human tibia bones. The first method involves vibratory modes of the bone, excited and measured by piezoelectric transducers and sensors respectively. The measured frequency response is validated using finite element simulation (FES), also used in the inverse problem for retrieval of the bone material properties. The principle of in-plane (IP) and out-of-plane (OP) mode splitting to determine the degree of the damage, that we developed in an earlier study, is applied to classify the bones according to their degree of damage. The second method involves the analysis of the guided wave phase velocities recovered using the reassigned Gabor spectrogram from signals measured along the diaphysis of the tibias. The frequency difference between the peaks of the split modes are then correlated to the phase velocities of the retrieved Lamb modes.

FRIDAY MORNING, 4 JULY 2008

P3-B, LEVEL 3, 9:40 TO 11:20 A.M.

\title{
Session 5aBBc
}

\section{Biomedical Ultrasound/Bioresponse to Vibration and Engineering Acoustics: Transducers for Medical Imaging and Therapy II (Poster Session)}

\author{
Jeffrey Ketterling, Cochair \\ Frederic L. Lizzi Center for Biomedical Engineering
}

Marc Lethiecq, Cochair

LUSSI

\begin{abstract}
All posters will be on display from 9:40 a.m. to 11:20 a.m. To allow contributors an opportunity to see other posters, contributors of odd-numbered papers will be at their posters from 9:40 a.m. to 10:30 a.m. and contributors of even-numbered papers will be at their posters from 10:30 a.m. to 11:20 a.m.
\end{abstract}

\section{Contributed Papers}

5aBBc1. High performance ultrasound arrays assessment through in vitro imaging performance. Mathieu Legros (Vermon SA, 180 rue Général Renault, 37000 Tours, France, m.legros@vermon.com), Guillaume Ferin (Vermon SA, 180 rue Général Renault, 37000 Tours, France, g.ferin@vermon.com), Leong Ratsimandresy (Vermon SA, 180 rue Général Renault, 37000 Tours, France, 1.ratsi@vermon.com), Rémi Dufait (Vermon SA, 180 rue Général Renault, 37000 Tours, France, r.duvait @ vermon.com)

Probes are well-known to be a capital element for ultrasound image quality. During design, many parameters can be tuned on the acoustic stack to optimise the electrical, electroacoustical and acoustical performance measurements. But the effects on image quality of these performances are not so well identified. To overcome such a limitation, we developed a quan- titative method for image quality assessment. A set of algorithms was developed to assess in vitro images. The goal of this investigation is to link the electroacoustical, acoustical performances and transducer parameters to the imaging performance. Ultrasound test objects were used to quantify the ultrasound images. The acquisition was carried out on a commercial scanner and imaging parameters were set constant in order to benchmark the probes in the same environment. From B-mode images, key parameters such as axial and lateral resolutions, contrast, statistic or metric parameters, and signal to noise ratio are established. Data from transducers, exhibiting different trade-offs on their performances (bandwidth, bandwidth shape, center frequency, elementary directivity) were characterized. Using the algorithms developed, all identified image properties were analysed with regard to these performances. The impact of each electroacoustical parameter on image quality have been identified and discussed. 
5aBBc2. Modeling of different transducer configurations with combined pseudospectral and finite-difference time-domain methods. Erwan Filoux (LUSSI, 10 Bd Tonellé, 37032 Tours, France, erwan.filoux@etu.univ-tours.fr), Franck Levassort (LUSSI, 10 Bd Tonellé, 37032 Tours, France, franck.levassort@univ-tours.fr), Samuel Callé (LUSSI, 10 Bd Tonellé, 37032 Tours, France, calle_s@med .univ-tours.fr), Marc Lethiecq (LUSSI, 10 Bd Tonellé, 37032 Tours, France, lethiecq@univ-tours.fr)

The numerical simulation of acoustic waves propagating in inhomogeneous media is often achieved using pseudospectral (PS) algorithms, which require few nodes per wavelength to converge, while complex piezoelectric structures are simulated with finite-difference (FD) or finite-element (FE) methods. A combination of the PS and FD algorithms, retaining their advantages, is presented in order to simulate the behavior of various piezoelectric transducers used in ultrasonic imaging with one single model. The theory is exposed and the algorithm is applied to simulate PZT resonators flooded into water. Perfectly matched layers are developed to absorb the mechanical waves at the borders of the computational domain, and space-shifted grids are used to reduce Gibbs phenomenon. The electrical impedance and various physical parameters (displacements, electric potentials) are calculated. Different high frequency transducer configurations have been modeled. In the case of a simple two-dimensionnal plate, described in Cartesian coordinates, the results are satisfactorily compared to those obtained with a commercial FE software. Then, simulations of an axisymmetrical single-element transducer are favorably compared to $\mathrm{FE}$ simulations and experimental measurements. The hybrid algorithm is also used to calculate the large radiation pattern of an annular array with little time-processing, which illustrates the efficiency of the method.

FRIDAY MORNING, 4 JULY 2008

P3-B, LEVEL 3, 9:40 TO 11:20 A.M.

\title{
Session 5aBBd
}

\section{Biomedical Ultrasound/Bioresponse to Vibration: General Topics in Biomedical Ultrasound/Bioresponse to Vibration I (Poster Session)}

\author{
Michael Bailey, Cochair \\ Center for Industrial and Medical Ultrasound, Applied Physics Lab. \\ Oleg Sapozhnikov, Cochair \\ Center for Industrial and Medical Ultrasound, Applied Physics Lab.
}

\begin{abstract}
All posters will be on display from 9:40 a.m. to 11:20 a.m. To allow contributors an opportunity to see other posters, contributors of odd-numbered papers will be at their posters from 9:40 a.m. to 10:30 a.m. and contributors of even-numbered papers will be at their posters from 10:30 a.m. to 11:20 a.m.
\end{abstract}

\section{Contributed Papers}

5aBBd1. $1 \mathrm{kHz}$ sound stimulates nitric oxide and prostaglandin E2 production by rat mesenchymal stem cells. Dorothée Bossis (Université Paris 6, Laboratoire d'Imagerie Paramétrique, 15, rue de l'Ecole de Médecine, 75006 Paris, France, dorothee.bossis@lip.bhdc.jussieu.fr), Frederic Padilla (Université Paris 6, Laboratoire d'Imagerie Paramétrique, 15, rue de l'Ecole de Médecine, 75006 Paris, France, Frederic.Padilla @ lip.bhdc.jussieu.fr), Bertrand David (Faculté de Médecine Paris 7- Denis Diderot, Laboratoire Biomécanique et Biomatériaux Ostéo-Articulaires B2OA, 10 avenue de Verdun, 75010 Paris, France, bertrand.david @ paris7.jussieu.fr), Hervé Petite (Faculté de Médecine Paris 7- Denis Diderot, Laboratoire Biomécanique et Biomatériaux Ostéo-Articulaires B2OA, 10 avenue de Verdun, 75010 Paris, France, hpetite@infobiogen .fr), Pascal Laugier (Université Paris 6, Laboratoire d'Imagerie Paramétrique, 15, rue de l'Ecole de Médecine, 75006 Paris, France, laugier @lip.bhdc.jussieu.fr)

Pulsed ultrasound has become a common therapy for delayed unions and non unions. Currently available clinical devices use modulated $(1 \mathrm{kHz}) 1.5$ $\mathrm{MHz}$ ultrasound stimulation that produces a $1 \mathrm{kHz}$ radiation force vibration. It was hypothesized that this radiation force is responsible for the stimulatory effect on bone formation. Therefore, we have investigated the effect of $1 \mathrm{kHz}$ stimulation in rat mesenchymal stem cells (MSCs) by measuring the production of on nitric oxide (NO) and prostaglandin E2 (PGE2). Continuous wave $1 \mathrm{kHz}$ low power sound was applied to cultured rat MSCs for 20 min. NO concentration was determined by amperometry and PGE2 was assayed by ELISA in the supernatant. The stimulation produced a significant increase in both NO and PGE2 concentration compared to controls. We conclude that rat MSCs respond to the mechanical stress produced by $1 \mathrm{kHz}$ continuous wave as evidenced by the increase in NO and PGE2 production. This study provides evidence for the action of $1 \mathrm{kHz}$ stimulation on bone cells.

5aBBd2. Accurate ultrasonic measurement of two-dimensional displacement of heart wall motion for estimation of myocardial regional strain rate. Yasunori Honjo (Department of Electronic Engineering, Graduate School of Engineering, Tohoku University, 6-6-05 Aramaki-azaAoba, Aoba-ku, 980-8579 Sendai, Japan, honjo@us.ecei.tohoku .ac.jp), Hideyuki Hasegawa (Department of Electronic Engineering, Graduate School of Engineering, Tohoku University, 6-6-05 Aramaki-azaAoba, Aoba-ku, 980-8579 Sendai, Japan, hasegawa@us.ecei.tohoku .ac.jp), Hiroshi Kanai (Department of Electronic Engineering, Graduate School of Engineering, Tohoku University, 6-6-05 Aramaki-aza-Aoba, Aoba-ku, 980-8579 Sendai, Japan, hasegawa@us.ecei.tohoku.ac.jp)

Methods for imaging of strain rate in the heart wall are useful for quantitative evaluation of regional heart function. We developed a method which can accurately measure the heart wall motion along an ultrasonic beam based on phase changes in rf echoes. However, there are some components in the wall motion which are not along each ultrasonic beam. Therefore, the measurement of motion in the direction perpendicular (lateral) to the beam has been required in addition to that in the axial direction, but some unsolved problems remain in estimation of lateral motion of the wall. In this study, two-dimensional displacement was estimated by 2D cross-correlation between rf echoes. Important parameters, the sizes of a region-of-interest and search region, which determine tracking accuracy, were adaptively op- 
timized by referring to instantaneous wall velocities, in the respective cardiac phases. The correlation coefficient between the lateral displacement estimated by the 2D tracking with optimized parameters in longitudinal-axis view and axial displacement in apical view (corresponding to lateral displacement in longitudinal-axis view) separately and accurately estimated by the $1 \mathrm{D}$ phase-based method was 0.93 . These results show possibility of this method for accurate measurement of two-dimensional heart motion to assess the regional myocardial strain rate.

5aBBd3. Flow-mediated change in viscoelasticity of radial arterial wall measured by 22-MHz ultrasound. Kazuki Ikeshita (Department of Electronic Engineering, Graduate School of Engineering, Tohoku University, 6-6-05 Aramaki-aza-Aoba, Aoba-ku, 980-8579 Sendai, Japan, ikeshita@us.ecei.tohoku.ac.jp), Hideyuki Hasegawa (Department of Electronic Engineering, Graduate School of Engineering, Tohoku University, 6-6-05 Aramaki-aza-Aoba, Aoba-ku, 980-8579 Sendai, Japan, hasegawa@us.ecei.tohoku.ac.jp), Hiroshi Kanai (Department of Electronic Engineering, Graduate School of Engineering, Tohoku University, 6-6-05 Aramaki-aza-Aoba, Aoba-ku, 980-8579 Sendai, Japan, hasegawa@us.ecei .tohoku.ac.jp)

The endothelial dysfunction is considered to be an initial step of atherosclerosis. Moreover, it was reported that the smooth muscle, which constructs the media of the artery, changes its characteristics due to earlystage atherosclerosis. Therefore, it is essential to develop a method for assessing the regional endothelial function and mechanical property of the arterial wall. There is an ultrasound-based conventional technique to measure the change in inner diameter of the brachial artery caused by flow-mediated dilation (FMD) after release of avascularization. In this study, the transient change in the mechanical property of the arterial wall was further revealed by measuring the stress-strain relationship during each heartbeat. For this measurement, the minute change in thickness (strain) of the radial artery was measured using the ultrasonic phased tracking method, together with the waveform of blood pressure (stress) which was continuously measured at the radial artery. From in vivo experiments, it has been founded the slope of the stress-strain hysteresis loop decreases due to FMD and the area increase depends on the ratio of elastic modulus and loss modulus (depends on viscosity). These results show a potential of the proposed method for thorough analysis of the transient change in viscoelasticity due to FMD.

5aBBd4. Experimental investigation of the scattering of sound by solid spheres in a liquid. Jason Bach (Georgia Tech Lorraine - G.W. Woodruff School of ME, UMI Georgia Tech - CNRS 2958, 2 rue Marconi, 57070 Metz, France, jsbach82@yahoo.com), Nico F. Declercq (Georgia Tech Lorraine - G.W. Woodruff School of ME, UMI Georgia Tech - CNRS 2958, 2 rue Marconi, 57070 Metz, France,nico.declercq@me.gatech.edu), David $\mathrm{Ku}$ (Georgia Tech Lorraine - G.W. Woodruff School of ME, UMI Georgia Tech - CNRS 2958, 2 rue Marconi, 57070 Metz, France, david.ku@me .gatech.edu)

The framework of this investigation is the characterization of spheres in a liquid by means of ultrasound. Experiments are performed to study the effectiveness of the use of bounded ultrasonic pulses in single transmission and in double through transmission to characterize size and shape of spheres. Special attention is paid to the influence of the beam width and frequency in comparison with the size of the sphere. The research is performed by means of a new generation polar c-scan apparatus. In a first step the interaction of sound with a single sphere is studied. In a second step two spheres are studied where one sphere crosses the sound path through the other sphere. Limitations for detection and characterization of spheres depending on their relative position and on the characteristics of the applied ultrasonic pulse are described in detail. The research is performed in the framework of the use of ultrasound for biomedical applications.

FRIDAY MORNING, 4 JULY 2008

P3-B, LEVEL, 9:40 TO 11:20 A.M.

\title{
Session 5aBBe
}

\section{Biomedical Ultrasound/Bioresponse to Vibration: Biomedical Applications of Acoustic Radiation Force I (Poster Session)}

\author{
Mostafa Fatemi, Cochair \\ Dep. of Physiology and Biomed. Eng., Mayo Clinic College of Medicine \\ Mathias Fink, Cochair \\ Laboratoire Ondes et Acoustique
}

\begin{abstract}
All posters will be on display from 9:40 a.m. to 11:20 a.m. To allow contributors an opportunity to see other posters, contributors of odd-numbered papers will be at their posters from 9:40 a.m. to 10:30 a.m. and contributors of even-numbered papers will be at their posters from 10:30 a.m. to 11:20 a.m.
\end{abstract}

\section{Contributed Papers}

5aBBe1. Comparative study of vibro-acoustography and B-mode ultrasound in prostate imaging. Azra Alizad (Dept. of Physiology and Biomed. Eng., Mayo Clinic College of Medicine, 200 First Street SW, Rochester, MN 55905, USA, aza@mayo.edu), Farid Mitri (Dept. of Physiology and Biomed. Eng., Mayo Clinic College of Medicine, 200 First Street SW, Rochester, MN 55905, USA, mitri.farid@mayo.edu), Brian Davis (Dep. of Radiation Oncology, Mayo Clinic College of Medicine, 200 1st St. SW, Rochester, MN 55905, USA, davis.brian@mayo.edu), James Greenleaf (Dept. of Physiology and Biomed. Eng., Mayo Clinic College of Medicine, 200 First Street SW, Rochester, MN 55905, USA, jfg@mayo.edu), Mostafa Fatemi (Dept. of Physiology and Biomed. Eng.,
Mayo Clinic College of Medicine, 200 First Street SW, Rochester, MN 55905, USA, fatemi@mayo.edu)

Vibro-acoustography (VA) is an imaging modality that uses the radiation force of ultrasound to vibrate tissue at a low frequency and records the resulting acoustic field to produce an image that is represents object characteristics at both ultrasound as well as low (audio) frequencies. Currently, B-mode ultrasound is used for prostate imaging. This imaging method has some limitations, including the speckle noise, which limit its ability in detection of lesions and calcifications. The purpose of this study is to explore the potentials of VA for prostate imaging. VA, B-mode ultrasound, and radiography were performed on 12 excised human prostate samples and the 
resulting images were compared. VA system parameters were, center frequency $3 \mathrm{MHz}, \Delta \mathrm{f}=50 \mathrm{kHz}$, image area $5 \times 5 \mathrm{~cm}$, pixel size $0.2 \times 0.2 \mathrm{~mm}$. Images evaluated in terms of visibility of anatomical and pathological structures and contrast. Results showed that prostate anatomical structures, mass lesions, and calcifications were visible in the VA images taken at different depths, and VA image quality was superior to B-mode. VA images exhibited significant contrast between the central and peripheral zones with enhanced borders. It is concluded that, with further development, VA may become suitable modality for in prostate imaging.

5aBBe2. Acoustic radiation forces in monitoring of milk composition Diana Priev (Hebrew University, HaGomeh 16/4, 98537 Maale Adumim, Israel, diana.priev@mail.huji.ac.il), Victor Ponomarev (NDT Instruments Ltd, HaGomeh 16/4, 98537 Maale Adumim, Israel, viktora @aaanet.ru), Aba Priev (Hebrew University, Hadassah Medical School, Ein Kerem, 91120 Jerusalem, Israel, abbap@ekmd.huji.ac.il)

High sensitivity of ultrasonic velocity and attenuation to composition and state of milk and other liquid food products is now well established. Unfortunately, existing devices include measurements of the acoustic properties of milk at different temperatures and therefore require waiting for temperature equilibration of the milk when the sample is heated or cooled and cannot be used for real-time monitoring. In this paper ultrasonic particles analyzer of NDT Instruments, AcoMilk-02, used for continuous monitoring of fat globules and somatic cells of raw milk in computerized milking station of cowsheds has been described. This device is based on high intensity standing wave for preliminary separation and concentration of the fat globules and somatic cells by the acoustic radiation forces and low intensity standing wave to measure their content. Testing of analyzer was carried out on 5 cows during one-month period. It was found that milk production level, stage of lactation, and outside temperature have significant influence on the milk composition. Continuous monitoring of milk fat and somatic cells count that typically have high day-to-day variation, provide a much-needed tool for dairy management and for veterinary diagnostic purposes.

5aBBe3. Ultrasound-enhanced latex immunoassay of pathogens in water. Danny Bavli (Hebrew University, Hadassah Medical School, Ein Kerem, 91120 Jerusalem, Israel, danny.bavli@mail.huji.ac.il), Yechezkel Barenholz (Hebrew University, Hadassah Medical School, Ein Kerem, 91120 Jerusalem, Israel, yb@cc.huji.ac.il), Noam Emanuel (Hebrew University, Hadassah Medical School, Ein Kerem, 91120 Jerusalem, Israel, noam.emanuel@mail.huji.ac.il), Victor Ponomarev (NDT Instruments Ltd,
HaGomeh 16/4, 98537 Maale Adumim, Israel, viktora@aaanet.ru),Aba Priev (Hebrew University, Hadassah Medical School, Ein Kerem, 91120 Jerusalem, Israel, abbap@ekmd.huji.ac.il)

Real-time knowledge of contaminants in water is an essential component of any potable water security system. Latex immunoassay is the basic technique in rapid identification of pathogens. We used acoustic radiation to accelerate the latex immunoassay and to bring about separation between free and bound antigen (rotavirus SA-11) in less than one minute. The ultrasonic cylindrical standing-wave device of NDT Instruments, UltraAssay 101, creates areas of maximum and minimum potential energy (nodes). Acoustic radiation forces acting on the pathogens drive them directly to the central node. In this manner, antibody-antigen complexes accumulate in the nodes in 40-60 sec. The nonbound antibodies are washed out of the separation area by the buffer flow. The forces responsible for separation of particles in the UltraAssay 101 depend on the frequency of the standing-wave resonator and on the density, compressibility and size of the immune complexes. Additionally, UltraAssay 101 is able to directly monitor water salinity, turbidity and specific gravity.

5aBBe4. Wave propagation modes and arterial stiffness. Miguel Bernal (Dept. of Physiology and Biomed. Eng., Mayo Clinic College of Medicine, 200 First Street SW, Rochester, MN 55905, USA, bernalrestrepo.miguel@mayo.edu), James Greenleaf (Dept. of Physiology and Biomed. Eng., Mayo Clinic College of Medicine, 200 First Street SW, Rochester, MN 55905, USA, jfg@ mayo.edu)

Arterial elasticity is an independent predictor of cardiovascular disease and mortality. Measurment of waves in thin shells can be used to estimate the circumferential and the longitudinal elastic moduli. Latex tubes and excised pig arteries were used as isotropic and anisotropic models. Waves were generated using focused ultrasound $(1 \mathrm{~ms}, 3 \mathrm{MHz})$ in different locations of the wall. The modes of propagation of the waves were detected with a laser vibrometer or an ultrasound pulse echo tool while the excitation was moved known distances. Bending waves were predominant when exciting in the center of the wall, while a combination of torsion and bending waves resulted from exciting off center. Wave speeds of $15 \mathrm{~m} / \mathrm{s}$ and $5 \mathrm{~m} / \mathrm{s}$ respectively, were measured for the bending waves. Using the Moens-Korteweg equation, values of 1.1 and $0.350 \mathrm{MPa}$ were found for the longitudinal elastic modulus in the tube and artery respectively. Generation of different modes in the tubes and arteries is possible using focused ultrasound. Elastic components of the tubes and vessels in the longitudinal and the circumferential direction can be estimated from the speed of propagation and the frequency of the bending and the torsional waves. 


\title{
Session 5aBBf
}

\section{Biomedical Ultrasound/Bioresponse to Vibration: Biomedical Applications of Acoustic Radiation Force II}

\author{
Mostafa Fatemi, Cochair \\ Dep. of Physiology and Biomed. Eng., Mayo Clinic College of Medicine, 200 First Street SW, Rochester, MN 55905, USA \\ Mathias Fink, Cochair \\ Laboratoire Ondes et Acoustique, ESPCI, Université Paris 7, CNRS, 10 rue Vauquelin, Paris, 75005, France
}

Invited Paper

11:00

\begin{abstract}
5aBBf1. Biomedical applications of radiation force generated in standing ultrasonic waves. Lev Ostrovsky (Zel Technol./Univ. of Colorado, 325 Broadway, R/PSD99, Boulder, CO 80305, USA, Lev.A.Ostrovsky@noaa.gov), Armen Sarvazyan (Artann Laboratories, 1753 Linvale-Harbourton, Lambertville, NJ 08350, USA, armen@artannlabs.com)

This talk presents an overview of physical basis for the action of acoustic radiation force on particles in standing waves in relation to biomedical applications. The effect itself is known since the 19th century (Kundt, 1974) and its biomedical significance was demonstrated in 1971 by Dyson et al. However, despite a long history, extensive studies of particle behavior in standing ultrasonic waves have started only during the last decade due to numerous emerging biomedical applications. The range of currently explored applications is broad: targeted drug and gene delivery, increasing sensitivity of biosensors and immunochemical tests, manipulating cells in suspensions, microstirring, and others. The presentation will focus on theoretical analysis and applications of radiation force acting on particles and bubbles in the standing ultrasonic fields. Dynamics of particle motion induced by ultrasonic radiation force as a function of frequency, intensity, and other variables will be considered. The principal difference in interaction of ultrasonic standing wave field with solid particles and with bubbles due to resonant properties and high compressibility of the latter will be quantitatively analyzed. Several specific biomedical applications of the analyzed phenomena will be discussed.
\end{abstract}

\section{Contributed Papers}

\section{1:20}

5aBBf2. Radiation force on spheres in acoustic beams and related aspects of scattering. Philip L. Marston (Washington State University, Physics and Astronomy Department, Pullman, WA 99164-2814, USA, marston@wsu.edu), David B. Thiessen (Washington State University, Physics and Astronomy Department, Pullman, WA 99164-2814, USA, thiessen@wsu.edu)

The close connection between the acoustic radiation force on objects in fluids and the angular distribution of the farfield scattering is especially useful in cases where dissipative effects are weak in the surrounding fluid. This connection also applies to objects illuminated by acoustic beams and has been used to extend the analysis of scattering by spheres in Bessel beams [P. L. Marston, J. Acoust. Soc. Am. 121, 753-757 (2007); 122, 247-252 (2007)] to the evaluation of the radiation force on spheres [P. L. Marston, J. Acoust. Soc. Am. 120, 3518-3524 (2006); 122, 3162-3165 (2007)]. The quantitative predictions (which include situations of negative radiation force) have been verified by nearfield analysis of the radiation force using the finite element method. We have also examined the radiation force on a sphere for a special case of co-propagating Bessel beams having unequal beam parameters. The scattering of a higher order Bessel beam by a sphere placed on the axis has also been analyzed. The backscattering in that case vanishes in agreement with prior predictions [B. T. Hefner and P. L. Marston, J. Acoust. Soc. Am. 106, 3313-3316 (1999)] for a general class of helicoidal acoustic beams. [Supported by NASA and ONR.]

\section{1:40}

5aBBf3. Modeling the modulated acoustic radiation force distribution in a viscoelastic medium driven by a spherically focused ultrasound transducer. Faik Can Meral (University of Illinois, 842 West Taylor St. MC 251, Chicago, IL 60607, USA, fmeral2@uic.edu), Thomas J. Royston (University of Illinois at Chicago, 842 W. Taylor St. ERF
1072, Chicago, IL 60607, USA, troyston@uic.edu),Richard L. Magin (University of Illinois, 842 West Taylor St. MC 251, Chicago, IL 60607, USA, rmagin@uic.edu)

Acoustic radiation force created by focused ultrasound transducers is gaining great interest in medical diagnosis. This study aims to clarify the acoustic power delivery by means of a modulated focused transducer and to predict the performance of such systems. A spherical-cap shaped transducer, made of piezoelectric material, is used to create ultrasonic waves at a focal point. Different modulation methods, given in the literature and reviewed here, are available for creating a concentrated alternating force due to the acoustic radiation pressure. The relationship between the voltage input to the piezoelectric transducer and its resulting mechanical deformation is examined using a finite element model (FEM) for high frequency harmonic excitation (3 - $10 \mathrm{MHz})$. The oscillating surface of the transducer drives the contacting media, which exerts an acoustic load on the transducer that is also considered in the FE analysis. Also, the motion of resulting acoustic waves in a lossy medium is studied for a more accurate estimation of the induced force distribution and energy dissipation within the medium. Ultimately, the intention of this work is to relate the electrical power input to the transducer to the resulting dynamic force generated in the coupling medium. [Research supported by NIH Grant \# EB004885.]

\section{2:00}

5aBBf4. Biomedical applications of acoustic radiation force based on somatosensory reception. Leonid R. Gavrilov (N.N. Andreyev Acoustics Institute, 4, Shvernik Street, 117036 Moscow, Russian Federation, gavrilov@akin.ru), Jeffrey W. Hand (Imperial College London, Hammersmith Campus, Ducane Road, W12 0NN London, UK, 
j.hand@imperial.ac.uk),Efim M. Tsirulnikov(I.M. Sechenov Institute of Evolutionary Physiology and Biochemistry, 44 Torez ave., 194223 St. Petersburg, Russian Federation, tsiruln@iephb.ru)

We have previously shown that short pulses of focused ultrasound may be used to stimulate locally receptor neural structures and thereby induce sensations that humans can perceive through the skin (for example tactile, warmth and cold, tickling, pain, etc.). Furthermore, ultrasound modulated by sound signals (tone, speech, music, etc.) can induce sound sensations corre- sponding to the nature of the modulation in persons with normal hearing. The mechanism underlying these effects that have been used in clinical diagnostic practice is related to the radiation force. We also showed recently that the phenomenon of the radiation force could be used in perspective robotic systems, sensors, and automated control systems, based on the use of tactile sensations in the human-machine interface, as well as in devices that may allow blind and visually impaired persons to perceive textual information presented on a tactile display. The research was supported by INTAS (05-1000008-7841) and RFBR (06-02-16079).

\section{Invited Paper}

\section{2:20}

5aBBf5. Ultrasound stimulated vibrometry for measuring tissue properties. James Greenleaf (Dept. of Physiology and Biomed. Eng., Mayo Clinic College of Medicine, 200 First Street SW, Rochester, MN 55905, USA, jfg@ mayo.edu), Shigao Chen (Dept. of Physiology and Biomed. Eng., Mayo Clinic College of Medicine, 200 First Street SW, Rochester, MN 55905, USA, chen.shigao@mayo.edu), Xiaoming Zhang (Dept. of Physiology and Biomed. Eng., Mayo Clinic College of Medicine, 200 First Street SW, Rochester, MN 55905, USA, Zhang.xiaoming@mayo.edu)

Harmonic or pulsed radiation pressure and subsequent measurement of the tissue response can be used to accurately quantitatively measure fundamental material properties of tissue. The measurement of shear wave dispersion can be used to estimate elastic shear moduli of tissue using an appropriate model. Fundamental model free properties such as complex modulus can also be measured. Ultrasound radiation pressure is used to induce free propagating shear waves. The measurable properties of the shear waves such as speed are sensitive to only the local material properties of the tissue under certain circumstances. A model relating the shear wave speed as a function of frequency is related to the elastic and viscous moduli within small regions of tissue according to, for instance, the Voigt model. Shear and elastic moduli in tissue are measured with high accuracy and precision given appropriate models of wave propagation within the geometry of the tissue. Complex modulus in liver of live pig and other tissues have been made. Careful use of shear wave propagation and subsequent measurements can provide fundamental quantitative measurements of tissue mechanical properties if models are accurate. An application of this method is the noninvasive measurement of liver stiffness as a surrogate for fibrosis.

\section{2:40-2:00 Lunch Break}

\section{Invited Papers}

\section{2:00}

5aBBf6. Supersonic shear imaging: a multiwave imaging example. Mathias Fink (Laboratoire Ondes et Acoustique, ESPCI, Université Paris 7, CNRS, 10 rue Vauquelin, 75005 Paris, France, mathias.fink@espci.fr), Mickael Tanter (Laboratoire Ondes et Acoustique, ESPCI, Université Paris 7, CNRS, 10 rue Vauquelin, 75005 Paris, France, michael.tanter@espci.fr), Jeremy Bercoff (Supersonic Imagine, 13857 Aix en Provence, France, jeremy.bercoff@supersonicimagine.fr)

A new imaging method that relies on the simultaneous use of both a low frequency shear wave and a high frequency ultrasound is described. The shear waves have typically centimetric wavelengths and they propagated at low velocity in tissues. They are progressively distorted by the viscoelastic inhomogeneities of encountered tissues. When coupled to an ultrafast ultrasound scanner (5000 images per second), it allows for the follow up of the propagation of these waves with a millimetric resolution over a large zone of interest. From the spatiotemporal evolution of the shear displacement fields, inversion algorithms are used to recover the shear modulus and viscosity map with submillimetric resolution. These techniques are no more diffraction limited because, the near field of the transient waves is directly observed. In this multivave technique, the shear wave gives the contrast while the ultrasonic wave gives the spatial resolution. Shear waves are generated leveraging the innovative use of the ultrasonic radiation force generated by an ultrasound probe. A supersonic shear source is remotely created in tissues. Such a moving source creates two plane shear waves propagating in a Mach cone. In vivo images obtained in breast, liver, muscles will be presented that show the great interest of this quantitative imaging technique.

5aBBf7. Image quality assessment: Vibro-acoustography versus B-mode. Mostafa Fatemi (Dept. of Physiology and Biomed. Eng., Mayo Clinic College of Medicine, 200 First Street SW, Rochester, MN 55905, USA, fatemi@mayo.edu)

Vibro-acoustography (VA) is based on conversion of ultrasound energy from high frequency to a low frequency. This conversion, which results from the nonlinear nature of wave equation, gives VA some key advantages over linear ultrasound imaging such as B-mode. Two key features of VA are analyzed: lack of speckle noise, and angle-independency, which is the ability of imaging specular reflectors regardless of their orientation. Both the speckle noise and angle dependency are major artifacts in B-mode. Lack of speckle noise results from the fact that VA images are constructed from the low frequency acoustic signal. Using an acoustical model, it is shown that the random scatterers within soft tissue do not promote speckle noise in VA as they do in B-mode; thus VA generally produces high contrast images. Angle-independency results from the broad beam pattern of the low-frequency acoustic field. A model is constructed describing the amplitude of the acoustic signal versus object orientation angle. It is shown that the signal amplitude is relatively insensitive to object orientation; thus VA may be suitable for imaging specular objects such as implants. It is concluded that VA may be advantageous in applications where speckle noise and angle dependency are of concern. 
5aBBf8. In vivo feasibility of local harmonic motion imaging and its use for thermal surgery control. Kullervo Hynynen (University of Toronto, Sunnybrook Health Sciences Centre, Suite S6 65b, 2075 Bayview Ave, Toronto, ON M4N 3M5, Canada, khynynen@sri.utoronto.ca), Laura Curiel (University of Toronto, Sunnybrook Health Sciences Centre, Suite S6 65b, 2075 Bayview Ave, Toronto, ON M4N 3M5, Canada, lcuriel@sri.utoronto.ca), Rajiv Chopra (University of Toronto, Sunnybrook Health Sciences Centre, Suite S6 65b, 2075 Bayview Ave, Toronto, ON M4N 3M5, Canada, chopra@sri.utoronto.ca)

A local harmonic motion can be generated within the tissues by the periodic induction of radiation force using a focused ultrasound (FUS) transducer. Tissue motion can then be tracked by collecting rf signals during the excitation using a separate transducer. Finally, displacement estimates can be obtained by cross-correlating the collected rf signals. The characteristics of the induced LHM depend on the local elastic properties of the tissues making it an attractive tool for imaging and therapy control applications. LHM measurements have been obtained in vivo on rabbit muscle and it was observed that the amplitude of the motion was significantly reduced after coagulation. LHM was successfully used to spatially detect the presence of the coagulation lesions within the tissues as a drop in LHM amplitude. It was also possible to detect the location of an implanted VX2 tumor when a spatial scan was performed as the LHM amplitude was lower inside the tumor because of an increased stiffness. Measurements of LHM during tissue heating using FUS reflected the changes in stiffness and revealed the apparition of coagulation showing the potential of these measurements as an alternative control for the FUS exposure. [Work supported by NIH Grant R33 CA102884 and the Canadian Research Chair Program.]

3:00

5aBBf9. Impulsive acoustic radiation force: imaging approaches and clinical applications. Kathryn Nightingale (Biomedical Engineering, Duke Univ., 136 Hudson Hall, Durham, NC 27708, USA, kathy.nightingale @ duke.edu), Mark Palmeri (Duke University, P.O. Box 90281, Durham, NC 27708, USA, mlp6@duke.edu), Liang Zhai (Duke University, P.O. Box 90281, Durham, NC 27708, USA, liang.zhai@duke.edu), Kristin Frinkley (Biomedical Engineering, Duke Univ., 136 Hudson Hall, Durham, NC 27708, USA, kdf2@duke.edu), Michael Wang (Duke University, P.O. Box 90281, Durham, NC 27708, USA, michael.h.wang@duke.edu), Jeremy Dahl (Duke University, P.O. Box 90281, Durham, NC 27708, USA, jjd@duke.edu), Brian Fahey (Duke University, P.O. Box 90281, Durham, NC 27708, USA, bjf8@duke.edu), Stephen Hsu (Duke University, P.O. Box 90281, Durham, NC 27708, USA, sjh6@duke.edu), David Bradway (Duke University, P.O. Box 90281, Durham, NC 27708, USA, david.bradway@duke.edu), Gregg Trahey (Duke University, P.O. Box 90281, Durham, NC 27708, USA, gregg.trahey@ duke.edu)

Focused acoustic radiation force can be used to locally mechanically excite tissue, and the tissue response can be monitored with conventional ultrasonic displacement estimation methods. Many groups are currently exploring the potential for radiation force based methods to derive information about tissue stiffness associated with different pathologies. These techniques can be implemented on a modified diagnostic ultrasound scanner, using the same transducer for both generating the radiation force excitation and monitoring the tissue response. Multiple locations within an imaging field of view can be interrogated sequentially, by electronically controlling the push and track apertures and beam locations. A variety of data processing and imaging approaches are under investigation. Images are generated of the tissue displacement magnitude within the region of excitation at a given time after radiation force application. These images portray relative differences in tissue stiffness, and provide interesting structural information that is well correlated with, and often exhibits improved contrast over, matched B-mode images. Methods for quantifying tissue stiffness through monitoring shear wave propagation, as originally proposed by Sarvazyan, are also under investigation. Results from ongoing clinical studies using these methods in a variety of organs (e.g., liver, prostate, breast, and heart) will be presented.

3:20

5aBBf10. Experimental validation of the amplitude-modulated harmonic motion imaging for tissues stiffness estimation. Caroline Maleke (Columbia University Dept. of Biomedical Engineering, 622 W 168th SSt, PH-7, Room 200 Center, New York, NY 10032, USA, cm2243@columbia.edu), Jianwen Luo (Columbia University Dept. of Biomedical Engineering, 622 W 168th St, PH-7, Room 200 Center, New York, NY 10032, USA, j12767@columbia.edu), Viktor Gamarnik (Columbia University Dept of Biomedical Engineering, 622 W 168th st, PH-7, room 200 center, New York, NY 10032, USA, vg2125@ columbia.edu), Elisa Konofagou (Dept. of Biomedical Engineering, Columbia Univ., 351 Engineering Terrace MC 8904, 1210 Amsterdam Ave., New York, NY 10027, USA, ek2191@columbia.edu)

It has been previously shown that amplitude-modulated harmonic motion imaging (AM-HMI) has the capability of induce and image tissue displacement during the application of an oscillatory radiation force. Here, we aim at validating theoretical HMI findings with experimental results on similar phantoms. A finite-element-method (FEM) was first used to model a dynamic response of phantoms with inclusions at different stiffnesses and sizes. The FEM and experimental results were compared and used to describe the behavior of the locally displaced tissue. The radiation force was generated by a $4.68 \mathrm{MHz}$ FUS transducer modulated at $50 \mathrm{~Hz}$ with acoustic pressure levels varied between 1.2 and $4 \mathrm{MPa}$. A 7.5 MHz pulse-echo transducer was placed through the center of the FUS transducer and used to image the displaced tissue. A 1D-cross-correlation method on successive RF signals was used to estimate the axialdisplacement. The FEM and experimental results displayed good agreement in displacement patterns, i.e., the highest localized displacement occurred at the focus and was symmetrically distributed. In addition, mechanical testing was performed to estimate the phantom-gels moduli. A linear relationship between HMI displacements and tissue Young's moduli was established within the range of 13-to-50kPa. Further studies will involve the implementation of 1D linear-array transducer for full-view imaging. 


\section{Contributed Papers}

3:40

5aBBf11. An integrated system to deliver impulsive radiation force and to image induced transient strain for monitoring focused ultrasound surgery. Gearoid Berry (Institute of Cancer Research, 15 Cotswold Road, Belmont, Sutton, SM2 5NG Surrey, UK, gearoid.berry@icr.ac.uk), Jeff Bamber (Institute of Cancer Research, 15 Cotswold Road, Belmont, Sutton, SM2 5NG Surrey, UK, jeff.bamber@icr.ac.uk), Yixin Ma (Institute of Cancer Research, 15 Cotswold Road, SM2 5NG Sutton, UK, yixin.ma@icr.ac.uk), Ian Rivens (Institute of Cancer Research, 15 Cotswold Road, SM2 5NG Sutton, UK, ian.rivens@icr.ac.uk), Gail Ter Haar (Institute of Cancer Research, 15 Cotswold Road, Belmont, Sutton, SM2 5NG Surrey, UK, gail.terhaar@icr.ac.uk)

Thermal coagulation of tissue causes an approximate three-fold increase in stiffness, which can be easily detected by various elasticity imaging methods. Advantages have been reported, for application to breast cancer diagnosis, of an elasticity imaging method that applies a highly localised transient stress deep within the tissue using a low frequency focused ultrasound radiation force impulse, and uses relatively high frequency echo imaging to measure the transient strain generated in the tissue placed between the transducers. In this paper we describe a new system that implements this concept using a focused ultrasound surgical transducer to apply the transient $(<10 \mathrm{~ms})$ load, synchronized by a customised field programmable gate array to a Zonare C5-2 probe to image the strain. Both transducers are on the same side of the tissue and locked together for scanning to create transient strain elastograms. Performance measurements, obtained using gelatine phantoms and ex vivo thermally ablated liver tissue, indicate an elastogram spatial resolution of $2 \mathrm{~mm}$, and acceptable contrast for detecting regions of thermally coagulated tissue. An advantage of this configuration is that it may be easily integrated into focused ultrasound therapy.

\section{4:00-4:20 Break}

\section{4:20}

5aBBf12. Ultrasonic imaging of displacements inside objects induced by continuously applied fluctuating acoustic radiation forces. Hideyuki Hasegawa (Department of Electronic Engineering, Graduate School of Engineering, Tohoku University, 6-6-05 Aramaki-aza-Aoba, Aoba-ku, 9808579 Sendai, Japan, hasegawa@us.ecei.tohoku.ac.jp), Yoshitaka Odagiri (Department of Electronic Engineering, Graduate School of Engineering, Tohoku University, 6-6-05 Aramaki-aza-Aoba, Aoba-ku, 9808579 Sendai, Japan, hasegawa@us.ecei.tohoku.ac.jp), Hiroshi Kanai (Department of Electronic Engineering, Graduate School of Engineering, Tohoku University, 6-6-05 Aramaki-aza-Aoba, Aoba-ku, 980-8579 Sendai, Japan, hasegawa@us.ecei.tohoku.ac.jp)

Recently, many studies are conducted to measure the response of soft tissue to applied ultrasonic acoustic radiation force. However, the change in position of a target is dominantly induced by a radiation force applied from only one direction, particularly when the target is much harder than surrounding medium. In this case, elasticity of the target cannot be evaluated because the change in position does not relate to target's elasticity. In this study, 1-MHz continuous-wave ultrasound whose envelope fluctuated at 5 $\mathrm{Hz}$ was used to apply the radiation force fluctuating at $5 \mathrm{~Hz}$. By applying two radiation forces from two opposite horizontal directions, the object would be effectively deformed (compressed) in the horizontal direction and vertically expanded due to its incompressibility. Resultant vertical displacements were measured by ultrasonic pulses at $22 \mathrm{MHz}$ in center frequency which can be easily separated from $1-\mathrm{MHz}$ ultrasound by filtering. In in vitro experiments using chicken muscle and porcine liver, displacements at $5 \mathrm{~Hz}$ were clearly imaged. Amplitudes of displacements in liver are similar to those in muscle, whereas applied radiation force was one-fifth. Furthermore, shear wave propagating in liver was clearly visualized because liver was much softer than muscle, i.e., the wavelength was much shorter.

\section{4:40}

5aBBf13. Viscoelastic properties of myocardium tissue with surface and shear wave methods. Xiaoming Zhang (Dep. of Physiology and Biomed. Eng., Mayo Clinic College of Medicine, 200 First Street SW, Rochester, MN 55905, USA, Zhang.xiaoming@mayo.edu), Shigao Chen (Dep. of Physiology and Biomed. Eng., Mayo Clinic College of Medicine, 200 First Street SW, Rochester, MN 55905, USA, chen.shigao@mayo.edu), Matthew Urban (Dep. of Physiology and Biomed. Eng., Mayo Clinic College of Medicine, 200 First Street SW, Rochester, MN 55905, USA, urban.matthew@mayo.edu), Randall Kinnick (Dep. of Physiology and Biomed. Eng., Mayo Clinic College of Medicine, 200 First Street SW, Rochester, MN 55905, USA, kinnick.randall@mayo.edu), James Greenleaf (Dep. of Physiology and Biomed. Eng., Mayo Clinic College of Medicine, 200 First Street SW, Rochester, MN 55905, USA, jfg@ mayo.edu)

Considerable interest in the elastic properties of soft tissue has increased in medicine due to their clinical relevance for monitoring various diseases as well as a biomarker for cancer. A recent emerging field is the use of acoustic radiation force for imaging and estimating the elastic properties of tissue. Newly developed imaging modalities include shear wave elasticity imaging, supersonic shear wave imaging, acoustic radiation force impulse imaging, and vibro-acoustography. In these new methods, the shear wave is used to estimate the elasticity of tissue. We have developed a novel surface wave method for noninvasively estimating the elasticity of tissue [X. Zhang et al., J. Acoust. Soc. Am., 122, 2522-2525 (2007)]. In this method, a localized ultrasound radiation force is remotely and noninvasively applied inside the tissue. The surface wave speed is used to estimate the elasticity of tissue. This method has been further developed and applied to estimate the viscoelasticity of animal myocardium tissue. In this abstract, we report our recent results of in vitro studies on animal myocardium tissue. Both the surface wave and shear wave are measured and analyzed. Noninvasive characterization of viscoelastic properties of myocardium tissue may be a very important tool for assessing heart function.

\section{Invited Papers}

5aBBf14. Use of radiation force for enhancing efficacy of molecular targeted ultrasound contrast agents - in vitro and in vivo. John A. Hossack (University of Virginia, Biomedical Engineering, MR5, 415 Lane Rd, Charlottesville, VA VA 22908-0759, USA, jh7fj@virginia.edu)

Intravenously injected modified ultrasound microbubbles possessing molecule specific targeting ligands hold significant promise as a method for "molecular imaging." Unfortunately, although microbubbles can achieve specific adhesion in regions of vascular pathology, the method breaks down in high blood flow regions where the bond formation process is more problematic. I present results indicating that acoustic radiation does increase the specific targeted adhesion of microbubbles by pushing the bubbles towards the periphery of a vessel. During in vitro studies, radiation force enhanced microbubble adhesion up to 60-fold. Microbubble adhesion is observed to increase approximately with the square of acoustic pressure between 25 and $122 \mathrm{kPa}$, but decreases at higher pressures as the bubbles rupture. Our in vivo studies involved using intravital microscopy to assess the adherence of P-selectin targeted microbubbles in the mouse cremaster microcirculation and femoral vessels. Acoustic radiation force enhanced microbubble retention four-fold in cremaster venules and in the femoral vein. A 20 -fold enhancement was observed in the femoral artery. I discuss the potential for derivatives of the technique to provide molecular targeted therapeutic contribution. 
5aBBf15. Assessment of tissue viscoelasticity using acoustically interrogated laser-induced microbubble. Stanislav Y. Emelianov (Applied Research Laboratories, The University of Texas, P.O. Box 8029, Austin, TX 78713-8029, USA, emelian@mail.utexas.edu), Salavat R. Aglyamov (Applied Research Laboratories, The University of Texas, P.O. Box 8029, Austin, TX 78713-8029, USA, aglyamov@mail.utexas.edu), Andrei B. Karpiouk (Applied Research Laboratories, The University of Texas, P.O. Box 8029, Austin, TX 78713-8029, USA, Andrei.Karpiouk@engr.utexas.edu), Yurii A. Ilinskii (Applied Research Laboratories, The University of Texas, P.O. Box 8029, Austin, TX 78713-8029, USA, yura@arlut.utexas.edu), Evgenia A. Zabolostkaya (Applied Research Laboratories, The University of Texas, P.O. Box 8029, Austin, TX 78713-8029, USA, zhenia@arlut.utexas.edu)

Lasers are used in many biomedical and clinical applications, ranging from diagnosis to therapy. In eye microsurgery, for example, a pulsed laser beam produces a localized surgical effect through the formation of a cavitation microbubble as a result of tissue photodisruption occurring in the focal zone. However, to insure successful presurgical planning, surgical procedure, and postoperative stages of pathology treatment, the mechanical properties of the tissue must be analyzed before selective laser intervention. Since microbubbles are already produced during laser surgery, we have developed an integrated approach utilizing these laser-induced microbubbles as reporters of tissue viscoelasticity. Specifically, we have derived a general model of gas bubble dynamics in viscoelastic media to describe both translation and oscillations of the microbubble exposed to the acoustic radiation force. Furthermore, an ultrasound method based on temporal measurement of passive acoustic emission from cavity during laser-tissue interaction and simultaneous active pulseecho ultrasound probing of the cavitation bubble was developed to detect and characterize the laser-induced microbubbles. The results of our theoretical, numerical, and experimental studies demonstrate that measurements of gas bubble behavior exposed to acoustic radiation force can be used to assess the mechanical properties of the surrounding tissue.

\section{Contributed Papers}

5:40

5aBBf16. Acoustic radiation force manipulation of PC12 cells In vitro. Robert Muratore (Frederic L. Lizzi Center for Biomedical Engineering, Riverside Research Institute, 156 William St. Fl 9, New York, NY 100382609, USA, muratore@ rrinyc.org), Erin Szulman (Frederic L. Lizzi Center for Biomedical Engineering, Riverside Research Institute, 156 William St. Fl 9, New York, NY 10038-2609, USA, erin.szul@gmail.com), Nina $\mathrm{Xu}$ (Dept. of Biomedical Engineering, Columbia Univ., 351 Engineering Terrace MC 8904, 1210 Amsterdam Ave., New York, NY 10027, USA, xu.nina@gmail.com), Melissa J. Simon (Dept. of Biomedical Engineering, Columbia Univ., 351 Engineering Terrace MC 8904, 1210 Amsterdam Ave., New York, NY 10027, USA, mjs2150@columbia.edu), Barclay Morrison (Dept. of Biomedical Engineering, Columbia Univ., 351 Engineering Terrace MC 8904, 1210 Amsterdam Ave., New York, NY 10027 , USA,bm2119@columbia.edu)

To understand and prevent brain injuries from head trauma, researchers study mechanically stressed neuronal tissue. To anticipate future application of controllable ultrasonic in-vivo stress, we investigated the effects of acoustic radiation force on in-vitro PC12 cells. Undifferentiated PC12 cells were serum-cultured in DMEM/F12 on poly-L-lysine-coated polystyrene. Some cultures were DAPI stained. The culture plates were placed on an inverted phase-contrast microscope. An f/1.1 ultrasound transducer with a waterfilled coupling cone was focused on the culture at a 45-degree angle-ofincidence, and excited with $30-\mathrm{ms}$ 4.7-MHz pulses. Acoustic power was 8 $\mathrm{W}$, and peak pressure was estimated at $300 \mathrm{kPa}$. Digital images were recorded before, during, and after insonification. Incident-light and fluorescence images revealed three populations: cells that were stationary (apparently outside the effective force field region), cells that elongated about $2 \mu \mathrm{m}$ under radiation force and returned to approximately their original shapes when the force was removed (apparently adhered to the substrate), and cells that moved about $50 \mu \mathrm{m}$ with each pulse and did not return (apparently free-floating). We conclude that cell morphology can be influenced reversibly with acoustic radiation force. (Support was provided by the Riverside Research Institute Fund for Biomedical Engineering Research and the Gatsby Initiatives in Brain Circuitry.)

\section{6:00}

5aBBf17. Enhancement of bead-based immunoassays by the use of acoustic radiation force. Martin Wiklund (Royal Institute of Technology, Biomedical and X-Ray Physics, KTH - AlbaNova, Roslagstullsbacken 21, SE-10691 Stockholm, Sweden, martin@biox.kth.se)

Radiation forces generated by ultrasonic standing waves have long been used for increasing the speed and sensitivity of bead-based immunoagglutination tests. More recently, detection methods based on confocal laser-scanning fluorometry and single-step homogeneous bead-based immunoassays show promise for fast, easy and sensitive bioanalytics. If such methods are combined with ultrasonic enhancement, very high sensitivity can be obtained. Here, we analyze and compare the characteristics, performance and limitations of ultrasonic enhancement used in agglutinationbased and fluorescence-based immunoassays. Both radiation force aspects, as well as reaction kinetics aspects, are discussed. Finally, we report on novel results where ultrasonic enhancement is performed in microtiter plates, using a fluorescence-based assay. Here, the ultrasound enriches the sample a factor $10^{6}-10^{7}$, and a detection limit of 60 femtomolar is obtained for a thyroid stimulating hormone (TSH) assay. Our results are compared with the results from a high-performance commercial instrument (Luminex xMAP technology). 


\title{
Session 5aED
}

\section{Education in Acoustics: General Topics in Education in Acoustics}

\author{
William M. Hartmann, Chair \\ Michigan State University, Dept. of Physics and Astronomy, East Lansing, MI 48824, USA
}

\section{Contributed Papers}

\section{8:00}

5aED1. Education for professional career in acoustics. Marion Burgess (Acoustics and Vibration Unit, University of NSW @ Aust Defence Force Academy, 2600 Canberra, Australia, m.burgess@adfa .edu.au), Matthew Stead (Bassett Acoustics, Level 6, 100 Pirie St, 5000 Adelaide, Australia, m.stead@bassett.com.au)

There is a demand around the world for staff for acoustical consulting companies as evidenced by the "positions available" listings. Companies would ideally like to hire staff with a good engineering, physics or building background plus an understanding of acoustics. Programs of study at University may deal with the principles of acoustics and vibration but there is usually a need for supplementary education and training for new staff in practical applications of those principles. While larger companies may provide this "in house" smaller companies do not have such resources and seek to supplement the basic knowledge with short courses or other educational opportunities. Most of these are available at specific times which may limit their applicability. In this paper we will discuss an approach to address this educational need with a fully flexible distance learning program. The program is loosely based on the UK Institute of Acoustics Diploma program The modules comprising the program have been developed with the assistance of the senior consultants and from the acoustic consulting industry body. The early experiences with implementing the program will be discussed.

\section{8:20}

5aED2. The need to integrate speech acoustics course work across the speech-language pathology curriculum. Kathleen Siren (Loyola College in Maryland, 4501 North Charles Street, Department of Speech-Language Pathology/Audiology, Baltimore, MD 21210, USA, ksiren@loyola.edu)

The profession of speech-language pathology faces two critical challenges: (1) the lack of sufficient evidence-based practice due to limited clinical research in the field and (2) the dramatic decline in recent years in the number of students pursuing PhDs. Although speech acoustics courses are a part of most undergraduate programs in speech-language pathology, these courses are often disassociated from students' additional undergraduate and graduate course work and clinical training. These isolated speech science courses may inadequately prepare speech-language pathology students for future research-based course work and clinical practice. This paper reports results of a survey of 140 former undergraduate students who took a speech acoustics course between 1994 and 2003. The survey asks students about additional speech acoustics course work, related research experience, and use of speech science information in clinical practice. The majority of students surveyed report that speech acoustics information is largely isolated in one undergraduate course. This paper concludes that programs must consider better harmonizing speech science course work with other undergraduate and graduate course work and clinical training to meet the current research related challenges to the profession.

\section{8:40}

5aED3. Interdisciplinary teamwork, "Soundscaping": A pedagogical challenge. Jan Tro (Norwegian University of Science and Technology, O.S. Bragstad plass 2B, NO-7491 Trondheim, Norway, tro@iet.ntnu.no)

For seven years the Norwegian University of Science and Technology, NTNU, has offered the Master course Interdisciplinary Teamwork
("Eksperter i Team", EiT). EiT is organized into "villages" (consisting of up to thirty students). The Acoustics Group, NTNU, is in charge of a village named "Soundscaping" mainly dealing with sound design in public areas. The students in each village are divided into teams of five. The team members may represent very different fields of study like history, philosophy, literature, religion, music, electronics, architecture, physics, medicine, chemistry, computer science and more. The paper will discuss aspects of interdisciplinary teamwork training in acoustics and gives visual and sound examples of very different and interesting results and sound products ranging from pure sound art installations and interactive educational programs to talking waste basket and new developed sound absorbers.

\section{9:00}

5aED4. Noise induced hearing loss amongst classical music students An audiometry study of students of the Royal Academy of Music. Georgia Zepidou (London South Bank University, FESBE, Borough Road, SE1 0AA London, UK, zepidoug@1sbu.ac.uk), Stephen Dance (London South Bank University, FESBE, Borough Road, SE1 0AA London, UK, dances@1sbu.ac.uk), Salvador Morant (London South Bank University, FESBE, Borough Road, SE1 0AA London, UK, salpelmo@hotmail.com)

Under the new UK guidance for Control of Noise at Work, entertainment noise now has to be considered for all employees. Health surveillance has been undertaken as part of the induction for all 1st year students studying at the Royal Academy of Music in London, as of September 2007. Seminars were arranged and details of the audiometric tests given prior to the health surveillance, so that only valid data was collected. A total of 350 students were asked to fill out questionnaires on their particular habits. Analysis of the audiograms showed double the incidence of health surveillance warning levels of hearing loss amongst men compared to women, although both had significantly better hearing than general population for their age. When early signs of noise induced hearing loss were considered, a loss of more than 20 $\mathrm{dB}$ at any high frequency, was indicated for more than half of the brass musicians.

\section{9:20}

5aED5. Problem based Masters Programme in Acoustics - ten years of experience. Sofus Birkedal Nielsen (Aalborg University, Fredrik Bajers Vej 7 B, 9220 Aalborg Ø, Denmark, sbn@es.aau.dk)

The Section of Acoustics at Aalborg University in Denmark has more that ten years of experience in offering a masters programme in Acoustics. The programme provides students with classical and modern aspects of physical acoustics, psychoacoustics and electro acoustics. Also signal processing which today is a major tool in understanding and utilizing of the newest sound technology is a compulsory part of the study. The language is English and the programme is open for all students with the appropriate background. So far half of the students have been non-Danish citizens coming from four continents. The pedagogical concept is problem based learning, with all students organized in groups of 4 - 6 students (having their own group rooms). Each semester has its own theme with relevant courses and suggested projects from the supervisors, industry or the students themselves. The project work is a considerable part of the workload at a semester, one could say that the courses give the theoretical background for the projects which are "learning by doing" with access to the best facilities, rooms and equipment. 


\title{
Session 5aMUa
}

\section{Musical Acoustics: Control of Natural and Synthetic Musical Sounds III}

\author{
Marcelo M. Wanderley, Cochair \\ Centre for Interdisciplinary Research in Music Media \& Technology (CIRMMT) - Schulich School of Music - McGill Univ., \\ 555 Sherbrooke Street West, Montreal, QC H3A1E3, Canada \\ Jean Kergomard, Cochair \\ Laboratoire de Mécanique et d'Acoustique CNRS UPR-7051, 31, Chemin Joseph Aiguier, Marseille Cedex 20, 13402, France
}

\section{Invited Papers}

8:00

5aMUa1. Indirect acquisition of flutist gestures: a case study of harmonic note fingerings. Vincent Verfaille (Centre for Interdisciplinary Research in Music Media \& Technology (CIRMMT) - Schulich School of Music - McGill Univ., 555 Sherbrooke Street West, Montreal, QC H3A1E3, Canada, vincent@music.mcgill.ca), Marcelo M. Wanderley (Centre for Interdisciplinary Research in Music Media \& Technology (CIRMMT) - Schulich School of Music - McGill Univ., 555 Sherbrooke Street West, Montreal, QC H3A1E3, Canada, marcelo.wanderley@mcgill.ca), Philippe Depalle (Centre for Interdisciplinary Research in Music Media \& Technology (CIRMMT) - Schulich School of Music - McGill Univ., 555 Sherbrooke Street West, Montreal, QC H3A1E3, Canada, depalle @ music.mcgill.ca)

The indirect acquisition of musicians' gestures consists in retrieving information about gestures from the sound. Inspired by previous studies investigating clarinetists' gestures, we focus on the flute, which features numerous playing modes and a rich palette of sounds. Our goal is to provide musicians with guidelines for using indirect acquisition. For a variety of playing modes, we collected information about gestures, body parts involved, and effects on the acoustical signal. We then designed signal processing algorithms for recognizing which gesture is involved in a given sound; later we will quantize related movement by combining direct acquisition, indirect acquisition and motion capture. Focusing on harmonic note fingerings, a first experiment investigated octave-related harmonic note fingerings with 1 nonexpert performer (ff dynamics and normal articulation). A target $F_{0}$-guided PCA on the amplitude of the first six harmonics of $F_{0} / 4$ from 1 semi-tone frequency bands retrieved the correct fingering with two principle components in all trials. A second experiment investigated octave and non-octave related harmonic note fingerings with four expert performers. A target $F_{0}$-guided PCA on the sum of energies around $i F_{0} / k(k=2, \ldots, 6 ; i=1, \ldots, 12)$ provided wider clusters, making more difficult to retrieve the correct fingering in some specific cases that we will discuss.

5aMUa2. Control parameters of a generalized vibrato model with modulations of harmonics and residual. Vincent Verfaille (Centre for Interdisciplinary Research in Music Media \& Technology (CIRMMT) - Schulich School of Music - McGill Univ., 555 Sherbrooke Street West, Montreal, QC H3A1E3, Canada, vincent@music.mcgill.ca), Catherine Guastavino (Centre for Interdisciplinary Research in Music Media \& Technology (CIRMMT) - School of Information Studies - McGill Univ., 3459 McTavish, Montreal, QC H3A1Y1, Canada, Catherine.Guastavino@mcgill.ca), Philippe Depalle (Centre for Interdisciplinary Research in Music Media \& Technology (CIRMMT) - Schulich School of Music - McGill Univ., 555 Sherbrooke Street West, Montreal, QC H3A1E3, Canada, depalle@music.mcgill.ca)

Our previous research focused on vibrato modeling of the harmonics of musical sounds. We demonstrated the need to account for fundamental frequency modulation, global amplitude modulation, but also spectral envelope modulation linked to brightness modulations. This may result from nonlinearities between the excitation signal and the resonating body, and/or from variable spectral sampling of the impedance function as $F_{0}$ varies. For instance on flute, vibrato results from air pressure modulations. We also observed in sounds with vibrato of wind instruments that the residual also exhibits amplitude modulation, somehow due to air pressure modulations that are responsible vibrato. In a pilot listening test on shakuhashi sounds (for which the residual is obviously modulated), we asked participants to discriminate between the original modulated harmonics plus the amplitude modulated residual, and the original modulated harmonics plus a non-modulated residual, in a AB comparison task (same/different test). Preliminary results show that participants can hear the difference, suggesting that the amplitude modulation on the residual is audible. Subsequently, we developed a generalized vibrato model incorporating modulations on harmonics and residual. This model is presented here with an emphasis on its control parameters. 


\title{
Contributed Papers
}

\section{8:40}

5aMUa3. Why practice scales? Details of fingering transitions in flute playing. André Almeida (University of New South Wales, Music Acoustics, School of Physics, NSW 2052 Sydney, Australia, aalmeida@phys.unsw.edu.au), Renee Chow (University of New South Wales, Music Acoustics, School of Physics, NSW 2052 Sydney, Australia, z3130148@student.unsw.edu.au), John Smith (University of New South Wales, Music Acoustics, School of Physics, NSW 2052 Sydney, Australia, john.smith@unsw.edu.au)

Mastering a wind instrument requires not only expert blowing and embouchure, but also many hours of practice of exercises that improve the fingering speed, regularity and coordination. Many note transitions involve the motion of several fingers, often in opposite directions. The order of finger movement can sometimes affect the transitions between notes and produce short, spurious notes between the original and destination notes. We adapted a transverse flute for real time measurement (with a resolution of $3 \mathrm{~ms}$ ) of all key positions using reflected light sensors. Beginner and advanced players were asked to perform a phrase including multifinger transitions. The detailed motion, average speed and intervals between finger motion were analysed. The comparison among players is not surprising: an experienced player was found to have more regular time intervals between fingers moving in a same direction (within $10 \mathrm{~ms}$ ) than an amateur (within $25 \mathrm{~ms}$ ). Contrary fingering motions are slightly less regular $(15 \mathrm{~ms}$ for an experienced player against $50 \mathrm{~ms}$ for an amateur), but still unperceptible to the ear in the case of the experienced musician. A range of details will be reported, for instance the influence of scale exercises.
9:00

5aMUa4. Gesture synthesis: basic control of a flute physical model. Nicolas Montgermont (Institut Jean Le Rond d'Alembert/LAM (UPMC/CNRS/Ministère Culture), 11, rue de Lourmel, 75015 Paris, France, montgermont@lam.jussieu.fr), Benoit Fabre (Institut Jean Le Rond d'Alembert/LAM (UPMC/CNRS/Ministère Culture), 11, rue de Lourmel, 75015 Paris, France, fabreb@ccr.jussieu.fr), Patricio De La Cuadra (Centro de Investigación en Tecnologias de Audio (CITA), Universidad Católica de Chile, Alameda 340, Oficina 13, Casilla 114-D Santiago, Chile, pcuadra@uc .cl)

In the flute family, the oscillation is due to the instability of a jet at the output of a channel coupled with an acoustic resonator. Recent physical models allows to simulate the behavior of the complete instrument, but we still lack a convincing way to drive them. The simulation of the isolated instrument must be completed with a modelization of the control exerted by the flutist. Depending of the instrument of the flute family, the number and type of control parameters are differents. For example, in a recorder the player blows inside a fixed channel built by the instrument maker and in the case of the transverse flute, the channel is shaped by the player's lips during the playing. This paper presents a simple model of flute player, based on measurements carried on instrumentalists playing on a recorder or a transverse flute. The model is generating the basic features of the instrument control in order to produce given pitches and dynamics. The coupling with a flute physical model allows to study its validity.

FRIDAY MORNING, 4 JULY 2008

P2-D, LEVEL 2, 9:40 TO 11:20 A.M.

\section{Session 5aMUb}

\section{Musical Acoustics: General (Mode Conversion, Radiation and Perception of Musical Sound) (Poster Session)}

\begin{abstract}
All posters will be on display from 9:40 a.m. to 11:20 a.m. To allow contributors an opportunity to see other posters, contributors of odd-numbered papers will be at their posters from 9:40 a.m. to 10:30 a.m. and contributors of even-numbered papers will be at their posters from 10:30 a.m. to 11:20 a.m.
\end{abstract}

\section{Contributed Papers}

5aMUb1. Subjective evaluations of the performance proficiency for fluctuating musical sounds using fluctuation strength or roughness. Nozomiko Yasui (Graduate School of Science and Technology, Ryukoku University, 1-5, Yokotani, Oe-cho, Seta, 520-2194 Otsu, Shiga, Japan, n-yasui@mail.ryukoku.ac.jp), Masafumi Kinou (Graduate School of Science and Technology, Ryukoku University, 1-5, Yokotani, Oe-cho, Seta, 520-2194 Otsu, Shiga, Japan, kino@rins.ryukoku.ac.jp), Masanobu Miura (Graduate School of Science and Technology, Ryukoku University, 1-5, Yokotani, Oe-cho, Seta, 520-2194 Otsu, Shiga, Japan, miura@rins .ryukoku.ac.jp)

The "Fluctuation Strength (FS)" and the "Roughness" were suggested as evaluation indices of hearing sensation concerning fluctuated sounds of low or high frequencies. Past studies have been investigated FS and roughness of modulated pure tones and broadband noise. However, few studies have been reported concerning FS and roughness of attenuating sounds produced by musical instruments and performance proficiency for them. Therefore, the tremolo played on the mandolin and the closed roll played on the snaredrum are employed here as fluctuating musical sounds of low or high frequencies. Introduced here is the FS and roughness as indices for the evaluation of the performance proficiency for tremolo and roll. Specifically, experts of mandolin or snare-drum are asked to play tremolo of various plucking rate (number of plucking per second) and closed roll of various stroking rate (number of stroking per second). An evaluation experiment using the $2 \mathrm{AFC}$ method is conducted to evaluate performance proficiency for tremolo and roll. Aesthetic performances of tremolo and roll are described as "smooth, or not fluctuated" by trained players. The amount of physical FS for tremolo and physical roughness for roll are calculated by an original procedure to investigate relations between physical indices and evaluation results.

5aMUb2. Musical perception within a highly reverberant room. Nelia Valverde (Universidad de Castilla-La Mancha, Campus Universitario, $16071 \quad$ Cuenca, Spain, Nelia.Valverde@uclm.es), Marcos D. Fernandez (Universidad de Castilla-La Mancha, Campus Universitario, $16071 \quad$ Cuenca, Spain, Marcos.Fernandez@uclm.es), Javier Ariza (Universidad de Castilla-La Mancha, Campus Universitario, 16071 Cuenca, Spain, Javier.Ariza@uclm.es), Jose A. Ballesteros (Universidad de Castilla-La Mancha, Campus Universitario, 16071 Cuenca, Spain, Josea.Ballesteros@uclm.es), Juan P. Ruiz (Universidad de Castilla-La 
Mancha, Campus Universitario, 16071 Cuenca, Spain, Juanpedro.Ruiz @uclm.es)

The listening of a musical composition generates a unique and different perception in every listener, but, at the same time, the specific acoustic conditions of the room chosen for its performance have a decisive influence in that perception, which can fit in, almost completely, with the acoustic qualification of the room. The aim of this work is to find out how the high reverberance of a room can have a strong influence on the perception of a musical composition. For that aim, the St. Peter's church (in Cuenca, Spain) with a circular plan, a reduced volume and a high reverberance time has been chosen for the performance. A musical composition for voice and electroacoustics has been created specifically for this church. This has been recorded within the church using a HATS in the listener position and a set of music experts have been asked a survey about their impressions on perception after listening the recording using headphones. The results of the psychoacoustic analysis reveal that the high reverberance of this church leads to an extremely low intelligibility and an unclear sound of the recording. This, together with an unbereable background noise, gives a general acoustic perception not very attractive.

5aMUb3. Foundations of sound therapy. Iegor Reznikoff (Université de Paris X, Département de Philosophie, 92001 Nanterre, France, dominiqueleconte@yahoo.fr)

In the practice of music therapy, the use of the sounds of a live naturally singing voice appears to be the most effective; in some cases, results are obtained whereas there are no results using musical sounds, and generally results are obtained in a much shorter time. Sounds and singing in just intonation are particularly efficient. This practice introduces to a deep understanding of sound therapy. Sketched here are the vocal sound/body relationship and the vocal sound / consciousness relationship, which are relevant in this therapy. Finally clinical examples are given (coma states, loss of speech, old persons, states close to death, mind handicapped persons, depression, etc.). Bibliography I. Reznikoff: On Primitive Elements of Musical Meaning, www.musicandmeaning.net, Journal of Music and Meaning 3 (Invited papers), 2005.

5aMUb4. Broadband directive sources modeling for acoustic discrete-time domain methods. José Escolano (University of Jaén,
Alfonso X, 28, E-23700 Linares, Spain, escolano@ujaen.es),José J. Lopez (Tech. Univ. of Valencia, Camino de Vera S/N, 46021 Valencia, Spain, jjlopez@dcom.upv.es)

The use of sources with complex directivities in discrete-time domain methods, such as the finite-difference time-domain method or the digital waveguide mesh, is a recently open topic. However, so far, the provided solutions work for discrete frequencies or frequency-independent sources. Both solutions are useful for very particular cases and in most cases, far from real sources. This paper provides an extension in the use of a monopole source combination for broadband directive sources in discrete-time domain simulations. This method results very effective for frequency-independent and dependent sources, even in cases where the directivity diagrams have considerable lobules. Finally, this method will be tested through several examples.

5aMUb5. Auditory resolution in virtual environments: Effects of spatialization algorithm, off-center listener positioning and speaker configuration. Georgios Marentakis (Centre for Interdisciplinary Research in Music Media \& Technology (CIRMMT) - Schulich School of Music - McGill Univ., 555 Sherbrooke Street West, Montreal, QC H3A1E3, Canada, gmarentakis@music.mcgill.ca), Nils Peters (Centre for Interdisciplinary Research in Music Media \& Technology (CIRMMT) - Schulich School of Music - McGill Univ., 555 Sherbrooke Street West, Montreal, QC H3A1E3, Canada, nils.peters@mcgill.ca), Stephen McAdams (Centre for Interdisciplinary Research in Music Media \& Technology (CIRMMT) Schulich School of Music - McGill Univ., 555 Sherbrooke Street West, Montreal, QC H3A1E3, Canada, smc@ music.mcgill.ca)

We present the results of an empirical study on the effects of room and off-center listener positioning on sound localization in two virtual environments, VBAP and Ambisonics. Localization accuracy has been assessed by estimating Minimum Audible Angles and Minimum Audible Movement Angles for the two spatialization algorithms and for three directions of sound incidence, in the studio and a concert hall for listeners in off-center listener positions, with two loudspeaker setups. A model of localization accuracy as a function of the experimental variables is presented, that can be used to quantify the effects of the room and off-center listening positions on sound localization and auditory movement detection. 


\title{
Session 5aMUc
}

\section{Musical Acoustics: Plucked and Struck Idiophones I (Poster Session)}

\author{
Thomas D. Rossing, Cochair \\ Stanford University \\ Charles Besainou, Cochair \\ Institut Jean le Rond d'Alembert
}

\begin{abstract}
All posters will be on display from 9:40 a.m. to 11:20 a.m. To allow contributors an opportunity to see other posters, contributors of odd-numbered papers will be at their posters from 9:40 a.m. to 10:30 a.m. and contributors of even-numbered papers will be at their posters from 10:30 a.m. to 11:20 a.m.
\end{abstract}

\section{Contributed Papers}

5aMUc1. Analysis of drum player's motion. Hiroshi Kawakami(Nihon University, 2-42-1 Asahigaoka, Nerima, 176-8525 Tokyo, Japan, hkawakami@eva.hi-ho.ne.jp), Yuki Mito (Nihon University, 2-42-1 Asahigaoka, Nerima, 176-8525 Tokyo, Japan, mitotic@hotmail.com), Reiji Watanuma (Nihon University, 2-42-1 Asahigaoka, Nerima, 176-8525 Tokyo,Japan,VFE16733@nifty.ne.jp), Mieko Marumo (Nihon University, 2-42-1 Asahigaoka, Nerima, 176-8525 Tokyo, Japan, marumo@rg8.so-net .ne.jp)

In this experiment, the motion of a drum player's arm was measured by the optical motion capture system in various performance types. From these actual movement data, we analyzed about the acceleration and sound energy flux of the stick before and after excitation. Moreover, the snap of a wrist which is very important at the time of a high drum performance and the relation of acceleration, and the relation of sound energy flux were also analyzed. From those results, it was suggested that the snap of the wrist was important at the time of a high drum performance, and it was concerned with sound power. Especially, the performance which player hit the drum speedy and repeatedly was concerned with the acceleration of stick before and after excitation

5aMUc2. Dampening vibration modes of the snare drum batter head Barry Larkin (Iowa State University, Department of Music, Music Hall, Ames, IA 50011, USA, blarkin@iastate.edu), Andrew Morrison (Illinois Wesleyan University, Department of Physics, 1312 N. Park St., Bloomington, IL 61701, USA, amorriso@iwu.edu)

Percussionists have always had to contend with an undesirable ringing sound while performing on the snare drum. It is usually referred to as the "edge ring." A common method to eliminate this sound comes from placing some type of dampening material on the edge of the drum head.
Manufacturers of drums have provided many ways of dealing with this problem including internal dampening devices, customized drums heads or material designed to be placed on the drum head. Using electronic television holography, it was revealed the source of this "ring" to be the third mode of vibration that produces a pitch approximately one octave and a half step above the fundamental [Larkin and Morrison, JASA, 122, \#5, p 3056]. In this update to the previous work, we will show the effect of dampening devices on the third and other modes.

5aMUc3. Finite element modelling of steelpan acoustics. Derek A. Gay (The University of The West Indies, Department Civil Engineering, St Augustine, Trinidad and Tobago, derek.gay@sta.uwi.edu)

In this paper the finite element method is used to model acoustic vibrations of steelpan shells. The steelpan surface is characterized as a threedimensional compound shell, comprising notes (surfaces with reverse curvature) on a concave ellipsoidal surface attached to a cylindrical shell (the skirt). In this model note and inter-note surfaces are defined by geometric parameters which can be varied to define complex surface geometries. The geometric mesh model is used develop tenor, cello and bass steelpans instruments and a 3D finite element shell vibration algorithm is used to demonstrate their vibration characteristics. Modes shapes and frequencies of the composite shell structures are computed for typical configurations of note and skirt geometry. The model demonstrates that there exist many composite natural modes of a playing surface involving the interaction between two or more notes. In addition, it is found that the frequency range of mode shapes associated primarily within skirt vibration overlaps with the musical range of the notes underscoring the potential for "skirt-note" coupling. The degree of frequency overlap was found to be largely dependent on skirt length and configuration. 


\title{
Session 5aMUd
}

\section{Musical Acoustics and Signal Processing in Acoustics: Signal Representations and Models of Musical Sounds I (Poster Session)}

\author{
James Beauchamp, Cochair \\ University of Illinois \\ Bertrand David, Cochair \\ Télécom Paristech (ENST) / TSI - CNRS LTCI
}

\begin{abstract}
All posters will be on display from 9:40 a.m. to 11:20 a.m. To allow contributors an opportunity to see other posters, contributors of odd-numbered papers will be at their posters from 9:40 a.m. to 10:30 a.m. and contributors of even-numbered papers will be at their posters from 10:30 a.m. to 11:20 a.m.
\end{abstract}

\section{Contributed Papers}

5aMUd1. The perceptual and cognitive nonlinearities underlying musical preference across multiple exposures. Elizabeth $H$. Margulis (University of Arkansas, Department of Music - MUSC 109, Fayetteville, AR 72701, USA, ehm@uark.edu), Anil K. Roy (Northwestern University, 2240 Campus Drive, Evanston, IL 60208, USA, a-roy@u.northwestern.edu), Patrick C. Wong (Northwestern University, 2240 Campus Drive, Evanston, IL 60208, USA, pwong@ northwestern.edu)

Repetition is a fundamental part of engagement with the auditory world. Repeated exposures influence what gets perceived as important and what features are singled out for attention. That repetition can also influence the mysterious phenomenon of aesthetic enjoyment is a longstanding observation to which much intellectual attention has been paid. A special case of repetition and perceptual learning is music, a domain in which people choose to be exposed to the identical stimulus again and again. This apparent preference for repeated exposures connects fundamentally to notions of human identity, affect, cognitive processing, and neural function. We report on a set of experiments that uses behavioral measures to assess perceptual changes across multiple exposures (five over one week) of a 20-minute piece for orchestra. Across the five exposures, we found an inverted U-shape preference response, which possibly is decomposable to the complex interaction of multiple nonlinear components, represented by performance on tasks relating to memory, attention, affect, syntax, and error detection We argue that musical preference has remained a puzzle because the behavior depends on an underlying complex system with numerous nonlinear cognitive components revealed by our results. [Work supported by NIH, NSF, and Northwestern University.]

5aMUd2. Single sensor singer/music separation using a source/filter model of the singer voice. Jean-Louis Durrieu (Télécom Paristech (ENST) / TSI - CNRS LTCI, 46, rue Barrault, 75634 Paris Cedex 13, France, durrieu@enst.fr), Bertrand David (Télécom Paristech (ENST) / TSI - CNRS LTCI, 46, rue Barrault, 75634 Paris Cedex 13, France, bertrand.david@enst.fr), Gaël Richard (Télécom Paristech (ENST) / TSI CNRS LTCI, 46, rue Barrault, 75634 Paris Cedex 13, France, gael.richard @enst.fr)

Separating the singer voice from polyphonic music signals has many useful applications, such as demixing/remixing, desoloing or audio indexing. In the works of Benaroya on single sensor blind source separation, the signals are modeled by Gaussian mixtures (GMM) such that each state is characterized by a spectral shape. The separation itself is then done by adaptive Wiener filtering. However, to better fit general signals, the number of states for the vocal model should be equal to the number of notes multiplied by the number of vowels (or canonical vocal tract shapes) that the singer uses. Therefore, in order to separate a singer voice from background music, we suggest a source/filter model for the singer signal, keeping the same models as used by Benaroya for the background music signal. Assuming the presence of only one singer, we separate the desired part from the rest by first estimating the sung melody thanks to the source part of our model and then re-evaluating the parameters of our model. This research is partly supported by the European Commission under contract FP6-027026-K-SPACE and by the French AII (Quaero project).

5aMUd3. What/when causal expectation modelling applied to percussive audio. Amaury Hazan (Pomeu Fabra University, Ocata 1, 08003 Barcelona, Spain, ahazan@iua.upf.edu), Paul Brossier (Pomeu Fabra University, Ocata 1, 08003 Barcelona, Spain, piem@piem .org), Ricard Marxer (Pomeu Fabra University, Ocata 1, 08003 Barcelona, Spain, rmarxer@iua.upf.edu), Hendrik Purwins (Pomeu Fabra University, Ocata 1, 08003 Barcelona, Spain, hpurwins@iua.upf.edu)

A causal system for representing a musical stream and generating further expected events is presented. Starting from an auditory front-end which extracts low-level (e.g., spectral shape, mel frequency cepstral coefficients) and midlevel features such as onsets and beats, an unsupervised categorisation process builds and maintains a set of symbols aimed at representing musical stream events using both timbre and time descriptions. The time events are represented using inter-onset intervals relative to the beats. These symbols are then processed by an expectation module based on predictive partial match, a multiscale technique derived from $\mathrm{N}$-grams. The system capacity to generate an expectation that matches its transcription is evaluated using drum recordings from the ENST-drums database. We show that the MFCC-based representation leads to a more compact set of symbols and a better match between transcription and expectation. Also, we suggest that the system is sensitive to exposure and illustrate some properties of the expectation entropy while attending percussive audio patterns.

5aMUd4. Dynamical hierarchical self-organization of harmonic and motivic musical categories. Ricard Marxer (Pomeu Fabra University, Ocata 1, 08003 Barcelona, Spain, rmarxer@iua.upf.edu), Piotr Holonowicz (Pomeu Fabra University, Ocata 1, 08003 Barcelona, Spain, pholonow@iua.upf.edu), Amaury Hazan (Pomeu Fabra University, Ocata 1, 08003 Barcelona, Spain, ahazan@iua.upf.edu), Hendrik Purwins (Pomeu Fabra University, Ocata 1, 08003 Barcelona, Spain, hpurwins@iua.upf.edu)

We introduce a generic model of emergence of musical categories during the listening process. The model is based on a preprocessing and a categorization module. Preprocessing results in a perceptually plausible representation of music events extracted from symbolic input. The categorization 
module lets a taxonomy of musical entities emerge according to a cognitively plausible online learning paradigm. We show the advantages of using a conceptual clustering method in the musical domain. The system extracts multilevel hierarchies and can be tuned to clustering at various resolutions. The potential of the model is exemplified by exposing it to two different datasets resulting in music harmonic and motivic categorization consistent with music theory.

5aMUd5. A comparison of molecular approaches for generating sparse and structured multiresolution representations of audio and music signals. Bob Sturm (University of California, Box 117, Department of Electrical and Computer Engineering, Santa Barbara, CA 93106, USA, boblsturm@ece.ucsb.edu), John J. Shynk (University of California, Box 117, Department of Electrical and Computer Engineering, Santa Barbara, CA 93106, USA, shynk@ece.ucsb.edu), Aaron McLeran (University of California, Media Arts and Technology Program, Santa Barbara, CA 93106, USA, amcleran@gmail.com), Curtis Roads (University of California, Media Arts and Technology Program, Santa Barbara, CA 93106, USA, clang@mat.ucsb.edu), Laurent Daudet (UPMC Univ Paris 06, LAM / IJLRA, 11 rue de Lourmel, 75015 Paris, France, daudet@lam.jussieu.fr)

The authors investigate the characteristics and performance of joint (single-step) and sequential (two-step) approaches to creating sparse and structured multiresolution representations of audio and music signals derived using sparse overcomplete methods. A joint approach, such as molecular matching pursuit, attempts to find structures in a signal as part of the decomposition process, while a sequential approach, such as agglomerative clustering, attempts to find structures in the completed decomposition of a signal. Each of these approaches have different benefits and drawbacks. For a joint approach, it is computationally convenient that the decomposition and structuring are done simultaneously, but usually only simple structural relations are possible. For a sequential approach, one is working in a parameter space of much smaller dimension than the original signal, but the computation is higher since the decomposition and the structure building are two separate processes. Results from these approaches using real audio and music signals will be compared and contrasted, and will contribute to our goal of creating an enhanced interface between the content of audio and music signals, e.g., onsets, notes, voices, and their multiresolution sparse atomic decompositions.

5aMUd6. Initialization, distances and local minima in audio applications of the non-negative matrix factorization. Nancy Bertin (Télécom Paristech (ENST) / TSI - CNRS LTCI, 46, rue Barrault, 75634 Paris Cedex 13, France, nbertin@enst.fr), Roland Badeau (Télécom Paristech (ENST) / TSI - CNRS LTCI, 46, rue Barrault, 75634 Paris Cedex 13, France, roland.badeau@enst.fr)

The use of the non-negative matrix factorization (NMF) as a decomposition technique has dramatically grown in various signal processing applications over the last years. Its computation, based on the iterative minimization of a cost function, relies on several choices, among which the distance involved in the cost function itself but also the initialization of the algorithm. These choices are all the more crucial as the usual algorithms, iterative, only ensure convergence to a local minimum. In this work, we compare three typical distances in the NMF problem (namely Euclidian, Kullback-Leibler divergence and Itakura-Saito distance) and their combination with different initializations, in an audio context: decomposition of the time-frequency representation for the transcription of polyphonic music. Both the existence of global and local minima, and the efficiency of transcription are examined. Moreover, NMF update rules can be formulated in a unified framework for the three aforementioned cost functions. This formulation allows figuring out new NMF algorithms which could address the local minima question.
5aMUd7. Automatic morphological description of sounds. Geoffroy G. Peeters (Ircam, 1, pl. Igor Stravinsky, 75004 Paris, France, peeters@ircam .fr), Emmanuel Deruty (Ircam, 1, pl. Igor Stravinsky, 75004 Paris, France, emmanuel.deruty@gmail.com)

Morphological description has been proposed by Pierre Schaeffer. It consists in describing sounds by identifying the temporal evolution of their acoustical properties to a set of profiles. This kind of description is especially useful for indexing sounds with unknown cause such as SoundFX. The present work deals with the automatic estimation of this morphological description from audio signal analysis. In this work, three morphological descriptions are considered: - dynamic profiles (ascending, descending, ascending/descending, stable, impulsive), - melodic profiles (asc., desc. fixed, up/down, down/up) - repetition profiles. For each case we present the most appropriate audio features (loudness, pitch, pitch salience, temporal increase/decrease, lag-matrix-periodicity, ...) and mapping algorithm (slope computed from spline approximations of temporal profiles, ...) used to automatically estimate the profiles. We demonstrate the use of these descriptions for automatic indexing (using decision trees) and search-by-similarity of SoundFX.

5aMUd8. Sound feature extraction to distinguish between a grand and an upright piano. Grigorios Plitsis (Greece, grigoriosplitsis@merseymail .com)

The sound of a grand piano differs from that of an upright piano. In order to find what characterizes the produced sound, an instrument recognition system capable of producing a model of the sound features should be created. Feature extraction can be performed with the use of the Mel frequency cepstral coefficients (MFCCs). This method is useful as it approximates the mechanism of human hearing. Statistical modeling can be further applied using the Gaussian mixture model (GMM) so as to train the system. In order to decide whether the under test instrument is a grand or an upright piano the log-likelihood should be calculated. This study could assist in the identification of the parameters defining the sound of a grand and an upright piano.

5aMUd9. Instrument sound description and modelisation in the context of computer aided orchestration. Damien Tardieu (Ircam, 1, pl. Igor Stravinsky, 75004 Paris, France, Damien.Tardieu@ ircam.fr), Grégoire Carpentier (Ircam, 1, pl. Igor Stravinsky, 75004 Paris, France, Gregoire .Carpentier@ircam.fr), Geoffroy G. Peeters (Ircam, 1, pl. Igor Stravinsky, 75004 Paris, France, peeters@ircam.fr)

We present a work related to the description of instrumental sound in the context of computer aided orchestration. We define the orchestration problem as the search of instrument sound combinations that sound close to a given target. In the presented approach, instrument sounds are described by a set of acoustic features that are extracted from large sound sample databases. The features are then modeled by a probabilistic distribution. Finally the models are combined to approximate the description of a combination of instrument sounds. We discuss the requirement induced by the orchestration problem on the choice of the acoustic features and detail the chosen set of features. In a second part we describe the method used to learn the probabilistic model of different instruments and playing techniques and show that it allows to learn the models from few samples and to deduce the models of some playing techniques, pitches or dynamics that are not available in the training set. Finally we evaluate the proposed method on classification tasks and describe its integration in a computer assisted orchestration program. 


\title{
Session 5aMUe
}

\section{Musical Acoustics and Signal Processing in Acoustics: Signal Representations and Models of Musical Sounds II}

\author{
James W. Beauchamp, Cochair \\ University of Illinois, 1002 Eliot Drive, Urbana, IL 61801, USA \\ Bertrand David, Cochair \\ Télécom Paristech (ENST) / TSI - CNRS LTCI, 46, rue Barrault, Paris Cedex 13, 75634, France
}

\section{Contributed Paper}

\begin{abstract}
11:00
5aMUe1. Early use of the Scott-Koenig phonautograph for documenting performance. George Brock-Nannestad (Patent Tactics, Resedavej 40, DK-2820 Gentofte, Denmark, pattac@image.dk), Jean-Marc Fontaine (Université UPMC - Ministère de la Culture - CNRS - IJRA LAM, 11, rue de Lourmel, F-75015 Paris, France, jmfontai@ccr.jussieu.fr)

Acoustics in the 1850s combined listening, observation and notation. This was "real-time", catching any phenomenon as it appeared. If it was repeatable, one could prepare for it. Continuous data rather than observation data enabled a very different analysis from observation and notebooks. Édouard-Léon Scott's invention of the phonautograph enabled this. A surface moved below a stylus that was vibrated by sound in air. Originally a
\end{abstract}

blackened glass plate, the scientific instrument maker Rudolph Koenig contributed by devising a very long axis representing time, because now blackened paper was wrapped around a cylinder on a threaded shaft. A twodimensional representation of the individual vibrations was obtained. Scott both deposited a sealed letter with the Paris Academy of Sciences in January, 1857 and filed a patent application in April, 1857. Later he deposited a further sealed letter and in 1859 he filed an application for patent of addition. Following the thoughts expressed and documented in his manuscripts here analyzed in context and comparing with Koenig's production it is feasible to see how they were dependent on each other, although they had different purposes in mind. The paper concentrates on Scott's interests in performance, and a number of original tracings are discussed.

\section{Invited Papers}

11:20

5aMUe2. Sparse representations of audio: from source separation to wavefield compressed sensing? Remi Gribonval (INRIA, IRISA, Campus de Beaulieu, 35042 Rennes Cedex, France, remi.gribonval@irisa.fr)

Sparse signal representations, which are at the heart of today's coding standards (JPEG, MPEG, MP3), are known to have had a substantial impact in signal compression. Their principle is to represent high-dimensional data by a combination of a few elementary building blocks, called atoms, chosen from a large collection called a dictionary. Over the last five years, theoretical advances in sparse representations have highlighted their potential to impact all fundamental areas of signal processing. We will discuss some current and emerging applications of sparse models in musical sound processing including: signal acquisition (Compressed Sensing - sampling wave fields at a dramatically reduced rate) and signal manipulation (e.g., source separation and enhancement for digital remastering). We will conclude by discussing the new algorithmic and modeling challenges raised by these approaches.

\section{1:40}

5aMUe3. Towards a hierarchically sparse model for audio signals. Laurent Daudet (UPMC Univ Paris 06, LAM / IJLRA, 11 rue de Lourmel, 75015 Paris, France, daudet@lam.jussieu.fr)

In this paper we discuss a major issue that arises when building sparse atomic models for music signals: in which space (/ dictionary) should we represent the signals? Having extremely redundant dictionaries is very informative for the locally most important atoms, but is irrelevant for the detail atoms that represent residual signals. Also, what are the advantages of dictionaries composed of learned atoms (which brings the issue of representativeness) compared to generic dictionaries? We here advocate for non-fixed dictionaries, with a multi-layered hierarchical decomposition: the first layer roughly describes the signal, in an extremely redundant, signaltailored, structured dictionary. This layer is also very sparse, most of the information being carried by the atoms' parameters, similarly to parametric representations. Subsequent layers give more and more details, increasing the data amount while reducing overcompleteness and structural model constraints. These techniques have obvious applications for audio coding, but are also useful for scalable music data mining. This research is supported by the French ANR (DESAM project).

12:00

5aMUe4. Bayesian inference in hierarchical non-negative matrix factorisation models of musical sounds. Ali Taylan Cemgil (University of Cambridge, Trumpington Street, CB2 1PZ Cambridge, UK, atc27@cam.ac.uk), Tuomas Virtanen (Tampere University of Technology, Inst. of Signal Processing, FI-33101 Tampere, Finland, tuomas.virtanen@tut.fi)

There has been a surge of interest to efficient audio and music modeling using tools from statistical machine learning. One such technique, that has been particularly successful, is non-negative matrix factorisation (NMF). However, a detailed theoretical understanding of this success is missing, as well as links to other modeling strategies such as sinusoidal or harmonic models. To fill this gap, 
we describe NMF in a statistical framework, as a hierarchical generative model consisting of an observation and a prior component. We show that particular choices lead to standard NMF algorithms as special cases, where parameter estimation is carried out via maximum likelihood. Starting from this view, we develop extensions that facilitate more realistic acoustic modeling (such as spectral smoothness or harmonicity of natural sounds) and alternative inference techniques via Gibbs sampling and variational Bayes, which allow us to do principled comparisons between alternative models via Bayesian model selection. Our novel construction, where we make use of Markov chains of Gamma random variables, retains conjugacy and enables us to develop models that fit better to real data while retaining attractive features of standard NMF such as fast convergence and easy implementation. We illustrate our approach on polyphonic pitch estimation.

12:20

5aMUe5. On sinusoidal modeling of nonstationary signals. Axel Roebel (IRCAM, 1, pl. Igor-Stravinsky, 75004 Paris, France, roebel@ircam.fr)

In this presentation we are going to give an overview over a number of techniques that have been developed in our group to improve the modeling of musical (nonstationary) signals using sinusoidal models. One of the basic problems with sinusoidal models is the fact that the underlying theory is derived assuming stationary sinusoids, while in the real world all sinusoidal components are non stationary. The two topics that will be covered main are, first a technique that allows to distinguish between nonstationary sinusoidal components, noise and transients, and second a new technique for parameter estimation of nonstationary sinusoids using frequency domain demodulation.

\section{2:40-1:40 Lunch Break}

\section{Invited Papers}

\section{$1: 40$}

5aMUe6. Advances in the tracking of partials for the sinusoidal modeling of musical sounds. Sylvain Marchand (LaBRI CNRS, University of Bordeaux 1, 351 cours de la Liberation, F-33405 Talence, France, sylvain.marchand@labri.fr)

Whereas sinusoidal modeling is widely used for sounds, polynomial models are still used for the model parameters, which can hardly handle modulations (vibrato and tremolo) present in musical sounds. Moreover, the partial tracking algorithms are often designed under stationarity assumptions. Advances in partial tracking may come out of the modeling of the partials themselves. We consider their parameters (frequencies and amplitudes) as predictable and slow time varying: First, the future of any partial can be determined from its past evolutions; second, no audible frequency should appear in the spectral content of these evolutions, otherwise it would question the perceptive consistency of the model. We then choose to handle nonstationary sinusoidal modeling by a deterministic approach based on linear prediction of the partial evolutions and partial discrimination based on the spectral properties of these evolutions. The underlying model for each partial is now a sum of sinusoids, thus leading to a two-level sinusoidal modeling, well suited for musical sounds, where modulations are important. The enhanced partial tracking algorithm also handles the case of crossing partials, without the need for any probabilistic approach. Better modeling the deterministic part of polyphonic sounds leads to enhanced source separation and time scaling algorithms.

5aMUe7. Adaptive subspace methods for high resolution analysis of music signals. Roland Badeau (Télécom Paristech (ENST) /TSI - CNRS LTCI, 46, rue Barrault, 75634 Paris Cedex 13, France, roland.badeau@enst.fr), Bertrand David (Télécom Paristech (ENST)/TSI - CNRS LTCI, 46, rue Barrault, 75634 Paris Cedex 13, France, bertrand.david@enst.fr)

In the field of music signals analysis, the tonal part of a broad variety of sounds is often represented as a sum of slowly varying sinusoids. The Fourier transform remains a prominent tool for estimating the parameters of this model, due to its robustness and to the existence of fast algorithms. Its main drawback relies in its spectral resolution, bounded by the length of the analysis window. Subspacebased high resolution (HR) methods are conversely not constrained by this limit, since they rely on the particular geometrical structure of the signal model. Nevertheless, they have been seldom used in audio signal processing, mainly due to their high computational cost. Based on recent advances in the field of subspace tracking, enhanced adaptive algorithms for HR analysis have thus been developed, leading to a high resolution time-frequency representation of the signal, called HR-ogram. The application of these algorithms to music signals, made difficult by the high dynamics and the presence of colored noise, has required the tuning of well-adapted preprocessing techniques. The whole tool is now mature, and allows a high quality separation of the tonal part of various musical sounds. This research is supported by the French ANR under contract ANR-06-JCJC-0027-01 (DESAM).

\section{2:20}

5aMUe8. Towards an adaptive subspace-based representation of musical spectral content. Bertrand David (Télécom Paristech (ENST)/TSI - CNRS LTCI, 46, rue Barrault, 75634 Paris Cedex 13, France, bertrand.david@enst.fr), Roland Badeau (Télécom Paristech (ENST)/TSI - CNRS LTCI, 46, rue Barrault, 75634 Paris Cedex 13, France, roland.badeau@enst.fr)

This study presents an algorithm based on an adaptive framework model for musical sound signals assumed to be composed of slowly varying frequency components surrounded by additive noise. These components appear as contours in a time-frequency representation, as for instance, a spectrogram. To extract these contours, an often used solution is to estimate the parameters (amplitude, frequency, and phase) of each component at each frame and then to link them from one to the next with the help of a distance measure or an HMM. Conversely, our method attempts to update the estimated values from one time instant to the next. It relies on principal subspace tracking (with respect to time) together with gradient descent to individually update each of the component parameters. Finally, each extracted contour, which represents the frequency and amplitude variation of a single component, is available for subsequent 
processing. Applications are demonstrated in the fields of harmonic plus noise decomposition and analysis/transformation/synthesis. This research is supported by the french Institut Telecom, TAMTAM project.

\section{$2: 40$}

5aMUe9. Damped sinusoids and subspace based approach for lossy audio coding. Olivier Derrien (Université de Toulon, Av Georges Pompidou, BP 56, 83162 La Valette du Var, France, olivier.derrien@univ-tln.fr), Gaël Richard (Télécom Paristech (ENST) /TSI - CNRS LTCI, 46, rue Barrault, 75634 Paris Cedex 13, France, gael.richard@enst.fr), Roland Badeau (Télécom Paristech (ENST)/TSI - CNRS LTCI, 46, rue Barrault, 75634 Paris Cedex 13, France, roland.badeau@enst.fr)

The new subspace-based techniques recently introduced appear to be well adapted for the parameters estimation of a damped sinusoids + noise signal model. These high-resolution (HR) methods have a better frequency resolution than the Fourier analysis, but they are rarely used in audio coding. Although HR methods would be suitable for parametric coding at low bitrates, we show that they are also efficient for high-bitrate coding where state-of the art codecs are usually transform-based. Our coding scheme first includes a 8-band PQMF filter-bank decomposition. Then, each subband signal is segmented in onsets and a maximum-order HR analysis is performed on each segment with the ESPRIT algorithm. For each component of the model, frequency, damping, amplitude and phase are quantized. The residual signal is not coded. This codec is compared to a MDCT framework, where transform and quantization are the same as in a MPEG-AAC but without inclusion of perceptual modelling and entropy coding. Preliminary objective and subjective tests show the potential of this approach which requires, on mostly tonal signals, significantly less bits than the traditionnal MDCT method for a given quality.

\section{3:00}

5aMUe10. Auditory model based analysis of polyphonic music. Anssi Klapuri (Tampere University of Technology, Korkeakoulunkatu 1, 33720 Tampere, Finland, anssi.klapuri@tut.fi)

This study is about the use of an auditory model to extract multiple pitches from polyphonic music signals. One goal was to identify the conditions where pitch analysis using an auditory model is advantageous over more conventional time or frequency domain approaches. It is shown that these conditions include especially the processing of band-limited signals or signals where important parts of the audible spectrum are corrupted by band-limited interference. An efficient implementation strategy is described which reduces the computational complexity of the auditory model roughly by factor 10 . Further prospects of bandwise processing and redundant signal representations in general are discussed.

\section{$3: 20$}

5aMUe11. On perceptual distortion measures and parametric modeling. Mads G. Christensen (Aalborg University, Niels Jernes Vej 12 A, DK-9220 Aalborg, Denmark, mgc@es.aau.dk)

Over the past two decades, there has been much interest in incorporating human sound perception in signal processing algorithms, often in the form of perceptual distortion measure or an approximation thereof. An example of this is MDCT-based audio coding where a good quality can be achieved at very low bit-rates by taking masking effects into account. More recently, the same principles have been applied to parametric modeling and coding of audio signals. We discuss the inherent tradeoffs in choosing a perceptual distortion measure and a parametric model and the pros and cons of various ways of implementing such perceptual distortion measures is discussed. An important question that we seek to answer is whether perception should be taken into account in the estimation of model parameters or this should be done in a separate step.

\section{Contributed Paper}

\section{3:40}

5aMUe12. Efficient coding of a xylophone sound using spikogram nonredundent coding. Rolf Bader (University of Hamburg, Institute of Musicology, Neue Rabenstr. 13, 20354 Hamburg, Germany, R_Bader@tonline.de)

Sensory systems try to use the incoming data most efficiently. Studies of Lewicki et al. lately showed, that a representation of a spike train just representing the sound and not having any redundancy is how the auditory system of the cat represents incoming sounds. To compare this theory with musical instruments, a xylophone sound was analyzed in terms of a spikogram.
Here, gammatones of a 64 channel filterbank with different attack and decay values are superposed in a way to reconstruct the original sound. Only those gammatones were used which are needed to result in the sound used as input and so no redundancy is present in the analysis of the sound. It was found, that the most reasonable fit of the gammatone shape with the empirical data indeed made the representation most efficient and so the xylophone sound is most easily represented by the auditory system. Although more analysis are needed here, musical instruments could show up to be built in a way to fit a most efficient coding by listeners and so fulfill a middle-of-the-road rule of not too much and not too few information so that listeners are interested in but not overtaxed by the sounds.

4:00-4:20 Break 


\section{Invited Papers}

5aMUe13. A frequency shifting model of pitch. Paris Smaragdis (Adobe Systems Inc., 275 Grove St., Newton, MA 02466, USA, paris@media.mit.edu)

We present a model useful for tracking of melodies of sounds that can have arbitrary harmonic structure (including inharmonic instruments and noise sources). This model is based on a spectral shift assumption which is capable of tracking melodic movements of an instrument regardless of the irregularity of its spectrum. This technique can be used to simultaneously estimate the spectral character of the instrument to be analyzed in addition to its melody. It is capable of dealing with multiple instances of the same instrument, thereby recognizing chords as well as notes, and can also extract multiple melodies in audio signals composed out of many instruments.

\section{4:40}

5aMUe14. Modeling vocal sounds in polyphonic musical audio signals. Masataka Goto (National Institute of Advanced Industrial Science and Technology (AIST), IT, 1-1-1 Umezono, Tsukuba, 305-8568 Ibaraki, Japan, m.goto@aist.go.jp), Hiromasa Fujihara (National Institute of Advanced Industrial Science and Technology (AIST), IT, 1-1-1 Umezono, Tsukuba, 305-8568 Ibaraki, Japan, h.fujihara@aist.go.jp)

This paper describes our research aimed at modeling vocal sounds (singing voices) in available music recordings and its applications to singer identification, singer similarity, and lyrics synchronization. Our predominant-F0 estimation method, PreFEst, can obtain the melody line by modeling the input sound mixture as a weighted mixture of harmonic-structure tone models (probability density functions) of all possible F0s and estimating their weights and the tone models by MAP estimation. Since the PreFEst was designed for general melodies, we extended it to specialize in vocal melodies by using vocal timbre models --- vocal and nonvocal GMMs. Those GMMs are trained beforehand and used to evaluate the vocal probability. The GMMs are also used to identify vocal regions, but its strategy should depend on applications. For singer identification and singer-similarity calculation, since the purpose is to model singer's identity by training each singer's vocal GMM, certainly reliable vocal regions should be identified even if most true regions were missed. On the other hand, for lyrics synchronization, since the purpose is to align each phoneme to the estimated vocal melody, vocal regions should be identified without missing any true regions. We achieved this by biasing log likelihoods provided by vocal and nonvocal GMMs.

\section{5:00}

5aMUe15. Music and speech signal processing using harmonic-temporal clustering. Jonathan Le Roux (University of Tokyo, Sagayama/Ono Laboratory, 7-3-1 Hongo, Bunkyo-ku, 113-8656 Tokyo, Japan, leroux @ hil.t.u-tokyo.ac.jp), Hirokazu Kameoka (NTT Communication Science Laboratories, NTT Corporation, 3-1 Morinosato wakamiya, 243-0198 Atsugi, Kanagawa, Japan, kameoka@eye.brl.ntt.co.jp), Nobutaka Ono (University of Tokyo, Sagayama/Ono Laboratory, 7-3-1 Hongo, Bunkyo-ku, 113-8656 Tokyo, Japan, onono@ hil.t.u-tokyo.ac.jp), Alain De Cheveigne (CNRS, Universite Paris 5, Ecole Normale Superieure, 29 rue d'Ulm, 75230 Paris, France, alain.de.cheveigne@ens.fr), Shigeki Sagayama (University of Tokyo, Sagayama/Ono Laboratory, 7-3-1 Hongo, Bunkyo-ku, 113-8656 Tokyo, Japan, sagayama@ hil.t.u-tokyo.ac.jp)

We present here the principle of the recently introduced harmonic-temporal clustering (HTC) framework and its applications in both music and speech signal processing. HTC relies on a precise parametric description of the harmonic parts of the power spectrum through constrained Gaussian mixture models. The model parameters of all the elements of the acoustical scene are estimated jointly by an unsupervised 2D time-frequency clustering of the observed power density. HTC is effective for multi-pitch analysis of music signals and F0 estimation of single and multiple speaker speech signals in various noisy environments. It also enables to perform extra processing of monaural music and speech signals, such as isolation or cancellation of a particular part, noise reduction and source separation.

5aMUe16. Timbre transposition based on time-varying spectral analysis of continuous monophonic audio and precomputed spectral libraries. James W. Beauchamp (University of Illinois, 1002 Eliot Drive, Urbana, IL 61801, USA, jwbeauch@uiuc.edu), Mert Bay (University of Illinois, 212 W. Healey, Apt. 303, Champaign, IL 61820, USA, mertbay@uiuc.com)

A sinusoidal model for solo musical sounds consisting of time-varying harmonic amplitudes and frequencies allows for convenient temporal and spectral modifications. With a harmonic model, analysis frames can be grouped by fundamental frequency (F0) and then clustered in terms of their harmonic spectra. The resulting cluster centroid spectra are used as spectral libraries. When continuous audio monophonic passages are analyzed in the form of harmonic components, F0 vs. time data are used to guide the extraction of parameters from the sound in order to find appropriate library spectra for resynthesis. Two methods for finding appropriate spectra are: (1) best rms match with the incoming spectra and (2) best spectral centroid match. These give similar results, but centroid matching yields smoother spectra over time. Timbre transposition is performed by using a library that belongs to another instrument. We have found that when the target instrument has a unique timbral quality based on its spectrum, the synthesis sounds mostly like that instrument. However, if the target instrument's spectral characteristic is not sufficiently differentiated from the source, the source timbral quality may dominate, probably due to its temporal behavior being transmitted. Results will be demonstrated by audio examples. 


\title{
Contributed Papers
}

\section{$5: 40$}

5aMUe17. Comparison of the sound of a grand and an upright piano using wavelets. Grigorios Plitsis (Greece, grigoriosplitsis@merseymail .com)

Wavelet analysis is useful for extracting patterns and thus analyzing signals. Although the Fourier analysis can reveal different features of a signal, it is less appropriate for describing transient phenomena and sudden sound changes. As a result, Fourier-based music reconstruction cannot exactly imitate the physical sound, as it is not possible to know at the same time a specific frequency as well as the time of occurrence of this frequency. Wavelet analysis is capable of highlighting different attributes of a signal. Different types of wavelets are thus used in the present study in order to compare the sound produced by a grand piano with that produced by an upright piano.

\section{6:00}

5aMUe18. Modeling of piano sounds using FEM simulation of soundboard vibration. Luis I. Ortiz-Berenguer (Universidad Politecnica de Madrid, Ctra.Valencia km7, 28031 Madrid, Spain, lortiz@diac .upm.es), Francisco J. Casajus-Quiros (Universidad Politecnica de Madrid,
Ctra.Valencia km7, 28031 Madrid, Spain, javier@gaps.ssr.upm.es),David Ibanez-Cuenca (Universidad Politecnica de Madrid, Ctra.Valencia km7, 28031 Madrid, Spain, dibanez@alumnos.euitt.upm.es)

Pattern-matching methods for polyphonic transcription of piano sounds require a set of patterns that can be obtained by modeling the piano-sound spectra. The modeling should take into account not only the string stiffness but also the effect of the soundboard impedance on the string vibration. Studies on that effect corresponding to a wide range of impedance values have previously been carried out by the authors. However, actual impedance values for real pianos must be used in the model. Although the impedance value of a few grand-pianos have been measured by the authors, these results are not significative enough to create a model. Thus, a FEM simulation of soundboard vibration is proposed to obtain nearly-actual impedance values. The simulation considers several cases of vibrating plates from the simplest rectangular one and increasing the similarity to real piano soundboards. The quality of the simulation is verified comparing the obtained results with either recognized theoretical results for the simplest cases or measured values for the more complex ones. The complexity of the simulated soundboard is limited to the case that produces only slight variations in the modeled spectrum. [This work has been supported by Spanish National Project TEC2006-13067-C03-01/TCM.]

FRIDAY MORNING, 4 JULY 2008

ROOM 202/203, 11:00 A.M. TO 12:00 NOON

\section{Session 5aMUf}

\section{Musical Acoustics: Plucked and Struck Idiophones II}

\author{
Thomas D. Rossing, Cochair \\ Stanford University, CCRMA, Department of Music, Stanford, CA 94305, 26464 Taaffe Rd, Los Altos Hills, CA 94022, USA \\ Charles Besnainou, Cochair \\ Institut Jean le Rond d'Alembert, Laboratoire d'Acoustique Musicale, 11, rue de Lourmel, Paris, 75015, France
}

\section{Contributed Papers}

11:00

5aMUf1. Characterizing the sound of an African thumb piano (kalimba). David M.f. Chapman (Scientific Consultant, 8 Lakeview Avenue, Dartmouth, NS B3A 3S7, Canada, dave.chapman@ns.sympatico.ca)

The kalimba is an African percussion instrument whose notes are generated by vibrating metal tines of various lengths attached to sound board or sound box. Unlike a vibrating string or organ pipe, the overtone structure is anharmonic, that is, the overtone frequencies (which determine sound quality) are not simple integer multiples of the fundamental frequency (which determines pitch). The ratio of the first overtone to the fundamental is in the range 5.3-5.9, depending on the tine geometry. The vibrating tines are modeled as a clamped-supported-free beam and the observed overtone structure is shown to be in accordance with this model. Audio examples will be provided.

\section{1:20}

5aMUf2. The jew's harp, experimental study and modeling. Charles Besnainou (Institut Jean le Rond d'Alembert, Laboratoire d'Acoustique Musicale, 11, rue de Lourmel, 75015 Paris, France, chbesnai@ccr .jussieu.fr), Joel Frelat (Institut Jean le Rond d'Alembert, Lab. d'Acoustique Musicale, 11, rue de Lourmel, 75015 Paris, France,
frelat@1mm.jussieu.fr),Adrien Mamou-Mani(Institut Jean le Rond d'Alembert, Lab. d'Acoustique Musicale, 11, rue de Lourmel, 75015 Paris, France,mamou-mani@1mm.jussieu.fr)

Under its archaic aspects jew's harp is a musical instrument highly subtle. Indeed, a metal blade (or wooden) attached to a rigid frame put into vibration by the musician, and coupled to the buccal resonator allow nice tune. The skilt of the jew's harp focuses on the conformations of this cavity whose function is to select the right components of the vibration to be amplify. In our study, we have modelled a playing technique which involves blowing during the blade vibrates. In the lake of breath, the spectre of sound produced by the blade is odd, i.e., it includes at first approximation odd components $(\mathrm{n}+1)$ multiple the fundamental. Whereas when the musician adds breath the spectrum turns into a spectrum containing all components of basic integer multiples (n). This work takes place in the context of studies of vibrating systems under prestress and loaded. In that case the load, and the prestress are generated by the musician breath by bending the blade On the other hand, experimental studies are compare with the model results.

\section{1:40}

5aMUf3. Sound of the HANG. Thomas D. Rossing (Stanford University, CCRMA, Department of Music, Stanford, CA 94305, 26464 Taaffe Rd, Los Altos Hills, CA 94022, USA, rossing@ccrma.stanford.edu), David 
Wessel(Center for New Music and Audio Technologies, 1750 Arch Street, Berkeley, CA 94720, USA, wessel@ cnmat.berkeley.edu.)

Previous studies of the HANG [T. D. Rossing, U. Hansen, F. Rohner, and S. Schärer, 142nd ASA meeting, December 2001; T. D. Rossing, A. Morrison, U. Hansen, F. Rohner, and S. Schärer, Proceedings of ISMA07, Barcelona, 2007] have discussed the modes of vibration and the sound radiation field of the HANG when note areas are excited by sinusoidal force. Since the HANG is a hand-played steel instrument, a wide variety of playing techniques are used by various performers. We report on the sound of the
HANG when played in various ways. Recordings of the HANG were made with a hemispheric microphone array. The instrument was excited by taking multiple samples of each of a number of striking techniques with the hand. These include single strokes from the soft part of the finger, as well as, the finger nail itself. Various grace note techniques like flams were also investigated. Damping was controlled by the time the finger remained in contact with the instrument, as well as, by using the palm. Force hammer measurements were also included. We report on these measurements and incorporate them in to an excited-resonance model of the instrument. Demonstrations of the real and modeled instrument will be provided.

FRIDAY MORNING, 4 JULY 2008

ROOM 250A, 8:00 A.M. TO 6:20 P.M.

\title{
Session 5aNSa
}

\section{Noise and EURONOISE: Soundscape \& Community Noise I}

\author{
Bennett Brooks, Cochair \\ Brooks Acoust. Corp., 27 Hartford Turnpike, Vernon, CT 06066, USA \\ Brigitte Schulte-Fortkamp, Cochair \\ TU Berlin, Institute of Fluid Mechanics and Engineering Acoustics, Einsteinufer 25, Sekr. TA 7, Berlin, D-10587, Germany
}

\section{Invited Papers}

8:00

5aNSa1. Soundscapes: from noise annoyance to the music of urban life. Catherine Guastavino (Centre for Interdisciplinary Research in Music Media \& Technology (CIRMMT) - School of Information Studies - McGill Univ., 3459 McTavish, Montreal, QC H3A1Y1, Canada, Catherine.Guastavino@mcgill.ca), Danièle S. Dubois (CNRS, LCPE/LAM 11 rue de Lourmel, 75015 Paris, France, ddubois@ccr.jussieu.fr)

Through an overview of empirical research over the past ten years, we present an interpretation of the evolution of the concept of soundscape. From a strictly acoustic definition in terms of physical descriptors, it evolved into a more complex notion integrating the effects of noise on citizens. Soundscape research therefore requires the contribution of human sciences (psychology of perception, cognitive psychology, sociology, anthropology of senses). There is converging evidence that people judgments of noise is related to the meaning given to the activities producing the noise. Consequently, physical descriptions in acoustics have to face the diversity of human reactions to noise correlated with the diversity of activities, the diversity of source producing noises within a diversity of physical environments (natural, architectural). This paper focuses on major emerging issues in soundscapes research, namely - the integration of such a diversity of pieces of knowledge within a general knowledge - the translation of these diverse conceptualizations into physical descriptions - the elaboration of convincing physical measurements for decision makers. We will draw consequences for further developments in the cooperative and pluridisciplinary research and for producing guidelines for new orientations in community policies.

8:20

5aNSa2. The soundscape of church bells - sound community or culture clash. Brenda H. Kiser (DL Acoustics, 14301 Middletown Lane, Westminster, CA 92683, USA, brendakiser@ix.netcom.com), David Lubman (DL Acoustics, 14301 Middletown Lane, Westminster, CA 92683, USA, dlubman@dlacoustics.com)

Church bells have long communicated important information to the surrounding community, creating a sound neighborhood. The community relied on the sound neighborhood to learn of events such as weddings, births and deaths, impending danger, church services, and the curfew. The bells thus provided communal identity. This paper reviews the sound neighborhood created by the bells of the church of St. Mary-le-Bow in the City of London, popularly known as "Bow Bells." The ringing of these bells has for centuries defined London's Cockneys and has featured in London's folklore and history since the 14th century. The current status of the soundscape is presented. Sound examples are given. This paper also discusses the future of the soundscape of church bells in light of current societal changes.

8:40

5aNSa3. How is hearing heard in second year architectural design education? Juergen Bauer (Waterford Institute of Technology, Cork Road, IRE - 00000 Waterford, Ireland, jbauer@wit.ie)

Architects are often accused of "not being interested in acoustics because it is invisible". Surely, architectural design, particularly at preliminary stage, has always been driven visually: architects usually develop a project by vision, not by hearing. On the other hand, designing architects have to deal creatively with more or less invisible parameters: sociocultural demands, climatic aspects as wind or temperature, or even daylight which can be considered to be visible but certainly is everything but constant. If an architect's design 
ability is about observation and awareness, and to give an example, if light is considered to be much more than just being bright or dark, how then could we implement early awareness that sound is much more than noise or silence, and that sound planning is much more than just using anti-noise-panels? We asked second year architecture students at Waterford Institute of Technology to (re-)think the architecture of schools and to question the sound qualities and their own sound recollection of such an environment. The paper will outline the teaching approach and present some student's responses to the given aims and brief. Finally, the lessons learnt by teaching staff will be summarized.

\section{9:00}

5aNSa4. Using the soundscape approach to develop a public space in Berlin - perception and evaluation. Brigitte Schulte-Fortkamp (TU Berlin, Institute of Fluid Mechanics and Engineering Acoustics, Einsteinufer 25, Sekr. TA 7, D-10587 Berlin, Germany, brigitte.schulte-fortkamp@tu-berlin.de), Rudi Volz (Advacoustics, Leibnizstr.21, Dr. Rudi Volz, 10625 Berlin, Germany, volz@advacoustics.de), Andre Jakob (TU Berlin, Institute of Fluid Mechanics and Engineering Acoustics, Einsteinufer 25, Sekr. TA 7, D-10587 Berlin, Germany, kontakt@advacoustics.de)

In Berlin the Soundscape Project "Nauener Platz" is carried out to rebuild a difficult public space into a space which is dominated by social freedom. A designed space related procedure is developed following the soundscape approach, which will provide acoustical indicators and parameters describing urban and other outdoor living areas with respect to physical conditions and their relevance for life. The measurement of outdoor sound quality will take into consideration not only the sound pressure but also the characteristics of the sounds which lead to specific human reactions, both positive and negative: calmness, inspiration, annoyance, discontentment, anxiety, etc. and the pathogenic effects. Qualitative evaluations through soundwalks and open interviews have been conducted introducing the new experts. Framework and procedure will be discussed. The Soundscape Project is a module of the Project "Nauener Platz - Remodelling for Young and Old"in the framework of the research program "Experimental Housing and Urban Development (ExWoSt)" of the "Federal Ministry of Transport, Building, and Urban Affairs (BMVBS)" by the "Federal Office for Building and Regional Planning (BBR)". It is related to the fields of research (ExWoSt) concerned with "Innovation of Urban Neighbourhoods for Families and the Elderly". The project executing organization is the Regional Office Berlin-Mitte.

\section{9:20}

5aNSa5. Using the soundscape approach to develop a public space in Berlin - measurement and calculation. Rudi Volz (Advacoustics, Leibnizstr.21, Dr. Rudi Volz, 10625 Berlin, Germany, volz@advacoustics.de), Andre Jakob (TU Berlin, Institute of Fluid Mechanics and Engineering Acoustics, Einsteinufer 25, Sekr. TA 7, D-10587 Berlin, Germany, kontakt@advacoustics.de), Brigitte Schulte-Fortkamp (TU Berlin, Institute of Fluid Mechanics and Engineering Acoustics, Einsteinufer 25, Sekr. TA 7, D-10587 Berlin, Germany, brigitte.schulte-fortkamp@tu-berlin.de)

In this contribution the Soundscape Project "Nauener Platz" in Berlin is subject to different measurement- and calculation methods to develop acoustical indicators and parameters into a database describing urban and other outdoor living areas with respect to physical conditions and their relevance for life. The "Nauener Platz" is carried out to rebuilt a difficult open space into a space which is dominated by social freedom. To obtain the physical quantities sound pressure levels in 1/3-octave bands were measured and artificial head recordings were taken to describe the current state. Combined with calculations using noise prediction software it was possible to verify the sound propagation data outside according to the measurements and allow predictions about planned arrangements. First estimations of these measurements and calculations will be presented. The Soundscape Project is a module of the Project "Nauener Platz - remodelling for young and old". This project is in the framework of the research program "Experimental Housing and Urban Development (ExWoSt)" of the "Federal Ministry of Transport, Building, and Urban Affairs (BMVBS)" by the "Federal Office for Building and Regional Planning (BBR)". It is related to the fields of research (ExWoSt) concerned with "Innovation of Urban Neighbourhoods for Families and the Elderly". The project executing organization is the Regional Office Berlin-Mitte.

9:40-11:00 Posters

Lecture sessions will recess for presentation of poster papers on various topics in acoustics. See poster sessions for topics and abstracts.

\section{Invited Papers}

\section{1:00}

5aNSa6. Applicability of advanced measurement techniques to soundscape studies. André Fiebig (HEAD acoustics GmbH, Ebertstrasse 30a, 52134 Herzogenrath, Germany, andre.fiebig@ head-acoustics.de), Klaus Genuit (HEAD acoustics GmbH, Ebertstrasse 30a, 52134 Herzogenrath, Germany, klaus.genuit@head-acoustics.de)

A wide range of measurement systems and analysis techniques were applied in previous soundscape studies to document and describe the physical conditions of the scrutinized area. Monaural and binaural recording systems positioned at different measurement points are frequently used, and the collected recordings are played back with headphones or sophisticated loudspeaker arrangements in specific surroundings for further analyses. In the paper, an overview of measurement and playback technologies applied in soundscape studies will be given and the immanent problems related to certain recording and playback systems will be discussed, e.g., measurement accuracy and expense, costs, etc. Furthermore, soundscape researchers have started to establish more and more a pool of specific analyses, which allows - at least partially - the detection of relevant (acoustical) patterns and features as well as a first classification of soundscapes. This allows even the detection of positively experienced environments. However, varying measurement technologies and procedures used in different soundscape studies lead to the problem that the studies and the obtained analysis results are often difficult to compare. The present paper takes up this problem and deals with developing a common basis of measurement techniques and procedures. 
5aNSa7. Analysis and evaluation of traffic sounds. Jens Guettes (TU Berlin, Institute of Fluid Mechanics and Engineering Acoustics, Modersohnstr.64, 10245 Berlin, Germany, jensguettes@gmx.de)

This work focuses on a residential area in Friedrichshain, a district close to the centre of Berlin. A soundscape analysis was made in this area, where the neighbours spent most of their free time, especially their leisure time. Basically, our measurements pointed out that immissions into the flats are mainly due to the low-frequency range under $200 \mathrm{~Hz}$, in particular in the case of closed windows. In addition, this low-frequency range initiates standing waves and vibrations within the flats, causing annoying secondary sounds. Furthermore, effects arose, caused by an impulsive signal structure and a discontinuity in the time structure of the source signals, depending on the street pavement and on the traffic situation. With regard to that point, different physical and perceptive measurement and analysis methods were compared and interviews within the neighbourhood were conducted. Moreover, we tried to correlate the results of the survey with those of our measurements in order to see advantages and disadvantages of the applied methods. The scope of A-weighted measurements was then critically considered. Different ways will be discussed about how the complex evaluation of traffic sounds in the future could be based more on neighbours' perception and needs.

\section{1:40}

5aNSa8. Soundscape design methods to acheive net zero environmantal noise impacts. Gary W. Siebein (Univ. of Florida, 231 Arch, P.O. Box 115702, Gainesville, FL 32611, USA, gsiebein@siebeinacoustic.com), Robert M. Lilkendey (Siebein Associates, Inc., 625 NW 60th Street, Suite C, Gainesville, FL 32607, USA, rlilkendey@ siebeinacoustic.com), Hyun Paek (Siebein Associates, Inc., 625 NW 60th Street, Suite C, Gainesville, FL 32607, USA, hpaek@siebeinacoustic.com)

Soundscape design methods were developed to approach net zero environmental noise impacts for significant building projects in complex settings. Auralization methods for various design alternatives were presented for stake holder review in a large auditorium and in a full size demonstration in the actual environment. Innovative acoustical design and analysis methods were developed to economically reduce source noise levels to the vicinity of the ambient sound level. Methods to determine the nature of the ambient sound level as it varies with time of day, time of year, and other natural and community factors included a series of long term average A-weighted sound levels as well as more detailed octave band measurements and calibrated aural recordings of specific acoustic events. Sound walks were conducted at various times of day to understand the dynamics of the acoustical environment and to identify issues. Focus group discussions among stakeholders and team members developed the long term plans for the community and appropriate architectural and acoustical design criteria for the project.

\section{2:00}

5aNSa9. The influence of subjective response on the choice of measurement for aircraft arrival noise at Nantucket Airport. Nancy Timmerman (Nancy S. Timmerman, P.E., 25 Upton Street, Boston, MA 02118-1609, USA, nstpe@ hotmail.com)

The Nantucket Airport in Massachusetts serves primarily turboprop commuter aircraft, and private or charter jets, with most of its operations in the summer. A seasonal homeowner was severely affected by arrival flight noise and requested noise measurements and noise control recommendations for this architect-designed residence. Time was spent with the homeowner to understand steps already taken, and problems still causing trouble. Standard measurement procedures for aircraft operations in the United States call for the use of A-weighted, slow response for the assessment of noise impact. Since the noise sources at this airport have strong tonal components (from the propellers), and noise control recommendations were sought, one-second 1/3-octave band levels were used to both document the observed problem, and to estimate the field transmission loss of the windows and doors. Measurements were made during a Friday afternoon, when arriving aircraft were mostly 1.5 to 2 min apart. A-weighted maximum and single event levels (SELs) were also measured for each overflight, for comparison with published aircraft data. A "short circuit" was found due to an exposed vent pipe, which resulted in no transmission loss in the $100 \mathrm{~Hz}$ band. Appropriate recommendations were made.

\section{Contributed Paper}

12:20

5aNSa10. Are perceived soundscapes within urban parks restorative? Sarah R. Payne (The University of Manchester, SED, MARC, Oxford Road, M13 9PL Manchester, UK, sarah.payne@postgrad.manchester.ac.uk)

The perceived quality of urban park soundscapes is starting to be explored, and attention restoration research has shown which environments are generally restorative. Yet the effect of perceived soundscapes on individuals' restorative experiences is hardly known. Natural environments, in general, provide restoration for people, including recovery from attentional fatigue and enabling reflection. Therefore the visually "natural" environment of ur- ban parks provides a useful setting to explore the role of varying soundscapes in restoration. Four hundred park users within Sheffield, UK, were surveyed as they left two urban parks. Data were collected on aspects of their park visit, along with measures of their current perceived restoration. Participants' perceived soundscapes were described by the amount of time they heard certain sound types in the park, and the volume at which they heard them. Sound levels [LAeq, $\mathrm{dB}(\mathrm{A})$ ] within the parks were also monitored on a number of days to provide contextual information. Results of the study will be discussed along with the importance of soundscape quality and individuals' restorative experiences in helping to provide a productive and positive quality of urban life. 


\section{Contributed Papers}

\section{2:00}

5aNSa11. Perceptive relevance of soundscape descriptors: a morpho-typological approach. Jean-Dominique Polack (Institut Jean le Rond d'Alembert, Laboratoire d'Acoustique Musicale, 11, rue de Lourmel, 75015 Paris, France, polack@ccr.jussieu.fr), Jacuqes Beaumont (INRETS Laboratoire Transports et Environnement, 25, avenue François Mitterrand, 69675 Bron Cedex, France, jacques.beaumont@inrets.fr), Christine Arras (Acouphen, 33 route de Jonage - BP 30, 69891 Pusignan Cedex, France, christine.arras@acouphen.fr), Mikael Zekri (Institut Jean le Rond d'Alembert, Laboratoire d'Acoustique Musicale, 11, rue de Lourmel, 75015 Paris, France, mikael.zekri@gmail.com), Benjamin Robin (INRETS - Laboratoire Transports et Environnement, 25, avenue François Mitterrand, 69675 Bron Cedex, France, benjamin.robin@inrets.fr)

For assessing the validity of a morpho-typological classification of urban sounds with respect to sound assessment, factor analysis was applied to a database compiled in the city of Lyon and lead to a four-classes classification based on the number of lanes and whether a street is one-way or not. Free categorisation was then carried out on sound excerpts recorded in different sites corresponding to different classes. The main results are: a corpus of $42 \mathrm{~h}$ of five-channel recordings of a choice of sites from the classification at different times of the day; the validation of the relevance of the morphotypological classification for perception; and a confirmation of the semantic oppositions between sources and background noise, as well as holistic and event sequences. Further, annoyance is controlled by the absence or the presence of human or nature sound sources, pleading for research toward automatic identification of sources. Last, categorisation confirms earlier investigations based on variance analysis: in narrow and large streets, traffic increase differently governs the increase of both noise level and annoyance.

\section{2:20}

5aNSa12. Environmental management of a large construction site in a densely populated urban area: an innovative approach to noise and vibration mitigation. Marco Masoero (Politecnico di Torino, Corso Duca degli Abruzzi 24, 10123 Torino, Italy, marco.masoero@polito.it), Carlo Alessandro Bertetti (Studio Progetto Ambiente s.r.l., Corso Rosselli 44, 10128 Torino, Italy, ac.bertetti@ progambiente.it)

An innovative approach to noise and vibration mitigation has been applied to the construction of a new underground railway. The aim is to overcome the difficulties in respecting noise level limits in densely populated areas, as specified by current legislation, even with state-of-the-art technology (e.g., low-emission machinery, noise barriers, etc.). The proposed approach aims at integrating the construction activity in the urban soundscape, placing maximum emphasis on construction organisation measures (e.g., construction activity scheduling and execution, training and information of contractor's personnel, etc.) and on communication and involvement of the exposed population. In this respect, two key figures have been identified: the first is an environmental engineer permanently assisting the construction manager in all the environmental impact issues; the second consists of a sample of population for which the subjective reaction to noise is monitored and fed back to the construction manager. Results are presented and discussed for one of the main construction sites in central Turin.

$$
\text { 2:40 }
$$

5aNSa13. The application of the soundscape approach in the evaluation of the urban public spaces. Monika Rychtarikova (Lab. ATF, Katholieke Universiteit Leuven, Celestijnenlaan 200D, B-3001 Leuven, Belgium, Monika.Rychtarikova@bwk.kuleuven.be), Gerrit Vermeir (Lab. ATF, Katholieke Universiteit Leuven, Celestijnenlaan 200D, B-3001 Leuven, Belgium, Gerrit.Vermeir@bwk.kuleuven.be), Markieta Domecka (Centre for Sociological Research, K.U. Leuven, Parkstraat 45, 3001 Leuven, Belgium, markieta.domecka@soc.kuleuven.be)

An important issues in the development of European cities is the design and renovation of the urban public areas. Typically, broad variety of approaches (sociological, ecological, environmental, physical, etc.) is needed. Earlier studies show the necessity of the transversal multidisciplinary approach in this issue. In order to study the acoustical dimension, the concept of soundscape needs to be proposed and elaborated. Soundscape approach differs from the classical statistical noise analysis in the evaluation of a context-related noise and in the extrapolation of environmental sounds in its complexity. Nowadays, even by using recently developed sophisticated acoustical and psycho-acoustical measurable and quantifiable parameters, it still remains difficult to grasp the complete meaning of a soundscape in words only or by numbers only. Our hypothesis is that the description of the city soundscape might be successfully done by combination of acoustical numbers and words. This article will propose a method for acoustical characterization of urban areas in the framework of the Belgian federal project: $\mathrm{SD} / \mathrm{TA} / 05 \mathrm{~A}$ Design and renovation of urban public spaces towards sustainable cities (DRUPSSuC).

\section{3:00}

5aNSa14. Subjective evaluation of community noise in Canada's National Capital Region and its relation to waking levels of salivary biomarkers. David S. Michaud (Health Canada, 775 Brookfield Road, Ottawa, ON, Ottawa, ON K1A1C1, Canada, dmichaud@hc-sc.gc.ca), Stephen E. Keith (Health Canada, 775 Brookfield Road, Ottawa, ON, Ottawa, ON K1A1C1, Canada, skeith@hc-sc.gc.ca), Jason Tsang (Health Canada, 775 Brookfield Road, Ottawa, ON, Ottawa, ON K1A1C1, Canada, jtsang@hc-sc.gc.ca), Anne Konkle (Health Canada, 50 Columbine Driveway, Tunney's Pasture, Ottawa, ON K1AOK9, Canada, akonkle@hc-sc.gc .ca)

Some research has suggested an association between long-term exposure to traffic noise and relative risk of cardiovascular disease (Babisch et al., 2005; Willich et al., 2006). It has been assumed that noise may act as a nonspecific stressor. Acute exposure to noise can evoke physiological and behavioural changes reminiscent of a stress response in rodents (Michaud et al., 2003), but it is equivocal that this occurs in humans chronically exposed to traffic noise. This pilot project examined annoyance to community noise and salivary biomarkers known to be influenced by stressor exposure. A face-to-face interview subjectively assessed community noise for 60 residents ( 30 males, 30 females; mean age $41.3, \mathrm{SD}=14.98)$. Traffic sound levels will be determined and respondents categorized into high ( $>65 \mathrm{dBA}$, Leq24) and low $(<50 \mathrm{dBA})$ noise areas. Participants also provided saliva samples upon awakening, 30-min after awakening, and prior to bedtime. Concentrations of salivary biomarkers of alpha-amylase and cortisol were spectrophotometrically determined using commercial enzyme-linked immunosorbant assays. Two-way (high-noise versus low-noise) mixed-model analyses of variance will examine differences in questionnaire and salivary responses. Sex differences will be evaluated with independent t-tests, and polynomial regression analyses will relate salivary biomarker levels to highor low-noise areas.

\section{$3: 20$}

5aNSa15. Processing of long-term acoustic measurements. Laurent Faiget (01dB-Metravib, 200 Chemin des Ormeaux, F-69578 Limonest, France, laurent.faiget@01db-metravib.com), Benoit Plassat (01dBMetravib, 200 Chemin des Ormeaux, F-69578 Limonest, France, benoit.plassat@01db-metravib.com), Stéphane Bloquet (01dB-Metravib, 200 Chemin des Ormeaux, F-69578 Limonest, France, stephane .bloquet@01db-metravib.com), Christine Aujard (01dB-Metravib, 200 Chemin des Ormeaux, F-69578 Limonest, France, christine.aujard@01dbmetravib.com)

Many standards and European directives relative to noise are used to assess an acoustic situation, based on long-term indicators such as Lday, Lnight or Levening. These indicators are defined mainly as the mean yearly equivalent acoustic level on a particular part of the day. Acoustic measurements are performed on reference periods representative of the typical acoustic situation. An Oper@ monitoring system performed measurements for two years on several points. An acoustic database of the one-second integration time measurement was created. Different kinds of calculations were performed on these data, the variant being the measurement time considered. This study evaluates the convergence of the classic equivalent level with these long-term indicators. 


\section{3:40}

5aNSa16. The association between residential quality of life and aircraft noise annoyance around Frankfurt Airport. Dirk Schreckenberg (ZEUS GmbH, Sennbrink 46, 58093 Hagen, Germany, schreckenberg@zeusgmbh.de)

A large field study on aircraft noise annoyance with 2312 residents from 66 areas within a radius of $40 \mathrm{~km}$ around Frankfurt Airport was performed between the announcement and implementation of the airport extension (4th runway). For the address of each participant aircraft noise levels were calculated. This presentation concentrates on results with regard to the relationship between perceived - not noise-related - environmental situation and social quality of life in the residential area (i.e. infrastructure, economic development, neighbours, attractiveness of the area) and aircraft noise annoyance. Furthermore the impact of actual perceived residential situation and the expected future situation after airport extension on noise annoyance before the change in aircraft noise exposure occurs is compared. Results of the analyses will be presented and discussed.

\section{4:00-4:20 Break}

4:20

5aNSa17. Ergonomics of intern environments: privacy and reactions to noise pollution. G. Mario Mattia (Bruel Acoustics S.r.l. - EuroAcustici, Viale Cesare Pavese 304, 00144 Roma, Italy, m.mattia@euroacustici.org) , Per V. Bruel (Bruel Acoustics S.r.l. - EuroAcustici, Viale Cesare Pavese 304, 00144 Roma, Italy, bruel@mclink.it)

The acoustical ergonomic is an important target for any room acoustics, intelligibility and community noise. The authors explain the experience on metrological and neuropsychological approach to the human reaction to noise. Environmental sound quality, room acoustics and interference of perceived sound (interference with the privacy) can start subjective effects and strong reaction by humans. The author explains experience and research of human reactions on noise interference, privacy, stress, ... annoying sounds (also with very low levels), rooms with stationary waves, long reverberation time, low acoustical building insulation, are the first cause of complain from the community. We suggest noise limits, international standards, neuropsychological interpretation of the acoustical annoyance.

\section{4:40}

5aNSa18. Noise annoyance indicators for various industrial noise sources: Results and comparisons. Marion Alayrac (EDF R\&D, 1 avenue général de Gaulle, 92141 Clamart Cedex, France, marion.alayrac@edf.fr), Stéphanie Viollon (EDF R\&D, 1 avenue général de Gaulle, 92141 Clamart Cedex, France, stephanie.viollon @edf.fr), Catherine Marquis-Favre (Université de Lyon, Ecole Nationale des Travaux Publics de l'Etat, CNRS, URA 1652, Département Génie Civil et Bâtiment, 3, rue Maurice Audin, 69120 Vaulx-en-velin, France, Catherine .MARQUIS@entpe.fr)

Characterizing the environmental impact of an industrial plant goes through a better understanding of the noise annoyance caused by industrial noise sources, which are numerous and various. A first step was to construct a perceptive typology of various industrial noise sources from a categorization test and based on perceived similarities. Now, laboratory tests are carried out, for each perceptive category separately, through the same experimental procedure, by testing the influence of the sound pressure level and of the noise spectral characteristics (low frequency noise, tonal noiseœ) on noise annoyance. The effect of spectral modifications due to the noise propagation on noise annoyance is also assessed. Subjects have also to judge the annoyance induced by an ambient noise exposure, built with a background noise and an industrial noise for which the emergence level is controlled. Different types of background noises are studied. Through a statistical analysis, indicators are developed for each perceptive category, based on classical indicators such as sound pressure level or loudness (for instance), or on indicators improved to suit spectral characteristics noticed. In this paper, the results of those listening tests are detailed and compared.

\section{5:00}

5aNSa19. Soundscape characterization in selected areas of Central London. Gianluca Memoli (Imperial College London, Department of Chemical Engineering, Exhibition road, SW7 2AZ London, UK, g.memoli@imperial.ac.uk), Alan Bloomfield (Greater London Authority (GLA), City Hall, The Queen's Walk, SE1 2AA London, UK, Alan.Bloomfield@london.gov.uk), Max Dixon (Greater London Authority (GLA), City Hall, The Queen's Walk, SE1 2AA London, UK, max.dixon @1ondon.gov.uk)

The Mayor of London's Ambient Noise Strategy 'Sounder City' is the first UK public policy document to promote, not just noise reduction, but positive soundscape management. For its prescriptions to be put into full effect, psychoacoustic methods are needed to characterize existing areas where action may be needed to counteract existing noise pollution. To this aim, the soundscape characterization of two different areas in Central London will be presented in this work, where the two selected parks have in common the presence of a heavily trafficked road nearby. The acoustical experience of passers-by will be mapped using an indicator related to the time history of sound energy, related in previous studies to people's perceptions. Comparison with the same description performed by classical psychoacoustic parameters and perspectives for innovative, positive soundscape based actions will be discussed.

\section{$5: 20$}

5aNSa20. Social survey on the road traffic noise effect on the quality of life in Moroccan cities. Weam Kharbaoui (Ecole Supérieure de Technologie de Salé, Laboratoire Energétique Matériaux et Environnement, Avenue du Prince Héritier, BP 227, 10000 Salé, Morocco, alex_weam @yahoo.es), Mohammed Garoum (Ecole Supérieure de Technologie de Salé, Laboratoire Energétique Matériaux et Environnement, Avenue du Prince Héritier, BP 227, 10000 Salé, Morocco, garoum1 @yahoo.fr), Abdelaziz Bahoussa (Ecole Supérieure de Technologie de Salé, Laboratoire Energétique Matériaux et Environnement, Avenue du Prince Héritier, BP 227, Salé, Morocco, abahoussa@yahoo.fr), Mohammed Rhachi (Ecole Supérieure de Technologie de Salé, Laboratoire Energétique Matériaux et Environnement, Avenue du Prince Héritier, BP 227, 10000 Salé, Morocco,mrhachi@hotmail.com)

Because of the fast growth of the urbanisation, the noise road traffic causes annoyance, and a wide range of negative effects on the health in many Moroccan urban areas. This problem becomes so serious that it is necessary to develop an adequate global noise policies, regulations, and laws. Unfortunately there is a lack of sufficiently technical, scientific, and social data allowing a precise evaluation of this pollution. The objective of this work is to complete our preliminary social survey and make a further step in the evaluation of the Moroccan urban community response to road traffic noise. The questionnaire used contains 55 closed and open questions and was performed using the ISO-15666 recommendations. The sample was composed of 1200 adults chosen randomly in Rabat, Salé and Tetouen cities. The targeted population consisted on persons of both sexes of 18 and more years old. According to the road traffic condition in Moroccan cities, results were analysed and compared with those obtained in several European cities.

\section{$5: 40$}

5aNSa21. Advanced acoustical material tuned for low frequency noise reduction: a case study. Kathleen Kondylas (NEVA Associates Noise Control, 15 Beck Street, Newburyport, MA 01950, USA, kondylas@earthlink.net), Natalia Levit (DuPont, 5401 Jefferson Davis Highway, Richmond, VA 23234, USA, natalia.v.levit@usa.dupont .com), Joseph A. King (DuPont, 5401 Jefferson Davis Highway, Richmond, VA 23234, USA, joseph.a.king@usa.dupont.com), Chris R. Fuller (Virginia Tech, 131 Durham Hall, Blacksburg, VA 24061, USA, christopher.r.fuller@nasa.gov)

Low frequency noise is a significant annoyance at very low amplitudes, particularly when outdoor community noise from mechanical equipment penetrates commercial and residential building structures. Mid and high frequencies are selectively attenuated by the exterior walls. However, mitigation of the low frequency noise has been very challenging due to the longer wavelength and higher energy. This work presents case studies of an ad- 
vanced acoustical material in typical metal panels to mitigate community noise from outdoor mechanical equipment. These advanced materials were developed with a unique combination of patented acoustical and vibration absorbing technology, based of distributed absorbers, layered with standard acoustic materials. The materials provide enhanced low frequency noise (below $300 \mathrm{~Hz}$ ) over state of the art $10 \mathrm{~cm}$ metal panels filled with the fiberglass absorber. The case studies demonstrate superior acoustic performance in low frequency without substantial increase in weight.

\section{6:00}

5aNSa22. A study on the adaptation of soundscape to covered spaces: Part 2. Asli Ozcevik (Yildiz Technical University Faculty of Architecture, Chair of Building Physics, Besiktas, 34349 Istanbul, Turkey, aozcevik@hotmail.com), Zerhan Yuksel Can (Yildiz Technical University
Faculty of Architecture, Chair of Building Physics, Besiktas, 34349 Istanbul, Turkey, karabi@yildiz.edu.tr)

This study is the second part of a research on the Adaptation of soundscape to covered spaces. A modern and a historical shopping center were compared and evaluated by means of subjective and objective evaluations. The consistency of the objective (time histories) and subjective (listening tests) findings showed that covered commercial areas may have a specific sound environment. The formation of streets is generally the basic spatial design concept that connects open and closed shopping areas. Therefore one of the studies that authors proposed at the previous paper was the comparison of open and closed shopping areas. In this study the soundscapes of a modern and a traditional street having shopping as a basic function will be determined by soundwalks and listening tests, and the findings will be compared with those of the previous studies.

FRIDAY MORNING, 4 JULY 2008

ROOM 251, 8:00 TO 10:00 A.M.

\title{
Session 5aNSb
}

\section{Noise, Architectural Acoustics, and EURONOISE: Noise, Vibration and Acoustics for Medical and Research Facilities and Their Occupants III}

\author{
James West, Cochair \\ Johns Hopkins University, Department of Electrical Engineering, 3400 North Charles Street, Baltimore, MD 21218, USA
}

Jack B. Evans, Cochair

JEAcoustics, 1705 West Koenig Lane, Austin, TX 78756, USA

Marc Asselineau, Cochair

Peutz \& Associés, 10 rue des Messageries, Paris, F75010, France

Erica Ryherd, Cochair

Woodruff School of Mechanical Engineering, Georgia Institute of Technology, Atlanta, GA 30332-0405, USA

\section{Invited Papers}

\section{8:00}

5aNSb1. Realistic floor vibration criteria for MRI operation: A compilation of case studies. Basel Jurdy (Sparling, 720 Olive Way Suite 1400, Seattle, WA 98101-1853, USA, bjurdy@sparling.com), Michael Yantis (Sparling, 720 Olive Way Suite 1400, Seattle, WA 98101-1853, USA, myantis@ sparling.com)

Vibration criteria published by various magnetic resonance imaging (MRI) manufacturers have been found to be too conservative with respect to allowable floor vibration resulting from MRI operation. The floor vibration produced by the MRI operation is greater than the published criteria. Previous experience by the authors has shown that there is an allowable threshold for MRI operation induced vibration, above which the MRI will not operate properly. This paper reports results of a survey of structural floor systems for $1.5 \mathrm{~T}$ and 3.0T MRIs that are working satisfactorily. The most common floor systems have been analyzed structurally using finite element analysis to determine the forced response to a standardized input. Measurement results for some of the floors during MRI operation is also included.

\section{$8: 20$}

5aNSb2. Vibration case study - design challenges for meeting medical imaging manufacturer specifications. Chris Papadimos (Papadimos Group, 818 Fifth Avenue, Suite 207, San Rafael, CA 94901, USA, chris@papadimosgroup.com)

Manufacturer vibration specifications for medical imaging equipment impose formidable challenges particularly when locating such equipment on elevated floors, which is increasingly desirable for work flow and proximity to operating rooms and other support spaces in healthcare facilities. Urban sites can further complicate matters as such sites tend to often be near busy roads that are sources of vibration. Other internal sources of vibration that must be taken into consideration include occupant activities and operating building mechanical systems. All these conflicting interests need to be properly balanced and clearly evaluated; developing solutions requires a diligent and experienced design team. Some of the challenges that had to be overcome in order to develop vibration designs to accom- 
modate medical imaging equipment such as CT Scanners and MRI's on elevated floors of a new healthcare facility are presented in this paper. Extensive site testing to evaluate design constraints and pre-existing conditions, avoidance of incompatible adjacencies and proper considerations in terms of space layouts, development of specialized floor designs and a clear understanding of imaging equipment manufacturer specifications are the key elements addressed in this paper.

\section{Contributed Paper}

8:40

5aNSb3. Noise in an Emergency treatment ward. Sergio Beristain (Mexican Institute of Acoustics, P.O. Box 12-1022, Narvarte, 03001 Mexico, D.F., Mexico, sberista@hotmail.com)

It is beign assessed the noise inside a typical social service Hospital in Mexico City, several sources and relevant areas have been identified and evaluated. This paper presents a detailed description of the Adults Urgency
Room, where all the emergency cases are received and attended arround the clock. In this section of the hospital, forty to sixty patients are attended at the same time in a large open plan room, separated only by curtains, with only a few private rooms for doctors in order to attend incoming patients, trying to solve each case immediatelly, allow him/her entrance into the main room for full emergency treatment, or find a bed inside the hospital for a longer treatment. Noise sourcees are all over the place, and noise variablity is large.

\section{Invited Papers}

\section{9:00}

5aNSb4. Study case of a centre for biomedical analyses: the importance of an acoustic analysis of the current situation and definition of the targets to be achieved. Fabien Krajcarz (Gamba Acoustique, 2 rue de la Découverte, BP 163, 31676 Labege Cedex, France, fabien.krajcarz@acoustique-gamba.fr)

The French regulations concerning the limits on noise levels in medical establishments include the acoustic performances of existing buildings used to accommodate defined medical activities and in which the regulations are normally observed. Nevertheless, there are cases which are either outside the list of the buildings covered, or merit special treatment. One example is described here: a centre for biomedical analyses in a large regional teaching hospital covering a technical area of several hundreds square meters. This concentration of human activities and technical equipment without any acoustic attenuation measures was the spark for a considerable labour movement. In this article we describe the challenges of these situations, the pitfalls to be avoided and any post-project and design stage measures to be implemented.

\section{9:20}

5aNSb5. Use of a versatile acoustic room for audiology applications. Chantal Laroche (University of Ottawa, 451 Smyth Road, room 3062, Guindon Hall, Ottawa, ON K1H 8M5, Canada, claroche@uottawa.ca), Christian Giguère (University of Ottawa, 451 Smyth Road, room 3062, Guindon Hall, Ottawa, ON K1H 8M5, Canada, cgiguere@uottawa.ca), Véronique Vaillancourt (University of Ottawa, 451 Smyth Road, room 3062, Guindon Hall, Ottawa, ON K1H 8M5, Canada, vaillancourt@mail.health.uottawa.ca)

For several decades, hearing tests have been carried out in audiometric test rooms which must meet acoustical requirements set forth in standards on ambient noise levels (i.e., ANSI S3.1-1999 (R2003)). It is however generally accepted that acoustical environments in which individuals with hearing loss function daily seldom correspond to environments found in audiometric rooms. Constraints regarding the sound attenuation by room walls (i.e., costs) can thus be minimized with a more rigorous control over certain acoustical characteristics within the sound room (i.e., reverberation time), thereby allowing to recreate more realistic environments of daily living. During the planning stage of the Research Unit on Noise and Communication at the University of Ottawa, the researchers opted for a versatile acoustic room with reversible panels that not only offers adequate sound attenuation by walls but also some control over reverberation time, thereby allowing to recreate more typical acoustic environments. The characteristics of this versatile acoustic room, as well as examples describing applications of use (effect of repetition on speech recognition, attenuation by headphones used with portable audio systems, effect of hearing protection on speech recognition within military noise environments) will be presented.

\section{Contributed Paper}

\section{9:40}

5aNSb6. NMR sensitivity to floor vibration. Yuval Ben-Dov (IDE, 68 Mazzeo Drive, Randolph, MA 02368, USA, yuval.ben-dov@ideworld .com), Peter Heiland (IDE, 68 Mazzeo Drive, Randolph, MA 02368, USA, peter.heiland@ideworld.com), Srinivasan Rajan (Novartis Institutes for BioMedical Research, 250 Massachusetts Avenue, Building \#600 Room \# 6C-172, Cambridge, MA 02139, USA, srinivasan.rajan@ novartis.com)

NMR is widely used in the determination of molecular structures in solution. Floor vibrations interfere with the NMR measurements and are coupled with the signal of interest. To minimize the effect of floor vibra- tions, NMR spectrometers are typically installed on a solid floor with minimal structurally induced vibrations. However, in some cases this is not possible, and the tool is exposed to structural vibrations. We study the NMR sensitivity to floor vibrations. A Bruker $500 \mathrm{MHz}$ installed on IDE active isolation platform is used. By exciting the platform in a controlled manner we can measure the noise as observed in the NMR spectrum due to the platform vibrations. The sensitivity highly depends on the excitation frequency, and is not well reflected in a typical requirements specification. Revised requirements are presented for the floor vibration both for a tool installed as is, and for a tool on top of our isolation platform. 


\title{
Session 5aNSc
}

\section{Noise and EURONOISE: Acoustic Performance of Energy Efficient Building Products I}

\author{
Anthony Hoover, Cochair \\ McKay Conant Hoover, Inc., 5655 Lindero Canyon Road, Suite 325, Westlake Village, CA 91362, USA \\ Jacques Roland, Cochair \\ Centre Scientifique et Technique du Bâtiment, 24 rue Joseph Fourier, Saint Martin d'Hères, 38400, France
}

\section{Invited Papers}

\author{
8:00
}

5aNSc1. Buildings thermal and acoustics insulation: friends or enemies. Jacques Roland (Centre Scientifique et Technique du Bâtiment, 24 rue Joseph Fourier, 38400 Saint Martin d’Hères, France, jacques.roland@cstb.fr)

The increasing concern on climate changes and greenhouse gas accumulation has urged most of countries to enforce new thermal regulation to improve the energy efficiency of existing and new buildings. This improvement shall not at least induce a reduction of the acoustic comfort, and possibly increase it by a careful choice of products and a relevant design. The new products and technologies involve the building envelop insulation, the thermal bridges suppression, the improvement of air conditioning efficiency, and the renewable energy sources attached to the building. The author will review these new products and technologies and show what are the expected benefit or threat for the acoustic performance of buildings. This will include triple glazing, bulk insulating materials, natural fibrous materials, vacuum insulation panels, phase change materials, thermal bridge isolators, integrated photovoltaic cells, light weight construction, passive house concept, heat pumps, and air conditioning with heat exchangers.

\section{$8: 20$}

5aNSc2. Acoustic behavior of triple glazings. Marc Rehfeld (Saint Gobain Glass, CRDC, B.P. 40103, 60777 Thourotte Cedex, France, marc.rehfeld@saint-gobain.com), David Fournier (Saint Gobain Glass, CRDC, B.P. 40103, 60777 Thourotte Cedex, France, david.fournier@saint-gobain.com)

Making of triple glazings is the only way to still improve thermal performances of Insulating Glass Units. Possible ways with double glazings are already in use: increase the space between glasses, use low emissivity coatings and special gas with lower thermal conductivity as argon or krypton. Specific acoustic weak point of double glazings is the resonance between the two panes which works as a mass spring mass system, and coupling of eigenmodes of panes through the air (gas) cavity. These phenomena are of course still more important with triple glazings, as there are two resonances. The paper will give all comparative data concerning thermal and acoustic performances, and describe a way to achieve the same single number values of sound transmission loss with triple glazing that with double glazing by adding absorption in the gas cavities.

\section{Contributed Paper}

\section{8:40}

5aNSc3. Acoustic design criteria for naturally ventilated buildings. Chris D. Field (Arup Acoustics, 901 Market Street, Suite 260, San Francisco, CA 94103, USA, chris.field@arup.com)

This paper proposes satisfactory indoor noise level criteria for office buildings which are compatible with achieving minimum natural ventilation standards set out in green building rating systems for sustainable building design. Indoor air quality standards related to the use of natural ventilation in buildings conflict with the control of ingress of external noise through ventilation openings to meet internationally recognized background noise limits for building use. These standards generally assume, however, that buildings are sealed and air conditioned to meet the stated recommended indoor noise levels. It is not feasible, however, that these noise standards can be expected or are appropriate to be achieved in naturally ventilated buildings. Therefore, to account for the thermal comfort benefit of natural ventilation and the ability to locally control natural ventilation and noise levels by closing of windows, an allowable exceedance of the recommended indoor noise levels is explored. The allowable deviation from existing background noise level guidelines is determined, which is considered to be an acceptable compromise for increased thermal comfort.

\section{Invited Paper}

\section{9:00}

5aNSc4. $\mathbf{R}+\mathrm{D}$ for the integration of high acoustic-thermal performances in spanish building products. Marta Fuente (LabeinTecnalia, C/Geldo - Parque Tecnológico de Bizkaia, Edificio 700, 48160 Derio, Spain, mfuente@labein.es)

Many EU countries are introducing new national regulations aimed at complying with the European Directive "Energy Performance of Buildings" (EPBD). Subsequently, in Spain a new normative has been recently approved (CTE-HE), compiling new requirements for energy saving in buildings. In this framework, energy efficiency considerations will have an increasing impact on the design of buildings and the choice of materials and products. In the same way, the acoustical quality of dwellings in Spain is going to be guaranteed with the compliance of the new Spanish Building Regulation (CTE-HR). Searching a higher level of comfort in dwellings the CTE is in- 
creasing its requirements and is considering the building as a product itself. These new developments involve efforts and resources for the upgrading of the constructive materials and systems, demanding investments for R\&D activities. Therefore manufacturers of the constructive sector in Spain are launching many innovative research strategies for the development of better products, addressing key factors for the energy efficiency of buildings.

\section{Contributed Paper}

\section{9:20}

5aNSc5. Sound insulation of traditional roof constructions considering energy efficiency requirements. Beáta Mesterházy (Budapest University of Technology and Economics, Lab. of Building Acoustics, Mûegyetem rakpart 3., 1111 Budapest, Hungary, bmester@mail.bme.hu), Frigyes Reis (Budapest University of Technology and Economics, Lab. of Building Acoustics, Mûegyetem rakpart 3., 1111 Budapest, Hungary, reis@mail.bme.hu), Gábor Szlávik (URSA Salgótarján zRt., Budapesti út 31, 3104 Salgótarján, Hungary, gabor.szlavik@uralita.com)

The efforts for energy efficiency result changes in building constructions, also in case of roof constructions. The most important constructional conclusion is the growth of the thickness of the heat insulating layer. Beside heat insulation, inner ventilation between the layers is the other important parameter, which has a great effect on sound insulation behaviour. In regular product information sheets, either in internet or in printed forms, the effect of these later parameter is not present and this way the laboratory data do not show the reality. For the purpose of modeling the reality, a new installation method has been developed, which considers the effects of ventilation usually used in our climate conditions. This new installation method has been realised and used in laboratory experiments. The results based on the new installation method approach the reality better and reflect the acoustical importance of the certain constructional components.

\section{9:40-10:40 Posters}

Lecture sessions will recess for presentation of poster papers on various topics in acoustics. See poster sessions for topics and abstracts.

\section{Invited Paper}

\section{0:40}

5aNSc6. Acoustic performance of membrane based multilayered systems with improved thermal inertia characteristics. Catherine Guigou-Carter (CSTB, 24, rue Joseph Fourier, 38400 Saint Martin D’Hères, France, catherine.guigou@cstb.fr), Hebert Sallee (CSTB, 24, rue Joseph Fourier, 38400 Saint Martin D’Hères, France, hebert.sallee@cstb.fr), Xavier Normand (IFTH, Avenue Guy de Collongue, 69134 Ecully Cedex, France, xnormand@ifth.org)

In this paper the development of double membrane based systems, including phase change materials (denoted-PCM) is discussed. The cavity between the two membranes is filled with a new type of absorbing material: a 3D, nonwoven, complex structure made of two or three fibrous mats that allows the inclusion of small granulated particles while being fabricated. Granulated phase change materials are therefore included in this $3 \mathrm{D}$, nonwoven structure in order to increase thermal properties by the heat storage/restoring principle. The PCMs introduced in this nonwoven structure act to store part of the heat when it is in excess, and then, to restore it when the temperature inside the room is too low. The intermediate temperature chosen to correspond to a desired comfort temperature (for example around 20 ${ }^{\circ} \mathrm{C}$ ) corresponds to the phase transition temperature of the PCM. Lightweight membranes are then fixed on each side of this complex 3D, nonwoven structure, including the PCMs. The acoustic performance of such systems is investigated in terms of the sound transmission loss, both experimentally and analytically. The thermal performance is also briefly presented.

\section{Contributed Paper}

\section{1:00}

5aNSc7. Sound transmission loss of vacuum insulation panels. Waldemar Maysenhölder (Fraunhofer Institute for Building Physics, Dept. of Acoustics, Nobelstraße 12, 70569 Stuttgart, Germany, maysenhoelder @ibp.fraunhofer.de)

The sound transmission loss of a variety of vacuum insulation panels (VIP) has been measured in the laboratory. Core materials of the VIP include silica, polyurethane, micro fleece, and glass fibers. Mass law behavior and minima due to bending-wave coincidence and thickness resonance have been observed. Destroying the vacuum may reduce the sound insulation drastically. Since in practical applications the VIP need protection, the predominant interest lies in the transmission loss of multilayered assemblies, e.g., VIP sandwiched between aluminum plates. Calculations performed by the software LAYERS show that with additional "decoupling" layers (air, rubber) the coincidence dip can be avoided or shifted to higher frequencies, where it is less critical. Preliminary experimental realizations indicate the important role of a proper gluing of the layers. According to further LAYERS calculations, attachment of VIP to massive walls and protection by a plaster layer does not change the transmission loss of the wall appreciably, except for the VIP with the soft polyurethane core material.

\section{Invited Papers}

5aNSc8. Vibration reduction of thermal break balcony connections. Martin Schneider (University of Applied Sciences, Schellingstr. 24, 70174 Stuttgart, Germany, martin.schneider@hft-stuttgart.de), Heinz-Martin Fischer (University of Applied Sciences, Schellingstr. 24, 70174 Stuttgart, Germany, heinz-martin.fischer@hft-stuttgart.de)

In energy efficient buildings concrete balconies have to be thermally insulated from the concrete floor to reduce heat loss and to increase surface temperature. This thermal break is obtained by polystyrene in combination with high-strength concrete thrust-force bearings and an arrangement of stainless steel straight and bent bars to act as tension and shear reinforcement. The acoustic performance of such thermal break units was investigated under laboratory conditions. Therefore the vibration level difference at different junctions 
between balcony and the thermal isolated concrete floor as well as the normalized impact sound pressure level of the floor when the balcony is excited with the standard tapping machine are measured. The influence of the number of tension and shear steel bars and the type of thermal insulating material was studied on typical sized balconies connected to small test floors. The vibration isolation at low frequencies was determined using modal testing.

\section{1:40}

5aNSc9. Using high light reflectance acoustical ceilings to increase the energy efficiency of buildings. Marsha S. Bischel (Armstrong Building Products, 2500 Columbia Ave, Lancaster, PA 17603, USA, msbischel@armstrong.com), William E. Beakes (Armstrong Building Products, Retired, 4057 Woodcrest Lane, Columbia, 17512, USA, beakeswe@embarqmail.com)

Acoustical ceilings with white, highly light-reflecting surfaces can be used as an effective, passive means to significantly improve the energy efficiency of a space. When a ceiling with high light reflectance is coupled with an indirect lighting system, illumination can be increased by $20 \%$, or the energy directly associated with lighting can be reduced by $20 \%$. In addition, lower lighting loads can reduce cooling costs by $7 \%$. A highly light reflective ceiling can also be used to reflect daylight into a space, increasing the amount of light in interior areas away from the windows by as much as $20 \%$; alternatively, the surface area of windows may be reduced by $14 \%$ while maintaining the same illumination levels. In all cases, the increased reflectance of light allows the number of fixtures to be decreased, further increasing the savings related to both energy and maintenance. The percentage of energy savings associated with lighting is proportional to the percentage increase in the light reflectance value; therefore, even small increases in light reflectance provide increases in energy efficiency. When the acoustical performance of two products is comparable, light reflectance could be a critical differentiator in determining total building performance and occupant comfort.

$$
\text { 12:00 }
$$

5aNSc10. High sound and thermal insulation constructions with dB-deck. Bart Ingelaere (BBRI, rue du Lombard, 42, B-1000 Brussel, Belgium, bart.ingelaere@bbri.be), Gerrit Vermeir (Lab. ATF, Katholieke Universiteit Leuven, Celestijnenlaan 200D, B-3001 Leuven, Belgium, Gerrit.Vermeir@bwk.kuleuven.be), Debby Wuyts (BBRI, rue du Lombard, 42, B-1000 Brussel, Belgium, debby .wuyts@bbri.be)

The new Belgian requirements demand $\mathrm{DnT}, \mathrm{w} \geqslant 58 \mathrm{~dB}$ and $\mathrm{L}$ 'nT, $w \leqslant 50 \mathrm{~dB}$ between apartments for enhanced acoustic comfort. At the same time the Energy Performance Regulations impose strict requirements for the thermal insulation between apartments. Higher demands on sound insulation can be realized in practice by using higher surface weights for walls and floors and by applying failure free floating floors. However requiring higher weights for floors is in contradiction with the technique of concrete hollow floor elements. A thermal and acoustical solution was found in the development of dB-deck, a prefab double floor element with intermediate supporting elastic pads, cutting structural vibration paths between superposed apartments. In combination with double walls without anchors (allowing for a high sound reduction index and no structural transmission in the horizontal sense), this construction technique allowed for DnT,w $>63 \mathrm{~dB}$ and $\mathrm{L}$ 'nT, $\mathrm{w}<47 \mathrm{~dB}$ without a floating floor in experimental setups This solution was developed by a team of the BBRI, KULeuven, and CDM for an important producer of hollow floor elements. This technique has now been used for real constructions. The paper presents technical details, the results of the acoustical measurements and the acoustical advantages and difficulties met in the construction of this apartment block.

\section{2:20}

5aNSc11. The effect of additional thermal lining on the acoustic performance of a wall. Jacek Nurzynski (Building Research Institute, Filtrowa Str. 1, 00-611 Warsaw, Poland, j.nurzynski@itb.pl)

External thermal insulation systems (ETICS) are commonly used in Poland mainly for thermorenovation of multifamily residential buildings constructed in seventies using industrialized methods. Additional layer improves thermal performance of a wall but also causes decrease of sound insulation in certain frequency range. Usually the acoustic effect of thermorenovation is unnoticed by inhabitants, but in the case of noisy locations it is sometimes perceived as an appearance of a new tonal outside noise source. The paper presents results of investigation on the influence of additional lining on the acoustic performance of massive wall based on laboratory tests. Resonance frequency, its location and influence on single number quantities is discussed. The problem of sound reduction index improvement prediction acc. to EN 12354-1, separate acoustical characteristics of lining and testing acc. to ISO 140-16 is also considered.

\section{2:40}

5aNSc12. Development of thermoacoustic floating floors for use between parking and dwellings. Catherine Guigou-Carter (CSTB, 24, rue Joseph Fourier, 38400 Saint Martin D’Hères, France, catherine.guigou@ @stb.fr), Jean-Baptiste Chene (CSTB, 84, Avenue Jean-Jaurès, 77447 Marne-la-Vallée Cedex 2, France, jean-baptiste.chene@cstb.fr)

The French thermal regulation (RT2005) is favoring thermal floating floor between spaces such as parking or stores, and dwelling units. However, these solutions do not fulfill the French acoustic regulation with regards to airborne noise. This paper discusses the development of solutions allowing fulfilling both the thermal and the acoustic regulation. To achieve this goal, a mixed approach combining measurements and numerical predictions is used. Furthermore, the laboratory characterization of such floating systems usually involves a concrete base floor $140 \mathrm{~mm}$ in thickness (following the NF EN 140-8 standard) and a floating concrete layer $40 \mathrm{~mm}$ in thickness. However, in situ the concrete base floor as well as the floating concrete layer can be thicker. Therefore, some laboratory measurements are performed on a thermal floating floor system combining a $200 \mathrm{~mm}$ thick concrete base floor, a thermal insulation layer (polystyrene or polyurethane based foam for example) and a $60 \mathrm{~mm}$ thick floating concrete layer. The prediction method shows that this type of thermal floating systems is not acceptable with respect to the acoustic regulation. The behaviour of such multilayered systems is investigated using a wave approach based prediction tool in order to develop solutions allowing fulfilling the acoustic regulation. 


\title{
Session 5aNSd
}

\section{Noise, ASA Committee on Standards, Architectural Acoustics, and EURONOISE: Classroom Acoustics I (Poster Session)}

\author{
Louis Sutherland, Cochair \\ Consultant in Acoustics \\ Luigi Maffei, Cochair \\ Built Environment Control Laboratory Ri.A.S., Second University of Naples
}

\begin{abstract}
All posters will be on display from 9:40 a.m. to 11:20 a.m. To allow contributors an opportunity to see other posters, contributors of odd-numbered papers will be at their posters from 9:40 a.m. to 10:30 a.m. and contributors of even-numbered papers will be at their posters from 10:30 a.m. to 11:20 a.m.
\end{abstract}

\section{Contributed Papers}

5aNSd1. Comparisons of predicted and measured values of speech, noise, reverberation and early reflections in optimized classrooms. Sooch San Souci (AiA - Audition, Intelligibility, Acoustics, 35 bis rue de Fay, 77140 St Pierre Les Nemours, France, s.sansouci@wanadoo.fr), Line Guerra (AiA - Audition, Intelligibility, Acoustics, 47 rue Le Corbusier, 92100 Boulogne, France, line.guerra@aiacoustique.com), Richard Campbell (Bang-Campbell Associates, 26-G Chilmark Drive, East Falmouth, MA 02536, USA, rhcamp@ rcn.com)

12 newly renovated classrooms have been studied to determine the accuracy of prediction software used to guide architects, acousticians and interior designers. Several variations in renovation techniques based on traditional precepts were compared. After the renovations were complete, measurements and recordings were made of speech, noise, and reverberation when the rooms were both occupied and nonoccupied by students. A systematic map of the results was compiled, which when presented on a seat by seat basis, defined values of merit for the presumed optimizations and were compared to the predicted values. The two principle indices chosen as targets were increases in total speech level and decreases in total noise level (including late reverberation). When referred to these targets, several novel strategies proved to offer higher value/cost ratios while a few traditional strategies proved to be deficient and in some cases detrimental to at least one of the principle indices. Emerging from this study is what appears to be evidence for two points: (1) that some common acoustic criteria are inadequate for classrooms and (2) there are several strategies that might offer significant advantages over some of the traditional guidelines for acoustic treatments for learning spaces.

5aNSd2. The use of U50 to calculate the optimal absorption in a classroom. Lau Nijs (Delft University of Technology, Berlageweg 1, 2628 BG Delft, Netherlands, L.Nijs@tudelft.nl), Monika Rychtarikova (Lab. ATF, Katholieke Universiteit Leuven, Celestijnenlaan 200D, B-3001 Leuven, Belgium, Monika.Rychtarikova@bwk.kuleuven.be), Gerrit Vermeir (Lab. ATF, Katholieke Universiteit Leuven, Celestijnenlaan 200D, B-3001 Leuven, Belgium, Gerrit.Vermeir@bwk.kuleuven.be)
Common measures for the speech intelligibility predict increasing values with increasing absorption in empty classrooms. Measurements confirm this relation, so it seems as if an anechoic room is the best place to teach. However, there are two problems: the sound pressure level at the back row is too low when compared to the levels on the front row or when compared to the noise level caused by pupils plus ventilation systems. A simple method is proposed based on U50, which gives the relation between the sound energy from direct sound (from a teacher or a pupil) plus early reflections on one side and late reflections plus noise on the other. Barron's formula for the sound level decrease in a concert hall is included to predict the sound level drop through the classroom. Model results indicate that the absorption coefficient should be in the order of 20 to $30 \%$ if the signal to noise ratio is in the range between +5 to $-5 \mathrm{~dB}$. These values seem low, but measurements in existing classrooms show low signal to noise levels as well.

5aNSd3. The Nordic voice ergonomic group: legislation and vocal health. Valdis Inigbjörg Jonsdottir (Tad er Malid, Furuvellir 13, 601 Akureyri, Iceland, valdisj@ismennt.is)

Research has revealed a number of factors which have a negative impact on voice function (e.g., activity noise, background sound, poor acoustics, long duration of voice usage, speaking distance). In these circumstances vocal loading increases, potentially giving rise to voice disorders. Currently, practical measures within society for the improvement of speakers' working conditions and the prevention of voice loading are not satisfactory. Voice experts from the five Nordic countries met in Reykjavik in May 2006 to inaugurate the Nordic Voice Ergonomic Group, with the aim of investigating the current situation within voice ergonomics, with a particular focus on sound-related problems. The group found that occupational safety and health legislation relating to speech communication in educational working environment differs between Nordic countries. Moreover, this legislation does not specifically deal with speech communication or the risk of voice disorders. This presentation will report on the details of the current legislation and the Group's suggestions for legislative change which might prevent disturbance in speech communication and decrease the risk of vocal disorder as an occupational hazard. 


\title{
Session 5aNSe
}

\section{Noise and EURONOISE: Examples of Noise Control I (Poster Session)}

\author{
Brigitte Schulte-Fortkamp, Cochair \\ TU Berlin, Institute of Fluid Mechanics and Engineering Acoustics \\ Pierre Etienne Gautier, Cochair \\ SNCF DIR
}

\begin{abstract}
All posters will be on display from 9:40 a.m. to 11:20 a.m. To allow contributors an opportunity to see other posters, contributors of odd-numbered papers will be at their posters from 9:40 a.m. to 10:30 a.m. and contributors of even-numbered papers will be at their posters from 10:30 a.m. to 11:20 a.m.
\end{abstract}

\section{Contributed Papers}

5aNSe1. Jet noise prediction using RANS CFD input. Magdi Omais (Airbus/ONERA, 64 avenue de Paris, 92320 Châtillon, France, magdi.omais@onera.fr), Stephane Redonnet (ONERA (French aerospace Center), CFD \& Aeroacoustics Department, BP 72, 29 avenue de la division Leclerc, 92322 Chatillon Cedex, France, stephane.redonnet @ onera.fr), Bastien Caruelle (Airbus S.A.S, Department of Acoustic \& Environment, 316 route de Bayonne, 31000 Toulouse, France, bastien.caruelle@airbus.com), Eric Manoha (ONERA (French aerospace Center), CFD \& Aeroacoustics Department, BP 72, 29 avenue de la division Leclerc, 92322 Chatillon Cedex, France, eric.manoha@onera.fr)

In the present work, a volume source model based on the SNGR approach, first proposed by Bailly, has been implemented and developed for jet noise prediction purposes. This method presenting the advantage of providing unsteady three-dimensional turbulent data from a RANS computation, it shall be compatible with industrial development processes. First, the theoretical aspects of the original SNGR model were revisited. More particularly, analytical developments showed that considerable modifications were needed to take local flow effects into account while generating unsteady turbulent data. Moreover, the way to include anisotropy effects in the model, as originally proposed by Billson, was improved by using a nonlinear extension of Boussinesq approximation to recover the local Reynolds stress tensor. In a second step, the potentialities of the stochastic tool for jet noise simulations have been evaluated against existing numerical and experimental databases. In addition to that concern, its sensitivity to a slight modification of the RANS input data was studied. As a last step, the possibility of accounting for high frequency dynamics in reasonable CPU times has been deeply investigated. The main results obtained from this study show that the stochastic approach should be a promising method for future applications to industrial nozzle configurations.

5aNSe2. The primary noise sources associated with turbulent Coanda wall jets. Caroline Lubert (James Madison University, Department of Mathematics \& Statistics, MSC 1911, Harrisonburg, VA 22807, USA, lubertcp@jmu.edu)

A wide variety of aeronautical and aerospace applications utilize the Coanda effect. However, due to the enhanced turbulence levels observed with devices using this effect, turbulent mixing noise is a primary noise source. For supersonic jets close to Coanda surfaces, shock-associated noise (both broadband and screech tones) is also a significant source of sound. A theory has been developed to predict the turbulent mixing noise emitted by unit-volume of jet-type shear-layer turbulence close to a rigid plane, and extended to a plane two-dimensional wall-jet. However, most flows of practical interest are three-dimensional and, in the case of the Coanda jets, exhibit significant curvature. This paper extends the previous models for turbulent mixing noise to predict the aeroacoustic characteristics of a three- dimensional turbulent flow over a Coanda surface. Details of experimental observations of the shock-cell structure associated with supersonic Coanda wall jets, and a method of modeling the corresponding shock-associated noise, is also discussed.

5aNSe3. Efficiency of shielding effect on installed double stream nozzles. Sébastien Aeberli (SNECMA - groupe SAFRAN, Villaroche Rond-point R. Ravaud, 77550 Moissy-Cramayel, France, sebastien.aeberli @ snecma.fr)

In spite of acoustic improvements on jet noise brought by innovative technologies such as chevrons, a way to ensure important acoustic gains is to optimize the power plant integration on aircrafts in order to benefit of the shielding effect due to wing, empennage and fuselage surfaces. In this presentation, these effects are illustrated thanks to a jet noise test campaign at 1/10th scale in an anechoic chamber as part of a European project. This campaign gives a better understanding of shielding effect by a wing and also of the acoustic impact of pylon azimutal direction on two representative BPR 9 nozzles (Baseline - smooth - and Low Noise - with chevrons). The efficiency of shielding effect on jet noise sources in these configurations is assessed.

5aNSe4. Automatic segmentation of traffic noise. Fernando José Mato-Méndez (Universidad de Vigo, E.T.S.I.Telecomunicación C./ Maxwell S/N, 36200 Vigo (Pontevedra), Spain, fmato@tsc.uvigo.es)

The state of the art in audio source separation reflects a growing interest towards developing tools oriented to speech and music applications. However, its application to the study of noise, and more specifically to the study of traffic noise, is scarce. The source separation in this work environment is a key process for subsequent classification and analysis, and has motivated the present research carried out. This paper is intended to present, therefore, the different strategies followed in the implementation of an automatic segmentator for traffic noise, focusing the research on two main aspects: The removal of components of noise unrelated with the sources of study and, by other side, the detection and separation of audio sources acquired. For this purpose, measures of traffic noise are made and results are analyzed for the running system, achieving a high level of performance in real work conditions.

5aNSe5. Real time evaluation of soft microphones on a local active noise control system. Stelios M. Potirakis (Intracom Defense Electronics, $21 \mathrm{~km}$ Peania-Markopoulo Street, GR-19400 Koropi - Attica, Greece, spoti@intracomdefense.com), Maria Rangoussi (T.E.I. Piraeus/Department of Electronics, 250, Thivon str., GR-12244 Athens-Aigaleo, Greece, 
mariar@teipir.gr),Stylianos Savvaidis(T.E.I. Piraeus/Department of Electronics, 250, Thivon str., GR-12244 Athens-Aigaleo, Greece, ssavaid@teipir.gr), Nikos Zafeiropoulos (T.E.I. Piraeus / Department of Electronics, 250, Thivon str., GR-12244 Athens-Aigaleo, Greece, nikoszafeiropoulos@yahoo.gr)

Systems for the active control of acoustic noise (ANC) rely on measurements of the noise signal in order to produce the required "quiet zone." Soft microphones (SM) are a virtual microphone alternative already proposed by the authors, employing estimates rather than measurements of the noise signal obtained from room impulse responses based on measurements. Therefore, a single microphone can be used to estimate the noise signal at multiple points. This has already been successfully applied on single microphone noise mapping. In the present work, the SM method is evaluated on a real time local ANC system. The proposed scheme is a filtered-s least mean square (FXLMS) based control structure, incorporating SM. Following the proposed system design-simulation with Matlab-Simulink, a three-dimensional enclosure (office-simulating) and a real electroacoustic ANC system based on a TI ${ }^{\circledR}$ TMS320C6713 DSP, developed for this purpose, were built in the laboratory to carry out the experiments. Different options of estimating the SM signals and exploiting them into the control structure are experimentally evaluated as to their ability to enlarge the silence zone and their noise reduction performance around the listener ear zone (using a head and torso simulator) and compared to the standard FXLMS solution.

5aNSe6. Application examples of beamforming method. Andrea Cerniglia (Euroacoustic srl, via Gandhi, 13, 10013 Avigliana, Italy, a.cerniglia@euroacoustic.com)

Acoustical beamfoming technique is a powerful method for noise source investigation, suitable for many different applications. Thanks to this technology is possible to identify where the noise is coming from, in a very clear and understandable form, both in static or dynamic situations. In addition on standard features, some advanced functions can helps to make correlation between subjective and objective parameters, for better understanding of acoustical phenomena. The paper describes some real applications of a very innovative beamforming system, based on a spherical antenna. The described measurements coming from several fields as automotive, enviromental noise, occupational noise, architectural acoustic, noise barriers investigation.

FRIDAY MORNING, 4 JULY 2008

P2-A, LEVEL 2, 9:40 TO 11:20 A.M.

\title{
Session 5aNSf
}

\section{Noise and EURONOISE: Soundscape \& Community Noise II (Poster Session)}

\author{
Bennett Brooks, Cochair \\ Brigitte Schulte-Fortkamp, Cochair \\ TU Berlin, Institute of Fluid Mechanics and Engineering Acoustics
}

\begin{abstract}
All posters will be on display from 9:40 a.m. to 11:20 a.m. To allow contributors an opportunity to see other posters, contributors of odd-numbered papers will be at their posters from 9:40 a.m. to 10:30 a.m. and contributors of even-numbered papers will be at their posters from 10:30 a.m. to 11:20 a.m.
\end{abstract}

\section{Contributed Papers}

5aNSf1. The influence of meteorological conditions on noise propagation outdoors and sustainable planning of roads and motorways. Barbara Joanna Lebiedowska (Warsaw University of Technology, ul. Lukasiewicza 17, 09-400 Plock, Poland, barbara_lebiedowska@yahoo.fr)

Adequate road planning that allows for the separation of built-up areas and travel routes is one of the basic prevention strategies of hazards resulting from road usage. If possible, the roads should never be routed across cities and residential districts. However, if for some reason a busy road must be located near a built-up area, long-term meteorological conditions, characteristic of the area, must be examined prior to the execution of any work. Road planning on the basis of the analysis of the "rose of conditions favourable" to noise propagation will help minimise the road's negative acoustic influence on the adjacent buildings and facilities. The acoustic profile of two selected sites (in Poland and in France) is examined in the paper. Conditions favourable to propagation differ significantly in both cases depending on the position of the source-to-receiver direction in relation to the directions of the world

5aNSf2. Community response to military shooting noise immissions Mark Brink (ETH Zürich, Center for Organizational and Occupational Sciences, LEO B 9.1, 8092 Zürich, Switzerland, brink@ethz.ch), Jean Marc Wunderli (EMPA - Materials Science and Technology, Ueberlandstr. 129, CH-8600 Duebendorf, Switzerland, jean-marc.wunderli@empa.ch), Hans Boegli (Federal Office for the Environment, BAFU, 3003 Bern, Switzerland, hans.boegli@bafu.admin.ch)
The assessment of the impact of any kind of noise exposure on the population is a fundamental step in the process of noise abatement. Data about the exposure-response relationship for annoyance due to noise emissions from military shooting grounds are rather scarce at the time. In order to establish new legal exposure limit values for shooting noise from heavy and light weapons, we conducted an extensive telephone interview survey in several - mostly rural - municipalities affected by military shooting noise in Switzerland. The exposure assessment for each respondent was calculated based on the source models of a range of weapons and ammunitions of the Swiss army. Preliminary results suggest that although the responses of the population to noise from military weapons are rather dispersed, data should be sufficiently consistent to establish an exposure-response relationship which can help in defining a binding exposure limit value.

5aNSf3. Investigation of traffic noise from additional tolling lanes at Portage toll plaza. Silas Bensing (Columbia College Chicago, 370 Millport Drive, Valparaiso, IN 46385, USA, silas.bensing@loop.colum.edu)

Electronic toll collecting systems quickly and efficiently move traffic through toll facilities by automatically collecting tolls from individual vehicle transponders. An ongoing study investigates the addition of three electronic toll lanes to the existing twelve lanes at the Portage toll plaza on the Indiana Toll Road (Portage, Indiana, USA), scheduled to be completed by December 2008. The study aims to predict noise levels of the plaza after the addition of the lanes, the hypothesis being that the additional lanes will have little or no effect on the toll plaza noise levels. Noise measurements are be- 
ing made at locations in the vicinity of the existing plaza with consideration for a nearby residential area. An outdoor noise propagation software (SoundPlan) is used to create a comprehensive traffic noise model for the area under investigation. Test data from the existing site will be compared to the model and used to predict future noise levels.

5aNSf4. Assessment of military shooting noise. Hans Boegli (Federal Office for the Environment, BAFU, 3003 Bern, Switzerland, hans.boegli @bafu.admin.ch), Jean Marc Wunderli (EMPA - Materials Science and Technology, Ueberlandstr. 129, CH-8600 Duebendorf, Switzerland, jean-marc.wunderli@empa.ch), Mark Brink (ETH Zürich, Center for Organizational and Occupational Sciences, LEO B 9.1, 8092 Zürich, Switzerland, brink@ethz.ch)

The assessment of the impact of noise exposure on the population is a fundamental step in noise abatement. It includes the establishing of an exposure-response relationship and the setting of an impact threshold that specifies the protection level for the population and triggers eventually mitigating measures to reduce noise exposure. In Switzerland, the impact thresholds should be set so that, in the light of current scientific knowledge and experience, noise exposure below these thresholds will not seriously disturb the well-being of the population. For most current noise sources such as roads, railways and airports there already exist impact thresholds as part of the noise abatement legislation. Yet, no impact thresholds for military shooting grounds have been specified so far. Therefore a study was carried out in order to assess the impact of military noise exposure. The research included the calculation of noise exposure of eight military shooting grounds ranging from small infantry shooting ranges to expanded artillery and tank training facilities and a survey at over 1000 residents in the neighbourhood of these installations. Preliminary results suggest that although the responses of the population to military noise are rather dispersed, data should be sufficiently consistent to establish an exposure-response relationship.

5aNSf5. Design and evaluation of novel barriers. George Bamnios (Electronics Dept. ATEI of Thessaloniki, P.O. Box 141, Sindos, $57400 \quad$ Thessaloniki, Greece, bamnios@el.teithe.gr), Ioannis Rekanos (Physics Devision, Dept. of Mathematics, Physics and Computational Sciences, School of Engineering, Aristotle Univ. of Thessaloniki Campus, 54124 Thessaloniki, Greece, rekanos@auth.gr), Konstantinos Zacharias (Physics Devision, Dept. of Mathematics, Physics and Computational Sciences, School of Engineering, Aristotle Univ. of Thessaloniki Campus, 54124 Thessaloniki, Greece, zachark@gen.auth.gr), Athanasios Trochidis (Physics Devision, Dept. of Mathematics, Physics and Computational Sciences, School of Engineering, Aristotle Univ. of Thessaloniki Campus, 54124 Thessaloniki, Greece, trohidis@gen.auth.gr)

This work presents the results of a project for the design of novel noise barriers with improved performance. The aim of the project was twofold. First, to optimize the performance of barriers by appropriately modifying both the shape and the acoustical conditions at the edge to suppress the sound pressure. Second, to quantify the acoustic performance of the novel barriers both mathematically and experimentally in a standardized, well controlled process. The insertion loss of different types of barriers with modified edge shapes and acoustical conditions were investigated systematically in comparison with conventional ones using a method based on BEM. The comparison highlighted the influence of shape and edge configuration on barrier efficiency and allowed the design of novel barriers with optimal performance. The novel barriers were tested and their efficiency was quantified experimentally using the Maximum Length Sequence (MLS) technique, which offers the advantage of in-situ measurements in the presence of extraneous noise. The experimental results are shown to be in good agreement with the experiments. Based on the aforementioned procedure, a unified framework was established, witch allows the design of potentially improved noise barriers.
5aNSf6. Nuisance control of leisure activities. Robert Barti(LEM Laboratory, Pol. Ind. Cova Solera, Av. Can Sucarrats, 110 nau 11, 08191 Rubi, Spain,robert@lem-sl.com)

In Spain there are more than 345000 leisure activities with more than $1.2 \mathrm{M}$ jobs. This economical sector produces the $2.2 \%$ of the PIB overall Spanish economic production. Tourism is a big source of money for many Spanish cities. Leisure activities is the most common economic activity related with this sector. Typical Spanish good weather let's people to remain for a long time up to early morning in the streets. Human activity produces always noise. Acoustic noise control measurements uses $d B(A)$ scale for evaluation. Low noise levels $\mathrm{dB}(\mathrm{A})$ has no correlation with acoustic perception of people because people can "follow" the rhythm of the music. Increase the TL of structures let's to reduce the noise from activities, but increasing the power amplifier the problem remains still in the same point. The solution checked is to use an acoustic limiter as a warranty of no contamination to neighbours from the activity. Some results about this technique are discussed in this paper. In order to solve a complain between neighbours an leisure activity, a political solution is more effective than technical solution is. The main objective is to set the correct balance between perception of sounds and acoustic comfort at home.

5aNSf7. Perspectives for a strategical mapping of soundscapes. Gianluca Memoli (Imperial College London, Department of Chemical Engineering, Exhibition road, SW7 2AZ London, UK, g.memoli@imperial .ac.uk), Gaetano Licitra (ARPAT - Dept. Firenze, Via Porpora, 22, 50144 Firenze, Italy, g.licitra@arpat.toscana.it), Mauro Cerchiai (ARPAT - Dept. Pisa, Via V. Veneto, 27, 56127 Pisa, Italy, m.cerchiai@arpat.toscana.it)

The transposition of the European Noise Directive by Member States gives a new way to action plans of urban areas, underlying (article 6 and Annex III) that dose-effect relations should be used to assess the effect of noise on populations. Particular interest, in this sense, has the relationship between annoyance and perceived soundscape: it is now almost accepted that a higher annoyance is not always proportional to higher noise levels. This paper will demonstrate that a way to move from mapping noise exposure to a map of annoyance passes through the characterization of the soundscapes present on the territory. A numerical indicator ("slope"), derived from the time history of the measured sound/noise, will in fact be used to characterize the evolution along the day of the soundscapes present in the city of Pisa (I). In particular, "slope" (correlated to annoyance in previous studies) will be used to prepare a map of the annoyance in selected areas of Pisa: predicted annoyance will be to be compared with the one extrapolated by noise levels. Differences, similarities and their effects on action plans will be discussed.

5aNSf8. Analysis and evaluation of noise reaction in open public spaces in Mexico City. Miriam German (Universidad Nacional Autonoma de Mexico-Facultad de Arquitectura, Circuito Interior S/N, Ciudad Universitaria, Delegacion Coyoacan, 02410 Mexico D.F., Mexico, lydmgg@yahoo .com.mx)

Mexico City, with a population estimated in 19 millions, is part of one of the largest metropolitan areas in the world. Unfortunately, scientific work on urban noise in Mexico City is scarce. A study on people reaction to urban noise carried out in open public spaces of two different zones of Mexico City will be presented. A previous survey in those zones showed that the noise levels exceeded the values recommended by international organizations to protect public health and welfare. The general objective of the study to be presented was to identify the sound sources perceived by the pedestrians, and to evaluate their response to urban noise pollution in the two mentioned areas. The results show that for most of the people (a) urban noise is not considered a relevant aspect to improve the quality in the city, although urban noise has a negative effect on the decision of using open public spaces; (b) the people with knowledge of the negative effects of noise pollution are more annoyed; (c) vehicular traffic is the most annoying noise source, contributing significantly the public transportation; (d) one of the principal strategies to confront urban noise is to get used to it. 


\title{
Session 5aNSg
}

\author{
Jérôme Defrance, Chair \\ $C S T B$
}

\section{Noise and EURONOISE: Propagation and Urban Noise I (Poster Session)}

\begin{abstract}
All posters will be on display from 9:40 a.m. to 11:20 a.m. To allow contributors an opportunity to see other posters, contributors of odd-numbered papers will be at their posters from 9:40 a.m. to 10:30 a.m. and contributors of even-numbered papers will be at their posters from 10:30 a.m. to 11:20 a.m.
\end{abstract}

\section{Contributed Papers}

5aNSg1. Perception of noise in suburban and urban areas. Elaine $\mathrm{C}$. Paz (Federal University of Parana, Centro Politécnico - Setor de Tecnologia, Bairro Jardim das Américas, 81531-990 Curitiba, Brazil, epaz@bol.com .br), Paulo Henrique Trombetta T. Zannin (Federal University of Parana, Centro Politécnico - Setor de Tecnologia, Bairro Jardim das Américas, 81531-990 Curitiba, Brazil, paulo.zannin@pesquisador.cnpq.br)

This work analyses, using a comparative approach, the environmental noise perception in the daily lives of inhabitants of a residential area, with that of a mixed (residential and commercial) area (downtown), in a large Latin America city. The goal was to confront an ideal urban environment with one acoustically polluted, as function of noise descriptors. Concomitant with the evaluation of noise perception (subjective analysis), noise levels were measured (objective analysis) in both areas. The single average equivalent noise level (Leq) found for downtown was $73 \mathrm{~dB}(\mathrm{~A})$, and $53 \mathrm{~dB}(\mathrm{~A})$ for the residential area. A random sample of the populations of both areas was taken, through a questionnaire. Questionnaire data was treated statistically through a factorial multivariate analysis. This analysis has generated three statistical indicators: time perception, atypical noise perception, and sources and disturbances. Over $50 \%$ of both populations sampled have the perception that high frequency noise levels are gradually increasing, with higher contribution from traffic noise. The dominant organic effects reported were irritability and loss of concentration ability, these last being precursors of hearing loss.

5aNSg2. Traffic noise in shielded urban areas: comparison of experimental data with model results. Ando Randrianoelina (TNO Science and Industry, Stieljesweg 1, 2628CK Delft, Netherlands, ando.randrianoelina@tno.nl), Erik Salomons (TNO Science and Industry, Stieljesweg 1,2628CK Delft, Netherlands, erik.salomons@tno.nl)

Noise maps of cities are commonly produced with rather simple engineering models for sound propagation. These models may be inaccurate in complex urban situations, in particular in situations with street canyons. Street canyons are urban areas that are partly or completely enclosed by buildings, for example a street between two buildings or a backyard surrounded by buildings. In this paper we study sound propagation from a city bus in a street canyon to a receiver in a nearby street canyon. Multiple reflections of sound waves in both the source canyon and the receiver canyon play an important role in the sound propagation. Experimental data are compared with three types of model results: (i) results of a numerical boundary element model, (ii) results of a 1:30 scale model experiment in a semianechoic room, and (iii) results of the Dutch standard engineering model, which is similar to the international standard ISO 9613-2. The data are in reasonable agreement with model results (i) and (ii), while the engineering model yields sound levels that are about $10 \mathrm{~dB}$ too low.

5aNSg3. Reflection of sound from a forest: comparison of experimental data to scattering theory. Erik Salomons (TNO Science and Industry,
Stieljesweg 1, 2628CK Delft, Netherlands, erik.salomons@tno.nl),Jean Marc Wunderli (EMPA - Materials Science and Technology, Ueberlandstr. 129, CH-8600 Duebendorf, Switzerland, jean-marc.wunderli@empa.ch)

Reflection of sound by a forest has been investigated by means of measurements near Thun in Switzerland. Sound pulses were generated at 50 and $150 \mathrm{~m}$ from the forest, and reflected sound was recorded at distances of 100 , 200 , and $300 \mathrm{~m}$ from the forest. By comparison with numerical calculations of reflection of sound from a hard wall, using a parabolic-equation model for sound propagation in an atmosphere with wind and temperature as recorded during the measurements, the attenuation of sound waves upon reflection at the forest was determined. It was found that the reflection attenuation decreases from about $20 \mathrm{~dB}$ at low frequency to about $10 \mathrm{~dB}$ at high frequency. The experimental results for the reflection attenuation are compared with results of a theoretical model for scattering of spherical sound waves by a single row of cylinders. The model employs an effective spacing between the cylinders, to account for trees that are not at the edge of the forest, i.e., inside the forest. The model results are in good agreement with the experimental results. The comparison indicates that the effective spacing decreases with decreasing frequency, as low-frequency waves penetrate deeper into a forest than high-frequency waves do.

5aNSg4. Reflection of sound from a forest: Effective tree spacing for a scattering model with a single line of cylinders. Jean Marc Wunderli (EMPA - Materials Science and Technology, Ueberlandstr. 129, CH-8600 Duebendorf, Switzerland, jean-marc.wunderli@empa.ch), Erik Salomons (TNO Science and Industry, Stieljesweg 1, 2628CK Delft, Netherlands, erik.salomons@tno.nl)

A scattering model for the reflection of sound at forest rims is presented by the authors in another contribution to this conference. The forest is thereby modeled by a single row of cylinders with the effective spacing as single free parameter. In the present article an engineering scheme for this effective tree spacing is presented. The scheme is based on results of numerical calculations with the Euler model for sound propagation in a model forest consisting of cylinders. The numerical results show that forests are widely transparent for incoming as well as reflected sound waves. Multiple reflections can be neglected. Reflections from trees deep inside the forest experience additional propagation attenuation and are therefore weakened relative to contributions from trees in the first row. Apart from geometrical spreading foliage attenuation is the most prominent effect. The penetration depth of the forest reflection is proportional to the wavelength. The frequency dependent penetration depth is derived by means of a statistical analysis for numerous situations. Assuming the effective tree spacing to be inversely proportional to the penetration depth, the engineering scheme for the effective tree spacing is derived. Comparisons of the frequency response with measurements show good agreement. 


\title{
Session 5aNSh
}

\section{Noise, Structural Acoustics and Vibration, Physical Acoustics and EURONOISE: Sound and Vibration from Explosions in Air I (Poster Session)}

\author{
Roger Waxler, Cochair \\ University of Mississippi \\ Keith Attenborough, Cochair \\ Open University
}

\begin{abstract}
All posters will be on display from 9:40 a.m. to 11:20 a.m. To allow contributors an opportunity to see other posters, contributors of odd-numbered papers will be at their posters from 9:40 a.m. to 10:30 a.m. and contributors of even-numbered papers will be at their posters from 10:30 a.m. to 11:20 a.m.
\end{abstract}

\section{Contributed Papers}

5aNSh1. Environmental noise reduction means of weapons. Rauno Pääkkönen (Finnish Institute of Occupational Health, P.O. Box 486, 33101 Tampere, Finland, rauno.paakkonen@ttl.fi)

Noise from hand-held weapons is generated by their muzzle blast, the noise of the bullet in flight and on impact. The muzzle blast of almost all powder guns exceeds $140 \mathrm{~dB}$ near the ear of the shooter, which is considered to be able to generate a risk of hearing defects. The muzzle brake decreases recoil, but at the same time it forces the sound sideways and backwards. One novel aspect of noise attenuation is the use of active noise cancelling system at shooting ranges and in hearing protectors. The noise data has been collected on different shooting ranges in Finland. Both front and reflection types of suppressors were used. Silencers that attenuate noise to the sides and back have a significant effect, where as the noise to the front is not significantly attenuated because of the bullet noise. It is also possible to reach an attenuation of a few decibels of the maximum level of the muzzle blast with ANC headsets. The noise created by shotguns is the most significant source of environmental noise at many shooting ranges. It would be especially important to attain new ideas and innovations in the noise prevention of shotgun ranges.

5aNSh2. The development of a bespoke gunfire noise model for a military training area and the vibration impacts through the air on ancient structures. Graham Parry (ACCON UK Limited, Unit B, Fronds Park, Frouds Lane, Aldermaston, RG7 4LH Reading, UK, graham.parry @accon-uk.com)

This paper describes the development of a bespoke weapons system noise model known as the gunfire noise analysis tool (GNAT). The requirements for such a model came out of an expansion of military training within a National Park and the need to demonstrate that noise impacts could be modeled with reasonable accuracy out to distances of $10 \mathrm{kms}$ and without the need for specialist acousticians or meteorologists to run the model. Additionally, the requirement was to ensure that noise modeling could be carried out within a matter of minutes thus allowing military training decisions to be made while protecting sensitive receptor locations from very high noise levels. The model utilised best practice and acoustic theory available at the time within the specific requirements of noise modeling outlined above. The model was validated against a number of noise measurements exercises carried out using controlled military noise events and for a live military exercise.
5aNSh3. Transducers for acoustical impulse measurements. Per Rasmussen (G.R.A.S. Sound \& Vibration A/S, Skovlytoften 33, 2840 Holte, Denmark, pr@gras.dk)

The measurement of impulsive noise signals as for example from gunshots or airbag deployments requires special considerations when selecting transducer. The high frequency content in short impulses requires high bandwidth to correctly capture high rise times and small dimensions of the transducer to avoid diffraction. For high level impulses with very rapid rise time, the wide frequency range requires the use of preamplifiers with high slew rate capabilities and the capacitance and length of cables must be considered carefully. Slew rate limitations and bandwidth limitations can drastically change the peak value of an impulse. It is thus necessary to consider the whole measurement chain from transducer to $\mathrm{AD}$-converter to ensure the correct measurement of the impulse.

5aNSh4. Large weapon source emission data protocols and results. Michael James (Blue Ridge Research and Consulting, 13 1/2 W. Walnut St., Asheville, NC 28801, USA, michael.james@blueridgeresearch .com), Micah Downing (Blue Ridge Research and Consulting, 13 1/2 W. Walnut St., Asheville, NC 28801, USA, micah.downing@blueridgeresearch .com), Pater Larry (U.S. Army Engineer Research and Development Center, 2902 Farber Drive, Champaign, IL 61821, USA, Larry.L.Pater@erdc .usace.army.mil)

The U.S. Army needs detailed information about the blast pressure field created by large weapons to avoid negative impacts on testing and training mission capabilities due to noise. The noise software tools BNOISE2 ${ }^{\mathrm{TM}}$ and RMTK Noise Tool, which the Department of Defense (DoD) uses to predict and assess impacts of large weapon noise, require accurate acoustic source emission models. A free-field representation of the sound source acoustical emission, containing no waveform signal perturbations due to the ground, other reflecting surfaces, ballistic waves, or propagation anomalies, is required. The measurement protocols have been developed to collect the free-field source emission data. These protocols are described along with acoustical data and emission source results for the $120 \mathrm{~mm} \mathrm{M-1} \mathrm{tank} \mathrm{gun,}$ the $105 \mathrm{~mm}$ Stryker gun, and various weights of C-4 explosives. In addition, the measured acoustic data are compared with an idealized Friedlander curve. The overall results demonstrate that the protocols do provide sufficient free-field blast waveforms. 


\title{
Session 5aNSi
}

\section{Noise and EURONOISE: Acoustic Performance of Energy Efficient Building Products II (Poster Session)}

\author{
Anthony Hoover, Cochair \\ McKay Conant Brooks, Inc. \\ Jacques Roland, Cochair \\ Centre Scientifique et Technique du Bâtiment
}

\begin{abstract}
All posters will be on display from 9:40 a.m. to 11:20 a.m. To allow contributors an opportunity to see other posters, contributors of odd-numbered papers will be at their posters from 9:40 a.m. to 10:30 a.m. and contributors of even-numbered papers will be at their posters from 10:30 a.m. to 11:20 a.m.
\end{abstract}

\section{Contributed Paper}

5aNSi1. Roof-top urban wind turbines in London. Stephen Dance (London South Bank University, FESBE, Borough Road, SE1 0AA London, UK, dances@1sbu.ac.uk), Linda Liviani (RBA Acoustics, 104 The Foundry Annexe, 65 Glasshill Street, SE1 0QR London, UK, lindalive2 @ hotmail.com)

With the Mayor of London's office decision to allow planning permission only if $10 \%$ of the building's energy consumption is from renewable sources, wind turbines are starting to be erected on rooftops of residential blocks. A Proven $6 \mathrm{~kW}, 6 \mathrm{~m}$ horizontal axis turbine was positioned on an 11 story 1960s concrete construction block of flats in the summer of 2007. Noise and vibration measurements were taken on the roof-top and in the nearest flat. In addition, energy performance was monitored, wind speed and direction recorded and weather noted. Results confirm that central London is a $24 \mathrm{~h}$ city and, given the right conditions, the turbine generators meaningful amounts of electricity.

FRIDAY MORNING, 4 JULY 2008

ROOM 251, 11:00 A.M. TO 3:40 P.M.

\section{Session 5aNSj}

\section{Noise and EURONOISE: Examples of Noise Control II}

\author{
Karl Hirsch, Cochair \\ Cervus Consult, Kolpingstrasse 13, Wuerselen, D-52146, Germany \\ Pierre Etienne Gautier, Cochair \\ SNCF DIR, 45, rue de Londres, Paris, 75379, France
}

\section{Contributed Papers}

11:00

5aNSj1. Monitoring of noise reduction from traffic speed control. J Jabben (RIVM, A. van Leeuwenhoeklaan 9, 3720 BA Bilthoven, Netherlands, jan.jabben@ rivm.nl), C. Potma (RIVM, A. van Leeuwenhoeklaan 9 , 3720 BA Bilthoven, Netherlands, charlos.potma@rivm.nl)

In November 2005, at the Hague, Amsterdam, Rotterdam and Utrecht, a $80 \mathrm{~km} / \mathrm{u}$ speed limit was introduced on sections of motorways in the Netherlands. The aim was to evaluate the effects on noise, air quality and the circulation of the traffic. RIVM investigated the effects of noise emission by direct measurement at the sections with speed limit. This paper gives the results from continuous monitoring of noise levels that was started at July 2005 and continued up to November 2006. In the presentation the average measured effects on the equivalent noise emissions at the different sites will be given. Also the effects of traffic speed reduction on dwellings in the neighborhood of the motorways were evaluated using noise maps. Furthermore, apart from the average equivalent noise levels, the statistical noise indicators L5, L10, L70, and L95 were continuously monitored. It was found that speed reduction induces more reduction on maximum noise levels than on the average (equivalent) noise levels, probably due to a more steady and even distribution of traffic speeds.

\section{1:20}

5aNSj2. Automatic classification of traffic noise. Manuel A. Sobreira-Seoane (University of Vigo, E.T.S.I de Telecomunicación, Rúa Maxwell s/n, 36310 Vigo, Spain, msobre@gts.tsc.uvigo.es), Alfonso Rodríguez Molares (University of Vigo, E.T.S.I de Telecomunicación, Rúa Maxwell s/n, 36310 Vigo, Spain, amolares@gts.tsc.uvigo.es), José Luis Alba Castro (University of Vigo, E.T.S.I de Telecomunicación, Rúa Maxwell s/n, 36310 Vigo, Spain, jalba@gts.tsc.uvigo.es)

When modeling a city or a secondary road to calculate a noise map, the information about the number of heavy/light vehicles and the average speed it is not always available. In this paper, a first approach to get an automatic classification of vehicles is presented. The system is based on the classifi- 
cation of the audio signal that a noise source produces. Some basic classifiers have been tested (k-nearest neighbours, FLD (Fischer linear discriminator) and principal components. As first approach, the aim of the job was to determine if the different classes (trucks, cars, and motorbikes) could be separable using different time and frequency characteristics: zero crossing ratios, spectral centroids, spectral rolloff, subband energies and mel frequency cepstral coefficients. The results shows that for some of the characteristics tested, the signals are separable, so a continuous traffic noise signal could be processed to get the information of the number of heavy trucks, cars, and motorbikes that passed by during the recording time. Information of a stereo recording could be used to get information of the direction of the vehicle. At this moment, combining three characteristics and FLD, errors bellow $9 \%$ can be reported.

\section{$11: 40$}

5aNSj3. Remarks on a blast sound propagation model for noise management purposes at military training areas. Karl Hirsch (Cervus Consult, Kolpingstrasse 13, D-52146 Wuerselen, Germany, hirsch@cervus .de)

Germany will introduce a daily shooting noise management at its military training areas. Roughly speaking, this management adopts the basic ideas of ISO $17201-5$ but uses in addition a rating scheme that allows 18 exceedance days per year $(5 \%)$. For management purposes, the underlying sound prediction scheme must support two decision (1) Whether or not, the noise load of the daily planned occupancy is within certain noise limits. (2) Whether or not, the daily changing occupancy is close to a low noise minimum for the requested training options. The propagation model must consider particular weather conditions and the terrain shielding to enable the operator to optimise the occupancy with respect to these important conditions on a daily basis. The prediction must be fast enough to run several alternatives within minutes. Therefore, the prediction could not base on a sophisticated physical propagation model but must be done along a reasonable technical scheme. This paper discusses a ray tracing approach that is expected to substitute the currently in-use formula based on fitted empirical parameters. This model will at least provide one parameter to describe the sound refraction (radius of curvature) and a rule on how to estimate up wind propagation

\section{2:00}

5aNSj4. Simulation of highway traffic noise with utilization of equivalent sound level of pass-by vehicle. Pichai Pamanikabud (King Mongkut U. of Technology-Thonburi, Dept. of Civil Engineering, Faculty of Engineering, Pracha-u-tid Road, Thungkru, 10140 Bangkok, Thailand, pichai.pam@kmutt.ac.th), Marupong Tansatcha (Dept. of Public Works and Town \& Country Planning, Ministry of Interior, Rama 9 Rd., Huai-Khwang, 10320 Bangkok, Thailand, t_marupong@yahoo.com)

This paper presents study and building of a highway traffic noise simulation model by utilizing equivalent sound level over $20 \mathrm{~s}$ of passing-by vehicular noise in the analysis of nine types of vehicular basic noise. The basic noise of each vehicle type is applied into the formulation of the main highway traffic noise simulation model. The coefficient of propagation and ground effect for this model is also estimated and applied to the highway traffic noise model. This highway traffic noise model is then tested for its goodness-of-fit to field observations. The testing result shows that this model provides an effective prediction for highway noise in Thailand.

\section{2:20}

5aNSj5. Identification of noise sources in centrifugal blower with acoustic camera. Jurij Prezelj (University of Ljubljana, Faculty of Mechanical Engineering, Aškerčeva 6, 1000 Ljubljana, Slovenia, jurij.prezelj@fs.uni-lj.si), Mirko Čudina (University of Ljubljana, Faculty of Mechanical Engineering, Aškerčeva 6, 1000 Ljubljana, Slovenia, mirko .cudina@fs.uni-lj.si)

A centrifugal blower represents the main noise source in a vacuum cleaner. Noise emitted by the centrifugal blower working as a suction unit has aerodynamic, mechanical, and electromagnetic noise origins. Noise emitted by a suction unit consists of airborne and structure-borne noise. The contribution of the individual noise source to the total noise level depends on the geometry of the suction unit, rotational speed and on the operating conditions. Operating conditions of a suction unit depend on the amount of dust particles in the dust bag and are constantly changing during operation until a zero flow rate is reached. In order to reduce noise of suction unit at broad range of operating conditions, an identification of noise sources needs to be performed. Identification of most important noise sources on the centrifugal blower was performed with an acoustic camera at the design and off-design operation conditions. From the analyses we can conclude that the rotational noise usually prevails at the design point of operation, and that the nonrotational noise prevails at off-design operation. The main source of noise within a suction unit can be attributed to the aerodynamically generated noise, at the design as well as at off-design operation.

\section{2:40-2:00 Lunch Break}

\section{Contributed Papers}

\section{2:00}

5aNSj6. Buzz-saw noise : propagation of shock waves in aero-engine inlet ducts. Rasika Fernando (Institut Jean Le Rond d'Alembert, Université Pierre et Marie Curie, Boites 161 et 162, 4 place Jussieu, 75252 Paris Cedex 05, France, fernando@1mm.jussieu.fr), Régis Marchiano (Institut Jean Le Rond d'Alembert, Université Pierre et Marie Curie, Boites 161 et 162, 4 place Jussieu, 75252 Paris Cedex 05, France, marchi@lmm .jussieu.fr), François Coulouvrat (Institut Jean Le Rond d'Alembert, Université Pierre et Marie Curie, Boites 161 et 162, 4 place Jussieu, 75252 Paris Cedex 05, France, coulouvr@ccr.jussieu.fr), Yann Druon (Airbus S.A.S, Department of Acoustic \& Environment, 316 route de Bayonne, 31000 Toulouse, France, yann.druon@airbus.com)

When high bypass ratio aircraft engines run at takeoff operating conditions, blade relative flow velocities can exceed sonic speed, thus generating forward propagating shock waves that spiral inside the intake before being radiated. "Buzz-saw" or "multiple pure tone" noise then occurs, and measured acoustic spectra close to the fan display tones at the blade passing frequency and its harmonics, along with those of the engine shaft rotation frequency. This work first attempts to reformulate McAlpine and Fisher's frequency domain model for the propagation of a sawtooth waveform spi- ralling inside a hard-walled and lined cylindrical duct with uniform flow. The nondissipative Burgers equation is solved, and modal attenuation and dispersion are added using a split-step computational method. In practice, shocks do not only occur at blade tips, but on a significant portion of the blade span. The plane wave hypothesis being no longer valid, a new threedimensional model is presented for a no-flow case. This model is based on the computation of the axial variations of the mode amplitudes under the influence of the nonlinear modal interactions. The formalism of this new model, along with the preliminary results are also presented.

\section{2:20}

5aNSj7. Numerical simulation of aerodynamical noise generated by a cylindrical cavity. Daniel-Ciprian Mincu (ONERA (French aerospace Center), CFD \& Aeroacoustics Department, BP 72, 29 avenue de la division Leclerc, 92322 Chatillon Cedex, France, dmincu@onera.fr), Stephane Redonnet (ONERA (French aerospace Center), CFD \& Aeroacoustics Department, BP 72, 29 avenue de la division Leclerc, 92322 Chatillon Cedex, France, stephane.redonnet@onera.fr), Ivan Mary (ONERA (French aerospace Center), CFD \& Aeroacoustics Department, BP 72, 29 avenue de la 
division Leclerc, 92322 Chatillon Cedex, France, ivan.mary @ onera.fr), Lionel Larcheveque (Universite de Provence, 29, avenue Robert Schuman, 13621 Aix-en-Provence, France, lionel.larcheveque @ polytech.univ-mrs.fr), Eric Manoha (ONERA (French aerospace Center), CFD \& Aeroacoustics Department, BP 72, 29 avenue de la division Leclerc, 92322 Chatillon Cedex, France, eric.manoha@onera.fr)

Cylindrical cavities are mounted at some positions on fuselage and wings of commercial transport aircraft for various service functions. Such cavities were identified as potential airframe noise sources generating high intensity whistles at frequencies situated in the audible range of $0.5-0.8 \mathrm{kHz}$. The present paper focuses on the numerical simulation of the far field noise generated by the unsteady flow passing over a cylindrical cavity with an aspect ratio (diameter/height) equal to unity, using a numerical methodology combining a LES and a Kirchhoff integration method. The LES computation was done using ONERA's cell-center/finite-volume/CFD code named FLU3M. A laminar boundary layer profile was applied upstream the computational domain, without forcing any turbulence. The LES results were coupled with a 3D Kirchhoff method in the frequency domain. The computed far field noise was compared to dedicated experimental data, showing very good agreement, especially regarding the emission frequency. The final paper will present in detail the involved numerical methods, the computational procedure, and several comparisons to experimental data. This study was completed in the framework of the AEROCAV (AEROacoustique d'une CAVité cylindrique) project funded by the FRAE (Fondation Recherche Aéronautique et Espace).

\section{2:40}

5aNSj8. Efficient method for harmonics estimation of car engine sounds: application to high quality real-time synthesis. Stephane Molla (Genesis S.A., Bâtiment Gérard Mégie, Domaine du Petit Arbois BP 69, 13545 Aix-en-Provence Cedex 4, France, stephane.molla @ genesis.fr), Florent Jaillet (Genesis S.A., Bâtiment Gérard Mégie, Domaine du Petit Arbois - BP 69, 13545 Aix-en-Provence Cedex 4, France, florent.jaillet@gmail.com), Benoît Gauduin (Genesis S.A., Bâtiment Gérard Mégie, Domaine du Petit Arbois - BP 69, 13545 Aix-en-Provence Cedex 4, France, benoit.gauduin@genesis.fr)

Additive synthesis is the most popular method for the resynthesis of sounds containing slowly evolving harmonics or partials, such as car engine noise and more generally rotating machines sounds. The most important step lies in the estimation of the amplitude and frequency of each sinusoidal component. It appears that insufficiently precise estimation often leads to unsatisfactory results in terms of sound fidelity. A method providing the time and frequency evolution of the amplitude of the harmonics of a recorded sound is presented. This technique is based on a joint time and frequency chaining of amplitude's extrema of amplitudes coming from a short-time Fourier transform, using forward and backward estimation. The proposed method is compared to a rough estimation, and to an estimation based on measured tachometric data, all carried out over the same car engine sound. This estimation method is implemented into the LEA software, which thus allows fast and precise computations of the parameters. Moreover, it also helps in estimating the frequency content of the "background" noise, which depends on the speed, allowing the extraction of the partials from the original sound in an easy and efficient way. Once these characteristics are found, a high quality real-time synthesis is possible.

\section{3:00}

5aNSj9. A simple acoustic model to characterize the internal sound field in centrifugal pumps originated by blade-tongue interaction. Jorge Parrondo (Universidad de Oviedo, Dep. de Energía, Campus de Viesques s/n, 33203 Gijón, Spain, parrondo@uniovi.es), Javier Perez (Universidad de Oviedo, Dep. de Energía, Campus de Viesques s/n, 33203 Gijón, Spain, javier.perez_castillo@siemens.com), Raul Barrio (Universidad de Oviedo, Dep. de Energía, Campus de Viesques s/n, 33203 Gijón, Spain, barrioraul @ uniovi.es)

Conventional centrifugal pumps with volute casing generate fluiddynamic noise particularly at the so-called blade-passing frequency, due to the interaction of the flow exiting the pump impeller with the volute tongue. The amplitude of the sound generated is very dependent on the pump operating point. Following previous studies by the authors, a methodology has been applied to quantify the generation of tonal noise for a given centrifugal pump, previously tested in laboratory. The procedure is based on a simple acoustic model for the pump, in which one or several ideal point sources are located at some arbitrary position in the volute. These ideal sources are assumed to radiate plane sound waves along the volute, which was considered to be composed by a succession of slices, each of them equivalent to a linear three-port acoustic system with sound transmission and reflexion coefficients according to the corresponding port areas. A series of tests was conducted to check the assumptions of the acoustic model, by applying external acoustic loads onto the pump outlet duct and measuring the noise reflected. The resulting reflection coefficient was in good agreement with the predictions of the acoustic model.

\section{$3: 20$}

5aNSj10. Performance evaluation method for high noise environment intercom headsets. Stelios M. Potirakis (Intracom Defense Electronics, $21 \mathrm{~km}$ Peania-Markopoulo Street, GR-19400 Koropi - Attica, Greece, spoti@intracomdefense.com), Yiannis Moisiadis (Intracom Defense Electronics, 21km Peania-Markopoulo Street, GR-19400 Koropi - Attica, Greece, gmoy@intracomdefense.com), Antonis Varagis (Intracom Defense Electronics, 21km Peania-Markopoulo Street, GR-19400 Koropi - Attica, Greece, avar@intracomdefense.com)

Intercoms' use in high noise environments (HNE) is typical since direct speech communication of individuals is difficult or even impossible. The headset selection for such an application is crucial since it serves both for noise attenuation and voice reproduction. It has been recently acknowledged in telecommunications (ITU-T/P.380) that headset electroacoustic measurements should be performed on head and torso simulator (HATS). However, in military applications where headsets are mainly used in HNE there is not a standard performance evaluation method but only for the earphone elements (MIL-PRF-25670B), thus excluding the acoustics of the earcup cavity, absorbing materials and face fitting quality. It is well known that the reproduced speech level should be of about $10 \mathrm{~dB}$ above noise level to achieve good intelligibility, so both sensitivity and noise reduction capability should be measured on a HATS to evaluate such a headset. In this work, a systematic methodology for measurement and performance evaluation of HNE headsets is proposed based on HATS. Critical aspects that may affect the test procedure, such as HATS response and the right headset placement are examined. Finally, a series of measurements prove the value of the proposed method. Large differences are revealed even for headsets following the same standard. 


\title{
Session 5aPAa
}

\section{Physical Acoustics: Infrasound I}

\author{
Henry E. Bass, Cochair \\ The University of Mississippi - NCPA, 1 Coliseum Drive, University, MS 38677, USA \\ Alexis Le Pichon, Cochair \\ CEA-DASE, Arpajon Cedex, Bruyères-le-Châtel, 91297, France
}

\section{Invited Paper}

\begin{abstract}
8:00
5aPAa1. Monitoring hurricanes using infrasound. Carrick L. Talmadge (The University of Mississippi - NCPA, 1 Coliseum Drive, University, MS 38677, USA, clt@olemiss.edu), Claus Hetzer (National Center for Physical Acoustics, University of Mississippi, University, MS 38677, USA, claus@olemiss.edu), Henry E. Bass (The University of Mississippi - NCPA, 1 Coliseum Drive, University, MS 38677, USA, pabass@olemiss.edu)

In spite of recent technological advances, forecasting the intensity of a hurricane remains a challenging problem. Knowledge of the location and the strength of the storm at the moment where it makes landfall is critical for civil defense planning and public safety. Part of the reason for this is that storms that are well away from land can only be monitored intermittently (e.g., three times per day). Infrasound signals are produced by the interaction of the hurricane with the sea-state and hold promise for characterizing sudden changes in hurricane strength on a time scale not possible with current sensing technologies. The University of Mississippi is leading a US national collaboration to monitor hurricanes using infrasound in the Gulf of Mexico and in the Western Atlantic Ocean. The results of measurements by from our group will be summarized, and the technical challenges associated with these measurements will be discussed.
\end{abstract}

\section{Contributed Papers}

8:20

5aPAa2. Infrasonic precursor of tropical cyclone. Konstantin A. Naugolnykh (University of Colorado/Zeltech, 325 Broadway, Boulder, CO 80305, USA, konstantin.naugolnykh@noaa.gov), Samuil A. Rybak (N. Andreev Acoustics Institute, 4 Schwernik St., 117420 Moscow, Russian Federation,am_rybak@hotmail.com)

Intense infrasonic emission was observed prior to the birth of a tropical cyclone (Netreba, 1991). It is connected apparently with instability of atmospheric layers in cyclone area. There are different mechanisms of this effect. Stratified compressional flow is unstable (Rybak, 2002), providing amplification of its potential component. Cooling of air in upward convection flow makes water vapor supersaturated. This is a nonequilibrium state of fluid where effect of instability can be developed. The equation of infrasonic propagation in such a medium is developed. (Naugolnykh, Rybak, 2006). Solutions of instability equation indicates the effect of radiation instability which produces infrasonic wave generation and its nonlinear evolution. Corresponding analyses of this equation make it possible to clarify the principally important aspects of cyclone infrasonic precursors. [Work supported by ESP.NR.NRCLG982524.]

\section{8:40}

5aPAa3. Origin of the sound prduced by volcanic eruptions. Sylvie Vergniolle (Institut de Physique du Globe, 4 Place Jussieu, 75252 Paris, France, vergniolle@ipgp.jussieu.fr)

Volcanic eruptions present different regimes, which can be understood and classified in the framework of a two-phase flow, either an annular flow such as in hawaiian fire fountains or a slug flow in strombolian explosions. The gas, exsolved at depth, carries physical information about the dynamics of strombolian activity, which in turn may lead to a better understanding of volcanic systems. The sound produced by volcanic eruptions is mostly infrasonic, although the landing of magma fragments on the ground is in the audible range. The first cause for sound generation is related to the gas overpressure when the sound waves are produced by a slug breaking at the surface. Gas overpressure induces oscillations of the gas volume and pushes passively the liquid film above the slug, and has been modeled accordingly. Sometimes the overpressure is small and the bubble vibration prior to breaking does not exist. In that case, the sound is produced by the gas escaping through a small hole on the bubble nose and is modeled as an Helmholtz resonator. The second class of sound source is related to the expulsion of a gas-ejecta mixture at a large velocity. Examples of volcanic sound waves will be presented.

\section{9:00}

5aPAa4. Implications from the analysis of meteoric infrasound. Laeslo G. Evers (Royal Netherlands Meteorological Institute (KNMI), PO Box 201, 3730 AE De Bilt, Netherlands, evers@knmi.nl), Hein Haak (Royal Netherlands Meteorological Institute (KNMI), PO Box 201, 3730 AE De Bilt, Netherlands, haak@knmi.nl), Alexis Le Pichon (CEA-DASE, Arpajon Cedex, 91297 Bruyères-le-Châtel, France, alexis.le-pichon@cea.fr), Karl Antier (CEA-DASE, Arpajon Cedex, 91297 Bruyères-le-Châtel, France, karl.antier@cea.fr)

Monitoring of infrasound has been widely applied in the past to detect nuclear tests. The Limited (Partial) Test Ban Treaty of 1963 confined nuclear test explosions to the underground marking the end of the global interest in infrasound. During the negotiation of the Comprehensive Nuclear-Test-Ban Treaty, in 1995-1996, it became gradually clear that infrasound monitoring should be become one of the four techniques for the treaty's verification system. The global interest in infrasound has increased significantly since the signature of the CTBT. Meteors are one of the few natural sources mimicking a nuclear. Therefore, it is an ideal object to test detection capability, phase identifiers, localization procedures and propagation models of infrasound and their processing techniques. In this presentation, we will show the results of studies on meteoric-generated infrasound and their implications for infrasound as verification technique. In addition, results of the 2007 .09 .15 meteor in Peru will be discussed which is one of the few meteoroids in recent times leaving an impact crater. 


\section{Invited Paper}

9:20

5aPAa5. Infrasound monitoring and global atmospheric dynamics. Elisabeth Blanc (Commissariat à l'Energie Atomique, DASE/SLDG/LSEG, Centre DAM-Ile de France, 91297 Arpajon Cedex, France, elisabeth.blanc@cea.fr), Alexis Le Pichon (CEA-DASE, Arpajon Cedex, 91297 Bruyères-le-Châtel, France, alexis.le-pichon@cea.fr), Lars Ceranna (Federal Institute of Geosciences and Natural Ressources, Section B3.11 Seismology, Stilleweg 2, 30655 Hannover, Germany, ceranna@sdac.hannover.bgr.de)

The development of the Infrasound International Monitoring System, used for the verification of the Comprehensive Test Ban Treaty, offers a powerful way to measure, atmospheric waves permanently and at a global scale. Infrasonic waves propagate in the channel formed by the temperature and wind gradients of the atmosphere. Long term observations provide information about the evolution of the propagation conditions and then of atmospheric parameters. The monitoring of continuous sources, as ocean swell, gives the characteristics of the stratospheric wave duct submitted to stratospheric warming effects. Large scale gravity waves, which are also observed by the network, produce a forcing of the stratosphere at low and middle latitudes and long-lived changes in the stratospheric circulation towards high latitudes, leading to fluctuations in the strength of the polar vortex. These fluctuations move down to the lower stratosphere with possible effects on the tropospheric temperature. Gravity wave monitoring in Antarctica reveals a gravity wave system correlated with the wind and the temperature gradients in the stratosphere. Gravity waves associated with magnetic storms are generally not observed. However, wave systems coming from North could reveal other processes related with the global dynamics of the stratosphere.

9:40-10:40 Posters

Lecture sessions will recess for presentation of poster papers on various topics in acoustics. See poster sessions for topics and abstracts.

\section{Contributed Paper}

10:40

5aPAa6. Locating infrasound events in wind with dense distributed arrays. Wheeler Howard (Mil-tec Research and Technology, 9 Industrial Park Dr, Oxford, MS 38655, USA, whoward@mil-tec.com), Kevin Dillion (Mil-tec Research and Technology, 9 Industrial Park Dr, Oxford, MS 38655, USA, kdillion@mil-tec.com), F. Douglas Shields (University of Mississippi/ NCPA, 1 Coliseum Dr, University, MS 38677, USA, dshields @olemiss.edu)
Applications for infrasound suffer due to the presence of wind noise in the sensor data. Several techniques have been and are being used to mitigate the influence of wind noise in locating infrasonic signals of interest. One of these methods is the averaging of data from multiple sensors in a distributed array. A distributed array was employed to study infrasonic signals from airborne sources. Wind noise during testing masked the location of the infrasonic signals in the time domain. Postprocessing techniques using statistical measures for dense arrays were employed to recover the onset of the infrasonic events. Data from these tests and an explanation of the postprocessing techniques will be discussed.

\section{Invited Papers}

\section{1:00}

5aPAa7. Detection and localization capability of the European infrasound network. Lars Ceranna (Federal Institute of Geosciences and Natural Ressources, Section B3.11 Seismology, Stilleweg 2, 30655 Hannover, Germany, ceranna@ sdac.hannover .bgr.de), Alexis Le Pichon (CEA-DASE, Arpajon Cedex, 91297 Bruyères-le-Châtel, France, alexis.le-pichon @ cea.fr), Julien Vergoz (CEA-DASE, Arpajon Cedex, 91297 Bruyères-le-Châtel, France, julien.vergoz@cea.fr), Laslo Evers (KNMI, PO Box 201, 3730 De Bilt, Netherlands, laslo.evers@knmi.nl)

The infrasound network of the International Monitoring Network (IMS) for the compliance with the Comprehensive Nuclear-TestBan Treaty (CTBT) is currently not fully established. However, it has demonstrated its capability for detecting and locating infrasonic sources like meteorites, as well as volcanic eruptions on a global scale. Such ground-truth events are rare; therefore regions providing a dense network of infrasound stations have to be considered to test and to calibrate detection and location procedures. In central Europe, several years of continuous infrasound waveform data are available for nine stations in Sweden, the Netherlands, France, and Germany, whereas only one of them is part of the IMS. This exquisite setting with an average interstation distance below $500 \mathrm{~km}$ allows the analysis of natural and artificial infrasonic activity in Europe. The results of the association of multiple arrays demonstrate the need of continuous infrasound monitoring on a regional scale to advance the development of automatic location procedures. Beside the seasonal variation of the network's detection capability, which is dominated by the prevailing stratospheric winds, dominant source regions showing repeating events will be discussed in detail considering the next recording stations.

5aPAa8. Continuous infrasound monitoring for atmospheric studies. Alexis Le Pichon (CEA-DASE, Arpajon Cedex, 91297 Bruyères-le-Châtel, France, alexis.le-pichon@cea.fr), Nicolas Brachet (CTBTO PTS/IDC Vienna International Centre, P.O. Box 1200, A-1400 Vienna, Austria, Nicolas.Brachet@ctbto.org), Lars Ceranna (Federal Institute of Geosciences and Natural Ressources, Section B3.11 Seismology, Stilleweg 2, 30655 Hannover, Germany, ceranna@sdac.hannover.bgr.de)

Infrasound data processing on a routine-basis provides a powerful tool to quantify relationships between infrasonic observables and atmospheric specification problems. In particular, studies of continuous sources of infrasonic waves provide further evidence that longrange propagation primarily depends on the seasonal variability of the prevailing zonal winds. It confirms that most of the detected infrasound energy propagates in the stratospheric waveguide. The observed detection capability of the IMS network is compared to the 
predicted one using near-real time atmospheric updates. Moreover, on regional scales, signals from volcanic activities open up new fields for investigations into inverse problems for atmospheric remote sensing in a range of altitude where routine ground based or satellite measurements are rare. With the increasing number of IMS and experimental arrays deployed around the globe, a valuable contribution can be anticipated in the understanding of the dynamics of the upper atmosphere. In return, such studies will help to optimize the siting of infrasound arrays with respect to both the number and configuration in order to monitor infrasonic sources of interest.

\section{1:40}

5aPAa9. Inversion of infrasound signals for atmospheric remote sensing. Douglas Drob (Naval Research Laboratory, Space Science Division, 4555 Overlook Avenue, Washington, DC 20375, USA, douglas.drob@nrl.navy.mil), Milton Garces (Infrasound Laboratory, University of Hawaii, 73-4460 Queen Kaahumanu Highway \#119, Kailua-Kona, HI 96740, USA, milton@isla.hawaii.edu), Robert Meier (Naval Research Laboratory, Space Science Division, 4555 Overlook Avenue, Washington, DC 20375, USA, robert.meier@nrl.navy.mil), Michael Picone (Naval Research Laboratory, Space Science Division, 4555 Overlook Avenue, Washington, DC 20375, USA, j.picone@nrl.navy.mil)

This paper investigates the utilization of observed long-range infrasound signals to improve our knowledge of upper atmospheric winds and temperatures. A series of numerical experiments designed to provide a mathematical framework for implementation of this idea is presented. The statistical performance of a realistic infrasonic upper atmospheric remote sensing network over a range of different environmental and propagation modeling assumptions, source types, and geophysical variables (season, local-time, latitude, etc) is quantified. For a series of fictitious events, infrasonic observables are calculated with 3D Cartesian ray tracer and a series of atmospheric profiles spanning the range of geophysical parameters. These synthetic measurements are then inverted to estimate the original atmospheric background fields.

\section{Contributed Papers}

12:00

5aPAa10. The influence of shallow atmospheric structure on tropospheric ducted infrasound from the Buncefield oil depot explosion. David N. Green (AWE Blacknest, Brimpton, RG7 4RS Reading, UK, dgreen@blacknest.gov.uk), Matthew Hort (Met Office, FitzRoy Road, EX1 3PB Exeter, UK, matthew.hort@metoffice.gov.uk), David Bowers (AWE Blacknest, Brimpton, RG7 4RS Reading, UK, bowers@blacknest.gov.uk)

The vapour cloud explosion which destroyed the Buncefield oil depot, UK, on 11th December 2005, has proven to be a benchmark ground truth event for infrasonic studies. The regional infrasonic returns, those that propagated in the stratosphere and thermosphere, have been analysed in detail elsewhere. Here, we present the results of a study into infrasound ducted in the troposphere, recorded within $250 \mathrm{~km}$ of the source as air-to-ground coupled waves by a dense seismometer network. These tropospheric arrivals exhibit large waveform differences across the UK, both in amplitude and waveform shape. We numerically model these infrasound arrivals using a wave number integration method, incorporating a velocity profile derived from the UK Met Office numerical weather prediction model. Although some of the waveform variability is due to ground conditions at the recording site, we show that consistent changes in waveform shape across a 200 $\mathrm{km}$ swath of stations are correlated with a change in the wind vector in the lowermost $2 \mathrm{~km}$ of the atmosphere. Also, small amplitude, high-frequency precursors to the dominant acoustic signal, which might be misinterpreted as evidence of a small initial explosion, are shown to be consistent with the dispersion expected from a thin, shallow wind jet.

\section{2:20}

5aPAa11. Regional and telesonic propagation of volcano-acoustic signals. Milton Garces (Infrasound Laboratory, University of Hawaii, 734460 Queen Kaahumanu Highway \#119, Kailua-Kona, HI 96740, USA, milton@isla.hawaii.edu), David Fee (Infrasound Laboratory, University of Hawaii, 73-4460 Queen Kaahumanu Highway \#119, Kailua-Kona, HI 96740, USA, dfee@isla.hawaii.edu)

Volcanic explosions are reliable sources for studying infrasonic propagation. Large amplitude acoustic explosions with known locations and onset times provide useful constraints for the signal changes induced by atmospheric variability. As part of the Acoustic Surveillance of Hazardous Eruptions (ASHE) project, two four-element infrasound arrays with collocated seismometers have been deployed 37 and $251 \mathrm{~km}$ from Tungurahua Volcano, Ecuador since February 2006. During this period Tungurahua has been in near-constant eruption, with energetic tremor signals and a multitude of energetic explosions. A combination of array processing and energy threshold detectors have been used to identify over 12000 explosions at the nearby array (RIOE, $37 \mathrm{~km}$ ), with the peak pressure of the largest explosion around $24 \mathrm{~Pa}$. Many of the larger explosions are recorded at the more distant LITE $(251 \mathrm{~km})$ array as well. To further supplement the dataset, select data from infrasonic sensors at distances of 3-5 $\mathrm{km}$ from the crater will be used. State-of-the-art wave propagation and atmospheric models will be used to assess the influence of long-range propagation on the volcanic signals. By selecting a data subset with high signal to noise ratios, it should be possible to infer statistically significant atmospheric propagation effects.

12:40-1:40 Lunch Break

\section{Contributed Papers}

\section{$1: 40$}

5aPAa12. Gravity and rumble of distant sources. Philippe Delorme (ONERA/DSNA, 29, avenue de la Division Leclerc, 92322 Chatillon, France, Philippe.delorme@onera.fr), Geraldine Menexiadis (ONERA/DSNA, 29, avenue de la Division Leclerc, 92322 Chatillon, France, geraldine.menexiadis@onera.fr)

The subject of this paper is the comprehension of this physical phenomenon: Let an atmospheric source emiting an impulsive signal (such as thunder, explosion...). The received signal at short distance is itself impulsive but at long distance one can hear a rumble which can last up to one minute. If one models the propagation of the sound by the traditional equation of the waves, as the Green function is a distribution whose support is the sphere of radius CT (C: speed of sound, T: the travel time), the received signal (calculated by convolution) should last only the duration of emission. If one uses ray tracings to take into account the heterogeneity of the medium, one obtains the same conclusion (only the time of arrival is modified) in contradiction with the experimental results such as those obtained for example during the First World War to detect the position of the enemy batteries. A similar phenomenon can be observed in the study of the propagation of a sonic boom (although less obvious because the support of the 
two-dimensional Green function is not a circle). We thus propose a critic of the physical models, and our conclusion lays on the need for taking into account gravity.

\section{2:00}

5aPAa13. Study of Infrasound Propagation from the Shuttle Atlantis using a large Seismic Network. Michael A. Hedlin (University of San Diego California, Scripps Institute of Oceanography, 9500 Gilman Drive, La Jolla, CA 92093, USA, hedlin@ucsd.edu), Catherine D. Degroot-Hedlin (University of San Diego California, Scripps Institute of Oceanography, 9500 Gilman Drive, La Jolla, CA 92093, USA, chedlin@ucsd.edu), Kris Walker (University of San Diego California, Scripps Institute of Oceanography, 9500 Gilman Drive, La Jolla, CA 92093, USA, walker@ucsd.edu), Douglas Drob (Naval Research Laboratory, Space Science Division, 4555 Overlook Avenue, Washington, DC 20375, USA, douglas.drob@nrl.navy.mil), Mark A. Zumberge (University of San Diego California, Scripps Institute of Oceanography, 9500 Gilman Drive, La Jolla, CA 92093, USA, mzumberge@ucsd.edu)

Inclement weather in Florida forced the shuttle Atlantis to land at Edwards Air Force base in southern California on June 22, 2007, passing near three infrasound stations and several hundred seismic stations in northern Mexico, southern California, and Nevada. The high signal-to-noise ratio, broad receiver coverage, and Atlantis' positional information allowed us to test our ability to model infrasound propagation through the atmosphere to regional distances. We predicted shadow zones and arrival times using rays launched at right angles to the conical shock front surrounding the shuttle, and traced through a standard atmospheric model, as well as a global G2S model. Comparison of predictions vs observations shows agreement over much of the study area for both atmospheric specifications. To the east of the shuttle trajectory, there were no detections beyond the primary acoustic carpet. Infrasound energy was detected hundreds of kilometers to the west and northwest of the shuttle trajectory, consistent with the predictions of ducting due to the westward summer-time stratospheric jet. Both atmospheric models predict regions of ensonification alternating with shadow zones to the northwest. However, infrasound energy was detected tens of kilometers beyond predicted zones of ensonification, possibly due to uncertainties in stratospheric wind speeds.

\section{2:20}

5aPAa14. Nonlinear propagation modeling of infrasound. David Norris (BBN Technologies, 1300 N. 17th St., Ste. 400, Arlington, VA 22209, USA, dnorris@bbn.com), Kevin Bongiovanni (BBN Technologies, 4 John Clarke Rd, Middletown, RI 02842, USA, kbongiov @bbn.com), James Masi (BBN Technologies, 4 John Clarke Rd, Middletown, RI 02842, USA, jmasi@bbn.com)

The significance of nonlinear propagation effects on infrasound is studied using the Nonlinear Progressive Wave Equation (NPE) [B. E. McDonald and W. A. Kupperman, J. Acoust. Soc. Am., 81, 1406--1417 (1987)]. The NPE model accounts for nonlinear effects associated with a weak shock front, including shock-driven energy loss and self refraction. Numerical implementation is accomplished using a pseudospectral approach, which provides excellent computational efficiency while still maintaining acceptably small numerical errors. The nonlinear effects are isolated by generating NPE predictions with the nonlinear terms turned on and off. With the nonlinear terms off, the NPE reduces to a standard linear PE formulation. Waveform predictions through a realistic atmosphere are compared to ground truth observations to evaluate the NPE model performance and assess the influence of the nonlinear effects along the propagation path.

\section{2:40}

5aPAa15. Misty picture: A unique experiment for the interpretation of the infrasound propagation from large explosive sources. Olaf Gainville (Commissariat à l'Energie Atomique, DASE/SLDG/LSEG, Centre DAM-Ile de France, 91297 Arpajon Cedex, France, olaf.gainville@ec-lyon.fr), Philippe Blanc-Benon (Ecole Centrale de Lyon, LMFA, UMR CNRS 5509, Ecully, 69134 Lyon, France, Philippe.Blanc-Benon@ec-lyon.fr), Elisabeth Blanc (Commissariat à l'Energie Atomique, DASE/SLDG/LSEG, Centre DAM-Ile de France, 91297 Arpajon Cedex, France, elisabeth.blanc@cea.fr), Roger Roche (Commissariat à l'Energie Atomique, DASE/SLDG/LSEG, Centre DAM-Ile de France, 91297 Arpajon Cedex, France, roger.roche @ cea.fr), Christophe Millet (Commissariat à l'Energie Atomique, DASE/SLDG/LSEG, Centre DAM-Ile de France, 91297 Arpajon Cedex, France, christophe.millet@cea.fr), Francoise Le Piver (Commissariat à l'Energie Atomique, DASE/SLDG/LSEG, Centre DAM-Ile de France, 91297 Arpajon Cedex, France, francoise.lepiver@cea.fr), Bruno Despres (Commissariat à l'Energie Atomique, DSSI, Centre DAM-Ile de France, 91297 Arpajon Cedex, France, bruno.despres@cea.fr), Pierre-Franck Piserchia (Commissariat à l'Energie Atomique, DASE/SLDG/LSEG, Centre DAM-Ile de France, 91297 Arpajon Cedex, France, pierre-franck .piserchia@cea.fr)

In the framework of the Comprehensive Nuclear-Test-Ban Treaty, the International Monitoring System develops a 60 micro-barometric stations network. These stations, which records infrasound, detect various powerful natural and artificial sources like long range explosions, oceanic swell, and volcano eruptions. For data analysis, the CEA, in collaboration with the LMFA, develops specific methods based on measurements, data processing and numerical simulation. The Misty Picture experiment is a high explosive event (4685 Tons of ANFO) realized in 1987 in New Mexico (US). Infrasounds were recorded by 22 sensors until a distance of $1000 \mathrm{~km}$ in a quiet background noise condition. Multireflected tropospheric, stratospheric and thermospheric phases are detected. Signals recorded near the source $(1 \mathrm{~km}$ away) and observed in the geometrical shadow zone (between $150 \mathrm{~km}$ and $250 \mathrm{~km}$ from the point source) are of particular interest. This reference experiment very well documented is used to improve our understanding of the atmospheric propagation of infrasound as well as to evaluate our models. Using various methods such as ray tracing, parabolic equation and finite differences, we investigate effects of the wind, atmospheric absorption, nonlinearity, refraction, (2D and 3D) and scattering by small atmospheric scales on observed phase kinds, their travel time and their waveform.

\section{Invited Paper}

5aPAa16. A low power and low noise infrasound sensor for temporary measurements. Damien Ponceau (CEA/DASE, BP12, Service TMG, 91680 Bruyères le Chatel, France, damien.ponceau@cea.fr)

Temporary infrasound measurements require low power consumption, reliable, and compact equipments. As a consequence, these equipments are not always as accurate as equipments operating on long term stations. Measurements quality can be affected. DASE has developed a portable infrasound sensor from MB2005 microbarometer. This new microbarometer consists in improved mechanics from MB2005 and in a movement transducer providing a voltage directly proportional to pressure derivate all over infrasound bandwidth and even more: sensor response phase is purely independent from environment (temperature, condensationœ) all over the same bandwidth. Moreover, thanks to its very simple design this sensor needs very low power and is lighter, smaller and cheaper than previous generation sensors. It reaches Infrasound Low Noise Model (IS LNM) down to $0.02 \mathrm{~Hz}$ and is very portable as it weights less than $3 \mathrm{~kg}$ with less than $1 \mathrm{~L}$. A batch of prototypes has been manufactured and evaluated in lab thanks to an infrasound generator used for routine calibration of microbarometers installed by DASE on some International Monitoring System stations (IMS). Sensors field evaluation is running. First results are promising. 


\title{
Session 5aPAb
}

\section{Physical Acoustics: Time Reversal Acoustics for Nonlinear Imaging I}

\author{
Alexander Sutin, Cochair \\ Stevens Institute of Technology, Castle Point on Hudson, Hoboken, NJ 07030, USA \\ Serge Dos Santos, Cochair \\ ENI Val de Loire, Université François Rabelais de Tours, LUSSI CNRS, rue de la Chocolaterie, Blois, 41034, France
}

\section{Invited Papers}

8:00

5aPAb1. Time reversal of acoustic waves in the nonlinear regime: basic physics and application to ultrasound contrast imaging. Mickael Tanter (Laboratoire Ondes et Acoustique, ESPCI, Université Paris 7, CNRS, 10 rue Vauquelin, 75005 Paris, France, michael.tanter@espci.fr), Olivier Couture (Laboratoire Ondes et Acoustique, ESPCI, Université Paris 7, CNRS, 10 rue Vauquelin, 75005 Paris, France, olivier.couture@espci.fr), Mathias Fink (Laboratoire Ondes et Acoustique, ESPCI, Université Paris 7, CNRS, 10 rue Vauquelin, 75005 Paris, France, mathias.fink@espci.fr)

Time reversal invariance of acoustic waves propagation remains valid even in the nonlinear regime in nondissipative media. This very interesting property that was extensively used in linear acoustics can also find potential applications in the nonlinear regime. Harmonic imaging coupled to the use of contrast agents is a topic of wide interest in ultrasonic medical imaging. Images are built at twice the excitation frequency that corresponds to the resonance frequency of the bubbles embedded in the medium. Consequently, the contrast becomes important between areas of low and high concentration of bubbles. However, at a high mechanical index, the harmonic components of backscattered echoes depends on the intrinsic nonlinear properties of tissues as well as the bubble's resonance. The focused beam is generating harmonics during its nonlinear propagation. It results in a degradation of the harmonic image contrast. Time reversal is an elegant way to find the emission codes allowing us to cancel the harmonic components due to nonlinear propagation of the ultrasonic beam. In the absence of bubbles, if the backscattered echoes are time-reversed and reemitted by the array, the harmonic components of the resulting wavefront are transferred back to the fundamental frequency during propagation. Experiments conducted with 1D-linear arrays illustrate these cancellation techniques.

\section{$8: 20$}

5aPAb2. Localized nonlinearity time reversal imaging with chaotic cavities. Olivier Bou Matar (IEMN, UMR CNRS 8520, avenue Poincaré, BP 60069, 59652 Villeneuve d'Ascq, France, olivier.boumatar@iemn.univ-lille1.fr), Yifeng Li (IEMN - LEMAC UMR CNRS 8520, Ecole Centrale de Lille, BP 48, Cite scientifique, 59651 Villeneuve D'Ascq, France, Yifeng.li@iemn.univ-lille1.fr), Vladimir L. Preobrazhensky (IEMN, UMR CNRS 8520, avenue Poincaré, BP 60069, 59652 Villeneuve d'Ascq, France, vladimir.preobrajenski@iemn.univ-lille1.fr), Philippe Pernod (IEMN, UMR CNRS 8520, avenue Poincaré, BP 60069, 59652 Villeneuve d'Ascq, France, philippe.pernod@iemn.univ-lille1.fr)

Recent results have shown that nonlinear elastic wave spectroscopy (NEWS) and time reversal (TR) techniques can be combined to localize defects. NEWS techniques can be used either as a post-treatment of TR used as a tool for generating strong localized stress (TR-NEWS), or as a pretreatment, of TR used as a tool for defect identification (NEWS-TR). The TR process can be implemented with a programmable multielements ultrasonic system. But recently, it has been demonstrated that a single PZT ceramic glued on the sample can also be used as a TR mirror. In this case the quality of the retrofocusing process, and so the microdamage localization, depends on the sample geometry. In this study, numerical and laboratory studies, performed in order to determine and enhance the possibility to localize microdamage based on a combination of a time reversal technique using an emitter with a chaotic shape and nonlinear elastic wave response, will be presented. The benefit of using such emitters is clearly found in breaking the symmetry of the problem, leading to an unambiguous retrofocusing. Moreover, chirp-coded excitation, instead of short pulses, is studied in order to transmit more energy on the defect without increasing the peak intensity of the excitation.

\section{$8: 40$}

5aPAb3. Landmine detection using nonlinear vibrations excited by time reversal techniques. Brad Libbey (U.S. Army RDECOM CERDEC NVESDS, 10221 Burbeck Rd, Fort Belvoir, VA 22060, USA, brad.libbey@us.army.mil), Alexander Sutin (Stevens Institute of Technology, Castle Point on Hudson, Hoboken, NJ 07030, USA, asutin@stevens.edu), Armen Sarvazyan (Artann Laboratories, 1753 Linvale-Harbourton, Lambertville, NJ 08350, USA, armen@artannlabs.com)

Time reversal focuses seismic waves and excites nonlinear surface vibrations that are large in the presence of a landmine. These soil vibrations provide an accurate localization cue when contrasted with measurements made without a mine. Traditionally nonlinear effects were investigated by measuring the interaction of harmonic waves and nonlinear resonance frequency shifts. These methods provide high signal to noise ratios, but required tuning of excitation frequencies resulting in test times unsuitable for field applications. Time reversal is capable of providing broad band excitation at amplitudes large enough to drive the nonlinear mechanisms at measurable levels while reducing the overall measurement time. Spatial and temporal focusing data will be presented for three different time reversal systems compromising speakers and vibrators for seismic excitation and a Doppler laser vibrometer and geophones for signal 
recording. The high amplitude signals generated by these systems adequately drive the nonlinear responses. Special processing techniques, phase inversion and varied amplitude, have been developed to extract the nonlinear responses from the broadband response by cancelling the linear part. The remaining nonlinear response shows greater mine/no mine contrast than the linear data. Practical implementations of the developed methods will be discussed.

9:00

5aPAb4. Separation of multiple scatterers in NEWS-TR experiments. Sigfried Vanaverbeke (K.U.Leuven Campus Kortrijk, E. Sabbelaan 53, 8500 Kortrijk, Belgium, Sigfried.Vanaverbeke@kuleuven-kortrijk.be), Lieven De Lathauwer (K.U.Leuven Campus Kortrijk, E. Sabbelaan 53, 8500 Kortrijk, Belgium, Lieven.DeLathauwer@kuleuven-kortrijk.be), Herbert De Gersem (K.U.Leuven Campus Kortrijk, E. Sabbelaan 53, 8500 Kortrijk, Belgium, Herbert.DeGersem@kuleuven-kortrijk.be), Koen Van Den Abeele (K.U .Leuven Campus Kortrijk, E. Sabbelaan 53, 8500 Kortrijk, Belgium, koen.vandenabeele@kuleuven-kortrijk.be)

Nonlinear elastic wave spectroscopy combined with acoustic time reversal (NEWS-TR) is a promising new methodology for detecting microdamage at an early stage. When dealing with structures which could potentially contain many pointlike nonlinear scatterers, there is a need to develop techniques for separately imaging the defects using a distributed sensor network which acts as a timereversal mirror. In this contribution, we present numerical simulations of a newly developed version of the DORT method for nonlinear imaging and also discuss the possibility of applying PARAFAC (parallel factor analysis) and ICA (independent component analysis) methods to solve the problem of separating multiple nonlinear scatterers in the time-frequency domain.

\section{9:20}

5aPAb5. Wide band time reversal acoustic nonlinear diagnostics. Tj Ulrich (EES-11 (Geophysics) - Los Alamos National Laboratory, MS D443, Los Alamos, NM 87545, USA, tju@lanl.gov), Alexandr Sutin (Stevens Institute of Technology, Castle Point on Hudson, Davidson Laboratory, Hoboken, NJ 07030, USA, asutin@stevens-tech.edu), Jim A. Tencate (EES-11 (Geophysics) - Los Alamos National Laboratory, MS D443, Los Alamos, NM 87545, USA, tencate@lanl.gov)

Time reversal acoustics (TRA) techniques provide a means to focus energy at a localized time and space, or at an unknown location (i.e., source or scatterer not known a priori) without the need to calculate time delays, as is necessary for phased arrays. The experiments demonstrate that time reversal focusing provides localized surface vibration with amplitude sufficient to induce measurable nonlinear effects and does so over a wide frequency band. Because the amplitude is spatially focused, time reversal enables local probing of this nonlinearity that can be used for nonlinear imaging. The nonlinear effects for wide band frequency signals were measured by phaseinversion method combining two signals of opposite sign in order to cancel the linear response and preserve quadratic nonlinearities. The method works effectively for nonlinear NDE of thin plates where laser Doppler Vibrometer can be used for measurements of the surface vibration and TRA focusing of acoustic waves. Presented results include crack and delamination diagnostic is epoxy graphite composites, diffusion bonded metallic disks and impact surface damage in glass. Scanning electron microscopy and optical images are also shown to demonstrate the types of damage features detected using this method.

\section{9:40-11:00 Posters}

Lecture sessions will recess for presentation of poster papers on various topics in acoustics. See poster sessions for topics and abstracts.

\section{Contributed Papers}

\section{1:00}

5aPAb6. Time reversal use in detection of buried cracks. Pierre-Yves Le Bas (EES-11 (Geophysics) - Los Alamos National Laboratory, MS D443, Los Alamos, NM 87545, USA, pylb@lanl.gov), Brian E. Anderson (EES-11 (Geophysics) - Los Alamos National Laboratory, MS D443, Los Alamos, NM 87545, USA, bea@lanl.gov), Michele Griffa (EES-11 (Geophysics) - Los Alamos National Laboratory, MS D443, Los Alamos, NM 87545, USA, mgriffa@lanl.gov), Tj Ulrich (EES-11 (Geophysics) - Los Alamos National Laboratory, MS D443, Los Alamos, NM 87545, USA, tju@lanl.gov), Paul A. Johnson (EES-11 (Geophysics) Los Alamos National Laboratory, MS D443, Los Alamos, NM 87545, USA, paj@lanl.gov)

Time reversal has the potential to become a powerful tool in nondestructive evaluation. Coupled with nonlinear properties of cracks in a technique known as time reverse nonlinear elastic wave spectroscopy (TRNEWS), it provides the means to detect defects in complex structures. This experimental study explores the capabilities of TR to focus energy inside a 3D medium in order to activate nonlinear features or defects. Special attention is given to buried cracks. The current challenge is introducing sufficient energy in order to excite the buried feature and produce nonlinear scattering. We will provide an overview of the problem.

\section{1:20}

5aPAb7. Computational time reversal acoustics imaging of embedded defects in solid media. Michele Griffa (EES-11 (Geophysics) - Los
Alamos National Laboratory, MS D443, Los Alamos, NM 87545, USA, mgriffa@lanl.gov), Brian E. Anderson (EES-11 (Geophysics) - Los Alamos National Laboratory, MS D443, Los Alamos, NM 87545, USA, bea@lanl.gov), Tj Ulrich (EES-11 (Geophysics) - Los Alamos National Laboratory, MS D443, Los Alamos, NM 87545, USA, tju@lanl.gov), Pierre-Yves Le Bas (EES-11 (Geophysics) - Los Alamos National Laboratory, MS D443, Los Alamos, NM 87545, USA, pylb@lanl.gov), Paul A. Johnson (EES-11 (Geophysics) - Los Alamos National Laboratory, MS D443, Los Alamos, NM 87545, USA, paj@lanl.gov), Lianjie Huang (EES-11 (Geophysics) - Los Alamos National Laboratory, MS D443, Los Alamos, NM 87545, USA, ljh@lanl.gov)

Time reversal acoustics (TRA) techniques can exploit the nonlinear processes emerging from the interaction between the incident waves and nonlinear scatterers in solid media in order to localize and characterize the scatterers themselves. When nonlinear scatterers are embedded, their localization can be obtained through a mixed experimental/numerical TRA procedure: the forward propagation is performed experimentally, while the time reversal (TR) backward propagation is simulated using computational codes and a reference model of the solid. The synergetic use of dedicated processing of the forward propagation signals collected at the time reversal mirror (TRM), for example with nonlinear elastic wave spectroscopy (NEWS) techniques, and the calculation of the backpropagated wave fields at each point within the specimen leads to imaging of the nonlinear scatterers. We show examples of nonlinear scatterer (macroscopic cracks, distributed microcracks) imaging by such a procedure, exploiting high per- 
formance (parallel) computational codes. We address issues in the imaging method related to the discrepancy between the reference model of the specimen and the real specimen itself. Finally, we address the implementation of the technique for non-destructive Evaluation (NDE) real-world applications using multi-threaded computational codes to be run on common multicore desktop computers.

\section{Invited Paper}

\section{1:40}

5aPAb8. Improving signal analysis for nonlinear time reversed acoustics: simulation results. Marco Scalerandi (Politecnico di Torino, Corso Duca degli Abruzzi 24, 10129 Torino, Italy, marco.scalerandi@infm.polito.it), Antonio S. Gliozzi (Politecnico di Torino, Corso Duca degli Abruzzi 24, 10129 Torino, Italy, antonio.gliozzi@polito.it)

Nonlinear time reversed acoustics have attracted attention as a tool to localize and identify nonlinear defects in solids [1-4]. The procedure is based on extracting the features due to the nonlinear scatterers from the signals received at the transducer array and backpropagate only the resulting portion of the signals. The usual approach is to filter the received signals to keep only contributions due to higher order harmonics or sidebands. As a result, the signals are very small in amplitude and can be not detectable if transducers are located far from the scatterers. It is evident the need for novel signal processing tools to increase the signal-to-noise ratio of the nonlinear contributions. We have recently proposed a scaling subtraction method [5], which is applied here to nonlinear time reversal virtual experiments, showing the improvements in the signal-to-noise ratio, with resulting better imaging of the nonlinear scatterer. Results will be presented for two approaches to nonlinear time reversal. [1] A. S. Gliozzi et al., J. Acoust. Soc. Am. 120, 2506-2517 (2006). [2] T. J. Ulrich et al., J. Acoust. Soc. Am. 119, 1514-18 (2006). [3] T. J. Ulrich et al., Phys. Rev. Lett. 98, 10430 (2007). [4] T. Goursolle et al., J. Acoust. Soc. Am. 122, 3220 (2007). [5] M. Scalerandi et al., submitted to Appl. Phys. Lett. (2008).

\section{Contributed Papers}

12:00

5aPAb9. 3D numerical study for defect detection with nonlinear elastic wave spectroscopy and time reversal techniques. Thomas Goursolle (ENI Val de Loire, Université François Rabelais de Tours, LUSSI CNRS, rue de la Chocolaterie, 41034 Blois, France, thomas.goursolle @univ-tours.fr), Samuel Callé (LUSSI, 10 Bd Tonellé, 37032 Tours, France, calle_s@med.univ-tours.fr), Serge Dos Santos (ENI Val de Loire, Université François Rabelais de Tours, LUSSI CNRS, rue de la Chocolaterie, 41034 Blois, France, serge.dossantos@univ-tours.fr), Olivier Bou Matar (IEMN, UMR CNRS 8520, avenue Poincaré, BP 60069, 59652 Villeneuve d'Ascq, France, olivier.boumatar@iemn.univ-lille1.fr)

Nonlinear elastic wave spectroscopy (NEWS) techniques have been developed to study the anomalously high level of "nonclassical" nonlinearity of cracked materials. Within AERONEWS project (http://www.kuleuvenkortrijk.be/aeronews), more recent innovative nondestructive NEWS techniques have been proposed to detect microinhomogeneities like cracks. It has been notably shown that NEWS and time reversal (TR) techniques can be combined to precisely localize cracks with a high sensitivity. NEWS techniques can be used either as a post-treatment of TR used as a tool for strong localized stress generation (TR-NEWS), or as a pre-treatment of TR used as a tool for defect (nonlinear source) identification (NEWS-TR). A 3D multiscale pseudo-spectral time domain (PSTD) code has been developed to simulate nonlinear acoustic propagation in heterogeneous nonlinear hysteretic solids. The hysteretic nonlinearity is introduced owing to a PM space model, based on a multiscale approach, extended in 3D owning to Kelvin notations. Using this algorithm, the influence of various parameters such as the defect position or the filtering method is particularly described for NEWS-TR and TR-NEWS methods. Finally, the sensitivity and feasibility of both methods are discussed.

\section{$12: 20$}

5aPAb10. Varied amplitude method in time reversal acoustic land mine. Laurent Fillinger (Artann Laboratories/ Stevens Institute of Technology, 711 Hudson St, Hoboken, NJ 07030, USA, laurent.fillinger @artannlabs.com), Alexander Sutin (Stevens Institute of Technology, Castle Point on Hudson, Hoboken, NJ 07030, USA, asutin@stevens.edu), Brad Libbey (U.S. Army RDECOM CERDEC NVESDS, 10221 Burbeck Rd, Fort Belvoir, VA 22060, USA, brad.libbey@us.army.mil), Armen Sarvazyan (Artann Laboratories, 1753 Linvale-Harbourton, Lambertville, NJ 08350, USA, armen@artannlabs .com)

Acoustic methods of land mine detection are being developed for nonmetal mines where conventional electromagnetic methods fail. One such method, based on time reversal acoustic principles, is the hhase-inversion method; which detects quadratic and even ordered harmonic nonlinearities in the surface vibration above a mine. While this has shown higher sensitivity than linear methods it cannot detect all nonlinear components. These components can be detected by the Varied Amplitude method that is based on wide band excitation of surface vibration using the principles of time reversal acoustics. The nonlinear effects are measured for surface vibrations with different amplitudes and the normalized received signals can then be subtracted to reveal all the nonlinear components. We conducted a set of experiments using a box with six loudspeaker and an array of spatially distributed geophones to measure the linear and nonlinear components for various types of mines in different soils. Information about nonlinear components' spatial distribution allows one to observe greater mine/no mine contrast than with linear and other nonlinear techniques. [Work was supported by the U.S. Army RDECOM CERDEC Night Vision and Electronic Sensors Directorate.] 


\title{
Session 5aPAc
}

\section{Physical Acoustics: Vibrations in Plates I}

\author{
Daniel Royer, Cochair \\ Laboratoire Ondes et Acoustique, ESPCI, Université Paris 7, CNRS, 10 rue Vauquelin, Paris, 75005, France \\ Olivier Poncelet, Cochair \\ LMP, UMR CNRS 5469, Université Bordeaux I, 351, cours de la Libération, Talence, 33405, France
}

\section{Contributed Papers}

\begin{abstract}
8:00
5aPAc1. Discussion on the validity domain of SEA. Alain Le Bot (CNRS, LTDS Ecole centrale de Lyon 36, av. Guy de Collongue, 69134 Ecully, France, alain.le-bot@ec-lyon.fr), Vincent Cotoni (ESI Group, 12555 High Bluff Dr., suite 250, San Diego, CA 92130, USA, vincent.cotoni @esi-group-na.com)

This paper is concerned with the validity domain of SEA which is defined in terms of four criteria. The mode count $\mathrm{N}$ and the modal overlap $\mathrm{M}$ must be high, the normalized attenuation factor $\mathrm{m}$ and the coupling strength gamma must be small. The application of the dimensional analysis on the governing equations of plates gives the space of dimensionless parameters in which the validity domain of SEA must be delimited. This domain is discussed on the basis of the geometric properties of the surfaces delimiting it. The diagrams of validity of SEA are introduced and proposed as a tool for a practical use of SEA.
\end{abstract}

\section{8:20}

5aPAc2. Lamb waves filtering by waveguides with linear varying section. Zahia Hamitouche (LOMC FRE-3102 CNRS, Groupe Ondes Acoustiques, University of Le Havre, Place Robert Schuman, BP 4006, 76610 Le Havre, France, zahia.hamitouche@univ-lehavre.fr), Mounsif Ech-Cherif El-Kettani (LOMC FRE-3102 CNRS, Groupe Ondes Acoustiques, University of Le Havre, Place Robert Schuman, BP 4006, 76610 Le Havre, France, elkettani@univ-lehavre.fr), Hakim Djelouah (Faculty of Physics, University of Science and Technology Houari Boumedienne, BP 32 El Allia, Bab-Ezzouar, 16000 Algiers, Algeria, djelouah_hakim @yahoo.fr), Jean-Louis Izbicki (LOMC FRE-3102 CNRS, Groupe Ondes Acoustiques, University of Le Havre, Place Robert Schuman, BP 4006, 76610 Le Havre, France, jean-louis.izbicki@univ-lehavre.fr)

This experimental and numerical work deals with the study of Lamb modes propagating in a plate with a linear variation of thickness. The varying section area exhibits a filtering phenomenon for Lamb modes having a frequency-thickness cutoff. If the incident Lamb wave, excited at a given frequency and propagating downslope, reaches its thickness cutoff in the varying section area, it is reflected and propagates upslope. Otherwise, it is transmitted outside the varying section domain with modes conversion. The S2 incident mode is a particular case: when it reaches its cutoff, it is converted into the S1 mode with negative groupe velocity and a downslope propagation; then the S1 Lamb wave is reflected at its cutoff. The experimental and numerical studies are in good agreements.

\section{8:40}

5aPAc3. Experimental study of lamb mode conversions at the junction of two joined plates. Taoufiq Belhoussine Drissi (LOMC FRE-3102 CNRS, Groupe Ondes Acoustiques, University of Le Havre, Place Robert Schuman, BP 4006, 76610 Le Havre, France, taoufiq_belhoussine_drissi@yahoo.fr), Bruno Morvan (LOMC FRE-3102
CNRS, Groupe Ondes Acoustiques, University of Le Havre, Place Robert Schuman, BP 4006, 76610 Le Havre, France, bruno.morvan@univ-lehavre.fr), Pascal Pareige (LOMC FRE-3102 CNRS, Groupe Ondes Acoustiques, University of Le Havre, Place Robert Schuman, BP 4006, 76610 Le Havre, France, pascal.pareige@univ-lehavre.fr)

In this study we are interested in a welded junction of two plates. Plates have the same thickness and are joined along their edges. Some previous theoretical studies have shown that guided Lamb waves are suitable for the characterisation of the interface between two plates [C. Scandrett and N. Vasudevan \& M.V Predoi and M. Rousseau]. In a first time a junction perpendicular to the plates is experimentally investigated. Aluminum, copper and steel plates are used. Incident S0 Lamb wave is excited by a contact piezocomposite transducer and the surface displacements of the plate are detected by use of a laser vibrometer on both side of the junction. Converted waves are observed. From the measured normal surface displacements, reflection and transmission energy coefficients of the incident S0 wave through the junction are calculated. These coefficients are in a good agreement with the theoretically predicted ones. In a second time, the studied junction is not perpendicular to the plate. Experimental results are presented and compare to numerical ones obtained with the $\mathrm{Comsol}^{\circledR}$ Finite Element code.

\section{9:00}

5aPAc4. Second order dispersive effect on zero-group velocity Lamb modes. Daniel Royer (Laboratoire Ondes et Acoustique, ESPCI, Université Paris 7, CNRS, 10 rue Vauquelin, 75005 Paris, France, daniel.royer@espci.fr), Dominique Clorennec (Laboratoire Ondes et Acoustique, ESPCI, Université Paris 7, CNRS, 10 rue Vauquelin, 75005 Paris, France, dominique.clorennec@espci.fr), Claire Prada (Laboratoire Ondes et Acoustique, ESPCI, Université Paris 7, CNRS, 10 rue Vauquelin, 75005 Paris, France, claire.prada-julia@espci.fr)

After a local and transient loading of a plate, the acoustic energy decay in the source area can be explained by three mechanisms: the energy transport phenomenon at the Lamb wave group velocity, the material damping and the second-order dispersive effect. Generally, the first mechanism dominates the other two. However, some dispersion curves of Lamb modes present a minimum frequency for a nonzero wave number. Since at these points the group velocity vanishes, no energy transport occurs, and the slower other two phenomena can be observed. Using laser-based ultrasonic techniques, we show that the mechanical response of various thin metallic plates to a laser pulse impact is dominated by the resonance of the zero group velocity $\mathrm{S}_{1}$-Lamb mode. In the first microseconds, we observed a $\mathrm{t}^{-1 / 2}$ decay of the local vibration amplitude, which cannot be explained by the material damping. We demonstrated that this effect is ascribed to the second-order term in the dispersion relation. After this power law decay, the 
mechanical displacement undergoes an exponential decay corresponding to the wave damping. Then, we show that the local attenuation of the plate material can be measured without any mechanical contact.

\section{9:20}

5aPAc5. Guided waves in plates with linear variation of thickness Mihai Valentin M. Predoi (University Politechnica of Bucharest, Department of Mechanics, 060032 Bucharest, Romania, predoi @ cat.mec.pub.ro), Mounsif Ech Cherif El Kettani (LOMC FRE-3102 CNRS, Groupe Ondes Acoustiques, University of Le Havre, Place Robert Schuman, BP 4006, 76610 Le Havre, France, mounsif.elkettani @ univ-lehavre.fr), Zahia Hamitouche (LOMC FRE-3102 CNRS, Groupe Ondes Acoustiques, University of Le Havre, Place Robert Schuman, BP 4006, 76610 Le Havre, France, zahia.hamitouche@univ-lehavre.fr) , Cristian Catalin Petre (University Politechnica of Bucharest, Department of Mechanics, 060032 Bucharest, Romania, cristian.petre@promteh.ro)

Guided waves represent promising nondestructive evaluation (NDE) techniques. Their advantage of long distance propagation is however hampered by complex wave scattering at each discontinuity along the investigated structure. These scattered waves can be used to locate and size possible defects. Theoretical investigation is a prerequisite step in the development of a NDE technique. Planar metallic structures with localized thickness variation imposed by design represent possible new industrial structures that could be tested using guided waves. The waves scattered at the region of variable thickness must be theoretically predicted and experimentally measured before any defect could be detected. Very few authors have investigated waveguides with continuous variation of thickness. Our previous numerical and experimental studies have proven the complexity of the scattering phenomenon in such cases. Among other remarks is the curved shape of the wave front. The present work investigates properties of the waves in the linear thickness variation region of the waveguide. A plain strain model, developed in polar coordinates is theoretically developed and numerically tested using specialized finite elements based algorithms. Specific properties such as stress and displacements fields are determined by numerical simulation at several frequencies and dihedral angles.

\section{9:40-10:40 Posters}

Lecture sessions will recess for presentation of poster papers on various topics in acoustics. See poster sessions for topics and abstracts.

\section{Contributed Papers}

\section{0:40}

5aPAc6. Refracting characteristics of backward propagating Lamb modes. Massimo Germano (Sapienza Università di Roma, Via A. Scarpa, 14, 00161 Rome, Italy, massimo.germano@uniroma1.it), Adriano Alippi (Sapienza Università di Roma, Via A. Scarpa, 14, 00161 Rome, Italy, adriano.alippi@uniroma1.it), Andrea Bettucci (Sapienza Università di Roma, Via A. Scarpa, 14, 00161 Rome, Italy, andrea.bettucci@uniroma1 .it), Annunziata D’Orazio (Sapienza Università di Roma, Via A. Scarpa, 14, 00161 Rome, Italy, annunziata.dorazio@uniroma1.it)

Lamb waves are acoustic modes propagating along thin plates or shells and result from the coupling of longitudinal waves and shear vertical waves (polarized along the thickness direction), taking place at the surface boundaries for satisfying the boundary conditions of the waveguide. Their nature being a composition of two differently polarized components, it permits that, for some combination of frequency and wavenumber, a Lamb mode is propagating which has its group velocity contradirected with respect to the phase velocity. These are the so-called "backward" Lamb modes, and one of them takes place at the starting region (low wave number) of the $S_{1}$ mode dispersion curve in aluminum. Peculiar features of a wave packet of a symmetric Lamb mode $S_{1}$, backward propagating in an aluminum waveguide, are observed and anomalous behavior in refraction, diffraction and interference of this peculiar mode is experimentally put in evidence. Particularly, contradirected refraction is put in evidence, as it takes place at the boundary where a forward propagating mode is coupled to a backward propagating one, and change from forward to backward mode is obtained in case of a wedge shaped laminar plate, which goes through a zero point of the group velocity.

\section{1:00}

5aPAc7. Conversion of a Scholte - Stoneley wave at the extremity of a fluid loaded plate: comparison between finite element method, radiation mode theory and experiments. Ebrahim Lamkanfi (Ghent University, Sint-Pietersnieuwstraat 41, 9000 Ghent, Belgium, Ebrahim.Lamkanfi @ugent.be), Nico F. Declercq (Georgia Tech Lorraine - G.W. Woodruff School of ME, UMI Georgia Tech - CNRS 2958, 2 rue Marconi, 57070 Metz, France, nico.declercq@me.gatech.edu), Wim Van Paepegem (Ghent University, Sint-Pietersnieuwstraat 41, 9000 Ghent, Belgium, wim.vanpaepegem@ugent.be), Joris Degrieck (Ghent University, SintPietersnieuwstraat 41, 9000 Ghent, Belgium, joris.degrieck@ugent.be)

The radiation theory has proved to produce results in agreement with experiments when the conversion is investigated of a Scholte - Stoneley wave at the extremity of a fluid loaded plate. The drawback of the radiation mode theory is that it is not universally applicable and is also very cumbersome. The advantage is that it is an exact method. There is a trend in acoustics to develop finite element models to describe the interaction of sound waves with materials. This investigation compares a newly developed finite element model to simulate the considered effect. Results are compared with the exact results obtained by the radiation mode theory [J. Acoust. Soc. Am. 101(3), 1347-1357, 1997] and experimental observations [J. Acoust. Soc. Am. 95(1), 13-20, 1994]. The study shows correspondence between the finite element analysis and earlier obtained experimental and theoretical results as a function of a relationship between mesh properties and the evanescence (rather than the wave length) of the considered Stoneley waves. 


\title{
Session 5aPAd
}

\section{Physical Acoustics: Time Reversal Acoustics for Nonlinear Imaging II (Poster Session)}

\author{
Alexander Sutin, Cochair \\ Stevens Institute of Technology \\ Serge Dos Santos, Cochair \\ ENI Val de Loire, Université François Rabelais de Tours, LUSSI CNRS
}

\begin{abstract}
All posters will be on display from 9:40 a.m. to 11:20 a.m. To allow contributors an opportunity to see other posters, contributors of odd-numbered papers will be at their posters from 9:40 a.m. to 10:30 a.m. and contributors of even-numbered papers will be at their posters from 10:30 a.m. to 11:20 a.m.
\end{abstract}

\section{Contributed Papers}

5aPAd1. Nonlinear phase conjugate ultrasonic waves in moving media. Vladimir L. Preobrazhensky (IEMN, UMR CNRS 8520, avenue Poincaré, BP 60069, 59652 Villeneuve d'Ascq, France, vladimir.preobrajenski @iemn.univ-lille1.fr), Philippe Pernod (IEMN, UMR CNRS 8520, avenue Poincaré, BP 60069, 59652 Villeneuve d'Ascq, France, philippe .pernod@iemn.univ-lille1.fr), Yuri Pyl'Nov (Moscow Institute of Radio Engineering, Electronics \& Automation, Vernadsky prosp., 78, 119454 Moscow, Russian Federation, pylnov@yandex.ru), Nikolay Smagin (Moscow Institute of Radio Engineering, Electronics \& Automation, Vernadsky prosp., 78, 119454 Moscow, Russian Federation, pylnov@yandex.ru)

The basic principle of acoustic wave phase conjugation provides compensation of phase incursions during back propagation of the phase conjugate waves (PCW) towards the source of the primary emission. This feature of PCW results from time reversal invariance of acoustic field in a stationary medium. In moving media or in the presence of flows the time reversal invariance is broken resulting in essential modification of PCW properties. The incident wave spatial distribution is not reproduced completely by the phase conjugate wave and the phase incursions are not compensated any more. In the present paper the general theory of PCW propagation in moving nonlinear media is developed. The theory is applied for description of PCW acoustic rays and calculation of noncompensated phase shift of PCW on the source of the primary probing wave. The treatment of the phase shift of the PCW is developed as a new method of diagnostics and imaging of flow velocity distributions in liquid. The method is approved experimentally on examples of laminar and vortex flows and immersed fluid jet in water. Improvement of quality of the images of flows using nonlinear modes of propagation and interaction of phase conjugate waves is demonstrated.
5aPAd2. Evaluation of local nonlinearity using time reversal acoustic focusing. Serge Dos Santos (ENI Val de Loire, Université François Rabelais de Tours, LUSSI CNRS, rue de la Chocolaterie, 41034 Blois, France, serge.dossantos@univ-tours.fr), Alexander Sutin (Stevens Institute of Technology, Castle Point on Hudson, Hoboken, NJ 07030, USA, asutin @ stevens.edu), Armen Sarvazyan (Artann Laboratories, 1753 LinvaleHarbourton, Lambertville, NJ 08350, USA, armen@artannlabs .com), Thomas Goursolle (ENI Val de Loire, Université François Rabelais de Tours, LUSSI CNRS, rue de la Chocolaterie, 41034 Blois, France, thomas.goursolle@univ-tours.fr)

Time reversal acoustic focusing can be used for local assessment of nonlinear properties of media [Sarvazyan A., Sutin A., JASA, 115 (5): 2595 (2004)]. Time Reversal focusing provides high acoustic pressure leading to a nonlinear response localized in space and time. Interaction area of two overlapping focused beams acts as a virtual localized source generating ultrasonic waves with combination frequencies. Measurements of the intensity of this signal allow estimation of the nonlinear parameter in the focal region. The first experiment was conducted in water with spatially varying acoustical nonlinearity produced by a microbubble column or contrast agent Optison. We used two synchronized TRA aluminium reverberators to focus ultrasonic waves with frequencies $660 \mathrm{kHz}$ and $776 \mathrm{kHz}$. Various parameters such as amplitude and frequency of the primary field, the position and number of emitters, choice of initial excitation (tone burst, binary, shirpcoded) were optimized for the nonlinear signature extraction. The second experiment was conducted in steel samples where surface vibration were measured by laser vibrometer. Excitation frequencies were between $490 \mathrm{kHz}$ and $860 \mathrm{kHz}$. These experiments show again that amplitude dependence of nonlinear generated components was slightly less than the product of primary component that is predicted by classical nonlinear theory. 


\title{
Session 5aPAe
}

\author{
Albert Migliori, Cochair \\ Los Alamos National Laboratory \\ Claude Boutin, Cochair \\ École Nationale des Travaux Publics de État
}

\section{Physical Acoustics: Ultrasonics: Transducers and Instruments I (Poster Session)}

\begin{abstract}
All posters will be on display from 9:40 a.m. to 11:20 a.m. To allow contributors an opportunity to see other posters, contributors of odd-numbered papers will be at their posters from 9:40 a.m. to 10:30 a.m. and contributors of even-numbered papers will be at their posters from 10:30 a.m. to 11:20 a.m.
\end{abstract}

\section{Contributed Papers}

5aPAe1. An ultrasonic technique using shear waves interactions to characterize new hybrid gels. Philippe Banet (ECIME Université de Cergy-Pontoise, rue d'Eragny, 95031 Neuville-sur-Oise, France, philippe.banet@u-cergy.fr), Pascal Griesmar (ECIME Université de CergyPontoise, rue d'Eragny, 95031 Neuville-sur-Oise, France, pascal.griesmar @u-cergy.fr), Frederic Vidal (LPPI - Université de Cergy-Pontoise, rue d'Eragny, 95031 Neuville-sur-Oise, France, frederic.vidal@u-cergy .fr), Jean-Yves Le Huerou (ECIME Université de Cergy-Pontoise, rue d'Eragny, 95031 Neuville-sur-Oise, France, jean-yves.lehuerou@u-cergy .fr), Claude Chevrot (LPPI - Université de Cergy-Pontoise, rue d'Eragny, 95031 Neuville-sur-Oise, France, claude.chevrot@u-cergy.fr), Dominique Teyssie (LPPI - Université de Cergy-Pontoise, rue d'Eragny, 95031 Neuville-sur-Oise, France, dominique.teyssie@u-cergy.fr), Stéphane Serfaty (ECIME Université de Cergy-Pontoise, rue d'Eragny, 95031 Neuville-sur-Oise, France, stephane.serfaty@u-cergy.fr)

Thickness shear mode transducer techniques using AT-cut quartz crystal can be used to measure viscoelastic parameters of soft materials (gels, colloidal suspensions $\propto$ ) during their formation. Today the control of hybrid materials synthesis and evolution has a great interest, particularly for biomedical applications such as drug delivery. With those materials, a challenge is to obtain new structures constituted by interpenetrating organic- inorganic polymers networks (IPN) to get novel therapeutic behaviours. In this paper, the monitoring of ultrasonic shear waves propagation in the material at 6 $\mathrm{MHz}$ is presented for different thermosensible hybrid (poly-Nisopropylacrylamide (PNIPAM) - silica). We show that at a mesoscopic scale it is possible to analyze mechanical interactions between different networks related to chemical bounds. The network evolution observed with our technique is in good agreement with the Fourier transform infrared analysis of NIPAM polymerization. As shown in previous work for silica gels, a precursor characteristic time of gelation process (in liquid phase) can be determined to describe the polymerization kinetic. By its nondestructive character and its simplicity of measurement, this technique allows an online optimization of new hybrid materials.

5aPAe2. Effects of materials conductivity on the viscosity measurement using a QCM. Stéphane Serfaty (ECIME Université de Cergy-Pontoise, rue d'Eragny, 95031 Neuville-sur-Oise, France, stephane.serfaty@u-cergy .fr), Emmanuel Caplain (ECIME Université de Cergy-Pontoise, rue d'Eragny, 95031 Neuville-sur-Oise, France, emmanuel.caplain@u-cergy .fr), Pascal Griesmar (ECIME Université de Cergy-Pontoise, rue d'Eragny, $95031 \quad$ Neuville-sur-Oise, France, pascal.griesmar@u-cergy .fr), Jean-Yves Le Huerou (ECIME Université de Cergy-Pontoise, rue
d'Eragny, 95031 Neuville-sur-Oise, France, jean-yves.lehuerou@u-cergy .fr), Marcel Gindre (ECIME Université de Cergy-Pontoise, rue d'Eragny, 95031 Neuville-sur-Oise, France, marcel.gindre@u-cergy.fr)

Quartz crystal microbalance (QCM) is commonly used to characterize the viscosity of soft materials. For biomedical applications the modified BVD model of QCM is unsuitable due to the conductivity of the biomaterial. In order to take into account the electrical effects, a new model including a static lossy capacitor is proposed. A theoretical study of the shear wave propagation in the quartz shows that these effects modify the static and the motional branches of the BVD circuit. The conductivity effects of the material at the surface of the QCM can be modeled by same parallel elements added in both branches. In the motional branch the electromechanical coupling factor is applied to these elements. To validate the new lumped element model measurements for $\mathrm{KCl}$ mixtures are achieved. The results show that an accurate extraction of viscosity $(<5 \%)$ can be obtained for a middle of conductivity less than $0.3 \mathrm{~S} / \mathrm{m}$. In addition for water/glycerol mixtures the resonant frequency shift and damping follow an accurate linear shape $(<5 \%)$ according to the square root of the liquid viscosity and density product. These results increase (up to 200 times) the validity domain of viscosity compared to the classical modified BVD model.

5aPAe3. Viscosimetry using a new electromagnetic-acoustic microbalance. Emmanuel Caplain (ECIME Université de Cergy-Pontoise, rue d'Eragny, 95031 Neuville-sur-Oise, France, emmanuel.caplain @ u-cergy.fr), Stéphane Serfaty (ECIME Université de Cergy-Pontoise, rue d'Eragny, $95031 \quad$ Neuville-sur-Oise, France, stephane.serfaty @u-cergy.fr), Pascal Griesmar (ECIME Université de Cergy-Pontoise, rue d'Eragny, 95031 Neuville-sur-Oise, France, pascal.griesmar@u-cergy .fr), Jean-Yves Le Huerou (ECIME Université de Cergy-Pontoise, rue d'Eragny, 95031 Neuville-sur-Oise, France, jean-yves.lehuerou @u-cergy.fr), Nicolas Wilkie-Chancellier (ECIME Université de CergyPontoise, rue d'Eragny, 95031 Neuville-sur-Oise, France, Nicolas.WilkieChancellier@u-cergy.fr)

The nanostructure evolution of gels, biomaterials or porous media can be characterized by its mechanical properties. Few nondestructive techniques are developed to investigate the viscosity evolution. This paper present a new electromagnetic-acoustic technique using a wireless thickness shear mode transducer. A suitable model of the measurement is also presented to characterize the viscosity of the nanostructure in contact with the transducer. This transducer is a double copper-clad PVDF substrate resonator, designed to operate over a wide radiofrequency range without lumped tuning capacitors. This architecture constitutes an alternative solution to design a high-Q ultrasonic microbalance. To characterize the material at the surface of the transducer, the evolution of the induced complex impedance is measured. From this evolution, the mechanical energy storage and dissipa- 
tion in the material can be extracted. In order to validate the lumped element model used, a series of glycerol/water mixtures is studied. We show that the resonant frequency shift and damping follow an accurate linear shape $(<5 \%)$ according to the square root of the liquid viscosity and density product. This result is in good agreement with the classical prediction of Martin and Kanazawa obtained with a quartz crystal microbalance.

5aPAe4. Functionally graded plates studied by laser ultrasonic technique. Yongdong Pan (Institute of Acoustics, Tongji University, 1239 Siping Road, 200092 Shanghai, China, ypan@mail.tongji.edu.cn), Qian Wang (Institute of Acoustics, Tongji University, 1239 Siping Road, 200092 Shanghai, China, wang_qian_@126.com), Zheng Zhong (School of Aerospace Engineering and Applied Mechanics, Tongji University, 1239 Siping Road, 200092 Shanghai, China, zhongk@mail.tongji.edu.cn), Clément Rossignol (LMP, UMR CNRS 5469, Université Bordeaux I, 351, cours de la Libération, 33405 Talence, France, c.rossignol@1mp.u-bordeaux1 .fr), Bertrand Audoin (LMP, UMR CNRS 5469, Université Bordeaux I, 351, cours de la Libération, 33405 Talence, France, b.audoin@1mp.ubordeaux1.fr)

In a functionally graded plate, the composition and structure change continuously along the thickness direction, resulting in corresponding changes of the acoustic impedance of the material. The study of the wave propagation in such a plate is important for both the nondestructive evaluation of properties of functionally gradient materials and engineering. In this work, the experimental system of laser ultrasonic measurement is introduced, and a theoretical model is proposed to predict the transient displacement field generated by laser in a functionally graded plate (FGP). After the laser line source is assumed as an idea transient force, and the FGP is considered as an inhomogeneous plate along its thickness direction, the transient displacement field is numerically solved by the Peano expansion method. Laser ultrasonic measurement was carried on several FGPs, and the direct arrivals of longitudinal and shear waves were observed in the experimental displacement field. The agreement between experimental and calculated theoretical waveforms provides a promise for both experimental and theoretical methods.

5aPAe5. Genetic manipulation engineers the elasticity of arabidopsis thaliana. Ari Salmi (Univ. of Helsinki / Dept. of Physical Sciences, POB 64 (Gustaf Hällströmin katu 2), 00014 Helsinki, Finland, edward.haeggstrom@helsinki.fi), Kimmo Mustonen (University of Helsinki, Gustaf Hällströmin Katu 2, 00015 Helsinki, Finland, kimmo.mustonen@helsinki.fi), Juha Immanen (Dept. of Biological and Environmental Sciences, P.O.B 65, 00014 Helsinki, Finland, juha.immanen@helsinki.fi), Yrjö Helariutta (Dept. of Biological and Environmental Sciences, P.O.B 65, 00014 Helsinki, Finland, yrjo.helariutta@helsinki.fi), Edward Hæggström (Electronics Research Unit, University of Helsinki, P.O.Box 64 (Gustaf Hällströmin katu 2), FIN00014 Helsinki, Finland, edward.haeggstrom@helsinki.fi)

Arabidopsis thaliana is a model species widely used in plant science studies. It grows fast and exhibits wood formation in its stem and root resembling softwood produced by angiosperm trees. The Arabidopsis model allows rapid testing of methods for wood composition modification possibly adaptable for tree breeding. We estimated ultrasonically the modulus of elasticity in Arabidopsis stems and roots in both radial and in-plane direction for two commonly used ecotypes (Columbia and Landsberg erecta). Plants were grown in greenhouse conditions and six inflorescence stems per ecotype were measured in vivo applying $4 \mathrm{MHz}$ out-of-plane and $2 \mathrm{MHz}$ in-plane ultrasonic tone bursts. The effects of AtCAD1 gene expression silencing in Col ecotype were tested using the same method. We recorded a $(10 \%$, $\mathrm{p}=0.1)$ decrease in the elastic modulus of the lignin-decreased Atcad 1 samples (10-20\% decrease in lignin content), and a significant $(29 \%, \mathrm{p}=0$ .02) difference between the sound velocities in the two ecotypes. In-plane elasticity results are also presented. The results are the first ultrasonically measured modulus values for this commonly used plant model species, creating a quantitative mechanical measure for the effects of wood composition modification.

5aPAe6. Nondestructive evaluation of heterogeneous materials using acoustic emission and ultrasound. Adil Faiz (ENSIM - LAUM, Université du Maine, rue Aristote, 72085 Le Mans, France, adil.faiz@univ-lemans.fr), Rachid El Guerjouma (ENSIM - LAUM, Université du Maine, rue Aristote, 72085 Le Mans, France, Rachid.El_Guerjouma@univ-lemans.fr), Mouras Bentahar (Université du Maine LAUM, Lab. d'Acoustique Université du Maine, UMR CNRS 6613, 72085 Le Mans Cedex 9, France, mourad.bentahar@univ-lemans.)

Heterogeneous materials as composites and concrete are advantageous as structural components in many applications. However, damage detection in such materials is difficult due to their heterogeneity and anisotropy. Furthermore, conventional non destructive technique as $\mathrm{x}$-radiographic is mostly not very sensitive to early damage and very time consuming and expensive. The purpose of this contribution is to study the capabilities of several methods, nondestructive and very sensitive for damage characterisation, as acoustic emission (AE) and ultrasonic for the structural health monitoring of heterogeneous materials as polymer based composite materials and concrete. These materials are instrumented by piezoelectric sensors in order to detect acoustic emission and to measure the ultrasonic velocity. The mechanisms of the damage events and their space-time localizations are identified from AE. Simultaneously, the longitudinal ultrasonic velocity is measured in situ by transmission through the composite thickness. The AE is very well correlated with the loss of stiffness determined from ultrasonic velocity measurements showing the potentiality of this combined approach for in situ structural monitoring.

5aPAe7. Development of a system using ultrasonic waves for assessing the quality of frying oil and salad oil during frying. Driss Izbaim (Ibn Zohr University, FS Agadir, 80000 Agadir, Morocco, driss_izbaim@yahoo.fr), Bouazza Faiz (Ibn Zohr University, FS Agadir, 80000 Agadir, Morocco, faizbou@hotmail.com), Adil Hamine (Ibn Zohr University, FS Agadir, 80000 Agadir, Morocco, adil.hamine @Gmail.com), Naima Taifi (Fac. des sciences; Univ. Chouaib Doukkali, B.P: 20, 24000 El Jadida, Morocco, taifinaima@yahoo.fr), Ali Moudden (Ibn Zohr University, FS Agadir, 80000 Agadir, Morocco, ali _moudden@yahoo.fr)

The design and construction of a real-time system is described with reference to applications in quality control of frying oil. This system, developed using the LabVIEW ${ }^{\mathrm{TM}}$ software, controls the ultrasonic velocity by receiving the signals backscattered by a recipient full of oil. Measurements on samples of frying oil and salad oil are described and the results of velocity measurements on a group of samples of varying frying time taken in $160^{\circ} \mathrm{C}$ are reported. The new system is built around three main elements: acquiring the oil signals, automating the signals control, and several calculations to determine in real-time the phase velocity of the oil. The results obtained show the feasibility of assessing the quality of oils using ultrasonic waves through monitoring the frying process. This system can be used also to control other industry products such as the canned fish oil, milk, and the cement.

5aPAe8. Influence of polymeric nanocomposite film on acoustic waves in piezoelectrics. Iren E. Kuznetsova (Institute of Radio Engineering and Electronics of RAS, Saratov Branch, Zelyonaya str., 38, 410019 Saratov, Russian Federation, kuziren@yandex.ru), Boris D. Zaitsev (Institute of Radio Engineering and Electronics of RAS, Saratov Branch, Zelyonaya str., 38, 410019 Saratov, Russian Federation, zai-boris@yandex.ru), Anastasia S. Kuznetsova (Institute of Radio Engineering and Electronics of RAS, Saratov Branch, Zelyonaya str., 38, 410019 Saratov, Russian Federation, tigrnasya@yandex.ru)

At present the attention of researchers is attracted by investigation of multilayer acoustical waveguides with using of well known as well as novel materials. Also in present time there exist a lot of papers devoted to novel nanocomposite polymeric materials. But the influence of such materials on parameters of acoustic waves in piezoelectrics did not study. In this connec- 
tion the paper is devoted to theoretical investigation of acoustic waves in structure "piezoelectric - polymeric nanocomposite film." The analysis was carried out by the example of polymeric nanocomposite film with various contents of $\mathrm{Fe}$ and $\mathrm{CdS}$ nanoparticles and lithium niobate plate or substrate. As a result of conducted calculation the velocity and attenuation per wavelength have been found for acoustic waves propagating in aforementioned structure. The conducted analysis has showed that for certain value of nano- composite film thickness $d$ the attenuation of investigated waves has the resonant behavior. The obtained results allow choose such values of $\mathrm{d}$ for which the acoustic wave does not practically attenuate in the presence of the film. This opens, for example, the prospects of development of undisturbed substrates of nanocomposite materials for thin piezoelectric plates. This work is supported by RFBR 06-08-01011, RNP.2.1.1.8014 (Russia), contract 2007-3-1.3-07-15-036 (Russia).

FRIDAY MORNING, 4 JULY 2008

P3-A, LEVEL 3, 9:40 TO 11:20 A.M.

\title{
Session 5aPAf
}

\section{Physical Acoustics: Infrasound II (Poster Session)}

\author{
Henry Bass, Cochair \\ The University of Mississippi - NCPA \\ Alexis Le Pichon, Cochair \\ CEA-DASE
}

\begin{abstract}
All posters will be on display from 9:40 a.m. to 11:20 a.m. To allow contributors an opportunity to see other posters, contributors of odd-numbered papers will be at their posters from 9:40 a.m. to 10:30 a.m. and contributors of even-numbered papers will be at their posters from 10:30 a.m. to 11:20 a.m.
\end{abstract}

\section{Contributed Papers}

5aPAf1. Close listening of a permanently degassing volcano: Yasur (Vanuatu). Christelle Zielinski (Institut de Physique du Globe, 4 Place Jussieu, 75252 Paris, France, zielinski@ipgp.jussieu.fr), Sylvie Vergniolle (Institut de Physique du Globe, 4 Place Jussieu, 75252 Paris, France, vergniolle@ipgp.jussieu.fr), Michel Lardy (IRD Center, BPA5, Cedex, 98848 Noumea, New Caledonia, lardy@noumea.ird.nc), Alexis Le Pichon (CEA-DASE, Arpajon Cedex, 91297 Bruyères-le-Châtel, France, alexis.le-pichon@cea.fr), Michel Frogneux (IPGS, 5, rue René Descartes, 67084 Strasbourg Cedex, France, michel.frogneux@eost.u-strasbg.fr)

Acoustic measurement is a very powerful technique to monitor gas volume and pressure at a safe distance of a volcano. Since eruptions are driven by a gas phase, acoustic recordings of eruptions can provide insights into the degassing processes. The permanent activity of Yasur volcano (Vanuatu) has been acoustically monitored continously to detect and understand the sudden increases in the volcanic degassing. In the absence of appropriate modeling of the sound source, we have used a dimensionless analysis of our infrasonic records close to the source to relate acoustic power and the velocity of the gas - ejecta mixture. Our one-year dataset at the source shows several sudden increases in gas flux over one week as well as more progressive evolution, over several months. Explosions are also detected using a method based on wavelet decomposition. This shows that most of the changes in gas flux are related to strong changes in the number of explosions. Understanding the origin of these variations has strong implications for hazards assessments.

5aPAf2. Low frequency airplane noise measurement. Xiao Di (National Center for Physical Acoustics, University of Mississippi, University, MS 38677, USA, xiaodi@olemiss.edu), Claus Hetzer (National Center for Physical Acoustics, University of Mississippi, University, MS 38677, USA, claus@olemiss.edu), Ronald A. Wagstaff (National Center for Physical Acoustics, University of Mississippi, University, MS 38677, USA, rwagstaf@olemiss.edu), Ali M. Mir (National Center for Physical Acoustics, University of Mississippi, University, MS 38677, USA, mali1 @ olemiss.edu)
Low frequency airplane noise during takeoff and landing periods is a serious environment hazard. Also, the low frequencies originating from the airplanes could potentially be an easy target for detection. Since there is very little attenuation at the low frequencies, the low frequency sound can propagate to relatively long distances. Several field experiments were conducted to identify the low frequency signal and noise content from many different airplanes. What is different in our experiment from most other noise experiments is that we will identify the very low frequency content of the airplane noise spectrum, from $200 \mathrm{~Hz}$ down to the infrasound range, $0.02 \mathrm{~Hz}$. During the experiments, two infrasound microphone systems were used: Chaparral 50, which has a bandwidth from 0.02 to $50 \mathrm{~Hz}$, and Chaparral 2.5, which has a bandwidth from 0.1 to $200 \mathrm{~Hz}$. In addition to the infrasound systems, a recording system with $\mathrm{B} \& \mathrm{~K}$ microphones was also used. The data were collected before, during, and after landing and takeoff periods. The low frequency signal and noise content during each of those periods will be analyzed and reported on.

5aPAf3. Experimental study on the effects of $\mathrm{N}$-wave sonic-boom signatures on window vibration. Yusuke Naka (Japan Aerospace Exploration Agency, 6-13-1 Osawa, Mitaka, 181-0015 Tokyo, Japan, ynaka@chofu.jaxa.jp), Yoshikazu Makino (Japan Aerospace Exploration Agency, 6-13-1 Osawa, Mitaka, 181-0015 Tokyo, Japan, makino@chofu.jaxa.jp), Takeshi Ito (Japan Aerospace Exploration Agency, 6-13-1 Osawa, Mitaka, 181-0015 Tokyo, Japan, ito@chofu.jaxa.jp)

For fulfillment of overland supersonic flight, it is important to understand the effects of sonic booms on buildings, since these effects, including vibration of walls and rattling noise from windows, are believed to be the main causes of annoyance in indoor spaces. In this study, the vibration of windows impacted by sonic booms is investigated through in-laboratory experiments by using a device developed at Japan Aerospace Exploration Agency (JAXA). Rattle is captured as high-frequency components of the acceleration of the windows. The effects of sonic booms having $N$-shape signatures with various values of peak overpressure, rise time, and duration are investigated. Results show general tendency of increase of rattle with increasing peak overpressure and decreasing rise time, as expected. However, 
the results also indicate a possibility that these parameters, as well as duration, affect the time-frequency structure of the acceleration (and hence rattle) of windows in a somewhat complicated manner.

5aPAf4. Correlating electromagnetical and infrasonic signals from thunderstorms. Jelle Assink (Royal Netherlands Meteorological Institute (KNMI), PO Box 201, 3730 AE De Bilt, Netherlands, jelle.assink@gmail.com), Laeslo G. Evers (Royal Netherlands Meteorological Institute (KNMI), PO Box 201, 3730 AE De Bilt, Netherlands, evers@knmi.nl), Iwan Holleman (Royal Netherlands Meteorological Institute (KNMI), PO Box 201, 3730 AE De Bilt, Netherlands, holleman@knmi $. n 1)$

During thunderstorm activity, electromagnetical and infrasonic signals are emitted due to the process of lightning and thunder. It is shown that correlating infrasound detections with results from a electromagnetical lightning detection network is successful up to distances of $50 \mathrm{~km}$ from the infrasound array. Infrasound recordings clearly show blast wave characteristics, relatable to CG discharges, with a dominant frequency between 1-5 Hz. Amplitude measurements can partly be explained by the beam pattern of a line source. The ability to measure thunderstorm activity with infrasound arrays has both positive and negative implications for infrasound verification purposes.

5aPAf5. Coherency of infrasound studied with a large aperture infrasound array in the Netherlands. Johan Mentink (Royal Netherlands Meteorological Institute (KNMI), PO Box 201, 3730 AE De Bilt, Netherlands, mentink@knmi.nl), Laeslo G. Evers (Royal Netherlands Meteorological Institute (KNMI), PO Box 201, 3730 AE De Bilt, Netherlands, evers @ knmi.nl)

A large aperture infrasound array (LAIA) is being constructed in the Netherlands as geophysical part of an astronomical low frequency array, i.e., the LOFAR project. This array extends the detection capabilities of infrasound up to the $\mathrm{mHz}$ range, including acoustic-gravity and gravity waves as well. Owing to its large aperture $(80 \mathrm{~km})$ and number of interstation distances, this 30 element microbarometer array is ideally suited to study the coherency of infrasound as function of frequency under various atmospheric conditions. Based on conventional and modern correlation techniques, the ultimate aim is to experimentally determine and quantify infrasound propagation characteristics. As a result, we gain understanding in the physical mechanisms responsible for decoherence, which in turn will be used to develop infrasound as a passive atmospheric probe. Knowledge on the coherency length as function of the state of the atmosphere also has direct implications for the design of optimal infrasound arrays. This study will outline the characteristics of LAIA and present the first results of the coherency studies.
5aPAf6. Modeling real porous tube infrasonic arrays. Timothy Marston (The Graduate Program in Acoustics, The Pennsylvania State University, PO Box 30, State College, PA 16804, USA, tmm357@psu.edu), Thomas Gabrielson (The Graduate Program in Acoustics, The Pennsylvania State University, PO Box 30, State College, PA 16804, USA, tbg3@psu.edu)

Measured values of acoustic resistance in porous hoses have been implemented into a previously developed porous tube model for infrasonic arrays [J. Acoust. Soc. Am. 122, 2960 (2007)]. Garden soaker hoses are commonly used in porous infrasonic arrays, and a simple method for the measurement the acoustic resistance of a segment of soaker hose will be presented. This experimentally determined resistance value can be implemented into the porous tube model for an improved array response prediction. It is anticipated that this analysis will give rise to improved phase and magnitude predictions for this type of array. Applications include design optimization of porous tube arrays, and infrasonic propagation through porous ducts. [Research funded by the Applied Research Laboratory Educational and Foundational Fund.]

5aPAf7. Finite difference time domain synthesis of infrasound propagation through an absorbing atmosphere. Catherine D. Degroot-Hedlin (University of San Diego California, Scripps Institute of Oceanography, 9500 Gilman Drive, La Jolla, CA 92093, USA, chedlin @ ucsd.edu)

Equations applicable to finite difference time domain (FDTD) computation of infrasound propagation through an absorbing atmosphere are derived and examined in this paper. It is shown that over altitudes up to $160 \mathrm{~km}$, and at frequencies relevant to global infrasound propagation, i.e., $0.02-5 \mathrm{~Hz}$, the acoustic absorption in $\mathrm{dB} / \mathrm{m}$ varies approximately as the square of the propagation frequency plus a small constant term. A second-order differential equation is presented for an atmosphere modeled as a compressible Newtonian fluid with low shear viscosity, acted on by a small external damping force. It is shown that the solution to this equation represents pressure fluctuations with the observed form for infrasound propagation, i.e., sound is attenuated as $\left(\beta+\gamma f^{2}\right)$. Increased dispersion is predicted at altitudes over $100 \mathrm{~km}$. The equation is separated into two partial differential equations that are first order in time for FDTD implementation. A numerical analysis of errors inherent to this FDTD method shows that the attenuation term imposes additional stability constraints on the FDTD algorithm. Comparison of FDTD results for models with and without attenuation shows that the predicted transmission losses for the attenuating media agree with those computed from the synthesized waveforms. 


\title{
Session 5aPAg
}

\section{Physical Acoustics: Ultrasonics Under Extreme Conditions I (Poster Session)}

\author{
Albert Migliori, Cochair \\ $L A N L$ \\ Frédéric Decremps, Cochair \\ IMPMC, Université Paris VI
}

\begin{abstract}
All posters will be on display from 9:40 a.m. to 11:20 a.m. To allow contributors an opportunity to see other posters, contributors of odd-numbered papers will be at their posters from 9:40 a.m. to 10:30 a.m. and contributors of even-numbered papers will be at their posters from 10:30 a.m. to 11:20 a.m.
\end{abstract}

\section{Contributed Papers}

5aPAg1. Guided modes modeling in multilayered composites plates. Morched Ben Amor (Faculté des Sciences de Sfax, Laboratoire de Physique des Matériaux, BP 802, 3018 Sfax, Tunisia, morched_benamor@yahoo.fr)

Due to the periodicity of the composite structure the Floquet wave approach seems well suited for medium characterization. The pass and stop band domains for [0/45/90/-45] cross ply composites have been plotted. Dispersive guided wave propagation through a lossy composite laminates immersed in water have been investigated. Our interest is focused on a guided mode in the frequency range from $1.6 \mathrm{MHz}$ to $5 \mathrm{MHz}$. This mode has been pointed out by both reflection coefficient and energy vector analysis in term of incident angle. In this paper it is investigated how by finetuning the frequency and the incident angle, internal displacement and stress field vary in the multilayer. When the frequecy is increased the mode changes from a plate mode to a surface mode. The description includes real Floquet wave numbers as well as complex wave numbers.

5aPAg2. Influence of the calorimetric system setup on the output acoustic power measurement results. Antonio Petosic (Faculty of Electrical Engineering and Computing, Unska 3, 10000 Zagreb, Croatia, antonio.petosic@fer.hr), Bojan Ivančević (Faculty of Electrical Engineering and Computing, Unska 3, 10000 Zagreb, Croatia, bojan.ivancevic@fer
.hr),Dragoljub Svilar(Brodarski Institut, Avenija Većeslava Holjevca bb, 10000 Zagreb, Croatia, dsvilar@hrbi.hr)

The problem of measuring an output acoustic power using calorimetric method has been considered in different regimes of transmitter working. The transmitter has been driven at different excitation electrical power levels and the influence of sonotrode tip position in the different calorimetric systems on the measurement results is considered. Two calorimetric systems with different volumes of loading liquid (water) and different geometries (having influence on thermodynamical losses) have been used in the experiments. The exponential experimental temperature-time curves have been fitted with theoretical and time constants, losses, output acoustic power and electroacoustic efficiency factor have been found assuming that all ultrasound energy is absorbed in the system. The pressure field in the box is complicated and radiation impedance (radiated power) depends on the position in the complex ultrasound field where the point source is located. In the nonlinear regime of working strong cavitation activity occur with oscillating bubles in the front of vibrating tips and the radiated power is decreased because the tip is only partially radiating due to radiation impedance variations. The electroacustic efficiency factor has been compared with equivalent circuit approach where parameters of transmitter have been found for unloaded and loaded transmitter. 


\title{
Session 5aPAh
}

\section{Physical Acoustics: Ducts and Waveguides I (Poster Session)}

\author{
Marc Deschamps, Cochair \\ LMP \\ Olivier Dazel, Cochair \\ Laboratoire d'Acoustique de I'Université du Maine
}

\begin{abstract}
All posters will be on display from 9:40 a.m. to 11:20 a.m. To allow contributors an opportunity to see other posters, contributors of odd-numbered papers will be at their posters from 9:40 a.m. to 10:30 a.m. and contributors of even-numbered papers will be at their posters from 10:30 a.m. to 11:20 a.m.
\end{abstract}

\section{Contributed Papers}

5aPAh1. The design and modeling of a lateral acoustic particle manipulator exhibiting quarter-wave operation. Peter Glynne-Jones (University of Southampton, School of Engineering Sciences, University Road, SO17 1BJ Southampton, UK, p.glynne-jones@soton ac.uk), Martyn Hill (University of Southampton, School of Engineering Sciences, University Road, SO17 1BJ Southampton, UK, m.hill@soton.ac.uk), Nicholas R. Harris (University of Southampton, Electronics and Computer Science, SO17 1BJ Southampton, UK, nrh@ecs.soton.ac.uk), Rosemary J. Townsend (University of Southampton, School of Engineering Sciences, University Road, SO17 1BJ Southampton, UK, R.J.Townsend@soton.ac.uk), Surendra K. Ravula (Sandia National Laboratories, Eubank Blvd, Albuquerque, NM 87123, USA, skravul@sandia .gov)

Prior work [Petersson et al., 2004] has demonstrated the operation of a half-wave acoustic particle manipulator, whose forces act in the plane of a silicon substrate. In a half-wave device particles are directed to the centre of a channel. Devices acting in plane have manufacturing advantages, and lend themselves to many microfluidic applications. We demonstrate, for the first time, such a device with a quarter-wave mode that is able to manipulate particles to the side of a channel in addition to a near half wave mode. The design utilises resonant "islands" to create the necessary pressure release boundary condition. The device is conventionally milled in brass, permitting cheaper and quicker fabrication than in silicon. Finite element modelling is presented to elucidate the operation of both half and quarter-wave devices. In contrast to essentially one-dimensional planar devices, the twodimensional distribution of the velocity and pressure fields result in particles being constrained to a line within the channel.

5aPAh2. A semianalytical finite element method for elastic guided waves propagating in helical structures. Fabien Treyssède (Nantes Atlantique University - LCPC, Division for Metrology and Instrumentation, Route de Pornic - BP 4129, 44341 Bouguenais, France, fabien.treyssede @1cpc.fr), Ahmed Frikha (Nantes Atlantique University - LCPC, Division for Metrology and Instrumentation, Route de Pornic - BP 4129, 44341 Bouguenais, France, ahmed.frikha@lcpc.fr)

Steel multiwire cables are widely used in civil engineering as loadcarrying members. The basic element of these cables is usually a simple straight strand made of a straight core and one layer of helical wires. Several difficulties arise in the understanding of guided ultrasonic waves in such structures, partly due to the helical geometry and the interwire coupling effects. In the context of nondestructive evaluation, this paper aims at theoretically investigating the propagation of elastic waves in helical waveguides. A numerical method is chosen based on a semianalytical finite element technique that relies on a specific nonorthogonal curvilinear coordinate system. This system is shown to be translationally invariant along the helix centerline so that a spatial Fourier transform can be explicitly performed along the axis and the problem is reduced to two dimensions. A single helical wire is first considered. The convergence and accuracy of the proposed method are assessed by comparing finite element results with reference solutions. The method is then extended to study a seven wire cable with simplified contact conditions. Dispersion curves are presented for wave numbers as well as energy velocities. Significant differences with the infinite straight cylinder are observed.

5aPAh3. Numerical simulation of acoustic wave propagation via a liquid with gas bubbles. Nikolai Kudryashov (Department of Applied Mathematics, Moscow Engineering and Physics Institute (State University), 31, Kashirskoe Shosse, 115409 Moscow, Russian Federation, kudryashov@mephi.ru), Nikolai Teterev (Department of Applied Mathematics, Moscow Engineering and Physics Institute (State University), 31, Kashirskoe Shosse, 115409 Moscow, Russian Federation, us_tet@mail.ru)

Wave propagation through a liquid with gas bubbles is numerically investigated, in $1 \mathrm{D}$ and $2 \mathrm{D}$ cases. The mathematical model is constructed in one-velocity, two-temperature approximation. The liquid is described by the empiric Tait equation. The bubble oscillations are described by RayleighPlesset-type equation. The heat exchange between bubbles and the liquid is taking into account. Comparison of analytical solutions to results of numerical simulation has shown, that the developed model adequately describes dynamics of the wave propagation in a gas-liquid mixture. In the 2D case different regimes of wave propagation through a liquid containing gas bubbles are found. The leading wave with damped wave train is formed in a gas-liquid mixture out of bell-shaped impulse. The pressure splash can appear behind, on and before initial solitary wave in a liquid with a bubble channel and exist for a long time. The heat exchange effect on wave propagation is investigated. It is shown that properties of the wave propagation and it structure depend on the gas volume content, wavelength and on the bubble area width.

5aPAh4. Transmission gaps and Fano resonances in a simple acoustic waveguide: Analytical model. El Houssaine El Boudouti (Faculté des Sciences, Département de Physique, Université Mohamed I, 60000 Oujda, Morocco, elboudouti@yahoo.fr), Tarik Mrabti (Faculté des Sciences, Département de Physique, Université Mohamed I, 60000 Oujda, Morocco, t.mrabti@yahoo.fr), Hosni Al-Wahsh (Faculty of Engineering, Benha University, 11241 Cairo, Egypt, alwahsh2003@yahoo.com), Bahram Djafari-Rouhani (IEMN, UMR CNRS 8520, avenue Poincaré, BP 60069, 59652 Villeneuve d'Ascq, France, Bahram.Djafari-Rouhani@univ-lille1 
.fr),Abdellatif Akjouj(IEMN, UMR CNRS 8520, avenue Poincaré, BP 60069, 59652 Villeneuve d'Ascq, France, abdellatif.akjouj@univ-lille1 .fr), Leonard Dobrzynski (IEMN, UMR CNRS 8520, avenue Poincaré, BP 60069, 59652 Villeneuve d'Ascq, France, leonard.dobrzynski@univ-lille1 .fr)

A simple acoustic device consisting of two dangling side resonators grafted at two sites on a slender tube is designed to obtain possibly transmission stop bands where the propagation of longitudinal acoustic waves is forbidden. Contrary to all known systems of this kind, a spectral transmission gap of nonzero width occurs here even with this simple structure. This is obtained by combining appropriately the zeros of transmission of the side resonators. Sharp resonant states inside the gaps can be achieved without introducing any defects in the structure. This results from an internal resonance of the structure when such a resonance is situated in the vicinity of a zero of transmission or placed between two zeros of transmission, the socalled Fano resonances. A general analytical expression for the transmission coefficient is given for these systems within the framework of the Green's function method. The amplitude and the phase time of the transmission are discussed as a function of frequency and it is shown that the width of the stop bands is very sensitive to the number of the side resonators. These results should have important consequences for the suppression of lowfrequency noise and for designing filters.

5aPAh5. Complex edge resonance in elastic waveguides. Vincent Pagneux (Laboratoire d'Acoustique de l'Université du Maine, UMR CNRS 6613, AV. O. Messiaen, 72085 Le Mans, France, vincent.pagneux@univlemans.fr)

The resonance for different configurations of elastic waveguides with a free edge is studied from the point of view of complex resonance. We determine numerically the variations of the real part and of the imaginary part of the complex resonance frequency as a function of the Poisson ratio. For three different configurations, semi-infinite 2D plates, elastic cylinder and circular hole through a plate, the results show similar behaviours which are: a real resonance frequency for a zero Poisson ratio and a real resonance frequency that corresponds to a Lamé mode at one positive value of the Poisson ratio. In all the configurations, these free edges have complex resonances which have very high quality factors. The two Poisson ratio where the complex resonance frequency has a zero imaginary part (infinite quality factors) corresponds to trapped modes for which there is a decoupling between the propagating waves and the evanescent waves. The first decoupling at zero Poisson ratio is due to the special structure of the elasticity equations there and the second decoupling is due to the decoupling between the propagating Lamé mode and the higher order evanescent modes.

5aPAh6. Measurements on quarterwavelength tubes and Helmholtz resonators. Alberto Soto-Nicolas (Nokia Co, Sinivaival 5, 33720 Tampere, Finland, alberto.soto-nicolas@nokia.com)

The aim of this work is to determine what are factors limiting the prediction of the resonance frequency in unflanged quarter wavelength tubes and cylindrical Helmholtz resonators. When Helmholtz resonators are cylindrical, the frequency resonance may be predicted by solving a frequency 4th order polynomial equation. This formula may be used in quarter wavelength tubes too by just eliminating the value of the internal end correction. Some measurements were carried out showing that the accuracy in the prediction of the frequency resonance is limited by three factors: The uncertainty of values of end corrections, the visco-thermal effects not considered by the formula and the error in the estimation of the sound speed.

FRIDAY MORNING, 4 JULY 2008

P3-A, LEVEL 3, 9:40 TO 11:20 A.M.

\title{
Session 5aPAi
}

\section{Physical Acoustics: General Topics in Physical Acoustics I (Poster Session)}

\author{
Walter Lauriks, Cochair \\ Katholicke Univsiteit Leuven \\ Andi Petsculescu, Cochair \\ University of Louisiana
}

\begin{abstract}
All posters will be on display from 9:40 a.m. to 11:20 a.m. To allow contributors an opportunity to see other posters, contributors of odd-numbered papers will be at their posters from 9:40 a.m. to 10:30 a.m. and contributors of even-numbered papers will be at their posters from 10:30 a.m. to 11:20 a.m.
\end{abstract}

\section{Contributed Papers}

5aPAi1. Focused borehole radiator. Dmitry Kas'Yanov( Radiophysical Research Inst., 25 Bolshaya Pecherskaya, 603950 Nizhny Novgorod, Russian Federation, vo-kak@yandex.ru)

A promising type of borehole acoustic radiators intended for both stimulating borehole geotechnological processes and investigating the borehole environment is considered. The basic difference of the radiators under consideration from the existing ones is the possibility of focusing the acoustic field into a preset region of borehole environment. Elastic fields produced by focusing borehole radiators in rock are studied. Limiting abilities of focus- ing borehole systems are investigated. Optimal type of focusing borehole radiator is shown for well stimulation. It is demonstrated that borehole radiators with a variable focal distance can be designed, which provides a real opportunity to control the distribution of elastic fields in the borehole environment. This property may be very important in developing the acoustic technologies for stimulating productive reservoirs of geotechnological boreholes.

5aPAi2. Experimental study of the convergence of two-point cross-correlation toward the Green's Function. Pierre Gouedard (LGIT 
- CNRS - Université Joseph Fourier, Maison des Géosciences, 1381 rue de la Piscine, BP 53, 38041 Grenoble, France, pierre.gouedard @ ujf-grenoble.fr), Philippe Roux (LGIT - CNRS - Université Joseph Fourier, Maison des Géosciences, 1381 rue de la Piscine, BP 53, 38041 Grenoble, France, philippe.roux@obs.ujf-grenoble.fr), Michel Campillo (LGIT - CNRS - Université Joseph Fourier, Maison des Géosciences, 1381 rue de la Piscine, BP 53, 38041 Grenoble, France, Michel.Campillo@obs.ujf-grenoble.fr), Arie Verdel (Shell International Exploration and Production B.V., Kessler Park 1, 2288 Rijswijk, Netherlands, arie.verdel@shell.com), Xander Campman (Shell International Exploration and Production B.V., Kessler Park 1, 2288 Rijswijk, Netherlands, xander.campman@shell.com)

Cross-correlation of seismic noise recorded at two points yields the Green's Function (GF) between the two points when seismic noise is spatially and temporally diffuse. Seismic events yield the same result (1) when direct waves are cross-correlated from sources that are azimuthally distributed or (2) when coda waves are cross-correlated whatever the source distribution. In this work, the question of convergence toward the GF is investigated with real data. From a high-resolution survey with dense arrays of geophones and sources, we select different sets of sources and time windows to compute the cross-correlation between two receivers. We compare correlations derivative with the actual GF, and show the influence of source locations and scattering on the GF reconstruction. For direct waves, sources located in the endfire lobes of the receiver pair have an essential contribution. With sources located outside the endfire lobes, the GF can nevertheless be retrieved using coda waves.

5aPAi3. Spectral properties of the surface SH waves in a vertically periodic half-space. Stanislav V. Golkin (LMP, UMR CNRS 5469, Université Bordeaux I, 351, cours de la Libération, 33405 Talence, France, s.golkin@1mp.u-bordeaux1.fr), Olivier Poncelet (LMP, UMR CNRS 5469, Université Bordeaux I, 351, cours de la Libération, 33405 Talence, France, o.poncelet@1mp.u-bordeaux1.fr), Alexander Shuvalov (LMP, UMR CNRS 5469, Université Bordeaux I, 351, cours de la Libération, 33405 Talence, France, a.shuvalov@1mp.u-bordeaux1.fr)

We consider shear horizontal (SH) waves propagating under a free surface of semi-infinite medium with vertically periodical continuous or discrete variation of material properties. This case is marked by certain special features. It is known that the dispersion spectrum embracing 'physical' and "non-physical" (in the sense of radiation condition) surface SH waves precisely coincides with another spectrum, which describes the SH waves in a single period with traction-free edges and which is relatively straightforward to calculate [1]. Of a particular interest for possible applications is that the distribution of those spectral bands, where the surface SH waves are "physical" and hence do exist, depends essentially on the profile of variation of material properties across the unit cell. In the present paper, we analyze the link between the periodic-inhomogeneity profile and the existence of surface $\mathrm{SH}$ waves in the frequency-wave number domain, and we show how this feature manifests itself in the time-space response. [1] A. L. Shuvalov, O. Poncelet, and A. N. Podlipenets, "On the guided and surface shear horizontal waves in monoclinic transversely periodic layers and halfspaces with arbitrary variation of material properties across the unit cell," Stud. Geophys. Geod. 50 381-398 (2006)

5aPAi4. SH surface wave in multicracked solids. Olivier Poncelet (LMP, UMR CNRS 5469, Université Bordeaux I, 351, cours de la Libération, 33405 Talence, France, o.poncelet@1mp.u-bordeaux1 .fr), Stanislav V. Golkin (LMP, UMR CNRS 5469, Université Bordeaux I, 351, cours de la Libération, 33405 Talence, France, s.golkin @lmp.u-bordeaux1.fr), Christophe Aristegui (LMP, UMR CNRS 5469, Université Bordeaux I, 351, cours de la Libération, 33405 Talence, France, c.aristegui@1mp.u-bordeaux1.fr), Mihai Caleap (LMP, UMR CNRS 5469, Université Bordeaux I, 351, cours de la Libération, 33405 Talence, France,
m.caleap@1mp.u-bordeaux1.fr),Alexander Shuvalov(LMP, UMR CNRS 5469, Université Bordeaux I, 351, cours de la Libération, 33405 Talence, France, a.shuvalov@1mp.u-bordeaux1.fr)

We focus on the dispersion spectra of surface coherent waves in damaged semi-infinite elastic solids. The damage region is located within the surface layer containing depth-depending concentration of parallel closed cracks. The dispersion equation is similar to that for the Love waves in a coated substrate, except that the layer's effective elastic properties, being deduced from multiple scattering theory, are frequency-dependent and complex. For healthy elastic solids, the grazing $\mathrm{SH}$ wave is nondispersive. The dispersion under study is induced by the presence of cracks. The effect of both the magnitude of crack-concentration and its profile into the depth is investigated. The reliability of the semianalytical predictions is also discussed through a comparison with the numerical results obtained by using a finite-difference code.

5aPAi5. The acoustical research of shear viscoelastic properties of nanoparticles suspensions. Bair Damdinov (Buryat State University, Smolina Str., 24A, 670000 Ulan-Ude, Russian Federation, dababa@rambler.ru), Tuyana Dembelova (Buryat State University, Smolina Str., 24A, 670000 Ulan-Ude, Russian Federation, 1mf@ofpsrv .bsc.buryatia.ru), Badmaev Badma (Buryat State University, Smolina Str., 24A, 670000 Ulan-Ude, Russian Federation, 1mf@ofpsrv.bsc.buryatia.ru)

The acoustical research of some physical properties of nanoparticles suspensions has been carried out in this work. Mechanical properties of the $\mathrm{SiO}_{2}$ and YAG particles suspensions have been investigated. Resonance method is used for measurement of viscoelastic properties (shear modulus and shear viscosity) of the suspensions. It was shown that the viscosity and elasticity depend on size and concentration of particles. Colloid suspension of nanoparticles of silica dioxide $\left(\mathrm{SiO}_{2}\right)$ and yttrium alumina garnet doped by neodymium (Nd:YAG) are synthesized. The shear viscoelastic properties of the colloid suspensions have been measured by acoustical method. The researching of colloid suspension of $\mathrm{SiO}_{2}$ nanoparticles of different concentration by the acoustical resonance method is shown that viscosity and shear modulus decrease with nanoparticle concentration increasing. The nonlinear behavior of viscosity and elasticity in depending on shear influence amplitude has been shown. Investigation of viscosity of suspensions of Nd:YAG nanoparticles in ethanol in depending on concentration has been carried out. The decreasing of shear modulus of the suspensions with concentration decrease has been shown. The work was supported by RFBR 05-02-16584a and 07-02-90103-Mong-a.

5aPAi6. Modelization of acoustic waves radiation from sources of complex geometry aperture. Rafik Serhane (Université des Sciences et de la Technologie Houari Boumediene; Faculté de Physique, BP 32, El-Alia, DZ-16111 Alger, Algeria, rafik_serhane@ @otmail.com), Tarek Boutkedjirt (Université des Sciences et de la Technologie Houari Boumediene; Faculté de Physique, BP 32, El-Alia, DZ-16111 Alger, Algeria, tarek_boutkedjirt@hotmail.com)

The Rayleigh integral giving the impulse response for the acoustic velocity potential cannot always be determined analytically for all types of transducer apertures. The shape of the transducer surface and the spatial distribution of the excitation on its surface can complicate the calculation. This makes using numeric methods indispensable. One of these methods consists in discritizing the aperture surface in polygonal shape elements. Our work makes use of both methods of Jensen and Faure [IEEE UFFC 39-2(1992) 262, Acta Acustica 2(1994)257]. In the former, the transducer surface is subdivided in triangles. The latter one uses additional virtual triangles one side of which is that of the physical element and its summit is the field point projection on the plane containing the considered physical element. Additionally, a rotation of the virtual triangle around the field point projection is performed. In this work, the orientations of those virtual triangles are considered according to Jensen method and their contributions to the impulse response are calculated according to the two situations described by Faure. The combination of the two methods is, then, generalized to the case of nonplanar complex surfaces such as concave phased arrays. 


\title{
Session 5aPAj
}

\section{Physical Acoustics: Scattering and Diffraction I (Poster Session)}

\author{
Michael L. Oelze, Cochair \\ University of Illinois at Urbana-Champaign \\ Jean-Marc Conoir, Cochair \\ Université Paris 6
}

\begin{abstract}
All posters will be on display from 9:40 a.m. to 11:20 a.m. To allow contributors an opportunity to see other posters, contributors of odd-numbered papers will be at their posters from 9:40 a.m. to 10:30 a.m. and contributors of even-numbered papers will be at their posters from 10:30 a.m. to 11:20 a.m.
\end{abstract}

\section{Contributed Papers}

5aPAj1. Time-domain acoustic pressure fields from axisymmetric impulse sources by Rayleigh's Integral. Stephen I. Warshaw (40 West 15th St. Loft 1C, New York, NY 10011, USA, siw1939@yahoo.com)

This paper shows how Rayleigh's Integral is used to efficiently and rapidly calculate time-domain pressure waveforms and wavefronts radiated from a planar baffle due to axisymmetric normal impulse accelerations that converge toward or expand away from a fixed center of symmetry. These accelerations are prescribed with simple functional forms and histories. The Rayleigh Integrals are evaluated by Gaussian integrations that seem peculiarly suited to the integrands. The acoustic fields are presented as "snapshots" of pressure contours of the evolving wavefront structures emerging from the baffle surface, and as functions of time at selected key points. As a bonus, significant insights are provided by graphs of slant range versus time that clearly identify key signal paths from the source points to the field points. The presented methods avoid the complexities inherent in more standard transform and harmonic source treatments. They also provide insights into actual acoustic fields generated by impulsive sources, as, e.g., above the ground surface over a deeply buried explosion. [J. R. Banister and W. V. Hereford, J. Geophys. Res. 96(D3), 5185-5193 (1991)] Simpler examples were reported earlier. [S. I. Warshaw, JASA 77, S60 (1985); JASA 79, S90 (1986); JASA 80, S106 (1986), and 12 ICA Toronto, L3-6 (1986)]

5aPAj2. Unified and stable scattering matrix formalism for acoustic waves in piezoelectric stacks. Victor Y. Zhang (IEMN-CNRS, Av. Poincare, Cite Scientifique, B.P. 60069, 59652 Villeneuve d'Ascq Cedex, France, victor.zhang@iemn.univ-lille1.fr), Vincent Laude (Institut FEMTOST/CNRS, 32 avenue de l'Observatoire, 25044 Besançon cedex, France, vincent.laude@femto-st.fr)

This paper presents a unified and full scattering matrix (s-matrix) formalism. A stable recursive algorithm is derived for computation of the total s-matrix of a multilayered stack in terms of the interface s-matrix, both referring to the eigen-modes amplitudes. The derivation is direct and succinct, the derived expressions of the s-matrix are terse and concise, and the recursion is efficient and convenient for implementation. By combining the s-matrix formalism and the surface impedance matrix of the external media, various terminations of the stack can be easily handled. Numerical examples are given to show its numerical superiority over other currently used matrix formalisms: including unconditional stability throughout large and small thicknesses and pole-free feature.

5aPAj3. Temperature-dependent diffusing acoustic wave spectroscopy with resonant scatterers. Valentin Leroy (Laboratoire Ondes et Acoustique, ESPCI, Université Paris 7, CNRS, 10 rue Vauquelin, 75005 Paris,
France, valeroy77@yahoo.fr),Arnaud Derode(Laboratoire Ondes et Acoustique, ESPCI, Université Paris 7, CNRS, 10 rue Vauquelin, 75005 Paris, France, arnaud.derode@ujf-grenoble.fr)

The influence of a slight temperature change on the correlation of multiply scattered acoustic waves is studied, and experimental results are discussed. The technique presented here, similar to diffuse acoustic wave spectroscopy, is based on the sensitivity of a multiple scattering medium to a slight change. Ultrasonic waves around $3 \mathrm{MHz}$ are transmitted through a sample made of steel rods in water and recorded by an array of transducers at different temperatures. The cross-correlations between highly-scattered signals are computed. The main effect of the temperature change is a simple dilation of the times of arrival, due to a change of the sound velocity in water. But the scatterers also play a role in the progressive decorrelation of waveforms. An analysis resolved in both time and frequency shows that at some particular frequencies, the resonant behavior of the scatterers are responsible for a significantly larger decorrelation. Interestingly, the experimental results allow one to detect the presence of a small resonance that was not detected earlier on the same scatterers with classical measurement of the scattering mean freepath. A simple model is proposed to interpret the experimental results.

5aPAj4. Ultrasonic wave propagation in heterogeneous solid media. Mathieu Rossat (LCND - Université de la Méditerranée, IUT Aix Provence, Avenue Gaston Berger, 13625 Aix en Provence Cedex, France, mathieu.rossat@univmed.fr), Jean-François Chaix (LCND - Université de la Méditerranée, IUT Aix Provence, Avenue Gaston Berger, 13625 Aix en Provence Cedex, France, jean-francois.chaix@univmed.fr), Vincent Garnier (LCND - Université de la Méditerranée, IUT Aix Provence, Avenue Gaston Berger, 13625 Aix en Provence Cedex, France, vincent.garnier @ univmed.fr)

To check up concrete structures "in situ" engineers often employ ultrasonic waves. The implementation of tests and analysis of results even inversion of the problem need good knowledge about propagation phenomena. These phenomena require understanding interaction between ultrasonic waves and scatters, such as granular, porosity, and cracks. This defect can come from fabrication or mechanical damage. The size and the shape of obstacle have an influence on the spatial scattering of ultrasonic waves. We propose to show you the possible using multiple scattering in homogeneous models of propagation and the last work on a sphere in a hard media. Our objective is to bring obstacle geometries closer to the reality. Consequently, we study spheroidal scatters with various aspect ratios. 
5aPAj5. Comparisons between multiple scattering methods and time-domain numerical simulations for elastic waves. Mathieu Chekroun (EDF R\&D - LCPC, LCPC centre de Nantes BP4129, 44341 Bouguenais, France, mathieu.chekroun@lcpc.fr), Loic Le Marrec (Institute of Mathematical Research of Rennes IRMAR, Campus de Beaulieu, 35042 Rennes, France, loic.lemarrec@univ-rennes1.fr), Bruno Lombard (Laboratoire de Mécanique et d'Acoustique LMA, 31 chemin Joseph Aiguier, 13402 Marseille, France, lombard@1ma.cnrs-mrs.fr), Odile Abraham (LCPC, Centre de Nantes BP4129, 44341 Bouguenais, France, odile.abraham@lcpc.fr), Joel Piraux (Laboratoire de Mécanique et d'Acoustique LMA, 31 chemin Joseph Aiguier, 13402 Marseille, France, piraux@1ma.cnrs-mrs.fr)

Propagation of elastic waves in heterogeneous medium composed of scatterers embedded in a homogeneous matrix is considered. Both matrix and scatterers are isotropic elastic media. The multiple scattering regime is assumed, and the focus is put on the coherent field obtained by averaging several equivalent realizations of disorder. Classical methods, such as Independent Scattering Approximation, Foldy or Waterman-Truell's model, provide expressions of the complex effective wave number of the coherent field, leading to an effective phase velocity and effective damping factor. Two-dimensional time-domain numerical simulations are performed for studying the validity of these analytical or semianalytical methods. To reduce spurious effects, such as numerical diffraction, to a negligible level, a high-order numerical scheme and an immersed interface method are used together. Comparisons between theoretical and numerical values of the effective phase velocity and damping factor are proposed and analyzed in terms of the frequency, the scatterer density, and the propagation length.

5aPAj6. Coherent elastic wave propagation through nonuniform spatial distributions of cracks. Christophe Aristegui (LMP, UMR CNRS 5469, Université Bordeaux I, 351, cours de la Libération, 33405 Talence, France, c.aristegui@1mp.u-bordeaux1.fr), Mihai Caleap (LMP, UMR CNRS 5469, Université Bordeaux I, 351, cours de la Libération, 33405 Talence, France, m.caleap@lmp.u-bordeaux1.fr), Olivier Poncelet (LMP, UMR CNRS 5469, Université Bordeaux I, 351, cours de la Libération, 33405 Talence, France, o.poncelet@1mp.u-bordeaux1.fr), Stanislav V. Golkin (LMP, UMR CNRS 5469, Université Bordeaux I, 351, cours de la Libération, 33405 Talence, France, s.golkin@lmp.u-bordeaux1 .fr), Alexander Shuvalov (LMP, UMR CNRS 5469, Université Bordeaux I, 351, cours de la Libération, 33405 Talence, France, a.shuvalov@1mp.ubordeaux1.fr)

Models for multiple scattering of elastic waves usually concern uniform distributions of scatterers. The aim of this work is to predict the propagation of SH coherent waves through nonuniform distribution of parallel closed cracks. The spatial variation of the distribution properties is taken into account via replacing heterogeneous media by stacks of effective homogeneous layers. Propagation in each layer is then governed by the effective acoustic impedance and the effective wave number, derived in the framework of Waterman and Truell approach. On this basis, the reflection and transmission coefficients of the nonuniform distributions are calculated by using the transfer matrix method. We focus especially on distributions of crack size and concentration. Impact of the distribution profiles is also investigated. The semianalytical predictions are compared with numerical results obtained by using a finite-difference code.

5aPAj7. New approach to the problem on long-wave sound scattering by a Rankine vortex. Ivan Belyaev (Central Aerohydrodynamics Institute (TsAGI), Acoustic Division, 17 Radio str., 105005 Moscow, Russian Federation, belyaev_iv@bk.ru), Victor Kopiev (Central Aerohydrodynamics Institute (TsAGI), Acoustic Division, 17 Radio str., 105005 Moscow, Russian Federation, vkopiev@mx.iki.rssi.ru)

The well-known problem of sound scattering by a Rankine vortex at small Mach number M is considered. Despite the long history of the problem (Pitaevskii 1958, O'Shea 1975 etc.), solutions obtained by different authors still are not free from essential objections. The main difficulty is that the slow decay of the mean velocity field at infinity hinders a correct for- mulation of the problem. Most authors use a plane wave as the incident field in the leading approximation in M (Sakov 1993, Ford and Smith 1999, Howe 1999), however to obtain a physically reasonable solution the second approximation in the incident field is to be chosen beforehand. It is shown that instead of the plane wave condition, the condition of a point source far from the vortex should be posed. In the new formulation the correct solution of the problem of nonresonant sound scattering by the Rankine vortex is determined from the matching procedure in M. This solution is compared with the previous results, and areas where these results hold are revealed. Existing solutions for resonant scattering (Kopiev and Leontiev1987, Sozou 1990) are analyzed too and a result unifying the previous ones is found.

5aPAj8. Scattering of seismic waves by a fracture zone containing randomly distributed frictional cracks. Mihai Caleap (LMP, UMR CNRS 5469, Université Bordeaux I, 351, cours de la Libération, 33405 Talence, France, m.caleap@lmp.u-bordeaux1.fr), Christophe Aristegui (LMP, UMR CNRS 5469, Université Bordeaux I, 351, cours de la Libération, 33405 Talence, France, c.aristegui@1mp.u-bordeaux1.fr)

An investigation is conducted how the geometrical properties of a crack distribution in a fault fracture zone and the frictional characteristics of the crack surface are reflected in the attenuation and dispersion of incident seismic waves. All cracks are assumed to be either aligned or randomly oriented. The crack width is assumed to obey a power law distribution, according to seismological knowledge. The crack surface is assumed to be stress-free, or to undergo viscous friction. To deal with cracks under high confining pressure, the latter case will be more realistic than the stress-free crack, due to the existence of fluid in the earth's crust and the viscoelastic response of contacting solid material to seismic waves under high confining pressure. When the crack distribution is statistically homogeneous, the calculated dispersion and attenuation exhibit that the variance of crack size affects in different way the coherent wave. The analysis on the effect of the friction shows that the crack scattering decreases as the viscosity increases, which is expected since for high viscosity, the crack faces remain almost welded to each other. The results obtained in this work will be applicable to the state close to the occurrence time in large earthquakes.

5aPAj9. Identification and characterisation of the scholte $A$ and circumferential So waves from the time-frequency analysis of an acoustic experimental signal backscattered by a tube. Rachid Latif (ESSI - ENSA, BP 1136, Ibn Zohr University, 80000 Agadir, Morocco, latif@ensa-agadir.ac.ma), Elhoucien Aassif (Ibn Zohr University, FS Agadir, 80000 Agadir, Morocco, aassif@hotmail.com), Mustapha Laaboubi (Ibn Zohr University, FS Agadir, 80000 Agadir, Morocco, laaboubi@gmail.com), Ali Moudden (Ibn Zohr University, FS Agadir, 80000 Agadir, Morocco, ali_moudden@yahoo.fr), Gerard Maze (LAUE, Université du Havre, Place Robert Schuman, F-76610 Le Havre, France, gerard.maze@univ-lehavre.fr)

The problem of the acoustic diffusion by a tube is a theoretical problem which had known since few years a considerable interest. A good comprehension of the acoustic diffusion makes it possible the study of the opposite problem. Many theoretical and practical works were made by a tube in the acoustic diffusion field. These works showed in particular that the resonance phenomenons are directly related to the physical and geometrical properties of the target. The interest of the study presented in this paper is the identification of the circumferential wave. Several frequential analysis techniques were applied to characterize the target. These analysis techniques currently used present the major disadvantage to not be able to highlight the temporal structure of the field diffused by the target. Reciprocally the acoustic temporal analysis of the target ca not always separate the successive arrivals of the various type waves in the signal and to have information on their frequential contents. The joint time-frequency representations provide this need. In this paper, the time-frequency of Wigner-Ville is applied to analyse the acoustic experimental signal backscattered by a steel tube with radii radio $\mathrm{b} / \mathrm{a}=0.97$ (a: external radius, $\mathrm{b}$ : internal radius) immersed in water. Through the Wigner-Ville image we have identified the scholte A and the circumferential S0 waves. 


\title{
Session 5aPAk
}

\section{Physical Acoustics: Vibrations in Plates II (Poster Session)}

\author{
Daniel Royer, Cochair \\ Université Paris 7 \\ Oliver Poncelet, Cochair \\ Université Bordeaux
}

\begin{abstract}
All posters will be on display from 9:40 a.m. to 11:20 a.m. To allow contributors an opportunity to see other posters, contributors of odd-numbered papers will be at their posters from 9:40 a.m. to 10:30 a.m. and contributors of even-numbered papers will be at their posters from 10:30 a.m. to 11:20 a.m.
\end{abstract}

\section{Contributed Papers}

5aPAk1. Study and development of a low-frequency acoustic sensor dedicated to the vibratory analysis and the mechanical characterization of the plates. Georges Nassar (IEMN - DOAE, Université de Valenciennes, Le Mont - Houy, 59313 Valenciennes, France, gnassar @univ-valenciennes.fr), Bassam Merheb (IEMN - DOAE, Université de Valenciennes, Le Mont - Houy, 59313 Valenciennes, France, bassam.merheb@univ-valenciennes.fr), Alain Skaf (IEMN - DOAE, Université de Valenciennes, Le Mont - Houy, 59313 Valenciennes, France, alain.skaf@univ-valenciennes.fr), Fabrice Lefebvre (IEMN - DOAE, Université de Valenciennes, Le Mont - Houy, 59313 Valenciennes, France, Fabrice.Lefebvre@univ-valenciennes.fr), Bertrand Nongaillard (IEMN DOAE, Université de Valenciennes, Le Mont - Houy, 59313 Valenciennes, France, Bertrand.Nongaillard@univ-valenciennes.fr)

A low-frequency acoustic method was implemented for the purpose of nondestructive control and evaluation of metal plates coating. In this method, a mechanical pulse (acoustic impact) is used to generate acoustic vibrations on a frequency band between $100 \mathrm{~Hz}$ to $20 \mathrm{kHz}$, and a compact acoustic sensor, constructed with by composite materials with an embedded ferroelectric disk, is used to receive the acoustic vibrations. The technique consists in setting in resonance the integrality of a reduced size mechanical structure. The modal frequencies of plates are calculated by two methods: analytically and by finite element method. Then, a numerical modeling of the acoustic behaviour of the sensor is presented. Finally, experimental trials are described and results showing the sensitivity of the method to evaluate coating of metal plates are analyzed.

5aPAk2. Construction of symmetrical and asymmetrical notches from elementary steps in isotropic plates. Farouk Benmeddour (UVHC, IEMN-DOAE, Le Mont-Houy, 59313 Valenciennes, France, farouk_benmeddour@yahoo.fr), Sébastien Grondel (UVHC, IEMNDOAE, Le Mont-Houy, 59313 Valenciennes, France, sebastien.grondel @univ-valenciennes.fr), Jamal Assaad (UVHC, IEMN-DOAE, Le MontHouy, 59313 Valenciennes, France, jamal.assaad@univ-valenciennes .fr), Emmanuel Moulin (UVHC, IEMN-DOAE, Le Mont-Houy, 59313 Valenciennes, France, emmanuel.moulin@univ-valenciennes.fr), Lucie Dupont (UVHC, IEMN-DOAE, Le Mont Houy, 59313 Valenciennes, France, lucie.dupont@univ-valenciennes.fr)

The interactions of the fundamental Lamb modes with discontinuities in an isotropic structure will be presented and analyzed in a simple and a fast way. The key point is to decompose the symmetrical or asymmetrical notch into two elementary steps. The fundamental antisymmetrical and symmetrical Lamb modes contributions are separated by means of the simple arithmetic operations of addition and subtraction. The power reflection and transmission coefficients are then computed with the well-known average power flow equation described in a previous work [NDT\&E international, Vol. 41, p.1-9 (2008)]. Using these coefficients, the symmetrical notch can be constructed from one elementary symmetrical step while the asymmetrical notch is constructed from the superposition of asymmetrical down- and upsteps. Mode conversions phenomena are observed and analyzed for the asymmetrical steps and notches case. Finally, an experimental device is realized to test aluminum plates with different notch depths. Good agreement is found between experimental and numerical results.

5aPAk3. Lamb and Stoneley wave interactions with a fluid droplet. Loic Martinez (ECIME Université de Cergy-Pontoise, rue d'Eragny, 95031 Neuville-sur-Oise, France, loic.martinez@u-cergy.fr), Nicolas Wilkie-Chancellier (ECIME Université de Cergy-Pontoise, rue d'Eragny, 95031 Neuville-sur-Oise, France, Nicolas.Wilkie-Chancellier@u-cergy .fr), Christ Glorieux (Lab. ATF, Katholieke Universiteit Leuven, Celestijnenlaan 200D, B-3001 Leuven, Belgium, christ.glorieux@fys .kuleuven.be)

In order to analyse wetting aspects from an acoustical point of view, the present work investigates the interaction of a liquid droplet with Lamb and Stoneley waves propagating on a plane plate. A horizontal plane plate is in contact with a liquid on its lower interface, air in contact with its upper interface, the droplet lying on the top interface. Lamb waves are generated by a pulse trough a contact transducer placed at one edge of the plate. This generates in a wide range the high wave numbers of the Lamb modes A0, A1, So and the fluid born Sholte Stoneley wave (A-wave). For low wave numbers, the transducer is placed at normal incidence, under both the plate and the droplet. Signals obtained by emission/reception with the same transducer $(2 \mathrm{MHz}$ ) clearly exhibits a coupling effect between acoustic modes inside the droplet and Lamb modes. Laser Doppler scans around the droplet show reflection/refraction/diffraction patterns of the incident line wavefront through the droplet. These experiments carried out with various wetting conditions point out a good correlation between the parameters of the acoustical resonances and the wetting properties, enabling an acoustic estimation of the wetting in a wide frequency range. 


\title{
Session 5aPAl
}

\section{Physical Acoustics: Ultrasonics: Transducers and Instruments II}

\author{
Albert Migliori, Cochair \\ Los Alamos National Laboratory, Los Alamos, NM, 87545, USA \\ Claude Boutin, Cochair \\ École nationale des travaux publics de l'État, Rue Maurice Audin, F- 69518 Vaulx-en-Velin Cedex, FRANCE
}

\section{Contributed Papers}

11:20

5aPAl1. Analysis of wavefronts for the piston source acoustic fields Dmitry V. Perov (Institute of Metal Physics, 18, Sofia Kovalevskaya St., GSP-170, 620041 Ekaterinburg, Russian Federation, peroff@imp.uran .ru), Anatoly B. Rinkevich (Institute of Metal Physics, 18, Sofia Kovalevskaya St., GSP-170, 620041 Ekaterinburg, Russian Federation, rin@imp .uran.ru)

The development of modern acoustic methods is based to a great extent on a thorough investigation of the structure of acoustic fields and on the refinement of methods of their analysis and calculation. The study of the local features of acoustic field and its space-time structure is an urgent problem of physical acoustics. This work is dedicated to analytical investigation of space-time structure of wavefronts corresponding to the acoustic fields which are radiated by the piston source into elastic media. It is assumed that the spatial distributions. Various spatial distributions of acoustic field over the surface of the transducer are taken into consideration.

\section{1:40}

5aPAl2. Miniature, high efficiency transducers for ultrasonic flow meter applications. Shrinivas G. Joshi (Marquette University, EECE Department, College of Engineering, P. O. Box 1881, Milwaukee, WI 532011881, USA, shri.joshi@mu.edu), Boris D. Zaitsev (Institute of Radio Engineering and Electronics of RAS, Saratov Branch, Zelyonaya str., 38, 410019 Saratov, Russian Federation, zai-boris@yandex.ru), Iren E. Kuznetsova (Institute of Radio Engineering and Electronics of RAS, Saratov Branch, Zelyonaya str., 38, 410019 Saratov, Russian Federation, kuziren @yandex.ru)

This paper is concerned with the development of novel, miniature, high efficiency transducers for use in ultrasonic flow meters. Theoretical and experimental work done in our laboratory has shown that a flat, planar transducer that has minimal protrusion into the flow pipe can be realized based on conversion of plate acoustic waves (PAWs) to bulk acoustic waves (BAWs) and vice versa. The transducer is essentially a thin plate of a suitable piezoelectric material on which intedigital transducers (IDTs) are fabricated to generate and detect plate acoustic waves. With proper design very efficient conversion of energy from plate waves in the piezoelectric materia to bulk waves in the fluid (and vice versa) can be achieved. For example, using lithium niobate as the piezoelectric material and water as the fluid, total conversion loss (PAW to BAW and back from BAW to PAW) of less than $2.5 \mathrm{~dB}$ has been obtained. An important advantage of plate waves is that since wave energy is present on both plate surfaces, the IDT can be on the surface opposite from that which is in contact with the fluid. Details of the theoretical analysis used, experimental results obtained, and potential applications will be presented.

\section{2:00}

5aPAl3. Free-field calibration of microphones: theoretical and experimental determination of the acoustic center. Dominique Rodrigues (Laboratoire Nationale de métrologie et d'Essais (LNE), 29 avenue Roger Hennequin, 78197 Trappes Cedex, France, dominique
.rodrigues@lne.fr),Jean-Noel Durocher(Laboratoire Nationale de métrologie et d'Essais (LNE), 29 avenue Roger Hennequin, 78197 Trappes Cedex, France, jean-noel.durocher@lne.fr), Michel_Bruneau (Laboratoire d'Acoustique de l'Université du Maine, Avenue Olivier Messiaen, 72085 Le Mans, France, michel.bruneau@univ-lemans.fr), Anne-Marie Bruneau (Laboratoire d'Acoustique de l'Université du Maine (LAUM, UMR CNRS 6613), Avenue Olivier Messiaen, 72085 Le Mans, France, anne-marie.bruneau@univ-lemans.fr)

The concept of acoustic center is closely linked to the free-field calibration of microphones and have a great influence on the final results for the free-field sensitivity of the calibrated microphones. The international standard IEC 1094-3 defines the acoustic center of a reciprocal transducer as the point from which spherical waves seem to be diverging when the transducer is acting as a source. In the literature, the principal procedure used to measure the acoustic center of a reciprocal transducer is based on the measurement of the modulus of electrical transfer impedance at various distances of three pairs of microphones coupled by a free field. This paper presents a new procedure to determine the acoustic center of a reciprocal transducer assuming a source without any acoustic center and measuring the modulus of the electrical transfer impedance at various distances between this source and the transducer to be tested. Finally, the position of the acoustic center is deduced from the inverse distance law. Experimental values are presented and compared with theoretical values obtained using a theoretical model of the laboratory standard microphones (mounted on a semi-infinite rod) derived from those available in the literature.

\section{2:20}

5aPAl4. Study of acoustoelasticity behavior of concrete material under uniaxial compression. Jean-François Chaix (LCND - Université de la Méditerranée, IUT Aix Provence, Avenue Gaston Berger, 13625 Aix en Provence Cedex, France, jean-francois.chaix@univmed.fr), Ivan Lillamand (LCND - Université de la Méditerranée, IUT Aix Provence, Avenue Gaston Berger, 13625 Aix en Provence Cedex, France, ivan.lillamand@univmed.fr), Marie-Aude Ploix (LCND - Université de la Méditerranée, IUT Aix Provence, Avenue Gaston Berger, 13625 Aix en Provence Cedex, France, marie-aude.ploix@univmed.fr), Vincent Garnier (LCND - Université de la Méditerranée, IUT Aix Provence, Avenue Gaston Berger, 13625 Aix en Provence Cedex, France, vincent.garnier@univmed.fr), Gilles Corneloup (LCND - Université de la Méditerranée, IUT Aix Provence, Avenue Gaston Berger, 13625 Aix en Provence Cedex, France, gilles.corneloup@univmed.fr)

The general subject concerns the non destructive testing of prestressed structures in civil engineering. In this topic we are interested in the feasibility of stress evaluation in concrete by ultrasonic methods. To do that, we use the acoustoelasticity theory which exploits the nonlinear behaviour of media to establish the link between stress and ultrasonic velocity. This theory has been validated in homogeneous media and used in steel (to evaluated stresses in steel bolt for example). Concrete is a heterogeneous medium where the ultrasonic velocity measurement is more difficult than in steel. The scattered waves induce uncertainty in the measured values but the nonlinear behaviour of concrete is more important than the one of steel. We 
show an application in high performance concrete submitted to uniaxial compression. We measure velocities of longitudinal and transversal transmitted ultrasonic waves in the direction and perpendicularly to the stress at different level of the stress test. The sensibility of the different velocities is observed and the potential to evaluate stress in concrete is shown. Prospects particularly concern extension of tested formulations and in situ measurements. This study is supported by ANR-ACTENA, a French research program.

\section{2:40-2:00 Lunch Break}

\section{Contributed Papers}

\section{2:00}

5aPA15. Analysis of the backscattered waves in an heterogeneous material: Application on concretes. Vincent Garnier (LCND - Université de la Méditerranée, IUT Aix Provence, Avenue Gaston Berger, 13625 Aix en Provence Cedex, France, vincent.garnier@univmed.fr), Stéphane Morières (LCND - Université de la Méditerranée, IUT Aix Provence, Avenue Gaston Berger, 13625 Aix en Provence Cedex, France, stephane.moriere@gadz.org), Jean-François Chaix (LCND - Université de la Méditerranée, IUT Aix Provence, Avenue Gaston Berger, 13625 Aix en Provence Cedex, France, jean-francois.chaix@univmed.fr), Gilles Corneloup (LCND - Université de la Méditerranée, IUT Aix Provence, Avenue Gaston Berger, 13625 Aix en Provence Cedex, France, gilles.corneloup @univmed.fr)

In heterogeneous materials, ultrasonic waves can be scattered forward or backward. The set of the backscattered contribution generates a wave that is attenuated over time. We make the measurement of this attenuation with the envelope of the backscattered signal. This information evolves with the nature, shape, and size of the scatterers so with the material state. First, we propose the simulation of the signal in which we introduce the attenuation dependence on the frequency. Second, we develop this application on concretes in which many scatterers contribute to the signal's generation. We apply the technique with differents tools to generate and exploit the backscattered waves. The attenuation evolutions with the material's nature, porosity, damage and saturation rate of the concrete are analysed.

\section{$2: 20$}

5aPAl6. Airborne phased array for airborne applications based on cellular polymer. Christian Degel (Fraunhofer IBMT, Ensheimer Strasse 48, 66386 Sankt Ingbert, Germany, christian.degel@ibmt.fraunhofer.de) , Franz Josef Becker (Fraunhofer IBMT, Ensheimer Strasse 48, 66386 Sankt Ingbert, Germany, franz.becker@ibmt.fraunhofer.de), Martin Heinz (Fraunhofer IBMT, Industriestrasse 5, 66280 Sulzbach, Germany, martin.heinz@ibmt.fraunhofer.de), Heinrich Fonfara (Fraunhofer IBMT, Ensheimerstrasse 48, $66386 \quad$ Sankt Ingbert, Germany, heinrich.fonfara@ibmt.fhg.de), Robert M. Lemor (Fraunhofer IBMT, Ensheimer Strasse 48, 66386 Sankt Ingbert, Germany, robert.lemor@ibmt.fhg .de)

Object recognition, advanced distance measurements and other inspection scenarios have an increasing demand in versatile airborne ultrasonic phased arrays for acoustic scanning without moving parts. Based on cellular polymer film with high piezoelectric effect we have realized an array structure with a pitch of $0.5 \mathrm{~mm}$ and element length of $10 \mathrm{~mm}$. The working frequency of the material was measured to $250 \mathrm{kHz}$. From pointspread simulation with the small ratio of pitch/wavelength of 0.35 we could expect good beam steering and focusing characteristics. A first test array was realized and characterized. There was good agreement between measurement results and simulations. Additionally a low frequency electronic beamformer system was developed for generating the first B-image of an airborne phased array. Measurements showed that cellular polymer is a well suitable material for airborne applications. It can easily be structured to the desired shape. It allows especially the realization of phased arrays for applications like surface or profile measurement, access control, attendance check, robot guidance etc. New airborne array types like linear, phased, curved, or circular arrays are now possible. Also single element transducers with varying apertures (rectangular, oval), shaped apertures [focusing (line- or pointfocusing), defocusing] or combinations of both are possible.

\section{2:40}

5aPA17. Nonlinear focal shift in focused ultrasonic transducers and its dependence on the Fresnel number. Yuri Makov (Moscow State University, Dept. of Acoustics, 46730 Moscow, Russian Federation, yuri_makov@mail.ru), Victor Sánchez-Morcillo (IGIC - Universitat Politècnica de València, Cra. Nazaret-Oliva S/N, E-46730 Gandia, Spain, victorsm@fis.upv.es), Francisco Camarena (IGIC - Universitat Politècnica de València, Cra. Nazaret-Oliva S/N, E-46730 Gandia, Spain, fracafe@fis.upv.es), Víctor Espinosa (IGIC - Universitat Politècnica de València, Cra. Nazaret-Oliva S/N, E-46730 Gandia, Spain, vespinos@fis .upv.es)

In this work we present a systematic study of the on-axis location of the maximum pressure and intensity points in the field radiated by a focused transducer. We report the motion of these characteristic points as the transducer voltage is increased. Different initial distributions, ranging from uniform to Gaussian cases, are considered. The numerical analysis is based on the solutions of the KZK equation with different initial conditions. Experimental results have been obtained for three different Fresnel number focused transducers in linear and nonlinear regime in order to analyze the focal shift effect. An analytical expression of the initial (linear) shift of the maximum pressure position is given. This expression, and the results of the numerical simulations, shows a good agreement with the experimental data. As a main conclusion, we demonstrate that the axial range of the nonlinear shift of pressure is larger for strong initial focal shifts, occurring for small Fresnel number transducers. Theoretical and numerical predictions of the focal shift effect in Gaussian beams are also presented. In this work we have established the relation between the focal shift (both in linear and nonlinear regime) with the Fresnel number of the transducer.

\section{3:00}

5aPAl8. Linking static and dynamic NDT through fractional derivatives. Ari Salmi (Univ. of Helsinki / Dept. of Physical Sciences, POB 64 (Gustaf Hällströmin katu 2), 00014 Helsinki, Finland, edward.haeggstrom@helsinki.fi), Tuomas Hintikka (Electronics Research Unit, University of Helsinki, P.O. Box 64 (Gustaf Hällströmin katu 2), FIN00014 Helsinki, Finland, tuomas.hintikka@yahoo.com), Pekka Saranpaa (Finnish Forest Research Institute METLA, Vantaa Research Unit, Vantaa, FI-01301 Helsinki, Finland, pekka.saranpaa@metla.fi), Timo Karppinen (Electronics Research Unit, University of Helsinki, P.O. Box 64 (Gustaf Hällströmin katu 2), FIN-00014 Helsinki, Finland, timo.karppinen@helsinki.fi), Edward Hæggström (Electronics Research Unit, University of Helsinki, P.O. Box 64 (Gustaf Hällströmin katu 2), FIN00014 Helsinki, Finland, edward.haeggstrom@helsinki.fi), Ritva Serimaa (Univ. of Helsinki / Dept. of Physical Sciences, POB 64 (Gustaf Hällströmin katu 2), 00014 Helsinki, Finland, ritva.serimaa@helsinki.fi)

Static testing of mechanical properties of materials is widely used and well documented. Several extensively employed NDT techniques relying on induced mechanic wave motion (dynamic strain) exist. Comparing results obtained with dynamic NDT techniques to each other and to certified static tests is problematic especially for polymer materials whose absolute mechanical moduli depend on loading frequency. We present a study performed on 102 block-shaped dry samples of Norwegian spruce wood (Picea Abies [L.] Karst., a complex natural polymer) using ultrasound propagated in the longitudinal wood direction with frequencies ranging from $50 \mathrm{kHz}$ to 8 MHz. The samples were also tested using a standardized three-point bending test providing longitudinal elasticity estimates at near-zero frequency (static MOE). We validate experimentally the Pritz[1] five-parameter fractional derivative model and we link together the static test and the ultrasonic NDT 
technique. Our results allow predicting the static MOE from the dynamic MOE obtained with ultrasonic test equipment, and permit comparison of data obtained with ultrasonic methods applying different frequencies. [1] $\mathrm{T}$. Pritz, Five-parameter fractional derivative model for polymeric damping materials, Journal of Sound and Vibration, 265(5), 935-952 (2003)

\section{3:20}

5aPA19. Influence of low temperature cycles on the integrity of pretreated 2024 T3 aluminium joints by an ultrasonic method. Martine Rousseau (Institut Jean Le Rond d'Alembert Université Paris 6 CNRS UMR 7190, 4, Place Jussieu, Case 162, 75252 Paris cedex 05, France, mrousse@ccr.jussieu.fr), Valentina Vlasie Belloncle (Institut Jean Le Rond d'Alembert Université Paris 6 CNRS UMR 7190, 4, Place Jussieu, Case 162, 75252 Paris cedex 05, France, vlasie_valentina@yahoo.fr)

Many authors have considered the problem of adhesive bond durability over a long period of time or under special environmental conditions, and all have conclued that surface preparation is of paramount importance. Here, 2024 T3 aluminium alloy bonded joints, whose metallic plates underwent phosphoric acid pretreatment prior to bonding, are investigated before and after exposure to $-40{ }^{\circ} \mathrm{C}$ for $67 \mathrm{~h}$. This procedure of ageing is repeated several times and the samples are examined by ultrasonic Lamb waves after each cycle. In particular, the position of the cutoff-frequencies of ultrasonic guided waves which propagate into the bonded aluminum/epoxy/aluminium structure allows us to calculate the stiffnesses of the metal/adhesive interfaces with a rheological model. Thus, after exposure, a progressive decrease in the value of the stiffnesses is observed even for pretreated surfaces. Two complementary mechanical explanations are proposed. First, the cold exposure of the sample and the difference between thermal dilatation coefficients of aluminum and epoxy produce a damage of the interfacial zone. Second, the cooling introduces microcracks in the epoxy then caracterized by a smaller Young modulus.

FRIDAY MORNING, 4 JULY 2008

ROOM 241, 8:00 TO 11:40 A.M.

\title{
Session 5aPPa
}

\section{Psychological and Physiological Acoustics: Cross-Spectral Auditory Integration: Physiological, Psychophysical, and Clinical Evidence I}

\author{
Blas Espinoza-Varas, Cochair \\ CommunicationSciences \& Disorders, OU Health Sciences Ctr., 825 NE 14th St., Oklahoma City, OK 73126-0901, USA
}

Alain De Cheveigne, Cochair

CNRS, Universite Paris 5, Ecole Normale Superieure, 29 rue d'Ulm, Paris, 75230, France

\section{Contributed Paper}

8:00

5aPPa1. Sensitivity of high-frequency inferior colliculus neurons to sinusoidal amplitude-modulation of low-frequency tones. Laurel $\mathrm{H}$. Carney (University of Rochester, Depts. of Biomedical Engineering and Neurobiology \& Anatomy, 601 Elmwood Ave., Box 603, Rochester, NY 14642, USA, Laurel.Carney@Rochester.edu)

Responses of neurons in the inferior colliculus of awake rabbit were studied using sinusoidally amplitude-modulated (SAM) tones with a wide range of carrier and modulation frequencies. Neurometric thresholds for detection of modulation were estimated by applying both average rate and temporal metrics to neural responses. For neurons tuned to high frequencies, neurometric thresholds for SAM detection were substantially lower for mid- to high-level carriers at frequencies in the tail of the tuning curves than for carriers near the characteristic frequency (CF). Sensitivity to SAM for low carrier frequencies was often superior to sensitivity for near-CF carriers at low sound levels. These results suggest that psychophysical SAM detection thresholds at sound levels above approximately $60 \mathrm{~dB}$ SPL may be supported by "tail" responses of neurons tuned octaves above the carrier frequency. Improvement of psychophysical SAM detection thresholds with sound level is thus consistent with the contribution of a large population of responsive and sensitive "off-CF" neurons as level is increased. The fact that many auditory neurons, in both the peripheral and central nervous system, respond across several octaves at mid-to-high SPLs must be taken into account when considering "across-channel" interactions in tasks that involve both simple and complex sounds.

\section{Invited Paper}

5aPPa2. Role of peripheral nonlinearities in across-frequency processing of envelope fluctuations in normal hearing and hearing impaired listeners. Jesko Verhey (Universität Oldenburg, Medizinische Physik, Carl-von-Ossietzky Str. 9-11, 26111 Oldenburg, Germany, jesko.verhey@ uni-oldenburg.de), Jesko Rennies (Universität Oldenburg, Medizinische Physik, Carl-von-Ossietzky Str. 9-11, 26111 Oldenburg, Germany, jan.rennies@uni-oldenburg.de), Stephan M. Ernst (Universität Oldenburg, Medizinische Physik, Carl-von-Ossietzky Str. 9-11, 26111 Oldenburg, Germany, stephan.ernst@uni-oldenburg.de)

A psychoacoustical phenomenon related to the ability of the auditory system to compare information across frequency is comodulation masking release (CMR). CMR describes the effect that signal detectability of a sinusoidal signal embedded in a masker at the signal frequency can be improved by simultaneously presenting additional maskers in off-frequency regions if the additional maskers and the on-frequency masker (OFM) component have the same temporal envelope. Recently it was proposed that peripheral nonlinear processes such as suppression may account for large proportions of the CMR in conditions with a large spectral distance between the masker components. The aim of the present study was to test this hypothesis by measuring suppression and CMR within the same subjects for various frequency-level combinations of the off-frequency masker component. Average data for normal hearing listeners 
show a large overlap between the existence regions for suppression and CMR. The hearing impaired subjects with a sensorineural hearing loss show a negligible suppression and CMR. The data support the hypothesis that peripheral nonlinearities play an important role in CMR experiments with large spectral distances and large level differences between the masker components.

\section{Contributed Paper}

\section{8:40}

5aPPa3. Frequency modulated harmonics induces informational masking of Chinese speech. Xihong $\mathrm{H}$. Wu (Dept. of Machine Intelligence, Speech and Hearing Research Center, 2 Science Building, Peking Univ., 5 Yeheyuan Road, Haidian District, 100871 Beijing, China, wxh@cis.pku.edu.cn), Jing Chen (Dept. of Machine Intelligence, Speech and Hearing Research Center, 2 Science Building, Peking Univ., 5 Yeheyuan Road, Haidian District, 100871 Beijing, China, chenj@cis.pku.edu.cn), Xuefei F. Zou (Dept. of Machine Intelligence, Speech and Hearing Research Center, 2 Science Building, Peking Univ., 5 Yeheyuan Road, Haidian District, 100871 Beijing, China, zouxf@cis.pku.edu.cn), Jingyu Y. Li (Dept. of Machine Intelligence, Speech and Hearing Research Center, 2 Science Building, Peking Univ., 5 Yeheyuan Road, Haidian District, 100871 Beijing, China, lijy@cis.pku.edu.cn), Lijuan J. Xu (Dept. of Psychology, Peking Univ., 5 Yeheyuan Road, Haidian District, 100871 Beijing, China, lijuan179021@yahoo.com.cn), Liang Li (Dept. of Psychology, Peking Univ., 5 Yeheyuan Road, Haidian District, 100871 Beijing, China, liangli@pku.edu.cn), Huisheng S. Chi (Dept. of Machine Intelligence,
Speech and Hearing Research Center, 2 Science Building, Peking Univ., 5 Yeheyuan Road, Haidian District, 100871 Beijing, China, chi@pku.edu.cn)

Informational masking (IM) occurs when target speech is co-presented with competing speech. It is important to know what acoustic characteristics of a speech masker are essential to induce IM. This study investigated whether frequency modulation (FM) of speech is associated with IM. Nonsense Chinese Mandarin sentences were spoken by a young female as target stimuli and maskers were tone harmonics whose fundamental frequency (F0) was modulated sinusoidally and number of harmonic streams was varied. The magnitude of IM was evaluated by measuring the precedenceinduced releasing effect of perceived spatial separation on target speech. The results show that the releasing effect reached the highest level when F0 became $252 \mathrm{~Hz}$, which was the mean pitch of target speech. In addition, the releasing effect was significantly higher when the masker contained two harmonic streams than when one stream. These results suggest that because frequency-modulated harmonics can be used to inducing IM of speech, the FM component of speech is an important acoustic factor underlying IM. [Supported by the NSFC 60435010; 60535030; 60605016; 30670704.]

\section{Invited Papers}

5aPPa4. Cross-spectral synergy and consonant identification. Thomas Ulrich Christiansen (Technical University of Denmark, Center for Applied Hearing Research, Ørsteds Plads, Building 352, 2800 Lyngby, Denmark, tuc@oersted.dtu.dk), Steven Greenberg (Technical University of Denmark, Center for Applied Hearing Research, Ørsteds Plads, Building 352, 2800 Lyngby, Denmark, steveng @ silicon-speech.com)

The auditory processing of consonants was investigated using an information-theoretic approach. Listeners identified eleven different Danish consonants spoken in a Consonant + Vowel + [1] environment. Each syllable was processed so that only a portion of the original audio spectrum was present. Three-quarter-octave bands of speech, with center frequencies of 750, $1500 \mathrm{and} 3000 \mathrm{~Hz}$, were presented individually and in combination with each other. Confusion matrices were computed, and from these the amount of information transmitted for each of three phonetic-features - voicing, manner and place of articulation - was computed for each condition. From such analyses one can determine whether information associated with any given phonetic feature combines linearly across the acoustic spectrum or not. Our results indicate that information associated with voicing and manner-of-articulation combines in quasilinear fashion across the frequency spectrum. In contrast, place-of-articulation cues are integrated synergistically - information associated with two or three channels combined is far greater than predicted from the amount of information associated with individual spectral bands. Because consonants are essential for understanding speech, and place-of articulation information is crucial for decoding consonants, spoken language perception is likely to reflect highly non-linear processes.

5aPPa5. Across-frequency integration of speech information in listeners with sensorineural hearing loss. Joseph W. Hall (University of North Carolina, School of Medicine, 1115 Bioinformatics. Bldg., CB7070, 130 Mason Farm Rd., Chapel Hill, NC 27599, USA, jwh@med.unc.edu), Emily Buss (University of North Carolina, School of Medicine, 1115 Bioinformatics. Bldg., CB7070, 130 Mason Farm Rd., Chapel Hill, NC 27599, USA, ebuss@med.unc.edu), John H. Grose (University of North Carolina, School of Medicine, 1115 Bioinformatics. Bldg., CB7070, 130 Mason Farm Rd., Chapel Hill, NC 27599, USA, jhg@med.unc.edu)

At very poor signal-to-noise ratios, good speech perception may depend critically upon the ability of the listener to combine relatively brief glimpses of the signal that are separated both in terms of frequency and time. This presentation will review past research that has addressed the ability of normal-hearing and hearing-impaired listeners to integrate speech information in this way. The presentation will also consider recent studies from our laboratory that have investigated spectral/temporal integration of speech signals in listeners with mild-to-moderate sensorineural hearing losses. One of these studies focused upon the integration of speech information that was asynchronous across frequency and the other focused upon the integration of synchronous information arising from two relatively narrow bands that were widely separated in frequency. The results from these studies indicated that although the hearing-impaired listeners exhibited some characteristics suggestive of poor processing of speech, there was little indication of essential deficits in the ability to integrate synchronous or asynchronous across-frequency speech information. 
Lecture sessions will recess for presentation of poster papers on various topics in acoustics. See poster sessions for topics and abstracts.

\section{Invited Papers}

11:00

5aPPa6. Cross-spectral integration of speech patterns by normal and impaired listeners. Eric W. Healy (University of South Carolina, Speech Psychoacoustics Laboratory, Department of Communication Sciences and Disorders, William Brice Bldg., 1621 Greene St., Columbia, SC 29208, USA, ewh@sc.edu)

According to the current session description, "Recent evidence suggests that speech recognition depends importantly on the ability to integrate information across the frequency spectrum, which is not accounted for by the sensitivity (information content) of the respective spectral regions." Indeed, temporal speech patterns that yield near zero intelligibility when presented individually can provide intelligibility in excess of $80 \%$ when presented in pairs. These and other recent data involving the integration of speech patterns across the spectrum by the normal auditory system will be described. In addition, "speech-recognition data supporting the existence of a crossspectral integration deficit in hearing-impaired patients" will be discussed and contrasted against similar experiments involving nonspeech stimuli.

11:20

5aPPa7. Integration of acoustic cues for consonant identification by cochlear implant users. Mario Svirsky (New York University, Dept. of Otolaryngology, NYUSOM, 550 First Ave., NBV-5E5, New York, NY 10016, USA, mario.svirsky@ nyumc.org), Elad Sagi (New York University, Dept. of Otolaryngology, NYUSOM, 550 First Ave., NBV-5E5, New York, NY 10016, USA, elad.sagi @med.nyu.edu)

Users of cochlear implants (CIs) obtain substantial benefit from their devices, but their speech perception is (on average) less than perfect and there are significant individual differences among patients. In particular, their consonant identification levels are lower than those of normal hearing listeners, or even most users of hearing aids. We have developed a simple quantitative model (multidimensional Phoneme Identification or MPI model) to predict consonant confusion matrices for individual cochlear implant users based on their discrimination of three consonantal acoustic cues: place of stimulation in the cochlea, silent gap duration, and percentage of energy above $800 \mathrm{~Hz}$. Despite using only three degrees of freedom (i.e., JND for each cue) the model can explain most of the consonant pairs that are confused (or not confused) by individual CI users. However, when a listener's measured JNDs are used as inputs to the model, the predictions that result tend to have a higher percentage of correct responses than shown by the listener. One possible explanation is that CI users' speech perception may be limited not only by their basic psychophysical capabilities but also by their imperfect integration of different acoustic cues. [Work supported by NIDCD (R01-DC03937).]

FRIDAY MORNING, 4 JULY 2008

ROOM 242A, 8:00 A.M. TO 12:20 P.M.

\section{Session 5aPPb}

\section{Psychological and Physiological Acoustics: Otoacoustic Emissions, from Cochlear Modeling to Experimental Techniques, and Back I}

Christopher A. Shera, Cochair

Eaton-Peabody Laboratory, 243 Charles St, Boston, MA 02114, USA

Arturo Moleti, Cochair

University of Roma Tor Vergata - Physics Department, Via della Ricerca Scientifica, 1, Roma, 00133, Italy

\section{Invited Papers}

8:00

5aPPb1. Testing coherent reflection in chinchilla. Christopher A. Shera (Eaton-Peabody Laboratory, 243 Charles St, Boston, MA 02114, USA, shera@epl.meei.harvard.edu), Arnold Tubis (Institute for Nonlinear Science, University of California, San Diego, La Jolla, CA 92093, USA, tubisa@aol.com), Carrick L. Talmadge (The University of Mississippi - NCPA, 1 Coliseum Drive, University, MS 38677, USA, clt@olemiss.edu)

Coherent-reflection theory explains the generation of stimulus-frequency and transient-evoked otoacoustic emissions by showing how they emerge from the coherent "backscattering" of forward-traveling waves by mechanical irregularities in the cochlear partition. Recent published measurements of stimulus-frequency otoacoustic emissions (SFOAEs) and estimates of near-threshold basilarmembrane $(\mathrm{BM})$ responses derived from Wiener-kernel analysis of auditory-nerve responses allow for comprehensive tests of the theory in chinchilla. Model predictions are based on: (1) an approximate analytic expression for the SFOAE signal in terms of the BM traveling 
wave and its complex wave number; (2) an inversion procedure that derives the wave number from BM traveling waves; and (3) estimates of BM traveling waves obtained from the Wiener-kernel data and local scaling assumptions. At frequencies above $4 \mathrm{kHz}$, predicted median SFOAE phase-gradient delays are in excellent agreement with the SFOAE measurements. At frequencies below $4 \mathrm{kHz}$, chinchilla SFOAEs show strong evidence of interference between short- and long-latency components. Approximate unmixing of these components, and association of the long-latency component with the predicted SFOAE, yields close agreement throughout the cochlea.

\section{$8: 20$}

5aPPb2. Two-tone suppression of stimulus frequency otoacoustic emissions in human ears with sensorineural hearing loss. Douglas H. Keefe (Boys Town National Research Hospital, 555 North 30th Street, Omaha, NE 68131, USA, keefe@boystown.org), John C. Ellison (Boys Town National Research Hospital, 555 North 30th Street, Omaha, NE 68131, USA, ellisonj@boystown.org), Denis F. Fitzpatrick (Boys Town National Research Hospital, 555 North 30th Street, Omaha, NE 68131, USA, fitzpatrickd@boystown.org)

Two-tone suppression (2TS) of stimulus frequency otoacoustic emissions (SFOAEs) recorded in normal-functioning human ears is a noninvasive measure of cochlear nonlinearity. This study investigates 2TS SFOAE response properties in 63 subjects with sensorineural hearing loss and normal middle-ear function. SFOAEs were measured using tones at frequencies $\left(f_{p}\right)$ from 0.5 to $8 \mathrm{kHz}$ and levels from 40-60 dB SPL. For interpreting SFOAEs, hearing status was classified at each frequency according to degree of hearing loss, with losses ranging from within normal limits to $95 \mathrm{~dB}$ HL. SFOAE suppression was measured as a function of suppressor frequency $\left(f_{s}\right)$ and level $\left(L_{s}\right)$, with results analyzed in terms of a SFOAE suppression tuning curve. A tip-to-tail difference (TTD) was defined between the $L_{s}$ needed to produce a criterion decrement in SFOAE level at $f_{s}$ one octave below $f_{p}$ and the $L_{s}$ needed to produce a criterion decrement near $f_{p}$. The TTD estimates cochlear-amplifier gain in normal-functioning ears, but its properties in impaired ears based on SFOAEs have not been examined. Results will be analyzed as a function of the hearing status to assess the relative impact of outer-hair cell dysfunction in the SFOAE source region at tonotopic place. [Work supported by NIH.]

\section{8:40}

5aPPb3. Species differences in low-level otoacoustic emissions may be explained by "hot regions" in the cochlea. Jon Siegel (Northwestern Univ, 2240 Campus Drive, Evanston, IL 60208, USA, j-siegel@ northwestern.edu)

Otoacoustic emissions evoked by low-level transients (TEOAE) and single tones (SFOAE) appear to excite the same emission mechanism, a concept originally formulated by Kemp and others and confirmed quantitatively by Kalluri and Shera (JASA 121, 2097-2110 (2007)). We have studied emissions evoked by single tones (SFOAE) measured using the suppression method. In humans, 50-70 dB SPL tones most readily suppress SFOAE evoked by lower-level tones when the suppressor is near the frequency of the evoking tone, suggesting that most of the emission originates near the peak of mechanical activity induced by the evoking tone. However, in chinchillas, Mongolian gerbils and mice, emission components originating basal to the peak appear to be relatively larger than in humans. The range of frequencies evoking the largest SFOAE correspond to those where spontaneous emissions are detected, from roughly $0.5-6 \mathrm{kHz}$ in humans, $4-12 \mathrm{kHz}$ in chinchillas, $15-30 \mathrm{kHz}$ in mice and still higher in bats. Stimulus tones below this "hot" frequency range appear to generate the largest SFOAE components basal to the peak. The existence of "hot regions" in the cochlea may explain the apparent species differences in emission behavior between humans and small mammals. (Supported by NIH grant DC-00419 and Northwestern University.)

\section{9:00}

5aPPb4. $5 \mathrm{mHz}$ oscillations in OAE intensity following sound exposure. David Kemp (UCL Ear Institute, UCL Centre for Auditory Research, 332 Gray's Inn Road, WC1X8EE London, UK, d.kemp@UCL.ac.uk), Oliver J. Brill (UCL Ear Institute, UCL Centre for Auditory Research, 332 Gray’s Inn Road, WC1X8EE London, UK, Oliver@ otodynamics.com)

Otoacoustic emission (OAE) intensity is highly stable in healthy ears but can be temporarily reduced following noise exposure. This reduction in OAE intensity is well correlated with temporary noise induced hearing threshold elevation and is presumed to be due to metabolic fatigue in the outer hair cells. These cells are responsible for the generation of OAEs and are essential for the maintenance of normal hearing threshold. The severity and recovery of post exposure OAE reduction in an individual is therefore of interest for hearing protection purposes. This paper concerns the regulation of outer hair cell status and the effect of overstimulation on that regulation. We measured OAE level fluctuations following mild over stimulation (e.g., $100 \mathrm{dBSPL}$ for $2 \mathrm{~ms}$ ) in order to observe the dynamics of OAE regulation. We propose this parallels outer hair cell physiological regulation. Under-damped oscillations in OAE level occur typically at a frequency of 5 milliHertz after sound exposure. We find oscillations are excited by both the onset and offset of overstimulation and a linear model explains our observations. The oscillation is clearly not itself a fatigue process but fatigue is revealed by the need for a small depression of the baseline of the OAE oscillations.

\section{9:20}

5aPPb5. Several properties of distortion product otoacoustic emissions can be modeled by simple input/output functions. Paul Fahey (University of Scranton, Dept. of Physics/Electrical Engineering, Scranton, PA 18510, USA, faheyp1@scranton.edu), Glen K. Martin (Jerry Pettis Memorial Veterans Medical Center, Research Service (151), 11201 Benton Street, Loma Linda, 92357, USA, glen.martin2@va.gov)

Distortion product otoacoustic emissions (DPOAEs) often exhibit regular patterns in both amplitude and phase as functions of both the levels and frequencies of the generating primary tones. Many of these patterns can be approximated when modeled by simple input/output functions. In particular, nulling patterns in rabbit DPOAEs and their modifications upon noise damage or fatiguing tonal exposures can be modeled at the level of input/output functions. Also, input/output functions can be used to propose the possible physics that underlies some complicated DPOAE patterns in level/frequency space that occur in the presence of a third tone. Both data and models will be reviewed. 


\title{
Invited Papers
}

\author{
11:00
}

5aPPb6. Using the frequency dispersive properties of otoacoustic emissions to facilitate separation of different components. Glenis R. Long (Graduate Center of the City University of New York, Speech-Language-Hearing Program, 365 Fifth Ave, New York, NY 10016, USA, glong@gc.cuny.edu), Carrick L. Talmadge (The University of Mississippi - NCPA, 1 Coliseum Drive, University, MS 38677, USA, clt@olemiss.edu)

Otoacoustic emissions (OAE) are frequency dispersive (different frequencies have different latencies), because of the properties of the basilar membrane. These frequency-dependent latency differences, are used to separate the transient otoacoustic emissions (TEOAE) from the stimulus, but have not been used with stimulus frequency (SFOAE) and distortion product (DPOAE) otoacoustic emissions. If continuously-sweeping tones are used to generate DPOAE and SFOAE, the frequency dispersive properties of these OAE cannot only be used to separate the evoking stimuli from the OAE, but also to separate OAE coming from different cochlear regions. Modifications of the LSF procedure to extract OAE (Long \&Talmadge, 1997: JASA, 102:2831-2848; Talmadge et al. 1999: JASA,105:275-292) permit not only the extraction of the different OAE components, but also provide an estimate of the latency of the OAE, which does not depend on group delay. DPOAE and SFOAE extracted using sweeping tones in normal and impaired adults and infants provide information about the nature of cochlea function in these populations

\section{1:20}

5aPPb7. Does overexposure modify the fine structure of distortion product otoacoustic emissions? Karen Reuter (Acoustics, Aalborg University, Fredrik Bajers Vej 7 B5, 9220 Aalborg Ø, Denmark, kr@es.aau.dk), Dorte Hammershøi (Acoustics, Aalborg University, Fredrik Bajers Vej 7 B5, 9220 Aalborg Ø, Denmark, dh@es.aau.dk)

It is investigated, whether the pattern of distortion product otoacoustic emission (DPOAE) fine structure (quasiperiodic variations across frequency) is altered by an acoustical overexposure. DPOAE fine structures are determined in 16 normal-hearing humans using a high frequency-resolution and primary levels of $\mathrm{L} 1 / \mathrm{L} 2=65 / 45 \mathrm{~dB}$. DPOAEs are measured both before and after the subjects are monaurally exposed to a $1 \mathrm{kHz}$ tone lasting for $3 \mathrm{~min}$ at an equivalent threshold sound pressure level of $105.5 \mathrm{~dB}$. After the exposure the DPOAE levels are shifted to lower values and recover to the initial levels within $20 \mathrm{~min}$. The DPOAE fine structure pattern is analyzed by an automatic classification algorithm, which determines ripple center frequency, ripple spacing, ripple height, and ripple prevalence. For some individuals, an alteration of some of the parameters could be observed after the exposure, i.e., either a more pronounced or a less pronounced fine structure could be observed after the exposure. Across subjects, no shift of the fine structure pattern in frequency could be observed, also none of the calculated parameters changed consistently after the exposure. The results do not support the idea that the DPOAE fine structure might contain more information about the state of hearing than the DPOAE level.

\section{1:40}

5aPPb8. Changes in distortion product otoacoustic emission (DPOAE) fine structure due to contralateral acoustic stimulation. Manfred Mauermann (Universität Oldenburg, Medizinische Physik, Carl-von-Ossietzky Str. 9-11, 26111 Oldenburg, Germany, manfred.mauermann@uni-oldenburg.de), Birger Kollmeier (Universität Oldenburg, Medizinische Physik, Carl-von-Ossietzky Str. 9-11, 26111 Oldenburg, Germany, birger.kollmeier@uni-oldenburg.de)

Contralateral acoustic stimulation (CAS) can cause changes in the amplitude of the $2 \mathrm{f}_{1}-\mathrm{f}_{2}$ DPOAE in humans - most probably mediated by the medial olivocochlear reflex. DPOAE amplitude changes due to CAS show large interindividual variability and large changes from suppression to enhancement for small changes of the primary levels. The underlying mechanisms of these effects are still not fully understood. We hypothesize that the two interacting DPOAE sources might be differently affected by the CAS. If so, CAS will cause specific changes in DPOAE fine structure. Therefore, DPOAE fine structures were measured using frequency-modulated primaries $\left(\mathrm{f}_{2}: 1500-3000 \mathrm{~Hz}, \mathrm{f}_{2} / \mathrm{f}_{1}: 1.2 ; \mathrm{L}_{2}: 60 \mathrm{~dB}\right.$ SPL; $\mathrm{L}_{1}: 58,63,68 \mathrm{~dB} \mathrm{SPL}$ ) without and with a broadband CAS (50 dB SPL). The fine structure changes and shifts according to CAS were analyzed in detail - including latency windowing to separate the contributions from the two interacting DPOAE sources. The results indicate, e.g., that there is no "true" enhancement in terms of enhanced cochlear activity during broadband CAS. Rather there are slight frequency shifts in DPOAE fine structure due to changes in the relative contribution of the two DPOAE sources. This shift can lead to observations of "pseudo" enhancement of up to $20 \mathrm{~dB}$ for isolated frequencies.

\section{Contributed Paper}

\section{2:00}

5aPPb9. Otoacoustic emission latency and cochlear mechanics. Arturo Moleti (University of Roma Tor Vergata - Physics Department, Via della Ricerca Scientifica, 1, 00133 Roma, Italy, moleti@roma2.infn.it), Renata Sisto (ISPESL - Occupational Hygiene Department, Via di Fontana Candida, 1, 00040 Monte Porzio Catone (RM), Italy, renata.sisto@ispesl.it)

Otoacoustic emission (OAE) latency data provide important information about several aspects of cochlear mechanics. Time-frequency analysis techniques, euther based on the wavelet transform or on Matching Pursuit algorithms, permit to get accurate estimates of the latency of transient evoked OAEs (TEOAEs). Wavelet estimates of the TEOAE latency of 14 young subjects, recorded at different stimulus levels from 60 to $90 \mathrm{~dB}$ pSPL, are used here to test several predictions of transmission line cochlear models. The experimental relation between latency, frequency, and stimulus level, is used to get estimates of cochlear tuning at different stimulus levels, which can be compared with behavioral and other OAE-based estimates. 
These estimates can be used to get information about the functional form of the cochlear amplifier nonlinearity. The comparison between the TEOAE latency and the auditory brainstem response (ABR) latency may help discriminating between different models for the OAE backward propagation.
Wavelet latency and phase-gradient delay may be estimated on the same TEOAE waveforms. The comparison between these two characteristic times permits, in principle, to identify the place-fixed or wave-fixed nature of the TEOAE generation mechanisms.

FRIDAY MORNING, 4 JULY 2008

P2-C, LEVEL 2, 9:40 TO 11:20 A.M.

\title{
Session 5aPPc
}

\section{Psychological and Physiological Acoustics: Otoacoustic Emissions, from Cochlear Modeling to Experimental Techniques, and Back II (Poster Session)}

\author{
Christopher Shera, Cochair \\ Eaton-Peabody Laboratory \\ Arturo Moleti, Cochair \\ University of Roma Tor Vergata - Physics Department
}

\begin{abstract}
All posters will be on display from 9:40 a.m. to 11:20 a.m. To allow contributors an opportunity to see other posters, contributors of odd-numbered papers will be at their posters from 9:40 a.m. to 10:30 a.m. and contributors of even-numbered papers will be at their posters from 10:30 a.m. to 11:20 a.m.
\end{abstract}

\section{Contributed Papers}

5aPPc1. Hemispheric Laterality and Peripheral Auditory Asymmetry studied by Transiently Evoked Otoacoustic Emissions. Annie M. Moulin (Neurosciences Sensorielles Comportement Cognition UMR5020 CNRS, Université de Lyon, 50, avenue Tony Garnier, 69366 Lyon Cedex 07, France, amoulin@olfac.univ-lyon1.fr), Jean-Bertrand Nottet (Hôpital du service de santé des Armées Desgenettes, Boulevard Pinel, 69000 Lyon, France, jean-bertrand.nottet@wanadoo.fr)

A peripheral left-right assymetry has been shown at the cochlear level: Transiently evoked otoacoustic emissions (TEOAEs) amplitudes are greater in the right ear than in the left ear of right-handed subjects, whereas no difference is obtained in left-handed subjects (Khalfa et al., 1998). This study sought to investigate a possible link between peripheral auditory assymetry and hemispheric laterality assessed by a dichotic listening task. Peripheral auditory laterality was investigated by comparing TEOAEs from both ears of each subject in 289 normally hearing subjects, with 94 left-handed subjects (52 women), and 194 right-handed subjects (129 women). Results show significantly greater OAE amplitude in right ear than in left ears for both right-handers $(12.93 \mathrm{~dB}, \mathrm{SD}=4.46$ versus $11.89, \mathrm{SD}=4.6)$ and lefthanders $(11.62 \mathrm{SD}=3.8$ versus $10.44, \mathrm{SD}=3.8$, whereas average hearing loss across frequencies did not show any significant difference. However, in the left-handers, only the subgroups of subjects presenting a right-ear advantage at the dichotic tasks, presented a significantly greater TEOAE amplitude in the right ear versus the left ear. Those results suggest a definite link between peripheral auditory assymetry and hemispheric laterality.

5aPPc2. Temporal suppression and augmentation of click-evoked otoacoustic emissions. Sarah Verhulst (Centre for applied hearing research, Technical University of Denmark, DTU, Bygn. 352, 2800 Lyngby, Denmark, sv@oersted.dtu.dk), James M. Harte (Centre for applied hearing research, Technical University of Denmark, DTU, Bygn. 352, 2800 Lyngby, Denmark, jha@oersted.dtu.dk), Torsten Dau (Centre for applied hearing research, Technical University of Denmark, DTU, Bygn. 352, 2800 Lyngby, Denmark, tda@oersted.dtu.dk)

This study investigates and models temporal suppression of clickevoked otoacoustic emissions (CEOAEs). This suppression-effect is created when a suppressor-click is presented close in time to a test-click. The analy- sis was carried out for short time-frames of short- and long-latency CEOAEs. The latter is defined as a CEOAE with duration greater than 20 $\mathrm{ms}$, typically observed for test subjects with spontaneous otoacoustic emissions (SOAEs). Previous studies have tended to exclude these test subjects but they are incorporated here. The results from six exemplary subjects demonstrate that temporal suppression is present in all CEOAEs for inter-click intervals (ICIs) less than $8 \mathrm{~ms}$. The long-latency CEOAEs showed augmentation (i.e., negative suppression) for ICIs of 6-7 ms which was not reported for the short-latency CEOAE at these ICIs. A phenomenological approach is adopted here to explain both temporal suppression and augmentation of CEOAEs. Recently, a feedback automatic gain control (AGC) system has been used to model CEOAE suppression. However, this system cannot account for augmentation and therefore another, dynamic nonlinear model was developed. Suppression and augmentation were modeled phenomenologically using compression or expansion of the system output. This was obtained by shifting the operating-point on the input/output-characteristic in relation to the ICI.

5aPPc3. A psychophysical test of the Kummer primary-level rule for measuring distortion-product otoacoustic emission input/output functions. Enrique A. Lopez-Poveda (University of Salamanca, Instituto de Neurociencias de Castilla y León, Avda. Alfonso X 'El Sabio' s/n, 37007 Salamanca, Spain, ealopezpoveda@usal.es), Peter T. Johannesen (University of Salamanca, Instituto de Neurociencias de Castilla y León, Avda. Alfonso X 'El Sabio's/n, 37007 Salamanca, Spain, peter@usal.es)

The standard procedure for measuring distortion-product otoacoustic emission (DPOAEs) input/output (I/O) curves involves using primaries whose levels conform to the rule of Kummer [Kummer et al., J. Acoust. Soc. Am. 103, 3431-44 (1998)]. The assumption is that the DP originates at the F2 cochlear site and primaries that conform to this rule produce equal excitation at that site, which maximizes the DP amplitude. These ideas were tested psychophysically by measuring temporal masking curves (TMCs) for masker frequencies equal to the primary frequencies. A TMC-based level rule was obtained by plotting the levels of the F1 masker against those for the F2 one for corresponding masker-probe intervals. DPOAE I/O curves were then measured using the Kummer rule, the TMC-based rule, and pri- 
mary levels optimized individually to maximize DP amplitude. DPOAEs for the TMC-based and the Kummer rule had statistically similar amplitudes, but they were both lower than those measured with individually-optimized levels. This undermines the assumption that maximum DP responses occur for primaries that produce equal excitation at the F2 cochlear site. Individually-optimized levels showed that L1 should be higher than prescribed by the Kummer rule. [Work supported by IMSERSO-131/06, PROFIT-CIT-390000-2005-4, and MEC-BFU-2006-07536, and The Oticon Foundation.]

5aPPc4. Cochlear nonlinearity in normal-hearing subjects as inferred psychophysically and from distortion-product otoacoustic emissions. Peter T. Johannesen (University of Salamanca, Instituto de Neurociencias de Castilla y León, Avda. Alfonso X 'El Sabio' s/n, 37007 Salamanca, Spain, peter@usal.es), Enrique A. Lopez-Poveda (University of Salamanca, Instituto de Neurociencias de Castilla y León, Avda. Alfonso X 'El Sabio’ s/n, 37007 Salamanca, Spain, ealopezpoveda@usal.es)

The aim was to investigate the correlation between estimates of compression ratio, compression threshold, and cochlear gain for normal-hearing subjects inferred from temporal masking curves (TMC) and distortionproduct otoacoustic emission (DPOAEs) input-output (I/O) curves. Special care was given to reduce the influence of DPOAE fine structure on the I/O curves. Data were collected for 10 subjects and for cochlear places with characteristic frequencies (CFs) of 0.5, 1, 2, and $4 \mathrm{kHz}$. Except at $4 \mathrm{kHz}$, little correlation was found between ratio estimates obtained with the two methods. One reason is that the DPOAE I/O curves show plateaus or dips which result in unexpectedly high compression estimates. Moderately high correlation was found between the compression-threshold estimates obtained with the two methods, although DPOAE-based values were systematically lower than those based on the TMCs. Both methods confirmed that compression ratio and threshold are approximately constant across the CF range considered. Cochlear gain could be estimated from TMCs only and it was found to increase slightly with increasing CF. It is discussed that DPOAE I/O curves may be inappropriate to infer precise individual nonlinear cochlear characteristics in normal-hearing listeners, particularly at low CFs [Work supported by IMSERSO 131/06, PROFIT CIT-390000-2005-4, MEC BFU-2006-07536, and The Oticon Foundation.]

5aPPc5. Recovery of distortion product otoacoustic emissions (DPOAE) with high time-resolution from a moderate monaural-exposure to 2-kHz in human subjects. Miguel Angel Aranda De Toro (Acoustics, Aalborg University, Fredrik Bajers Vej 7 B5, 9220 Aalborg Ø, Denmark, maat@es.aau.dk), Rodrigo Ordoñez (Acoustics, Aalborg University, Fredrik Bajers Vej 7 B5, 9220 Aalborg $\varnothing$, Denmark, rop@es.aau.dk), Karen Reuter (Acoustics, Aalborg University, Fredrik Bajers Vej 7 B5, 9220 Aalborg Ø, Denmark, kr@es.aau.dk), Dorte Hammershøi (Acoustics, Aalborg University, Fredrik Bajers Vej 7 B5, 9220 Aalborg Ø, Denmark, dh@es.aau.dk)

The amplitude of distortion product otoacoustic emissions (DPOAE) decreases temporarily after exposure to a sound of moderate level. These changes show similarities to the changes observed in absolute hearing thresholds after similar sound exposures. This paper presents the experimental protocol to study how DPOAEs in human subjects are affected after a monaural exposure of ten minutes to a pure tone of $2 \mathrm{kHz}$. The experimental protocol allows to measure fine structures of the DPOAE with high timeresolution in a limited frequency range. Thus, the results give a detailed description of the DPOAE recovery process and can be used to develop a mathematical model of the recovery. This is the first approximation to study the recovery of more complex exposures. [Work supported by the Danish Research Council for Technology and Production.]

5aPPc6. Analysis of time-frequency fine structure of transiently evoked otoacoustic emissions to study the effects of exposure to GSM radiofrequency fields. Alessia Paglialonga (Institute of Biomedical
Engineering CNR - Polytechnic Univ. Milan, Piazza Leonardo da Vinci, 32, I-20133 Milan, Italy, alessia.paglialonga@polimi.it), Gabriella Tognola (Institute of Biomedical Engineering CNR - Polytechnic Univ. Milan, Piazza Leonardo da Vinci, 32, I-20133 Milan, Italy, gabriella.tognola@polimi.it), Marta Parazzini (Institute of Biomedical Engineering CNR - Polytechnic Univ. Milan, Piazza Leonardo da Vinci, 32, I-20133 Milan, Italy, marta.parazzini@polimi.it), Federica Sibella (Institute of Biomedical Engineering CNR - Polytechnic Univ. Milan, Piazza Leonardo da Vinci, 32, I-20133 Milan, Italy, federica.sibella@polimi.it), Steven L. Bell (University of Southampton, University Road, Highfield/ISVR, SO17 iBJ Southampton, UK, alessia.paglialonga@polimi.it), Mark E. Lutman (University of Southampton, University Road, Highfield / ISVR, SO17 iBJ Southampton, UK, mel@isvr.soton.ac.uk), Gyorgy Thuroczy (National Research Institute for Radiobiology and Radiohygiene NIRR, Department of Non-Ionizing Radiation, Anna u. 5, 1775 Budapest, Hungary, alessia.paglialonga@ polimi .it), Paolo Ravazzani (Institute of Biomedical Engineering CNR - Polytechnic Univ. Milan, Piazza Leonardo da Vinci, 32, I-20133 Milan, Italy, paolo .ravazzani@polimi.it)

Mobile phones have become very commonly used throughout the world within a short period of time. To date there is only limited knowledge about interaction between electromagnetic fields (EMFs) emitted by mobile phones and the auditory function. Moreover, there is widespread concern that there may be potential for harm. The aim of this study, performed in the framework of the European Commission Project GUARD "Potential adverse effects of GSM cellular phones on hearing" (5FP, QLK4-CT-200100150, 2002-2004), was to assess potential subtle changes in cochlear function of normal hearing subjects by measuring the time-frequency fine structure of TEOAEs after exposure to low-intensity EMFs emitted by GSM mobile phones. TEOAEs were recorded in 27 healthy young adults before and after $10 \mathrm{~min}$ of real or sham exposure in a double-blind design. TEOAE data were analyzed both globally (broadband analysis) and using the wavelet transform (analysis of time-frequency fine structure). The broadband analysis revealed no significant effect on TEOAEs related to exposure, confirming results of previous studies; in addition, no significant change was detected in the analysis of the elementary wavelet components, suggesting that a 10-min exposure to EMFs emitted by GSM mobile phones has no immediate after-effect on TEOAE time-frequency fine structure.

5aPPc7. Simulating electrically evoked emissions in the cochlea. Julien Meaud (University of Michigan, 2250 G G Brown Bldg, 2350 Hayward St., Ann Arbor, MI 48109, USA, jmeaud@umich.edu), Karl Grosh (University of Michigan, 2250 G G Brown Bldg, 2350 Hayward St., Ann Arbor, MI 48109, USA, grosh@umich.edu)

Electrical stimulation of the cochlea is a means for investigating the electromechanics of the organ. In this study, we use a mechanical-electricalacoustic cochlear model to make predictions of the effect of intracochlear bipolar electrical stimulation on the basilar membrane velocity and stapes velocity. Bipolar stimulation provides for a localized means of stimulating the outer hair cells $(\mathrm{OHC})$ and is a well-established technique for evoking otoacoustic emissions. We show that both fast, fluid-borne, and slow, fluidstructure waves are possible. Whether the fast or slow waves dominate the overall response depends on the level of activity in the cochlea, the frequency and location of excitation, and the boundary conditions at the stapes and round window. We show that forward propagating traveling waves may dominate the basilar membrane response even with intracochlear stimulation apical to the measurement site. These results bear direct impact on the interpretation of noninvasive measurements using otoacoustic emissions.

5aPPc8. Otoacoustic emissions in sheep (Ovis aries): Sex differences and prenatal androgen effects. Dennis McFadden (University of Texas, Psychology Dept, SEA 4.110, Austin, TX 78712-0187, USA, mcfadden@psy.utexas.edu), Edward Pasanen (University of Texas, Psychology Dept, SEA 4.110, Austin, TX 78712-0187, USA, pasanen 
@ psy.utexas.edu),Michelle D. Valero(University of Texas, Department of Biology, San Antonio, TX 78249, USA, bonfire_2002@hotmail.com), Eila K. Roberts (University of Michigan, Department of Psychology and Reproductive Sciences Program, Ann Arbor, MI 48109, USA, ekrobert@umich.edu), Theresa M. Lee (University of Michigan, Department of Psychology and Reproductive Sciences Program, Ann Arbor, MI 48109, USA, terrilee@umich.edu)

Otoacoustic emissions (OAEs) were measured in Suffolk sheep (Ovis aries), the first ruminant species tested for OAEs to our knowledge. Some sheep had been administered testosterone or estradiol during prenatal development, some had been gonadectomized after birth, and some had been allowed to develop normally. The click-evoked otoacoustic emissions (CEOAEs) exhibited by the female control group $(\mathrm{N}=11)$ were stronger than those in the male control group $(\mathrm{N}=15)$, which is the same direction of effect reported for other mammals, including humans. The females administered testosterone prenatally $(\mathrm{N}=13)$ had substantially weaker (masculinized) CEOAEs than control females. Both outcomes suggest that prenatal exposure to androgens weakens the cochlear amplifiers. The CEOAEs of males administered testosterone prenatally $(\mathrm{N}=5)$ were not different from those of control males, which is contrary to expectation, but in accord with data from similarly treated rhesus monkeys. Seven males (of 24) and seven females (of 34) had no measurable DPOAEs at any frequency or level of primary tones tested, even though all did have normal-appearing CEOAEs. No spontaneous otoacoustic emissions (SOAEs) were found in any ears, a common finding in non-human species. [Supported by NIDCD (DM) and NICHD (TML).]

5aPPc9. Potential adverse effects of UMTS electromagnetic fields generated by mobile phones on the human inner ear: A European project (EMFNEAR). Annie M. Moulin (Neurosciences Sensorielles Comportement Cognition UMR5020 CNRS, Université de Lyon, 50, avenue Tony Garnier, 69366 Lyon Cedex 07, France, amoulin@olfac.univ-lyon1 .fr), Marta Parazzini (Institute of Biomedical Engineering CNR - Polytechnic Univ. Milan, Piazza Leonardo da Vinci, 32, I-20133 Milan, Italy,
marta.parazzini@polimi.it),Mark E. Lutman(University of Southampton, University Road, Highfield/ISVR, SO17 iBJ Southampton, UK, mel@isvr.soton.ac.uk), Gyorgy Thuroczy (National Research Institute for Radiobiology and Radiohygiene NIRR, Department of Non-Ionizing Radiation, Anna u. 5, 1775 Budapest, Hungary, alessia.paglialonga@polimi .it), Mariola Sliwinska-Kowalska (Nofer Institute of Occupational Medicine, PL-90-950 Lodz, Poland, marsliw@imp.lodz.pl), Georgy Tavarkiladze (National Research Centre for Audiology and Hearing Rehabilitation, 117513 Moscow, Russian Federation, gtavartkiladze@audiology .ru), Ingrida Uloziene (Kaunas University of Medicine, LT44307 Kaunas, Lithuania, uloziene@kmu.lt), Virgilius Uloza (Kaunas University of Medicine, LT44307 Kaunas, Lithuania, uloza@kmu.lt), Shrikanta Mishra (University of Southampton, University Road, Highfield / ISVR, SO17 iBJ Southampton, UK, sm2@isvr.soton.ac.uk), Lionel Collet (Univ. Lyon 1 - Lab. Neurosciences, Service Pr Collet, Pavillon U, Hôpital Edouard Herriot, F-69003 Lyon, France, iakhoun@olfac.univ-lyon1.fr), Paolo Ravazzani (Institute of Biomedical Engineering CNR - Polytechnic Univ. Milan, Piazza Leonardo da Vinci, 32, I-20133 Milan, Italy, paolo.ravazzani @ polimi.it)

To answer concerns as to the potential effects of electromagnetic fields (EMF) generated by third generation mobile phones on the inner ear, the European Commission funded project "EMFNEAR" assessed potential changes in auditory function induced by UMTS field both in humans and in laboratory animals. The purpose of this paper is to present the final outcomes of the project in humans. Normally hearing subjects (18-30 years old) were exposed to $20 \mathrm{~min}$ UMTS or sham exposure in a double-blind controlled study. Two different EMF field strengths were used: SAR of 0.069 $\mathrm{W} / \mathrm{kg}$ using a commercially available phone and SAR of $1.75 \mathrm{~W} / \mathrm{kg}$ using a patch antenna. Audiological assessment, involving pure tone audiometry, distortion product otoacoustic emissions, effects of contralateral acoustic stimulation on Transiently evoked otoacoustic emissions and late cognitive potentials (P300), were performed before and immediately after real or sham exposures. Results showed no effects of acute UMTS electromagnetic fields exposure on the inner auditory system of humans. 


\title{
Session 5aPPd
}

\section{Psychological and Physiological Acoustics: General Topics in Psychological and Physiological Acoustics VII (Poster Session)}

\author{
Elizabeth Strickland, Cochair \\ Purdue University
}

Armin Kohlrausch, Cochair

Philips Research Europe

\author{
Alain De Cheveigne, Cochair \\ CNRS, Universite Paris 5, Ecole Normale Superieure
}

\begin{abstract}
All posters will be on display from 9:40 a.m. to 11:20 a.m. To allow contributors an opportunity to see other posters, contributors of odd-numbered papers will be at their posters from 9:40 a.m. to 10:30 a.m. and contributors of even-numbered papers will be at their posters from 10:30 a.m. to 11:20 a.m.
\end{abstract}

\section{Contributed Papers}

5aPPd1. Phase interactions between auditory filter and complex stimuli: psychophysical evidence for level dependence in cochlear phase response. Yi Shen (Indiana Univ., 200 S. Jordan Ave., Dept. of Speech and Hearing Sciences, Bloomington, IN 47405-7000, USA, shen2@indiana.edu), Jennifer Lentz (Indiana Univ., 200 S. Jordan Ave., Dept. of Speech and Hearing Sciences, Bloomington, IN 47405-7000, USA, jjlentz@indiana.edu)

Harmonic complexes with the same power spectrum and different phase spectra lead to significant differences in their efficiency in masking puretone signals. This process is presumably due to phase interactions between the masker and the cochlea, leading to internally represented waveforms with different amounts of modulation. The present study applied this idea to estimate the phase characteristic of the auditory system at different stimulus levels. Two masking experiments were conducted for estimating whether auditory-filter phase curvatures are level dependent at 2 and $4 \mathrm{kHz}$. Curvatures were measured at fixed masker levels ranging between 50 and $90 \mathrm{~dB}$ SPL in one experiment and at fixed signal levels ( $25-50 \mathrm{~dB}$ SPL) in a second experiment. Results from both experiments suggest a level dependence in the auditory-filter phase curvature at both frequencies, which shifts from a negative value towards zero with increasing excitation level. A plausible explanation for this observed level dependence was proposed with the use of a nonlinear cochlear model. This modeling approach indicated that the behaviorally measurable phase curvature is affected by level dependent alterations in the magnitude and phase response of the basilar membrane.

5aPPd2. Is auditory intensity discrimination a comparison of entropy changes? Iftikhar Nizami (1312 Grayson Place, Decatur, GA 30030, USA nizamii2@aol.com)

In JASA $(\mathbf{9 7}, 1995)$, Wong \& Norwich presented a Weber-fraction equation derived from McConville, Norwich, \& Abel (Int J Biomed Comput 27, 1991). The latter modeled two-alternative forced-choice discrimination between stimuli of identical spectra and identical durations " $\mathrm{t}$ " but differing intensities I and $\mathrm{I}+\Delta \mathrm{I}$. Discrimination depended on $\Delta \mathrm{H}(\mathrm{I}, \mathrm{t})$, the change in the information-theoretic entropy over $\mathrm{t}$ and $\Delta \mathrm{I}$. $\Delta \mathrm{H}(\mathrm{I}, \mathrm{t})$, assumed constant over intensities, emerged as one of five unknowns in a Weber-fraction equation, $(\Delta \mathrm{I}) / \mathrm{I}=f\left(\mathrm{t}_{0}, \mathrm{t}_{\mathrm{W}}, \mathrm{n}, \beta, \Delta \mathrm{H}(\mathrm{I}, \mathrm{t})\right)$. McConville et al. presumed values for $\mathrm{n}$ and $\mathrm{t}_{\mathrm{w}}$. Using $(\Delta \mathrm{I}) / \mathrm{I} \approx \Delta \mathrm{H}(\mathrm{I}, \mathrm{t}) /\left(\mathrm{I} \Delta \mathrm{t}\left[\partial^{2} \mathrm{H} / \partial \mathrm{I} \partial \mathrm{t}\right]\right)$ and setting $\Delta \mathrm{t}=\mathrm{t}$, $f\left(\mathrm{t}_{0}, \mathrm{t}_{\mathrm{W}}, \mathrm{n}, \beta, \Delta \mathrm{H}(\mathrm{I}, \mathrm{t})\right) \quad$ was approximated as $g(\mathrm{n},(\beta / \mathrm{t}), \Delta \mathrm{H}(\mathrm{I}, \mathrm{t}))$ $=\Delta \mathrm{H}(\mathrm{I}, \mathrm{t}) /\left(\mathrm{It}\left[\partial^{2} \mathrm{H} / \partial \mathrm{I} \partial \mathrm{t}\right]\right)$, which was then curvefitted to one listener's Weber fractions. The obtained $\Delta \mathrm{H}(\mathrm{I}, \mathrm{t})$ alone was substituted back into $f\left(\mathrm{t}_{0}, \mathrm{t}_{\mathrm{W}}, \mathrm{n}, \beta, \Delta \mathrm{H}(\mathrm{I}, \mathrm{t})\right)$, which was then substantiated by curvefitting to the data to reveal the remaining unknowns, $\mathrm{t}_{0}$ and $\beta$. McConville et al. had to curvefit, because they model only a single, unspecified forced-choice trial, making $\Delta \mathrm{I}$ any intensity change; and none of the unknowns could legitimately be presumed. The curvefitting was flawed: the assumption $\Delta \mathrm{H}(\mathrm{I}, \mathrm{t})=$ constant affirms Fechner's postulate; and $\partial^{2} \mathrm{H} / \partial \mathrm{I} \partial \mathrm{t}<0$, giving a negative Weber fraction. McConville et al. fail to explain auditory intensity discrimination as a comparison of entropy changes, casting doubt on Wong \& Norwich (1995).

5aPPd3. Does Norwich's Entropy Theory of Perception avoid the use of mechanisms, as required of an information-theoretic model of auditory primary-afferent firing? Iftikhar Nizami (1312 Grayson Place, Decatur, GA 30030, USA,nizamii2@aol.com)

Norwich et al.'s Entropy Theory (1975-present) stipulates that (1) the auditory receptor is uncertain about the stimulus' intensity, (2) uncertainty, given by the "entropy" $\mathrm{H}$, relates to primary afferent neuronal firing-rate $\mathrm{F}$ as $\mathrm{F}=$ constant $\times \mathrm{H}$. $\mathrm{H}$ was calculated according to Garner \& Hake (Psychological Review 58, 1951; after Shannon, Bell System Technical Journal 27, 1948) using Information Theory. $\mathrm{H}$ is available any time events, their outcomes, and the appropriate probabilities of occurrence are known, precisely because Information Theory ignores mechanisms, such as sensory transduction. Hence, any "information-theoretic" hearing model must avoid physiological mechanisms, and Norwich et al. claim to comply. But do they? The Entropy Theory was scrutinized for avoidance of physiological mechanisms. Norwich et al. stipulated throughout that (1) "We can envisage a steady sensory stimulus as a stationary stochastic sequence of microscopic sensory events" (Norwich \& Wong, Annales de la Fondation Louis de Broglie 22, 1997), (2) those events (representing different intensities) are repeatedly sampled by the receptor. Sampling is a mechanism, not separable from transduction, the event's (presumed) "outcome". Also, Norwich et al.'s attempts to separate events from outcomes produces inconsistent identification of "events." The Entropy Theory is not an information-theoretic model of primary-afferent firing.

5aPPd4. Slopes of psychometric functions for forward-masked tones with and without a suppressor. Ifat Yasin (UCL Ear Institute, 332 Gray's Inn Road, WC1X 8EE London, UK, i.yasin@ucl.ac.uk), Christopher J. Plack (Psychology Department, Lancaster University, LA1 4YF Lancaster, UK, c.plack@lancaster.ac.uk)

The compressive nonlinearity defined by the basilar membrane (BM) input-output (I/O) function is evident in the slopes of psychometric 
functions for forward-masked tones. A suppressor tone may reduce compression by linearizing the compressive portion of the BM I/O function, or reduce cochlear gain without affecting the value of maximum compression. The present study compared slopes of psychometric functions obtained for a fixed low-level signal in the presence of a variable masker with or without a suppressor gated with the masker. Thresholds and psychometric functions were obtained for four normal-hearing listeners using a 2I-AFC task. The sinusoidal signal was a low-level 4-kHz tone (0-ms steady state, $2-\mathrm{ms}^{2} \cos ^{2}$ ramps) masked by a sinusoidal masker (100-ms steady state, 2 -ms $\cos ^{2}$ ramps). The maskers were either on frequency $(4 \mathrm{kHz})$ or off frequency $(2.2$ $\mathrm{kHz}$ ) and were presented at masker-signal intervals of 20-100 ms. Initial analyses suggest that as masker levels increase, the slopes of psychometric functions become increasingly shallow (on-frequency masker) or remain relatively steep (off-frequency masker). The effect of a suppressor on the slopes of the psychometric functions appears to be minimal. Results will be discussed in the context of a cochlear model of peripheral nonlinearity.

5aPPd5. An effect of top-down processing on intensity discrimination in the sensory-trace mode. Ervin R. Hafter (University of California, Department of Psychology, 3210 Tolman Hall, Berkeley, CA, CA 94720-1650, USA, hafter@berkeley.edu), Anne-Marie Bonnel (University of California, Department of Psychology, 3210 Tolman Hall, Berkeley, CA, CA 947201650, USA, AMBonnel@berkeley.edu), Swapan Gandhi (University of California, Department of Psychology, 3210 Tolman Hall, Berkeley, CA, CA 94720-1650, USA, swapangandhi@berkeley.edu)

A traditional measure of intensity discrimination presents a standard stimulus followed by a test. In one perceptual strategy, the subject compares the test to a coded version of the standard in long-term "context-coded memory" while, in another, the comparison is to a copy of the standard held in short-term "trace memory" [Durlach and Braida, J. Acoust. Soc. Amer, 46, 372-383 (1969)]. Subjects can be induced to use trace memory by roving the level of the standard randomly from trial to trial. It is well known that roving lowers overall performance, but by parsing individual standards we find that the loss is not uniform. Rather, an inverse correlation with the roved levels shows a severe departure from Weber's Law, with slopes of about $-6 \mathrm{~dB}$ over $20 \mathrm{~dB}$ of roving. This can be modeled in terms of an additive noise that, unlike the multiplicative noise implied by Weber's Law, is constant across levels. We believe this represents top-down processes involved in the use of stimuli held in rehearsal memory.

5aPPd6. Contribution of very low modulation frequencies to speech intelligibility in a competing speech task. Christian Füllgrabe (University of Cambridge, Department of Experimental Psychology, Downing Street, CB2 3EB Cambridge, UK, cf277@cam.ac .uk), Michael A. Stone (University of Cambridge, Department of Experimental Psychology, Downing Street, CB2 3EB Cambridge, UK, mas19@cam.ac.uk), Brian Moore (University of Cambridge, Department of Experimental Psychology, Downing Street, CB2 3EB Cambridge, UK, bcjm@cam.ac.uk)

Vocoder studies have demonstrated that low-rate temporal-envelope cues (below $\sim 50 \mathrm{~Hz}$ ) in a few frequency bands are sufficient for good speech identification in quiet. From the effects of low pass and high pass filtering of the temporal envelopes extracted from band pass filtered sentences, Drullman et al. [J. Acoust. Soc. Am. 95, 2670-2680 (1994); J. Acoust. Soc. Am. 95, 1053-1064 (1994)] argued that modulation frequencies between 4 and 16 $\mathrm{Hz}$ are the most important for speech identification in a steady background noise. However, Stone and Moore (JASA, in press) found that modulation frequencies below $2 \mathrm{~Hz}$ were also beneficial when the speech signal was processed through an 8-channel (but not a 12-channel) noise vocoder. The present study investigated speech identification for nine normal-hearing listeners using IEEE sentences presented with a competing talker; the mixed signal was processed using a 6-, 9-, or 15-channel noise vocoder. Low-rate temporal-envelope cues were manipulated using a compressor for each channel. Compression speed varied from slow to fast, so as to progressively degrade modulation frequencies below $4 \mathrm{~Hz}$. Independent of the spectral resolution of the vocoder, mean intelligibility decreased as the compression speed increased; even modulation frequencies below $1.3 \mathrm{~Hz}$ contributed significantly to speech intelligibility.

5aPPd7. Comodulation masking release and its representation in human auditory cortex. Stephan M. Ernst (Universität Oldenburg, Medizinische Physik, Carl-von-Ossietzky Str. 9-11, 26111 Oldenburg, Germany, stephan.ernst@uni-oldenburg.de), Jesko Verhey (Universität Oldenburg, Medizinische Physik, Carl-von-Ossietzky Str. 9-11, 26111 Oldenburg, Germany, jesko.verhey@uni-oldenburg.de), Stefan Uppenkamp (Universität Oldenburg, Medizinische Physik, Carl-vonOssietzky Str. 9-11, 26111 Oldenburg, Germany, stefan.uppenkamp@unioldenburg.de)

The aim of the present study was to find a correlate of the psychoacoustical effect of comodulation masking release (CMR) in human auditory cortex using functional magnetic resonance imaging (fMRI). The target stimulus was a sinusoidal signal embedded in a band limited white noise which was either unmodulated or multiplied with an irregular square-wave modulator with mean modulation frequency of $40 \mathrm{~Hz}$ (comodulated condition). For all listeners participating in the fMRI study, the average psychoacoustic threshold difference between the unmodulated and the comodulated condition (i.e., the CMR) was $20 \mathrm{~dB}$. Similar to the results from a previous fMRI study on pure-tone masking (Ernst, Verhey, Uppenkamp, ARO abstracts 30 (2007), p. 302(A)), a spatial dissociation of changes of overall level and signal-to-noise ratio in auditory cortex was found. The comparison of the fMRI activation maps for a signal presented in modulated and in unmodulated noise reveals that those regions in the antero-lateral part of Heschl's gyrus previously shown to represent the audibility of a target (rather than overall level) exhibit a stronger activation for the modulated than for the unmodulated conditions. This result is interpreted as a physiological correlate of the psychoacoustical CMR effect at the level of the auditory cortex.

5aPPd8. Behavioral evidence for off-frequency compression at $4 \mathbf{k H z}$. Dhany Arifianto (University of Zagreb, Psychology Department, Lancaster University, LA1 4YF Lancaster, UK, arifianto@ieee.org), Christopher J. Plack (Psychology Department, Lancaster University, LA1 4YF Lancaster, UK, c.plack@lancaster.ac.uk)

Most behavioral techniques for estimating the basilar-membrane response rely on the assumption that the response to an off-frequency masker is linear at the signal place. To test this assumption, we modified the additivity of forward masking technique. The signal was a 4-kHz, 10-ms pure tone, presented at $10 \mathrm{~dB}$ sensation level. The maskers were $500-\mathrm{Hz}$-wide bands of noise centered on 1.5, 2, or $4 \mathrm{kHz}$. Masker 1 had a duration of 200 $\mathrm{ms}$ and was followed immediately by masker 2 with a duration of $20 \mathrm{~ms}$. The masker 2-signal silent interval ranged from 10 to $60 \mathrm{~ms}$. For each interval, the masker level required to mask the signal was determined, for each masker presented individually, and for the two maskers combined. In the combined case, the levels of the two maskers were set to be roughly equally effective, and adaptively varied together. Combining two equally effective maskers should produce a 3-dB reduction in masker level at threshold if the system is linear. A reduction greater than this indicates compression. Although compression was greatest for the $4-\mathrm{kHz}$ maskers, compression was also observed at 1.5 and $2 \mathrm{kHz}$, suggesting that the assumption of a linear off-frequency response may not valid.

5aPPd9. Multiple looks in spectrotemporal integration. Evelyn Hoglund (The Ohio State University, 110 Pressey Hall, 1070 Carmack Road, Columbus, OH 43210, USA, hoglund.1@ osu.edu), Lawrence Feth (The Ohio State University, 110 Pressey Hall, 1070 Carmack Road, Columbus, OH 43210, USA, feth.1@ osu.edu)

The multiple looks hypothesis was originally developed as an explanation for temporal integration and resolution. Our preliminary work supports its extension to spectral integration as well spectrotemporal integration. The current study was designed to measure detection of tonal signals that vary in both spectral and temporal dimensions. Preliminary work began by measuring thresholds in quiet. Elementary signals consisted of $10 \mathrm{~ms}$ tone bursts centered on one of eight frequencies spaced 1 ERB apart. Complexes were constructed by adding two to eight individual tone bursts along the temporal or spectral dimension, or both. The signals varied along both dimensions in several ways to focus on the relative salience of each dimension for 
detection. In quiet, the spectrotemporal integration conditions showed threshold improvement to be limited by spectral integration. Surprisingly, even trial-by-trial randomization of frequencies did not negatively influence detection. For this study, the same tone complexes from the preliminary work were used to measure thresholds in a wide band energetic masker. As expected, threshold improvement was most dependent on increasing the number of tones, with less effect from other variables. Subtle differences between quiet and masked integration performance will be discussed. Research supported by a grant from NIH/NIDCD R01-DC006879.

5aPPd10. Estimating the basilar-membrane input/output-function in normal-hearing and hearing-impaired listeners using forward masking. Morten L. Jepsen (Centre for Applied Hearing Research, Technical University of Denmark, DTU, Bygn. 352, 2800 Lyngby, Denmark, mlj@oersted.dtu.dk), Torsten Dau (Centre for Applied Hearing Research, Technical University of Denmark, DTU, Bygn. 352, 2800 Lyngby, Denmark,tda@oersted.dtu.dk)

To partly characterize the function of cochlear processing in humans, the basilar membrane (BM) input-output (I/O) function can be estimated. In recent studies, forward masking has been used to estimate BM compression. If an on-frequency masker is processed compressively, while an off-frequency masker is transformed more linearly, the ratio between the slopes of growth of masking (GOM) functions provides an estimate of BM compression at the signal frequency. In this study, this paradigm is extended to also estimate the knee-point of the I/O-function between linear processing at low levels and compressive processing at medium levels. If a signal can be masked by a low-level on-frequency masker such that signal and masker fall in the linear region of the I/O-function, then a steeper GOM function is expected. The knee-point can then be estimated in the input level region where the GOM changes significantly. Data were collected from eight normal-hearing $(\mathrm{NH})$ and five hearing-impaired (HI) listeners with mild to moderate sensorineural hearing loss. Both groups showed large inter-subject but low intrasubject variability. When the knee-point could be estimated for the HI listeners it was shifted towards higher input levels and compression was similar to that of $\mathrm{NH}$ listeners.

5aPPd11. Unpredictable interruption can enhance the auditory continuity illusion. Maori Kobayashi (R.I.E.C., Tohoku University, 2-1, Katahira, Aoba-ku, 980-8577 Sendai, Japan, maori@ais.riec.tohoku .ac.jp), Makio Kashino (NTT Communication Science Laboratories, NTT Corporation, 3-1, Morinosato Wakamiya, 243-0198 Atsugi, Japan, kashino @avg.brl.ntt.co.jp)

It was examined whether the predictability of interruption timing affects the limit of perceiving the continuity illusion. The stimulus consisted of a $500-\mathrm{Hz}$ sinusoidal inducee and a 200-ms, 500-Hz, 1/3-octave noise band inducer, which were alternated repeatedly, with a fixed interval $(400 \mathrm{~ms})$ between adjacent inducers (regular condition) or with random intervals (irregular condition). The continuity limit (CL) was measured in terms of the maximum level of the inducee for the illusory continuity to be perceived while maintaining the inducer level at $60 \mathrm{~dB}$ SPL. In Experiment 1, the listeners' task was to judge whether the inducee appeared continuous or discontinuous. In Experiment 2, the task was to discriminate illusory and physical continuity in a two-interval forced choice paradigm. In both experiments, the mean CLs in the irregular condition were significantly higher than those in the regular condition. In Experiment 3, when an identical irregular interval pattern was used repeatedly in successive trials, the mean CL gradually declined, and reached the level of the regular condition in approximately 80 trials. These results suggest that unpredictability, not mere irregularity, in interruption timing promotes the continuity illusion, implying the involvement of the short-term plasticity of the auditory system.

5aPPd12. A filterbank model of human nonlinear auditory frequency selectivity. Almudena Eustaquio-Martín (University of Salamanca, Instituto de Neurociencias de Castilla y León, Avda. Alfonso X 'El Sabio' s/n, 37007 Salamanca, Spain, aeustaquio@usal.es), Enrique A.
Lopez-Poveda(University of Salamanca, Instituto de Neurociencias de Castilla y León, Avda. Alfonso X 'El Sabio' s/n, 37007 Salamanca, Spain, ealopezpoveda@usal.es)

Filterbank models of human nonlinear auditory frequency selectivity are useful in a variety of applications. Existing filterbanks are almost certainly flawed because they do not account for the recent observation that compression extends to a wider range of stimulus frequencies in apical than in basal cochlear sites [Lopez-Poveda et al. (2003), J. Acoust. Soc. Am. 113, 951960]. Here, we present a filterbank based on the forward-masking data from which this observation was made. A forward-masking model was constructed by cascading a linear "outer/middle-ear" filter, followed by a dualresonance nonlinear (DRNL) filter, followed by a linear temporal window. The temporal window was assumed to be identical across listeners and cochlear places. Only the parameters of the DRNL filter were adjusted to simulate the absolute threshold and the masking data of three listeners for probe frequencies of $0.5,1,2,4$, and $8 \mathrm{kHz}$. A bank of DRNL filters was then constructed by linear or quadratic regression of the optimum parameters. It is discussed that this filterbank is the best possible approximation of human level-dependent auditory frequency selectivity to date. Additionally, the results support the linearity of the temporal window. [Work supported by IMSERSO 131/06, PROFIT CIT-390000-2005-4, MEC BFU-2006-07536.]

5aPPd13. Object's width and distance distinguished by the blind using auditory sense while they are walking. Takahiro Miura (Graduate School of Information Science and Technology, University of Tokyo, 7-3-1 Hongo, Bunkyo-ku, 113-8654 Tokyo, Japan, miura@human.rcast.u-tokyo .ac.jp), Kazutaka Ueda (Research Center for Advanced Science and Technology, University of Tokyo, 4-6-1 Komaba, Meguro-ku, 153-8904 Tokyo, Japan, ueda@human.rcast.u-tokyo.ac.jp), Teruo Muraoka (Research Center for Advanced Science and Technology, University of Tokyo, 4-6-1 Komaba, Meguro-ku, 153-8904 Tokyo, Japan, muraoka@human.rcast.u-tokyo .ac.jp), Shuichi Ino (Research Center for Advanced Science and Technology, University of Tokyo, 4-6-1 Komaba, Meguro-ku, 153-8904 Tokyo, Japan, ino@human.rcast.u-tokyo.ac.jp), Tohru Ifukube (Research Center for Advanced Science and Technology, University of Tokyo, 4-6-1 Komaba, Meguro-ku, 153-8904 Tokyo, Japan, ifukube@ human.rcast.u-tokyo.ac.jp)

Mobility aid has recently become important for the blind because of increasing their outgoing opportunity. It is necessary for the blind to acquire "obstacle sense" by which they can recognize surrounding objects auditorily. In particular, it is indispensable to investigate the characteristics of the obstacle sense while they are walking. However, training method for the mobility has not been systematically proposed yet because factors regarding the obstacle sense while walking remain unknown. Final goal of this study is to propose a systematic training method for their mobility using the obstacle sense. In this study, the authors particularly focused on a relation of interaural differences between both ears to the ability of the obstacle sense while walking toward the obstacles. In the experiments, the blind subjects were asked to answer when they find the obstacle made of wooden plate with various widths in both conditions of head moving and head fixing. Auditory discrimination ability of the obstacles was measured as a function of the obstacle's width and the distance from the blind subject. From the experimental results, it was found that blind people generally can recognize the obstacles better in the head moving case than the head fixing case.

5aPPd14. Amplitude modulation depth discrimination in hearing-impaired and normal-hearing listeners. Stephan D. Ewert (Carl von Ossietzky Universität Oldenburg, Ammerländer Heerstraße 114-118, 26111 Oldenburg, Germany, stephan.ewert@uni-oldenburg.de), Jutta Volmer (Universität Oldenburg, Medizinische Physik, Carl-von-Ossietzky Str. 9-11, 26111 Oldenburg, Germany, jutta.volmer@uni-oldenburg .de), Torsten Dau (Centre for applied hearing research, Technical University of Denmark, DTU, Bygn. 352, 2800 Lyngby, Denmark, tda@oersted.dtu.dk), Jesko Verhey (Universität Oldenburg, Medizinische Physik, Carl-von-Ossietzky Str. 9-11, 26111 Oldenburg, Germany, jesko .verhey@uni-oldenburg.de)

The processing of amplitude modulations (AM) of sounds is assumed to be crucial for decoding and understanding of speech in humans. Since hearing-impaired (HI) listeners often suffer from severely hampered speech intelligibility, particularly in reverberant or noisy environments, they might also show degraded performance in AM processing tasks. However, several 
studies indicated a similar or even better performance in AM detection tasks for sensorineural HI listeners than for normal hearing $(\mathrm{NH})$ listeners when reduced audibility was compensated. In addition to AM detection, this study investigates the differential processing of amplitude modulation depth in $\mathrm{HI}$ and $\mathrm{NH}$ listeners. AM-depth discrimination of a 4-, 8-, and $30-\mathrm{Hz}$ sinusoidal AM, imposed on a 1- or 4-kHz pure-tone carrier, was measured. The AM of the standard ranged from being well detectable to near threshold. AM-depth discrimination thresholds strongly varied among HI listeners and were elevated in comparison to $\mathrm{NH}$ for high standard depths. A model of AM processing is suggested incorporating an individually adjusted simulation of the auditory periphery. To account for the data of HI listeners, however, the key element appeared to be an increased internal noise in the AM-depth domain. Consequences for speech perception are discussed.

5aPPd15. Exploring the psychophysical temporal effect in masking using a model of the auditory periphery. Skyler Jennings (Purdue University, 500 Oval Drive, West Lafayette, IN 47907, USA, sgjennin@purdue.edu), Michael Heinz (Purdue University, 500 Oval Drive, West Lafayette, IN 47907, USA, mheinz@purdue.edu), Elizabeth Strickland (Purdue University, 500 Oval Drive, West Lafayette, IN 47907, USA, estrick@purdue.edu)

Under some conditions, normal hearing individuals can detect a short duration signal in a simultaneous masker at a lower signal-to-masker ratio when there is preceding stimulation (either a precursor or an extension of the masker). This effect has been referred to as overshoot or the temporal effect. It has been hypothesized the neural adaptation may account for some but not all of the temporal effect. Recent psychophysical data suggest that cochlear gain may decrease over the course of the masker and improve detection. This could be mediated by the medial olivocochlear reflex (MOCR). In the present study, a model of peripheral auditory processing [Zilany and Bruce, J. Acoust. Soc. Am. 120, 1446-1466 (2006)] using a d'type metric was used to assess the relative contributions of these processes. Although the model includes neural adaptation, initial results indicate that it does not predict a temporal effect. The potential role of the MOCR will be simulated by decreasing the gain of the cochlear amplifier, to see if this will produce a temporal effect in the model predictions. [Work supported by NIH.]

5aPPd16. Intelligibility of whispered speech interleaved with speech or noise maskers. Nandini Iyer (AFRL, 2610 Seventh Street, WPAFB, OH 45433, USA, nandini.iyer@wpafb.af.mil), Douglas S. Brungart (AFRL, 2610 Seventh Street, WPAFB, OH 45433, USA, douglas.brungart @wpafb.af.mil), Brian D. Simpson (AFRL, 2610 Seventh Street, WPAFB, OH 45433, USA, Brian.Simpson@wpafb.af.mil)

When an interrupted speech signal is alternated with a noise masker, performance is generally improved relative to the condition where the speech and noise are presented continuously. However, when two interrupted speech signals are alternated, performance declines relative to the continuous presentation condition. One possible explanation for this result is that listeners in the alternated speech and noise condition use the contrast between the periodic temporal structure of voiced speech and the random temporal structure of noise to segregate the time intervals associated with the speech target and noise masker. In the current experiment, a whispered speech signal was either presented continuously in the presence of a noise masker or alternated with a noise masker at an $8-\mathrm{Hz}$ rate. The results show near perfect performance in the alternated whisper and noise condition, suggesting that harmonic structure due to voicing is not necessary to segregate a speech signal from an interleaved random-noise masker. Indeed, when whispered speech was interleaved with voiced speech, performance decreased relative to the continuous condition when the target talker was voiced but not when it was whispered, suggesting that listeners are better at selectively attending to unvoiced intervals and ignoring voiced intervals than the converse.

5aPPd17. Temporal integration functions of amplitude modulation depth discrimination: can multiple-looks model explain this? Jungmee Lee (Univ. of Arizona, 1131 E. 2nd Street, Tucson, AZ 85721, USA, jmlee6@email.arizona.edu), Derek Edwards (Univ. of Arizona, 1131 E. 2nd Street, Tucson, AZ 85721, USA, derek09@email.arizona.edu), Jennifer Andrews (Univ. of Arizona, 1131 E. 2nd Street, Tucson, AZ 85721, USA, andrewsj@email.arizona.edu), Heather Murray (Univ. of Arizona, 1131 E. 2nd Street, Tucson, AZ 85721, USA, hmurray@email.arizona.edu)

Lee and Bacon (1994) applied a multiple looks model (Viemeister and Wakefield, 1990) as a mechanism of AM depth discrimination when the carrier and the modulator were presented at the same time (gated condition). The model provided a reasonable prediction, but the performance (d') was underpredicted when the number of modulation cycles changed from two to four. They speculated that onset information loss due to gating with two modulation cycles lead to a greater improvement with four modulation cycles. Following this, one would expect that AM depth discrimination can be better predicted by the multiple-looks model for the condition where there is no loss of modulation information at onset. This study compared psychometric functions of AM depth discrimination with different modulation cycles for the gated and onset-only conditions (carrier was presented for $250 \mathrm{~ms}$ prior to the modulation to preserve onset information). The slop of the psychometric functions with two modulation cycles was shallower than those with greater modulation cycles for the gated condition, while the slop of the psychometric functions was similar for all modulation cycles for the onset-only condition. The data will be discussed with multiple looks model. [This work was supported by NIDCD Grant No. R03 DC06605-02.] 


\title{
Session 5aPPe
}

\section{Psychological and Physiological Acoustics and Computational Acoustics: Computational Auralization I (Poster Session)}

\author{
Durand Begault, Cochair \\ NASA Ames Research Center \\ Lauri Savioja, Cochair \\ Helsinki University of Technology
}

\begin{abstract}
All posters will be on display from 9:40 a.m. to 11:20 a.m. To allow contributors an opportunity to see other posters, contributors of odd-numbered papers will be at their posters from 9:40 a.m. to 10:30 a.m. and contributors of even-numbered papers will be at their posters from 10:30 a.m. to 11:20 a.m.
\end{abstract}

\section{Contributed Papers}

5aPPe1. Effect of sound spatialisation on multitasking in remote meetings. Stuart N. Wrigley (University of Sheffield, Regent Court, Dept. of Computer Science, 211 Portobello Road, s1 4dp Sheffield, UK, s.wrigley@dcs.shef.ac.uk), Simon Tucker (University of Sheffield, Dept. of Information Studies, Regent Court, 211 Portobello Street, S1 4DP Sheffield, UK, s.tucker@dcs.shef.ac.uk), Guy J. Brown (University of Sheffield, Dept. of Computer Science, Regent Court, 211 Portobello Street, S1 4DP Sheffield, UK, g.brown@dcs.shef.ac.uk), Steve Whittaker (University of Sheffield, Dept. of Information Studies, Regent Court, 211 Portobello Street, S1 4DP Sheffield, UK, s.whittaker@shef.ac.uk)

Participants in a teleconference often multitask during meetings, e.g., they work on a text-based "foreground" task whilst listening in the 'background' for a topic of interest to appear. Audio material should therefore be presented in a manner that has the smallest possible impact on the foreground task without affecting topic detection. Here, we ask whether spatialised audio presentation of a meeting is less disruptive than the singlechannel mixture of talkers that is normally used in teleconference audio. Head-related impulse responses are used to synthesise a stereo signal in which each meeting participant is placed at a spatially distinct location. A number of talker location configurations are used, and we examine how these impact upon a text-based foreground task: finding all letter "e" occurrences in a block of text as quickly as possible. We also examine the effect of listening task (e.g., "listen for keyword x" versus "listen for keyword x spoken by talker on left") and record listener preferences for audio presentation style. Our results suggest that single-channel and spatialised audio are equally disruptive when listeners are unaware of the audio presentation style. We also report studies in which presentation type and target direction/gender are cued.

5aPPe2. The use of virtual acoustics in the evaluation and development of binaural hearing aid algorithms. Monika Rychtarikova (Lab. ATF, Katholieke Universiteit Leuven, Celestijnenlaan 200D, B-3001 Leuven, Belgium, Monika.Rychtarikova@bwk.kuleuven.be), Tim Van Den Bogaert (ExpORL, Dept. Neurosciences, K.U. Leuven, Herestraat 49 bus 721, O. \& N2, B-3000 Leuven, Belgium, tim.vandenbogaert@med.kuleuven .be), Gerrit Vermeir (Lab. ATF, Katholieke Universiteit Leuven, Celestijnenlaan 200D, B-3001 Leuven, Belgium, Gerrit.Vermeir@bwk .kuleuven.be), Koen Eneman (ExpORL, Dept. Neurosciences, K.U. Leuven, Herestraat 49 bus 721, O. \& N2, B-3000 Leuven, Belgium, koen.eneman@med.kuleuven.be), Walter Lauriks (Lab. ATF, Katholieke Universiteit Leuven, Celestijnenlaan 200D, B-3001 Leuven, Belgium,
Walter.Lauriks@fys.kuleuven.be),Marc Moonen(ESAT/SISTA, K.U. Leuven, Kasteelpark Arenberg 10, B-3001 Leuven, Belgium, marc.moonen@esat.kuleuven.be), Jan Wouters (ExpORL, Dept. Neurosciences, K.U. Leuven, Herestraat 49 bus 721, O. \& N2, B-3000 Leuven, Belgium, jan.wouters@med.kuleuven.be)

The development of noise reduction algorithms for hearing aids (HA) is not longer only related to the improvement of signal to noise ratio, but also to the quality of hearing, e.g., binaural aspects of hearing. This is very important for the recognition of the localization of sound sources but also for an improved speech intelligibility in noisy situations due to spatial release from masking effects. New design and signal processing algorithms for binaural HA's need to be tested and validated in different acoustical scenarios. As it is too laborious and time consuming to perform sufficient numbers of perceptual evaluations in different rooms with different acoustical parameters, advanced acoustic modeling of different virtual acoustical environments might be needed. Virtual acoustics in our research relates to the convolution of the measured or simulated binaural signals (head related transfer functions - HRTF's) with the impulse response generated from a computer model of a room (using ODEON ${ }^{\circledR}$ software) to simulate binaural sounds. This study investigates the usage of virtual acoustics in the framework of developing algorithms for binaural hearing aids. It evaluates and quantifies the fidelity of binaural signals generated by commercially available virtual acoustics software with respect to the localization of sound and speech intelligibility in different acoustical scenarios.

5aPPe3. Auralization of urban soundscaping designs using the Arup SoundLab. Seb Jouan (Arup Acoustics, Bergius House, 11-12 Claremont Terrace, G3 7XR Glasgow, UK, seb.jouan@arup.com)

Noise is unwanted sound (from an urban planning point of view). The standard practice to control noise, while valuable, is a negative process (i.e., reducing unwanted "noise"). In response, there is a drive by several authorities to take a more positive approach to improve and manage soundscapes in cities and civic spaces. Urban soundscape design consists of planning, shaping and managing the sound to fit each area of the masterplan in terms of civic, cultural, and social character. Arup uses its SoundLab, a 3D sound facility (ambisonic), to design and auralise (the sound equivalent to visualisation) soundscapes with planners, architects or artists and demonstrate to Local Planning Authorities or clients the results of the design. An example of urban soundscaping design is Dark Neville Street, Leeds for Leeds City Council where architectural, lighting design, acoustical soundscaping design were integrated together with an artistic sonic piece by HansPeter Kuhn which was auralised in the Arup SoundLab. An example of "indoor" sonic art and soundscape design installation is "Harmonic Bridge" by 
Bill Fontana and Arup Acoustics in the Turbine Hall of the Tate Modern (June-August 2006) where the sonic art piece was composed in the SoundLab.

5aPPe4. Salience of spatial attributes on quality evaluation of multichannel audio processing devices. Inyong Choi (Seoul National University, ENG 420-025, Gwanak PO Box 34, 151-600 Seoul, Republic of Korea, inyongchoi@gmail.com), Jung-Hun Seo (Seoul National University, ENG 420-025, Gwanak PO Box 34, 151-600 Seoul, Republic of Korea, pollini@acoustics.snu.ac.kr), Koeng-Mo Sung (Applied Acoustics Lab., INMC, 132-302, Seoul National University, San 56-1, Sillim-dong, Kwanak-gu, 151-742 Seoul, Republic of Korea, kmsung@snu.ac.kr)

Objective quality assessment methods for audio signal processing devices, e.g. ITU-R BS.1387-1, measure perceived quality by using various types of test excerpts and incorporating a number of psychoacoustic metrics for evaluating various attributes of quality degradations. As many multichannel audio processing devices are being developed, recent quality assessment methods include not only timbral features but also spatial features, such as analysis on interaural disparities. In this paper, the importance of the newly proposed spatial - interaural - features are studied for different types of multichannel test signals. The spatial features have bigger salience on directly recorded sound sources than artificially manipulated multichannel contents.

5aPPe5. Assessment of simulation quality of three different auralization procedures. Stephan Paul (Lab. of Vibrations and Acoustics, Fed. Univ. of Santa Catarina, CTC, EMC, Campus Trindade, 88040 Florianópolis, Brazil, stephan.paul.acoustic@gmail.com)

When acoustical problems occur in architectural spaces acousticians are often requested to help. The help is often complicated by very different knowledge in acoustics and problems in communication between architect, musicians and acousticians. Auralization of sound sources is considered to be a powerful tool to solve the problem, making acoustic information accessible for hearing. The present research assessed the quality of the output of different simulation procedures by subjective assessment of similarity with a reference sound, considering different perceptual dimensions (localization, reverberation, and timbre). The reference sound was obtained by binaural recording in the room that was auralized using ray-tracing, mirror sources or a hybrid pyramid tracing method. Two different types of sound sources, human speech and a guitar, were auralized. The results show that the subjective quality of the simulation procedure depends on the type of sound source and the perceptive dimension that was assessed. In general the mirror source method performed best for localization of speech, whereas ray-tracing was the preferred method for the guitar sound. Overall mirror sources also outperform the other methods when good localization is required. Independent from the source the hybrid method was considered the best for the reverberation dimension.

5aPPe6. Effects of hardware on optimal filter segmentations for the segmented convolution. Frank Wefers (Institute of Technical Acoustics, RWTH Aachen University, Neustrasse 50, 52066 Aachen, Germany, Frank .Wefers@akustik.rwth-aachen.de)

The segmented convolution is a commonly used method for the auralisation in interactive virtual acoustic environments. Typically the auralisation of a virtual scene is done by convolution of the scenes' sound sources signals with impulse responses, that describe the sound transmission paths. The segmented convolution algorithm allows an efficient computation of the convolution in real-time, by segmentation of the impulse response into several parts. In order to allow the realistic simulation of complex acoustical scenes, the convolutions' computational effort must be minimised. It is a fact that the segmentation of the impulse response is a key parameter to the algorithm and has great effects on its computational load, stability and even realisability in real-time. Considerations on optimal filter segmentations mainly founded on a theoretical point, like the runtime complexity of operations or the number of required floating point operations. However, practical systems behave differently: Calculations on real hardware suffer cache losses and memory bandwidth. In this contribution, the influence of real hardware on optimal filter segmentations is examined. An optimisation algorithm is presented, that allows to gain optimal filter segmentations for a dedicated target system. The consequences of efficiency losses to the structure of optimal filter segmentations are discussed. 


\title{
Session 5aPPf
}

\section{Psychological and Physiological Acoustics and Speech Communication: Acoustic Features and Speech Perception I (Poster Session)}

\author{
Jont Allen, Cochair \\ University of Illinois \\ Sarah Hawkins, Cochair \\ University of Cambridge
}

\begin{abstract}
All posters will be on display from 9:40 a.m. to 11:20 a.m. To allow contributors an opportunity to see other posters, contributors of odd-numbered papers will be at their posters from 9:40 a.m. to 10:30 a.m. and contributors of even-numbered papers will be at their posters from 10:30 a.m. to 11:20 a.m.
\end{abstract}

\section{Contributed Papers}

5aPPf1. English speech intelligibility test among persons of Arabic native language. Mohammed Abd-Elbasseer (National Institute of Standard (NIS) - Acoustics Department, Teras Street/Al Ahram Street/Giza, 202 Cairo, Egypt, mbacir@yahoo.com), Sanaa Barakat (National Institute of Standard (NIS) - Acoustics Department, Teras Street/Al Ahram Street / Giza, 202 Cairo, Egypt, s43223@yahoo.com)

English language as a non-native language is the most common language over the world, it is the language of conferences, business and the airports. While the Arabic language is the native language of Egypt, the English language is considered as the second language. This research will conduct to investigate the effect of speech level, rate and repetition on the intelligibility of English language among some highly educated Egyptian persons. 34 males and females subjects are participated in this test. 40 sentences of BKB sentences were used with three scored words per sentence. First, they are subjected to the speech rate of about 220, 190, 160, and 100 words per minute at the speech level of about $60 \mathrm{dBA}$, which is considered as the normal level. Second,they are subjected to the speech level of 60,70 , and $80 \mathrm{dBA}$ at speech rate of 190 words per minute, which is considered as the normal rate. It was found that the intelligibility score increased with decreasing the rate and increasing the level respectively. It was found that scores of the worst scored sentence for the first 15 subjects did not change, whatever we repeat the tested sentence at normal rate and level.

5aPPf2. Spectral masking release with HiRes120 sound processing in cochlear implant users. Julie Bestel (Advanced Bionics, 76 rue de Battenheim, 68170 Rixheim, France, julieb@abionics.fr), Olivier Sterkers (Hôpital Beaujon, service ORL, 100 bd du Général Leclerc, 92118 Clichy, France, olivier.sterkers@bjn.ap-hop-paris.fr), Bruno Frachet (Hôpital Avicenne, Service ORL et de chirurgie cervico-faciale, 125 rue de Stalingrad, 93009 Bobigny, France, bruno.frachet@club-internet .fr), Bernard Meyer (Hôpital Saint-Antoine, Service ORL, 184 rue du Faubourg St Antoine, 75012 Paris, France, bernard.meyer@sat .ap-hop-paris.fr), Eric Truy (Hôpital Edouard Herriot, Service ORL, Place d'Arsonval, 69003 Lyon, France, eric.truy@chu-lyon.fr), Patrick Boyle (Advanced Bionics, 76 rue de Battenheim, 68170 Rixheim, France, patrickb@abionics.fr), Christian Lorenzi (Univ Paris Descartes, CNRS, Ecole Normale Superieure, DEC, 29 rue d'Ulm, 75005 Paris, France, lorenzi @ens.fr)

Current sound processors in cochlear implants show poor frequency resolution compared to normal auditory processes. They smooth spectral gaps within background maskers, thereby preventing implant users from experiencing release from masking (i.e., improved speech identification in maskers showing spectral gaps compared to maskers with continuous spectra). The Advanced Bionics' HiRes120 coding scheme aims to increase the transmis- sion of fine-grain spectral information using a strategy based upon current steering. If HiRes 120 improves the precision of spectral coding compared to the standard HiRes strategy, listeners should show improved release from masking for the slowest spectral modulations in a noise masker. This hypothesis is tested in newly implanted adults who alternately use HiRes and HiRes120 sound processing for a period of 10 months. Every two or three months, consonant identification is measured in each implantee in the presence of a stationary speech-shaped noise (SSN) masker at a fixed signal-tonoise ratio yielding $30-40 \%$ correct identification with unmodulated noise. The SSN masker is either unmodulated or spectrally modulated to produce periodic, three ERB-wide "spectral gaps" in the noise masker. Masking release scores defined as the change in consonant identification performance in modulated versus unmodulated noise will be presented and discussed.

5aPPf3. Comparison of speech vs speech-analogue temporal encoding in human: An auditory brainstem response and categorical perception assessment. Idrick Akhoun (Univ. Lyon 1 - Lab. Neurosciences, Service Pr Collet, Pavillon U, Hôpital Edouard Herriot, F-69003 Lyon, France, iakhoun@olfac.univ-lyon1.fr), Céline Richard (Univ. Lyon 1 - Lab. Neurosciences, Service Pr Collet, Pavillon U, Hôpital Edouard Herriot, F-69003 Lyon, France, iakhoun@olfac.univ-lyon1.fr), Annie Moulin (Univ. Lyon 1 - Lab. Neurosciences, Service Pr Collet, Pavillon U, Hôpital Edouard Herriot, F-69003 Lyon, France, iakhoun@olfac.univ-lyon1.fr), Evelyne Veuillet (Univ. Lyon 1 - Lab. Neurosciences, Service Pr Collet, Pavillon U, Hôpital Edouard Herriot, F-69003 Lyon, France, iakhoun@olfac.univ-lyon1 .fr), Stéphane Gallégo (Univ. Lyon 1 - Lab. Neurosciences, Service Pr Collet, Pavillon U, Hôpital Edouard Herriot, F-69003 Lyon, France, iakhoun@olfac.univ-lyon1.fr), Christian Berger-Vachon (Univ. Lyon 1 Lab. Neurosciences, Service Pr Collet, Pavillon U, Hôpital Edouard Herriot, F-69003 Lyon, France, iakhoun@olfac.univ-lyon1.fr), Lionel Collet (Univ. Lyon 1 - Lab. Neurosciences, Service Pr Collet, Pavillon U, Hôpital Edouard Herriot, F-69003 Lyon, France, iakhoun@olfac.univ-lyon1.fr), Hung Thai-Van (Univ. Lyon 1 - Lab. Neurosciences, Service Pr Collet, Pavillon U, Hôpital Edouard Herriot, F-69003 Lyon, France, iakhoun@olfac.univlyon1.fr)

Temporal speech cues, such as periodicity and voice onset time (VOT), are particularly salient information for speech intelligibility. In addition, speech elicited auditory brainstem response (Speech ABR) to consonant vowels $(\mathrm{CV})$ has been shown to mimic the temporal periodicity of the acoustic signal very accurately. Consequently, Speech ABR has been described as a potential objective tool to explore the temporal integration of speech excerpts in human brainstem. We aimed at putting in evidence the specialization of temporal encoding of speech vs nonspeech signals through clinical and behavioral measurements. A sum of five sinusoids, at the five 
formant frequencies, modulated by the corresponding $\mathrm{CV}$ temporal envelope, was taken as speech-analogue. Accordingly, Speech and Analogue ABRs components were compared, although a priori due to be similar. Furthermore, categorical perception assessment was processed to both analogue and full-speech continuums. If confirmed, these preliminary results (seven normal hearing adults for physiological ABRs and behavioral categorical perception) would bring further evidence of an early specialization of temporal processing of speech, in line with previous research [Kraus and Nicol, Trends in Neuroscience 28(4), 176--81 (2005)]. [Work supported by CNRS, Cochlear \& Univ.Lyon 1.]

5aPPf4. Modulation of the voice-cuing effect on releasing speech from informational masking. Lijuan J. Xu (Dept. of Psychology, Peking Univ., 5 Yeheyuan Road, Haidian District, 100871 Beijing, China, lijuan179021@yahoo.com.cn), Jingyu Y. Li (Dept. of Machine Intelligence, Speech and Hearing Research Center, 2 Science Building, Peking Univ., 5 Yeheyuan Road, Haidian District, 100871 Beijing, China, lijy@cis.pku.edu.cn), Ying Huang (Dept. of Psychology, Peking Univ., 5 Yeheyuan Road, Haidian District, 100871 Beijing, China, innhuang@gmail.com), Xihong H. Wu (Dept. of Machine Intelligence, Speech and Hearing Research Center, 2 Science Building, Peking Univ., 5 Yeheyuan Road, Haidian District, 100871 Beijing, China, wxh@cis.pku.edu.cn), Liang Li (Dept. of Psychology, Peking Univ., 5 Yeheyuan Road, Haidian District, 100871 Beijing, China, liangli@pku.edu.cn)

In cocktail-party environments, familiarity or knowledge of target talker's voice is useful for reducing speech-on-speech masking (Yang et al., Speech Communication, 49, 892-904, 2007). In addition, the onset asynchrony between target speech and masking speech is an effective cue for unmasking target speech. The present study examined whether the voicecuing effect can be modulated by either the degree of familiarity/knowledge of target talker's voice or the speech onset asynchrony. When target speech started one second after masking speech, prepresenting a priming sentence voiced by the target talker significantly improved the recognition of the target speech which was copresented with masking speech. However, reinforcing the familiarity/knowledge of the target-talker's voice did not further improve the recognition. When target speech and masking speech started at the same time, a single presentation of voice-priming speech did not change participants' speech recognition against masking speech unless the familiarity/knowledge of target-talker's voice was reinforced by either a learning procedure or repeated presentation of the target-talker's voice before testing. These results suggest that the voice-cuing effect on releasing speech from informational masking is graded, depending on both the degree of familiarity/knowledge of the target-talker's voice and the modulation by other cues such as speech-onset asynchrony.

5aPPf5. Consequences of regional accent differences for speech perception studies. Richard Wright (University of Washington, $1417 \mathrm{NE}$ 42nd Street, Seattle, WA 98105, USA, rawright@u.washington .edu), Pamela Souza (University of Washington, 1417 NE 42nd Street, Seattle, WA 98105, USA, psouza@u.washington.edu), Stephanie Bor (University of Washington, 1417 NE 42nd Street, Seattle, WA 98105 , USA, stebor@u.washington.edu), Star Reed (University of Washington, 1417 NE 42nd Street, Seattle, WA 98105, USA, starreed@u.washington .edu)

Sociophonetic research has demonstrated effects of regional accent on vowels. However, little attention is paid to regional accent in hearing and speech perception studies that use standard vowel stimuli. This poster illustrates the importance of these factors to perception. A library of synthetic vowels from nine different regions of the U.S. (e.g., southern California, upper Midwest) was generated according to published formant values. The vowels were categorized into three groups (near, medium, far) according to Euclidean distance in an F1 by F2 space relative to Pacific Northwest (PNW) vowels. Based on several experimental factors, two vowel pairs representing the high back and low front vowels were chosen as stimuli. The near, medium and far exemplars and PNW exemplars were presented to native PNW listeners in a forced-choice identification task. Dependent variables were identification accuracy and reaction time. Task difficulty was manipulated using background noise level. Results indicate that recognition scores were poorer, and reaction time was longer, for vowels that were spec- trally distant from the listener's regional accent. These data suggest that researchers should take into account regional accent in selecting vowel stimuli or generating synthetic vowels for general-use speech perception tests. Supported by NIDCD (DC00033, RO1 DC006014).

5aPPf6. Factors affecting recognition of vocoded speech: Effect of envelope cutoff frequency and carrier type. Pamela Souza (University of Washington, 1417 NE 42nd Street, Seattle, WA 98105, USA, psouza@u.washington.edu), Stuart Rosen (UCL, Wolfson House, 4, Stephenson Way, NW12HE London, UK, stuart@phon.ucl.ac.uk)

Multiband vocoded signals are used to study temporal cues as well as to simulate information available to cochlear implant wearers, but different implementations of these signals may affect results. This series of experiments varied envelope cutoff frequency $(30$ or $300 \mathrm{~Hz}$ ), carrier type (tone or noise) and number of frequency bands (2-5) for vocoded speech presented to normal-hearing subjects. Dependent measures were consonant recognition (vowel-consonant-vowel syllables), vowel recognition (/b/-vowel-/d/ words), and sentence recognition. Results indicated better performance with a high envelope cutoff for tone-vocoded signals, and no effect of envelope cutoff for noise-vocoded signals. When only low-frequency envelope cues were available, performance was better for noise-vocoded than for tonevocoded signals. When higher-frequency envelope cues were available, performance was better for tone-vocoded than for noise-vocoded signals. To understand the source of the observed differences, a follow-up study tested the relative contributions of comodulation across bands (as in the 30- and $300-\mathrm{Hz}$ tone-vocoded conditions) and spectral sidebands (as in the $300-\mathrm{Hz}$ tone-vocoded conditions). Those data indicated that comodulation across bands did not contribute to performance. The presence of sidebands which provided more spectral detail improved performance over a sparser spectrum. [Supported by the Bloedel Hearing Research Center and NIH RO1 DC006014.]

5aPPf7. When and how disyllables are contracted into monosyllables in Taiwan Mandarin? Chierh Cheng (University College London, Department of Phonetics and Linguistics, Gower Street, WC1E 6BT London, UK, chiacheng.f186@nctu.edu.tw), Yi Xu (University College London, Wolfson House, 4 Stephenson Way, NW1 2HE London, UK, yi.xu@ucl.ac.uk)

In Taiwan Mandarin, a disyllabic word or phrase is often contracted into a monosyllabic unit in conversational speech, just as "do not" is often contracted into "don't" in English. The goal of this research is to a) test the hypothesis that contraction is directly related to time pressure, and so it can be elicited in the laboratory from nonsense disyllabic units, and b) search for the underlying mechanisms of contraction. We devised four groups of nonsense disyllabic combinations according to the level of obstruction in the intervocalic consonant: (1) zero obstruction $-\mathrm{CV}+\mathrm{V} ; \mathrm{CV}+\mathrm{VN}$; $\mathrm{CV}+\mathrm{VV}$, (2) nasal consonant - $\mathrm{CVN}+\mathrm{V}$; $\mathrm{CV}+\mathrm{NV}$, (3) non-nasal consonant $\mathrm{CV}+\mathrm{CV}$, where $\mathrm{C}$ is a stop, fricative or affricate, and 4) nasal + non-nasal consonant-CVN $+\mathrm{CV}$. These disyllabic units were embedded in carriers that would impose different degrees of time pressure on them. Four speakers of Taiwan Mandarin read aloud these sentences at three articulatory modes: clear, natural and fast. Preliminary examinations showed that contraction occurred more frequently as speech rate increased and as intervocalic obstruction decreased. Detailed acoustic analyses are being conducted to search for evidence of the underlying mechanism of such contraction, and the results will be reported.

5aPPf8. Estimation model for the speech-quality dimension "Noisiness." Lu Huo (Institute for Circuit and System Theory, ChristianAlbrechts-University of Kiel, Kaiserstrasse 2, 24143 Kiel, Germany, lhu@tf.uni-kiel.de), Marcel Wältermann (Deutsche Telekom Laboratories, Berlin Institute of Technology, Ernst-Reuter-Platz 7, 10587 Berlin, Germany, marcel.waeltermann@telekom.de), Ulrich Heute (Institute for Circuit and System Theory, Christian-Albrechts-University of Kiel, Kaiserstrasse 2, 24143 Kiel, Germany, uh@tf.uni-kiel.de), Sebastian 
Möller(Deutsche Telekom Laboratories, Berlin Institute of Technology, Ernst-Reuter-Platz 7, 10587 Berlin, Germany, sebastian.moeller@telekom .de)

State-of-the-art assessment method of speech-transmission quality (e.g., PESQ or TOSQA) predict the mean-opinion score (MOS) quite accurately, but cannot provide diagnostic information, which is, however, highly desirable for system developers. In our research project, we aim at the development of an attribute-based speech-quality measure, which provides estimates of different attributes of speech samples and then maps them to one integralquality estimate. Three dominant, mutually orthogonal perceptual dimensions were firstly identified by auditory experiments and multidimensional analysis (MDA) for narrow-band speech transmission: "directness/ frequency content," "continuity," and "noisiness." The present paper focuses on the further decomposition and measurement of the global dimension "Noisiness." Therefore, an auditory test including samples degraded by different kinds of noises has been conducted. The subsequent MDA indicates that at least two sub-dimensions (SD), "Speech Contamination" and (perceived) "Additive-Noise Level," are further describing the global dimension "Noisiness." The first SD characterizes the degree the noise distorts the speech signal as such, whereas the second SD reflects the degree the additive circuit or background noise itself annoys the listener. The instrumental estimation methods for both SDs and the mapping to the integralquality ratings are presented in this paper.

5aPPf9. Across-ear grouping of speech bands in quiet and in the presence of interference. Erol Ozmeral (Boston University Hearing Research Center, 677 Beacon Street, Boston, MA 02215, USA, ozmeral@bu.edu), Virginia Best (Univ. of Sydney, Dept. of Physiology, NSW 2006 Sydney, Australia, ginbest@physiol.usyd.edu.au), Jaime McCoin (Boston University Hearing Research Center, 677 Beacon Street, Boston, MA 02215, USA, jmsushigirl@gmail.com), Barbara Shinn-Cunningham (Boston University Hearing Research Center, 677 Beacon Street, Boston, MA 02215, USA, shinn@cns.bu.edu)

Speech was filtered into 16 frequency bands. Three bands were randomly selected on each trial as the LOW (two low-frequency bands) and HIGH (one high-frequency band) target components. LOW and HIGH were either presented to the same (SAME) or opposite (SPLIT) ears. Four randomly selected, nonoverlapping frequency bands from a different utterance were time reversed, and summed. When present, this masker was either played to the same ear as LOW and HIGH (SAME-MASK), same ear as LOW with HIGH opposite (SPLIT-MASKLOW), or same ear as HIGH with LOW opposite (SPLIT-MASKHIGH). Intelligibility was best in the SAME and SPLIT conditions and much better than with either LOW or HIGH presented alone. Thus, in quiet, listeners can perceptually integrate information across target components with little spectro-temporal correlation, even when spatial cues promote segregation of these components. Performance in the SAME-MASK condition was generally better than either SPLITMASKLOW or SPLIT-MASKHIGH conditions (although worse than in SAME and SPLIT conditions). Furthermore, performance in the two SPLITMASK conditions was comparable to performance with LOW or HIGH alone. Thus, when spatial cues oppose grouping of the target and there is a competing off-frequency interference, listeners have difficulty integrating information across target components.

5aPPf10. The role of consonant duration and amplitude processing on speech intelligibility in noise. Jeffrey J. Digiovanni (Ohio University, W218 Grover Center, Athens, OH 45701, USA, digiovan@ohio.edu), Ashley K. Stover (Ohio University, W218 Grover Center, Athens, OH 45701, USA, as357803@ ohio.edu)

The effects of hearing loss on speech intelligibility have led researchers to search for methods to ameliorate these effects. Clear speech has been studied extensively to determine the specific acoustic and phonetic differences between speech spoken in a conversational versus a clear-speech mode. Two characteristics of clear speech, increased consonant duration and selective-consonant amplification were investigated. These components were applied individually to Hearing-in-Noise Test sentences and consonant-vowel $(\mathrm{CV})$ pairs. Stimuli were presented in sound field to hearing-impaired and normal-hearing individuals in the presence of speech- shaped noise. Percent correct was measured for HINT sentences and confusion matrices were developed for the CV pair intelligibility tests. An information transmission analysis was performed. During HINT sentence presentations, subjects benefited most from lengthening the duration of the consonant, maximally in the $30 \mathrm{~ms}$ condition, beyond which significant degradation in intelligibility was observed. Consonant specific amplification of $10 \mathrm{~dB}$ resulted in improved intelligibility for sentences. These data suggest that the two processing components are viable to improve speech intelligibility and they, however, also suggest that there is a limit to the processing whereby benefits are no longer observed.

5aPPf11. The role of combined consonant duration and amplitude processing on speech intelligibility in noise. Jeffrey J. Digiovanni (Ohio University, W218 Grover Center, Athens, OH 45701, USA, digiovan@ohio.edu), Jessica A. Wolfanger (Ohio University, W218 Grover Center, Athens, OH 45701, USA, jw241702@ ohio.edu)

Reduced speech intelligibility in noise, especially consonant perception, is a well documented phenomenon. Research in clear speech has revealed many acoustic and phonetic differences between speech spoken in a conversational versus clear-speech mode. In the present study two components were tested in combination, namely increases in consonant duration (and respective decreases in adjacent vowel duration) and selective-consonant amplification. Conditions in which intelligibility improvements were observed for the individual processing of duration and amplitude modifications were chosen and tested in combination. The components were applied to Hearing-in-Noise Test sentences and consonant-vowel (CV) pairs. Stimuli were presented in sound field to normal-hearing individuals in the presence of speech-shaped noise. Percent correct was measured for HINT sentences and confusion matrices were developed for the $\mathrm{CV}$ pair intelligibility tests. An information transmission analysis was performed. Results will be discussed with regard to the greater body of research in clear speech.

5aPPf12. Effect of amplification on the intelligibility of speech in hearing impaired children with and without dead regions in the cochlea. Alicja N. Malicka (The University of Manchester, School of Psychological Sciences, Ellen Wilkinson Building, M13 9PL Manchester, UK, alicja.malicka@manchester.ac.uk), Kevin J. Munro (The University of Manchester, School of Psychological Sciences, Ellen Wilkinson Building, M13 9PL Manchester, UK, kevin.j.munro@manchester.ac.uk), Thomas Baer (University of Cambridge, Department of Experimental Psychology, Downing Street, CB2 3EB Cambridge, UK, tb107@cus.cam.ac.uk)

Adults with high-frequency (HF) sensorineural hearing impairment with and without dead regions (DRs) in the cochlea differ in benefit from amplification of speech presented in quiet [Vickers et al., J. Acoust. Soc. Am. 110, 1164-1175 (2001)]. Subjects with HF DRs showed no improvement in speech intelligibility when spectral components of the speech above about 1.7 times the edge frequency of the DR were amplified according to a hearing-aid prescription formula while performance of those without DRs showed improvement with addition of amplified frequency components up to $7.5 \mathrm{kHz}$. In the present study we tested a group of six children (8-12 years old) who were experienced hearing-aid users with moderate-to-severe sensorineural hearing impairment. The presence of DRs was diagnosed using the TEN(HL) test and "fast" psychophysical tuning curves. Four children showed evidence for DRs (two unilateral and two bilateral). The vowelconsonant-vowel stimuli (65-dB SPL) were subjected to the frequency-gain characteristic prescribed by the DSL prescription formula then low-pass filtered with various cutoff frequencies and presented via headphones. The results showed that in ears with or without DRs the performance improved with increasing cutoff frequency up to $7.5 \mathrm{kHz}$.

5aPPf13. Acoustic correlates of fricative perception. Shira Katseff (University of California, Department of Linguistics, 1203 Dwinelle Hall, Berkeley, CA 94720, USA, skatseff@berkeley.edu)

A number of spectral attributes have been proposed as correlates of fricative place of articulation. However, these attributes have difficulty distinguishing fricatives in noncanonical speech, e.g., from children. Because child speech is intelligible, it is possible that some of these characteristics 
are not used in speech perception. This study collected a corpus of /s/ and /S/-containing words from children between 3 and 5. Adults categorized fricatives extracted from this corpus. A series of regressions are used to compare the ability of spectral attributes to predict (1) human categorization of /s/ and /S/ productions, and (2) the intended fricative production. The analysis reveals that mistakes in perception are driven by overuse of a single spectral attribute, spectral slope. This result suggests caution in equating methods of computational separation with methods of human perception, and provides a simple method for investigating relationships between the two.

FRIDAY MORNING, 4 JULY 2008

P2-C, LEVEL 2, 9:40 TO 11:20 A.M.

\title{
Session 5aPPg
}

\section{Psychological and Physiological Acoustics: Cross-spectral Auditory Integration: Physiological, Psychophysical, and Clinical Evidence II (Poster Session)}

\author{
Blas Espinoza-Varas, Cochair \\ CommunicationSciences \& Disorders, OU Health Sciences Ctr. \\ Alain De Cheveigne, Cochair \\ CNRS, Universite Paris 5, Ecole Normale Superieure
}

\begin{abstract}
All posters will be on display from 9:40 a.m. to 11:20 a.m. To allow contributors an opportunity to see other posters, contributors of odd-numbered papers will be at their posters from 9:40 a.m. to 10:30 a.m. and contributors of even-numbered papers will be at their posters from 10:30 a.m. to 11:20 a.m.
\end{abstract}

\section{Contributed Papers}

5aPPg1. Stimulus effects on spectral shape discrimination. Mini N. Shrivastav (University of Florida, 336 Dauer Hall, Dept. of Communication Sciences and Disorders, Gainesville, FL 32611, USA, mnarendr@csd.ufl.edu), David A. Eddins (University of Rochester, Department of Otolaryngology, Rochester, NY 14618, USA, David_Eddins @URMC.Rochester.edu)

For a single stimulus such as shaped broadband noise, spectral-shape discrimination can vary based on the nature of the spectral feature that is being detected. For example, Shrivastav et al. (2006) found a significant difference in thresholds for broadband noise with a narrow localized spectral peak and those with a broad overall spectral change. They also found a moderate association between discrimination thresholds and speechidentification scores in older hearing-impaired listeners, but only for the stimuli with a narrow spectral peak. It is not clear whether these stimulusdependent effects were associated with the bandwidth of the spectral peak, the slope of the peak, or a combination of these features. In the present study, spectral shape discrimination thresholds and speech recognition scores will be measured for ten young normal-hearing and ten older hearingimpaired listeners. The broadband noise will vary systematically in the bandwidth and the slope of a single spectral peak. The results will determine if and how spectral-shape perception changes with the nature of the stimulus paradigm used to measure it. Further, the results will also indicate if the association between spectral shape perception and speech identification is observed for all stimuli or only to a subset.

5aPPg2. Resolution, spectral weighting, and integration of information across tonotopically remote cochlear regions: hearing-sensitivity, sensation level, and training effects. Blas Espinoza -Varas (CommunicationSciences \& Disorders, OU Health Sciences Ctr., 825 NE 14th St., Oklahoma City, OK 73126-0901, USA, blas-espinozavaras@ouhsc.edu)

This paper examines how listeners resolve, weight, and integrate redundant sensory information from tonotopically remote cochlear regions, one in the mid $(\mathrm{m})$ the other in the high $(\mathrm{h})$ frequencies. Subjects listened to twocomponent complexes and attempted to resolve and integrate simultaneous differences in frequency (DFm) at $\approx 1000 \mathrm{~Hz}$ and in duration (DTh) at $\approx 3500 \mathrm{~Hz}$. Discrimination performance was studied as a function of hearing sensitivity, sensation level (SL) and training. Normal hearing listeners tend to resolve both DFm and DTh and integrate the information if the component SLs are equal, but resolve only the louder component difference if the SLs are very unequal; i.e., spectral weighting is biased and integration is limited or nil. Much the same pattern obtains when the low SL in H results from high-frequency impaired sensitivity (IS). Once established, equating the component SLs (e.g., by high-frequency amplification) is often ineffective to rectify the weighting bias; doing so requires extensive discrimination training with the low SL component. With IS, an anomalous trend obtains when the SL is much lower for (m) than for (h): the resolution of DFm is equal to or better than that of DTh, and integration is quite efficient, possibly reflecting tonotopic map reorganization.

5aPPg3. Pitch discrimination of simultaneous and nonsimultaneous complexes across spectral regions. Elizabeth Borchert (University of Minnesota, Department of Psychology, 75 E. River Road, Elliott Hall N218, Minneapolis, MN 55455, USA, olsen064@umn.edu), Andrew J. Oxenham (University of Minnesota, Department of Psychology, 75 E. River Road, Elliott Hall N218, Minneapolis, MN 55455, USA, oxenham@umn.edu), Christophe Micheyl (University of Minnesota, Department of Psychology, 75 E. River Road, Elliott Hall N218, Minneapolis, MN 55455, USA, cmicheyl@umn.edu)

Detecting differences in fundamental frequency (F0) across different spectral regions may be important in the perceptual organization of both simultaneous and sequential sounds, but it is unknown whether the same mechanisms are involved in these two cases. Here we directly compare the detection of pitch differences between pairs of complex tones presented either simultaneously or sequentially. 28 normal-hearing listeners heard two pairs of complex tones in each trial and indicated the pair in which the pitches of the two tones differed. The complex tones in each pair were bandpass filtered to have nonoverlapping spectra, but at least some resolved harmonics in all cases. Performance was generally better in the simultaneous than in the sequential condition. In a follow-up condition, it was found that introducing an onset asynchrony between the two spectral regions in the si- 
multaneous condition resulted in impaired performance, approaching that found in the sequential case, despite the duration of the simultaneous portion remaining the same. The results suggest that perceived fusion, rather than an explicit F0 extraction and comparison may underlie the detection of F0 differences between simultaneous groups of harmonics in distinct spectral regions. [Work supported by NIH grant R01 DC 05216.]

5aPPg4. Across-frequency processes involved in auditory detection of coloration. Jörg M. Buchholz (CAHR, Department of Electrical Engineering, DTU, Ørsteds Plads, Bygning 352, 2800 Kgs. Lyngby, Denmark, jb@oersted.dtu.dk), Paris Kerketsos (CAHR, Department of Electrical Engineering, DTU, Ørsteds Plads, Bygning 352, 2800 Kgs. Lyngby, Denmark, paris_kerketsos@hotmail.com)

When an early wall reflection is added to a direct sound, a spectral modulation is introduced to the signal's power spectrum. This spectral modulation typically produces an auditory sensation of coloration or pitch. Throughout this study, auditory spectral-integration effects involved in coloration detection are investigated. Coloration detection thresholds were therefore measured as a function of reflection delay and stimulus bandwidth. In order to investigate the involved auditory mechanisms, an auditory model was employed that was conceptually similar to the peripheral weighting model [Yost, JASA, 1982, 416-425]. When a "classical" gammatone filterbank was applied within this spectrum-based model, the model largely underestimated human performance at high signal frequencies. However, this limitation could be resolved by employing an auditory filterbank with narrower filters. This novel filterbank was designed to approximate auditory filter-shapes measured by Oxenham and Shera [JARO, 2003, 541-554], derived from forward masking data. The results of the present study demonstrate that a "purely" spectrum-based model approach can successfully describe auditory coloration detection even at high signal frequencies.

5aPPg5. Phoneme recognition as a function of the number of auditory filter outputs. Frederic Apoux (University of South Carolina, Speech Psychoacoustics Laboratory, Department of Communication Sciences and Disorders, William Brice Bldg., 1621 Greene St., Columbia, SC 29208, USA, apoux@sc.edu), Eric W. Healy (University of South Carolina, Speech Psychoacoustics Laboratory, Department of Communication Sciences and Disorders, William Brice Bldg., 1621 Greene St., Columbia, SC 29208, USA, ewh@sc.edu)

It has been proposed that listeners take advantage of brief "coups d'oeil" when processing speech in noise. These glimpses can be characterized both in time and frequency. The obligatory role of the auditory filters in determining the nature of any further processing suggests that the frequency extent of a glimpse should be equivalent to that of an auditory filter. Accordingly, it is hypothesized that the spectral characteristics of glimpses primarily relate to the available number of auditory channels. The present study investigated the number of auditory filter outputs needed to identify phonemes in quiet. Stimuli were first restricted to $80-8000 \mathrm{~Hz}$ and then split into 30 contiguous auditory filter width bands. Normal-hearing listeners were presented with $\mathrm{N}$ bands whose spectral location was selected randomly from trial to trial. No signal was presented in the other bands. Consistent with previous studies, performance gradually increased with the number of bands. An asymptote was reached with 24 and 16 bands for vowels and consonants, respectively. While high levels of speech understanding are typically observed with as few as 4 channels of spectral information, our results indicate that accurate phoneme recognition requires combination of a much larger number of auditory filter outputs.

\title{
Session 5aSAa
}

\section{Structural Acoustics and Vibration, ASA Committee on Standards, and EURONOISE: Ground Vehicle Noise and Vibration I}

\author{
Donald B. Bliss, Cochair \\ Duke University, Mechanical Engineering and Materials Science, 148B Hudson Hall, Durham, NC 27708, USA \\ Paul De Vos, Cochair \\ DHV BV, Postbus 1132, Amersfoort, NL 3800 BC, Netherlands
}

Invited Papers

8:00

5aSAa1. Contribution of the tyre to further lowering tyre/road noise. Ernst-Ulrich Saemann (Continental AG, Jaedekamp 30, 30419 Hannover, Germany, Ernst-Ulrich.Saemann@ conti.de)

The tyre is the only part of a vehicle that must be originally and primarily designed to transmit forces to outside the vehicle. At the same time the amount of energy a tyre consumes per kilometre in operation and for production has to be minimized. On dry, wet, and snow-covered roads, the safety of traffic can only be ensured by a sufficient capability for acceleration and braking deceleration, cornering stability, tracking on acceleration and braking, steering precision and direction stability. The high requirements on safety, economy, and durability limit the possible reduction in the emission of tyre/road noise. Nevertheless, nowadays patterned tires can be constructed which are not more than 1-3 dB(A) louder than smooth tires. As a further reduction of tire excitation by tread pattern optimization cannot be expected and a soft tread compound cannot be used due to the customer's requirements, the only possibility to build tyres with less sound radiation is to lower the noise already from the blank tyre. Considerably more tyre/road noise reduction can be gained if the excitation by road is addressed, too. 
5aSAa2. Prediction and audio synthesis of vehicle pass-by noise. Estelle Bongini (SNCF DIR, 45, rue de Londres, 75379 Paris, France, estelle.bongini@sncf.fr), Stephane Molla (Genesis S.A., Bâtiment Gérard Mégie, Domaine du Petit Arbois - BP 69, 13545 Aix-en-Provence Cedex 4, France, stephane.molla@genesis.fr), Cédric Herviou (SNCF DIR, 45, rue de Londres, 75379 Paris, France, cedric.herviou@gmail.com), Dominique Habault (LMA CNRS, 31 chemin Joseph Aiguier, 13402 Marseille, France, habault@1ma.cnrs-mrs.fr), Franck Poisson (SNCF DIR, 45, rue de Londres, 75379 Paris, France, franck.poisson@sncf.fr)

The European project SILENCE is dedicated to the reduction of railway and roadway noise in urban areas. Within this context, SNCF and LMA (Laboratoire de mécanique et d'acoustique, Marseille) collaborate into the subproject B to develop a pass-by sound simulation software. This global modelling tool will support parametric studies on the reduction of the noise of a train or a car pass-by, by providing standard indicators (time signature, equivalent sound pressure level) and sound samples. These sound samples are computed in B-Format, which allows, after a short postprocessing, to provide various listening formats (mono-aural, binaural, œ). The software is now operational and the sources characterisation has been carried out on an AGC bi-mode BOMBARDIER train and a passenger car. Equivalent sources have been defined in various operating conditions. Simulated pass-by indicators (signature, Leqtp(A)) in various configurations have been compared to measured one and show very good agreement. A perceptive validation has been carried out with listening tests, by indirectly comparing recorded samples corpus and corresponding simulated samples corpus. It shows that the arrangement made by the listeners of the simulated corpus and the arrangement of the recorded corpus are closely the same and are based on the same perceptive criterion.

\section{Contributed Papers}

\section{8:40}

5aSAa3. Dependence of the contact area on the velocity of a rolling tire. Hong Hai Nguyen (ENPC, UR Navier, 6 et 8 Avenue Blaise Pascal, Cité Descartes, Champs sur Marne, 77455 Marne la Vallée, France, nguyen.hong-hai@lami.enpc.fr), Julien Cesbron (Université d’Evry - Val d'Essonne, Laboratoire de Mécanique d'Evry, EA3332, 40, rue du Pelvoux, 91020 Evry Cedex, France, cesbron.julien@neuf.fr), Fabienne Anfosso-Ledee (Laboratoire Central des Ponts et Chaussées, BP 4129, 44341 Bouguenais Cedex, France, fabienne.anfosso@lcpc.fr), Hai Ping Yin (ENPC, UR Navier, 6 et 8 Avenue Blaise Pascal, Cité Descartes, Champs sur Marne, 77455 Marne la Vallée, France, yin@lami.enpc.fr), Silvano Erlicher (Université Paris-Est, 6 et 8 avenue Blaise Pascal, Cité Descartes - Champs sur Marne, 77455 Marne la Vallée Cedex 2, France, erlicher@lami.enpc.fr), Denis Duhamel (ENPC, UR Navier, 6 et 8 Avenue Blaise Pascal, Cité Descartes, Champs sur Marne, 77455 Marne la Vallée, France, duhamel@lami.enpc.fr)

It is known that the eigenfrequencies of a rolling tire depend on the velocity of rotation. We distinguish two causes for the stiffness increase: the frequency dependence of the complex modulus of the materials and the geometrical stiffness. The real part of the Young's modulus is monotonic according to the frequency. It contributes for an important part to the stiffening. The geometrical stiffness also increases with the rotational velocity. A consequence of these effects is the modification of the size of the contact area for different velocities of a rolling tire. Here we first present experimental results estimating the size of the contact area for a tire in statics and for different rolling velocities. Differences of $20 \%$ can be observed. Then the viscoelastic behaviours of the tire materials are presented and experimental results showing the frequency dependence of the complex modulus of the tire constitutive materials are given. Then finite element computations are presented with a real distribution of materials in the tire section and the size of the contact area is estimated and compared to experimental measurements. These results could improve the modelling of tire road interaction for tire noise predictions.

\section{9:00}

5aSAa4. Deploying successfully Laser Doppler Vibrometry techniques within the automotive NVH process. Mehdi Batel (Polytec, 32 rue Delizy, 93694 Pantin Cedex, France, 1.korn@ polytec.fr)

Laser Doppler Vibrometry is becoming today an established technique and the gold-standard for noncontact measurements in the automotive industry. These methodologies are widely used to characterize the mechani- cal behaviour of structures in vibration or strain, with always zero mass loading. They provide high precision results with very low operating costs as well as optimized setup times. The applications of laser vibrometry in the automotive industry are extremely various, and are helping today automotive professionals to improve dramatically the NVH performance of components and full vehicles. This paper explains how these methodologies are fitting today in the everyday challenge of automotive NVH Test \& CAE engineers: providing best in class noise and vibration quality with mass reductions constraints. A new laser scanning technique including the use of a $3 \mathrm{D}$ robot is also presented, providing an innovative way to characterize the dynamics of body structures with very high precision and rapidity. The paper will present a measurement performed on a full vehicle (43 robot positions done in $1 \mathrm{~h} 30$ hour), with 1094 points of vibration data at $1 \mathrm{~Hz}$ resolution.

\section{9:20}

5aSAa5. Application of digital speckle interferometry for vibration analysis of a statically loaded vehicle tyre. Dan Borza (National Institute of Applied Sciences of Rouen, INSA Rouen, LMR, Ave l'Universite, BP8 76800 Rouen, France, dan.borza@insa-rouen.fr), Ioana Nistea (Institut National des Sciences Appliquées de Rouen, BP8 avenue de l'Université, 76801 Saint-Etienne du Rouvray, France, ioana.nistea@insa-rouen.fr)

Vehicle noise has, as one of its main sources, tyres and their interaction with the road. Experimental analysis of tyre vibroacoustic behaviour may provide useful information on the contributions of various regions of the tyre to the resultant noise. Digital speckle interferometry represents a reliable and efficient choice for vibration analysis, providing full field and real time information on surface displacement. The optical configuration used for this study is a standard out-of-plane sensitive setup, based on a continuous wave YAG laser. A 4 bucket phase stepping algorithm is applied, with the use of a piezo actuator, in order to eliminate the random phase difference between the object and the reference wave. The measurement results are images of the tested tyre, covered with interference fringes, representing contour maps of out of plane vibration amplitudes. This paper presents measurements for the case of a tyre subjected to a point excitation at the limit of the contact surface with the road, under statical load of $4 \mathrm{kN}$. The principal resonant modes and frequencies are presented, then difficulties related to sensibility vector variation over the test surface and coupled mode resonances are discussed. 


\section{Contributed Papers}

\section{1:00}

5aSAa6. An energy-based updated modal approach for the efficient analysis of large trimmed models. Gregory Lielens (Free Field Technologies, Rue Emile Francqui 1 - batiment B - AXIS PARC LLN, 1435 Mont-Saint-Guibert, Belgium, gregory.lielens@fft.be), Benoit Van Den Nieuwenhof (Free Field Technologies, Rue Emile Francqui 1 - batiment B AXIS PARC LLN, 1435 Mont-Saint-Guibert, Belgium, benoit.vandennieuwenhof@fft.be), Fabien Acher (Free Field Technologies, Rue Emile Francqui 1 - batiment B - AXIS PARC LLN, 1435 Mont-SaintGuibert, Belgium, fabien.acher@fft.be), Jean-Pierre Coyette (Free Field Technologies, Rue Emile Francqui 1 - batiment B - AXIS PARC LLN, 1435 Mont-Saint-Guibert, Belgium, jean-pierre.coyette@fft.be)

Large trimmed models, widely encountered in the automotive industry, generally involve a body-in-white structure coupled to an acoustic cavity and covered by a set of trim components. Due to the large number of degrees of freedom of such models, the direct analysis in physical coordinates, though theoretically correct, is not feasible on current computers. The paper presents an alternative and efficient solution strategy in modal coordinates that relies on an update of the modal parameters of the car body and the acoustic cavity, based on the energetic behaviour of the trim components. The description of the trim component in terms of an energetic database simplifies the data exchange between the automotive manufactor and the trim provider. Furthermore, it enables a fast frequency analysis of various loadcases/trim scenarios and makes optimisation possible. The updated modal approach implemented in Actran/Trim is applied on a simplified car model on which various trim components are applied. The application shows how the stiffening, added mass and damping effects of each trim component can be derived from the energetic database and can guide an optimisation process.

\section{$11: 20$}

5aSAa7. Critical assessment of operational path analysis: mathematical problems of transmissibility estimation. Peter Gajdatsy (LMS International, Interleuvenlaan 68, 3001 Leuven, Belgium, peter.gajdatsy@1msintl.com), Karl Janssens (LMS International, Interleuvenlaan 68, 3001 Leuven, Belgium, Karl.Janssens@1msintl.com), Ludo Gielen (LMS International, Interleuvenlaan 68, 3001 Leuven, Belgium, ludo.gielen@1ms.be), Peter Mas (LMS International, Interleuvenlaan 68, 3001 Leuven, Belgium, peter.mas@1ms.be), Herman Van Der Auweraer (LMS International, Interleuvenlaan 68, 3001 Leuven, Belgium, Herman .VanderAuweraer@1msintl.com)

Classical transfer path analysis (TPA) is a widely used and reliable method for tackling noise and vibration problems. But due to its complexity and time-consuming measurement procedure there is an ongoing research for simpler and faster methods. One such method, most often referred to as operational path analysis (OPA), was presented two years ago and has quickly gained popularity in the NVH field for its speed and ease of use. To estimate path contributions transmissibilities are calculated from the measured operational reference and target accelerations and sound pressures. This new method is now critically examined and compared to a reliable classical TPA measurement. The results of this examination reveal three significant weaknesses. This paper focuses on the problems related to the estimation of transmissibilities which mostly arise from the limited amount of orders present in the signal and the coherence between inputs. It is shown that despite the advantages of the method, it is not applicable in many situations and has to be used with care for it can easily give misleading results.

\section{1:40}

5aSAa8. IC engine velocity fields, spherical harmonics and evanescent waves. Igor Machetta (Centro Ricerche FIAT, Strada Torino, 50, 10043 Orbassano (TO), Italy, igor.machetta@fptpowertrain.crf.it), Eugenius Nijman (Centro Ricerche FIAT, Strada Torino, 50, 10043 Orbassano (TO), Italy, eugenius.nijman@fptpowertrain.crf.it)

Spherical nearfield acoustical holography (SNAH) has been used to reconstruct the volume acceleration distribution close to the surface of an IC engine from the measured sound pressure distribution on a sphere enclosing the engine. Such reconstruction frequently requires the calculated target spheres to intercept the strongly nonspherical powertrain structure violating, at first sight, the conventional rules of SNAH. In this paper the SNAH results are compared with the results of two alternative, i.e., an inverse and a power based, source reconstruction methods. Notwithstanding the alleged violation of the rules it is shown that surprisingly good results may be obtained provided the spherical spectrum components associated with evanescent waves are omitted from the back propagation calculation.

\section{2:00}

5aSAa9. Influence of bolted items on modal analysis performed on a car body. Miguel Colomo (Universidad Politécnica de Madrid, Avda. Constitución $\mathrm{n}^{\circ} 621^{\circ} \mathrm{D}, 28931$ Móstoles, Spain, miguelcolomo @ gmail.com), Andrzej Pietrzyk (Volvo Car Corporation, NVH CAE Noise \& Vibration Center Dept 91620, PV2C2, 40531 Gothenburg, Sweden, apietrzy@volvocars.com), Wolfgang Kropp (Chalmers University of Technology, Division of Applied Acoustics, SE-41296 Gothenburg, Sweden, wolfgang.kropp@chalmers.se)

When performing Modal Analysis testing on a BIG (body-in-gray), some bolted items are included to better take into account their influence on body stiffness. However, their contribution to the stiffness is not relevant in the frequency range accessible for modal analysis (usually up to $70 \mathrm{~Hz}$ on a BIG). On the other hand, these bolted items increase the dispersion between results obtained for nominally identical test objects. The question which arises is whether the items should be included in the BIG definition to perform modal analysis and, in this case, which is their influence on the results? MIMO (multi-input-multi-output) measurements were carried out over three, nominally identical, Volvo S80 BIGs. Several configurations were measured for each BIG, starting from the complete body the bolted items were progressively removed. A version of LMS PolyMAX method was programmed by Matlab to analyze the measured data. Conclusions about bolted items influence are drawn based on the study of stabilization diagrams and modal parameters. The poles selection by the stabilization diagrams is one of PolyMAX method keys. The method understanding obtained from programming allows studying the ins and outs of poles selection. Polynomial order plays an important role in physical poles identification, especially for closely spaced modes. Results are shown to highlight its relevance.

\section{2:20}

5aSAa10. Analysis of structure borne transmission in a railway vehicle using Energy Flow Method. Pascal Bouvet (Vibratec, 28 Chemin du Petit Bois, BP 36, 69131 Ecully Cedex, France, pascal.bouvet@vibratec.fr)

The Energy Flow Method (EFM) is a computationally efficient method, by which the results of a Finite Element Analysis (FEA) may be postprocessed to form energy flow models. It can be used to study structure borne transmission in structures, based on modal descriptions of the structure and the internal cavity. The EFM allows to form a Energy Influence Coefficient model (EIC) or an inverse SEA model. A first issue consists in partitioning the FE model into SEA subsystems, with respect to the SEA assumptions (particularly the weak coupling assumptions). An automatic technique based 
on cluster analysis, can be used to determine an appropriate SEA substructuring, before calculating the Coupling Loss Factors (CLF) between mechanical subsystem. A second issue is to calculate the CLF between structural and acoustic subsystems. This paper presents an industrial application of these techniques, for the analysis of structure borne transmission inside a tramway vehicle. The EFM technique is used to calculate the vibroacoustic transfer of the car body of the tramway, and comparison with measurements on a vehicle are presented.

\title{
Session 5aSAb
}

\section{Structural Acoustics and Vibration and EURONOISE: Active Noise Control: New Strategies and Innovative Concepts I}

\author{
Alain Berry, Cochair \\ Univ. de Sherbrooke, Mechanical Engineering Depart., 2500 Boulevard de l'Université, Sherbrooke, QC J1K 2R1, Canada \\ Marie-Annick Galland, Cochair \\ Centre Acoustique du LMFA, Ecole Centrale de Lyon, 36 avenue Guy de Collongue, Ecully cedex, 69134, France
}

\section{Invited Papers}

8:00

\begin{abstract}
5aSAb1. Active structural-acoustic control of laminated cylindrical panels using vertically/obliquely reinforced 1-3 piezoelectric composite. Manas C. Ray (Indian Institute of Technology, Mechanical Engineering Department, 721302 Kharagpur, India, mcray @mech.iitkgp.ernet.in)

The performance of active constrained layer damping (ACLD) treatment for active structural-acoustic control of vibrating thin laminated cylindrical panels has been investigated. The constraining layer of the ACLD treatment has been considered to be made of vertically/obliquely reinforced 1-3 piezoelectric composite material. A finite element model has been developed for the laminated panels integrated with the patches of ACLD treatment to describe the coupled structural-acoustic behavior of the panels enclosing an acoustic cavity. Both velocity and pressure rate feedback controls have been implemented to activate the patches. Symmetric and antisymmetric cross-ply and antisymmetric angle-ply panels have been considered for evaluating the numerical results. Emphasis has also been placed on investigating the effect of the piezoelectric fiber orientation in the constraining layer and the shallowness angle of the cylindrical panels on the performance of the patches.
\end{abstract}

\section{8:20}

5aSAb2. Decentralized control of sound radiation from an aircraft-style panel using iterative loop recovery. Noah H. Schiller (NASA Langley Research Center, 2 North Dryden Street, Hampton, VA 23681, USA, noah.h.schiller@ nasa.gov), Randolph H. Cabell (NASA Langley Research Center, 2 North Dryden Street, Hampton, VA 23681, USA, randolph.h.cabell@nasa.gov), Chris R. Fuller (Virginia Tech, 131 Durham Hall, Blacksburg, VA 24061, USA, christopher.r.fuller@nasa.gov)

A decentralized LQG-based control strategy is designed to reduce low-frequency sound transmission through periodically stiffened panels. While modern control strategies have been used to reduce sound radiation from relatively simple structural acoustic systems, significant implementation issues have to be addressed before these control strategies can be extended to large systems such as the fuselage of an aircraft. For instance, centralized approaches typically require a high level of connectivity and are computationally intensive, while decentralized strategies face stability problems caused by the unmodeled interaction between neighboring control units. Since accurate uncertainty bounds are not known a priori, it is difficult to ensure the decentralized control system will be robust without making the controller overly conservative. Therefore, an iterative approach is suggested, which utilizes frequency-shaped loop recovery. The approach accounts for modeling error introduced by neighboring control loops, requires no communication between subsystems, and is relatively simple. The control strategy is validated using real-time control experiments performed on a built-up aluminum test structure representative of the fuselage of an aircraft. Experiments demonstrate that the iterative approach is capable of achieving $12 \mathrm{~dB}$ peak reductions and a $3.6 \mathrm{~dB}$ integrated reduction in radiated sound power from the stiffened panel.

\section{8:40}

5aSAb3. Experimental tests on smart panels for the reduction of sound radiation. Paolo Gardonio (ISVR, University of Southampton, Highfield, SO17 1BJ Southampton, UK, pg@isvr.soton.ac.uk), Cristobal Gonzalez Diaz (ISVR, University of Southampton, Highfield, SO17 1BJ Southampton, UK, cgd@isvr.soton.ac.uk), Neven Alujevic (ISVR, University of Southampton, Highfield, SO17 1BJ Southampton, UK, na2@isvr.soton.ac.uk), Yohko Aoki (ISVR, University of Southampton, Highfield, SO17 1BJ Southampton, UK, ya@isvr.soton.ac.uk)

This paper presents a comparative experimental study carried out on four types of smart panels for the reduction of sound radiation. The four panels are equipped with decentralized velocity feedback control units that are designed to generate active damping. In this way the frequency averaged response and sound radiation produced by stochastic disturbances can be effectively reduced at low audio 
frequencies. The first smart panel is composed of a $4 \times 4$ array of square piezoelectric patch actuators with accelerometer sensors at their centres. The second smart panel is composed of sixteen triangularly shaped piezoelectric patch actuators with base edges evenly distributed along the perimeter of the panel and accelerometer sensors located on the top vertices of the actuators. The third panel is composed of five electrodynamic inertial actuators with accelerometer sensors located under their footprints. The fourth panel is equipped with a light honeycomb trim panel mounted on four stiff mounts. A set of nine reactive electrodynamic actuators is located in the shallow cavity between the two panels. Accelerometer sensors are mounted at the two footprints of each actuator. The sensoractuator pairs mounted in the four panels are used to implement analogue local feedback loops.

\section{9:00}

5aSAb4. Extremal harmonic active control of power. Philippe Micheau (Univ. de Sherbrooke, Mechanical Engineering Depart., 2500 Boulevard de l'Université, Sherbrooke, QC J1K 2R1, Canada, Philippe.Micheau@USherbrooke.ca), Jean-Philippe Gauthier (Univ. de Sherbrooke, Mechanical Engineering Depart., 2500 Boulevard de l'Université, Sherbrooke, QC J1K 2R1, Canada, JeanPhilippe.Gauthier@cta-brp-udes.com)

The extremal harmonic active control of power consists to define an energetic criterion at the main harmonic and to extremalize it on-line by commanding a secondary source of power. The term extremal means that the optimal criterion value is reached with an on-line optimization algorithm. Without loss of originality, the context of rotating machines oscillation in synchronization with rotor position is used to illustrate this approach. A second commanded torque source, attached to the shaft, is used to control speed oscillation due to torque disturbance. Such configuration can be found in hybrid automotive for example. The energetic criterion can be the kinetic energy (for conventional active speed oscillation cancelling), the reactive power (to add a virtual flywheel on the shaft) or the active power (for energy harvesting to charge a battery). The optimization algorithm is the method of steepest descent implemented with implicit or explicit gradient computation. But, in the case of reactive power, the implicit controller is not practically achievable. The experimental results with a test bed show that the explicit controllers converge at the extremum of each criterion. To conclude, the presented explicit extremal controller is a good candidate to be used for energetic criterion control.

\section{9:20}

5aSAb5. Control of harmonic distortion in a motor driven subsonic acoustic source. Marty Johnson (Virginia Tech, Mechanical Engineering, 143 Durham 0238, Blacksburg, VA 24061, USA, martyj@vt.edu), Alessandro Toso (Virginia Tech, Mechanical Engineering, 143 Durham 0238, Blacksburg, VA 24061, USA, aletoso@vt.edu), Vincent Blandea (ISVR, University of Southampton, University Road, Highfield, S017 1BJ Southampton, UK, vblandea@gmail.com)

This paper presents a comparison of active control strategies used to reduce the harmonic distortion of a motor driven subsonic source. The source consists of a DC motor that drives a pair of speaker diaphragms through a belt drive system which in turn drives a pair of passive radiators through an acoustic enclosure. The motor drive system is inherently nonlinear with significant losses in force at polarity changes. A number of control strategies were tested both theoretically and experimentally in order to determine the best strategy for linearizing the output from the speaker. The methodologies tested were: (i) an FXLMS based adaptive time domain controller using a linear plant model, (ii) an FXLMS based adaptive frequency domain harmonic controller using a linear plant model, (iii) an FXLMS based adaptive frequency domain harmonic controller using a nonlinear plant model and (iv) a simplex based adaptive frequency domain harmonic controller. The time domain system is shown to suffer from slow adaption rates for the higher harmonics, the model based FXLMS harmonic controllers were shown to suffer from the nonstationarity of the nonlinear plant and the simplex method was shown to provide good results while being robust to slow variations in the plant.

9:40-11:00 Posters

Lecture sessions will recess for presentation of poster papers on various topics in acoustics. See poster sessions for topics and abstracts.

\section{Invited Papers}

\section{1:00}

5aSAb6. Variable area jet nozzle for noise reduction using shape memory alloy actuators. James Mabe (Boeing Phantom Works, M/C 42-51, PO BOX 3707, Seattle, WA 98124-2207, USA, james.h.mabe@boeing.com)

A significant reduction in noise and improved fuel consumption can be achieved by varying the area of a commercial jet engine's fan nozzle. A larger diameter at takeoff and approach can reduce jet velocity reducing noise. Adjusting the diameter in cruise, to account for varying Mach number, altitude, etc, can optimize fan loading and reduce fuel consumption. Boeing recently tested a scaled variable area jet nozzle capable of a $20 \%$ area change. Shape memory alloy actuators were used to position 12 interlocking panels at the nozzle exit. A closed loop control system was used to maintain a range of constant diameters with varying flow conditions and to vary the diameter under constant flow conditions. Acoustic data by side line microphones and flow field measurements at several cross-sections using PIV was collected at each condition. In this paper the variable area nozzle's design is described. The effect of the nozzle's diameter on its acoustic performance is presented for a range of Mach numbers and mass flow rates. Flow field data is shown including the effects of the joints between the interlocking panels. 
5aSAb7. Concept of active reverberation box. Hisaharu Suzuki (Kyushu University, 4-9-1, Shiobaru, Minamiku, 815-8540 Fukuoka, Japan, hisaha@souldsp.jp), Akira Omoto (Kyushu University, 4-9-1, Shiobaru, Minamiku, 815-8540 Fukuoka, Japan, omoto @ design.kyushu-u.ac.jp)

A quite small reverberation box with active controller system is proposed. If we try to measure the characteristics of acoustic materials, such as glass wool, in a small sized enclosure, there are few dominant modes which cause the condition that the incident wave to target material is biased toward the specific direction. As a result, the measured result shows the irregular distribution which depends on measuring point or evaluating method such as the reverberation time reading. The proposed system which utilized the active control enables us to create a plane wave with arbitrary direction in the enclosure. Therefore it has possibilities of solving the problems caused by insufficient incident condition. Also, in this study, the two evaluating strategies that the traditional reverberation time method and the steady state analysis method are concerned.

\section{1:40}

5aSAb8. Active control of engine noise transmitted into cavities: simulation, experimental validation and sound quality assessment. Leopoldo P. De Oliveira (KU Leuven, Celestijnenlaan 300B, Departement Werktuigkunde - PMA, 3001 Leuven, Belgium, leopoldo.deoliveira@mech.kuleuven.be), Paul Sas (KU Leuven, Celestijnenlaan 300B, Departement Werktuigkunde - PMA, 3001 Leuven, Belgium, Paul.Sas@ mech.kuleuven.be), Wim Desmet (K.U.Leuven - Dept. of Mechanical Engineering, Celestijnenlaan 300B - bus 2420, 3001 Heverlee, Belgium, Wim.Desmet@mech.kuleuven.be), Karl Janssens (LMS International, Interleuvenlaan 68, 3001 Leuven, Belgium, Karl.Janssens@1msintl.com), Peter Gajdatsy (LMS International, Interleuvenlaan 68, 3001 Leuven, Belgium, peter.gajdatsy@1msintl.com), Herman Van Der Auweraer (LMS International, Interleuvenlaan 68, 3001 Leuven, Belgium, Herman .VanderAuweraer@1msintl.com)

Active control has been proposed as a possible solution to cope with low frequency noise reduction in vehicles. Active noise control systems tend to be designed with a target on the sound pressure level reduction. However, the perceived control efficiency for the occupants can be more accurately assessed if psychoacoustic metrics are taken into account. The aim of this paper is to evaluate, numerically and experimentally, the effect of a collocated velocity feedback controller on the sound quality of engine noise in a vehicle mockup. The simulation scheme is described and experimentally validated. The engine excitation is provided by a sound quality equivalent engine simulator, running on a real-time platform that delivers harmonic excitation in function of the driving condition. The controller performance is evaluated in terms of sound quality metrics such as specific loudness and roughness. As a result of the control action, loudness is significantly reduced and roughness slightly spread, with an overall improvement on the perceived engine sound.

\section{2:00}

5aSAb9. Reproduction of loud low-frequency soundfields with Boundary Surface Control. Emmanuel Friot (Laboratory for Mechanics and Acoustics CNRS, 31 chemin Joseph Aiguier, 13009 Marseille, France, friot@lma.cnrs-mrs.fr), Régine Guillermin (Laboratory for Mechanics and Acoustics CNRS, 31 chemin Joseph Aiguier, 13009 Marseille, France, guillermin@lma .cnrs-mrs.fr), Cédric Pinhède (CNRS - LMA, 31 Chemin Joseph Aiguier, 13009 Marseille, France, pinhede@1ma.cnrs-mrs.fr)

Many research works have focused on multichannel soundfield reproduction during the past decade, and complete systems are now commercially available for 3D sound control e.g., with Wave Field Synthesis. However, the usual underlying approximation of free-field propagation is not valid when reproducing high level low frequency noise, such as a sonic boom, inside a closet; a specific sound control strategy is required in this case. In this context the paper presents an experiment of low-frequency soundfield reproduction with openloop control of the acoustic pressure at a set of 30 microphones enclosing a listening area. It is shown that this so-called Boundary Surface Control strategy allows an accurate reproduction of 3D soundfields inside the LMA sonic boom closet, equipped with 16 wallembedded noise sources, at frequencies from 3 to $200 \mathrm{~Hz}$.

\section{2:20}

5aSAb10. Experimental assessment of the performance of a "smart foam" absorber. Pierre M. Leroy (Laboratoire de Mécanique et d'Acoustique - CNRS, 31 chemin Joseph Aiguier, 13402 Marseille, France, leroy@lma.cnrs-mrs.fr), Philippe Herzog (Laboratoire de Mécanique et d'Acoustique - CNRS, 31 chemin Joseph Aiguier, 13402 Marseille, France, herzog@lma.cnrs-mrs.fr), Alain Berry (Univ. de Sherbrooke, Mechanical Engineering Depart., 2500 Boulevard de l'Université, Sherbrooke, QC J1K 2R1, Canada, alain.berry@usherbrooke.ca), Noureddine Atalla (Univ. de Sherbrooke, Mechanical Engineering Depart., 2500 Boulevard de 1'Université, Sherbrooke, QC J1K 2R1, Canada, Nouredinne.Atalla@Usherbrooke.ca)

The signal processing implementation of a hybrid passive/active absorber (smart foam) made up from the combination of a passive absorbent (poroelastic melamine foam) typically efficient at the high frequencies, and a bonded, curved PVDF film, to enhance low frequency performance is considered. Three methods for obtaining the control signal are experimentally compared in the case of a plane wave excitation. A prototype of such smart foam has been built and tested in a waveguide (rectangular impedance tube) at frequencies between $100 \mathrm{~Hz}$ and $1000 \mathrm{~Hz}$. The performances in term of the ratio of reflected to incident sound pressure are presented and discussed, comparing the three methods. The first method uses estimations of the transfer functions between the sources and two microphones in the tube to calculate off-line an optimal filter in the frequency domain. It is then compared with numerical simulations, based on a 3D FE model of the smart foam. The two other methods are based on a real-time adaptive control using a FXLMS algorithm, a unidirectional microphone as error sensor, and the primary source signal as reference signal. The control filter is obtained after the initial convergence, using either pure tone disturbance (secnd method) or broad band disturbance (third method). 


\section{Contributed Papers}

2:00

5aSAb11. Active vibration reduction applied to the compressor of an air-conditioning unit for trams. Joachim Bös (Fraunhofer Institute for Structural Durability and System Reliability LBF, Bartningstr. 47, 64289 Darmstadt, Germany, joachim.boes@lbf.fraunhofer.de), Enrico Janssen (Fraunhofer Institute for Structural Durability and System Reliability LBF, Bartningstr. 47, 64289 Darmstadt, Germany, enrico.janssen@lbf.fraunhofer.de), Michael Kauba (Fraunhofer Institute for Structural Durability and System Reliability LBF, Bartningstr. 47, 64289 Darmstadt, Germany, michael.kauba@lbf.fraunhofer.de), Dirk Mayer (Fraunhofer Institute for Structural Durability and System Reliability LBF, Bartningstr. 47, 64289 Darmstadt, Germany, dirk.mayer@lbf fraunhofer.de)

Within the framework of the European Integrated Project InMAR (intelligent materials for active noise reduction) active vibration control and active structural acoustic control approaches are applied to an HVAC (heating, ventilation, and air conditioning) unit used to air-condition the driver's cab of a tram. Measurements previously performed by the manufacturer of the unit indicated that annoying tonal noise in the $50 \mathrm{~Hz}$ and 100 $\mathrm{Hz} 1 / 3$ octave bands inside the driver's cab is mainly caused by the vibration of the compressor mounted in the HVAC unit on the roof of the tram. Therefore, two different concepts for the design of active compressor mounts were developed that are used to reduce the vibration excitation of the HVAC unit's housing. The first one is an active tuned vibration absorber whose natural frequency can be adapted by means of piezoelectric patch actuators and which behaves as a vibration compensator at higher frequencies. The second one is an active mount based on four piezoelectric stack actuators and an elastomer part that deflects the effective direction of the piezo stack displacement by 90 degrees while amplifying the displacement by a factor of approximately 30 . These two concepts are compared with each other.

\section{2:20}

5aSAb12. Training by research - program of, and experiences in, the Smart Structures Research Training Network project. Fülöp Augusztinovicz (Budapest University of Technology and Economics, BME Dept. of Telecommunications, Magyar tudósok körútja 2, H-1117 Budapest, Hungary, fulop@hit.bme.hu)

Smart Structures is a Marie Curie Research Training network (RTN) named "A Computer Aided Engineering Approach to Smart Structures Design". The overall objective of the RTN is to develop an educational and research framework for developing macroscale noise control applications based on intelligent material systems. The scientific and technological objective of the network is to advance the state-of-the-art in smart materials research to the level of system integration and industrial applicability, with a specific focus on solutions for noise and vibration reduction and for vibration-based damage detection. The paper gives a short overview of the project partners' activity and summarizes the experiences of the researchers and their tutors after the first 18 months of the network.

\section{2:40}

5aSAb13. Intelligent interfaces for sound insulation: Numerical and experimental optimisation. Cedric Batifol (Centre Acoustique du LMFA, Ecole Centrale de Lyon, 36 avenue Guy de Collongue, 69134 Ecully cedex, France, cedric.batifol@ec-lyon.fr), Marie-Annick Galland (Centre Acoustique du LMFA, Ecole Centrale de Lyon, 36 avenue Guy de Collongue, 69134 Ecully cedex, France, marie-annick.galland@ec-lyon.fr), Mohamed Ichchou (Centre Acoustique du LMFA, Ecole Centrale de Lyon, 36 avenue Guy de Collongue, 69134 Ecully cedex, France, mohamed.ichchou@eclyon.fr)

Sound insulation is actually achieved thanks to passive multilayers. These ones are classically made of elastic, acoustic and poroelastic media. Good results are obtained in medium and high frequencies, but low frequencies disturbances still remain a problem. Indeed, local and global resonant behaviours of the panel, such as plate bending mode or the double-leaf phenomenon, occur and lower the performances. An active concept has already been proposed and validated. Piezoelectric components are added to the interface and act a secondary source aiming at minimising the sound transmitted. In this contribution, a double-plate system is studied. A reduced finite element model of an elementary cell is presented. This one relies on modal projection and classical component mode synthesis (CMS) procedures. This model is first updated and validated thanks to measurements performed on a dedicated test bench. The use of active control allows a $15 \mathrm{~dB}$ enhancement of sound insulation at low resonant frequencies. $\mathrm{Nu}-$ merical experiments are designed. Response surfaces are obtained thanks to kriging procedures. Finally, the configuration of the optimal interface is discussed in a physical point of view.

3:00

5aSAb14. Practical implementation of low-latency DSP for feedback control of sound in research contexts. Edgar Berdahl (Stanford Univ., Center for Computer Research in Music and Acoustics (CCRMA), Dept. of Music, Stanford, CA 94305-8180, USA, eberdahl@ccrma.stanford.edu), Nelson Lee (Stanford Univ., Center for Computer Research in Music and Acoustics (CCRMA), Dept. of Music, Stanford, CA 94305-8180, USA, nalee@stanford.edu), Guenter Niemeyer (Stanford Univ., Mech. Eng., Bldg. 530, Stanford, CA 94305, USA, gunter.niemeyer@stanford.edu), Julius O. Smith (Stanford Univ., Center for Computer Research in Music and Acoustics (CCRMA), Dept. of Music, Stanford, CA 94305-8180, USA, jos@ccrma.stanford.edu)

Feedback control of sound requires low-latency signal processing. In addition, because the human range of hearing extends roughly from $20 \mathrm{~Hz}$ to $20 \mathrm{kHz}$, controller hardware must process signals with relatively large bandwidths in comparison with common control applications. Over the past decade, the appropriate embedded hardware has become a niche product, so its cost has actually increased. The open source community has developed an excellent alternative: a general-purpose computer runs Linux with the realtime application interface (RTAI). Open source drivers (see www.comedi .org) enable software to communicate efficiently with data acquisition cards. For the first time, we describe in detail from start to finish how to configure such a system. In particular, we explain how to run control code from user space while still disabling interrupts. We further explain how to reserve a processor for running only feedback control code. This configuration achieves less than one sample of total system delay at sampling rates as high as $50 \mathrm{kHz}$. Other processors remain free to run standard Linux programs, allowing researchers to control sound with calls to many generic software libraries. We present detailed measurements of system jitter for various configurations and provide an example open-source toolbox demonstrating all these aspects at http://ccrma.stanford.edu/ eberdahl/Projects/TFCMI.

\section{$3: 20$}

5aSAb15. From the electrical shunting of a loudspeaker to active impedance control. Hervé Lissek (Ecole Polytechnique Fédérale de Lausanne, EPFL STI LEMA, Station 11, CH 1015 Lausanne, Switzerland, herve.lissek@epfl.ch), Florian Sandoz (Ecole Polytechnique Fédérale de Lausanne, EPFL STI LEMA, Station 11, CH 1015 Lausanne, Switzerland, florian.sandoz@epfl.ch)

Variable acoustic properties can be obtained on an electroacoustic transducer's voicing face by very basic control strategies, among which are the shunting of the loudspeaker (shortcut, variable electric load, or negative resistance disposals). It is proven that better performances with enhanced controllability can be obtained by way of hybrid feedback control, consisting in a double feedback loop, one on acoustic pressure at the diaphragm and the other on its velocity, leading to a global acoustic impedance control. The present work describes the theory of the hybrid feedback control, by way of block diagrams aiming at visualizing the control principle, starting from the shunted loudspeaker. Simulations of performances obtained on Matlab/Simulink are presented, and compared to experimental results obtained on analog prototypes mounted at the end of a dedicated impedance tube. A last, a discussion on stability issues follows, leading to concluding remarks on the disposal behavior and possible means of enhancements. 


\section{3:40}

5aSAb16. Active-passive multilayered panels for reduction of both acoustic reflection and transmission. Azzedine Sitel (Centre Acoustique du LMFA, Ecole Centrale de Lyon, 36 avenue Guy de Collongue, 69134 Ecully cedex, France, azzedine.sitel@ec-lyon.fr), Ying Hu (Centre Acoustique du LMFA, Ecole Centrale de Lyon, 36 avenue Guy de Collongue, 69134 Ecully cedex, France, ying.hu@ec-lyon.fr), Marie-Annick Galland (Centre Acoustique du LMFA, Ecole Centrale de Lyon, 36 avenue Guy de Collongue, 69134 Ecully cedex, France, marie-annick.galland@eclyon.fr)

The design of panels reducing both acoustic reflection and transmission for a wide frequency range is a problem of considerable practical interest for building or transport industries. Classically, these panels are made up of elastic plates bonded to poroelastic layers. Such structures are efficient in the middle and high frequency range but exhibit a lack of performance at low frequency. Hybrid passive/active cells previously developed at the LMFA have proved their efficiency for global noise reduction. They combine passive means (elastic plates, poroelastic material) and active control through a piezoelectric actuator to ensure high panel performance throughout the whole frequency range. In this paper, two systems are described. The first one is a multilayered panel combining two cells: the first cell on the emission side ensures high absorption, the second cell located at the rear face aims at reducing transmission. The second structure consists of a double-plate system using a microperforated plate on the emission side and an active plate on the transmission side. The performance of the two systems are examined through analytical or numerical simulations developed for plane waves and diffuse field conditions, and through experiments carried out for plane waves under normal incidence.

FRIDAY MORNING, 4 JULY 2008

P2-B, LEVEL 2, 9:40 TO 11:20 A.M.

\title{
Session 5aSAc
}

\section{Structural Acoustics and Vibration and EURONOISE: Active Noise Control: New Strategies and Innovative Concepts II (Poster Session)}

\author{
Alain Berry, Cochair \\ Univ. de Sherbrooke \\ Marie-Annick Galland, Cochair \\ Centre Acoustique du LMFA
}

\begin{abstract}
All posters will be on display from 9:40 a.m. to 11:20 a.m. To allow contributors an opportunity to see other posters, contributors of odd-numbered papers will be at their posters from 9:40 a.m. to 10:30 a.m. and contributors of even-numbered papers will be at their posters from 10:30 a.m. to 11:20 a.m.
\end{abstract}

\section{Contributed Papers}

5aSAc1. New multichannel modified filtered-x algorithms for active noise control using the dichotomous coordinate descent method. Felix Albu (Politehnica University of Bucharest, Bd. Iuliu Maniu, no. 1-3, 061102 Bucharest, Romania, felix_albu@ieee.org), Constantin Paleologu (Politehnica University of Bucharest, Bd. Iuliu Maniu, no. 1-3, 061102 Bucharest, Romania, pale@ comm.pub.ro)

In this paper, several multichannel modified filtered-x algorithms for active noise control or acoustic equalization systems using the dichotomous coordinate descent method (DCD) are introduced. This multiplierless and divisionless method is used for avoiding the matrix inversion that appears in adaptive algorithms such us fast transversal filters (FTF), recursive least square (RLS) based algorithms, affine projection (AP) or its fast versions. The study is focused on the important computational savings given by the use of DCD method, the effect on the convergence properties and stability of the investigated algorithms. A comparison of their convergence performance in case of using non-ideal noisy acoustic plants is also given. It is proved by simulations that the use of the dichotomous coordinate descent method can be an interesting option for reducing the computational cost of practical multichannel algorithms for ANC or acoustic equalization algorithms.

5aSAc2. Use of peak filter for online secondary path modeling in a feedforward active ship-noise cancellation system. Xueshan Bao (Department of Electronics and Telecommunications, Norwegian
University of Science and Technology, Sem Sælands vei 5, 7491 Trondheim, Norway, xbao@iet.ntnu.no)

The injected auxiliary noise used in online secondary path modeling method often disturbs the convergence of control filter and increases the residual sound field energy of the active noise cancellation (ANC) system. To mitigate this problem, an improved online secondary path modeling method is proposed, in which the transfer function of secondary path is reconstructed by a peak filter. The frequency response of new transfer function remains unchanged at the frequency band of the noise to be cancelled; while the other bands are attenuated deeply. With this system the simulation experiment of active ship-radiated noise cancellation is accomplished. The result shows that new system's noise cancellation capability is improved and residual sound field energy (including channel response to injected auxiliary noise) is smaller than conventional system without peak filter because of the simplified transfer function of secondary path and reduced energy disturbance between control filter and modeling filter.

5aSAc3. Adaptive active noise control incorporating with a transfer function method for reducing acoustic feedback in a duct. Yun-Hui Liu (Southern Taiwan University, 1 Nan-Tai St, Yung-Kang City, 710 Tainan Hsien, Taiwan, yhliu@ mail.stut.edu.tw)

In most practical applications of active noise control, the acoustic feedback is a major problem that often interferes with the operation of the control system and even renders it unstable. We propose a frequency-domain method based on transfer function to reduce the influence of acoustic feedback based on plane wave transmission theory of sound in a duct. The origi- 
nal signal of primary noise is obtained from the measured signals of reference microphone and error microphone which are transformed to frequency domain by FFT and operated by the proposed method. In this study, Filtered-X LMS algorithm is applied to carry out the active noise controller incorporating with this transfer function method. Experimental results of active noise control show that the system has achieved $18.5 \mathrm{~dB}$ maximal attenuation in the frequency band $200-600 \mathrm{~Hz}$. On the contrary, it is only attenuate $3-4 \mathrm{~dB}$ if acoustic feedback is present. Therefore, it was to verify that the proposed method of acoustic feedback cancellation incorporating with the Filtered-X LMS algorithm can effectively reduce the influence of acoustic feedback and obtains a better performance of active noise control in a duct.

5aSAc4. Secondary source distribution effect on active noise control robustness. Mahdi Azarpeyvand (Institute of Sound and Vibration Research, University of Southampton, University Road, SO17 1BJ Southampton, UK, ma@isvr.soton.ac.uk)

Arrangement of secondary sources and microphones play an important role in effectiveness and robustness of any active noise control (ANC) system. This paper concerns effective distribution of secondary sources for a typical ANC system so that provides a robust and acceptable level of noise reduction for different types of primary source, namely monopole, dipole and multipole, radiating at $100 \mathrm{~Hz}$ and $250 \mathrm{~Hz}$. Two sets of secondary sources are considered here: planar distribution, and dome-type distribution. Results have shown that utilization of planar distribution model leads to more effective and stable noise reduction for most of the cases, while using a dome-type set of secondary sources is not able to effectively reduce noise from dipole and multipole primary sources. In addition, it was found that implementation of dome-type arrangement is more difficult than the planar distribution and is also very likely to become unstable for some particular source arrangements (i.e., or mathematically ill-conditioned).

5aSAc5. Improvement of acoustic transmission loss by active modal mass control. Vincent Lhuillier (Laboratoire Vibrations Acoustique INSA Lyon, 25 bis avenue Jean Capelle, Bâtiment Saint-Exupéry, F-69621 Villeurbanne cedex, France, vincent.lhuillier@insa-lyon.fr), Charles Pezerat (Laboratoire Vibrations Acoustique - INSA Lyon, 25 bis avenue Jean Capelle, Bâtiment Saint-Exupéry, F-69621 Villeurbanne cedex, France, charles.pezerat@insa-lyon.fr), Simon Chesne (LaMCoS - INSA-Lyon CNRS UMR5259, 18-20, rue des Sciences, Bâtiment Jean d'Alembert, F-69621 Villeurbanne, France, simon.chesne@insa-lyon.fr), Luc Gaudiller (LaMCoS - INSA-Lyon - CNRS UMR5259, 18-20, rue des Sciences, Bâtiment Jean d'Alembert, F-69621 Villeurbanne, France, luc .gaudiller@insa-lyon.fr)

This paper deals with an alternative modal control approach to reduce the sound transmission through a structure excited by an acoustic wave. Active control enables to conserve the characteristics of lightness while improving acoustic performances. For light and small structures having small modal overlap, the "modal mass damping control" is proposed. The aim of this control is to modify the modal distribution of high radiation efficiency modes with modal virtual mass and modal virtual damping. The interest of this approach is that at low frequencies, the active virtual mass effects shift down eigen frequencies to less audible frequency range while reducing vibration amplitudes in a broad frequency range. The modal virtual mass control appears to be a good complement to a standard damping control which is exclusively efficient at resonances. In order to detail the concept of the proposed method, an application is presented on a double panel equipped with piezoelectric patches. The structure is excited by a distributive acoustic plane wave. Acoustic transmission loss factors of the simulated controlled and the non-controlled smart structure are shown and optimization is discussed.

5aSAc6. A two-dimensional ANC system - from simulation to application. Christian Kleinhenrich (Univ. of Wuppertal, RainerGruenter-Str. 21, 42119 Wuppertal, Germany, c.kleinhenrich @uni-wuppertal.de), Detlef Krahé (Univ. of Wuppertal, Rainer-GruenterStr. 21, 42119 Wuppertal, Germany, krahe@uni-wuppertal.de), Arndt Niepenberg (WaveScape Technologies GmbH, Lise-Meitner-Str. 1-9, 42119 Wuppertal, Germany, niepenberg@wavescape-technologies.com)

An existing two-dimensional active noise control (ANC) system attenuates a given noise (primary field) inside a certain area and frequency range by an additionally generated sound (secondary field). The two-dimensional ANC system consists of several modules containing a pair of microphones and a line array of loudspeakers each. The configuration of the modules depends on the acoustical environment and the desired shape of the protection area. Planning this is supported by a tool based on simulating the superposition of both sound fields. This spatial, close to reality simulation for predicting the achievable noise attenuation and determining important system parameters is implemented in MATLAB. With this planning tool it is possible to place all required acoustic sources and sinks into a virtual room and analyze the properties of the resulting sound field concerning real application scenarios. This paper draws a comparison between the results given by simulation and the subsequently realized ANC system within the scope of a typical application.

5aSAc7. Active noise control in light jet aircraft. Oliver Pabst (Helmut-Schmidt-Universität - Universität der Bundeswehr Hamburg, Holstenhofweg 85, 22043 Hamburg, Germany, opabst@ hsu-hh.de), Thomas Kletschkowski (Helmut-Schmidt-Universität - Universität der Bundeswehr Hamburg, Holstenhofweg 85, 22043 Hamburg, Germany, thomas .kletschkowski@hsuhh.de), Delf Sachau (Helmut-Schmidt-Universität Universität der Bundeswehr Hamburg, Holstenhofweg 85, 22043 Hamburg, Germany, sachau@hsuhh.de)

Active systems for noise reduction are especially of interest when considering applications in which low-frequency noise is a main source of disturbance and only limited amounts of installation space and payload are available. This makes the adaptation and implementation of such systems plausible in vehicles such as automobiles and aircraft where passive reduction methods are restricted. In order to achieve effective active control in these environments, aspects such as the control method and actuator- and sensor-type as well as positioning must be considered. The noise characteristics are often known beforehand or are easily accessible by measurement. Using this data, an upper bound for possible noise reduction may be determined, e.g., by means of linear prediction methods. A current research project is aimed at developing an audiosystem for the cabin area of a light jet aircraft which, at the same time, should also function as an effective noise reduction system in order to enhance the cabin comfort as well as the audio quality. Using data from a measurement flight in a typical light jet aircraft, limitations of active control are determined. Furthermore, the testbed, an acoustic mockup, is presented, currently beholding a multichannel ANC- Audio system for tonal and broadband noise. 


\title{
Session 5aSAd
}

\section{Structural Acoustics and Vibration, ASA Committee on Standards, and EURONOISE: Ground Vehicle Noise and Vibration II (Poster Session)}

\author{
Donald Bliss, Cochair \\ Duke University \\ Paul De Vos, Cochair \\ $D H V B V$
}

\begin{abstract}
All posters will be on display from 9:40 a.m. to 11:20 a.m. To allow contributors an opportunity to see other posters, contributors of odd-numbered papers will be at their posters from 9:40 a.m. to 10:30 a.m. and contributors of even-numbered papers will be at their posters from 10:30 a.m. to 11:20 a.m.
\end{abstract}

\section{Contributed Papers}

5aSAd1. An hybrid method for sound transmission inside trains. Philippe A. Jean (CSTB, 24 rue Joseph Fourier, 38400 Saint-Martind'Hères, France, philippe.jean@cstb.fr), Michel Villot (CSTB, 24 rue Joseph Fourier, 38400 Saint-Martin-d'Hères, France, michel.villot@cstb.fr)

Hybrid methods mixing ray-tracing and energy approaches have been implemented and validated in the case of sound transmission inside a train. Generally, validation is proposed against measurements where it may be difficult to assess precisely the importance of the various assumptions and simplifications. In this paper, the proposed hybrid approach is compared to more precise numerical computations based on BEM and analytic models This allows studying the importance of assumptions made on the computations of incident power and transmitted power. The case of a train in a tunnel has also been analysed where the sound field in the tunnel is computed by means of 2.5D BEM

5aSAd2. Critical assessment of operational path analysis: effect of coupling between path inputs. Peter Gajdatsy (LMS International, Interleuvenlaan 68, 3001 Leuven, Belgium, peter.gajdatsy@lmsintl.com), Karl Janssens (LMS International, Interleuvenlaan 68, 3001 Leuven, Belgium, Karl.Janssens@1msintl.com), Ludo Gielen (LMS International, Interleuvenlaan 68, 3001 Leuven, Belgium, ludo.gielen@1ms.be), Peter Mas (LMS
International, Interleuvenlaan 68, 3001 Leuven, Belgium, peter.mas @ lms.be), Herman Van Der Auweraer (LMS International, Interleuvenlaan 68,3001 Leuven, Belgium, Herman.VanderAuweraer@1msintl.com)

Classical transfer path analysis (TPA) is a widely used and reliable method for tackling noise and vibration problems. But due to its complexity and time-consuming procedure the industry is constantly seeking for simpler and faster methods. One such method, often referred to as operational path analysis (OPA), was presented in 2006 and has quickly gained popularity in the NVH field. The method works with measured operational input and output accelerations and sound pressures and the transmissibilities calculated from these. The claim for its accuracy is based on being able to reproduce the original output signal by summing the calculated partial contributions but it has not yet been compared to other TPA methods. This new method is now critically examined and compared to a reliable classical TPA measurement. The results of this examination reveal three significant weaknesses. This paper deals with the effect of the cross-coupling between the input signals. Due to modal behavior a single force will cause vibrations at all inputs. Thus, there is not a simple one-to-one relationship between loads and inputs. This coupling then can easily lead to false identification of significant paths in case of the OPA method. 


\title{
Session 5aSCa
}

\section{Speech Communication: Speaker Identification by Machine}

\author{
Jaime Hernández-Cordero, Cochair \\ US Department of Defense, PO BOX 183, Odenton, MD 21113, USA \\ Louis-Jean Boë, Cochair \\ GIPSA-lab, Université Stendhal, 1180, avenue Centrale, 38031 Grenoble CEDEX 9, France
}

\section{Contributed Papers}

\section{8:00}

5aSCa1. Adaptive threshold estimation for speaker verification systems. Eduardo Castilllo-Guerra (University of New Brunswick, P.O. Box 4400, 15 Dineen Dr., D36 Head Hall, Fredericton, NB E3B 5A3, Canada, ecastill@unb.ca), Roberto Díaz-Amador (Central University of Las Villas, Carr. a Camajuaní km 5.5, 50100 Santa Clara, Cuba, ramador@uclv.edu.cu), Cárdenas-Barreras L. Julian (Central University of Las Villas, Carr. a Camajuaní km 5.5, 50100 Santa Clara, Cuba, jcardenas @uclv.edu.cu)

This paper describes an adaptive threshold estimation mechanism for speaker authentication systems. The mechanism estimates speakerdependent thresholds based on successful verifications considering the minimization of a relation-based cost function. Speaker authentication systems commonly use a threshold to decide whether a claimed identity matches a voice-print previously enrolled. Speaker independent threshold is a common option but it does not consider specific speaker characteristics that are relevant to achieve better system performance. Speaker dependent threshold on the contrary, uses speaker-specific data to estimate individual thresholds but the system performance can also suffer from suboptimal threshold conditioned by limited number of true scores. The algorithm reported in this paper starts with the speaker dependent threshold and use an adaptive algorithm to perform online re-estimation of the initial threshold based on speakerdependent data. The threshold is re-estimated in each successful authentication transaction according to a custom-made confidence score. The reported technique keep the voice print up-to-date while is less sensitive to score outliers than traditional speaker dependent threshold. The algorithm provided a performance enhancement of up to $36.2 \%$ when compared to traditional speaker independent or dependent threshold using an ad-hoc database involving cell and land-line utterances from male and female speakers.

\section{$8: 20$}

5aSCa2. Estimation of target-to-interferer ratio using the Auditory Image Model. Michael Carlin (Air Force Research Laboratory, 525 Brooks Road, Rome, NY 13441, USA, Michael.Carlin@rl.af.mil)

Performance in speech processing applications such as speaker recognition becomes severely degraded when the short-time audio being analyzed contains more than one speaker. However, it has been shown that if the audio is only minimally-corrupted by the interfering speech, i.e., the target-tointerferer ratio (TIR) is sufficiently large, then accurate recognition results can still be achieved. During phonation, estimation of TIR is especially critical since uncorrupted vowel sounds contain important speakerdiscriminating information. This research investigates a method to estimate the relative intensity of interfering speech using the Auditory Image Model (AIM) of Patterson et al. (J. Acoust. Soc. Am., 98, 1890-1894 (1995)). The proposed TIR estimator attempts to exploit both the apparent high resolution in the simulated Neural Activity Pattern and variation in cross-channel Strobe Point correlation when observing overlapping vowel sounds. Experiments were conducted for five canonical male vowels which were perceptually-scaled using the STRAIGHT algorithm (Chapter in Speech Separation by Humans and Machines, P. Divenyi, ed., Kluwer Academic Publishers, 2005) and superimposed at varying levels of TIR. Results sug- gest that the proposed approach is a promising step towards both detecting the presence and relative intensity of an interfering speaker.

\section{8:40}

5aSCa3. Extraction of likelihood-ratio forensic evidence from the formant trajectories of diphthongs. Geoffrey Stewart Morrison (Australian National University, School of Language Studies, Building 110, ACT 0200 Canberra, Australia, geoff.morrison@anu .edu.au), Phil Rose (Australian National University, School of Language Studies, Building 110, ACT 0200 Canberra, Australia, philip.rose@anu.edu.au), Yuko Kinoshita (University of Canberra, School of Languages and International Studies, ACT 2601 Canberra, Australia, yuko.kinoshita@canberra.edu.au)

The likelihood-ratio approach to forensic speaker recognition seeks to determine the likelihood that one would observe the evidence, the acoustic difference between suspect and offender speech samples, under the hypothesis that they were produced by the same speaker versus under the hypothesis that they were produced by different speakers. Before the results of a scientific forensic technique can be presented in court, it is necessary to demonstrate its efficacy. This presentation tests the efficacy of extracting information from the formant trajectories of diphthongs. Differences in physiology and learned motor patterns could potentially lead to different speakers producing quite different formant trajectories which could in turn lead to strong forensic evidence. The data tested were /aI/, /av/, /eI/, /"open o"I/, /ov/, /i"schwa"/, and / $\epsilon$ "schwa"/ tokens produced in several phonetic contexts by 27 male speakers of Australian English. Cubic polynomials were fitted to each vowel token, and the coefficient values were used in a multivariate-kernel-density procedure which calculated likelihood ratios. Cross-validated same-speaker and different-speaker comparisons were made, resulting in a series of same-speaker and different-speaker likelihood ratios for each vowel phoneme. Results indicated that substantial strength of evidence with respect to speaker identity can be extracted from diphthong formant trajectories.

\section{9:00}

5aSCa4. Identifying speaker-dependent acoustic parameters in Spanish vowels. Victoria Marrero (Universidad Nacional Educación a Distancia, Desp. 707A. C/ Senda del Rey, 7, 28040 Madrid, Spain, vmarrero@flog.uned.es), Elena Battaner (URJC, C/Tulipan s/n, 28345 Móstoles (Madrid), Spain, elena.battaner@urjc.es), Juana Gil (Universidad Nacional Educación a Distancia, Desp. 707A. C/ Senda del Rey, 7, 28040 Madrid, Spain, mgil@ flog.uned.es), Joaquim Llisterri (UAB, Filologia. Edifici B. Campus Bellaterra, 08193 Barcelona, Spain, Joaquim .Llisterri@uab.cat), María Machuca (UAB, Filologia. Edifici B. Campus Bellaterra, 08193 Barcelona, Spain, MariaJesus.Machuca@uab .es), Montserrat Marquina (UAB, Filologia. Edifici B. Campus Bellaterra, 08193 Barcelona, Spain, Montserrat.Marquina@campus.uab.es), Carme De La Mota (UAB, Filologia. Edifici B. Campus Bellaterra, 08193 Barcelona, Spain, Carme.delaMota@uab.cat), Antonio Rios (UAB, Filologia. Edifici B. Campus Bellaterra, 08193 Barcelona, Spain, Antonio.Rios@uab.cat)

In the frame of VILE Projects (Inter-and-Intra-Speaker-Variation-inSpanish for automatic speaker identification), we try to identify what 
vowel's acoustic parameters depend more on the individual characteristics of the speaker and less of the linguistic variables. Variations on standard deviation (SD), when grouping together parameters by speaker or by phoneme, are analysed. 30 speakers (from AHUMADA database) read the same text in three sessions. Mean value $(\mathrm{Hz})$ of four formants $(\mathrm{F} 1-\mathrm{F} 2-\mathrm{F} 3-\mathrm{F} 4)$ and fundamental frequency (F0) are analysed in Spanish vowels (except/u/), surrounded by unvoiced stops or /s/ (1850 samples). Hypothesis: Individual parameters will show less SD when grouping by speaker/session; vowel quality parameters, when grouping by phoneme. F1 and F2 are timbredependent parameters. F3 and F4 are speaker-dependent parameters. F0 has characteristics of both. The most variable parameter is F2. The opposite is F4. -No significant differences grouping by session or by speakers in none of the parameters. -F0 has the highest variability between vowel qualities, even if stressed and unstressed vowels are separated. -When clustering data by speaker/sessión (all vowels together), by comparison with clustering by vowel (all speakers together), SD is $* 50 \%$ higher in $\mathrm{F} 1-\mathrm{F} 2 * 75 \%$ lesser in $\mathrm{F} 4 * 66 \%$ lesser in F0 ${ }^{*} \mathrm{~F} 3$ shows no significant differences

\section{9:20}

5aSCa5. The question of disguised voice. Patrick Perrot (Telecom Paris Tech, 46 rue Barrault, 75013 Paris, France, perrot@tsi.enst.fr), Gerard Chollet (Telecom Paris Tech, 46 rue Barrault, 75013 Paris, France, chollet @enst.fr)

State of the art automatic speaker recognition systems show very good results in the discrimination between different speakers under controlled recording conditions. In a forensic context, the conditions are uncontrolled and voice can be disguised. In cases of terrorism claim, extortion or kidnapping, it is of great interest for offenders to conceal their identity. Voice disguise is an important constraint to speaker discrimination. Some disguises produce a great variation of parameters and change the perception of an identity. The main risk is to confound a disguised voice and a normal voice and accuse an innocent individual. This paper proposes on one hand to present the impact of voice disguise on automatic speaker recognition and, on the other hand a statistical study in order to detect and identify four disguises among the most common. The first step consists in extracting features and the second step to classify them. MFCC (Mel Frequency Cepstral Coefficient) are considered as features and different classification algorithms have been tested. The studied disguises are based on a deliberated and non electronic way. The proposed analysis of disguised voice classification provides interesting results in detection by the use of SVM (Support Vector Machine) and in identification by the use of GMM (Gaussian mixture models).

\section{9:40}

5aSCa6. Speaker identification on the SCOTUS corpus. Jiahong Yuan (University of Pennsylvania, 609 Williams Hall, Philadelphia, PA 19104, USA, jiahong@ling.upenn.edu), Mark Liberman (University of Pennsylvania, 609 Williams Hall, Philadelphia, PA 19104, USA, myl@cis .upenn.edu)

This paper reports the results of our experiments on speaker identification in the SCOTUS corpus, which includes oral arguments from the Supreme Court of the United States. Our main findings are as follows: 1) a combination of Gaussian mixture models and monophone HMM models attains near-100\% text-independent identification accuracy on utterances that are longer than one second; (2) the sampling rate of $11025 \mathrm{~Hz}$ achieves the best performance (higher sampling rates are harmful); and a sampling rate as low as $2000 \mathrm{~Hz}$ still achieves more than 90\% accuracy; (3) a distance score based on likelihood numbers was used to measure the variability of phones among speakers; we found that the most variable phone is the phone UH (as in good), and the velar nasal NG is more variable than the other two nasal sounds $\mathrm{M}$ and $\mathrm{N}$; 4.) our models achieved "perfect" forced alignment on very long speech segments (one hour). These findings and their significance are discussed.

\section{0:00}

5aSCa7. Dependency of recognition rate on number of words for text-independent speaker recognition using vector quantization. Hidenori Shimizu (Div. of Electronic Eng. and Computer Sci., Kanazawa Univ., Kakuma-machi, 920-1192 Kanazawa-shi, Japan, shimizu@oak .ec.t.kanazawa-u.ac.jp), Tetsuo Funada (Div. of Electronic Eng. and Computer Sci., Kanazawa Univ., Kakuma-machi, 920-1192 Kanazawa-shi, Japan,funada@t.kanazawa-u.ac.jp)

In this research, we discuss speaker recognition using the Kohonen feature map. The map is constructed for each speaker, and it is trained by using log-power and fourteenth-order mel-frequency cepstral coefficients (MFCC) and their temporal difference. The quantization distortion is computed between the input speech and a specific vector on the feature map of each speaker. We conduct speaker recognition experiment based on VQ distortion. Utterances of prefectural name in Japan are used as speech data. We examine particularly the dependency of recognition rate on number of words used for recognition. According to our experiments of speaker identification, this system correctly recognizes $98.9 \%$ by using a single word for 40 male speakers, while it attains $100 \%$ by using more than three words. Moreover, we confirmed superiority of using VQ over HMM under the same experimental conditions. 


\title{
Session $5 \mathrm{aSCb}$
}

\section{Speech Communication: Cross-Language Speech Perception and Production}

\author{
Yue Wang, Cochair \\ Simon Fraser Univ., RCB 9224, 8888 Univ. Dr., Burnaby, BC V5A 1S6, Canada \\ Dawn Behne, Cochair \\ Norwegian University of Science and Technology, Psychology Dept, Trondheim, NO 7491, Norway
}

\section{Invited Papers}

\author{
8:40
}

5aSCb1. Sound structure and function of English as a global language. Ann Bradlow (Northwestern University, Department of Linguistics, 2016 Sheridan Road, Evanston, IL 60208, USA, abradlow@ northwestern.edu)

Many English conversations across the globe today involve non-native speakers. To understand the sound structure of English in a global context, we asked native and non-native listeners to rate the similarity of various languages $(n=17)$ to English. In general, the native and non-native listeners' distance-from-English ratings were positively correlated; but, the non-natives tended to rank languages as more distant from English than the natives. Native and non-native listeners then rated the accents of native and non-native speakers. Again, the native and non-native listener ratings were positively correlated, but the non-native listeners generally rated both native and non-native speakers as more foreign-accented than did the native listeners. Together, these data suggest that both native and non-native English generally sound less "marked" to native listeners than to non-native listeners. As a means of understanding how English functions as a global language, we examined communicative efficiency in task-oriented dialogues between various combinations of natives and non-natives. All pairs successfully completed the task; yet, when one or more non-native talkers were involved, the task took about twice as long to complete as when both were native talkers. Together, these studies help characterize the structure and function of English as a twenty-first century lingua franca.

\section{9:00}

5aSCb2. Maintainance vs. "loss" of the perceptual bias favoring natural reference vowels. Ocke-Schwen Bohn (Aarhus University, English Department, J.-C.-Skous Vej 5, DK-8000 Århus C, Denmark, engosb@hum.au.dk), Linda Polka (McGill University, School of Communication Sciences and Disorders, Beatty Hall, 1266 Pine Avenue West, Montreal, QC H3G 1A8, Canada, linda.polka @ mcgill.ca)

Previous infant vowel discrimination studies (Polka \& Bohn, 2003) have shown that babies are perceptually biased to favor vowels with more extreme articulations such that a change from a more peripheral vowel ("natural reference vowel" - NRV) to a vowel located more centrally in the vowel space is less discriminable than a change presented in the reverse direction. Recent studies of adult vowel perception suggest that the universal bias favoring NRVs is maintained in the absence of specific language experience and but "lost" if native or non-native language experience causes a reset of the perceptual default favoring NRVs. This presentation reports on vowel discrimination experiments which examined whether absence or presence of specific vowel experience is related to maintenance or loss of this perceptual bias in Danish infants (age 6-12 months). For two Danish vowel contrasts, asymmetries were observed for younger infants, but not for infants older than 10 months. For an English vowel contrast that does not exist in Danish, the asymmetry was maintained in infants up to 12 months and in adult Danish listeners. Results confirm the assumption that the perceptual bias favoring NRVs is maintained throughout the lifespan unless specific vowel experience causes on override. [Work supported by the Danish Research Council for the Humanities.]

$$
\text { 9:20 }
$$

5aSCb3. Cross-language auditory-visual speech perception development. Denis Burnham (MARCS Auditory Laboratories, University of Western Sydney, Locked Bag 1797, Penrith South, NSW, 1797 Sydney, Australia, d.burnham@uws.edu.au), Kaoru Sekiyama (Kumamoto University, Division of Cognitive Psychology, Faculty of Letters, 861-0000 Kumamoto, Japan, sekiyama@kumamoto-u.ac.jp), Dogu Erdener (MARCS Auditory Laboratories, University of Western Sydney, Locked Bag 1797, Penrith South, NSW, 1797 Sydney, Australia, d.erdener@uws.edu.au)

Speech perception is auditory-visual (AV), but there is more visual information use by English than Japanese language perceivers (Sekiyama \& Tohkura, 1993). This raises two questions: (1) when and (2) why do such differences emerge? In Experiment 1 with English- and Japanese-language children $(6,8,11$ years) and adults, AV speech perception (AVSP) was equivalent in English and Japanese 6-year-olds, significantly increased between 6 and 8 years in English- but not Japanese-language children, and remained stable over all ages for the Japanese. The origin of the Japanese-English adult difference in English language children's dramatic rise in AVSP between 6 and 8 years was investigated in Experiment 2. English-language children (5 to 8 years) and adults were tested for AVSP, 
reading, articulation, and language-specific speech perception (focus on native and away from non-native speech sounds, a known correlate of reading, Burnham, 2003). Regressions showed that language specific speech perception reliably predicted AVSP. It appears children use extra information (AVSP) in the face of challenging linguistic hurdles (learning to read), but only when the language is phonetically complex and the phoneme-to-grapheme conversion rules of the script are not transparent (English, but not Japanese).

\section{Contributed Paper}

9:40

5aSCb4. Dependence of corresponding phonetic categories in native bilingual speakers and in monolingual overhearers of English and French. Carol A. Fowler (Haskins Laboratories, 300 George St, Suite 900, New Haven, CT 06511, USA, carol.fowler@haskins.yale.edu)

Our research addresses two main questions. First, among phonetic categories that correspond, but are not identical, in the two languages of native bilingual speakers, are there cross-language influences on speech production? Specifically, are the voiceless voice-onset times (VOTs) of bilingual speakers of English and French in Montreal longer in their French speech and shorter in their English speech than the VOTs of monolingual speakers of the two languages in Montreal? Our findings suggest a positive answer. Second, does mere overhearing of a language that one does not speak have an impact on production of phonetic categories? To answer this, we examined the voiceless VOTs of monolingual speakers of French and English in Montreal (who hear substantial unaccented English and French, respectively) with those, respectively, of monolingual speakers of French in Paris and of English in Connecticut. Our answer here was no. I discuss the implications of these findings for an understanding of the phonetic knowledge systems of monolingual and natively bilingual speakers.

\section{Invited Paper}

10:00

5aSCb5. Plurality and plasticity of neural representation for speech sounds. Yang Zhang (University of Minnesota, Dept. of Speech-Language-Hearing Sci. \& Center for Neurobehavioral Development, Minneapolis, MN 55455, USA, zhang470@umn.edu)

High-density event-related potential (ERP) and magnetoencephalography (MEG) data are presented here to illustrate the plurality and plasticity of speech representation in the spatio-temporal dynamics of neural responses. First, there is evidence for both continuous and discrete processing of speech sounds depending on the acoustic parameters and experimental conditions. Speech discrimination and neural sensitivity measures suggest that the perceptual organization of within-category variations is based on both phonetic knowledge and acoustic analysis. Second, attention plays an important role in determining the neural response patterns. Focused attention significantly changes the hemispheric laterality and strength of neural activation for speech processing. Selectively attending to the different aspects (phonetic vs social-indexical information) of the same speech stimuli shows different regional recruitment, hemispheric involvement and temporal characteristics. Third, the nature of language input and experience can significantly affect learning outcome. Enriched exposure alters the neural representations for speech sounds and plasticity of the system. Theoretical and practical implications for language learning and treatment are discussed. 


\title{
Session 5aSCc
}

\section{Speech Communication: General Topics in Speech Communication IV (Poster Session)}

\author{
Linda Polka, Cochair \\ McGill University \\ Christine Mooshammer, Cochair \\ Haskins Lab and MIT Research Lab of Electronics \\ Suzanne Boyce, Cochair \\ Department of Communication Sciences and Disorders, University of Cincinnati
}

\begin{abstract}
All posters will be on display from 10:20 a.m. to 12:00 a.m. To allow contributors an opportunity to see other posters, contributors of odd-numbered papers will be at their posters from 10:20 a.m. to 11:10 a.m. and contributors of even-numbered papers will be at their posters from 11:10 a.m. to 12:00 p.m.
\end{abstract}

\section{Contributed Papers}

5aSCc1. Call early in the evening on a spring day. Maxine Eskenazi (Language Technologies Institute Carnegie Mellon University, 4619 Newell Simon Hall, 5000 Forbes Ave, Pittsburgh, PA 15213, USA, max@cs.cmu.edu), Antoine Raux (Language Technologies Institute Carnegie Mellon University, 4619 Newell Simon Hall, 5000 Forbes Ave, Pittsburgh, PA 15213, USA, antoine@cs.cmu.edu)

The CMU Let's Go Spoken Dialogue System has been used daily for about three years to answer calls to the Pittsburgh Port Authority for bus information in the evening and on weekends. This has resulted in a database of over 50000 spoken dialogues as of January 2008, one of the largest publicly available sets of this type of data. While retraining the system with part of this data, it became apparent that there are times of the day, of the week and of the year when the average number of successful calls is significantly higher. We will present evidence, using these three measures of time (hour, day of week, month of year) and criteria such as signal-to-noise ratio, estimated success rate, number of turns per dialogue, number of nonunderstandings per dialogue, and barge-in rate to detect the regular, predictable appearance of high and low success rates and to suggest methods for palliating this effect in order to increase overall dialogue success rates.

5aSCc2. Duration modeling for English letters embedded in Chinese speech. Wen-Hsing Lai (National Kaohsiung First University of Science and Technology, No. 2, Jhuoyue Rd., Nanzih District, 811 Kaohsiung, Taiwan,1wh@ccms.nkfust.edu.tw)

A review of existing multilingual TTS (text-to-speech) systems shows that the secondary language inserted into the primary language sounds more like isolated individual words in an alien language environment and not congruous with the primary language's prosody. Since the letter-by-letter spelling of English words or acronyms appears in Chinese speech quite often, a duration modeling approach for English letters embedded in Chinese speech is proposed to make the English congruous with the primary language's tempo. It takes several major factors as additive factors and estimates all model parameters by an EM (expectation-maximization) algorithm. Experimental results showed that the standard deviation of the duration from the test set was greatly reduced from 59.82 to $9.37 \mathrm{~ms}$ by the duration modeling while eliminating effects from factors. The root mean squared error between the original and estimated durations was $9.35 \mathrm{~ms}$ for the open tests. Experimental results have confirmed its effectiveness on isolating several main fac- tors that seriously affects the duration. Moreover, the estimated value of the factors agreed well to our prior linguistic knowledge. Besides, the hidden state labels produced by the EM algorithm were linguistically meaningful.

5aSCc3. Modeling perception of breathy voice quality in vowels using data obtained in an adaptive matching task. Rahul Shrivastav (University of Florida, Dauer Hall, P.O. Box 117420, Dept. of Communication Sciences and Disorders, Gainesville, FL 32611, USA, rahul@csd.ufl.edu), Arturo Camacho (University of Florida, Dauer Hall, P.O. Box 117420, Dept. of Communication Sciences and Disorders, Gainesville, FL 32611, USA, arturocl@yahoo.com), Sona A. Patel (University of Florida, Dauer Hall, P.O. Box 117420, Dept. of Communication Sciences and Disorders, Gainesville, FL 32611, USA, sona09@ufl.edu), David A. Eddins (University of Rochester, Department of Otolaryngology, Rochester, NY 14618, USA, David_Eddins@URMC.Rochester.edu)

A computational model of breathy voice quality has been developed based on perceptual judgments of synthetic /a/ vowels [Shrivastav, Camacho, and Patel, JASA 120(5), 3248]. This model is based on the ratio of the loudness of aspiration noise ("noise loudness;" NL) to the loudness of the periodic components of the vowel when masked by the aspiration noise ("partial loudness;" PL) [Shrivastav and Sapienza, JASA, 114(1), 2218-2224 (2005)]. Results showed that the model accounted for a large amount of variance in perceptual ratings of training data $(\mathrm{R}$-square $=0.92)$ but not for testing data $(\mathrm{R}$-square $=0.59)$. It was hypothesized that this reduction was partly related to the use of a magnitude estimation task to obtain perceptual judgments, since magnitude estimates are biased by the range of the quantity measured and are prone to inconsistencies in how subjects assign numbers to items [Poulton, 1989; Guilford, 1954]. To minimize such biases, a matching task may be used to obtain ratio level estimates of breathiness [Patel, Shrivastav and Eddins, JASA, 119(5), 3340 (2006)]. The present study describes a model to predict breathy voice quality derived from perceptual judgments obtained using a matching task.

5aSCc4. The effect of training in noise on foreign language consonant acquisition. M. Luisa Garcia Lecumberri (University of the Basque Country, Paseo de la Universidad 5, Facultad de Filologia, 01006 Vitoria, Spain, garcia.lecumberri@ehu.es), Martin Cooke (Sheffield University, Computer Science Department, Regent Court, 211 Portobello St., S1 4DP Sheffield, UK, m.cooke@dcs.shef.ac.uk) 
Formal exposure to second language sounds normally takes place in clean, laboratory conditions, but at issue is the transfer of such learning to everyday situations. Categories learned in non-natural settings may be fragile, raising the question as to whether learning in noise leads to greater category robustness. The current study compared two groups of Spanish learners of English who were trained in either quiet or noise backgrounds on a 24 consonant discrimination task. Learners' performance was measured in preand post-tests and monitored over the course of nine weeks' training. Both groups showed continual improvement during training with similar overall gains of 6-7 percentage points, suggesting that training in adverse conditions is as effective as in quiet. Tests involving consonant identification in quiet and noise revealed no significant differences in pre-post improvement between the two groups. However, voiceless obstruents benefited more from training in noise while voiced obstruents experienced more improvement when trained in a quiet background regardless of the testing condition (quiet vs noise). The noise-trained disadvantage is consistent with masking of voicing in noise, while the quiet-trained deficit for voiceless obstruents may derive from overgeneralisation during the acquisition of new contrasts.

5aSCc5. Analyze effects of the flow on the vocalic reduction and the coarticulation in sequences $\mathbf{C V}$ of pharyngal Arabic. Leila Falek (USTHB, Faculté d'électronique et d'Informatique, 16111 Algiers, Algeria, lilalcpts@yahoo.fr), Othmane Bouferroum (USTHB, Faculté d'électronique et d'Informatique, 16111 Algiers, Algeria, lilalcpts@yahoo.fr), Amar Djeradi (USTHB, Faculté d'électronique et d’Informatique, 16111 Algiers, Algeria, lilalcpts@yahoo.fr)

The degree of coarticulation and the vocalic reduction (RV) are indices related to a good engine control (Gay, 1978). Fowler (1998) explains why locus equation (LE) is used to characterize, at the same time, the place of articulation and the degree of coarticulation between consonants and vowels: a strong slope $(\mathrm{m}=1)$ indicates a maximum coarticulation between consonants and vowels (i.e., minimal resistance of the coarticulation), while a weak slope $(\mathrm{m}=0)$ indicates absence of coarticulation between consonants and vowels (maximum resistance of the coarticulation). The bond between the degree of coarticulation and the RV can be explained according to the linear relation between F2onset and F2milieu: the modifications of values of F2milieu will affect those of F2onset and consequently those of the slopes of. In this study, the analysis of the vocalic reduction and slopes of the equations of locus, carried out on CV (extracts starting from sentences) in standard Arabic pronounced by speakers having different mother tongues (near to Arabic standard and very far away from standard Arabic), and at speed of variable elocution, revealed a vocalic reduction and a variation of the slope of the locus equation, specific to each speaker, who seems to be related to his mother tongue. El Tamimi (2006) carried out a similar study with normal flow, in dialectical Arabic and in French, with normal flow, an influence of the mother tongue showed on the vocalic reduction and the slope of the equation of locus.

5aSCc6. A comparison between two cases of voicing neutralization: Final devoicing and voicing assimilation in obstruent clusters. Rebeka Campos-Astorkiza (Ohio State University, Dept. Spanish \& Portuguese, 298 Hagerty Hall, 1775 College Rd, Columbus, OH 43210, USA, camposastorkiza.1@osu.edu)

This study compares two processes that result in voicing neutralization, namely final obstruent devoicing and regressive voicing assimilation in obstruent clusters in Lithuanian. The goal is to find out whether both neutralizing environments present similar acoustic patterns. Acoustic data was collected from native speakers of Lithuanian in order to analyze $/ \mathrm{k} /$ and $/ \mathrm{g} /$ in word final position (final devoicing) and in the word medial clusters /gs/, $/ \mathrm{ks} /, / \mathrm{kz} /$ and $/ \mathrm{gz} /$ (voicing assimilation). Three main acoustic cues to obstruent voicing were measured: obstruent closure duration, voicing during closure and duration of preceding vowel. The statistical results show that, although both final devoicing and voicing assimilation result in incomplete voicing neutralization, they use the acoustic cues differently. In the case of voicing assimilation, there are statistically significant differences in closure duration, voicing during closure and preceding vowel duration between the stops in $/ \mathrm{ks}, \mathrm{gz} /$ and their assimilated counterparts in $/ \mathrm{gs}, \mathrm{kz} /$. As for final devoicing, underlyingly voiceless obstruents and devoiced obstruents show differences in their closure duration and voicing during closure but not in their preceding vowel duration. These results suggest that final devoicing and voicing assimilation have different acoustic realizations in Lithuanian, supporting their analysis as two different processes.

5aSCc7. Effectiveness of prosodic features in the perception of mandarin utterance-final particles. Bin $\mathrm{Li}$ (Department of Chinese, Translation \& Linguistics, City University of Hong Kong, 83 Tat Chee Ave. Kowloon Tong, 220 Hong Kong, China, binli2@cityu.edu.hk), Jinping Zhu (TsingHua University, 104 Main Acadamic building, 100084 Beijing, China, yx@abcd.edu.cn)

Utterance-final particles (UFPs) in Mandarin are regarded as discourse markers, whose "elusive" meanings and functions have been investigated in different perspectives such as pragmatics and semantics. Our study focuses on two most frequently used UFPs: NE and A, both of which can occur in identical utterances. The difference lies in that NE implies a shared common ground between the speaker and the hearer, whereas such commonness lacks for A. A preliminary acoustic analysis found that prosodic features of an utterance were more salient when a UFP was not used. The current study aims at the effectiveness of these prosodic features in perceiving these particles. Native speakers of Mandarin are asked to listen to sentences, which may originally contain a UFP that is removed. Their tasks are to decide whether a UFP, and which one, is needed in a given sentence. It is hypothesized that without textual cues, speakers will reply on prosodic features in their judgment; therefore, they will perform better in sentences where UFPs are not used originally and where prosodic cues are more salient.

5aSCc8. The effect of learning on listening to ultra-Fast speech. Takuya Nishimoto (Graduate School of Information Science and Technology, The University of Tokyo, 7-3-1 Hongo, Bunkyo-ku, 113-8656 Tokyo, Japan, nishi@ hil.t.u-tokyo.ac.jp), Yukika Kariya (Department of Communication, Tokyo Woman's Christian University, 2-6-1 Zenpukuzi, Suginamiku, 167-8585 Tokyo, Japan, g04c043@cis.twcu.ac.jp), Takayuki Watanabe (Department of Communication, Tokyo Woman's Christian University, 2-6-1 Zenpukuzi, Suginami-ku, 167-8585 Tokyo, Japan, nabe@cis .twcu.ac.jp)

We investigated the intelligibility of ultrafast speech which may be used for screen reader for persons with visual disability. The subjects were 35 women who are university students and are not visually/hearing impaired. They were divided into four groups and they listened to 150 words with the speed of approximately $20 \mathrm{morae} / \mathrm{s}$. The vocabulary contained the tasks of high and low familiarity words, and the orders of tasks were different by the groups. Four morae Japanese words from the FW03 database were used as the vocabulary of the recall test. As a result, significant learning effect was observed in cases where the subject listened the high familiarity words in succession. This indicates that the learning effect to the ultrafast speech is promoted when the mental lexical access is easy. We also investigated the mental workloads of the listening task using the NASA-TLX method. As the results, significantly high workload scores were observed at the listening of low familiarity words. The results also suggested that the mental workload decreases when the subject was aware that the mental lexical access was difficult.

5aSCc9. Speech segmentation in monolingual and bilingual infant learners of Canadian English and Canadian French. Linda Polka (McGill University, School of Communication Sciences and Disorders, Beatty Hall, 1266 Pine Avenue West, Montreal, QC H3G 1A8, Canada, linda.polka@mcgill.ca), Jennifer Proulx (McGill University, School of Communication Sciences and Disorders, Beatty Hall, 1266 Pine Avenue West, Montreal, QC H3G 1A8, Canada, Jennifer.proulx@mail.mcgill .ca), Megha Sundara (UCLA Department of Linguistics, 3125 Campbell Hall, Los Angeles, CA 90095-1543, USA, megha.sundara@humnet.ucla .edu)

Speech segmentation skills emerging in infancy are influenced by the infant's native language. English infants favor a stress-based strategy (Jusczyk et al., 1999) whereas French infants favor a syllable-based strategy (Nazzi et al., 2006). Cross-linguistic findings from our lab, based on between group comparisons, show that monolingual 8-month-olds learning either Canadian English or Canadian French segment bisyllabic words in their native lan- 
guage but not in a rhythmically-different non-native language. Canadian French infants fail to segment Canadian English; Canadian English infants fail to segment Canadian French. Bilingual infants (exposed to both languages) were tested in each language on separate days. Although they appear to segment in both languages, attrition has high in the second test session. To assess cross-language segmentation more directly we tested monolingual and bilingual 8-month-olds using a task designed to assess segmentation in both languages in the same infant within a single test session. Findings confirm that monolingual 8-month-olds fail to segment bisyllabic words in a rhythmically-different non-native language. Preliminary data show that bilingual 8-month-olds segment only in the language that is favored in their language input. Thus, in early stages of speech processing all infants appear to develop speech segmentation strategies that are optimal for one language.

5aSCc10. Clear speech intelligibility and accentedness ratings for native and non-native talkers and listeners. Rajka Smiljanic (Northwestern University, Department of Linguistics, 2016 Sheridan Road, Evanston, IL 60208, USA, rajka@ northwestern.edu), Ann Bradlow (Northwestern University, Department of Linguistics, 2016 Sheridan Road, Evanston, IL 60208, USA, abradlow@ northwestern.edu)

This study investigated how native language background (L1) interacts with speaking style in determining levels of speech intelligibility. In four experiments, we explored whether native and non-native hyper-articulation clear speech strategies provide similar intelligibility benefits for native and high proficiency non-native listeners. The sentence-in-noise perception results revealed that native speech was preferred over non-native speech by both listener groups even when non-native talkers and listeners shared the same L1. Clear speech was shown to be beneficial for both the native and fluent non-native listeners. However, non-native clear speech enhanced intelligibility less than native clear speech, supporting the hypothesis that clear speech production strategies involve enhancement of language-specific phonological contrasts. In order to assess the relationship between objective intelligibility measures and subjective accentedness ratings, we obtained accentedness ratings of native and non-native conversational and clear speech by native and non-native listeners. The results showed that objective intelligibility and subjective accentedness were independent. Overall, these results provide strong evidence that clear speech involves language-specific modifications. Nevertheless, native and high proficiency non-native clear speech modifications are generally helpful for both native and high proficiency non-native listeners even when the objective intelligibility and subjective accentedness levels diverged for various listener and talker groups.

5aSCc11. Vietnamese monophthong vowel production by native speakers and American adult learners. Matthew Winn (University of Maryland College Park, Department of Hearing \& Speech Sciences, 0100 Lefrak Hall, College Park, MD 20742, USA, mwinn@hesp.umd.edu), Allison Blodgett (University of Maryland College Park, Center for Advanced Study of Language, 7005 52nd Ave, College Park, MD 20742, USA, ablodgett@casl.umd.edu), Jessica Bauman (University of Maryland College Park, Center for Advanced Study of Language, 7005 52nd Ave, College Park, MD 20742, USA, jbauman@hesp.umd.edu), Anita Bowles (University of Maryland College Park, Center for Advanced Study of Language, 7005 52nd Ave, College Park, MD 20742, USA, abowles@casl.umd.edu), Lykara Charters (University of Maryland College Park, Center for Advanced Study of Language, 7005 52nd Ave, College Park, MD 20742, USA, lykarac@mac.com), Anton Rytting (University of Maryland College Park, Center for Advanced Study of Language, 7005 52nd Ave, College Park, MD 20742, USA, crytting@casl.umd.edu), Jessica Shamoo (University of Maryland College Park, Center for Advanced Study of Language, 7005 52nd Ave, College Park, MD 20742, USA, jshamoo@casl.umd.edu)

This study provides new native speaker data regarding the vowel space and duration contrasts of Vietnamese monophthongs. These data add to existing empirical accounts and raise questions about descriptions aimed at adult language learners. In addition, specific acoustic measures target difficulties that English-speaking adults encounter when producing Vietnamese vowels. These measures include the rounding distinction for back vowels, height distinction for central vowels, and duration distinction between short and long vowels. Results show that adult learners exhibit greater variability and overall less accurate production than native speakers for the high-back unrounded vowel u', with slightly better performance for the mid-back unrounded vowel o', perhaps because it is similar to English / $\Lambda$ /. Additionally, these learners demonstrate varying levels of success with regard to the production of central vowel height but generally fail to distinguish the two vowel duration categories exhibited by native speakers.

5aSCc12. The role of segmental and intonational cues in dialect discrimination. Chad Vicenik (UCLA Department of Linguistics, 3125 Campbell Hall, Los Angeles, CA 90095-1543, USA, cvicenik@humnet.ucla.edu), Megha Sundara (UCLA Department of Linguistics, 3125 Campbell Hall, Los Angeles, CA 90095-1543, USA, megha .sundara@humnet.ucla.edu)

Research indicates that adult listeners are able to use intonation to discriminate between two languages when one of the languages is familiar (Ramus and Mehler, 1999; Pijper, 1983). In this paper, we test adults to determine whether they use segmental or intonational cues to distinguish their native dialect from a foreign one. In three experiments, American English listeners were asked to categorize American and Australian English sentences when (a) segmental and supra-segmental cues are available, (b) sentences are re-synthesized with flat intonation, leaving only segmental cues, (c) segmental information is stripped away, leaving only intonation. Results will be discussed in the context of infant research demonstrating that five month olds are able to distinguish different dialects of the same language (Nazzi, Jusczyk, and Johnson 2000).

5aSCc13. Effects of syllable structure on reaction times in a delayed naming task. Christine Mooshammer (Haskins Lab and MIT Research Lab of Electronics, 300 George Street Suite 900, New Haven, CT 06511, USA, tine@ haskins.yale.edu), Louis Goldstein (Haskins Laboratories, 300 George St., Suite 900, New Haven, CT 06511, USA, goldstein@haskins.yale.edu), Mark Tiede (Haskins Lab and MIT Research Lab of Electronics, 300 George Street Suite 900, New Haven, CT 06511, USA, tiede@ haskins.yale.edu), Hosung Nam (Haskins Laboratories, 300 George St., Suite 900, New Haven, CT 06511, USA, nam@haskins.yale.edu), Man Gao (Haskins Laboratories, 300 George St., Suite 900, New Haven, CT 06511, USA, gao@ haskins.yale.edu)

Syllable complexity has been found to affect the time the speaker needs for planning and initiating utterance production. Shorter latencies for complex onsets $(\mathrm{CCV})$ as compared to simple onsets $(\mathrm{CV})$ have been explained by effects of segment-specific biomechanical constraints at the level of motor execution, and by neighborhood density at the planning level. Within the framework of Articulatory Phonology, shorter planning latencies for CV syllables (compared to $\mathrm{VC}$ ) have been attributed to quicker stabilization for tighter gestural coupling hypothesized for in-phase coupling of the onset consonant and release with the vowel. We attempted to test both onset complexity (C vs $\mathrm{CC}$ ) and coda complexity (open vs. closed syllables) within a single experiment, so that we could evaluate the relative magnitudes of these effects and uncover potential interactions. To do so, American English monosyllabic words varying in syllable structure were presented using a delayed naming paradigm. The results replicated both effects, showing additive effects of roughly comparable magnitudes. Whether these effects are due to planning or motor execution will be tested by further simple naming and picture naming experiments.

5aSCc14. Breathy and whispery voicing in White Hmong. Sean Fulop (California State Univ. Fresno, 5245 N. Backer Ave., Linguistics PB92, Fresno, CA 93705, USA, sfulop@csufresno.edu), Chris Golston (California State Univ. Fresno, 5245 N. Backer Ave., Linguistics PB92, Fresno, CA 93705, USA, chrisg@ csufresno.edu)

Two phonatory strategies are acknowledged to involve a superposition of periodic vocal cord vibration with excessive airflow generating turbulent noise. Breathy voice has the vocal folds vibrating along their entire length, but incomplete closure allows a constant airflow. Whispery voice has the vocal folds vibrating modally along an anterior segment, while the arytenoids maintain a posterior hole which allows a constant airflow. In linguistic phonetics, these are standardly regarded as variant strategies of a 
single "breathy voice" specification, and have never been noted to coexist in one language in an important way. They do in White Hmong. One of the seven "tones" of White Hmong is breathy. Syllables bearing this tone display breathy voicing (of the first type) on the vowel. White Hmong also has a stop $\left[\mathrm{d}^{\mathrm{h}}\right]$, which sounds like whispery voicing during the release phase. In this study, the breathy tone is shown to have a higher $\mathrm{H} 1$ amplitude relative to H2 (compared to modal voice), while the whispery voiced stop is even more extreme in this measure. Whispery voiced stops are also shown to display less harmonicity than the breathy tone, which in turn has less harmonicity than modal voicing in a similar syllable.

5aSCc15. Developing a bilingual communication aid for a Japanese ALS patient using voice conversion technique. Akemi Iida (School of Media Science, Tokyo University of Technology, 1404-1, Katakura-cho, Hachiouji, 192-0982 Tokyo, Japan, ake@media.teu.ac.jp), Shimpei Kajima (Dept. of Electrical and Electronics Engineering, Sophia University, 7-1 Kiyoi-cho, Chiyoda-ku, 102-8554 Tokyo, Japan, s.kajima@gmail.com), Keiichi Yasu (Dept. of Electrical and Electronics Engineering, Sophia University, 7-1 Kiyoi-cho, Chiyoda-ku, 102-8554 Tokyo, Japan, k-yasu@sophia.ac.jp), John M. Kominek (Language Technologies Institute, Carnegie Mellon University, 5000 Forbes Ave. West, Pittsburgh, PA 15213, USA, jkominek@cs.cmu.edu), Yasuhiro Aikawa (1-36-2, Shinjuku, Shinjuku-ku, Shinjuku dai-nana-biru 7F, 160-0022 Tokyo, Japan, aikawa@loopedpicture.com), Takayuki Arai (Dept. of Electrical and Electronics Engineering, Sophia University, 7-1 Kiyoi-cho, Chiyoda-ku, 1028554 Tokyo, Japan, arai@sophia.ac.jp)

A bilingual communication aid for a Japanese amyotrophic lateral sclerosis (ALS) patient has been developed. From our previous research, a corpus-based speech synthesis method was ideal for synthesizing speech with voice quality identifiable as the patient's own. However, a recording of a large amount of speech, which is a burden for the patient, is required for such system. In this study, a voice conversion technique was applied so that a smaller amount of recording is needed for synthesis. An English speech synthesis system with the patient's voice was developed using Festival, a corpus-based speech synthesizer with voice conversion technique. Two methods for Japanese speech synthesis were attempted using HTS toolkit. The first used an acoustic model built from all 503 recordings of the patient. The second used an acoustic model built from 503 wavefiles of which voice was converted to the patient's from a native speaker's. The latter method requires fewer recordings of the patient's. The result of the perceptual experiment showed that the voice synthesized with the latter was perceived to have a closer voice quality to the patient's natural speech. Last, GUI on windows was developed for the patient to synthesize speech by typing in the text.

5aSCc16. Individual differences in perception of emotions from nonsense speech. Sona A. Patel (University of Florida, Dauer Hall, P.O. Box 117420, Dept. of Communication Sciences and Disorders, Gainesville, FL 32611, USA, sona09@ufl.edu), Rahul Shrivastav (University of Florida, Dauer Hall, P.O. Box 117420, Dept. of Communication Sciences and Disorders, Gainesville, FL 32611, USA, rahul@csd.ufl.edu)

Identification of the acoustic cues used to perceive emotions in speech is important for a number of applications including rehabilitation, natural speech modeling, and speech synthesis. In a recent experiment, Patel, Shrivastav, Harnsberger, and Shrivastav (2007) found that a fourdimensional solution accounted for $90 \%$ of the variance in similarity judgments for 19 emotional categories in nonsense speech. This solution was determined for averaged judgments across twelve listeners. The present study investigated individual differences in the perception of emotions for speech devoid of semantic information but rich in suprasegmental cues. Six male and six female listeners participated in a same-different discrimination test of a set of nonsense sentences produced in nineteen emotional contexts by two actors. Nonsense sentences were used in order to avoid any biases caused by semantics. The perceptual distance between each stimulus pair was computed in terms of d' values for each listener. These distances were submitted to a multidimensional scaling analysis using the INDSCAL algorithm. The INDSCAL analysis reports the best fitting solution for all listeners as a group, along with the weights assigned to each dimension by individual listeners. The results of this analysis will be presented.

5aSCc17. Phonological representation of negation in Sakizaya. Wen-Chi Shen (Grad. Inst. of Linguistics, National Taiwan Univ., 1, Roosevelt Rd. Sec. 4, 106 Taipei, Taiwan, r94142007@ntu.edu.tw), Wen-Yu Chiang (Graduate Institute of Linguistics, Grad. Inst. of Linguistics, National Taiwan Univ., 1, Roosevelt Rd. Sec. 4, 106 Taipei, Taiwan, countryroad0509@hotmail.com)

This paper aims at investigating phonological representation of negation in Sakizaya, an endangered Formosan language in Taiwan. Defying YaegerDror's "Cognitive prominence principles" (2003), Chiang (2006) examines negators in Saisiyat, a SVO language as English, and finds the sentential subjects are more prominent acoustically rather than negators. Thus, I will adopt Chiang's modal (2006) and analyze phonological representation of six negators in Sakizaya to see where the pitch accent falls. Besides, if falling on the negators, I will see whether it is because of either cognitive prominence properties or its sentence-initial position? Six informants (three male and three female), from 50-74 years old, participated in the experiment, recorded by DAT, analyzed by Praat and digitized at a $22050-\mathrm{Hz}$ sampling rate. Affirmative and negative sentences are asked in Mandarin while the informants are instructed to translate them into Sakizaya. Besides, they are asked to produce iterative -ay sentences since the negator ca'ay is the most frequently used negator with higher prominent syllable -ay. The results show negators are more prominent in Sakizaya compared to sentential subjects. I conclude it is word order that influences the prominence of pitch contour since Saisiyat is an SVO language and Sakizaya a VSO one.

5aSCc18. The effect of vowel duration on formant frequencies - data from Hakka Chinese. Wai-Sum Lee (Phonetics Lab, Dept. of Chinese, Translation and Linguistics, City University of Hong Kong, 83 Tat Chee Avenue, Kowloon Hong Kong, Hong Kong, w.s.lee@ cityu.edu.hk), Eric Zee (Phonetics Lab, Dept. of Chinese, Translation and Linguistics, City University of Hong Kong, 83 Tat Chee Avenue, Kowloon Hong Kong, Hong Kong, eric.zee@cityu.edu.hk)

In the target undershoot model, vowel duration is considered as the main determinant of vowel reduction, resulting in undershoot in formant frequencies relative to the "bull's-eye formant pattern" (Lindblom, 1963). The model predicts more schwa-like formant frequencies as vowel duration is shortened. In Hakka Chinese, vowel phonemes /i e a o u/ may be realized as long [i: e: a: o: u:] in the CV: syllables and short [i e a o u] in the CVS syllables ( $\mathrm{S}=\mathrm{a}$ stop consonant). Results of a formant frequency analysis of the long and short vowel sets in Hakka Chinese from ten male and ten female speakers show that (i) in all the short vowels there is a displacement of vowel formant frequencies away from the target frequencies associated with the long vowels; and (ii) relative to the target frequencies, F1F2 do not become more schwa-like for all the short vowels, with $[\mathrm{u}]$ being the only vowel that undergoes centralization in the F1F2 plane and with [i e o a] displaying a significant rise in $\mathrm{F}$, [i e] an insignificant decrease in F2, and [o] an insignificant increase in F2. It appears that the Hakka data do not support the target undershoot model.

5aSCc19. An experimental and modeling study of anticipatory coarticulation in VCV sequences. Liang Ma (Labaratoire Parole et Language, 29, Avenue Robert Schuman, 13621 Aix-en-Provence, France, liang.ma@lpl.univ-aix.fr), Pascal Perrier (ICP/GIPSA-lab, INPG, 46 Avenue Félix Viallet, 38031 Grenoble Cedex 01, France, Pascal.Perrier@gipsa-lab.inpg.fr), Jianwu Dang (Japan Advanced Institute of Science and Technology, 1-1 Asahidai, Nomi, 923-1292 Ishikawa, Japan, jdang@jaist.ac.jp)

Anticipatory coarticulation within V1CV2 sequences is studied for EMMA data in French and in Mandarin Chinese. The corpus was designed consistently for both languages. V1 and V2 were /i, a, u/ and C was either $/ \mathrm{t} /$ or $/ \mathrm{k} /$. The influences of $\mathrm{V} 2$ on tongue position of $\mathrm{V} 1$ and of $\mathrm{C}$ were analyzed for three French and two Chinese speakers. For French speakers vowel V2 influences the whole sequence V1CV2, while its influence is strictly limited to the syllable CV2 for Mandarin speakers. These suggested 
that speech planning strategies in French and in Chinese are different and that differences could arise from differences in the status of the syllable in the analyzed languages. A speech production model has been then used for testing quantitatively these conclusions. It involves a forward model and optimization techniques to infer optimal motor commands from the phonemic targets in the perceptual space. Different hypotheses about planning and execution of movement were tested on this model. The comparison of simulations and data supports the hypothesis that the syllable could be a basic unit for planning for Mandarin speakers, while it suggests that larger sequences such as VCV should be considered in the planning of French sequences.

5aSCc20. Psychoacoustic measures of blind audio source separation performance. Mingu Lee (Applied Acoustics Lab., INMC, 132-302, Seoul National University, San 56-1, Sillim-dong, Kwanak-gu, 151-742 Seoul, Republic of Korea, klugh34@acoustics.snu.ac.kr), Inseok Heo (Applied Acoustics Lab., INMC, 132-302, Seoul National University, San 56-1, Sillim-dong, Kwanak-gu, 151-742 Seoul, Republic of Korea, hisherz@acoustics.snu.ac.kr), Nakjin Choi (Applied Acoustics Lab., INMC, 132-302, Seoul National University, San 56-1, Sillim-dong, Kwanak-gu, 151-742 Seoul, Republic of Korea, nakjin@acoustics.snu.ac.kr), Koeng-Mo Sung (Applied Acoustics Lab., INMC, 132-302, Seoul National University, San 56-1, Sillim-dong, Kwanak-gu, 151-742 Seoul, Republic of Korea, kmsung@snu.ac.kr)

In this paper, an improved method for evaluating the performance of blind audio source separation (BASS) is discussed. In previous studies, such as described in E. Vincent et al., IEEE Transactions on Speech and Audio Processing, 2006, several computation methods for measuring quality of BASS algorithms e.g., defined by source-to-distortion ratio (SDR), sourceto-interferences ratio (SIR), sources-to-noise ratio (SNR) and sources-toartifacts ratio (SAR) are introduced. However, those methods do not take human auditory system into consideration. An improved method is developed by applying preprocessing and using weighted-inner product in frequency domain instead of simple inner-product in time domain. The proposed method incorporates well-known psychoacoustic characteristics e.g., masking effect and equal loudness contours. In comparison with the conventional quality measures, the proposed method shows better correlation with the results of carefully designed listening tests.

5aSCc21. The acoustic manifestation of consonant gradation in Northern Sami. Fredrik Karlsson (Umeå University, Department of Language Studies, SE90187 Umeå, Sweden, fredrik.karlsson@ling .umu.se), Kirk P. Sullivan (Umeå University, Department of Language Studies, SE90187 Umeå, Sweden, kirk.sullivan@ling.umu.se), Mikael Svonni (Umeå University, Department of Language Studies, SE90187 Umeå, Sweden, mikael.svonni@samiska.umu.se), Jan Van Doorn (Umeå University, Department of Clinical Sciences, SE90187 Umeå, Sweden, jan.vandoorn@logopedi.umu.se), Ola Wennstedt (Institute for Language and Folklore, Onomastics and Folklore Research in Umeå, Länsmansvägen 5, SE90420 Umeå, Sweden, ola.wennstedt@ sofi.se)

Consonant gradation is a feature of Sami and is realised in Northern Sami in changes from nominative singular to genitive plural form, e.g., "heavdni" (spider) and "heavnni-t" (spiders). The data examined in this paper was collected in 2007 and represents the first acoustic analysis of consonant gradation in Northern Sami. The data was collected in Övre Soppero in Northern Sweden using an elicitation technique that was designed to collect phonetically controlled material for analysis. The nouns were elicited in singular or plural form using picture prompts and the participants were asked to give the word in the carrier sentences "Lea okta _ govas" ("There is a __ in the picture") and "Leat guokte _ govas" ("There are two __ in the picture"). The recordings were labeled in Wavesurfer at a phonetic level and the segments relating to consonant gradation extracted for acoustic analysis. In the paper we present our initial findings.
5aSCc22. The use of fundamental frequency information by cochlear implant patients with residual low-frequency hearing. Stephanie Herf (Arizona State University, Dept. of Speech and Hearing Science, Box 87-0102, Tempe, AZ 85287-0102, USA, spitzer@asu.edu), Julie Liss (Arizona State University, Dept. of Speech and Hearing Science, Box 87-0102, Tempe, AZ 85287-0102, USA, julie.liss@asu.edu), Anthony Spahr (Arizona State University, Lattie F. Coor Hall, Room 3462, Tempe, AZ 85287, USA, Tony.Spahr@Asu.Edu), Michael Dorman (Arizona State University, Dept. of Speech and Hearing Science, Box 87-0102, Tempe, AZ 85287-0102, USA, michael.dorman@asu.edu)

Recent studies have demonstrated significant benefits of complementing the electric hearing provided by a cochlear implant (CI) with residual lowfrequency acoustic hearing. It has been suggested that the benefit of lowfrequency hearing $(<250 \mathrm{~Hz})$ is due to improved representation of fundamental frequency (F0). It was hypothesized that removing F0 information would negatively impact speech perception in bimodal CI patients (EAS) more so when they are using both modes of perception. It was further hypothesized that these patients' more effective use of F0 information would result in higher intelligibility scores and less unpredicted lexical boundary errors (LBEs) in a speech transcription task. Six EAS patients transcribed normal and F0-flattened phrases. Results indicate EAS patients demonstrated a significant benefit from the residual low-frequency acoustic hearing and perform better in this mode. Five of the six EAS patients were able to transcribe the normal phrases with greater accuracy than the flat F0 phrases in both conditions. These patients correctly parsed lexical boundaries and transcribed phonemic targets with greater accuracy in the bimodal condition. These results demonstrate the importance of pitch and low frequency spectral information in the perception of speech.

5aSCc23. Digital acquisition of ultrasound imaging of the tongue. Aude Noiray (Haskins Laboratories, 300 George St., New Haven, CT 06511, USA, aude.noiray@ haskins.yale.edu), Khalil Iskarous (Haskins Laboratories, 300 George St., New Haven, CT 06511, USA, iskarous@ haskins.yale.edu), D.h. H. Whalen (Haskins Laboratories, 300 George St., New Haven, CT 06511, USA, whalen@ haskins.yale.edu)

One of the goals of speech production research is to understand the functioning of the tongue in the production of overlapping lingual segments. This is especially important since all vocalic and most consonantal contrasts involve a tongue gesture. An obstacle to progress in this area has been the difficulty of observing tongue motion in enough spatial and temporal detail to develop theory on its linguistic functioning. Ultrasound has become increasingly popular in tongue imaging, since it allows the researcher to observe most of the tongue dynamically, while being clinically safe. However, a limitation of this technique is the slow $30 \mathrm{~Hz}$ rate of analog acquisition. We have developed a new method for digital acquisition of ultrasound data at over $100 \mathrm{~Hz}$, with simultaneous synchronized acquisition of audio and Optotrak data. Acquisition of the ultrasound digital video data is externally triggered using a novel pulse sequence that is simultaneously captured by the audio and Optotrak acquisition device. We will illustrate the detail of the dynamics of the tongue in an experiment on the coproduction of rhotic and vocalic segments (seven American English subjects), where digital ultrasound was captured at $127 \mathrm{~Hz}$ (Funded by NIH DC-02717).

5aSCc24. Vocal tract normalization in articulatory space using thin-plate spline method. Jianguo Wei (LTCI/CNRS, TSI/ENST, DB407, 37/39, rue Dareau, 75014 Paris, France, wei@enst.fr), Jianwu Dang (Japan Advanced Institute of Science and Technology, 1-1 Asahidai, Nomi, 9231292 Ishikawa, Japan, jdang @jaist.ac.jp)

Inter-subject normalization is a key issue of group analysis of articulatory data to obtain a general description of kinematic properties of human speech production. Multisubject articulatory study however is scarce due to the difficulty of normalization in articulatory domain. In order to reduce intersubject variations among articulatory space, a simple normalization procedure was proposed using a Thin-plate spline method. The purpose of this normalization processing is to reduce the morphological differences of vocal tracts such as shape and size among different subjects. Nonlinear factors of the reduction are considered in this normalization procedure. The electromagnetic Articulographic (EMMA) data were used in our experiments, 
which were obtained from the NTT EMMA database for three subjects data included. A physiological articulatory model has been used to serve as the template. The landmarks were defined consistently in vocal tract space over the template and all subjects. The evaluations showed that the variances over subjects have been reduced $2.1 \mathrm{~mm}$ for consonants and $2.3 \mathrm{~mm}$ for vowels averaged over all tongue pellets.

5aSCc25. A comparison of coarticulation in conversational and clear speech. Jean E. Andruski (Wayne State University, 207 Rackham Bldg, 60 Farnsworth Ave., Department of Communication Sciences and Disorders, Detroit, MI 48202, USA, ag0611@wayne.edu)

Coarticulation may either hinder speech perception by increasing variability and altering the distinctive features of speech sounds, or enhance speech perception by providing additional cues to nearby sounds and spreading these cues out over time. This study examines the clear and conversational speech of 16 English speakers ( 8 females and 8 males) to compare the amount of coarticulation in clear and conversational speech when distinctive features are changed, as opposed to when nondistinctive features are changed. Devoicing of voiced fricatives and voicing of $/ \mathrm{t} / \mathrm{are}$ investigated as examples of distinctive feature changes. Vowel nasalization and lip rounding in $/ \mathrm{s} /$ and $/ \mathrm{z} /$ are examined as examples of nondistinctive feature changes. Percentage of voicing during frication noise is used as a measure of fricative devoicing; voice onset time and percentage of voicing during the closure are used as measures of voicing in $/ \mathrm{t} /$; amplitude of the nasal formant and nasal formant onset time are used as measures of vowel nasalization; and center of gravity of the final $50 \%$ of the frication noise is used as a measure of lip rounding in $/ \mathrm{s} /$ and $/ \mathrm{z} /$. Results are compared across clear and conversational speech and across speaker gender. Individual speaker differences are also examined.

5aSCc26. The development of measurable speech rhythm during second language acquisition. Francisco Gutiérrez Díez (Universidad de Murcia, Departmento de Filología Inglesa, C/ Santo Cristo 1, 30009 Murcia, Spain, fgdiez@ono.com), Volker Dellwo (UCL, Wolfson House, 4, Stephenson Way, NW12HE London, UK, v.dellwo@ucl.ac.uk), Núria Gavaldà (Universitat de Barcelona, Departament de Filologia Anglesa i Alemanya, Granvia de les Corts Catalanes 585, 08007 Barcelona, Spain, nuria.gavalfe@gmail.com), Stuart Rosen (UCL, Wolfson House, 4 Stephenson Way, NW12HE London, UK, stuart@phon.ucl.ac.uk)

It has been demonstrated repeatedly that durational characteristics of consonantal $(\mathrm{C})$ and vocalic $(\mathrm{V})$ intervals are robust acoustic correlates of rhythm class (stress-timed, syllable-timed, mora-timed). Here, we investigate how objective rhythm measures develop during the acquisition of a second language. In a longitudinal study, nine native speakers of Spanish were recorded reading a text in English before and after a year of English language training at university level. A control group of nine native English speakers read the same text. Automatic forced alignment of speech segment boundaries using hidden Markov models allowed the calculation of $\mathrm{C}$ and $\mathrm{V}$ interval durations. Standard rhythm metrics (\%V, deltaC, deltaV, PVI) were calculated for all recordings. First results show that durational $\mathrm{C}$ interval characteristics between native English and Spanish English (pre- and posttraining) do not differ. However, V interval characteristics (deltaV, nPVI, $\% \mathrm{~V}$ ) are lowest for English natives, higher for the Spanish post training group and highest for the Spanish pretraining group. The results suggest that (a) deficits of speech rhythm competence in a second language are mostly revealed on a vocalic level and (b) an increase in competence in a second language is reflected well by measurable speech rhythm.

5aSCc27. Transforming modal voice into irregular voice by amplitude scaling of individual glottal cycles. Tamás Bőhm (Dept. of Telecommunications and Media Informatics, Budapest Univ. of Technology and Economics, Magyar Tudósok krt. 2, H-1117 Budapest, Hungary, bohm@tmit.bme.hu), Nicolas Audibert (GIPSA-lab, Speech \& Cognition Dept (ICP), INPG/UJF/Stendhal/CNRS UMR 5216, Université Stendhal, 1180 av. Centrale, 38040 Grenoble Cedex 9, France, Nicolas.Audibert @gipsa-lab.inpg.fr), Stefanie Shattuck-Hufnagel (Massachusetts Institute of Technology, Research Laboratory of Electronics, Speech Communication Group, 77 Massachusetts Ave., Cambridge, MA 02139, USA, stef@speech.mit.edu), Géza Németh (Dept. of Telecommunications and Media Informatics, Budapest Univ of Technology and Economics, Magyar Tudósok krt. 2, H-1117 Budapest, Hungary, nemeth@tmit.bme .hu), Véronique Aubergé (GIPSA-lab, Speech \& Cognition Dept. (ICP), INPG/UJF/Stendhal/CNRS UMR 5216, Université Stendhal, 1180 av. Centrale, 38040 Grenoble Cedex 9, France, Veronique.Auberge@icp.inpg.fr)

Irregular phonation can serve as a cue to segmental contrasts and prosodic structure as well as to the affective state and identity of the speaker. Thus algorithms for transforming between voice qualities, such as regular and irregular phonation, may contribute to building more natural sounding, expressive and personalized speech synthesizers. We describe a semiautomatic transformation method that introduces irregular pitch periods into a modal speech signal by amplitude scaling of the individual cycles. First the periods are separated by windowing, then multiplied by scaling factors, and finally overlapped and added. Thus, amplitude irregularities are introduced via boosting or attenuating selected cycles. The abrupt, substantial changes in cycle lengths that are characteristic of naturally-occurring irregular phonation can be achieved by removing (scaling to zero) one or more consecutive periods. A freely available graphical tool has been developed for copying stylized pulse patterns (glottal pulse spacings and amplitudes) from an irregular recording to a regular one, allowing the scaling factors to be refined and the waveform regenerated interactively. We present the effects of the transformation on harmonic structure, and perceptual test results showing that transformed signals are similar to natural irregular recordings in both roughness and naturalness.

5aSCc28. Speech transcription for Embodied Conversational Agent animation. Leila Zouari (Telecom Paris Tech, 46 rue Barrault, 75013 Paris, France, zouari@enst.fr), Gerard Chollet (Telecom Paris Tech, 46 rue Barrault, 75013 Paris, France, chollet@enst.fr)

This article investigates speech transcription within a framework of Embodied Conversational Agent (ECA) animation by voice. The idea is to detect some pronounced expressions/keywords in order to animate automatically the face and the body of an avatar. Extensibility, speed and precision are the main constraints of this interactive application. So after defining the set of the relevant words (to the application), a fast large vocabulary speech recognition system was developped and the keyword detection was evaluated. In order to fasten the recognition system without decreasing its efficiency, the acoustic models have been shortened by an original process. It consists in decreasing the number of shared central states of context dependant models which are considered stationary. The shared states situated in the border of the models remain inchanged. Then all the models are retrained. The system is evaluated on an hour of the ESTER database (a French broadcast news database). The experiments show that reducing the number central states of triphones is advantageous. Indeed, the length of models is reduced by $20 \%$ with no loss of accuracy.

5aSCc29. A cross-dialect acoustic description of vowels: Peruvian versus European Spanish. Katerina Chladkova (Palacky University Olomouc, Dept. of English and American Studies, Krizkovskeho 10, 77180 Olomouc, Czech Republic, katerina.chladkova@seznam.cz), Paola Escudero (University of Amsterdam, Institute of Phonetic Sciences, Spuistraat 210, 1012 VT Amsterdam, Netherlands, P.R.EscuderoNeyra @uva.nl), Paul Boersma (University of Amsterdam, Institute of Phonetic Sciences, Spuistraat 210, 1012 VT Amsterdam, Netherlands, P.P.G.Boersma @uva.nl)

This paper aims to provide acoustic description and comparison of vowel properties of two major dialects of Spanish: Peruvian and European. The method of data collection and acoustic analyses reported in Escudero et al. (submitted) for Portuguese is replicated in order to allow for future comparisons across languages. Each of the five Spanish monophthongs /i, e, a, $\mathrm{o}, \mathrm{u} / \mathrm{was}$ produced in stressed position in the first syllable of a CVCV (consonant-vowel-consonant-vowel) frame. The CVCV words were read both in isolation and in a carrier sentence. Vowel tokens were examined between voiceless stops /p, t, k/ and fricatives /f, s/, which supplied five 
variable consonantal contexts. We analyzed the vowels' first and second formants (F1 and F2) as well as their fundamental frequency and duration. Unsurprisingly, a main effect of vowel identity and gender on formant values was found. Most importantly, unlike Morrison and Escudero (2007), we found for Spanish an interaction between dialect and vowel identity, implying that the $\mathrm{F} 1$ and $\mathrm{F} 2$ values of some vowels are significantly different between dialects.

5aSCc30. Contextual influences: Perception of sentences in noise is facilitated similarly in young and older listeners by meaningful semantic context; neural correlates explored via functional magnetic resonance imaging (fMRI). Heather Macdonald (Queen's University, Dept Psychology, 62 Arch Street, Kingston, ON K7L 3N6, Canada, hpmacdonald@gmail.com), Matthew H. Davis (MRC CBU, 15 Chaucer Rd., CB2 7EF Cambridge, UK, matt.davis@mrc-cbu.cam.ac.uk), Kathy Pichora-Fuller (University of Toronto Mississauga, 3359 Mississauga rd N., Mississauga, ON L5L 1C6, Canada, k.pichora.fuller@utoronto.ca), Ingrid S. Johnsrude (Queen's University, Dept Psychology, 62 Arch Street, Kingston, ON K7L 3N6, Canada, ingrid.johnsrude@queensu.ca)

Meaningful semantic context has been demonstrated to improve comprehension of spoken sentences by young and old adults, especially in difficult listening conditions. Evidence for this benefit is based largely on data collected using SPIN sentences, highly structured sentences with a predictable or unpredictable final word. We asked young (14 participants, aged 18-25) and older adults (20 participants, aged 60-75) to report entire sentences which were less structured in nature and contained either a meaningful or anomalous global semantic context. Sentences were mixed with signalcorrelated noise, at 9 signal-to-noise ratios ( -6 to $+2 \mathrm{~dB}$ ), and also presented without noise. Comprehension by both groups benefited from meaningful context, without a clear overall difference in the amount of benefit obtained. We used fMRI to look at neural activity associated with deriving benefit from meaningful context. Whole-brain EPI data were acquired from young (16 participants, aged 19-26) adults using a sparse imaging design. Wordreport data obtained in the scanner were used as intelligibility measures in data analysis. In addition to activity associated with meaningful context, we observe a large left inferior-frontal region in which BOLD signal correlated more strongly with the intelligibility of anomalous compared to coherent prose, presumably reflecting challenged semantic integration processes.

5aSCc31. Landmark-based analysis of sleep-deprived speech. Suzanne Boyce (Department of Communication Sciences and Disorders, University of Cincinnati, Mail location 0394, Cincinnati, OH 45267, USA, boycese@email.uc.edu), Joel Macauslan (S.T.A.R. Corporation, 54 Middlesex Tnpk, Bedford, 01730, USA, joelM@starspeech.com), Ann Bradlow (Northwestern University, Department of Linguistics, 2016 Sheridan Road, Evanston, IL 60208, USA, abradlow@northwestern.edu), Rajka Smiljanic (Northwestern University, Department of Linguistics, 2016 Sheridan Road, Evanston, IL 60208, USA, rajka@northwestern.edu)

The speech of sleep-deprived persons has been variously described as more "slurred" or "tired-sounding" than speech by the same speakers in a rested condition. Although sleep deprivation is a major focus of research for public safety and health reasons, there has been notably little research on the connection between sleep deprivation and speech. Using an automatic system for detecting "landmarks", i.e., important perceptual and articulatory events in the acoustic signal, we compared sleep-deprived and rested speech recorded during two well-controlled studies of sleep deprivation. Our results show that sleep-deprived speech is significantly different from rested speech for both the number and pattern of landmarks detected. These results are similar in detail to differences previously found between clear and conversational styles of speech. The relevance of these findings for speech as a marker of sleep-deprivation will be discussed, along with potential applications in public safety and health. [Research supported by NIH.]
5aSCc32. Vowel duration as a cue for coda voicing and the perception of second-language vowel quantity. Vaclav Jonas Podlipsky (Dept. of English and American Studies, Palacky Univ., Krizkovskeho 10, 77180 Olomouc, Czech Republic, vaclavjonaspodlipsky@centrum.cz)

When acquiring the phonology of a second language (L2), a learner may have to dissociate a perceptual cue from what it marked in the first language (L1) and attach it to another linguistic entity. This study examined the acquisition of Czech vowel quantity by native speakers of American English. In Czech, vowel duration is reserved for cuing short/long vowel (V/V:) contrasts, while in English it is an important cue for coda obstruent voicing. It is not certain whether in Czech, as in many other languages, voiced codas lengthen the preceding vowels; if they do, a vowel should be more likely to be perceived as short before a voiced obstruent and as long before a voiceless one. A perceptual $\mathrm{V} / \mathrm{V}$ : categorization experiment showed that for Czech listeners $(\mathrm{n}=54)$ the $\mathrm{V} / \mathrm{V}$ : boundary came slightly $(3.3 \mathrm{~ms})$ but consistently $(\mathrm{p}<0.001)$ later before a voiced coda. If English learners transfer L1 perceptual strategies to their L2 Czech, the V/V: boundary shift should be even larger. However, this study found no significant effect of coda voicing on the perception of vowel quantity for the non-native listeners. It is concluded that the learners redefined the value of vowel duration as a perceptual cue.

5aSCc33. Talker-specific and accent-general perceptual adaptation to Korean-accented English. Sabrina K. Sidaras (Dept. of Psychology, Emory University, 532 Kilgo Cir, Atlanta, GA 30322, USA, ssidara@emory.edu), Jessica E. Alexander (Dept. of Psychology, Emory University, 532 Kilgo Cir, Atlanta, GA 30322, USA, jessica.alexander@emory.edu), Lynne C. Nygaard (Dept. of Psychology, Emory University, 532 Kilgo Cir, Atlanta, GA 30322, USA, lnygaar@emory .edu)

The present study investigated the relative contribution of talker-specific and accent-general learning to the perceptual adaptation of accented speech. Adult native English-speaking listeners were asked to transcribe Koreanaccented English words in a brief, high variability perceptual learning paradigm. During training, listeners transcribed individual words produced by six Korean-accented speakers and were given feedback on their transcription performance. Controls were either trained with native English speakers or were given no training. At test, listeners were presented with Korean-accented speech produced by both familiar and unfamiliar talkers. The results suggest that listeners learned accent-general properties of nonnative speech. Listeners showed better transcription performance for both familiar and unfamiliar accented talkers relative to English and no training controls. However, talker-specific properties such as individual talker familiarity and baseline intelligibility also influenced transcription performance at test. These findings suggest that although listeners are sensitive to multiple sources of variation in speech, they appear to engage in accent-general perceptual adaptation to non-native speech.

5aSCc34. Effect of musical experience on Mandarin tone and vowel discrimination and imitation. Terry L. Gottfried (Lawrence University, P. O. Box 599, Dept. of Psychology, Appleton, WI 54912-0599, USA, gottfrit@lawrence.edu), Yangqing Xu (Lawrence University, P. O. Box 599, Dept. of Psychology, Appleton, WI 54912-0599, USA, xuy@lawrence .edu)

Recent studies have demonstrated a relation between musical experience and accuracy in speaking and perceiving unfamiliar speech contrasts. For example, Gottfried [in Bohn and Munro, Eds., John Benjamins, 221-237 (2007)) found that native speakers of American English with musical training perform better than non-musicians when discriminating and imitating the four lexical tones of Mandarin Chinese. In this study, four native speakers of Mandarin (Chengdu region) produced target words in a fixed carrier sentence. Their productions of high unrounded /i/ and high rounded /u/ were significantly affected by the initial consonant, especially /u/ (which is often transcribed as two different vowels in pinyin, $\mathrm{u}$ and $\ddot{u}$, according to the initial consonant). Vowels that followed consonants with more anterior articulation (pinyin j, q, x) had lower F1 and higher F2 than vowels that followed more posterior consonants (pinyin $\mathrm{zh}, \mathrm{ch}, \mathrm{sh}$ ). The F0 of the word immediately preceding the target words (kan4) was significantly affected by 
the tone of the target word. Current research tests whether musicians and nonmusicians differ in their ability to discriminate and imitate these phonemic contrasts according to native speakers' phonemic categories, which allow contextual variation in acoustic information.

5aSCc35. Expanding the set of acoustic features of the post-vocalic voicing contrast in English. Blake Rodgers (University of Wisconsin, 1168 Van Hise, 1220 Linden Drive, Madison, WI 53706, USA, brodgers@wisc.edu), Thomas Purnell (University of Wisconsin, 1168 Van Hise, 1220 Linden Drive, Madison, WI 53706, USA, tcpurnell@wisc.edu), Joseph Salmons (University of Wisconsin, 1168 Van Hise, 1220 Linden Drive, Madison, WI 53706, USA, jsalmons@wisc.edu)

The literature on post-vocalic voicing contrasts indicates that no single acoustic characteristic or perceptual cue captures the phonological distinction [e.g., Nittrouer 2004]. Previous perceptual studies examined such acoustic characteristics as vowel duration, percent and duration of closure voicing, formant, and F0 transitions. Detailed acoustic examination reveals additional variation. It is hypothesized that these understudied acoustic characteristics also play a role in the family of voicing cues. In the present study, two laryngeal characteristics are measured and modeled: glottalization (here, an abrupt drop in rate of vocal fold vibration by tensed vocal folds) and a sharp amplitude drop (due to abrupt spreading of vocal folds). Examination of American English data reveals that speakers tend to have at least one of these two characteristics in their final voiceless obstruents, but generally not both. Results suggest a process of laryngeal enhancement of final voiceless obstruents in addition to the traditional cues of vowel duration, formant transitions, etc. Implications for the categorization of laryngeal features and trading relations within the family of final voicing cues are discussed.

5aSCc36. Providing simultaneous visual feedback may ameliorate speech disruptions caused by exposure to delayed auditory feedback. Jeffery Jones (Wilfrid Laurier University, 75 University Ave. West, Waterloo, ON N2L 3C5, Canada, jjones@wlu.ca), Danielle Striemer (Wilfrid Laurier University, 75 University Ave. West, Waterloo, ON N2L 3C5, Canada,dstriemer@gmail.com)

Speakers exposed to delayed auditory feedback (DAF) are often dysfluent. We investigated the possibility that providing visual feedback in addition to DAF would reduce speech disruption. Participants repeated sentences while they heard their auditory feedback delayed with and without simultaneous visual feedback. Replicating previous work, DAF led to increased sentence durations and an increased number of speech disruptions. Results showed that visual feedback did not reduce DAF effects on duration, however, a nonsignificant trend was observed that indicated fewer speech disruptions occurred when visual feedback was provided. This trend was significant in speakers who were overall less affected by DAF. These results suggest the possibility that speakers can strategically use alternative sources of feedback.

5aSCc37. Acoustic characteristics of Swedish dorsal fricatives. Ryan Shosted (University of Illinois, 4080 FLB, 707 S Mathews Ave; MC-160, Urbana, IL 61801, USA, rshosted@uiuc.edu)

The identity of ["hooktop heng"], the "voiceless dorso-palatal/velar fricative" or "simultaneous" ["esh"] and [x], is controversial (IPA 2009: 140, 203). The present study attempts to acoustically differentiate [“esh"], $[\mathrm{x}]$, and (non-labialized) ["hooktop heng"]. A female speaker of Stockholm Swedish produced VCV nonsense words with balanced V and alternating C. Windowed spectra were obtained and averaged at various locations in the fricatives. ["esh"] can be reliably differentiated from $[\mathrm{x}]$ and ["hooktop heng"] based on center of gravity measures in the middle of the fricative. However, center of gravity differences between [x] and ["hooktop heng"] are not revealing, confirming Lindblad's x-ray tracings (1980: 89). The most robust acoustic difference between the two dorsal fricatives is the presence of erratic, high-amplitude disturbances during $[\mathrm{x}]$. These may be attributed to sudden explosions of saliva and ephemeral contact with the uvula, more likely for slightly-retracted [x] than for ["hooktop heng"]. It seems unlikely that ["hooktop heng"] is produced with a second simultaneous constriction that is of acoustic relevance. International Phonetic Association. 1999. Handbook of the International Phonetic Association. Cambridge: Cam- bridge University Press. Lindblad, P. 1980. Svenskans sje- och tje-ljud i ett Allmänfonetisk perspektiv. Travaux de l'Institut de linguistique de Lund 16. Lund: LiberLäromedel/Gleerup.

5aSCc38. Audiovisual speech perception in children with autism spectrum disorders and typical development. Julia Irwin (Haskins Laboratories, 300 George Street, New Haven, CT 06511, USA, julia.irwin @ haskins.yale.edu)

For typically developing perceivers, visual speech information influences what listeners hear. When the place of articulation of visual and auditory speech tokens are incongruent, perceivers often report hearing a visually influenced response (the "McGurk effect", McGurk and MacDonald, 1976). Children with autism spectrum disorders (ASD) appear to be less susceptible to the McGurk effect than their typically developing peers (e.g., Mongillo, Irwin, Whalen, Klaiman, Carter and Schultz, in press). Given the gaze aversion characteristic of children with ASD, eye-tracking methodology was employed to extend our previous research on the McGurk effect to examine: (1) sensitivity to mismatched auditory and visual speech (McGurk), (2) visual gain in the presence of auditory noise, and (3) detection of auditory and visual asynchrony. Children with ASD and their chronological age-matched and verbal mental age-matched typically developing controls (ranging in age from 7-12 years) were assessed. Implications for the development of audiovisual speech processing in typically developing and children with ASD will be discussed. [Work supported by NIH.]

5aSCc39. Attentional modulation of the perception of illusory vowels and sound onsets: A functional magnetic resonance imaging (fMRI) study. Antje Heinrich (University of Cambridge, Department of Linguistics, Sidgwick Avenue, CB3 9DA Cambridge, UK, ah540@ cam.ac.uk), Matthew H. Davis (MRC CBU, 15 Chaucer Rd., CB2 7EF Cambridge, UK, matt.davis@mrc-cbu.cam.ac.uk), Robert P. Carlyon (MRC CBU, 15 Chaucer Rd., CB2 7EF Cambridge, UK, bob.carlyon@mrc-cbu.cam.ac.uk), Ingrid S. Johnsrude (Queen's University, Dept Psychology, 62 Arch Street, Kingston, ON K7L 3N6, Canada, ingrid.johnsrude@queensu.ca)

In a previous study, we exploited the Gestalt principle of closure to create illusory vowel sounds and examined the neural correlates of their perception using fMRI. When two formants of a synthetic vowel are presented in an alternating pattern, filling the gaps in each formant with bursts of noise causes the formants to be heard as continuous and more vowel-like. When this "Illusion" condition was modified by increasing the formant-to-noise ratio (FNR), the formants were heard as interrupted ("Illusion Break" condition) and less vowel-like. BOLD signal in the Middle Temporal Gyrus (MTG) was greater for Illusion than for Illusion-Break stimuli, reflecting the difference in speechlikeness. Primary auditory areas (PAC) exhibited the opposite pattern, probably because Illusion-Break stimuli contain more perceived sound onsets than the Illusion stimuli. In the current study we examine whether the neural activation to illusory vowels and sound onsets is modulated by attention. Participants were scanned while listening to Illusion, Illusion Break, and two types of intact vowels and simultaneously directing their attention either to the vowel stimuli or to auditory or visual distractors. Preliminary analyses suggest that activation to intact and illusory vowels in MTG, and to sound onset in PAC, is modulated by attention.

5aSCc40. Effect of head covers on directivity pattern of human head. Dejan Todoroviæ (Dirigent Acoustics, Takovska 45A, 11000 Beograd, Serby,dgtdejan@yahoo.com)

In this paper are presented the experimental results of changes in directivity patterns of artificial head with three different types of head covers. The mathematical model of human head approximated by radially vibrating spherical cap set in a sphere is discussed. For the purpose of this research, the physical model of a human speaker head is constructed, and the far-field detailed directivity patterns of the model with and without head covers is measured. Hair as porous absorber, cap and straw hat, as sound barriers, are discussed, and their influence on sound wave propagation is considered. Measured detailed directivity pattern changes affected by head covers in far 
field are calculated in step of ten degrees for spherical coordinates (polar angle and azimuth) and presented in form of two- and three-dimensional polar plots.

5aSCc41. English and Polish glides compared. Anna Balas (Adam Mickiewicz University, al. Niepodleglosci 4, 61-874 Poznan, Poland, abalas @ifa.amu.edu.pl)

This paper compares acoustic features of (1) Polish and English glides before vowels, and (2) Polish glides in vowel-plus-glide sequences with English diphthong offglides. Contrary to traditional articulatory descriptions, the results presented, based on acoustic measurements and vector length calculations, do not reveal that Polish glides in vowel-plus-glide sequences are characterized by more extreme formant values than English diphthong offglides. The difference between English diphthongs and Polish vowelplus-glide sequences is attributed to timing properties, which are shown to be relatively easily acquired by Polish learners of English, probably because of a universal tendency for the first element to be longer than the second one. Preliminary results, however, point to acoustic differences between Polish glides before and after vowels, the former being more consonantal than the latter.

5aSCc42. Correlations among three audiovisual (AV) speech tasks Julia Irwin (Haskins Laboratories, 300 George Street, New Haven, CT 06511, USA, julia.irwin@ haskins.yale.edu), Lawrence Brancazio (Southern Connecticut State University and Haskins Laboratories, 300 George Street, New Haven, CT 06511, USA, brancaziol @ southernct.edu)

Visual speech information influences what listeners hear. When the place of articulation of visual and auditory speech tokens are incongruent, perceivers often report hearing a visually influenced response (the "McGurk effect", McGurk and MacDonald, 1976). However, individual differences in this visual influence are poorly understood. Extending work by Grant \& Seitz (1998) and Conrey and Pisoni (2006), we examined correlations between susceptibility to the "McGurk effect" and performance on three related audiovisual tasks. (1) AV speech in noise: we assessed visual gain by comparing word identification in audio-only and AV conditions. (2) AV asynchrony detection: Participants made asynchrony judgments of speech and nonspeech stimuli with asynchronies ranging from $+250 \mathrm{~ms}$ visual to $+250 \mathrm{~ms}$ auditory lead. The speech stimuli were CV syllables and the nonspeech stimuli consisted of lissajous circles paired with sine waves. In one set of nonspeech stimuli, the lissajous was modeled on the lip aperture of the CV and the sine wave, amplitude and frequency were derived from the CV. For the other set, the lissajous and sine wave were derived from clapping hands. (3) Speechreading: Participants identified isolated words presented visually Factors associated with a strong McGurk effect will be discussed. [Work supported by NIH.]

5aSCc43. Factors influencing French schwa deletion and duration: A corpus-based study. Audrey Bürki (Laboratoire de Psycholinguistique, Université de Genève, 40, Bvd Pont d'Arve, 1201 Geneva, Switzerland, audrey.buerki@pse.unige.ch), Mirjam Ernestus (Radboud University Nijmegen \& Max Planck Institute for Psycholinguistics, P.O. Box 310, 6500 Nijmegen, Netherlands, Mirjam.Ernestus@mpi.nl), Cécile Fougeron (Laboratoire de Phonétique et Phonologie, UMR 7018, CNRSParis 3/Sorbonne Nouvelle, 19 rue des Bernardins, 75005 Paris, France, cecile.fougeron@univ-paris3.fr), Cédric Gendrot (Laboratoire de Phonétique et Phonologie, UMR 7018, CNRS-Paris 3/Sorbonne Nouvelle, 19 rue des Bernardins, 75005 Paris, France, cedric.gendrot@univ-paris3.fr), Ulrich H. Frauenfelder (Laboratoire de Psycholinguistique, Université de Genève, 40, Bvd Pont d'Arve, 1201 Geneva, Switzerland, ulrich.frauenfelder@pse unige.ch)

Numerous factors have been advanced to account for the alternance of schwa with zero in French, such as lexical frequency, sonority, syllable structure, phonotactic constraints, etc. ... In the present study we use a recently developed statistical modeling technique, mixed effects models with crossed random effects, to capture the relative contributions of multiple linguistic factors on both, schwa deletion and schwa's physical duration when present. This study is based on the analysis of 4300 occurrences, extracted from the production of more than 300 speakers in a broadcast corpus. The implications of these findings for the debate on the nature of the processes involved in schwa deletion as well as for the nature of lexical representations will be discussed.

5aSCc44. Perceptual clarity of speech modulates activity in left temporal-lobe regions: fMRI correlates of top-down influences. Conor Wild (Queen's University, Centre for Neuroscience Studies, 62 Arch Street, Kingston, ON K7L3N6, Canada, 9cw13@queensu.ca), Matthew H. Davis (MRC CBU, 15 Chaucer Rd., CB2 7EF Cambridge, UK, matt.davis@mrc-cbu.cam.ac.uk), Alexis G. Hervais-Adelman (Centre for the Neural Basis of Hearing, Department of Physiology, Development and Neuroscience, University of Cambridge, Downing Site, CB23EG Cambridge, UK, alexis.hervais-adelman@mrc-cbu.cam.ac.uk), Ingrid S. Johnsrude (Queen's University, Dept Psychology, 62 Arch Street, Kingston, ON K7L 3N6, Canada, ingrid.johnsrude@queensu.ca)

Behavioral evidence supports the idea that perception is guided by mechanisms that compute an input's most probable interpretation. For example, four-band noise-vocoded speech, which is largely unintelligible to naive listeners, becomes perceptually clear when listeners possess prior knowledge of the signal content - a phenomenon we call "pop-out". Feedback connections within and among auditory cortical regions may allow cognitively "higher" levels of processing to predict and thus "explain" the bottom-up signal. In the present study, we use fMRI to investigate the network underlying pop-out. We use written primes (matching or nonmatching text strings) to manipulate a subject's perception of single noise-vocoded words, thereby creating acoustically matched conditions that elicit the perceptions of intelligible or unintelligible speech. Whole brain fMRI data were gathered from 21 subjects using a sparse-imaging procedure. Preliminary analysis reveals regions of statistically significant signal change along the left superior and middle temporal gyri and superior temporal sulcus for matching trials that induce perceptual popout. Functional connectivity analysis will allow us to investigate coupling among these auditory regions, and how connectivity is modulated depending on the perceptual coherence of a noise-vocoded utterance.

5aSCc45. Some acoustic cues in the detection of the Nepalese aspiration. Rajesh Khatiwada (LPP UMR CNRS- 7018 Université Sorbonne Nouvelle, 19 rue des bernardins, 75005 Paris, France, rajesh .khatiwada@free.fr)

In terms of laryngeal setting, most languages have only two types of stops. In the Nepalese sound inventory there are four types of stops: voiceless unaspirated, voiced unaspirated, voiceless aspirated, and voiced aspirated (or breathy voiced). The main objective of this paper is to present the prominent and consistent acoustic cues that differentiate the aspirated segments from their unaspirated counterparts of the Nepalese coronals - the dental-alveolar (t,th,d,dh), retroflex (T,Th,D,Dh) and affricates (ts,tsh,dz,dzh). VOT (Voice Onset Time) (Lisker and Abramson 1964) is the familiar model used to characterize three of these phonation types - voiced, voiceless, and aspiration stops. Some authors have pointed out that this method is not able to distinguish the plain voiced and voiced aspirated stops. An alternative segmentation model proposed by Mikuteit and Reetz (2007) is adopted here. Beside the analysis of the closure duration of segments, measuring the beginning of the burst or its release, we use other acoustic cues to characterize Nepalese aspiration such as F0 lowering, the structure of the lower harmonics and spectral tilt (Hanson 1997, Stevens 1998). This experimental work is based on the corpus based on four native speakers of Nepali.

5aSCc46. Prosodic realization of focus on verbal phrases in two Chinese dialects. Yiya Chen (LUCL, Phonetics Laboratory, Cleveringaplaats 1, P. O. Box 9515, 2300 RA Leiden, Netherlands, yiya.chen@let .leidenuniv.nl)

A well-established observation in West-Germanic languages is that when a verbal phrase (e.g., likes movies) is uttered as new information (i.e., focused), speakers often accent (via F0 changes) the noun only (e.g., Ladd 1980 and 1996). Listeners, in turn, find that a single accent on noun sounds as appropriate as when both verb and object are accented (e.g., Gussenhoven 
1987, Birch and Clifton 1995, Welby 2003). This study will report results of an experiment which was designed to examine the prosodic realization of focus on verbal phrases in two tonal dialects - Beijing Mandarin and Shanghai Chinese. Both dialects use F0 changes to indicate word meanings, but they differ in tone sandhi patterns. That is, they differ in how words group into phrasal tonal domains and how lexical tones undergo changes within the domain. Two types of focus were elicited: One provides new information to Wh-questions and the other corrections of wrong information in the preceding discourse. The goal of the study is to contribute to the development of a cross-linguistic theory of the prosodic realization of focus. [Work supported by the VENI research grant from the Netherlands Organization for Scientific Research.]

5aSCc47. On nasal onset time (NOT) duration of French nasal vowels in function of the preceding consonant types. Julie Montagu (Laboratoire de Phonétique et Phonologie UMR 7018CNRS/Université Paris 3, 5, rue des Bernardins, 75005 Paris, France, julie _montagu@yahoo.com)

Aerodynamic requirement, a high intra-oral air pressure (Pio), for the production of stop and fricative consonants delays the nasalization (velum opening) of the subsequent nasal vowel. We call this time delay between the onset of the nasal vowel and that of its nasalization as nasal onset time (NOT). The NOT was expected to be longer after voiceless consonants (higher Pio) than after voiced counterparts. The measurement of NOT on simultaneous recordings of speech and nasal signals for 24 Parisian speakers have unexpectedly shown that a greater NOT after voiced stops $(38 \mathrm{~ms}$ in average corresponding to $17 \%$ of total nasal vowel duration) and voiced fricatives $(7 \%)$ than after voiceless ones, respectively, $14.5 \%$ for stops and $4.1 \%$ for fricatives. This order is observed regardless of changes in speech rate: NOT is $11.1 \%$ with the voiced and $9.3 \%$ with the voiceless stops in slow, $17 \%$ and $14.5 \%$ in normal, and $22.4 \%$ and $18.6 \%$ in rapid rate. These results suggest that not only the Pio, but also some other factors are involved in the determination of NOT. We shall discuss possible such factors in perception and in production.

5aSCc48. Sustained and transient brain activations in bilingual control. Yapeng Wang (State Key Laboratory for Cognitive Neuroscience and Learning, Beijing Normal University, 100875 Beijing, China, Institute for Learning and Brain Sciences, University of Washington, Seattle, WA 98115, USA, yapengw@u.washington.edu), Patricia K. Kuhl (University of Washington, Dept. of Speech \& Hearing Sciences, and Institute for Learning \& Brain Sciences, Box 357988, Seattle, WA 98195, USA, pkkuhl@u.washington.edu), He Li (State Key Laboratory for Cognitive Neuroscience and Learning, Beijing Normal University, 100875 Beijing, China, Institute for Learning and Brain Sciences, University of Washington,
Seattle, WA 98115, USA, her2003@163.com),Qi Dong(State Key Laboratory for Cognitive Neuroscience and Learning, Beijing Normal University, 100875 Beijing, China, Institute for Learning and Brain Sciences, University of Washington, Seattle, WA 98115, USA, dongqi@bnu.edu.cn)

Behavioral and neuroimaging evidence shows that bilinguals experience interference and competition during bilingual processing. The neural basis of bilingual language control is not well understood. Using mixed blocked and event-related design, the present study explored the sustained and transient activations during bilingual control. 15 Chinese-English bilingual speakers were scanned when they performed language switching tasks. The results showed that, compared to single language condition, the mixed language condition (sustained control) induced the activation in the bilateral prefrontal (BA6/BA8/BA10), middle frontal (BA45/46) and parietal lobes (BA7/ BA40/BA49); In contrast, relative to the no switch condition, language switching (transient control) activated the left superior, inferior parietal lobe (BA2/ BA40) and middle frontal (BA11/46). These results suggested that sustained and transient language control induced differential lateral activation patterns.

5aSCc49. Automatic acoustic analysis of substitute speech characteristica by speech recognition technique. Maria Schuster (Dpt. Phoniatrics and Pedaudiology, Bohlenplatz 21, 91054 Erlangen, Germany, maria.schuster@uk-erlangen.de), Andreas Maier (Dpt. Phoniatrics and Pedaudiology, Bohlenplatz 21, 91054 Erlangen, Germany, maier@informatik.uni-erlangen.de), Tino Haderlein (Dpt. Phoniatrics and Pedaudiology, Bohlenplatz 21, 91054 Erlangen, Germany, tino.haderlein@informatik.uni-erlangen.de), Ulrich Eysholdt (Dpt. Phoniatrics and Pedaudiology, Bohlenplatz 21, 91054 Erlangen, Germany, ulrich.eysholdt@uk-erlangen.de), Elmar Noeth (Dpt. for Pattern Recognition, Martensstr. 5, 91052 Erlangen, Germany, noeth@informatik.unierlangen.de)

Tracheo-esophageal (TE) substitute speech is often used after total laryngectomy as it resembles normal speech more than other substitute speech methods. However, TE speech still shows restrictions and is perceived as hoarse, rough, with strain, and usually monotone, e.g., due to augmented irregularity of the voice signal. Commonly used diagnostic tools to describe the acoustic properties of TE speech are mostly not applicable. Before, automatic speech recognition has been shown to serve as a appropriate acoustic tool for the quantification of TE speech intelligility. We now applied an automatic speech recognition system with prosody module on speech data of 41 laryngectomees. The prosody module extracted 150 parameters. Factor analysis revealed five principal components. They show high correlation to perceptively described TE speech characteristica. Automatic prosody analysis by speech recognition technique allows to detect and quantify characteristics of highly disordered speech. 


\title{
Session 5aUWa
}

\section{Underwater Acoustics, Signal Processing in Acoustics, and ECUA: Broadband Underwater Communications I}

\author{
Heechun Song, Cochair \\ MPL, Scripps Institution of Oceanography, University of California, San Diego, La Jolla, CA 92093-0238, USA \\ Sergio Jesus, Cochair \\ ISR, Universidade do Algarve, Faro, PT-8005-139, Portugal
}

\section{Invited Papers}

\author{
8:00
}

5aUWa1. Multiinput multioutput OFDM for shallow-water UWA communications. Yunus Emre (Arizona State University, Dept. of Electrical Engineering, Tempe, AZ 85287-5706, USA, yunus.emre@ asu.edu), Vinod Kandasamy (Arizona State University, Dept. of Electrical Engineering, Tempe, AZ 85287-5706, USA, vinod.kandasamy@asu.edu), Tolga M. Duman (Arizona State University, Dept. of Electrical Engineering, Tempe, AZ 85287-5706, USA, duman@asu.edu), Paul Hursky (HLS Research, Inc., 3366 N. Torrey Pines Ct., Ste. 310, La Jolla, CA 92037, USA, paul.hursky@hlsresearch.com), Subhadeep Roy (Qualcomm Flarion Technologies, Bridgewater, NJ 08807, USA, subhadee@qualcomm.com)

We investigate performance of turbo coded multiple-input multiple-output (MIMO)-OFDM systems with layered space time (LST) architectures for underwater acoustic (UWA) channels by using simulations and results from the AUVfest experiment performed in June 2007. MIMO systems have been shown to be promising in the sense that they increase the reliable transmission rates significantly without consuming additional bandwidth and power. Robustness of OFDM systems with cyclic prefix or zero padding to ISI channels are also well known; so the combination of MIMO techniques and OFDM can be regarded as a promising technology for shallow water UWA communications which is characterized by severe bandwidth limitations and long intersymbol interference (ISI) spans. The paper reviews necessary components of a MIMO-OFDM communication system, including, time and frequency synchronization, channel estimation, and tracking of the varying channel parameters. Modifications necessary to make the system suitable for UWA channels are summarized. Results of the AUVFest 2007 experiment are very promising; for instance, $2 \times 2$ MIMO-OFDM can reach up to $60 \mathrm{Kbps}$ transmission for a bandwidth of $16 \mathrm{KHz}$ with simple receiver structures for a range of $2000 \mathrm{~m}$. In addition to the coherent system, differential and unitary space-time coded MIMO-OFDM scenarios are also considered.

8:20

5aUWa2. High rate acoustic communications based on orthogonal frequency division multiplexing. Milica Stojanovic (Massachusetts Institute of Technology E38-376, 292 Main St., Cambridge, MA 02139, USA, millitsa@mit.edu)

Orthogonal frequency division multiplexing (OFDM) offers simplicity of FFT-based implementation with frequency-domain channel equalization, and has emerged as a standard in many terrestrial systems. Its application to underwater systems, however, is challenged by the motion-induced Doppler distortion, which creates nonuniform frequency offset in a wideband acoustic signal. To counteract this problem, recent research has focused on synchronization methods, demonstrating the feasibility of wideband OFDM in initial sea trials. We consider the design of a complete OFDM receiver based on three key techniques: adaptive carrier tracking, spatial diversity combining, and sparse channel estimation. In particular, we focus on time-domain channel estimation, which provides a natural platform for channel sparsing. Sparsing of the impulse response, or discarding of its insignificant coefficients, is shown to offer performance gains on acoustic channels, since they are often naturally sparse. Decision-directed operation, which is made possible by adaptive carrier tracking, yields a low overhead, and further improves the performance of channel estimation beyond that of nonadaptive pilot-based schemes. The technique proposed is applied to experimental data recorded in a shallow water channel over $1 \mathrm{~km}$. Results show excellent performance of coded OFDM signals, transmitted in the $19-31 \mathrm{kHz}$ band with a varying number of subcarriers.

$8: 40$

5aUWa3. Experimental assessment of time-reversed OFDM underwater communications. Joao Gomes (ISR - Instituto Superior Tecnico, Av. Rovisco Pais, Torre Norte 7.22, 1049-001 Lisboa, Portugal, jpg @isr.ist.utl.pt), Antonio Silva (ISR, Universidade do Algarve, PT-8005-139 Faro, Portugal, asilva@ualg.pt), Sergio Jesus (ISR, Universidade do Algarve, PT-8005-139 Faro, Portugal, sjesus@ualg.pt)

OFDM communication has recently been demonstrated in underwater channels at rates exceeding $7 \mathrm{kbps}$. This is a popular modulation in wireless communications due to its flexibility for spectrum management and the simplicity of transmitters/receivers. Despite widespread interest, experimental data on the performance of underwater OFDM are scarce. This work aims to contribute to a better understanding of this technique by examining results from the UAB'07 experiment, conducted in Norway in September 2007. Data were transmitted in a fjord using several formats with bandwidths of 3 and $7.5 \mathrm{kHz}$, and recorded at a range of $800 \mathrm{~m}$ in a 16-hydrophone array. Significant multipath was observed over at least $40 \mathrm{~ms}$, which would call for a rather large OFDM prefix/guard interval and hence drastically reduce the data rate. Passive time reversal is used here as a computationally inexpensive scheme to shorten the channel length 
to less than $10 \mathrm{~ms}$, so that moderate guard intervals can be used with a conventional OFDM receiver. The same preamble/postamble signals used for synchronization and Doppler precompensation are reused as probes for passive time reversal, so the latter entails no loss in efficiency. The performance of time-reversed OFDM is compared with plain QPSK modulation using either time reversal or multichannel equalization at the receiver.

\section{9:00}

5aUWa4. Linear and circular adaptive beamforming arrays for high bandwidth acoustic communications. Jeffrey Neasham (Newcastle University, School of Electrical, Electronic and Computer Engineering, Merz Court, NE1 7RU Newcastle upon Tyne, UK, j.a.neasham@ncl.ac.uk), David Howarth (Tritech International, Morecambe Road, Ulverston, LA12 9BN Cumbria, UK, davidhowarth@tritechulverston.co.uk), Bayan Sharif (Newcastle University, School of Electrical, Electronic and Computer Engineering, Merz Court, NE1 7RU Newcastle upon Tyne, UK, bayan.sharif@ncl.ac.uk), Oliver Hinton (Newcastle University, School of Electrical, Electronic and Computer Engineering, Merz Court, NE1 7RU Newcastle upon Tyne, UK, oliver.hinton@ncl.ac.uk)

This paper will discuss the development of a new generation of high bandwidth (up to $32 \mathrm{kbits} / \mathrm{s}$ ) underwater communication products for ranges up to $4 \mathrm{~km}$. Multipath propagation is the dominant factor in system performance in both shallow water, horizontal channels and deep water, vertical channels. The properties of such channels will be analysed and the design of both linear and circular receiver arrays will be discussed to maximise reliability. We will then describe how these arrays are combined with the latest "software" receiver principles to create a powerful and flexible system, incorporating adaptive signal processing with minimal hardware complexity. Finally the performance of this system will be illustrated with results from experimental trials.

\section{9:20}

5aUWa5. Channel estimation for underwater acoustic communications: sparse channels, soft input data, and Bayesian techniques. James Preisig (Woods Hole Oceanographic Institution, Bigelow 404, MS\#9, Woods Hole, MA 02543, USA, jpreisig@whoi.edu), Ballard Blair (Woods Hole Oceanographic Institution, Bigelow 404, MS\#9, Woods Hole, MA 02543, USA, bjblair@mit.edu), Weichang Li (Woods Hole Oceanographic Institution, Bigelow 404, MS\#9, Woods Hole, MA 02543, USA, wli @ whoi.edu)

The problem of estimating the time-varying impulse response of the communications channel is central to creating reliable and high-rate communications links. Past work has shown that by jointly accounting for channel dynamics and sparse channel characteristics, the accuracy of the channel impulse response estimate is improved with a corresponding improvement in communications systems performance. Following a survey of the basic techniques that have been developed, these basic techniques are extended in two ways. The first allows for a more complete accounting for the channel characteristics via apriori distributions on the probabilities of the complex channel tap values and optimized basis functions for sparse channel structure. The second allows for the use of soft input data in the channel estimation process thus making the techniques feasible for iterative channel estimation and data estimation algorithms. The extensions are developed and their performance compared using data from recent shallow water acoustic communications experiments.

\section{Contributed Paper}

\section{9:40}

5aUWa6. Investigation of modal processing for low frequency acoustic communications in shallow water. Andrey Morozov (Woods Hole Oceanographic Institution, Bigelow 404, MS\#9, Woods Hole, MA 02543, USA, amorozov@whoi.edu), James Preisig (Woods Hole Oceanographic Institution, Bigelow 404, MS\#9, Woods Hole, MA 02543, USA, jpreisig@whoi.edu), Joseph Papp (Woods Hole Oceanographic Institution, Bigelow 404, MS\#9, Woods Hole, MA 02543, USA, jpapp@whoi.edu)

Acoustic receptions at a vertical line array collected during the SW06 experiment were processed to show the feasibility of broadband mode decomposition as a preprocessing method to shorten time-spread and concentrate received signal energy in a small number of independent channels. The vertical array spanned the water column from $12 \mathrm{~m}$ depth to the bottom.
PSK m-sequence modulated signals with different carrier frequencies were transmitted from a distance of $19.2 \mathrm{~km}$. Signals were processed for both the case of ordinary internal waves activity and the case with abnormally strong internal wave solitons. The measured sound velocity and known bottom properties were accounted for. Mode filtering was based on a broadband pseudo-inverse processing of the received VLA signals. The broadband mode filtering decomposed the received signal into a number of independent signals with a reduced time-spread. The constellation of signals from the output of mode filters showed that a simple demodulator can achieve a high quality reception. Even during strong internal waves activity the acoustic energy was concentrated in a small number of the first acoustical modes. The receiver estimated the mode-time intensity distribution and used the strongest modes for demodulation. High quality reception is demonstrated with the data.

10:00-11:00 Posters

Lecture sessions will recess for the presentation of poster papers on various topics on acoustics. See poster sessions for topics and abstracts. 


\section{Contributed Papers}

\section{1:00}

5aUWa7. Basin-scale time reversal communications. Heechun Song (MPL, Scripps Institution of Oceanography, University of California, San Diego, La Jolla, CA 92093-0238, USA, hcsong@ucsd.edu), William A. Kuperman (MPL, Scripps Institution of Oceanography, University of California, San Diego, La Jolla, CA 92093-0238, USA, wkuperman@ucsd.edu), William Hodgkiss (MPL, Scripps Institution of Oceanography, University of California, San Diego, La Jolla, CA 920930238, USA,wsh@mpl.ucsd.edu)

During November 1994, broadband acoustic signals were transmitted from a $75-\mathrm{Hz}$ source to a 20 -element, $700-\mathrm{m}$ vertical array at approximately $3250 \mathrm{~km}$ range in the eastern North Pacific Ocean as part of the Acoustic Engineering Test (AET) of the Acoustic Thermometry of Ocean Climate (ATOC) program [Worcester et al., J. Acoust. Soc. Am. 105, 3185-3201 (1999)]. The AET tomography signal can be treated as a binary-phase shiftkeying (BPSK) communication signal with an information rate of 37.5 bits/s. With the multipath arrivals spanning 5-8 s, this data represents an extreme case of intersymbol interference (ISI). The AET array data are processed using time reversal combined with frequent channel updates to accommodate channel variations over the 20-min long reception, followed by a single channel decision-feedback equalizer (DFE). The almost error-free performance using all 20 array elements demonstrates the feasibility of time reversal communications. Further, comparable performance of single receive element communications integrating over multiple transmissions indicates that the ocean provided temporal diversity as well as the spatial diversity provided by the array.

\section{1:20}

5aUWa8. Coherent time reversal communications in a shallow multipath environment. Slawomir Jastrzebski (University of Technology and Life Sciences, ul. Kordeckiego 20, 85-225 Bydgoszcz, Poland, sj@utp .edu.pl)

Underwater acoustic channel is one of the less reliable communication channels due to its reverberant properties produced by the surface and the bottom of the sea and Doppler spreading caused by the transmitter and the receiver movements. Strong intersymbol interference (ISI) caused by timevarying multipath environments and relatively fast channel variations are two of the major challenges for practical implementation of coherent underwater acoustic communications. In this paper a phase conjugation is considered as a method for mitigating intersymbol interference in coherent communication and this technique reducing the complexity of underwater receivers. Phase conjugation uses time reversal to remove intersymbol interferences. This method performs the time reversal operation in the computer at the receiver instead of time reversed propagation through the sea. This paper presents numerical simulations results of coherent communications using this technique. Phase conjugation processing in acoustic communications was demonstrated with the transmission of BPSK and QPSK modulation schemes. Different messages were sent simultaneously to different depths at different ranges in $100 \mathrm{~m}$ deep shallow water. Simulation results suggest that the phase conjugation technique may be used as a potential application to undersea communications, especially in an environment with significant multipath.

\section{1:40}

5aUWa9. Very broadband high frequency underwater acoustic communications. Lee Freitag (Woods Hole Oceanographic Inst., 266 Woods Hole Road, MS 18, Woods Hole, MA 02543, USA, 1freitag@whoi.edu), Sandipa Singh (Woods Hole Oceanographic Inst., 266 Woods Hole Road, MS 18, Woods Hole, MA 02543, USA, ssingh@whoi.edu), Keenan Ball (Woods Hole Oceanographic Inst., 266 Woods Hole Road, MS 18, Woods Hole, MA 02543, USA, kball@whoi.edu)

Many new applications for underwater observatories and sensor systems do not require long ranges, but do require very power-efficient, highthroughput communications. Recent work in phase-coherent underwater acoustic communications has shown that signal bandwidths of $10-40 \mathrm{khz}$ at carrier frequencies from 80 to $120 \mathrm{khz}$ may be used to achieve throughputs of up to $80 \mathrm{kbps}$ at ranges of $100-500 \mathrm{~m}$ in shallow water. The work has included channel impulse response measurements over time and with respect to range, and application of the adaptive decision feedback equalizer using signals at multiple bandwidths. The broadband, high-frequency propagation environment is characterized, and its impact on the reliability of very wide band signals (relative to the signal carrier) is presented.

\section{2:00}

5aUWa10. Experiments of wideband color image transmission. Hiroshi Ochi (JAMSTEC, 2-15, Natsushima-cho, 237-0061 Yokosuka, Japan, ochi@jamstec.go.jp), Yoshitaka Watanabe (JAMSTEC, 2-15, Natsushima-cho, 237-0061 Yokosuka, Japan, yoshitakaw@jamstec .go.jp), Takuya Shimura (JAMSTEC, 2-15, Natsushima-cho, 237-0061 Yokosuka, Japan, shimurat@jamstec.go.jp), Takehito Hattori (Nippon Marine Enterprises, Ltd., 14-1, Ogawa-cho, 238-0004 Yokosuka, Japan, hattorit @ nme.co.jp)

In JAMSTEC, we have been studying about a high-speed underwater acoustic communication for color image transmission from underwater vehicle of deep water. The objective of our research is to transmit a JPEG compressed color image within once a second. Experiments were carried out at the depth of $1000 \mathrm{~m}$ area. The distance of transmission was $250-600 \mathrm{~m}$. Four omni-directional hydrophones were used for receiving. QPSK and 8PSK were used as its modulation method. The four-channel decision feedback equalizer with phase compensator was applied for the demodulation algorithm. Because of this experiment was carried out at deep sea environment, the communication channel characteristics was relatively simple. In these experiment, when the SNR was higher than $12 \mathrm{~dB}$, error free communication was carried out in case of QPSK. And also when the SNR was higher than $20 \mathrm{~dB}$, error free communication was carried out in case of 8PSK

\section{2:20}

5aUWa11. Robust time-division channel-access approach for an ad hoc underwater network. Kae Yeet Foo (University of Birmingham, Department of Electronic, Electrical and Computer Engineering, Edgbaston, B15 2TT Birmingham, UK, kyf740@bham.ac.uk), Philip Atkins (University of Birmingham, Department of Electronic, Electrical and Computer Engineering, Edgbaston, B15 2TT Birmingham, UK, p.r.atkins@bham.ac.uk), Steve A. Pointer (Qinetiq, Winfrith Technology Centre, DT2 8XJ Dorchester, UK, sapointer@qinetiq.com), Carl P. Tiltman (Dstl, Winfrith Technology Centre, DT2 8WX Dorchester, UK, cptiltman@dstl.gov.uk)

An ad hoc underwater network is formed by a cluster of stationary nodes that can act as a source, destination, or a relay, in which data packets usually travel across multiple hops. Using a time-division scheme where the propagation delay between the nodes is used as a packet queuing buffer optimizes throughput. This requires accurate knowledge of the relative ranges of all the nodes, a high level of accuracy in time-synchronization, and restricts changes in the position of the nodes especially in the absence of a central server or master node within the network. A method that offers greater robustness is by using a time-slotted approach, where each node is offered a time-slot sufficient for one transmission to reach its maximum effective range. A passive acknowledgment scheme is implemented where a node listens for acknowledgment when the transmitted packet is being relayed. Nodes monitor other time-slots for the opportunity to contend for idle slots in order to enhance throughput. Results obtained from simulations demonstrate that this method is robust in supporting changes in the relative distances between the nodes, and can typically operate with a time synchronization error of up to $1 \mathrm{~s}$. 


\title{
Session 5aUWb
}

\section{Underwater Acoustics and ECUA: Environmental Impact on Propagation}

\author{
Bashar Mohammad, Cochair \\ Heriot Watt University, School of Engineering and Physical Sciences, Riccarton, Edinburgh, EH14 4AS, UK \\ Jinshan $\mathrm{Xu}$, Cochair \\ MIT Mechanical Engieerning Department, 77 Massachusett Ave., Cambridge, MA 02139, USA
}

\section{Contributed Papers}

\begin{abstract}
8:00
5aUWb1. Spatial and temporal variations in acoustic propagation in Dabob Bay during PLUSNet'07 Exercise. Jinshan Xu (MIT Mechanical Engieerning Department, 77 Massachusett Ave., Cambridge, MA 02139, USA, jinshan@mit.edu), Pierre Lermusiaux (MIT Mechanical Engieerning Department, 77 Massachusett Ave., Cambridge, MA 02139, USA, pierrel@MIT.EDU), Patrick Haley (MIT Mechanical Engieerning Department, 77 Massachusett Ave., Cambridge, MA 02139, USA, phaley@MIT.EDU), Wayne Leslie (MIT Mechanical Engieerning Department, 77 Massachusett Ave., Cambridge, MA 02139, USA, wgleslie@MIT.EDU), Oleg Logutov (MIT Mechanical Engieerning Department, 77 Massachusett Ave., Cambridge, MA 02139, USA, logutov @ mit.edu)
\end{abstract}

We present spatial and temporal variability of the acoustic field in Dabob Bay during the PLUSNet'07 (Persistent Littoral Undersea Surveillance Network) Exercise. The study uses a 4D (3D in space plus 1D in time) dataassimilative numerical ocean model to provide inputs to an acoustic propagation model. The Havard-Ocean-Prediction-System with in-situ CTD measurement assimilation provided output forecasts with a 300-m and 1-to-5-m resolution in the horizontal and vertical direction, for a 3-h interval within a 15-day period. This environmental data as the input to acoustic modeling allowed prediction and study of the (semi-)diurnal temporal variations of the acoustic field, as well as the varying spatial structures of the field. Using the one-way coupled-normal-mode code, along- and across-sections in the Dabob Bay acoustic field structures at 100,400 , and $900 \mathrm{~Hz}$ were forecasts and studied for various source depths. Interesting propagation effects such as the acoustic transmission-loss fluctuations with respect to the source depth and frequency as a result of the regional ocean variability, wind forcing, and tidal effects are discussed. The novelty of this work lies in the accuracy of the acoustic transmission-loss prediction in the littoral region by physically coupling the real-time ocean prediction system to acoustic modeling. This work also offers a potential extension to a $4 \mathrm{D}$ acoustic modeling.

\section{8:20}

5aUWb2. On shallow water ocean acoustic tomography system based on parametric arrays physical properties evaluation. Andrew Semenov (Acad. N.N. Andreev's Acoustics Institute, Russ. Acad. of Sci., 4 Shvernik Street, 117036 Moscow, Russian Federation, asemen@akin.ru)

Unsophisticated acoustic tomography system properties using envelope of radiated acoustic signal for ocean long-range inhomogeneities control, presumably, in most unfavorable, in the author view, shallow water regions, are evaluated. Unique solution to be used there is a narrow directional parametric radiation array. When pump wave signal decay range to array Frauengopher zone dimension ratio exceeds pump wave frequency to radiated signal frequency ratio, parametric radiation array operation model turns to "horn" model instead of "traveling wave" model conventionally used for consumed power prediction. For utmost distance achievement both ends of tomography system should be designed as narrow directional parametric arrays working on optimal frequency basic propagation (first) mode. For instance, signal optimum frequency $40 \mathrm{~Hz}$. is shown to be necessary for mode inhomogeneity (100 m long cylinder) control on 500-km radiation array dis- tance in water layer of depth $200 \mathrm{~m}$ with unfavorable attenuation properties. Major lobe solid angle should be chosen not wider than 0,03 radians. Array frequency ratio is advised to be of an order of 10, while array length - 100 half wavelength for pump sound field frequency. Then estimate of power consumption looks like $180-200 \mathrm{kWt}$ of pump signal acoustic power.

\section{8:40}

5aUWb3. The analysis of underwater environmental factors in relation to the harbor protection in Tzoin-Kaohsiung areas. Jin-Yuan Liu (Department of Marine Environmental Informatics/National Taiwan Ocean University, 2 Pei-Ning Road, 202 Keelung, Taiwan, jimliu@faculty.nsysu.edu.tw), Chen-Fen Huang (Department of Marine Environmental Informatics/National Taiwan Ocean University, 2 Pei-Ning Road, 202 Keelung, Taiwan, chenfen@mail.ntou.edu.tw)

Harbor is an important shipping traffic area. The intrusion or threatening by outsiders in the harbor area will certainly cause great panic of the society, and therefore, the protection of harbor and its nearby water is utterly important. The Tzoin harbor is primarily serviced for navy, and is under planning for further expansion. Therefore, a complete control of the TzoinKaohsiung marine environment, both geographical and acoustical, is necessary for military applications. The purpose of this study is to conduct a complete survey of the environment in the designated area, covering the first seaway of the Kaohsiung harbor and off coast water of the Si-Tze bay area. The survey of the marine environment includes seafloor topography, morphology, subbottom profile, waves, currents, and wind fields. In acoustic aspect, the survey emphasizes the harbor ambient noise measurement. The data obtained shall be thoroughly examined and analyzed. The results provide a foundation for future underwater experimental studies, including target localization, geoacoustic inversion, and active sonar reverberation.

\section{9:00}

5aUWb4. The effects of a shallow-water acoustic channel on Right whale vocalisations. Bashar Mohammad (Heriot Watt University, School of Engineering and Physical Sciences, Riccarton, EH14 4AS Edinburgh, UK, mb41@hw.ac.uk), Ron McHugh (Heriot Watt University, School of Engineering and Physical Sciences, Riccarton, EH14 4AS Edinburgh, UK, r.mchugh@hw.ac.uk), Judith Bell (Heriot Watt University, School of Engineering and Physical Sciences, Riccarton, EH14 4AS Edinburgh, UK, j.bell @ hw.ac.uk)

For mitigation and monitoring of Right whales, identifying their presence from their vocalisations is a key research issue. Their vocalisations are characterized as frequency modulated up-sweeps with duration of $\sim 1 \mathrm{~s}$ and a frequency range from $50 \mathrm{~Hz}$ to $200 \mathrm{~Hz}$. Acoustic methods to classify these received calls are assessed by the variation in the received data set. As well as the natural variation in vocalisation within the species, the received acoustic signals are also influenced by the effect of the acoustic channel. The shallow water of Cape Cod Bay is one of the favoured habitats for the Northern Right whale. Such waters act as an acoustic waveguide where multiple reflections off boundaries cause calls to become dispersive in nature. In this paper we discuss the effects of channel environmental parameters such as water depth and sediment type on first the FM deviation and second on 
the time difference of arrival between the first and second modes, which in turn influences acoustic range estimation. Such channel effects were studied using the normal mode acoustic propagation model (PROSIM). An analysis of real acoustic data recorded in Cape Cod Bay (2001 obtained from IFAW) will be also presented in terms of dispersion results.

\section{9:20}

5aUWb5. Development of an ocean surveillance system. V. Ananthakrishnan (Department of Electronics, Cochin University of Science \& Technology, Thrikkakara, 682022 Ernakulam, India, ananthanmattathil@gmail.com), C Prabha (Department of Electronics, Cochin University of Science \& Technology, Thrikkakara, 682022 Ernakulam, India, prabhasuma@cusat.ac.in), Supriya M. Hariharan (Department of Electronics, Cochin University of Science \& Technology, Thrikkakara, 682022 Ernakulam, India, supriya@cusat.ac.in), Saseendran Pillai (Department of Electronics, Cochin University of Science \& Technology, Thrikkakara, 682022 Ernakulam, India, prspillai@cusat.ac.in)
Now-a-days, underwater sensor networks are gaining considerable importance, for Ocean Surveillance applications. A minimally configurable three-node sensor network is found to be capable of performing localization as well as tracking of underwater targets. Each node comprises of a surface buoy having processing modules, controller hardware and support electronics for estimating the direction of arrival. The development of the node system comprising of controller and processing modules is presented in this paper. The controller hardware, consisting of a gear assembly, magnetic compass and a precision digital signal controller, helps in sampling the ocean by way of steering the hydrophone arrays and capturing the target emanations, while the processing module performs the computation of the direction of arrivals. The target emissions picked up by the hydrophone arrays are analyzed and processed for computing the direction of signal arrival with reference to the geomagnetic meridian. The direction of maximum signal arrivals computed by the three nodes, lead to the estimation of the target position. Localization of the target is carried out by effectively utilizing the information furnished by Global Positioning System incorporated in the node hardware, which has an efficient power management mechanism, as well.

FRIDAY MORNING, 4 JULY 2008

ROOM 342A, 8:00 A.M. TO 12:40 P.M.

\title{
Session 5aUWc
}

\section{Underwater Acoustics and ECUA: High Frequency Scattering I}

\author{
Anthony P. Lyons, Cochair \\ Applied Research Laboratory, The Pennsylvania State University, P.O. Box 30, State College, 16804, USA \\ Philippe Blondel, Cochair \\ University of Bath, Department of Physics, Claverton Down, Bath, BA2 7AY, UK \\ Nicholas Pace, Cochair \\ Department of Physics, University of Bath, Bath BA2 7AY, UK
}

\section{Contributed Paper}

\section{8:00}

5aUWc1. Classification of angular backscattered responses obtained at sea with a forward looking sonar system. Pierre Cervenka (Institut Jean le Rond D'Alembert, UMR 7190, 2 Place de la Gare de Ceinture, 78210 Saint Cyr L’Ecole, France, cvk@ccr.jussieu.fr), Jacques Marchal (Institut Jean le Rond D'Alembert, UMR 7190, 2 Place de la Gare de Ceinture, 78210 Saint Cyr L’Ecole, France, jmarchal@ccr.jussieu.fr)

The prototype of a multibeam front-scan sonar has been developed within the frame of a MAST contract ( $n^{\circ}$ MAS3-CT97-0090 DG12-ESCY, acronym COSMOS). A large amount of data has been collected at sea. With the forward looking geometry of acquisition, the foot-prints of successive pings overlap largely, so that most parts of the surveyed areas are seen under a large range of incidence angles. It gives the capability to derive the local angular response of the bottom, which is indeed an important information for characterizing the nature of the seafloor. This presentation addresses the successive steps that were undergone to classify the backscattered responses: Data are conditioned to perform principal component analyzis; an original clustering identification process is also described; resulting maps are finally presented.

\section{Invited Papers}

\section{$8: 20$}

5aUWc2. A comparison between modeled and measured high frequency bottom backscattering. Dick G. Simons (Delft University of Technology, P.O. Box Postbus 5048, 2600 GA Delft, Netherlands, d.g.simons@tudelft.nl), M. Snellen (Delft Institute of Earth Observation and Space Systems, Delft University of Technology, Kluyverweg 1, 2629 HS Delft, Netherlands, M.Snellen@tudelft .nl)

Compared to taking many sediment grabs, classification techniques based on acoustic remote sensing allow for significant cost reductions, providing the required information at much denser spatial scale. An attractive approach hereto is to use the multibeamechosounder (MBES) which measures sediment backscatter strengths as a function of angle. By maximizing the match between this backscatter strength and model results, sediment properties can be derived. The backscatter model used here accounts for both surface roughness and volume scattering. MBES measurements $(300 \mathrm{kHz})$ were taken in the Cleaver-Bank area (North Sea), characterized by a wide variety of sediment types for which a large number of grabs is collected. For the majority of the sediment types occurring, well-defined single-sediment-type areas can be discerned. Based on MBES measurements in these areas and corresponding geotechnical 
sediment parameters provided by grab grain size analysis, calibration curves are derived, representing the mismatch between modeled and measured backscatter strengths. These curves are found to be similar for all sediment types considered. By applying these correction curves to the MBES measurements, they show good agreement with the model for all observed sediment types. It is shown that after this correction the model can be used for sediment classification purposes.

\section{8:40}

5aUWc3. Acoustic scattering by deformed elongated objects: bent or rough finite cylinders, bent edges, and other stuff. Timothy K. Stanton (Woods Hole Oceanographic Institution, Applied Ocean Physics \& Engineering Department, 98 Water Street, MS \#11, Woods Hole, MA 02543, USA, tstanton@whoi.edu), Dezhang Chu (NOAA/NMFS/NWFSC/FRAMD, Montlake Blvd., E. Seattle, WA 98112, USA, dchu@whoi.edu), Guy Norton (Naval Research Laboratory, Code 7181, Stennis Space Center, MS 39529, USA, guy.norton@nrlssc.navy.mil)

Predicting acoustic scattering by deformed elongated objects remains a challenge as there are no exact analytical solutions. Numerical solutions are also challenged through various computational issues. In this research, we have developed and applied an approximate analytical approach for predicting scattering by various deformed elongated objects, including bent or rough finite cylinders and deformed edges. The formulation is based on a line integral in which the scattering or diffraction per unit length of the exact solution to an infinitely long, straight undeformed object is integrated along the length of the finite-length deformed object, accounting for variations along the length of phase and certain local properties of the object. Predictions concerning various types of deformed finite cylinders are first discussed (including Stanton, J. Acoust. Soc. Am. 86, 691-705 (1989) and subsequent papers), followed by recent predictions concerning deformed finite edges (Stanton et al., J. Acoust. Soc. Am. 122, 3167-3176 (2007)). These latter predictions using the approximate line integral compare favorably with predictions based on the T-matrix numerical method, which is formally exact. Also discussed are: dependences upon length, orientation, bend, and roughness; comparisons with experimental data concerning machined objects and marine life; and range of validity of the approach.

\section{9:00}

5aUWc4. High frequency scattering from discrete inclusions in marine sediments. Anatoliy N. Ivakin (Applied Physics Laboratory, University of Washington, 1013 NE 40th Street, Seattle, WA 98105, USA, ivakin@apl.washington.edu)

A model of high frequency scattering from sediments with discrete inclusions (such as shells and shell fragments) having an arbitrary size-depth distribution is developed. The model assumes also knowledge of a depth-dependent individual scattering function of inclusions. It is more general than in previous models and includes discrete scatterers located both below and on the water-sediment interface (partially buried). Some simple results are obtained for the case of high enough frequencies using approaches of geometry acoustics. Frequency-angular dependencies of the bottom backscattering strength are calculated for sediments with different size-depth distributions of inclusions. Inputs for the size distribution of inclusions (shell fragments) are obtained from granulometric analysis of coarse fractions of the sediment samples taken at the SAX99/SAX04 site (near Ft Walton Beach, Florida). It is shown that taking into account partially buried shells is important and can significantly enhance estimates of the bottom scattering strength especially at grazing angles below critical (about 30 degrees for sand). [Work supported by ONR, Ocean Acoustics].

\section{Contributed Paper}

9:20

5aUWc5. A review of the scattering properties of suspended sandy sediments for the application of acoustics to sediment transport studies. Peter D. Thorne (Proudman Oceanographic Laboratory, Joseph Proudman Building, 6, Brownlow Street, L3 5DA Liverpool, UK, pdt@pol.ac.uk), Ramazan Meral (Kahramanmaras Sutcu Imam University, Faculty of Agriculture, Department of Agricultural, Structures and Irrigation, 46060 Kahramanmaras, Turkey, rmeral@ksu.edu.tr)

Multi-frequency acoustics backscattering has been used for over a decade, to quantitatively measure, in the marine environment, near-bed profiles of suspended sediment particle size and concentration. Central to obtaining the sediment parameters from the backscattered signal, is a description of the scattering properties of irregularly shaped particles randomly distributed in space. Formulations are therefore required for both the attenuation and backscattering properties of suspensions of sedimentary particles with size and acoustic frequency. There is no rigorous analytical solution or single formulation for these scattering properties and different researchers have used somewhat different expressions. However, all these expressions are based on a variation of sphere scattering, modified to fit available scattering data. Here we bring together four decades of published data on the acoustic scattering properties of suspensions of sandy sediments. These data are reformulated in terms of the usual acoustic scattering nomenclature, that is the form function and the normalised total scattering cross-section and simple heuristic generic expressions, based on a sphere scattering model, are formulated to describe the sediment scattering properties. The expressions are not limited to sand particles, but should have broad applicability to irregularly shaped particle scattering.

\section{Invited Paper}

\section{9:40}

5aUWc6. On the angular dependence of sound scattering from natural sand in turbulent suspensions. Alex E. Hay (Dalhousie University, Department of Oceanography, Halifax, NS B3H 4J1, Canada, alex.hay@dal.ca), Stephanie A. Moore (Dalhousie University, Department of Oceanography, Halifax, NS B3H 4J1, Canada, Stephanie.Moore@phys.ocean.dal.ca), Wesley Paul (Dalhousie University, Department of Oceanography, Halifax, NS B3H 4J1, Canada, Wes.Paul@Dal.Ca)

We report on measurements of the variation with scattering angle of the differential scattering cross section of particles embedded in turbulence. The experimental apparatus consists of a turbulent round water jet carrying the particles, and a pair of broadband $\mathrm{MHz}$ frequency transducers operating as transmitter and receiver and arranged in a bistatic geometry centred on the jet axis. The purpose of the jet is to confine the scatterers within a restricted range interval and thereby eliminate the need to correct the signal for attenuation due to scattering along the transmit and receive paths. Measurements were made at scattering angles between 90 and 170 degrees, for 
both natural sand grains and spherical glass beads in narrow size distributions with median diameters ranging from 200 to $500 \mu \mathrm{m}$. Particle size distributions were determined using different methodologies to obtain both volumetric and projected area estimates of equivalent spherical size. The results are compared to the theory for scattering by a solid sphere. Implications regarding the choice of equivalent sphere size for sound scattering by natural sand grains in suspension are discussed.

10:00-11:00 Posters

Lecture sessions will recess for presentation of poster papers on various topics in acoustics. See poster sessions for topics and abstracts.

\section{Contributed Papers}

11:00

5aUWc7. Measurements of the scattering characteristics of sediment suspensions having broad particle size distributions. Benjamin D. Moate (Proudman Oceanographic Laboratory, Joseph Proudman Building, 6, Brownlow Street, L3 5DA Liverpool, UK, bdm@pol.ac.uk), Peter D. Thorne (Proudman Oceanographic Laboratory, Joseph Proudman Building, 6, Brownlow Street, L3 5DA Liverpool, UK, pdt@pol.ac.uk)

Profiles of suspended sediment concentration and mean size can be measured in underwater environments using Acoustic Backscatter Systems (ABS). Inversion of ABS measurements into sediment size and concentration requires knowledge of the backscattering form function, $\mathrm{f}$, and the total normalised scattering cross section, $\chi$. Previous studies have measured these parameters for sediments sieved over narrow size ranges only. Narrow size ranges are unrealistic compared to the broad Particle Size Distributions (PSD) that typically occur in nature however, and it is known that $\mathrm{f}$ and $\chi$ are significantly altered by changes from narrow to broad PSDs. Theoretically, these changes can be accounted for in ABS inversions by taking values of $\mathrm{f}$ and $\chi$ obtained from narrow size ranges, and integrating them over the suspended PSD, though this theory has not been validated for real suspended sediments. Here, we compare the results of this integration with measured values of $\mathrm{f}$ and $\chi$ for suspensions of (irregularly shaped) sandy sediments having broad PSDs. The results show that as the standard deviation of the size distribution increases, acoustic scattering is increased in the Rayleigh regime whilst being reduced in the geometric regime. These changes significantly affect acoustic estimates of suspended sediment concentration and size.

\section{1:20}

5aUWc8. Acoustic scattering from double-diffusive microstructure. Andone Lavery (Woods Hole Oceanographic Institution, Applied Ocean Physics \& Engineering Department, 98 Water Street, MS \#11, Woods Hole, MA 02543, USA, alavery@whoi.edu), Tetjana Ross (Dalhousie University, Department of Oceanography, 1355 Oxford Street, Halifax, NS B3H 4JI, Canada, tetjana.ross@dal.ca)

Laboratory measurements of high-frequency broadband acoustic backscattering (200-600 kHz) from the diffusive regime of double-diffusive microstructure have been performed. This type of microstructure, which was characterized using direct microstructure and optical shadowgraph techniques, is identified by sharp density and sound speed interfaces separating well-mixed layers. Acoustic backscattering measurements were performed for a range of physical parameters controlling the double-diffusive microstructure. The echoes have been analyzed in both the frequency domain, providing information on the spectral response of the scattering, and in the time domain, using pulse compression techniques. High levels of variability were observed, associated with interface oscillations and turbulent plumes, with many echoes showing significant spectral structure. Acoustic estimates of interface thickness, obtained for the echoes with exactly two peaks in the compressed pulse output, were in good agreement with estimates based on direct microstructure and optical shadowgraph measurements. Predictions based on a one-dimensional weak-scattering model that includes the actual density and sound speed profiles agree reasonably with the measured scattering. A remote-sensing tool for mapping oceanic microstructure, such as high-frequency broadband acoustic scattering, could lead to a better understanding of the extent and evolution of double-diffusive layering, and to the importance of double diffusion to oceanic mixing.

\section{Invited Papers}

\section{1:40}

5aUWc9. Clustered scatterers: the effect on the mean acoustic field. Thomas C. Weber (University of New Hampshire, Ctr. for Coastal and Ocean Mapping, 24 Colovos Road, Durham, NH 03824, USA, weber@ccom.unh.edu)

Clustering, or spatially dependent correlation in the fluctuating number density of discrete scatterers of sound, is commonly found to occur in nature. In the ocean, clustering can occur as a result of scatterer entrainment in fluid flows, as in the case of particles and bubbles, or, in the case of fish, as a result of the behaviour of the scatterer. In either case, the presence of clustering can dramatically alter the effect of the scatterers on acoustic fields from that which would be predicted from the same scatterer population but without clustering. The effect of scatterers on the mean acoustic field is often treated using an effective medium wave number. In this paper, a modification to the effective medium number that accounts for clustering is presented and examined for the case of propagation through bubble clouds. Attenuation predictions over a broad range of frequencies (10-350 kHz) using observations of bubble clustering under oceanic breaking waves will be discussed.

\section{2:00}

5aUWc10. A very shallow water ray propagation model. Lucie Pautet (7A/01 Attemsgasse, 1220 Vienna, Austria, luciepautet@yahoo.com), Alessandra Tesei (NATO Undersea Research Centre, Viale San Bartolomeo 400, 19126 La Spezia, Italy, tesei@nurc.nato.int)

For applications such as minehunting, communications, high frequency time-reversal, there has been a need for reliable and fast propagation models able to correctly treat scattering at the rough waveguide boundaries. Data and scattering models have shown that as signal frequency or surface roughness increases, the scattering pattern from the ocean bottom and ocean surface evolves from specular to quasi omni-directional. Ray propagation models are fast but, a priori, they would treat surface bounces as perfectly specular which would not account for the angular spread observed on multipath structures. The idea followed by the authors over the past few years has 
been to introduce in the Bellhop ray tracing model a reflection kernel including deterministic properties of the sea surface and bottom. Simulations using this kernel display a significant effect of the multipath structure similar to the angular spread observed in in-situ data. Simulation results are compared with a recent experimental study at 10-20 KHz (BARRIER'04 at NURC) on the influence of wind strength on surface bounce spreading and subsequent TR-focus spread.

12:20

5aUWc11. Broadband acoustic scattering from nonlinear internal solitary waves. Andone Lavery (Woods Hole Oceanographic Institution, Applied Ocean Physics \& Engineering Department, 98 Water Street, MS \#11, Woods Hole, MA 02543, USA, alavery@ whoi .edu)

High-frequency broadband (150-600 kHz) acoustic scattering techniques have been used to obtain high-resolution images of nonlinear internal solitary waves (ISWs) propagating shoreward over the New Jersey continental shelf. In contrast to traditional singlefrequency measurements, broadband acoustic scattering allows the frequency spectrum of the scattering to be determined, which, in combination with scattering models and direct ground-truthing measurements, allows regions in which the scattering is dominated by biology versus microstructure to be distinguished. Multiple ISWs were tracked and imaged acoustically at different stages of their generation, propagation, and dissipation. Simultaneous microstructure and zooplankton net sampling measurements were performed. Multiple scattering layers and distinct scattering patches were often associated to the ISWs, often exhibiting distinct frequency responses. Clear day/night differences in the scattering from the ISWs were observed due to the daily vertical migration of zooplankton. Though high turbulence levels are associated with ISWs, the strongest scattering returns were not always associated to the highest turbulence levels. Instead, the strongest scattering, at some frequencies, was sometimes associated to large temperature gradients. In regions in which the scattering was dominated by either microstructure or zooplankton, acoustic inversions have been performed for the dissipation rate of turbulent kinetic energy and zooplankton size and abundance.

FRIDAY MORNING, 4 JULY 2008

ROOM 342B, 8:00 TO 11:40 A.M.

\title{
Session 5aUWd
}

\section{Underwater Acoustics and ECUA: Low-Frequency and High-Frequency Synthetic Aperture Sonar}

\author{
Peter T. Gough, Cochair \\ University of Canterbury, Private Bag 4800, Christchurch, 8022, New Zealand \\ Roy E. Hansen, Cochair \\ Norwegian Defence Research Establishment, P O Box 25, Kjeller, NO-2027, Norway

\section{Invited Paper} \\ 8:00
}

\begin{abstract}
5aUWd1. Ten key papers in synthetic aperture sonar. Peter T. Gough (University of Canterbury, Private Bag 4800, 8022 Christchurch, New Zealand, peter.gough@ canterbury.ac.nz), Michael Hayes (University of Canterbury, Private Bag 4800, 8022 Christchurch, New Zealand, michael.hayes@ canterbury.ac.nz)

There are now hundreds of patents and papers on synthetic aperture sonar; some of them of key importance whereas many others are of peripheral or minor interest. Here we take what we consider to be the top ten publications in synthetic aperture sonar and explain why we believe they are important and how they have made a significant contribution to the developing discipline or have made some leap of imagination in the area. This paper is more than a catalogue of the top ten citations since not all the real advances in the field have been published in academic peer-review journals.
\end{abstract}

\section{Contributed Papers}

\section{8:20}

5aUWd2. Multifrequency interferometry on synthetic aperture sonar images. Torstein Olsmo Sæbø (Norwegian Defence Research Establishment, P O Box 25, NO-2027 Kjeller, Norway, torstein-olsmo.sabo @ ffi.no), Roy E. Hansen (Norwegian Defence Research Establishment, P O Box 25, NO-2027 Kjeller, Norway, roy-edgar.hansen@ffi.no), Hayden J. Callow (Norwegian Defence Research Establishment, P O Box 25, NO2027 Kjeller, Norway, hayden-john.callow@ffi.no)

Interferometric processing on synthetic aperture sonar (SAS) images can provide accurate bathymetric maps with very high spatial resolution. Often, bathymetric precision is limited by two-pi phase ambiguities in the interferogram. Several phase-unwrapping algorithms exist, but they all depend on some type of continuity throughout the interferogram. The locations of the phase ambiguities are dependent on the carrier frequency of the transmitted signal. Dividing the frequency band into multiple subbands provides multiple interferograms, each with a unique carrier frequency and thus differing ambiguity positions. In this paper, we investigate how to take advantage of subband interferograms to reduce phase ambiguities at the expense of accuracy. This is performed by using the phase differences between the individual subband interferograms. We also present a method were we use the differences in subband interferograms to eliminate the phase ambiguities in both the original interferogram, and in the subband interferograms themselves. Both methods are tested on simulated data and field data collected by the HUGIN autonomous underwater vehicle carrying the Kongsberg HISAS 1030. 


\section{8:40}

5aUWd3. Circular synthetic aperture sonar results from autonomous underwater vehicle trials. Roy E. Hansen (Norwegian Defence Research Establishment, P O Box 25, NO-2027 Kjeller, Norway, roy-edgar.hansen @ffi.no), Torstein Olsmo Sæbø (Norwegian Defence Research Establishment, P O Box 25, NO-2027 Kjeller, Norway, torstein-olsmo.sabo @ffi.no), Hayden J. Callow (Norwegian Defence Research Establishment, P O Box 25, NO-2027 Kjeller, Norway, hayden-john.callow@ffi.no)

The principle of synthetic aperture sonar (SAS) is to combine successive pings coherently from different observation angles in order to increase the azimuth resolution. By collecting data along a circular track, a circular SAS (CSAS) image, or tomographic image, can be made. The image reconstruction can be done either incoherently, fully coherently, or partially coherently where each subaperture of coherent processing consists of a section of the circle. In target classification, CSAS has several benefits: the object is observed from all aspect angles giving a better perception; the resolution in the image increases. In this paper, we calculate required accuracy in navigation, bathymetry and sound velocity for successful circular SAS. Finally, we show circular SAS images of small targets from real data collected by the HUGIN autonomous underwater vehicle carrying the Kongsberg HISAS 1030. We test different beamforming strategies, and show the effect of coherent and incoherent tomographic imaging.

\section{9:00}

5aUWd4. Autofocus of synthetic aperture sonar data using the phase adjustment by contrast enhancement algorithm. Hayden J. Callow (Norwegian Defence Research Establishment, P O Box 25, NO2027 Kjeller, Norway, hayden-john.callow@ffi.no), Torstein Olsmo Sæbø (Norwegian Defence Research Establishment, P O Box 25, NO-2027 Kjeller, Norway, torstein-olsmo.sabo@ffi.no), Roy E. Hansen (Norwegian Defence Research Establishment, P O Box 25, NO-2027 Kjeller, Norway, roy-edgar.hansen@ffi.no)

Imagery from synthetic aperture systems often suffers from imperfect image formation due uncertainties in the collection geometry or environmental parameters. Autofocus techniques aim to estimate and subsequently eliminate the effect of these uncertainties---automatically adjusting focus parameters to obtain a "better" image. Typically, autofocus algorithms will optimise for reduced Doppler phase gradients or improved image contrast. The phase adjustment by contrast enhancement (PACE) algorithm belongs to the second class and was first proposed for use in synthetic aperture radar (SAR) autofocus. The algorithm is somewhat unusual for a contrast optimisation in that it avoids lengthy computation through directly solving the equations for optimum contrast. This advance allows for rapid autofocus without the need for complicated iterative optimisers. We compare results from using the PACE algorithm on local region of strip-map synthetic aperture sonar (SAS) data collected with the HUGIN AUV and compare against a benchmark phase gradient autofocus (PGA) algorithm. We also demonstrate the effect of using the algorithm in circular SAS imagery, something not currently possible with standard PGA-based autofocus.

\section{9:20}

5aUWd5. Planar synthetic aperture processing (P-SAS) to real acoustic data of underwater imaging. Adel Jammali (Faculté des Sciences de Monastir $\mu$ Ei Lab Tunisia, Avenue de l'environnement, 5019 Monastir, Tunisia, adel.jam@voila.fr), Afif Belkacem (Faculté des Sciences de Monastir $\mu$ Ei Lab Tunisia, Avenue de l'environnement, 5019 Monastir, Tunisia, afif_bel@yahoo.fr), Kamel Besbes (Faculté des Sciences de Monastir $\mu$ Ei Lab Tunisia, Avenue de l'environnement, 5019 Monastir, Tunisia, Kamel.Besbes@fsm.rnu.tn), Manell E. Zakharia (French Naval Acadamey, BP 600, 29240 Brest-Armees, France, manell.zakharia@ecole-navale.fr)

Planar synthetic aperture technique has been validated on both simulated and tank data. It showed a significant improvement of 3D representation of bottom and subbottom. In this paper we present its application to real data acquired during sea experiments on a dump site. Data were acquired during the SITAR (Seafloor Imaging and Toxicity: Assessment of Risks caused by buried waste) project. The transmitter was a parametric array (TOPAS). Two central frequency were explored, $10 \mathrm{kHz}$ and $20 \mathrm{kHz}$. The transmitter was fixed on a ROV which position was monitored. As P-SAS algorithm was designed for data obtained on a regular planar grid important modification were required to handle real sea data and "realistic" navigation conditions (irregular grid): a "re-arrangement" algorithm was designed for preprocessing actual data and correct for trajectory disturbances (in 2D). This algorithm is the re-projection of data to a new (virtual) regularly grid. The algorithm was validated on tank experimental data prior to application to sea data. Both conventional and SAS data will be presented. A strata representation technique was used for analyzing the seafloor, the subbottom and the localization of buried objects on a dump site in the Baltic sea.

\section{9:40-11:00 Posters}

Lecture sessions will recess for presentation of poster papers on various topics in acoustics. See poster sessions for topics and abstracts.

\section{Contributed Paper}

\section{1:00}

5aUWd6. Towards an understanding of how a synthetic aperture sonar images buried targets. Peter T. Gough (University of Canterbury, Private Bag 4800, 8022 Christchurch, New Zealand, peter.gough@canterbury .ac.nz), Michael Hayes (University of Canterbury, Private Bag 4800, 8022 Christchurch, New Zealand, michael.hayes@canterbury.ac.nz)

One of the useful features of synthetic aperture sonar (SAS) is that in the reconstructed image, the cross-track resolution is inversely proportional to the bandwidth and the along-track resolution is proportional to the transducer's along-track extent and that these are more or less independent of the center frequency. An unexpected aspect of SAS imaging so far is that objects buried in sand/sediment are sometimes visible even at geometries where the incident angle far exceeds the critical angle for the bulk materials. It is surmised that the roughness of the sea/sea-floor interface is the significant contributing factor. So as an adjunct to the main Kiwi SAS programme, a seafloor "document" camera colocated with a three-transducer active sonic probe has been designed and deployed in controlled circumstances. The intention is to estimate the critical parameters that best describes the roughness of the sea floor and perhaps how these parameters relate to the way the SAS images shallow buried targets. In regions of reasonable visibility, the camera is used to provide ground-truth. The paper details the construction of the camera and sonic probe as well describes some preliminary results using smooth and raked sand. 


\title{
Invited Paper
}

11:20

5aUWd7. Imaging buried objects using synthetic aperture processing. Steven Schock (Florida Atlantic University, Dept of Ocean Engineering, 777 Glades Rd, Boca Raton, FL 33431, USA, schock@oe.fau.edu)

FM sonar generates images of buried objects using synthetic aperture processing. A spherical projector illuminates objects buried in the seabed out to the range associated with the critical angle. Scattering off buried targets is measured with one meter long line hydrophone arrays embedded in sonar vehicle wings. The histories of the projector and hydrophones positions, used for compensating acoustic data during synthetic aperture processing, are calculated based on navigation data provided by an inertial measurement unit and Doppler velocity log. Three orthogonal image projections of the seabed containing 5, 7.5, 10, and $15 \mathrm{~cm}$ diameter cylinders, buried up to $30 \mathrm{~cm}$ in seabeds consisting of sand and sandy mud in three meters of water, show the burial depth and orientation of the cylinders. The temporal resolution of the imagery is approximately $6 \mathrm{~cm}$. The azimuthal resolution varies between 5 and $20 \mathrm{~cm}$ and is a function of target range and relative bearing with respect to the sonar vehicle. This imaging technique can be applied to marine archeological investigations in water depths of two meters and greater.

FRIDAY MORNING, 4 JULY 2008

ROOM 362/363, 8:00 TO 9:40 A.M.

\section{Session 5aUWe}

\section{Underwater Acoustics and ECUA: Monitoring Systems and Ambient Noise}

\author{
Mark K. Prior, Cochair \\ CTBTO, Vienna International Centre, P.O. Box 1200, Vienna, 1400, Austria \\ David Deveau, Cochair \\ Naval Undersea Warfare Center - AUTEC, PSC 1012 Box 701, FPO, AA, FL 34058, USA
}

\section{Contributed Papers}

\begin{abstract}
8:00
5aUWe1. An optimization approach to the automatic identification of signals originating from underwater explosions. Mark $\mathrm{K}$. Prior (CTBTO, Vienna International Centre, PO Box 1200, 1400 Vienna, Austria, Mark.Prior@CTBTO.ORG)

Data recorded by the International Monitoring System (IMS) of the Comprehensive Test-Ban Treaty Organization are used to illustrate the different types of signal that are routinely received on IMS hydrophone stations. It is shown that automated methods for characterizing the source of these signals sometimes fail to identify signals arising from underwater explosions. A new approach to the automatic identification of such signals is presented. The approach uses Receiver Operating Characteristic (ROC) curves to investigate the trade-off between false-alarm rate and probability of detection. It applies a threshold to a parameter developed as a measure of the likelihood of a signal being associated with an explosion. A numerical Measure of Performance (MoP) is derived from the ROC curves and the definition of the likelihood parameter is tuned so as to maximize this MoP. Optimization of the parameter definition is achieved using an approach based on genetic algorithms.
\end{abstract}

\section{8:20}

5aUWe2. Seafloor Geodetic Networks for monitoring tectonic plate motion and deformation. Stavroula Peroutsea (School of Rural and Surveying Engineering, National Technical University of Athens, Ch. Mantika 1, 11362 Athens, Greece, ROK2POK5G@INTERNET.GR), Efstratios Doukakis (School of Rural and Surveying Engineering, National Technical University of Athens, Ch. Mantika 1, 11362 Athens, Greece, EDOUK @CENTRAL.NTUA.GR)

This paper argues that seafloor geodetic networks, monitoring tectonic plate motion and deformation, supplementing terrestrial geodetic networks, can provide important information for the understanding of crustal processes, globally. The proposed monitoring system consists of three main components: real-time kinematic-differential GPS ship positioning, precise acoustic ranging between ship and seafloor transponders - which establish the network nodes- and near-bottom direct acoustic measurements of the network baselines. The coordinates of the transponders in a goedetic reference system can be determined from these observations and a sound-speed structure model. The motion and deformation of the tectonic plate can be traced as a change of coordinates and/or the internal geometry of the network. Stochastic and mathematic models, algorithms and data analysis are crucial for achieving the necessary accuracy in such a demanding process regarding seafloor position estimation.

\section{8:40}

5aUWe3. Confined deep water acoustic noise study within the Bahamian TOTO. David Deveau (Naval Undersea Warfare Center - AUTEC, PSC 1012 Box 701, FPO, AA, FL 34058, USA, david.deveau@autec navy.mil)

The three-sided deep coral reef that borders Andros Island, Bahamas is populated with deep water acoustic sensors that presents a unique opportunity to study the impact of wind and wave in a physical environment with limited man-made noise sources over a wide acoustic band $(50-40 \mathrm{kHz})$. This area is known as the "Tongue of the Ocean" (TOTO) for its unique satellite viewed shape. The region's deep acoustic channel $(\sim 1550 \mathrm{~m})$ is surrounded by steep walls and only one outlet suggesting a highly reverberant cavity where weather produced ambient noise signatures may persist over greater periods producing levels greater than typically observed in the open ocean. In this study, daily acoustic spectra are gathered on two sensors spaced $20 \mathrm{~km}$ apart with accompanied wind and weather information. The spectra will be correlated to advancing and receding weather fronts in an effort to relate the surface changes to the deep water ambient noise levels. This study also serves as a precursor to a shallow water acoustic noise intensity study where similar weather comparisons will be made and compared to the deep water counterpart. 
9:00

5aUWe4. Historical ambient noise levels in a topographically isolated basin. D. Benjamin Reeder (Naval Postgraduate School, Department of Oceanography, 833 Dyer Road, Rm 328, Monterey, CA 93943-5738, USA, dbreeder@nps.edu), Susan M. Mach (Naval Undersea Warfare Center Detachment AUTEC, P.O. Box 24619, West Palm Beach, FL 33416, USA, Susan.Mach@autec.navy.mil), Edmond S. Sheffield (Naval Undersea Warfare Center Detachment AUTEC, P.O. Box 24619, West Palm Beach, FL 33416, USA, Edmond.Sheffield@autec.navy.mil)

During the late 1970s and early 1980s, careful measurements of ambient noise were made in the Tongue of the Ocean (TOTO) in the Bahamas. The measurements were made using a vertical array of five omni-directional hydrophones moored in $2200 \mathrm{~m}$ water depth with an average hydrophone depth of $122 \mathrm{~m}$. Data were collected within a $20 \mathrm{~Hz}-20 \mathrm{kHz}$ band at ten specific $1 / 3$ octave band frequencies, and then reported in spectrum level as a function of surface wind speed. Records contaminated by anthropogenic sources (e.g., local vessel traffic), biological sources (e.g., marine mammal vocalizations) and other sources (e.g., rain) were eliminated to allow analysis of background ocean ambient noise only. TOTO is a deep basin, topographically isolated from the Atlantic Ocean, and therefore largely acoustically decoupled from the Atlantic Ocean deep sound channel. The basin's geographical isolation and the controlled data collection methodology employed over a four-year period make this three-decade-old data set a potential proxy for preindustrial era ocean noise levels. The environmental setting, data collection methodology, analysis methodology and results are presented and discussed within the context of historical ocean ambient noise levels.
9:20

5aUWe5. Analysis of wind generated shallow water ambient noise. Ari Poikonen (Finnish Naval Research Institute (FNRI), P.O. Box 116, FIN02631 Espoo, Finland, ari.poikonen@mil.fi), Seppo Madekivi (Finnish Naval Research Institute (FNRI), P.O. Box 116, FIN-02631 Espoo, Finland, seppo.madekivi@mil.fi)

Underwater ambient noise measurements were carried out in very shallow water (10-20 m) in the archipelago of the Gulf of Finland during the full year. Weather conditions varied from calm sea to near gale winds. The lowest spectral levels were obtained under the ice cover during the coldest winter months. No seasonal effect was observed in the measured spectra. The role of water depth was modeled with several bottom sediments in order to identify possible channel effects at lower frequencies of the noise spectra. The ambient noise spectra are typically bandlimited. A bandpass filter model was thus fitted to the noise spectra. The filter model provides several useful parameters to characterize the effect of wind speed on the noise spectra. The cutoff frequency of the high-pass part of the spectra decreases from ca 400 $\mathrm{Hz}$ down to below $200 \mathrm{~Hz}$ as wind increases from light breeze to near gale force. The corresponding spectral slopes get steeper with increasing winds saturating to the value of $12 \mathrm{~dB} /$ oct already in fresh breeze. The other parameters used in the analysis are high frequency (low-pass) spectral slope, the frequency of maximum spectral level, noise bandwidth and total power in the band.

FRIDAY MORNING, 4 JULY 2008

P3-C, LEVEL 3, 9:40 TO 11:20 A.M.

\title{
Session 5aUWf
}

\section{Underwater Acoustics, Signal Processing in Acoustics, and ECUA: Broadband Underwater Communications II (Poster Session)}

\author{
Heechun Song, Cochair \\ $M P L$ \\ Sergio Jesus, Cochair \\ $I S R$
}

\begin{abstract}
All posters will be on display from 9:40 a.m. to 11:20 a.m. To allow contributors an opportunity to see other posters, contributors of odd-numbered papers will be at their posters from 9:40 a.m. to 10:30 a.m. and contributors of even-numbered papers will be at their posters from 10:30 a.m. to 11:20 a.m.
\end{abstract}

\section{Contributed Papers}

5aUWf1. Basic at-sea experiment for long horizontal time-reversal communication in deep ocean. Takuya Shimura (JAMSTEC, 2-15, Natsushima-cho, 237-0061 Yokosuka, Japan, shimurat@jamstec .go.jp), Yoshitaka Watanabe (JAMSTEC, 2-15, Natsushima-cho, 237-0061 Yokosuka, Japan, yoshitakaw@jamstec.go.jp), Hiroshi Ochi (JAMSTEC, 2-15, Natsushima-cho, 237-0061 Yokosuka, Japan, ochi@jamstec .go.jp), Takehito Hattori (Nippon Marine Enterprises, Ltd., 14-1, Ogawacho, 238-0004 Yokosuka, Japan, hattorit@nme.co.jp)

In our research program, a long cruising autonomous underwater vehicle (AUV) has been developed and a project on a second AUV is planned, for wide-areal observation in the deep ocean. In such AUV operation, it is preferred to ensure acoustic communication with AUV even at low data- transmission rate. Time-reversal techniques have a possibility to achieve such long horizontal communication. We have proposed a method of combining time reversal and an adaptive equalizer to realize communication even with a sparse time-reversal array (TRA) and communication with a moving target. To verify the performance of such time-reversal communication, at-sea experiments were executed at the various distances. In the experiment of $10 \mathrm{~km}$ at a water depth of $1000 \mathrm{~m}$, bidirectional time-reversal communication between the focus point and TRA was achieved successfully and the effects of the TRA depth and its channel number are investigated. In the experiments of $20,30,40 \mathrm{~km}$ at the slight sloping area, the performance on the distances are discussed. In the experiment of $100 \mathrm{~km}$ at a water depth of $4000 \mathrm{~m}$, the convergence by passive time reversal was verified. 
5aUWf2. High data rate coherent underwater acoustic communications during KauaiEx and MakaiEx. Aijun Song (University of Delaware, College of Marine and Earth Studies, S. College Street, Newark, DE 19716, USA, ajsong@udel.edu), Mohsen Badiey (University of Delaware, College of Marine and Earth Studies, S. College Street, Newark, DE 19716, USA, badiey@udel.edu), Heechun Song (MPL, Scripps Institution of Oceanography, University of California, San Diego, La Jolla, CA 92093-0238, USA, hcsong@ucsd.edu), William Hodgkiss (MPL, Scripps Institution of Oceanography, University of California, San Diego, La Jolla, CA 92093-0238, USA,wsh@mpl.ucsd.edu)

During two acoustic communications experiments conducted around Kauai Island, Hawaii (KauaiEx, 2003 and MakaiEx, 2005), various coherent communications data with concurrent environmental measurements were collected under different experimental settings. The collected communications data are processed by a newly proposed receiver, which consists of time-reversal multichannel combining followed by a single channel DFE. Continuous channel updates along with Doppler tracking are used prior to time reversal combining to combat fast channel variations. The receiver can successfully demodulate different types of coherent communications signals, including phase shift keying (PSK) and quadrature amplitude modulation (QAM) signals, at different symbols rates for different source/receiver settings, such as fixed source/receiver, drifting source/receiver, and towed source. Selected high data rate communication results will be presented to show the effectiveness of the receiver. The receiver performance in relation to the environmental variability also will be shown.

5aUWf3. Optimal placement of array elements for time-reversal mirror. Jae Hoon Joo (LIG Nex1 Co., Ltd, 102-18 Mabuk-dong Giheunggu, 446-912 Yongin, Republic of Korea, jhjub@lignex1.com), Jea Soo Kim (Dept. of Ocean Engineering, Korea Maritime Univ., 1 Dongsam-dong Youngdo-ku, 606-791 Busan, Republic of Korea, jskim@hhu.ac.kr)

In the application of time-reversal mirror (TRM) to the underwater acoustic communication, the optimal placement of the transmit/receive array can enhance the signal-to-noise ratio at the foci when a number of array elements is limited. In this presentation, the optimal location of individual elements is determined by maximizing contrast between the focal location and the area of interest using genetic algorithm. As a result of optimization, the contrast as well as the intensity at foci is increased when compared to the conventional TRM. The optimal number of array elements is also investigated based on the objective function of genetic algorithm in connection with finding optimal placement of array. Numerical examples for TRM and contrast-maximization are presented and discussed.

5aUWf4. Experimental analysis of statistical characteristics of a very shallow underwater acoustic channel. Sea-Moon Kim (MOERI/KORDI, 171 Jang-dong, Yuseong-gu, 305-343 Daejeon, Republic of Korea, smkim@moeri.re.kr), Sung-Hoon Byun (MOERI/KORDI, 171 Jang-dong, Yuseong-gu, 305-343 Daejeon, Republic of Korea, byunsh@moeri.re.kr), Yong-Kon Lim (MOERI/KORDI, 171 Jang-dong, Yuseong-gu, 305-343 Daejeon, Republic of Korea, yklim@moeri.re.kr)

Underwater acoustic channel is characterized as a time-varying and multipath environment. Not only each propagation path changes randomly but also the scattered waves from the surface and bottom deteriorate signal transmission resulting in fading channels and poor communication performance. In order to design communication algorithms and determine modem parameters the characteristics of random ocean channel must be analyzed in advance. In this paper we investigate the statistical channel properties with experimental data gathered in a very shallow water near the southern coast of South Korea using band-limited signals with center frequencies of $20-40 \mathrm{kHz}$. The impulse responses at several distances up to $4 \mathrm{~km}$ are estimated and their statistical characteristics in the complex domain are analyzed. We found that the statistical properties are highly dependent on the channel impulse response and the carrier frequency.
5aUWf5. Characterization of long-range time-varying underwater acoustic communication channels. Morten Smedsrud (FFI, Karljohansvern, P.O. Box 115, 3192 Horten, Norway, morten.smedsrud@ffi.no), Trond Jenserud (FFI, Karljohansvern, P.O.Box 115, 3192 Horten, Norway, trond .jenserud@ffi.no)

We present communication channel characterizations performed on insitu measurements from the Baltic and North Sea. The communication channels were probed using pseudorandom binary sequences (PRBS), obtaining time-variant channel impulse responses through wideband matched filtering. Characteristics central to communications were obtained. Included are: scattering functions, coherence-times and coherence bandwidths. The validity of the quasi wide sense stationary uncorrelated scattering (quasi-WSSUS) assumption was also investigated for the measured channels. Measurements were acquired in the joint European project "UUV Covert Acoustic Communications." The project aims to design an acoustic communication system between an unmanned underwater vehicle (UUV) and a support mother ship. The first sea trials of the project focused on the acquisition of noise and long-range, low-frequency communication channel data for the explicit purpose of building an acoustic channel simulator.

5aUWf6. A novel architecture for multihops routing ad hoc underwater acoustic sensor networking. Roee Diamant (Technion - Israel Institute of Technology, RAFAEL, POB 2250, 31021 Haifa, Israel, diamantr@rafael.co.il), Azriel Sinai (Technion - Israel Institute of Technology, RAFAEL, POB 2250, 31021 Haifa, Israel, azrielsi@rafael.co.il)

Underwater acoustic communication networks are an innovative issue, which faces difficult medium problems, such as long propagation time and dynamic network topology. In addition, due to high error probabilities typical for this channel it is preferred to use anti collision network mechanism. Because of the long propagation time, methods such as CTS/RTS, requiring transmissions of pre-messages, achieve relatively low network efficiency. Moreover, TDMA methods do not utilize the network resources allowing reuse in the code domain (using CDMA) and in the spatial domain in case of nonhomogenic network topology which includes obstructions in the acoustic line of sight. In this paper we introduce a network concept based on the CDMA method confronting the acoustic underwater network challenges, such as the "near-far" problem, fast time varying topology, multihops routing and message distribution protection. Using this concept, a sea trial including broadcast, multicast and unicast transmissions of SMS and navigation control messages was carried out in various scenarios including multihops near the shores of Israel. We present results from this sea trial demonstrating the efficacy of the proposed networking scheme.

5aUWf7. Capacity estimations for underwater acoustic communication systems with source power constraints. Haibin Wang (National Laboratory of Acoustics, Institute of Acoustics, Chinese Academy of Sciences, NO .21, Northwest 4th Ring Road, 100080 Beijing, China, whb@mail.ioa.ac.cn), Di Meng (National Laboratory of Acoustics, Institute of Acoustics, Chinese Academy of Sciences, NO.21, Northwest 4th Ring Road, 100080 Beijing, China, whb@mail.ioa.ac.cn), Hua Yang (National Laboratory of Acoustics, Institute of Acoustics, Chinese Academy of Sciences, NO.21, Northwest 4th Ring Road, 100080 Beijing, China, yh@mail.ioa.ac.cn)

Channel capacity is an important parameter of underwater acoustic communication (UWAC) systems. For a certain channel and source power, water-filling algorithm of information-theory can be used to estimate the capacity. However, in practice, there are many constraints of the source transmission power. One of the most important constraints is that many communication nodes may exist in a local area, which means that the source power of each node should be confined in order to keep all nodes working normally. Considering this constraint and applying acoustic propagation modeling, this paper estimates the capacities of an UWAC system in some typical propagation conditions. The results are very useful for analyzing the maximum data rate of a practical acoustic channel and providing the optimal parameters of an UWAC system. 
5aUWf8. Synchronisation of underwater communication receivers by means of broadband sweep-spread pulses. Konstantin G. Kebkal (EvoLogics GmbH/Technical University Berlin, Ackerstrasse 76 (ACK1), 13355 Berlin, Germany, kebkal@bionik.tu-berlin.de), Rudolf Bannasch (EvoLogics GmbH/Technical University Berlin, Ackerstrasse 76 (ACK1), 13355 Berlin, Germany, bannasch@evologics.de), Alexey G. Kebkal (EvoLogics GmbH/Technical University Berlin, Ackerstrasse 76 (ACK1), 13355 Berlin, Germany, kebkal@gmail.com)

Using sweep spread pulses a variety of multipath components of a receive signal can be often resolved in time-frequency domain. In result of synchronisation of the receiver with the most powerful component, other multipaths can be effectively suppressed by means of a matched filter. Owing to exact synchronisation, the difference frequency will be equal to zero and outputs of matched filters will be good for estimation of (information) parameters. However, the synchronous component (as well as others) can occasionally turn out to be composite and to contain energy of several nonresolved multipaths. In this case, the synchronisation time of the receiver will be not exact and the difference frequency of demodulated signal will differ from zero. This effect will cause a systematic error in estimation of the information parameter. This paper represents the results of mathematic modeling of the systematic error (for receivers, utilising the sweep-spread carrier technology), as well as, experiments on synchronisation of the receiver by means of wideband sweep spread pulses.
5aUWf9. Measurement of angular power profile at an array receiver in a shallow underwater acoustic channel. Sung-Hoon Byun (MOERI/KORDI, 171 Jang-dong, Yuseong-gu, 305-343 Daejeon, Republic of Korea, byunsh@moeri.re.kr), Sea-Moon Kim (MOERI/ KORDI, 171 Jang-dong, Yuseong-gu, 305-343 Daejeon, Republic of Korea, smkim@moeri.re.kr), Yong-Kon Lim (MOERI/KORDI, 171 Jang-dong, Yuseong-gu, 305-343 Daejeon, Republic of Korea, yklim@moeri .re.kr), Woojae Seong (Seoul National University, Room. 306, Bd. 34, San 56-1, Sillim-dong, Kwanak-gu, College of Engineering, Dept. of Naval Architecture and Ocean Engineering, 151-744 Seoul, Republic of Korea, wseong@snu.ac.kr)

In the design of broadband underwater acoustic communication system, proper analysis of underwater acoustic channel characteristics is required to determine communication system parameters. Especially for high channel capacity transmissions such as multiinput multioutput (MIMO) systems, the spatial correlation characteristics among multiple sensors determine communication performance and they are affected by the angular power distribution of the incoming acoustic waves. In this study, we suggest a model-based array signal processing technique for measuring the angular power profile and apply it to experimental data gathered from very shallow water of 20 to $30 \mathrm{~m}$ water depth near the southern coast of South Korea. At first we show the variation of the angular power profile over different transmitter/receiver ranges and then its impact on the spatial correlation characteristics between two different array elements. Finally we discuss the expected spatial diversity effects from the viewpoint of underwater acoustic channel modeling.

FRIDAY MORNING, 4 JULY 2008

P3-C, LEVEL 3, 9:40 TO 11:20 A.M.

\title{
Session 5aUWg
}

\section{Underwater Acoustics and ECUA: High Frequency Scattering II (Poster Session)}

\author{
Anthony Lyons, Cochair \\ Applied Research Laboratory \\ Philippe Blondel, Cochair \\ University of Bath \\ Nicholas Pace, Cochair \\ SACLANT Undersea Research Centre
}

\begin{abstract}
All posters will be on display from 9:40 a.m. to 11:20 a.m. To allow contributors an opportunity to see other posters, contributors of odd-numbered papers will be at their posters from 9:40 a.m. to 10:30 a.m. and contributors of even-numbered papers will be at their posters from 10:30 a.m. to 11:20 a.m.
\end{abstract}

\section{Contributed Papers}

5aUWg1. Modeling high-frequency reverberation and propagation loss in support of a submarine target strength trial. Boris Vasiliev (DRDC Atlantic, 9 Grove St., Dartmouth, NS B2Y 3Z7, Canada, boris.vasiliev@drdc-rddc.gc.ca), Art Collier (DRDC Atlantic, 9 Grove St., Dartmouth, NS B2Y 3Z7, Canada, art.collier@drdc-rddc.gc.ca)

The presentation reviews the environmental modeling conducted in support of a submarine acoustic target strength trial. The measurements at 20-40 $\mathrm{kHz}$ were collected at a shallow site in North West Atlantic. The site bottom was gravel; surface winds were $2-6 \mathrm{~m} / \mathrm{s}$. For ranges less than $1.8 \mathrm{~km}$, the $\mathrm{CW}$ reverberation at 24, 28, and $39 \mathrm{kHz}$ agreed with CASS-GRAB predictions; beyond $1.8 \mathrm{~km}$, the model output underestimated the measurements. The predictions were computed for APL-UW surface and bottom models appropriate for trial conditions: negligible surface reflection loss and scattering strength, low bottom reflection loss and high bottom scattering strength. The LFM reverberation at 21, 28, and $36 \mathrm{kHz}$ for $2 \mathrm{kHz}$ bandwidth signals matched the $\mathrm{CW}$ reverberation after compensation for the pulse range resolution and frequency differences in the beam responses and volume attenuation. Agreement among CASS-GRAB prediction and data verified the assumed sonar and environmental parameters used in propagation loss modeling and increased confidence in the target strength estimates. The latter were consistent with previously reported values. 
5aUWg2. Performances of a multistatic model of sound scattering by rough surfaces. Virginie Jaud (E3I2 - EA3876, 2 rue François Verny,29806 Brest Cedex, France, virginie.jaud@ensieta.fr), Cédric Gervaise (E3I2 - EA3876, 2 rue François Verny, 29806 Brest Cedex, France, cedric.gervaise@ensieta.fr), Ali Khenchaf (E3I2 - EA3876, 2 rue François Verny, 29806 Brest Cedex, France, ali.khenchaf@ensieta.fr)

The ocean floor is far from being a smooth and perfectly rigid surface. That is why its sound scattering properties are a useful input to the analysis of this medium as for acoustic data inversion. Thus, multi-static scattering coefficients have been investigated in a frequency range of order of $10 \mathrm{kHz}$ to $300 \mathrm{kHz}$. The Kirchhoff Approximation and the Small Perturbation Method could be cited respectively in the case of dimensions of a rough surface larger and smaller than the wavelength. Nevertheless, for those frequencies, hence for those wavelengths, a rough surface should be considered at different scales compared to the wavelength. As a part of the incident wave is transmitted to the seabed medium, it is also important to know the effect of the scattering coming from the volume. Jackson's scattering model takes these considerations into account. The aim of the study is first to show an improvement of the surface scattering method using the Small Slope Approximation and keeping the initial method of Jackson's model to describe the scattering from the volume. Comparisons with well-known models are presented to show the performances of this new approach and comparisons between different multistatic geometries are analyzed to show the most useful configuration of the model.
5aUWg3. Complex interfaces of granular media: multiscale characterization and effective medium modeling. Yves Le Gonidec (Géosciences Rennes, Bât. 15, Campus de Beaulieu, 35042 Rennes, France, yves.legonidec@univ-rennes1.fr)

Seafloor is a complex geological interface : the acoustic reflectivity strongly depends on the sonar frequency which can be from few to hundreds $\mathrm{kHz}$. In order to understand the physical processes involved, we have developed a multiscale acoustic method based on the wavelet transform properties: we introduce this theoretical approach first applied on homogeneous discontinuities. Then, we have developed acoustic experiments where the discontinuties are complex interfaces: we present the experimental setup and describe the results measured on granular media made of monodisperse glass beads. For this seafloor modeling, where all parameters are known, we put in evidence five frequency ranges corresponding to particular acoustic regimes: we give physical interpretations based on the analysis of effective medium modeling. From low to high frequencies, a strong transition is observed between propagation and scattering phenomena. Going further in this study, we illustrate the results on a bimodal glass bead distribution where high frequency scattering can be isolated using an original experimental approach.

FRIDAY MORNING, 4 JULY 2008

ROOM 341, 11:00 A.M. TO 12:40 P.M.

\title{
Session 5aUWh
}

\section{Underwater Acoustics and ECUA: Sensor Coalition}

\author{
Henrik Schmidt, Cochair \\ MIT, 77 Mass Ave, 5-204, Cambridge, MA 02139, USA \\ Stéphane Jespers, Cochair \\ Délégation Générale de l'Armement, 7-9 rue des Mathurins, Bagneux, 92220, France
}

\section{Contributed Paper}

\section{1:00}

5aUWh1. A review of approaches towards effective underwater sensor networks. Michel Leonard (NURC, Viale San Bartolomeo 400, $19121 \mathrm{La}$ Spezia, Italy, leonard@nurc.nato.int), Diego Merani (NURC, Viale San Bartolomeo 400, 19121 La Spezia, Italy, merani@ nurc.nato.int), Alessandro Berni (NURC, Viale San Bartolomeo 400, 19121 La Spezia, Italy, merani @ nurc.nato.int)

The still largely unexplored vastness of the ocean, covering about twothird of the surface of earth, has fascinated humans for as long as we have records for. Its currents, chemical composition, and ecosystems are all highly variable at different locations and times. New paradigms for the monitoring of the undersea environment, based on autonomous underwater sensor nodes organized in ad-hoc coalitions, offer a promising approach towards the development of scalable networks for underwater sensing, moni- toring, reconnaissance and surveillance. This promise has however to be balanced against important challenges, which need to properly addressed in order to devise a credible system concept. Some challenges worth mentioning are the difficulty in ensuring a low error-rate end-to-end path between source and destination for the duration of the communications session, the negative impact of communications performance characteristics at applications level, and energy constraints for battery-operated nodes. The requirement is therefore to design new protocols to provide network flexibility and reliability through self-organization and reconfiguration, as well as new energy-efficient routing schemes, including geographic and data-centric routing, to maximize the operational lifespan of autonomous powerconstrained nodes. In this paper we will present the state of the art and approaches being investigated at NURC. 


\section{Invited Papers}

\section{1:20}

5aUWh2. Nested Autonomy - Adaptive and collaborative sensing with hybrid sensing networks. Arjuna Balasuriya (MIT, 77 Mass Ave, 5-204, Cambridge, MA 02139, USA, arjunab@mit.edu), Henrik Schmidt (MIT, 77 Mass Ave, 5-204, Cambridge, MA 02139, USA, henrik@mit.edu), Michael B. Benjamin (MIT, 77 Mass Ave, 5-204, Cambridge, MA 02139, USA, mikerb@csail.mit .edu)

Control of undersea sensing networks is restricted by the limited acoustic communication capacity and the need for surfacing "datamules" for communicating with the operators. Adaptive sampling of episodic events must therefore be performed fully autonomously. Similarly, collaboration between nodes is limited by the communication horizon. These constraints favor the use of a nested operational paradigm, with clusters of highly autonomous nodes capable of detecting, classifying, and localizing episodic events, if possible collaborating with neighboring nodes without operator intervention. Such a Nested Autonomy operational paradigm is being developed for capturing episodic oceanographic events by AUVs and gliders in the NSF ORION ocean observatory. Based on the portable, opensource MOOS-IvP autonomous control framework, the same paradigm is being applied for distributed acoustic surveillance networks in the ONR PLUSNet program. In either case the nested autonomy enables the autononomous exploitation of the environmental and tactical situation, and collaborative tracking of targets or oceanographic events. The operator control is limited to very simple deploy and prosecute commands. The performance of the nested autonomy paradigm is demonstrated by examples using a high-fidelity simulation environment, and by results from recent field experiments. [Work supported by ONR.]

\section{1:40}

5aUWh3. Performance modeling and tradeoffs studies for hybrid sensing networks. Stéphane Jespers (Délégation Générale de l’Armement, 7-9 rue des Mathurins, 92220 Bagneux, France, stephane.jespers@ dga.defense.gouv.fr)

The transformation of naval forces in support of expeditionary operations requires substantial endurance enhancement of their existing undersea reconnaissance and surveillance functions. Concepts of operations for distributed sensor fields - to be deployed in littoral waters - are now being proposed, encouraged by the favourable outlook for network technologies. The renewed attention for multistatic sonar is one example of this trend. Predicting the performance of distributed sensor fields for ASW applications, with sensors involving the use of acoustics and non-acoustics is complex. Though it is a great chance for optimizing the use of assets, the impact of sensor platforms' mobility (AUVs or gliders) adds yet another level of space-time complexity. Prior to any large scale test or development, system and operational studies must be able to determine optimal architectures and node placement as function of mission requirements. A major constraint is that of the limitations imposed on autonomous and covert data exchange in the maritime and underwater domains. This speeks for the design of hybrid sensor network architectures, as they appear to be well suited in dealing with substantial sensor data and command control data (e.g., backseat-driver paradigm) communication requirements.

\section{Contributed Paper}

12:00

5aUWh4. Acoustics for underwater neutrino telescopes. Miguel Ardid (IGIC - Universitat Politècnica de València, Cra. Nazaret-Oliva S/N, E-46730 Gandia, Spain, mardid@fis.upv.es), Manuel Bou-Cabo (IGIC Universitat Politècnica de València, Cra. Nazaret-Oliva S/N, E-46730 Gandia, Spain, maboca3@ doctor.upv.es), Juan Antonio Martínez-Mora (IGIC Universitat Politècnica de València, Cra. Nazaret-Oliva S/N, E-46730 Gandia, Spain, jmmora@fis.upv.es), Francisco Camarena (IGIC - Universitat Politècnica de València, Cra. Nazaret-Oliva S/N, E-46730 Gandia, Spain, fracafe@fis.upv.es), Víctor Espinosa (IGIC - Universitat Politècnica de València, Cra. Nazaret-Oliva S/N, E-46730 Gandia, Spain, vespinos@fis .upv.es)
In this communication we present the research activities in underwater acoustic sensors and arrays used in underwater neutrino telescopes. These activities are developed in two areas. First, in optical based underwater neutrino telescopes, an acoustic positioning system is used in order to monitor the position and orientation of the optical modules in deep sea. In the second field, there is the idea to build underwater telescopes composed of arrays of acoustic sensors in order to detect the weak thermoacoustic pulse which will be produced when a very high energy neutrino interacts in the water. For both, a challenging and very specific acoustic system is needed. After the description of these systems and a brief review of the status of the field, our work in the area in the frames of ANTARES and KM3NeT neutrino collaborations is shown.

\section{Invited Paper}

\section{$12: 20$}

5aUWh5. The applicability of a small autonomous vehicle towed array system to ocean acoustic measurements and signal processing. William M. Carey (College of Enginering, Boston University, 110 Cummington St, Boston, MA 02215, USA, wcarey @bu.edu), Jason D. Holmes (BBN Technologies, 10 Moulton St., Cambridge, MA 02318, USA, jholmes@bbn.com), Edmund J. Sullivan (EJS Consultants, 46 Lawton Brook Lane, Portmouth, RI 02871, USA, PADDYPRIEST@aol.com), James F. Lynch (Woods Hole Oceanographic Institution, 98 Water Street, Bigelow 203A, MS-11, Woods Hole, MA 02543, USA, jlynch@whoi.edu)

An array was developed to demonstrate, and quantify the performance characteristics of an autonomous-vehicle towed-array system. This technology provides for a cost effective tool for the measurement of coherent signal propagation, depth dependent and directional noise fields and to establish quantitative limits on array performance. The tangential drag on a 40-m length array composed of a reinforced tube with an outer diameter of $2.8 \mathrm{~cm}$ is extrapolated to be between 20-28 N for diameter Reynold's number of approximately 4104 . The hydrophone- preamplifier groups have a sensitivity of $-174 \pm 1.5 \mathrm{~dB}$ re $1 \mu \mathrm{Pa} / \mathrm{V}$ between $100 \mathrm{~Hz}$ to $10 \mathrm{kHz}$. with an adjustable spacing between 0.5 and $1.5 \mathrm{~m}$. The system tow stability enabled the use of the synthetic Hankel transform to estimate the modal 
horizontal wave number spectrum and the identification of interface wave speeds at frequencies up to $1000 \mathrm{~Hz}$. The formation of synthetic apertures combined with model based extended Kalman filter techniques was found to demonstrate both narrow band and broadband tracking. Finally the system is shown to provide a unique measurement capability for directional noise measurement in shallow water environments.

FRIDAY MORNING, 4 JULY 2008

ROOM 342B, 11:40 A.M. TO 1:00 P.M.

\title{
Session 5aUWi
}

\section{Underwater Acoustics and ECUA: Synthetic Aperture Sonar and Radar Convergences}

\author{
James Candy, Cochair \\ P.O. Box 808, L-156, Univ. of California, Lawrence Livermore Natl. Lab., Livermore, CA 94551, USA \\ Hugh Griffiths, Cochair \\ Defense college of, management and Technology, Academy of United Kingdom, Cranfield University, Swindon, UK \\ René Garello, Cochair \\ ENST-Bretagne, Dpt ITI, CS 83818, Brest Cedex 03, 29238, France
}

\section{Contributed Paper}

11:40

5aUWi1. Several imaging algorithms for synthetic aperture sonar and forward looking gap-filler in real-time and post-processing on IXSEA's "Shadows" sonar. Frédéric Jean (IXSEA, 46, quai François Mitterrand, 13600 La Ciotat, France, frj@ixsea.com)

SHADOWS is a new sonar system developed by IXSEA SAS. It is a towed composed of a synthetic aperture side-scan sonar and a forwardlooking sonar. The system produces SAS images with a constant resolution $15 \mathrm{~cm}$ from $30 \mathrm{~m}$ to $300 \mathrm{~m}$ on each side. It also fills the nadir gap from 0 to $30 \mathrm{~m}$ with images having $40 \times 15 \mathrm{~cm}$ resolution. The images are provided in real-time and are georeferenced. The synthetic aperture sonar algorithm uses
INS data combined with the Displaced Phase Center Algorithm (DPC). Differences between INS and DPC navigations can be used to compute a topography profile of the ground. The post processing and real-time modes differ on the weight given between INS and DPC, and on the approximations to be done. The real-time beam-forming algorithm used is the timedomain fast factorized back projection which can be pushed to an exact back projection in the post processing mode. The forward-looking sonar uses a patented "sectorized emission" architecture. The images are side-scanlike. The real-time algorithm can be customized to make some incoherent integration on several pings increasing the contrast but slightly decreasing the resolution. A post processing algorithm can also provide an animation on a specific contact on the floor.

\section{Invited Paper}

\section{2:00}

5aUWi2. Passive synthetic aperture as an experimental tool. Edmund J. Sullivan (EJS Consultants, 46 Lawton Brook Lane, Portmouth, RI 02871, USA, PADDYPRIEST@aol.com), William M. Carey (College of Enginering, Boston University, 110 Cummington St, Boston, MA 02215, USA, wcarey@bu.edu), Jason D. Holmes (BBN Technologies, 10 Moulton St., Cambridge, MA 02318, USA, jholmes@bbn.com), James F. Lynch (Woods Hole Oceanographic Institution, 98 Water Street, Bigelow 203A, MS-11, Woods Hole, MA 02543, USA, jlynch@whoi.edu)

The use of autonomous undersea vehicle towed arrays offer an inexpensive way to carry out oceanographic measurements. Due to the small size and limited power of such vehicles, the physical aperture of the array is necessarily limited and the tow speed is low. However, there is an advantage in that short arrays at low speeds can have no flow noise. In addition the use of passive synthetic aperture (PSA) to provide spatial resolution and gain can play a major role. Here, we describe the use of two forms of passive synthetic array processing used in a shallow-water experiment. A six-element towed array, with $0.75 \mathrm{~m}$ spacing, was used to estimate the horizontal wave numbers of a propagation channel. A narrow-band form of PSA will be described which was used to generate coherent spatial gain by generating an aperture hundreds of wavelengths and provided accurate estimates of the horizontal modal wave numbers at frequencies les than $600 \mathrm{~Hz}$. A broadband form of PSA was used to provide bearing estimation of a ferry passing through the area. The ferry's broadband propulsion noise, which was in the $900 \mathrm{~Hz}$ region, was used as the source. An overall increase in gain is demonstrated. 


\title{
Contributed Papers
}

12:20

5aUWi3. Radar and sonar interferometry. René Garello (ENSTBretagne, Dpt ITI, CS 83818, 29238 Brest Cedex 03, France, rene.garello@telecom-bretagne.eu), Christophe Sintes (ENST-Bretagne, Dpt ITI, CS 83818, 29238 Brest Cedex 03, France, christophe.sintes @enst-bretagne.fr), Didier Gueriot (Telecom Bretagne, Dept iTi - Technopole Brest-Iroise, CS 83818, 29238 Brest, France, didier.gueriot @ telecom-bretagne.eu), Jean-Marie Nicolas (Ecole Nationale Supérieure des Télécommunication de Paris, Telecom ParisTech, Département TSI, 46 rue Barrault, 75634 Paris Cedex 13, France, nicolas@enst.fr)

This paper is an attempt to compare two interferometric processings. The first one is applied to traditional space-borne radar (SAR) and the second on recent interferometric sonar data. Few comparisons between those tech-niques have already been made, despite the fact that they share many similar principles, only a. Thus, the key idea of this article is to present both techniques with assets, drawbacks and specific "tricks" used in dataprocessing. The first part introduces briefly both sensors and compares signal and processing techniques used for both of them. The second part deals with interferometry, and more precisely with underwater and satellite interferometry. Then a noise-pollution analysis is performed on both techniques followed by bias removal methods for getting interferometric information. The conclusion summarizes the similarities between sonar and radar processing, pointing at the techniques that can applied to both.
12:40

5aUWi4. Interferometric synthetic aperture processing: a comparison of sonar and radar. Michael Hayes (University of Canterbury, Private Bag 4800, 8022 Christchurch, New Zealand, michael.hayes @ canterbury.ac.nz), Peter T. Gough (University of Canterbury, Private Bag 4800,8022 Christchurch, New Zealand, peter.gough@canterbury.ac.nz)

Interferometric aperture synthesis is an inverse problem that attempts to form an elevation map of the earth (in the case of radar) or a bathymetric map of the seafloor (in the case of sonar). In both cases, a pair of (nominally) vertically displaced transducers is configured as an interferometer. After aperture synthesis is performed to produce a pair of images, the height of each resolvable scatterer can be estimated using time delay estimation between the image pairs and knowledge of the system geometry. While interferometric synthetic aperture sonar (InSAS) seems like an obvious extension of the methods of interferometric synthetic aperture radar (InSAR), the height estimation algorithms are surprisingly different. In this paper we start with the principle of generalised correlation for optimal time delay estimation. This filters the signals to maximise their coherence since the accuracy of the time delay estimates, and thus the height estimates, strongly depends upon the signal coherence. We then consider the fundamental differences between InSAR and InSAS; namely the relative signal bandwidth, aperture sampling rate, and geometry and show how application of generalised correlation time delay estimation leads to the differences in how InSAS and InSAR signals are processed.

FRIDAY AFTERNOON, 4 JULY 2008

ROOM 242B, 3:20 TO 6:20 P.M.

\section{Session 5pAAa}

\section{Architectural Acoustics and Musical Acoustics: New Measurement Parameters in Performing Arts Spaces II}

\author{
Lily M. Wang, Cochair \\ University of Nebraska - Lincoln, 1110 S. 67th St., Omaha, NE 68182-0681, USA \\ Brian F. Katz, Cochair \\ LIMSI-CNRS, B.P. 133, Orsay, 91403, France
}

Invited Paper

3:20

5pAAa1. Determining acoustical parameters using cochlear modeling and auditory masking. Jasper Van Dorp Schuitman (University of Technology Delft, Lorentzweg 1, 2628 CJ Delft, Netherlands, J.vanDorpSchuitman@tudelft.nl), Diemer De Vries (University of Technology Delft, Lorentzweg 1, 2628 CJ Delft, Netherlands, d.devries@tudelft.nl)

The acoustical qualities of a concert hall or any other room are generally expressed using acoustical parameters determined from impulse responses. From microphone array measurements it turned out that these parameters can fluctuate severely over small distances, whereas the perceptual cues for which these parameters are supposed to be a measure remain constant. This means that a local parameter value has a very low predictive value for acoustic quality. In this research, cochlear modeling techniques and simulations of auditory masking effects have been applied to model human hearing. These techniques together model various stages in the auditory path, like the movement of the basilar membrane inside the cochlea and mechanisms inside the brains. It turns out that determining acoustical parameters using this representation leads to results which show much less spatial fluctuations, and are closer to human perception. 


\title{
Contributed Papers
}

$3: 40$

5pAAa2. Sound strength and reverberation time in small concert halls. Marc Aretz (RWTH Aachen University, Institute for Technical Acoustics, Neustraße 50, 52066 Aachen, Germany, Marc.Aretz@akustik.rwth-aachen .de), Raf Orlowski (Arup Acoustics, St. Giles Hall Pound Hill, CB3 OAE Cambridge, UK, Raf.Orlowski@arup.com)

Many small concert halls are being built in music departments in schools and universities and these have to cater for a wide variety of musicalensembles ranging from orchestras to solo performers. Such diverse musical forces require different acoustic conditions in terms of reverberation and loudness and so variable acoustics are frequently provided. However, introducing absorption decreases reverberation and loudness and so a careful balance needs to be struck between controlling loudness and maintaining reverberation. In the course of this study a series of measurements was carried out in six small concert halls in Cambridge, UK, which accommodate a range of sizes of musical ensembles from quartets to orchestras, in order to determine the range of reverberation times and sound strengths, including changes due to variable absorption. The measured strength levels were compared to values derived from traditional and revised theory on strength calculations in order to assess the accuracy of the theories for small chamber music halls. The measured values of strength levels were observed to be mostly lower than the predicted ones. In order to account for this difference (particularly in spaces with added absorption) a combination of Barron's revised theory and the Vorländer correction factor is proposed.

\section{4:00}

5pAAa3. Proposition of new acoustical parameters to analyze the 3D spatial composition of sound in music spaces. Alban A. Bassuet (Arup Acoustics, 155 Avenue of the Americas, New York, NY 10013, USA, alban .bassuet@arup.com)

From acoustic measurements conducted in more than 100 renowned historical music spaces, for the Constellation Project, this paper proposes new acoustical parameters created to better describe the 3D spatial composition of sound in music spaces. Using B-format recordings, the author is proposing a visualization algorithm to plot the acoustic intensity at specific time frames and ranges. The acoustic energy is decomposed into relevant space segments and energy ratio parameters LH (lateral frontal high versus lateral frontal low), and FR (lateral rear high versus lateral rear low) are deduced and proposed for analyzing the distribution of sound and the envelopment characteristics of the room. Examples of intensity plots and of the proposed 3D acoustical parameters are given for various room types ranging from small to large concert halls, small to large opera houses, famous organ churches and Roman basilicas.

\section{4:20-4:40 Break}

\section{Invited Paper}

\begin{abstract}
$4: 40$
5pAAa4. Early reflection surfaces in Concert Halls - a new quantitative criterion. Yann Jurkiewicz (Kahle Acoustics, 188 avenue Molière, 1050 Brussels, Belgium, yjurkiewicz@kahle.be), Eckhard Kahle (Kahle Acoustics, 188 avenue Molière, 1050 Brussels, Belgium, kahle@kahle.be)

A new acoustic parameter has been defined for the acoustic brief of the Philharmonie de Paris Concert Hall. With a seating capacity of 2400 and the audience enveloping the performers on all sides, the new hall will be at the upper limit of the ideal range for symphonic music, and an efficient acoustic design was called for. In order to relate architectural design to acoustic efficiency, and based on quantitative study of existing halls, an early efficiency parameter was developed. For the Paris Philharmonie the brief requested a total area of $1400 \mathrm{~m}^{2}$ of surfaces being able to create early reflections, with $500 \mathrm{~m}^{2}$ being less than $15 \mathrm{~m}$ from the stage. The studies leading to the definition and the justificantion of the parameter will be presented. Another, more accurate definition expresses the early efficiency parameter in terms of the solid angle for a source on stage, allowing generalization of the new criterion for all hall sizes.
\end{abstract}

\section{Contributed Paper}

\section{5:00}

5pAAa5. Diffuseness and intensity analysis of spatial impulse responses. Tapio Lokki (Helsinki University of Technology, P.O. Box 5400, 02015 TKK, Finland, Tapio.Lokki@tkk.fi)

Spatial impulse responses, meaning responses measured with a microphone grid, were measured in seven concert halls. The microphone array consisted of 12 omni-microphones, enabling a construction of three intensity pairs (in $\mathrm{x}, \mathrm{y}$, and $\mathrm{z}$ directions) with $1 \mathrm{~cm}$ spacing and three intensity pairs with $10 \mathrm{~cm}$ spacing. In each hall impulse responses were measured with at least three loudspeaker and four microphone positions. They were analyzed with directional audio coding methodology, which enables analysis of diffuseness and instantaneous intensity as a function of time and frequency. In other words, with this analysis it is possible to analyze the directions of early reflections and to estimate diffuseness of sound field in a measurement position. Preliminary results indicate that diffuseness is quite similar in different positions in one hall, but it varies more between halls. The directions of early reflections are hard to visualize, however some example videos are shown to get an idea about the possibilities of such an analysis technique.

\section{Invited Paper}

\section{$5: 20$}

5pAAa6. Experiments with the orchestral impulse response. Gary W. Siebein (Univ. of Florida, 231 Arch, PO Box 115702, Gainesville, FL 32611, USA, gsiebein@siebeinacoustic.com), Robert M. Lilkendey (Siebein Associates, Inc., 625 NW 60th Street, Suite C, Gainesville, FL 32607, USA, rlilkendey@ siebeinacoustic.com), Hyun Paek (Siebein Associates, Inc., 625 NW 60th Street, Suite C, Gainesville, FL 32607, USA, hpaek@ siebeinacoustic.com), Chris Jones (Siebein Associates, Inc., 625 NW 60th Street, Suite C, Gainesville, FL 32607, USA, cjones@ siebeinacoustic.com), Joshua Fisher (Siebein Associates, Inc., 625 NW 60th Street, Suite C, Gainesville, FL 32607, USA, jfisher@ siebeinacoustic.com), Youngmin Kwon (Univ. of Florida, 231 Arch, PO Box 115702, Gainesville, FL 32611, USA, ymkwon@ hotmail.com)

Experiments were conducted in a large multipurpose performance hall to examine the differences in listening quality and physical acoustical measurements that resulted from using a single dodecahedral loudspeaker as the sound source and an array of multiple 
directional loudspeakers spread across the stage to simulate the various sections of an orchestra. Significant differences in listening qualities were recorded by listeners at three locations in the hall. The impulse responses recorded using the two systems varied dramatically in structure and in some of the typical acoustic metrics measured. However, there were also similarities among many measurements using the two systems. The research indicates the need for new measurement parameters to express the significant architectural features of the room and the physical acoustical difference that caused the perceived qualities of the sound field to vary. Preliminary analysis of the existing and proposed parameters will be presented.

\section{Contributed Papers}

\section{5:40}

5pAAa7. Relevance of acoustic parameters for musician communication. Anne Guthrie (1926 5th Ave, Troy, NY 12180, USA, guthra2@rpi.edu), Jonas Braasch (Rensselaer Polytechnic Institute, Greene Bldg., 110 8th St., Troy, NY 12180, USA)

In situations of indeterminate musical performance (particularly in telepresence, where acoustic degradation is a frequent concern), autonomous musical communication, both practical and artistic, forms the crux of the musical material. The relevance of stage acoustic and psychoacoustic parameters to contemporary performance situations must be re-examined with regards to the heightened importance of communication. Parameters developed by A. C. Gade, J. Meyer, and D. Brungart are starting points for this examination. Experiments are conducted with four instrumentalists playing excerpts from a composition by Christian Wolff (open notation allows for measurable variations depending on communication quality), communicating telematically between two virtual environments. Parameters determined by questionnaire to have the strongest effect on quality and efficiency of communication are varied at intervals and evaluated by the performers. Five parameters are tested: Self-to-Others Ratio, Commonality of Aural Space, Masking of Individual Voices, Visual-Audio Synchrony, and Position/ Directivity. The performances are recorded and analyzed for variations in musical content, such as dynamics, rhythm, register, and density. The three sets of data (objective parameters, performer evaluation, and musical analysis) are compared to determine the effects of the selected parameters on musical communication.

\section{6:00}

5pAAa8. A qualitative and quantitative analysis of impulse responses from balloon bursts. Dominique J. Cheenne (Columbia College Chicago,
Department of Audio Arts \& Acoustics, 33 East Congress, Suite 601, Chicago, IL 60605, USA, dcheenne @ colum.edu), Mauricio Ardila (Columbia College Chicago, Department of Audio Arts \& Acoustics, 33 East Congress, Suite 601, Chicago, IL 60605, USA, mardila@colum.edu), Connie G. Lee (Columbia College Chicago, Department of Audio Arts \& Acoustics, 33 East Congress, Suite 601, Chicago, IL 60605, USA, miss.connie.lee @gmail.com), Ben Bridgewater (Columbia College Chicago, Department of Audio Arts \& Acoustics, 33 East Congress, Suite 601, Chicago, IL 60605, USA, captain.cranium@gmail.com)

Anechoic recordings of balloon bursts were systematically acquired for various conditions of balloon diameters, puncture location, and inflation pressure. The recordings were analyzed to derive the average frequency spectrum over the effective duration of the acoustic impulse. Although the data show the well-known limitations for the impulse responses (in terms of repeatability and directional behavior) when viewed at high resolution, the results are quite consistent when averaged over one-third octave bands and reveal that the diameter factor (the ratio between the diameter of the inflated balloon to that of its stated maximum), rather than the overall diameter of the balloon, is a good indicator of the sound pressure level, especially above $200 \mathrm{~Hz}$. The study proposes some simple empirical formulas to predict the quantitative sound pressure level and the qualitative spectral response (using the spectral centroid and skewness) from balloon bursts, based on the inflation factor as a variable. The study also offers suggestions to maximize the value of the balloon-burst methodology in building acoustics measurements by describing an effective way to measure reverberation time while simultaneously acquiring useful directional information associated with the reflected sound. 


\title{
Session 5pAAb
}

\section{Architectural Acoustics: Coupled Volume Acoustics II}

\author{
Jason E. Summers, Cochair \\ U.S. Naval Res. Lab., Acoust. Div., Code 7142, 4555 Overlook Ave. SW, Washington, DC 20375, USA \\ Alexis Billon, Cochair \\ Universite de Liege, INTELSIG group - Département E.E.I., B28 Sart-Tilman, Liege, 4000, Belgium
}

\section{Invited Papers}

\author{
3:40
}

5pAAb1. Soundfields in coupled rooms: A theoretical and phenomenological synopsis. Jason E. Summers (U.S. Naval Res. Lab., Acoust. Div., Code 7142, 4555 Overlook Ave. SW, Washington, DC 20375, USA, jason.summers@ nrl.navy.mil)

In systems of acoustically coupled rooms, energy in the reverberant field is exchanged between constituent rooms via transondentboundaries. Energy in the direct field can be distributed between rooms by the same mechanism. These exchanges of energy have been able to explain the phenomenon of multiple-slope decay curves. Likewise, they result in spatial and spectral variations in steady-state SPL and decay-curve shape. The basic form of the decay curve is governed by the gross locations of the source(s) and receiver: which room(s) they occupy, in addition to properties of the rooms themselves: volume, surface area, and absorption. Historically, these basic dependencies have been well explained by statistical-acoustics (SA) models. More subtle variations in decay-curve shape result from the fine-scale locations of source(s) and receivers relative to one another and boundary regions through which energy is exchanged (e.g., apertures). By accounting for radiation from boundaries, and propagation delays within and between rooms, more sophisticated SA models can reproduce these effects. Even so, these models fail when SA assumptions are violated or energy transfer becomes so great (e.g., large aperture areas) that room boundaries are ambiguous. In these cases, newer computational models yield accurate predictions and physical insight. [Work supported by ONR.]

\section{4:00}

5pAAb2. On the use of diffusion equations to model the acoustics of coupled rooms. Alexis Billon (Universite de Liege, INTELSIG group - Département E.E.I., B28 Sart-Tilman, 4000 Liege, Belgium, abillon@ulg.ac.be), Vincent Valeau (Laboratoire d'Etudes Aérodynamiques (LEA), Université de Poitiers - ENSMA - CNRS, Bâtiment K, 40 Avenue du Recteur Pineau, F-86022 Poitiers, France, vincent.valeau @lea.univ-poitiers.fr), Judicaël Picaut (Lab. Central des Ponts et Chaussées, Division Entretien, Sécurité et Acoustique des Routes, Route de Bouaye - BP 4129, 44341 Bouguenais Cedex, France, Judicael.Picaut@lcpc.fr), Cédric Foy (CEBTP-SOLEN, 12 Avenue Gay Lussac, ZAC La Clef Saint Pierre, 78990 Elancourt, France, c.foy@cebtp.fr), Anas Sakout (LEPTIAB Université de La Rochelle, Avenue Michel Crépeau, 17042 La Rochelle Cedex 01, France, asakout@univ-lr.fr)

The acoustics of coupled rooms are characterized by energy exchanges through apertures and/or partition walls. The use of systems of diffusion equations allows to predict the temporal and spatial energy distributions in these configurations quite accurately. In this presentation, the diffusion formalism for room acoustics-prediction is summarized. The systems of equations to be solved in the case of coupling through an aperture and through a partition wall are presented. For two rooms coupled through an aperture (two classrooms connected through an open door), the results obtained with the diffusion model are compared to experimental data, in terms of sound pressure levels and sound decays. On the other hand, for the case of two classrooms connected through a partition wall, the diffusion model is compared to experimental data in terms of sound pressure level difference only. Finally, an engineering application is presented in the configuration involving a workroom including multiple sound sources (e.g., machines) connected to offices through open and closed doors.

\section{4:20}

5pAAb3. Modeling and analysis of acoustically coupled spaces using a diffusion equation model. Yun Jing (Rensselaer Polytechnic Institute, Greene Building, School of Architecture, 110 8th Street, Troy, NY 12180, USA, jingy@rpi.edu), Ning Xiang (Rensselaer Polytechnic Institute, Greene Building, School of Architecture, 110 8th Street, Troy, NY 12180, USA, xiangn@ rpi.edu)

Acoustically coupled spaces have been studied and applied to concert halls due to a number of interesting phenomena inside the spaces, including nonexponential energy decays, which are believed to benefit both desired clarity and reverberance. A diffusion equation model has been recently applied to acoustically coupled spaces to predict both steady-state and time-dependent sound field [A. Billon, et. al., J. Acoust. Soc. Am., 120, 2006, pp. 2043-2054], good agreements between simulations and experimental measurements have been found. In this paper, the diffusion equation along with a recently proposed modified boundary condition [Y. Jing and N. Xiang, J. Acoust. Soc. Am., 123, 145-153 (2008)] is used, to reveal intriguing characteristics of coupled spaces, including the sound pressure level distribution along the aperture, energy flow in both rooms, and location dependence of the acoustic source on energy decay characteristics. Experimental results are employed to verify the model, and show the capability of the diffusion equation model for guiding the design of coupled spaces. 
5pAAb4. Low frequency evaluation of steady-state pressure distribution and reverberation time in two-room coupled system. Miroslaw Meissner (Institute of Fundamental Technological Research PAS, Swietokrzyska 21, 00-049 Warsaw, Poland, mmeissn@ippt .gov.pl)

A modal expansion method supported by a numerical implementation has been used for studying acoustic properties of coupled room system composed of two connected rectangular enclosures. In a theoretical model a low frequency limit was considered, where modes are lightly damped, thus they were approximated by eigenmodes of a hard-walled room. Eigenfunctions and eigenfrequencies were computed numerically via an application of the forced oscillator method. Calculation results have shown a great influence of absorbing material location and sound source position on the distribution of acoustic pressure and sound decay inside enclosures. As was shown it is the result of a modal localization caused by a generation of modes with eigenfrequencies very close to frequencies of modes in rectangular prisms having the same dimensions as enclosures. When one of enclosures was much more absorbent than the other one, calculation data have demonstrated an interaction of modes during a sound decay that produces reverberant curves with a rapid initial decay and a shallow late decay slope. As was found a "sagging" appearance of decay curves occurs when a late sound decay is dominated by a decay of eigenmodes localized in an enclosure with a weak sound damping.

\section{Contributed Paper}

\section{5:00}

5pAAb5. The application of acoustically coupled spaces in concert hall design. Christopher Jaffe (167 East Rocks Road, Norwalk, CT 06851, USA, ADK117@GMAIL.COM)

At mid-twentieth century, a hall having a reverberation decay curve with a late arriving extended second slope was considered an acoustical failure. It was assumed that that the level of the extended reverberation in the hall would interfere with the ongoing running music of the ensemble and reduce orchestral clarity, definition and transparency. To my knowledge, only two practitioners pioneered the utilization of physical acoustic coupling in concert shell and concert hall design during those early years. One was Russell Johnson, when he was with Bolt Beranek and Newman and later with his own firm Artec. The other was me with both my firms Stagecraft Corporation and Jaffe Acoustics. This paper discusses how the need to improve the concert hall environment of American multi-use theatres led to the application of coupling techniques in these halls and how both Mr. Johnson and I later applied physical acoustic coupling in single room concert spaces.

\section{Invited Paper}

5pAAb6. Multivariable analysis of energy decay in coupled volume rooms: How can we objectively describe what we perceive in coupled volume performance spaces? Todd L. Brooks (Artec Consultants Inc, 114 W 26th ST FL 12, New York, NY 10001, USA, tlb@artecconsultants.com), Ted Pyper (Artec Consultants Inc, 114 W 26th ST FL 12, New York, NY 10001, USA, tap@artecconsultants.com), Kelly A. Aston (Artec Consultants Inc, 114 W 26th ST FL 12, New York, NY 10001, USA, ka@artecconsultants.com), Damian J. Doria (Artec Consultants Inc, 114 W 26th ST FL 12, New York, NY 10001, USA, dd @artecconsultants.com)

The behavior of coupled volumes in room acoustics is commonly characterized by multiple slopes observed in sound energy decay curves, as measured (or modeled) using an omnidirectional receiver. This characterization is often limiting and can not adequately explain the change in character of sound energy decay with time that we perceive as listeners in music performance spaces that employ coupled volumes. By investigating energy decay as a function of several independent variables, including time, frequency, and direction of energy arrival, we seek to forge new objective measures that relate to what a listener actually hears in a coupled volume concert hall. We will discuss relevant geometry and materials of several coupled volume performance spaces designed by Artec Consultants Inc, describe subjective phenomena we have observed in these halls, and present results of our ongoing investigation of new objective metrics geared to better characterize coupled volume music performance spaces.

\section{Contributed Paper}

\section{$5: 40$}

5pAAb7. Theoretical considerations in the prediction of decay times for the Philharmonie de Paris. Thomas Scelo (Marshall Day Acoustics LTD, P O Box 5811, Wellesley St., 1000 Auckland, New Zealand, thomas.scelo@marshallday.co.nz), Harold Marshall (Marshall Day Acoustics LTD, P O Box 5811, Wellesley St., 1000 Auckland, New Zealand, harold.marshall@marshallday.co.nz), Joanne O. Valentine (Marshall Day Acoustics LTD, P O Box 5811, Wellesley St., 1000 Auckland, New Zealand, joanne.valentine@marshallday.co.nz)

The brief for the Philharmonie de Paris includes the requirement that the hall should combine great clarity with high reverberation. The proposed solution, which won the design competition, consists of two nested chambers: an inner space producing acoustical intimacy and an outer space with its own architectural and acoustical presence. The interaction between these two spaces gives the possibility for the full range of acoustical adaptability required in the acoustical brief. This paper reports on some theoretical modelling work for the hall where the geometry considered is first described in the context of coupled space modelling. The predicted range of the variability achieved in the design by closure of the coupling openings is then presented. Finally, the paper discusses the appropriateness of these models when predicting the decay times in such a complex geometry. 


\title{
Invited Paper
}

6:00

5pAAb8. Adjustable acoustics --- Coupled volumes in Artec concert halls: an extravagance or necessity? Tateo Nakajima (Artec Consultants Inc, 114 W 26th ST FL 12, New York, NY 10001, USA, tn@artecconsultants.com), Damian J. Doria (Artec Consultants Inc, 114 W 26th ST FL 12, New York, NY 10001, USA, dd@artecconsultants.com), Edward Arenius (Artec Consultants Inc, 114 W 26th ST FL 12, New York, NY 10001, USA, ea@artecconsultants.com), Andrew Morgan (Artec Consultants Inc, 114 W 26th ST FL 12, New York, NY 10001, USA, ajm@artecconsultants.com)

Led by its founder, Russell Johnson, Artec has developed an unequalled body of experience in the practical application of coupled volumes in the design and construction of concert halls. This paper will present a survey of selected past and future Artec projects from the point of view of the artists and venue managers that perform and work in these halls on a daily basis. Are adjustable acoustics an extravagance or a necessity? What practical problems have been encountered and are they inherent in the concept of adjustability or can they be avoided? How have the musicians reacted? And what is the future of adjustable acoustics?

FRIDAY AFTERNOON, 4 JULY 2008

ROOM 343, 1:40 TO 4:20 P.M.

\section{Session 5pAO}

\section{Acoustical Oceanography and ECUA: Acoustical Tomography and Long Range Propagation}

\author{
Timothy Duda, Cochair \\ Woods Hole Oceanographic Institution, 98 Water Street, Bigelow 107, MS-12, Woods Hole, MA 02543, USA \\ Yann Stephan, Cochair \\ SHOM, 13 rue du Chatellier, CS 92803, Brest cedex 2, 29228, France
}

\section{Contributed Papers}

\begin{abstract}
1:40
5pAO1. Ocean acoustic tomography using a double-beamforming algorithm. Ion Iturbe (GIPSA-lab, dep. DIS, 961, rue de la Houille Blanche, 38402 St Martin d'Hères, France, ion.iturbe@gipsa-lab.inpg .fr), Philippe Roux (LGIT - CNRS - Université Joseph Fourier, Maison des Géosciences, 1381 rue de la Piscine, BP 53, 38041 Grenoble, France, philippe.roux@obs.ujf-grenoble.fr), Barbara Nicolas (GIPSA-lab, dep. DIS, 961, rue de la Houille Blanche, 38402 St Martin d'Hères, France, barbara.nicolas@gipsa-lab.inpg.fr), Jérôme I. Mars (GIPSA-lab, dep. DIS, 961, rue de la Houille Blanche, 38402 St Martin d'Hères, France, jerome .mars@gipsa-lab.inpg.fr)
\end{abstract}

Since Munk and Wunsch proposed the basis for ocean acoustic tomography, many experiments have been performed to estimate sound speed fluctuations in the ocean, using ray identification and measurement of their travel times. However, technical limitations appeared such as the precision of the arrival time measurements or the number of ray arrivals that can be extracted from the signal. Recently, technical improvements allowed more complete experiments using two vertical arrays of sensors (source array and hydrophone array). In this configuration, the signals between each source and receiver are recorded which greatly improve the available information to identify the acoustic rays. One way to increase the number of rays in the tomography algorithm is to perform double-beamforming on the source and receive arrays. With double-beamforming, ray arrivals are separated by emission angle, reception angle and arrival time. Thus, we solve more ray arrivals than with a single beamforming or with a point-to-point approach. In order to avoid previous limitations and to explore acoustical limitations, we study two simple cases through the double-beamforming algorithm: with simulated data and with ultrasonic small-scale experimental data.

\section{2:00}

5pA02. A simulation study of shallow water tomography for coastal monitoring. Olivier Carrière (Université libre de Bruxelles (U.L.B.) Environmental hydroacoustics lab, av. Franklin D. Roosevelt 50, CP 194/5, 1050 Bruxelles, Belgium, ocarrier@ulb.ac.be), Jean-Pierre
Hermand(Université libre de Bruxelles (U.L.B.) - Environmental hydroacoustics lab, av. Franklin D. Roosevelt 50, CP 194/5, 1050 Bruxelles, Belgium, jhermand@ulb.ac.be), Yann Stephan (SHOM, 13 rue du Chatellier, CS 92803, 29228 Brest cedex 2, France, yann.stephan@shom.fr)

Developing operational oceanographic models for coastal environment is an exciting challenge for the next decades. The typical sparsity of assimilated in-situ observations often creates biases in the model predictions reducing the overall accuracy of the forecasting. In such a highly dynamic environment, acoustic tomography can be a good candidate to provide synoptic measurements over wide areas while a range-dependent inversion scheme allows to achieve a reasonable spatial resolution. In this work, we present simulation results of a Kalman-based assimilation of ocean-acoustic data for a basic model of the Ushant front west off Britanny. In a first part, a single vertical slice tomography experiment is simulated for a static front model to study in which way the modal propagation of a multifrequency acoustic signal is affected by the characteristics of the front (position, intensity). In a second part, the problem of assimilating full-field acoustic data into a dynamic model and tracking of the range-dependent sound-speed field is addressed.

\section{2:20}

5pA03. Sound-speed estimation from RAFOS transmissions. Emmanuel Skarsoulis (FORTH / IACM, N. Plastira 100, Vasilika Voutes, GR-70013 Heraklion, Greece, eskars@iacm.forth.gr), George Piperakis (FORTH / IACM, N. Plastira 100, Vasilika Voutes, GR-70013 Heraklion, Greece, piperak@iacm.forth.gr)

Acoustic navigation of Lagrangian (moving) floats is carried out by measuring travel times from a number of fixed stations/moorings. A minimum of two fixed stations are needed for location estimation in the horizontal, whereas an additional fixed station is commonly used to remove leftright ambiguity. The signals (RAFOS) and sampling schemes used in ocean acoustic navigation are characterized by limited time resolution (order of $200 \mathrm{msec}$ ), much smaller than the resolution used in travel-time tomography (order of 1-10 msec). The possibility of combining navigation signals (travel 
times) from three fixed stations to multiple moving floats for simultaneous sound-speed estimation and float localization is examined here. The redundant travel-time information in this case offers a significant advantage and makes the accurate estimation of the speed of sound feasible. It is shown that the estimation error for the sound speed decreases with the number of floats, and thus the estimation accuracy improves as the set of floats grows larger. This procedure also leads to improved location estimates for the individual floats. A number of numerical experiments are used to demonstrate the performance of the method. [Work supported by EU/FP6 Damocles project.]

\section{2:40}

5pA04. Using Seagliders for acoustic receiving and communication. Bruce M. Howe (Applied Physics Lab., Univ. of Washington, 1013 Northeast 40th St., Seattle, WA 98105, USA, howe@apl.washington .edu), Michael L. Boyd (Applied Physics Lab., Univ. of Washington, 1013 Northeast 40th St., Seattle, WA 98105, USA, mike@apl.washington.edu)

Underwater gliders are beginning to be used as tools in ocean acoustics and acoustical oceanography. Results from several experiments conducted in summer 2006 with Seagliders equipped with acoustic modems and receivers are described. Off Kauai, a glider received signals from the Acoustic Thermometry of Ocean Climate/North Pacific Acoustic Laboratory $75 \mathrm{~Hz}$ source; subsequent coherent processing showed close to theoretical gain for 12 min records while moving away from the source at ranges $>100 \mathrm{~km}$ with velocity $20 \mathrm{~cm} / \mathrm{s}$ (measured by travel time, Doppler, and dead reckoning). In the Monterey Bay MB06 experiment, two-way communications between other subsea platforms and shore via the acoustic modem-equipped glider was demonstrated (albeit with latency). The results support the future use of gliders as precision navigated platforms, communication and time distribution nodes, and thermometry/tomography mobile receivers. Work supported by the Office of Naval Research.

\section{3:00}

5pA05. A decade of acoustic thermometry in the North Pacific Ocean: Using long-range acoustic travel times to test gyre-scale temperature variability derived from other observations and ocean models. Peter Worcester (MPL, Scripps Institution of Oceanography, University of California, San Diego, La Jolla, CA 92093-0238, USA, pworcester@ucsd .edu), Brian D. Dushaw (Applied Physics Lab., Univ. of Washington, 1013 Northeast 40th St., Seattle, WA 98105, USA, dushaw@apl.washington .edu), Rex K. Andrew (Applied Physics Lab., Univ. of Washington, 1013 Northeast 40th St., Seattle, WA 98105, USA, randrew@apl.washington .edu), Bruce M. Howe (Applied Physics Lab., Univ. of Washington, 1013 Northeast 40th St., Seattle, WA 98105, USA, howe@apl.washington .edu), James A. Mercer (Applied Physics Lab., Univ. of Washington, 1013 Northeast 40th St., Seattle, WA 98105, USA, mercer@apl.washington .edu), Robert C. Spindel (Applied Physics Lab., Univ. of Washington, 1013 Northeast 40th St., Seattle, WA 98105, USA, spindel@apl.washington .edu), Bruce Cornuelle (MPL, Scripps Institution of Oceanography, University of California, San Diego, La Jolla, CA 92093-0238, USA, bdc@ucsd .edu), Matthew Dzieciuch (MPL, Scripps Institution of Oceanography, University of California, San Diego, La Jolla, CA 92093-0238, USA, mad @ ucsd.edu), Theodore G. Birdsall (Univ. of Michigan, Electrical Engineering and Computer Science Dept., 1301 Beal Ave., Ann Arbor, MI 48109 2122, USA, birdsall@umich.edu), Kurt Metzger (Univ. of Michigan, Electrical Engineering and Computer Science Dept., 1301 Beal Ave., Ann Arbor, MI 48109-2122, USA, metzger@umich.edu), Dimitris Menemenlis (Jet Propulsion Laboratory, California Institute of Technology, 4800 Oak Grove Dr., Pasadena, CA 91109, USA, menemenlis@jpl.nasa.gov)

Large-scale, range- and depth-averaged temperatures in the North $\mathrm{Pa}$ cific Ocean were measured by long-range acoustic transmissions over the decade 1996-2006. Acoustic sources off central California and north of Kauai transmitted to receivers throughout the North Pacific. Even though acoustic travel times are spatially integrating, suppressing mesoscale variability and providing a precise measure of large-scale temperature, the travel times sometimes vary significantly on time scales of only a few weeks. The interannual variability is large, with no consistent warming or cooling trends. Comparison of the measured travel times with travel times derived from (i) the World Ocean Atlas 2005 (WOA05), (ii) an upper ocean tem- perature estimate derived from satellite altimetry and in situ profiles, (iii) an analysis provided by the Estimating the Circulation and Climate of the Ocean (ECCO) project, and (iv) simulation results from a high-resolution configuration of the Parallel Ocean Program (POP) show similarities, but also reveal substantial differences. The differences suggest that the data can provide significant additional constraints for numerical ocean simulations. The acoustic data show that WOA05 is a much better estimate of the timemean hydrography than either the ECCO or POP estimates and provide significantly better time resolution for large-scale ocean variability than can be derived from satellite altimetry and in situ profiles.

\section{3:20}

5pA06. Coherence of tracked arrivals in SPICEX. Matthew Dzieciuch (MPL, Scripps Institution of Oceanography, University of California, San Diego, La Jolla, CA 92093-0238, USA, mad@ucsd.edu), Bruce Cornuelle (MPL, Scripps Institution of Oceanography, University of California, San Diego, La Jolla, CA 92093-0238, USA, bdc@ucsd.edu), Peter Worcester (MPL, Scripps Institution of Oceanography, University of California, San Diego, La Jolla, CA 92093-0238, USA, pworcester@ucsd.edu)

In the fall of 2004, $250 \mathrm{~Hz}$ broadband signals were received at $500 \mathrm{~km}$ and $1000 \mathrm{~km}$ ranges on a near full water-column vertical array in the North Pacific ocean. Individual ray arrivals of very high SNR could easily be identified and tracked using a turning-point filter (time-delay beamforming accounting for channel structure), thus providing accurate vertical coherence estimates. The observations can be compared to standard Monte Carlo estimates of coherence made using accurate parabolic-equation acoustic propagation in an ensemble of ocean states consistent with the standard GarrettMunk ocean internal-wave spectrum. Acoustic coherence can also be expressed as a depth- dependent structure function. This is naturally estimated by the full-wave travel-time sensitivity kernel (TSK) which provides a linearized transformation from the internal wave spectrum to the structure function. Environmental measurements were conducted almost concurrently with the acoustic trials, constraining the acceptable ocean variability. Allowances must be made for scattering by sound-speed variability along isopycnals (spiciness) in the upper mixed layer. The most important conclusion to date is that the vertical coherence is depth-dependent but this statement must be qualified by the ability of the beamformer to separate arrivals.

\section{$3: 40$}

5pA07. Travel-time sensitivity kernels in long-range propagation. Emmanuel Skarsoulis (FORTH/IACM, N. Plastira 100, Vasilika Voutes, GR-70013 Heraklion, Greece, eskars@iacm.forth.gr), Bruce Cornuelle (MPL, Scripps Institution of Oceanography, University of California, San Diego, La Jolla, CA 92093-0238, USA, bdc@ucsd .edu), Matthew Dzieciuch (MPL, Scripps Institution of Oceanography, University of California, San Diego, La Jolla, CA 92093-0238, USA, mad @ucsd.edu)

The effect of increasing range on the sensitivity of finite-frequency travel-time observables to sound-speed perturbations is studied using the notion of wave-theoretic sensitivity kernels based on the first Born approximation of the Green's function and the notion of peak arrivals. Travel-time sensitivity kernels are examined in a range-independent background ocean environment. While at medium ranges the kernels exhibit zero-sensitivity cores on the eigenrays, these cores shrink and disappear at long ranges due to refraction and the kernels converge in the vertical towards the corresponding eigenrays; this behavior is not observed in free space. On the other hand the kernels expand in the horizontal cross-range direction and attain their maximum extent at the midpoint between source and receiver, similar to the behavior of the Fresnel volume in free space. Thus, stratification affects the shape of the sensitivity kernel in the vertical preventing expansion with increasing range but not in the horizontal such that the sensitivity kernel takes the form of a folded rug expanding the eigenray in the horizontal cross-range direction. [Work supported by ONR.] 


\section{4:00}

5pA08. High-frequency broadband acoustic current tomography in shallow water. Jing Luo (University of Delaware, College of Marine and Earth Studies, S. College Street, Newark, DE 19716, USA, luojing@udel .edu), Entin A. Karjadi (College of Marine and Earth Studies, University of Delaware, Newark, DE 19716, USA, karjadi@udel.edu), Mohsen Badiey (University of Delaware, College of Marine and Earth Studies, S. College Street, Newark, DE 19716, USA, badiey@udel.edu)

To study current tomography in very shallow water regions a simultaneous oceanographic and broadband (1-25 kHz) acoustic experiment was conducted in the Delaware Bay. The mean water depth was $15 \mathrm{~m}$ and the source-receiver range was $760 \mathrm{~m}$. In this paper, we discuss the feasibility of using reciprocal acoustic transmission for current tomography applications. A beamforming technique is used to resolve the arrival time of direct and surface-bounced rays since in shallow water the received acoustic signals are more complicated due to multiple interactions with bottom and sea surface. Using the experimental data, the accuracy of travel time measurements for variable environmental conditions is examined for different center frequencies and bandwidths. The current velocity prediction results are compared with ADCP measurements to determine the feasibility of current tomography in shallow water.

FRIDAY AFTERNOON, 4 JULY 2008

ROOM 353, 2:00 TO 5:20 P.M.

\title{
Session 5pBB
}

\section{Biomedical Ultrasound/Bioresponse to Vibration: General Topics in Biomedical Ultrasound/Bioresponse to Vibration II}

\author{
Michael R. Bailey, Cochair \\ Center for Industrial and Medical Ultrasound, Applied Physics Lab., University of Washington, 1013 NE 40th St., Seattle, \\ WA 98105, USA \\ Oleg A. Sapozhnikov, Cochair \\ Center for Industrial and Medical Ultrasound, Applied Physics Lab., University of Washington, 1013 NE 40th St., Seattle, \\ WA 98105, USA
}

\section{Contributed Papers}

\section{2:00}

5pBB1. Dual confocal ultrasound system for shear wave elastography. Michael D. Gray (Georgia Institute of Technology, Mechanical Engineering, 771 Ferst Drive, Atlanta, GA 30332-0405, USA, michael.gray@me .gatech.edu), James S. Martin (Georgia Institute of Technology, Mechanical Engineering, 771 Ferst Drive, Atlanta, GA 30332-0405, USA, james.martin@me.gatech.edu), Peter H. Rogers (Georgia Institute of Technology, Mechanical Engineering, 771 Ferst Drive, Atlanta, GA 30332-0405, USA, peter.rogers@me.gatech.edu)

A dual confocal transducer system for ultrasound-based elastography is presented. The system is intended to noninvasively measure the complex shear speed in cetacean head tissues, including brain, jaw fat, and melon. The system instrumentation features a pair of dual-element confocal ultrasound transducers, one of which is used to remotely generate low frequency $(100-1000 \mathrm{~Hz})$ shear waves in soft tissues via radiation force, and the other is used to measure the resulting shear wave displacements using Doppler techniques. One transducer is configured as an open ring into which the other transducer can be placed and translated. The relative positions of the transducers are mechanically manipulated in order to measure short-path propagation and estimate shear wave speed and loss. Work supported by ONR.

\section{$2: 20$}

5pBB2. Simulated response of the human lung to low-frequency underwater sound using a finite-element-based thoracic model. Mark S. Wochner (Applied Research Laboratories, The University of Texas, P.O. Box 8029, Austin, TX 78713-8029, USA, mwochner@mail.utexas .edu), Yurii A. Ilinskii (Applied Research Laboratories, The University of Texas, P.O. Box 8029, Austin, TX 78713-8029, USA, yura@arlut.utexas .edu), Mark F. Hamilton (Applied Research Laboratories, The University of Texas, P.O. Box 8029, Austin, TX 78713-8029, USA, hamilton@mail .utexas.edu), Evgenia A. Zabolotskaya (Applied Research Laboratories, The University of Texas, P.O. Box 8029, Austin, TX 78713-8029, USA, zhenia@arlut.utexas.edu)

In a previous paper, inhomogeneity within the lungs and its influence on the lung's response to low-frequency underwater sound using a finiteelement-based model was discussed [Wochner, et al., J. Acoust. Soc. Am. 122, 2957 (2007)]. Here we report an extension of the previous work that adds surrounding organs to the finite element model. It is hypothesized that the significant damage that can occur at relatively low amplitudes when the lung is in resonance is due primarily to the shear stresses that can occur in the lung through its interactions with surrounding organs. In particular, the heart, diaphragm, and ribs, all of which have considerably different material properties compared to lung, may have a sizable effect on the lung's response to low-frequency underwater sound. This paper will discuss the resonance frequency, motion, and stress and strain fields produced using this new finite-element-based thoracic model. [Work supported by ONR and ARL:UT IR\&D.]

\section{2:40}

5pBB3. Dual apodization technique for improved contrast. Jesse Yen (University of Southern California, 1042 Downey Way, Los Angeles, CA 90089, USA, jesseyen@usc.edu), Chi Seo (University of Southern California, 1042 Downey Way, Los Angeles, CA 90089, USA, chiseo@usc .edu)

We propose a method to use dual apertures or dual apodization functions to reduce side lobes and clutter for ultrasound imaging. Using a common transmit aperture but different receive apodizations or apertures, we create two point spread functions with very similar main lobes and different sidelobe and clutter signals. Main lobe signals can be distinguished from clutter signals using normalized cross-correlation of the raw radio-frequency data. The normalized cross-correlation coefficient is used as a pixel-by-pixel weighting to pass main-lobe signals and suppress side lobe and clutter 
signals. Main lobe signals will have a high cross-correlation coefficient near 1 and clutter signals will have coefficients between 0 and -1 . Point target simulations show a narrowing of the main beam compared to conventional beamforming at beamwidths $-20 \mathrm{~dB}$ and lower. Using a $5 \mathrm{MHz} 128$-element linear array, improvements of contrast-to-noise ratio $(\mathrm{CNR})$ of an anechoic cyst compared to delay and sum beamforming exceed $130 \%$ in both simulations and experiments. We also evaluated this method for robustness in the presence of phase aberration. Aberrators ranging from 25-45 ns rms with correlation lengths of 3-5 mm were used. Here, improvements in CNR also exceed $100 \%$ in many cases.

\section{3:00}

5pBB4. Nonlinear ultrasonic imagery of high contrast objects. Régine Guillermin (Laboratory for Mechanics and Acoustics CNRS, 31 chemin Joseph Aiguier, 13009 Marseille, France, guillermin@1ma.cnrs-mrs .fr), Philippe Lasaygues (Laboratory for Mechanics and Acoustics CNRS, 31 chemin Joseph Aiguier, 13009 Marseille, France, lasaygues@1ma.cnrsmrs.fr)

This study is concerned whith the ultrasonic imagery of elastic materials like cylinders or tubes by diffraction tomography technic. The aim of this work is then to solve a nonlinear inverse scattering problem. Various methods can be employed, generally involving a minimization of the differences between modeling data and measurements. The Distorted Born Iterative (DBI) method belongs to the class of algebraic reconstruction algorithms and have therefore been investigated in literature. Very promising results have been obtained both on synthetic and experimental data especially for electromagnetic inverse diffraction problems, but as far as the authors know few ultrasonic experimental results are available. This method was developed to increase the domain of application of the Born approximation to high contrast targets. Iterations are performed numerically solving a forward and an inverse problems at every iteration. This yields quantitative information about the scatterer, such as the speed of sound. Inversions of both numerical and experimental data are presented.

\section{$3: 20$}

5pBB5. Inverse scattering in modern ultrasound imaging. Francesco Simonetti (Imperial College London, Department of Mechanical Engineering, South Kensington Campus, SW7 2AZ London, UK, f.simonetti @imperial.ac.uk)

Progress in solid state electronics and sensor manufacturing has led to the rapid development of ultrasound arrays over the last decade resulting in prototypes with thousands of transreceivers. Ultrasound scanners that use this technology are widely used in medical imaging and are based on beamforming techniques. In a similar fashion to an optical lens, the array forms an aperture which can focus and steer an ultrasound beam in space as it is done by microscopes and telescopes. The beamforming process can be seen as an inverse scattering problem whereby the scattering measurements are used to reconstruct the structure of the object being probed. To achieve this, a model that describes the interaction of the probing wave with the object is required. Beamforming assumes that scattering events occurring at different locations within the object are independent of each other, thus neglecting multiple scattering. Here, it is argue that accounting for more accurate wavematter interaction models in the inverse scattering problem leads to greater image quality than that obtained with conventional beamforming. Experimental images with unprecedented resolution beyond the classical diffraction limit are presented along with tomographic reconstructions of a complex 3D breast phantom that show striking similarities with $\mathrm{x}$-ray CT.

\section{3:40}

5pBB6. Deconvolution of freehand 3D ultrasound data using improved reconstruction techniques in consideration of ultrasound point spread functions. Holger J. Hewener (Fraunhofer IBMT, Ensheimer Strasse 48, 66386 Sankt Ingbert, Germany, holger.hewener@ibmt.fraunhofer .de), Robert M. Lemor (Fraunhofer IBMT, Ensheimer Strasse 48, 66386 Sankt Ingbert, Germany, robert.lemor@ibmt.fhg.de)

Medical ultrasound data suffers from blur caused by the volume expansion of the pressure field of the mechanical wave. This blur is dependent on the used excitation pulse and focusing of the ultrasonic wave and can there- fore be examined. In order to improve the overall system resolution for $3 \mathrm{D}$ ultrasound reconstructions we have to know this signal degeneration to compensate it using deconvolution techniques or multicode compounding during the volume reconstruction step. Looking at the ultrasound transfer function we can focus on the simulation and measurement of the "point spread function" especially in the lateral and elevational direction. To understand its effects on a 3D reconstruction we compute a simulation of freehandultrasound slices based on synthetic phantom structures and given US parameters. Computing a 3D reconstruction of these simulated slices we are able to optimize the reconstruction algorithm itself to archive better resolution in the volume data sets considering ultrasound parameters like beamforming and the excitation pulses.

\section{4:00-4:20 Break}

4:20

5pBB7. Noninvasive monitoring of mesenchymal stem cells by $1.2 \mathrm{GHz}$ acoustic microscopy. Moritz Von Buttlar (Institute of Experimental Physics II, University of Leipzig, Linnéstr. 5, 04103 Leipzig, Germany, vbuttlar@physik.uni-leipzig.de), Evgeny Twerdowski (Institute of Experimental Physics II, University of Leipzig, Linnéstr. 5, 04103 Leipzig, Germany, twerdowski@physik.uni-leipzig.de), Reinhold Wannemacher (Institute of Experimental Physics II, University of Leipzig, Linnéstr. 5, 04103 Leipzig, Germany, wannemacher@physik .uni-leipzig.de), Wolfgang Grill (Institute of Experimental Physics II, University of Leipzig, Linnéstr. 5, 04103 Leipzig, Germany, grill@physik.unileipzig.de)

Cell-based therapies can benefit from noninvasive and marker-free monitoring techniques for living cells. For this purpose a phase-sensitive scanning acoustic microscope operating at a frequency of $1.2 \mathrm{GHz}$ was combined with a commercial confocal laser scanning microscope. The system is equipped with a live-support system for the long-term observation of living cells. Confocal acoustic imaging with phase and magnitude contrast and confocal laser scanning microscopy can be performed simultaneously. Both techniques are used in reflection from opposing sides of the object. Timelapsed acoustic microscope images of ovine mesenchymal stem cells are presented. For this purpose, a pseudo-3D representation is generated by encoding the unwrapped phase in the height and the magnitude in the brightness. In the case of highly reflective substrates and sufficiently low reflection from the interface between the cells and the surrounding fluid the echo from the top of the cells can be neglected and the phase contrast image can be transformed to a time-of-flight image. In the same approximation the magnitude image provides information about the gradual extinction of the echo signal due to absorption in the cells. The two images can be combined to generate a new form of contrast representing the product of the absorption coefficient and the velocity of sound inside the observed cells.

\section{4:40}

5pBB8. Transverse vibration of prestressed beams: An experimental technique for the determination of dynamic viscoelastic material properties of tissue mimicking materials. Yigit Yazicioglu (Middle East Technical University, Orta Dogu Teknik Universitesi, Makine Muhendisligi Bolumu B-313, 06531 Ankara, Turkey, yigit@metu.edu.tr), Bryn A. Martin (University of Illinois at Chicago, 2923 W. 71st Street, Woodridge, IL 60517, USA, flux@ebryn.com), Karen Navarro-Castillo (University of Illinois at Chicago, 842 W. Taylor St. ERF 1072, Chicago, IL 60607, USA, knc_001@yahoo.com.mx), Umit Kutluay (Tubitak-Sage, Samsun Yolu 25 .Km, Tilkicak Tepe Mevkii P.K.16, 06261 Ankara, Turkey, ukutluay@sage.tubitak.gov.tr), Thomas J. Royston (University of Illinois at Chicago, 842 W. Taylor St. ERF 1072, Chicago, IL 60607, USA, troyston @uic.edu)

An experimental dynamic material property identification technique is presented that is based on the theoretical formulations of a vibrating prestressed beam. The technique determines the viscoelastic material properties of tissue mimicking materials that govern their dynamic behavior. Results are presented for silicone-based materials (Sylgard 184, Dow Corning, Midland, MI) that are formed in the lab using a range of mixing ratios and cast in the form of a thick string held between fixed supports under tensile pre- 
stress. The specimens are excited through the transverse harmonic displacement of the boundary. Transverse vibration at an arbitrary location is measured and compared with theory to identify material viscoelastic moduli valid up to at least several hundred Hertz. The presented technique can aid in providing accurate viscoelastic parameter values for phantoms that are used in the development of a range of medical diagnostic techniques that attempt to identify pathology or tissue differentiation via changes in mechanical stiffness and viscosity.

\section{5:00}

5pBB9. Air-borne and tissue-borne sensitivity of skin-radiation acoustic sensors. Matias Zanartu (School of Electrical and Computer Engineering, Purdue University, 206 South Martin Jischke Drive, West Lafayette, IN 47907, USA, mzanartu@purdue.edu), Julio C. Ho (Weldon School of Biomedical Engineering, Purdue University, 206 South Martin Jischke Drive, West Lafayette, IN 47907, USA, hoj@purdue.edu), Steve Kraman (Department of Internal Medicine, University of Kentucky, 740 South Limestone Street, Lexington, KY 40536, USA, sskram01@email.uky.edu), Hans Pasterkamp (Department of Pediatrics and Child Health, University of Manitoba, CS516-840 Sherbrook St, Winnipeg, MB R3A 1S1, Canada, pasterkamp@umanitoba.ca), Jessica E. Huber (Department of Speech, Language, and Hearing Sciences, Purdue
University, 500 Oval Drive, West Lafayette, IN 47907, USA, jhuber@purdue.edu), George R. Wodicka (Weldon School of Biomedical Engineering, Purdue University, 206 South Martin Jischke Drive, West Lafayette, IN 47907, USA, wodicka@purdue.edu)

Measurements of body sounds on the skin surface have been widely used in the medical field and continue to be a topic of current research, ranging from the diagnosis of the respiratory and cardiovascular diseases to the monitoring of voice dosimetry. These measurements are normally acquired by means of light-weight accelerometers and/or air-coupled microphones attached to the skin. Such recordings can be corrupted by air-borne sounds that are generated by the subject or by other sources of background noise. In this project, the sensitivity of various commonly used bioacoustic sensors to airborne sounds was evaluated and compared with their sensitivity to tissueborne body sounds. To delineate the sensitivity to each pathway, the sensors were first tested in-vitro, and then on human subjects. The results indicated that in many cases the air-borne sensitivity is sufficiently high to significantly corrupt body sound signals. The effectiveness of different air-borne insulation devices was also evaluated. Spectral analysis showed that simple acoustic insulators (e.g., passive hearing protectors) provide significant attenuation within the range of frequencies of interest for most applications, particularly when using air-coupled microphones.

FRIDAY AFTERNOON, 4 JULY 2008

ROOM 252A, 2:00 TO 6:20 P.M.

\title{
Session 5pNSa
}

\section{Noise, ASA Committee on Standards, Architectural Acoustics, and EURONOISE: Classroom Acoustics II}

\author{
Louis C. Sutherland, Cochair \\ Consultant in Acoustics, 27803 Longhill Dr., Rancho Palos Verdes, CA 90275-3908, USA
}

Luigi Maffei, Cochair

Built Environment Control Laboratory Ri.A.S., Second University of Naples, Abazia di S. Lorenzo, Aversa, 81031, Italy

David Lubman, Cochair

DL Acoustics, 14301 Middletown Lane, Westminster, CA 92683, USA

\section{Invited Paper}

5pNSa1. Are classrooms in historical buildings compatible with good acoustics standards? Luigi Maffei (Built Environment Control Laboratory Ri.A.S., Second University of Naples, Abazia di S. Lorenzo, 81031 Aversa, Italy, luigi.maffei@unina2.it), Gino Iannace (Built Environment Control Laboratory Ri.A.S., Second University of Naples, Abazia di S. Lorenzo, 81031 Aversa, Italy, gino.iannace@unina2.it), Massimiliano Masullo (Built Environment Control Laboratory Ri.A.S., Second University of Naples, Abazia di S. Lorenzo, 81031 Aversa, Italy, ing.masullo@libero.it)

Many surveys and researches have underlined that the acoustic characteristics of classrooms are strictly connected to performances of students and to the stress of the teachers during lessons. In standard classrooms sound quality can be easily reached without sound amplification but introducing appropriate sound absorbing/scattering materials at the ceiling and/or at the vertical walls. Nevertheless in historical buildings with vaults or trusses, high walls and many architectural restrictions imposed by district superintendent, it could be very difficult to achieve good acoustics standards with widespread solutions. In this paper the acoustic performances of different classrooms in an historical Monastery actually center of the Faculty of Architecture of the Second University of Naples are analysed. After these analysis, compatible architectural and acoustic solutions to improve the sound quality were developed and tested in laboratory measurements and then applied in some classrooms to verify the benefits. 


\section{Contributed Paper}

5pNSa2. Results of acoustical treatments in existing classrooms Arianna Astolfi (Politecnico di Torino, Department of Energetics, Corso Duca degli Abruzzi, 24, 10129 Turin, Italy, arianna.astolfi@polito .it), Alessia Griginis (Onleco srl, Via Pigafetta, 3, 10129 Turin, Italy, griginis@onleco.com)

In 2001, 2002, and 2005, the Department of Energetics of the Politecnico di Torino has carried out in-field objective and subjective surveys with the aim of evaluating the acoustical quality in secondary-school classrooms of the Province of Turin (Italy). From the results the following main problems emerged: high reverberation times, high background noise levels caused mainly by low sound insulation between classrooms and corridors and between adjacent classrooms, low façade sound insulation, excessive teachers' vocal efforts. In this work results of acoustical treatments in some of these existing classrooms are reported. Measurements are made before and after the restorations. The case studies are chosen with reference to different building typologies and urban contexts, and consisted of improvements in sound absorption, sound insulation of internal partitions, doors and façades, and acoustic bridges elimination. They are divided in "light" and "heavy" types. The first, less expensive, with the aim to obtain good acoustical conditions, the latter, more expansive, focused to obtain optimal conditions. In fact, mainly in restoration of public schools, a good level of acoustical quality with contained costs is requested. The treatments are carried out with the aim to constitute a repertory of solutions to apply primarily in school restoration.

\section{Invited Papers}

5pNSa3. Speech perception in classroom noise and reverberation by children with typical and impaired hearing - Preliminary results. Frank Iglehart (Clarke School for the Deaf, 47 Round Hill Road, Northampton, MA 01060, USA, figlehart@clarkeschool .org)

A child's ability to perceive speech in the classroom influences academic progress. In this study, students with a various degrees of hearing loss perceive spoken words in sentences in a classroom. The room contains multiple noise levels and reverberation times (RTs). Classroom reverberation is measured using standardized procedures (with slight modifications to ASTM C423-02a: X2). RTs are controlled by quantities of acoustic panels in the room. Using the BKB-SIN Test, the speech-to-noise ratio at which the students perceive $50 \%$ words correctly (i.e., SNR-50) are measured in three reverberant conditions. Data collected to date indicate that students with severe-to-profound hearing loss $(\mathrm{n}=15$; ages 8-16 years) demonstrated average SNR-50s of $+12 \mathrm{~dB}(\mathrm{SD}=4),+13 \mathrm{~dB}(\mathrm{SD}=4)$, and $+17 \mathrm{~dB}(\mathrm{SD}=4)$ for conditions of $0.3,0.6$ and $0.9 \mathrm{~s}$ RT, respectively. Students with typical hearing ( $\mathrm{n}=14$; ages 8-16 years) had SNR-50s that averaged $-4 \mathrm{~dB}(\mathrm{SD}=2),-3 \mathrm{~dB}(\mathrm{SD}=2)$ and $-2 \mathrm{~dB}(\mathrm{SD}=2)$, respectively. Performance/intensity curves are also calculated in order to estimate minimal optimal listening conditions for each RT. This is an ongoing study. The session presentation will include emerging data on children with other degrees of hearing loss.

\section{3:00}

5pNSa4. Vocal symptoms in preschool teachers and the working environment. Valdis Inigbjörg Jonsdottir (Tad er Malid, Furuvellir 13, 601 Akureyri, Iceland, valdisj@ismennt.is)

Research has shown that teachers experience a high risk of developing voice problems. Noise levels in classrooms with young children are higher than in classrooms with older students, indicating preschool teachers' voices may be at greater risk. Questionnaires were sent to 88 preschool teachers in five preschools in Akureyri, and the parents of the 424 children in daycare. The study was aimed to obtain information about (1) teachers: experienced vocal symptoms, occurrence of vocal symptoms, opinions on working environment; air quality, noise, heat, acoustics. (2) parents: children's hearing problems. The study also aimed to ascertain noise levels and reverberation time in pre-school classrooms, and to compare teachers' reported ages, teaching experience, vocal symptoms and frequency with findings in other studies. Sound and reverberation-time measurements were taken by the Icelandic Department of Occupational Health and Safety. Reported vocal symptoms were more common among preschool teachers than other teachers, even though their youth and lack of teaching experience was marked. They appeared to be workrelated. $40 \%$ of the children had a history of hearing problems. Noise levels measured were very high. Correlation was found between voice fatigue and high temperature, bad indoor air, and poor acoustics.

5pNSa5. Acoustical requirements of classrooms and new concepts of teaching. Kurt Eggenschwiler (Empa, Laboratory of Acoustics, Ueberlandstrasse 129, CH-8600 Duebendorf, Switzerland, kurt.eggenschwiler@empa.ch), Markus Cslovejcsek (School of Teacher Education at the University of Applied Sciences FHNW, Kuettigerstrasse 42, CH-5000 Aarau, Switzerland, markus.cslovjecsek @ fhnw.ch)

In recent years acoustic requirements for classrooms were published in various guidelines. Of course it is important that the present knowledge will be implemented, but how should this be done? And what is the correspondence of acoustic requirements and new concepts of learning? These questions are the basis of a cooperation of the Laboratory of Acoustics at Empa, Dübendorf and the School of Teacher Education, Aarau in Switzerland. The cooperation itself is one possibility to transfer knowledge. In addition it was planned but not yet realized to develop concepts of knowledge transfer by student work (diploma thesis). Classroom acoustics were discussed in seminars and there were completed some diploma theses. One project dealt with the acoustic problems of a new teaching concept. Part of the concept is that teaching of four groups of pupils takes place in three classrooms and in the corridor. The doors of the classrooms remain open and at the same time pupils work individually, in groups, and a small group is instructed by a teacher. There were found suggestions for improving the acoustics, but for different reason the realization was not possible. 
5pNSa6. An Ambiophonics system for the study of intelligibility in the virtual classrooms. Nicola Prodi (Engineering Dept. Univ. of Ferrara, Via Saragat, 1, 44100 Ferrara, Italy, nicola.prodi@ unife.it), Andrea Farnetani (Engineering Dept. - Univ. of Ferrara, Via Saragat, 1, 44100 Ferrara, Italy, andrea.farnetani@unife.it), Patrizio Fausti (Engineering Dept. - Univ. of Ferrara, Via Saragat, 1, 44100 Ferrara, Italy, patrizio.fausti@unife.it), Roberto Pompoli (Engineering Dept. - Univ. of Ferrara, Via Saragat, 1, 44100 Ferrara, Italy, roberto.pompoli@unife.it)

In recent years the investigations on the intelligibility has profited by the increasing efficiency of the simulation and rendering technologies of virtual acoustics. In particular the latest systems are well suited to reproduce sound fields whose tridimensional characteristics are quite close to the real ones both objectively and subjectively. In this framework the Ambiophonics system, consisting of the merging of a double stereo-dipole and of an first order Ambisonics system, has gained particular attention. In the configuration used in this work the system employs twelve loudspeakers and is fed either with measured binaural and B-format data or by simulated ones. Based on this apparatus listening tests have been prepared in the Italian language to investigate the acoustics of classrooms and to focus on the effect of the directional characteristics of noise (i.e., fan-coils or tapping from the upper floor) on the intelligibility of words. The whole apparatus is driven by a Labview application which has also the aim of collecting the responses by the testers.

\section{4:00-4:20 Break}

5pNSa7. Noise - A stress factor? Acoustic ergonomics at schools. Gerhart Tiesler (University of Bremen/ISF, Grazer Str. 4, D-28359 Bremen, Germany, tiesler@uni-bremen.de), Markus Oberdoerster (Saint-Gobain Ecophon GmbH, Taschenmacherstr. 8, D-23556 Luebeck, Germany, markus.oberdoerster@ecophon.de)

Description of teaching reality is the main topic of this interdisciplinary examination about "acoustic ergonomics of schools." In the first step we analyse how different teaching methods affect basic noiselevel and working noise level. Which effect has an altered room acoustics on the sound levels in the context of each teaching method. The data records available as timeseries allows for the first time analysing single phases of lessons, which are characterized by certain pedagogical characteristics or individual instruction phases. Is it correct to speak of "noise stress" or is this stress an emotional reaction to the kind of? Based on recordings of teachers heartrate we analyse in the third step the effects of noise level on the workload of the teachers as a stress reaction. A distinction is made on the subject of different teaching methods, and on the basis the different room acoustic conditions. In one primary school we had four classes with a RT $>0.5 \mathrm{~s}$ and four further classes with a RT $<0.5 \mathrm{~s}$. At a second primary school we analysed the effect of the room acoustic refurbishment. Finally we investigate factors of fatigue. Which effect has $\mathrm{CO}_{2}$ in the classroom on all people, and the working noise level?

\section{4:40}

5pNSa8. Acoustical evaluation of nonclassroom university learning spaces. Murray Hodgson (The University of British Columbia, Department of Electrical and Computer Engineering, 2332 Main Mall, Vancouver, BC V6T 1Z4, Canada, mhodgson@interchange.ubc.ca), Jorge Garcia Moreno (The University of British Columbia, Department of Electrical and Computer Engineering, 2332 Main Mall, Vancouver, BC V6T 1Z4, Canada, 123calabaza@gmail.com)

This paper reports the results of an acoustical evaluation of non-classroom learning spaces at UBC. In twelve buildings, 25 indoor spaces -- a restaurant, a cafeteria, libraries, dedicated study spaces, building atria, etc. -- used for learning activities by at least 50 people were studied. The evaluation involved physical and acoustical (reverberation time, sound propagation, Speech Intelligibility Index) measurements, and occupant activity and satisfaction questionnaires. Questionnaires were administered three times (morning, lunchtime, and afternoon) on one day. The questionnaires asked about satisfaction with, and the effects of, the acoustical and nonacoustical environments. The acoustical measurement results were compared with established acceptability criteria. Questionnaires were analyzed for differences between times of day, test space, etc. The questionnaire responses and acoustical-measurement results were correlated. Using both as possible predictors, multivariable-regression models for predicting and explaining occupant satisfaction with, and the effects of, the acoustical environment were developed.

\section{5:00}

5pNSa9. The effect of amplification on children's performance in the classroom. Bridget M. Shield (London South Bank University, Borough Road, SE1 0AA London, UK, shieldbm@1sbu.ac.uk), Julie E. Dockrell (Institute of Education, 25 Woburn Square, WC1H 0AA London, UK, j.dockrell@ioe.ac.uk)

The use of amplification systems in the classroom has the potential to reduce the impact of poor classroom acoustics for typically developing pupils and those with special educational needs (SENs). The immediate benefits of amplified acoustic signals on the performance of 253 primary school children, including 24 children with special needs, were examined. All participants were familiar with the use of the amplification systems. Children's performance was assessed on two verbal measures (spelling and oral comprehension) and one non-verbal measure, using a balanced repeated measures design. It was predicted that the effects of amplification would be evident for spelling and oral language comprehension, but that there would be no discernable effect on speed of processing. Children with SEN were expected to have an added advantage with amplification. The predictions were partially supported. Both gain score analysis and ANOVAs of performance scores with amplification, controlling for performance without amplification, revealed an effect on spelling only for the typically developing children, while children with SENs benefited in both the spelling and the oral comprehension tasks. When installed appropriately, amplification improves children's ability to decode single words; SEN children also benefit in terms of their ability to process oral input. 
5pNSa10. Soundfield amplification is a poor substitute for good classroom acoustics. David Lubman (DL Acoustics, 14301 Middletown Lane, Westminster, CA 92683, USA, dlubman@dlacoustics.com), Louis C. Sutherland (Consultant in Acoustics, 27803 Longhill Dr., Rancho Palos Verdes, CA 90275-3908, USA, lou-sutherland@juno.com)

Soundfield (amplification) systems are widely and often aggressively marketed for small classrooms. In June 2006, the Acoustical Society of America (ASA) issued a public position statement on the use of sound amplification in typical small classrooms http://asa aip.org/amplification.pdf. This paper attempts to explain why the ASA found that soundfield systems are poor substitutes for good acoustics. Good acoustics for learning requires unoccupied classroom noise levels of $35 \mathrm{dBA}$ or less and midfrequency reverberation times of $0.6 \mathrm{~s}$ or less. At the same time, it is recognized that centralized amplifiers and sound distribution systems can provide valuable educational benefits in small classrooms with good acoustics. Such systems are useful for multimedia presentations and voice reinforcement. The authors urge that this message be brought to the attention of educators and educational facility decision makers.

\section{Contributed Papers}

\section{$5: 40$}

5pNSa11. Subjective evaluation of acoustical quality of lecture rooms with respect to the quality of the sound reinforcement system and the level of background noise. Sanja Grubesa (Faculty of EE and Computing, Unska 3, Department of Electroacoustics, HR-10000 Zagreb, Croatia, sanja.grubesa@fer.hr), Marko Horvat (Faculty of EE and Computing, Unska 3, Department of Electroacoustics, HR-10000 Zagreb, Croatia, marko.horvat@fer.hr), Hrvoje Domitrovic (Faculty of EE and Computing, Unska 3, Department of Electroacoustics, HR-10000 Zagreb, Croatia, hrvoje .domitrovic@fer.hr)

As a part of a general questionnaire on the quality of lecturing on the Faculty of EE and Computing in Zagreb, Croatia, the students have to grade the acoustical quality of lecture rooms in an indirect way by giving answers to several questions included in that questionnaire. As the sound reinforcement system in two largest lecture halls is in rather bad condition, it is to be replaced in the near future with a new one of significantly better quality. The goal of this paper is to examine whether this change is reflected in the grades of acoustical quality given by the students. In the second part of the paper, the students' grades on acoustical quality of two smaller lecture rooms of identical size and acoustic treatment are compared. The comparison is made with respect to the position of these rooms relative to the nearby street as the source of traffic noise. The overall level and spectral content of noise are measured in those lecture rooms. The correlation is then examined between the results of these measurements and the grades given by the students.

\section{6:00}

5pNSa12. Education vs cost - Difficulties in implementing acoustical design standards in classrooms. Patricia M. Scanlon (Cerami \& Associates, 404 Fifth Avenue, New York, NY 10018, USA, pscanlon @ ceramiassociates.com), James Perry (Cerami \& Associates, 404 Fifth Avenue, New York, NY 10018, USA, jperry@ ceramiassociates.com), Victoria J. Cerami (Cerami \& Associates, 404 Fifth Avenue, New York, NY 10018, USA, vcerami@ceramiassociates.com)

Good acoustical design standards for classrooms are established - quiet background noise levels due to mechancial systems and exterior sources, use of absorptive finishes to control reverberation, walls, doors and floors able to control airborne and impact noise transmission. So why do so many schools fail to follow these guidelines? Are private schools even less likely to adhere to these standards? What are the construction and equipment options? We will discuss various educational standards, as well as the reticence of school planners to comply. We will discuss what schools often build, versus what one might say they should build, together with cost comparison. We will review case studies where guidelines were, and were not, followed - examples that reflect the acoustician's mission to educate educators on the value of acoustical design considerations in classrooms. 


\title{
Session 5pNSb
}

\section{Noise, Structural Acoustics and Vibration, Physical Acoustics, and EURONOISE: Sound and Vibration from Explosions in Air II}

\author{
Roger Waxler, Cochair \\ University of Mississippi, NCPA, 1 Coliseum Drive, University, MS 38677, USA \\ Keith Attenborough, Cochair \\ Open University, Department of Design, Development, Materials and Environment, Walton Hall, Milton Keynes, MK7 6AA, UK
}

\section{Invited Papers}

2:00

5pNSb1. High-altitude infrasound calibration experiments. Henry E. Bass (The University of Mississippi - NCPA, 1 Coliseum Drive, University, MS 38677, USA, pabass@olemiss.edu), Eugene T. Herrin (Southern Methodist University, P. O. Box 750395, Dallas, TX 75275, USA, herrin@passion.isem.smu.edu), Paul Golden (Southern Methodist University, P. O. Box 750395, Dallas, TX 75275, USA, pgolden@mail.smu.edu), Robert Woodward (Incorporated Research Institutions for Seismology, 1200 New York Avenue, NW, Suite 800, Washington, DC 20005, USA, woodward@iris.edu), Douglas Drob (Naval Research Laboratory, Space Science Division, 4555 Overlook Avenue, Washington, DC 20375, USA, douglas.drob@nrl.navy.mil), Michael A. Hedlin (University of San Diego California, Scripps Institute of Oceanography, 9500 Gilman Drive, La Jolla, CA 92093, USA, hedlin@ucsd.edu), Catherine De Groot-Hedlin (University of San Diego California, Scripps Institute of Oceanography, 9500 Gilman Drive, La Jolla, CA 92093, USA, cdghedlin@gmail.com), Kris Walker (University of San Diego California, Scripps Institute of Oceanography, 9500 Gilman Drive, La Jolla, CA 92093, USA, walker@ucsd.edu), Milton Garces (Infrasound Laboratory, University of Hawaii, 73-4460 Queen Kaahumanu Highway \#119, Kailua-Kona, HI 96740, USA, milton@isla.hawaii.edu), Curt Szuberla (University of Alaska, 903 Koyukuk Drive, Fairbanks, AK 99775, USA, cas@gi.alaska.edu), Rod Whitaker (Los Alamos National Laboratory, EES-2 MS J577, Los Alamos, NM 87545, USA,rww@lanl.gov)

At the 152nd Meeting of the Acoustical Society of America, Andre and Bass reported an infrasound experiment conducted at White Sands Missile Range during the 2005-2006 time frame. The experiment consisted of exploding a $22.4 \mathrm{~kg}$ charge at altitudes from 31.3 $\mathrm{km}$ to $49.6 \mathrm{~km}$ then recording the waveforms at 30 infrasound arrays (not all at the same time) at distances up to $1200 \mathrm{~km}$ from the source. The analysis is not yet complete but some preliminary observations have been reported in the most recent issue of Acoustics Today. This talk will summarize the findings published in Acoustics Today and offer suggestions to others who might want to access and analyze the data.

$$
\text { 2:20 }
$$

5pNSb2. Infrasonic and seismic signals from explosions in Northwestern Europe. Laeslo G. Evers (Royal Netherlands Meteorological Institute (KNMI), PO Box 201, 3730 AE De Bilt, Netherlands, evers@knmi.nl), Hein Haak (Royal Netherlands Meteorological Institute (KNMI), PO Box 201, 3730 AE De Bilt, Netherlands, haak@knmi.nl)

Large explosions often generate both seismic and infrasound signals that can be detected over large ranges, i.e., hundreds of kilometers. Ground truth of such explosions is available from direct observations and/or seismic signals and can be used to get insight in the propagation of infrasound. Long range infrasound propagation is controlled by the state of the atmosphere. Tropospheric, stratospheric, and thermospheric ducts might exist and have implications on the signal characteristics and their detectability. In this study, we will show results of studies on explosions in Northwestern Europe using both seismic and infrasound data. These observations are used to derive source characteristics like: location, origin time and yield. Furthermore, the propagation characteristics of infrasound will be addressed.

\section{2:40}

5pNSb3. Infrasound propagation in the zone of silence. Paul Golden (Southern Methodist University, P. O. Box 750395, Dallas, TX 75275, USA, pgolden@mail.smu.edu), Petru Negraru (Southern Methodist University, P. O. Box 750395, Dallas, TX 75275, USA, pnegraru@smu.edu), Eugene T. Herrin (Southern Methodist University, P. O. Box 750395, Dallas, TX 75275, USA, herrin@passion .isem.smu.edu)

Two controlled source experiments were conducted in Nevada in 2006 and 2007 to study infrasound signal propagation at distances less than $300 \mathrm{~km}$ from the source. In 2006 three temporary infrasound arrays were deployed at distances of 76, 108, and 157 from the source. In 2007 the site at $157 \mathrm{~km}$ was reoccupied, and data was also recorded at $288 \mathrm{~km}$ from the source. Interesting results were derived from the travel time analysis. In 2006 the site at $76 \mathrm{~km}$ recorded both tropospheric and stratospheric arrivals, while at 108 and $157 \mathrm{~km}$ only stratospheric arrivals were recorded. In 2007 the site at $157 \mathrm{~km}$ recorded both tropospheric and stratospheric arrivals, while at $288 \mathrm{~km}$ both stratospheric and thermospheric arrivals were recorded. Atmospheric modeling with the InfraMAP software failed to predict returning rays or pressure levels similar to the observed data. Because of the large amplitude variations we attempt to estimate the yields of the explosions using the predominant frequency content of the signals. The physical basis for such a method is found in an increased acoustic transit time of the explosion blast radius with increased yield. Preliminary results suggest this is possible. 
5pNSb4. Validating upper-wind models using infrasound from active volcanoes. Alexis Le Pichon (CEA-DASE, Arpajon Cedex, 91297 Bruyères-le-Châtel, France, alexis.le-pichon@cea.fr), Karl Antier (CEA-DASE, Arpajon Cedex, 91297 Bruyères-leChâtel, France, karl.antier@cea.fr), Sylvie Vergniolle (Institut de Physique du Globe, 4 Place Jussieu, 75252 Paris, France, vergniolle@ipgp.jussieu.fr), Michel Lardy (IRD Center, BPA5, Cedex, 98848 Noumea, New Caledonia, lardy@noumea.ird.nc), Douglas Drob (Naval Research Laboratory, Space Science Division, 4555 Overlook Avenue, Washington, DC 20375, USA, douglas.drob@nrl.navy.mil)

Known and quasipermanent infrasonic sources are needed to evaluate and improve upper-wind models. Infrasounds generated by active volcanoes offer a unique opportunity for atmospheric studies. The Yasur volcano in the Vanuatu archipelago is an outstanding source of infrasonic waves due to its regular activity. This volcano is permanently monitored by the IS22 infrasound station located in New Caledonia and by one microbarometer installed close to its crater. A five-year monitoring period of Yasur at short and large propagation range provides new insights on quantitative relationships between infrasonic observables and atmospheric specifications. This experimental setting is proposed to validate consistently the Naval Research Laboratory Ground to Space (NRL-G2S) semi-empirical atmospheric model up to the stratosphere. The propagation modeling results accurately explain seasonal changes as well as small shorttimescale variations of the infrasonic observables. This study demonstrates that the use of appropriate propagation tools along with the NRL-G2S specifications provides accurate enough results for most of the long-range observations for the purpose of operational infrasound monitoring.

5pNSb5. Semianalytical modeling of plate flexural waves generated by laser-initiated air shock waves. Vasil B. Georgiev (Loughborough University, Department of Aeronautical and Automotive Engineering, Ashby Road, LE11 3TU Loughborough, UK, V.Georgiev@lboro.ac.uk), Victor V. Krylov (Loughborough University, Department of Aeronautical and Automotive Engineering, Ashby Road, LE11 3TU Loughborough, UK, V.V.Krylov@lboro.ac.uk), Qin Qin (University of Hull, Department of Engineering, Cottingham Road, HU6 7RX Hull, UK, q.qin@hull.ac.uk), Keith Attenborough (Open University, Department of Design, Development, Materials and Environment, Walton Hall, MK7 6AA Milton Keynes, UK, Keith.Attenborough@ioa.org.uk)

The paper describes the results of the semianalytical modeling of the interaction of laser-initiated air shock waves with an infinite elastic plate. The impact of the incident shock wave on the plate has been approximated by an equivalent cylindrically diverging surface force resulting from the combined surface pressure of the incident and reflected shock waves. This force has been then represented in the wave number-frequency domain by means of Hankel and Fourier transforms which have been carried out numerically - and the problem has been solved using the Green's function method applied to an infinite plate. The resulting frequency spectra and time histories of the generated flexural wave pulses have been calculated for different values of laser pulse energy and for different heights of the laser beam focusing above the plate surface. The obtained theoretical results have been compared with the results of the reducedscale model experiments on shock wave interaction with the ground in which large plastic and wooden plates have been used to represent the ground surface. The comparison shows that the obtained semi-analytical results are in good agreement with the experimental ones.

\section{$3: 40$}

5pNSb6. Ground effects on sound and vibration from explosions. Christian Madshus (NGI, P.O. Box 3930 Ullevaal Stadion, Sognsveien 72, 0806 Oslo, Norway, cm@ngi.no), Finn Løvholt (NGI, P.O. Box 3930 Ullevaal Stadion, Sognsveien 72, 0806 Oslo, Norway, flo@ngi.no), Ra Cleave (NGI, P.O. Box 3930 Ullevaal Stadion, Sognsveien 72, 0806 Oslo, Norway, rc@ngi.no), Karin Rothschild (NGI, P.O. Box 3930 Ullevaal Stadion, Sognsveien 72, 0806 Oslo, Norway, kmr@ngi.no), Zenon Cetina-Medina (NGI, P.O. Box 3930 Ullevaal Stadion, Sognsveien 72, 0806 Oslo, Norway, zmc@ngi.no)

Low frequency sound from military activity and explosions do propagate over large distances. The sound pressure may induce substantial vibration in the ground and particularly in buildings. Such vibrations turn out to be a major cause of complaints among neighbouring communities around training fields and blast sites. We will present investigations on long range propagation of low frequency sound and sound-induced vibration, based on a substantial amount of data collected during a series of full scale tests performed in Norway over the last 14 years. All data are assembled in the NORTRIAL database, which is now publicly available. Meteorology and ground interaction largely influence the sound propagation and vibration response. At low frequency meteorological- and ground effects may intricately interact and lead to large, apparently random variability in sound pressure at large distances. Statistically based investigations on the sound propagation and its variability will be presented. Particular focus will be on the ground interaction effects, and a new and more extensive system for ground classification, based on cartographic data, empirical and numerical modeling will be introduced. Findings on building response to outdoor low frequency sound and transfer mechanisms from outdoor pressure to indoor sound and vibration will be presented.

\section{4:00-4:20 Break}

5pNSb7. Deducing ground structure using seismic pulses originating from an outdoor explosive source. Shahram Taherzadeh (The Open University, Faculty of Mathematics, Computing and Technology, Walton Hall, MK7 6AA Milton Keynes, UK, s.taherzadeh@open.ac.uk), Keith Attenborough (Open University, Department of Design, Development, Materials and Environment, Walton Hall, MK7 6AA Milton Keynes, UK, Keith.Attenborough@ioa.org.uk)

Near-surface layering of ground soil can influence propagation of acoustic and seismic pulses originating from above-surface sources. Simultaneous recording of acoustic air pressure and seismic radial and vertical particle velocities resulting from a small, above ground explosion is used to obtain information about soil structure near the surface. Assuming nonlinear effects to be small at the ranges 
of interest here, a numerical model called Fast Field Program for Layered Air Ground Systems (FFLAGS), developed originally for continuous sound sources above a porous elastic ground is used to model a porous elastic layered ground system. Suitable optimisation methods were used to predict a set of best fit parameters for the near-surface ground structure. It is shown that the model can explain multiple seismic arrivals and give a reasonable prediction of wave speeds and layer depths while the pressure pulse can predict permeability of the surface soil.

\section{4:40}

5pNSb8. Predictions for the influence of the nocturnal jet on the long range propagation of impulsive signals. Roger Waxler (University of Mississippi, NCPA, 1 Coliseum Drive, University, MS 38677, USA, rwax@olemiss.edu), Kenneth E Gilbert (National Center for Physical Acoustics, University of Mississippi, University, MS 38677, USA, kgilbert@olemiss.edu), Carrick L. Talmadge (The University of Mississippi - NCPA, 1 Coliseum Drive, University, MS 38677, USA, clt@olemiss.edu), Xiao Di (National Center for Physical Acoustics, University of Mississippi, University, MS 38677, USA, xiaodi@olemiss.edu)

On clear nights, inland over flat ground, one generally finds a temperature inversion in the first hundred meters or so of the atmosphere. Near the ground, winds tend to be light, increasing with altitude. Above the temperature inversion one finds a stiff geostrophic wind known as the nocturnal jet. Theoretical predictions, based on ray theory and expansions in vertical modes, for the effect of the nocturnal jet on the long range propagation of impulsive signals are presented. For sufficiently short ranges, less than $3 \mathrm{~km}$ or so, the nocturnal jet has no effect. At these ranges only the temperature inversion and the light winds in the inversion play a role. At longer ranges the nocturnal jet significantly alters the arrival structure of the propagated signal. It is predicted that, due to coincident arrivals from the inversion and from the nocturnal jet, there is a segment of ranges about $1 \mathrm{~km} \mathrm{long}$, beginning at about $4 \mathrm{~km}$, in which the amplitude of the first arrival becomes anomalously large and then splits into two distinct arrivals.

\section{5:00}

5pNSb9. The physics of pulse propagation in the nocturnal atmospheric boundary layer: measurement and theory. Carrick L. Talmadge (The University of Mississippi - NCPA, 1 Coliseum Drive, University, MS 38677, USA, clt@olemiss.edu), Roger Waxler (University of Mississippi, NCPA, 1 Coliseum Drive, University, MS 38677, USA, rwax@olemiss.edu), Kenneth E Gilbert (National Center for Physical Acoustics, University of Mississippi, University, MS 38677, USA, kgilbert@olemiss.edu), Jin So (National Center for Physical Acoustics, University of Mississippi, University, MS 38677, USA, jso@ olemiss.edu), Rommel Stribling (National Center for Physical Acoustics, University of Mississippi, University, MS 38677, USA, eercs@olemiss.edu), Xiao Di (National Center for Physical Acoustics, University of Mississippi, University, MS 38677, USA, xiaodi@olemiss.edu)

A series of experiments designed to probe the effect of the nocturnal atmosphere on low-frequency (10- $500 \mathrm{~Hz})$ sound propagation will be discussed, and their ramifications explored. These experiments involve detecting arrivals from a propane cannon on a vertical array of microphones $1-3 \mathrm{~km}$ from the source, which were collected simultaneously with meteorological experiments designed to measure the vertical temperature and horizontal wind velocity profiles. Chief among the results of these experiments is the observation of a nocturnal model structure that has a significant surface wave component at frequencies below $150 \mathrm{~Hz}$. At higher frequencies and longer propagation distances $(>1.5 \mathrm{~km})$, the surface wave is not observed due to attenuation from its interaction with the ground. At higher frequencies, the model structure displays a characteristic "quiet height" first described in Waxler et al. (2006). The potential application of these results for remote sensing the atmospheric boundary layer are discussed, and contrasted with other methods of measurement of the atmospheric profile.

\section{Contributed Paper}

\section{$5: 20$}

5pNSb10. Nonlinear parabolic equation model for finite-amplitude sound propagation in an inhomogeneous medium over a nonflat, finite-impedance ground surface. Thomas Leissing (CSTB, 24 rue Joseph Fourier, 38400 Saint-Martin-d'Hères, France, thomas.leissing@cstb .fr), Philippe A. Jean (CSTB, 24 rue Joseph Fourier, 38400 Saint-Martind'Hères, France, philippe.jean@cstb.fr), Jérôme Defrance (CSTB, 24 rue Joseph Fourier, 38400 Saint-Martin-d'Hères, France, jerome.defrance @ cstb.fr), Christian Soize (Université de Marne la Vallée, 5, Boulevard Descartes, 77454 Marne la Vallée, France, soize@univ-mlv.fr)

A nonlinear parabolic equation (NPE) model for weakly nonlinear sound propagation in an inhomogeneous medium is described. The model being formulated in the time domain, complex impedances cannot be used to simulate ground surfaces. A second NPE model is thus derived to include the medium in the computational system. Based on a nonlinear extension of the Zwikker-Kosten model for rigidly-framed porous media, it allows to include Forchheimer's nonlinearities. Both models are then adapted to terrainfollowing coordinates, and used together with an interface condition, allow to simulate finite-amplitude sound propagation over a nonflat, finiteimpedance ground surface. Numerical examples show that the NPE model is in good agreement with the solutions of the frequency domain boundary element method. Applications of this model to the simulation of sound propagation from explosions in air are then discussed. 


\title{
Invited Papers
}

$5: 40$

5pNSb11. Mean vs event sound-level prediction: obtaining consistency between atmospheric data inputs, propagation models, and the predictand. David K. Wilson (U.S. Army Engineer Research and Development Center, 72 Lyme Rd., Hanover, NH 037551290, USA, D.Keith.Wilson@usace.army.mil), Chris L. Pettit (U.S. Naval Academy, Aerospace Engineering Dept., 590 Holloway Rd., MS 11-B, Annapolis, MD 21402, USA, petitcl@usna.edu), Vladimir E. Ostashev (NOAA/Earth System Research Laboratory, 325 Broadway, Boulder, CO 80305, USA, vladimir.ostashev@noaa.gov), Matthew S. Lewis (U.S. Army Engineer Research and Development Center, 72 Lyme Rd., Hanover, NH 03755-1290, USA, Matthew.S.Lewis@usace.army.mil)

The following, deceptively challenging, questions are addressed: What are the most suitable atmospheric data resources and propagation models for predicting event (explosion and other short duration) sound-exposure levels? Do these differ from those most suitable for predicting mean sound levels? Atmospheric data typically consist either of single, "snapshot" samples of the vertical profiles, as from a weather balloon, or average vertical profiles, as from climatology or a numerical weather model. Recent statistical studies, based on high-resolution atmospheric simulation, demonstrate the superiority of mean profiles for prediction of both mean and event sound levels, even when single-sample profiles are synchronized to and collected along the path of the propagation event. Running propagation models "blind" to the nature of the atmospheric inputs is shown to be hazardous: predictions from mean profiles lack turbulent scattering, thus underestimating sound levels near the ground, whereas predictions from single-sample profiles implicitly assume the turbulence has infinite horizontal extent, thus overestimating sound levels. Some desirable consistency results from numerically solving parabolic equations (PEs) for statistical moments of the sound pressure, rather than conventional deterministic PEs. The moment PEs directly predict mean sound levels or the expected value and variability of event sound-exposure levels.

$$
\text { 6:00 }
$$

5pNSb12. Beam-forming and dispersion measurements at the edge of a pine forest. Michael J. White (US Army Engineer Research and Development Center, 2902 Newmark Drive, Champaign, IL 61826, USA, Michael.J.White@usace.army.mil), Michelle E. Swearingen (Norwegian Defense Research Establishment/US Army, Postboks 25, 2027 Kjeller, Norway, michelle.swearingen@ffi.no)

Beam-forming analyses were performed on four-microphone linear arrays placed just within the interior of a pine forest to separate signal arrivals by direction from an impulsive sound source in an open field. The arrays were deployed in three configurations: transverse, longitudinal, and vertical. The arrays spacings were organized in a pattern that provides six baselines with four microphones. Longitudinal and transverse configurations show arrivals scattered from trunks, and the vertical configurations indicate some refraction and scattering by the canopy. Signal dispersion curves developed using the four-microphone arrays had greater variation in trace velocity at higher frequencies in each configuration.

FRIDAY AFTERNOON, 4 JULY 2008

ROOM 251, 4:20 TO 6:20 P.M.

\section{Session 5pNSc}

\section{Noise and EURONOISE: Propagation and Urban Noise II}

\author{
Jérôme Defrance, Chair \\ CSTB, 24 rue Joseph Fourier, Saint-Martin-d'Hères, 38400, France
}

\section{Contributed Papers}

$4: 20$

5pNSc1. Road traffic noise from viaducts in mountainous areas Jérôme Defrance (CSTB, 24 rue Joseph Fourier, 38400 Saint-Martind'Hères, France, jerome.defrance@cstb.fr), Matthieu Beyret (CSTB, 24 rue Joseph Fourier, 38400 Saint-Martin-d'Hères, France, matthieu.beyret @ cstb.fr), Marine Baulac (CSTB, 24 rue Joseph Fourier, 38400 SaintMartin-d'Hères, France, marine.baulac@cstb.fr), Philippe A. Jean (CSTB, 24 rue Joseph Fourier, 38400 Saint-Martin-d'Hères, France, philippe.jean @ cstb.fr)

Mountainous zones in Europe, such as the Alps, represent huge areas where many viaducts are built, most of them for motorways. The way the sound grazes the asphalt surface from the low and high traffic equivalent sources up to the road edges, and how it then diffracts towards dwellings is a complex mechanism. The standard approaches are suited to plain situations but fail in predicting finely sound propagation behaviour for such geometries. In this paper, one gives the main trends of received noise levels from viaducts as a function of both their geometry and the receiver location. A 2D Boundary Element Method is used for predictions since meteorological effects can be neglected for the short propagation (a few hundreds meters). This assumption makes sense since the viaduct considered in this work is sufficiently high $(20 \mathrm{~m})$ and the ground effect is weakly affected by refraction. Different configurations are then simulated in order to address and discuss several geometrical effects, such as: platform elevation, low height barriers addition, complex shape barriers and presence of a central gap in the platform.

\section{$4: 40$}

5pNSc2. Characteristics of road traffic noise level statistics for shielded areas. Jens Forssén (Division of Applied Acoustics, Chalmers University of Technology, 41296 Göteborg, Sweden, jens.forssen@chalmers .se), Maarten Hornikx (Applied Acoustics, Chalmers University of Technology, Sven Hultins Gata 8a, SE-41296 Gothenburg, Sweden, maarten .hornikx@chalmers.se)

For noise immission, it is of interest to study other noise level statistics besides the long-term equivalent levels and maximum levels. By further analysis of the time variations of the noise level, an improved description of the negative effects of the noise may be achieved, for instance concerning perceived annoyance. Here, noise level histograms, i.e., probability density 
functions of sound pressure levels, from controlled recordings have been investigated. This has been made for a situation of special interest, which is a courtyard shielded from a dominating road traffic noise source. It has been reported previously that many shielded urban areas show levels that are considerably higher than the equivalent level, described as an upward tail of the histogram, which is not a usual characteristic of directly exposed areas. From the analysis made here, it is shown that the upward tail, i.e., the higher levels, of the shielded area is caused by locally occurring, unshielded road traffic events. It is concluded that the upward tail as a common characteristic of shielded urban areas may well be due to locally occurring noise events, for instance due to local road traffic.

\section{5:00}

5pNSc3. Use of the transmission line matrix method for the sound propagation modeling in urban area. Gwenaël Guillaume (Lab. Central des Ponts et Chaussées, Division Entretien, Sécurité et Acoustique des Routes, Route de Bouaye - BP 4129, 44341 Bouguenais Cedex, France, Gwenael.Guillaume@1cpc.fr), Judicaël Picaut (Lab. Central des Ponts et Chaussées, Division Entretien, Sécurité et Acoustique des Routes, Route de Bouaye - BP 4129, 44341 Bouguenais Cedex, France, Judicael.Picaut@1cpc.fr), Guillaume Dutilleux (Lab. Régional des Ponts et Chaussées, 11, rue Jean Mentelin, BP 9, 67035 Strasbourg Cedex 2, France, Guillaume.Dutilleux@equipement.gouv.fr)

This paper deals with the sound propagation modeling in urban area. This problematic requires to take into account many phenomena that can have a substantial impact as well in semienclosed spaces as on long-range outdoor sound propagation, such as reflections and absorption on the frontages and on the ground, atmospheric attenuation, sound velocity variations related with wind and temperature vertical gradients, atmospheric turbulences. The numerical method used is the TLM (transmission line modeling), which has been originated in electromagnetism and adapted for acoustics applications. It consists in a physical rendering of the waves propagation based on the Huygens' principle. It is established on a spatiotemporal discretization of the domain using an iterative temporal process for sound pressure propagation, instead of the resolution of mathematical equations. The TLM model has then been developed in two and three dimensions allowing to combine all the phenomena affecting the sound propagation in urban area. Numerical simulations are given for canyon streets.

\section{$5: 20$}

5pNSc4. Predictions of sound pressure levels in streets using a diffusion model: numerical validations and experimental comparisons. Alexis Billon (Universite de Liege, INTELSIG group - Département E.E.I., B28 Sart-Tilman, 4000 Liege, Belgium, abillon@ulg.ac.be), Judicaël Picaut (Lab. Central des Ponts et Chaussées, Division Entretien, Sécurité et Acoustique des Routes, Route de Bouaye - BP 4129, 44341 Bouguenais Cedex, France, Judicael.Picaut@1cpc.fr)

Predictions of sound propagation in urban areas have attracted a considerable over the years. If the sound energy is assimilated to particles with a constant energy, their movement can be described by a transport equation. In canyon streets, this transport equation can be reduced to a diffusion equation whose expression is more simple. In this presentation, sound absorption at the boundaries (buildings facades and ground), as well as atmospheric sound attenuation are introduced. The problem is then solved numerically using a finite elements method for the configuration of a canyon street. A systematic validation of the obtained model is carried out in terms of sound pressure level by comparison to numerical simulations taken from the literature. Comparisons with experimental data are then conducted. Finally, applications in more complex configurations are presented.

\section{$5: 40$}

5pNSc5. A cellular automaton for urban traffic noise. Erik Salomons (TNO Science and Industry, Stieljesweg 1, 2628CK Delft, Netherlands, erik.salomons@tno.nl)

Propagation of traffic noise in a city is a complex phenomenon, due to multiple reflection, diffraction, and scattering at irregular facades of buildings. These effects may be calculated with computer models based on numerical integration of the basic acoustic equations, but in practice these models can be applied only to small urban regions due to limited computer power. Here we propose a new approach for simulating urban traffic noise: a cellular automaton (CA) based on simple update rules for the configuration of cars in a city, and simple rules for propagation of sound to receivers. An example is presented for a square model city of $25 \mathrm{~km}^{2}$, consisting of $10^{6}$ square cells. The CA employs a time integration step of $0.3 \mathrm{~s}$, and includes noise contributions from all cars in the city. The fluctuating sound level is computed for a period of $24 \mathrm{~h}$, both for a receiver along a street and for a receiver that is screened by buildings. While the sound level at the first receiver shows sharp peaks corresponding to passages of cars, the sound level fluctuations at the screened receiver are much smaller as most of the sound energy comes from distant cars in this case.

\section{6:00}

5pNSc6. Numerical prediction of the effect of traffic lights on the vehicle noise at urban street intersections. Jorge Parrondo (Universidad de Oviedo, Dep. de Energía, Campus de Viesques s/n, 33203 Gijón, Spain, parrondo@uniovi.es), Ruben Zurita (Universidad de Oviedo, Dep. de Energía, Campus de Viesques s/n, 33203 Gijón, Spain, ZURITA@1si.uniovi.es), Jose A. Corrales (Universidad de Oviedo, Dep. de Energía, Campus de Viesques s/n, 33203 Gijón, Spain, JA@1si.uniovi.es), Joaquin Fernandez (Univ. de Extremadura, Escuela de Ing. Industriales, 06071 Badajoz, Spain, ffrancos@unex.es)

Intersections of urban streets are particularly noisy locations due to the addition of the noise from vehicles at different streets, a long period of noise emission from queuing vehicles with traffic lights in red and the noise from accelerating vehicles. Besides, the traffic lights impose a modulation in the passage of the vehicles, so that the subsequent noise variability along time contributes to increase the annoyance degree. This paper presents a code especially developed to simulate both the spatial and temporal distribution of the sound pressure levels induced by the vehicle traffic in street intersections. The algorithm combines a traffic model with vehicles in dynamic motion through the domain with a model for sound propagation, based on the method of virtual images in which the determination of the location of the successive image sources was reduced to a reference horizontal plane. Sound emission from each vehicle was established according to the Harmonoise guidelines. After verifying the code predictions by comparison with measurements at several street intersections, the method was applied systematically to investigate the effect of varying the regulation parameters of the traffic lights on the Leq and L10 distributions for some particular cases. 


\title{
Session 5pPAa
}

\section{Physical Acoustics: Ducts and Waveguides II}

\author{
Marc Deschamps, Cochair \\ LMP, Lab. de Mécanique et Physique (LMP), 351, Place de la Libération, Talence, 33405, France \\ Olivier Dazel, Cochair \\ Laboratoire d'Acoustique de l'Université du Maine, Avenue Olivier Messiaen, Le Mans, 72085, France
}

\section{Contributed Papers}

\begin{abstract}
2:00
5pPAa1. A higher order parabolic equation for predicting in-duct propagation in high frequencies. Patrice Malbéqui (ONERA, 29 avenue de la Division Leclerc, 92322 Châtilllon, France, patrice.malbequi@onera .fr)

Complementary methods are required to predict the in-duct propagation in a large frequency range including the linear absorption and the flow effects. Numerical methods solving the Euler's equations are pertinent for rotational flow but limited to the low frequencies. The Boundary Element Method is applicable in a large frequency range assuming a homogeneous flow. The ray-model is valid in high frequencies but scattering effects are difficult to implement. This paper presents the capabilities of a Higher-Order Parabolic Equation (HOPE) to handle duct propagation in the high frequency range. In contrast with the so-called standard PE and the wide-angle $\mathrm{PE}$, the HOPE improves the accuracy of the solution due to its wider propagation aperture angle, especially close to the cutoff frequency. Several duct propagation configurations including flow and liner are considered. Using a marching algorithm, the HOPE computes in a very short CPU time the sound propagation and represents an attractive alternative to the ray-model in the high frequency range. [Work supported by Airbus-France.]
\end{abstract}

\section{$2: 20$}

5pPAa2. An integrated multimodal acoustic particle manipulator and optical evanescent field waveguide. Peter Glynne-Jones (University of Southampton, School of Engineering Sciences, University Road, SO17 1BJ Southampton, UK, p.glynne-jones@ soton.ac.uk), Martyn Hill (University of Southampton, School of Engineering Sciences, University Road, SO17 1BJ Southampton, UK, m.hill@soton.ac.uk), Rosemary J. Townsend (University of Southampton, School of Engineering Sciences, University Road, SO17 1BJ Southampton, UK, R.J.Townsend@soton.ac .uk), Nicholas R. Harris (University of Southampton, Electronics and Computer Science, SO17 1BJ Southampton, UK, nrh@ecs.soton.ac.uk), James S. Wilkinson (University of Southampton, Optoelectronics Research Centre, SO17 1BJ Southampton, UK, jsw@ecs.soton.ac.uk), Fan Zhang (University of Southampton, Optoelectronics Research Centre, SO17 1BJ Southampton, UK, faz@orc.soton.ac.uk), Tracy Melvin (University of Southampton, Optoelectronics Research Centre, SO17 1BJ Southampton, UK, tm@ecs.soton.ac.uk)

A new acoustic/optical/microfluidic system is presented for the manipulation of bead-tagged DNA molecules. Acoustic radiation forces are used to manipulate microspheres into and away from the evanescent field of a laser coupled waveguide that is integrated into the reflector of the acoustic chamber. With suitable fluorophores the presence of the target DNA can be detected with a fluorescence microscope enabling large populations of beads to be examined simultaneously. The integrated waveguide and multimodal acoustic chamber are presented here, with results showing that the microspheres can be successfully detected as they are brought into the evanescent field using a quarter-wave acoustic configuration. It is also shown that by measuring the time of flight of a microsphere between the half- and quarter- wave nodal planes the bead size can be determined, providing a means of multiplexing the detection (detecting a range of different target DNA sequences).

\section{2:40}

5pPAa3. Trapped wave in plan waveguides including gaussian varying section domain. Patrick Marical (LOMC FRE-3102 CNRS, Groupe Ondes Acoustiques, University of Le Havre, Place Robert Schuman, BP 4006, 76610 Le Havre, France, patrick.marical368@univ-lehavre .fr), Mounsif Ech-Cherif El-Kettani (LOMC FRE-3102 CNRS, Groupe Ondes Acoustiques, University of Le Havre, Place Robert Schuman, BP 4006, 76610 Le Havre, France, elkettani@univ-lehavre.fr), Zahia Hamitouche (LOMC FRE-3102 CNRS, Groupe Ondes Acoustiques, University of Le Havre, Place Robert Schuman, BP 4006, 76610 Le Havre, France, zahia.hamitouche@univ-lehavre.fr), Mihai Valentin M. Predoi (University Politechnica of Bucharest, Department of Mechanics, 060032 Bucharest, Romania, predoi@ cat.mec.pub.ro)

In previous works on plan waveguides including an area of varying section of Gaussian shape, we have observed experimentally and numerically the existence of a trapped wave in the Gaussian varying section domain in the case of the A1 incident Lamb mode. The purpose of this work is to highlight the existence of this trapped wave, depending on the equation of the Gaussian profile of the varying section domain. This study is carried out numerically using FEM, as our numerical model has been previously validated. The results obtained show that the phenomenon of trapped wave is strongly linked to the Lamb wave conversion phenomenon: when the incident Lamb wave gives rise to a trapped wave, it is systematically converted into other Lamb waves transmitted outside the area of varying section. Otherwise, it is totally transmitted without any conversion and in this case, any trapped wave exists. The conversion phenomenon is quantified and we show that it is important and depend on the breaking symmetry of the incident Lamb mode by the varying section area.

\section{3:00}

5pPAa4. Intermodal coupling in a dissipative fluid filling a rough-walled waveguide. Tony Valier-Brasier (Laboratoire d'Acoustique de l'Université du Maine, Avenue Olivier Messiaen, 72085 Le Mans, France, tony.valier-brasier.etu@univ-lemans.fr), Catherine Potel (Laboratoire d'Acoustique de l'Université du Maine, Avenue Olivier Messiaen, 72085 Le Mans, France, catherine.potel@univ-lemans .fr), Michel Bruneau (Laboratoire d'Acoustique de l'Université du Maine, Avenue Olivier Messiaen, 72085 Le Mans, France, michel.bruneau@univ-lemans.fr), Claude_Depollier (Laboratoire d'Acoustique de l'Université du Maine, Avenue Olivier Messiaen, 72085 Le Mans, France, claude.depollier@univ-lemans.fr)

The present study follows recent works dealing with the analytical model of an acoustic field in fluid-filled waveguides with rough walls. In these works, the acoustic field is obtained from the coupling between Neumann eigenmodes of the regularly shaped waveguide which bounds outwardly the rough walls of the waveguide considered, using integral formulation with suitable Green function. The effect of the roughness is expressed 
in such a way that two intermodal coupling mechanisms are highlighted: a bulk coupling and a surface coupling, the first one depending on the depth of the roughness and the second one depending in addition on the local slope. Moreover, a phonon relation is involved when the rough profile is periodic. The aim of the present study is to account for the thermo-viscous boundary layer effects through eigenmodes which satisfy appropriate mixed boundary conditions, leading to a better understanding of the physical mechanisms when resonances and phonon relationship are involved.

\section{3:20}

5pPAa5. Evaluation of the lined duct performances based on a 3D two port scattering matrix. Mohamed Taktak (Université de Technologie de Compiègne, Centre de Recherche Royallieu, BP20529, 60205 Compiègne, France, mohamed.taktak@utc.fr), Jean Michel Ville (Université de Technologie de Compiègne, Centre de Recherche Royallieu, BP20529, 60205 Compiègne, France, jean-michel.ville@utc.fr), Mohamed Haddar (Unité de Modélisation, Mécanique et de Production (U2MP), Ecole Nationale d'Ingénieurs de Sfax, BP 3038, 3038 Sfax, Tunisia, mohamed.haddar@enis.rnu.tn), Félix Foucart (Université de Technologie de Compiègne, Centre de Recherche Royallieu, BP20529, 60205 Compiègne, France, felix.foucart@utc.fr)

The scattering matrix constitutes a good tool to characterize a lined duct. In fact, this matrix relates the incoming modal pressures to the out coming ones and contains detailed information and per mode about the transmission, reflection, and conversion properties of the duct. It depends only on the geometric and acoustic properties of the duct. The two port acoustic dissipation and attenuation interest the designers of lined duct like aircraft engine manufacturers to evaluate the duct performances. These values can be deduced from the two port scattering matrix and from the vector of incoming modal pressures. In this work, a study about the two port acoustic dissipation and attenuation computed from the scattering matrix and for different cases of incoming modal pressures are presented. Scattering matrices used in this study are measured by an experimental procedure developed at the University of Technology of Compiègne based upon the experimental setup realized during the European Project DUCAT. The experimental acoustic power dissipation and attenuation are computed for different cases of modal structure on the both side of the duct. Then, these results were confronted with ones given by a theorical study of the problem based on the finite element method.

\section{3:40-4:00 Break}

\section{4:00}

5pPAa6. Elaboration of a sacttering matrix measurement procedure using the p-v probe. Yamen Kchaou (Université de Technologie de Compiègne, Centre de Recherche Royallieu, BP20529, 60205 Compiègne, France, yamen.kchaou@yahoo.fr), Mohamed Taktak (Université de Technologie de Compiègne, Centre de Recherche Royallieu, BP20529, 60205 Compiègne, France, mohamed.taktak@utc.fr), Jean Michel Ville (Université de Technologie de Compiègne, Centre de Recherche Royallieu, BP20529, 60205 Compiègne, France, jean-michel.ville@utc .fr), Mohamed Haddar (Unité de Modélisation, Mécanique et de Production (U2MP), Ecole Nationale d'Ingénieurs de Sfax, BP 3038, 3038 Sfax, Tunisia, mohamed.haddar@enis.rnu.tn), Félix Foucart (Université de Technologie de Compiègne, Centre de Recherche Royallieu, BP20529, 60205 Compiègne, France, felix.foucart@utc.fr)

The scattering matrix which relates traveling waves amplitudes as state variables has been shown to be more attractive than transfer or mobility matrices since it reflects the fundamental duct nature: it gives a more complete description of the transmission, reflection, and conversion properties of the duct. In the University of Technology of Compiègne, an experimental procedure was developed to measure this matrix: a p-p probe mounted on a setup designed during DUCAT project is used to measure pressures at two cross sections on the both side of the test lined duct, then by using a modal decomposition and separation techniques, the scattering matrix is deduced. In this paper, a method to measure the multimodal scattering matrix based on the use of a p-v probe getting simultaneously the acoustic pressure and velocity at one section on the both side of the test duct is developed. A com- parison of some acoustics values (scattering matrix coefficients, acoustic powersœ) of a hard wall duct straight duct obtained by each technique with the theory is presented to evaluate its advantages and limitations.

$4: 20$

5pPAa7. Axisymmetrical and nonaxisymmetrical guided waves propagating in a solid elastic cylinder embedded in a solid medium. Slah Yaacoubi (LCPC, Lab. Central des Ponts et chaussées (LCPC), Route de Bouaye-BP 4129, 44341 Bouguenais, France, slah.yaacoubi@1cpc.fr), Laurent Laguerre (LCPC, Lab. Central des Ponts et chaussées (LCPC), Route de Bouaye-BP 4129, 44341 Bouguenais, France, laurent.laguerre@1cpc.fr), Eric Ducasse (LMP, Lab. de Mécanique et Physique (LMP), 351, Place de la Libération, 33405 Talence, France, e.ducasse@1mp.u-bordeaux1.fr), Marc Deschamps (LMP, Lab. de Mécanique et Physique (LMP), 351, Place de la Libération, 33405 Talence, France, m.deschamps@1mp.u-bordeaux1.fr)

For NDT of rods and pipes, fundamental characteristics of guided waves are to be known, especially dispersion relations between frequency and wave number. A necessary step before detecting defects is to be able to calculate the propagated elastodynamical field in healthy waveguides. Thus, the goal of this work is the calculation of this field propagating in a cylindrical stratified waveguide. The incident field is generated at the end of the cylinder by a force or velocity source which is off-axis and Gaussian distributed. First, Vector Hankel transform and Fourier series are combined to decompose this field with respect to the angular position. Second, each component is decomposed into an infinite sum of rays, i.e., elementary generalized conical waves. These waves undergo multiple reflections with the guiding surface of the waveguide. Third, we use Generalized Debye Series (GDS) for calculating the global reflection coefficients resulting from these multiple reflections. Finally, the total field is synthesized by the summation of the incident and reflected rays. Many outputs of this code can be exploited like velocity field, stress field, energy field in 2D or 3D spatiotemporal or frequential simulations. Diagrams obtained by this code are compared with results from DISPERSE software.

\section{4:40}

5pPAa8. Coupling transfer matrix method to finite element method for the analysis of hollow body networks with passive or reactive elements. Fabien Chevillotte (Groupe d'Acoustique de l'Université de Sherbrooke, 2500, Boul. de l'Université, Département de génie mécanique, Sherbrooke, QC J1K-2R1, Canada, fabien.chevillotte@usherbrooke.ca), Raymond Panneton (Groupe d'Acoustique de l'Université de Sherbrooke, 2500, Boul. de l'Université, Département de génie mécanique, Sherbrooke, QC J1K2R1, Canada, raymond.panneton@usherbrooke.ca), Hakim Bougrab (Groupe d'Acoustique de l'Université de Sherbrooke, 2500, Boul. de l'Université, Département de génie mécanique, Sherbrooke, QC J1K2R1, Canada, Hakim.Bougrab@USherbrooke.ca), Christophe Chaut (Henkel Technologies, Acoustics Center, 58203 Cosne sur Loire, France, Christophe.Chaut@fr.Henkel.com), Jean-Luc Wojtowicki (Henkel Technologies, Acoustics Center, 58203 Cosne sur Loire, France, Jean-Luc .Wojtowicki@fr.Henkel.com)

This work shows how to couple transfer matrix method to finite element method with a view to analyze the acoustic response of hollow body structures with a minimum of memory requirements and computational time. An hollow body structure is made up from a series of elongated rigid-walled fluid partitions (i.e., waveguides). These fluid partitions are separated by any passive (e.g., multilayered sound barrier) or reactive elements (e.g., expansion chamber). In the proposed hybrid model, the elongated fluid partitions are modeled using 1D fluid finite elements, and the passive or reactive elements using transfer matrices. From the weak integral formulation of the acoustic problem, it is shown how the coupling with the transfer matrix is taken into account through a mixed boundary condition. After discretization of the acoustic pressure and application of the variational principle, the finite element matrix system is obtained, where only the nodal pressures in the fluid partitions remain. The transfer matrix has been converted into a kind of admittance matrix, where no additional degrees of freedom are necessary to account for the passive or reactive elements. The method is used to predict the acoustic response of a real hollow body structure. Good correlations are obtained with experimentations. 


\title{
Session 5pPAb
}

\section{Physical Acoustics: Ultrasonics Under Extreme Conditions II}

\author{
Albert Migliori, Cochair \\ NHMFL, Los Alamos Natl. Lab. Los Alamos, NM 87545, USA \\ Frédéric Decremps, Cochair \\ IMPMC, Université Paris VI, 140, rue de Lourmel, Paris, 75015, France
}

\section{Invited Papers}

\author{
3:20
}

5pPAb1. Gigahertz ultrasonic interferometry at high pressure and temperature: Geophysical implications. Anastasia P. Kantor (Bayerisches Geoinstitut, University Bayreuth, 95440 Bayreuth, Germany, Anastasia.Kantor@Uni-Bayreuth.de), Steven D. Jacobsen (Department of Earth and Planetary Sciences, Northwestern University, Evansto, IL 60208-2150, USA, steven@earth.northwestern.edu), Innokenty Y. Kantor (Bayerisches Geoinstitut, University Bayreuth, 95440 Bayreuth, Germany, Innokenty.Kantor@uni-bayreuth.de), Leonid S. Dubrovinsky (Bayerisches Geoinstitut, University Bayreuth, 95440 Bayreuth, Germany, Leonid.Dubrovinsky@uni-bayreuth.de), Hans Josef Reichmann (Geoforschungszentrum Potsdam, Telegrafenberg, Division 4, 14473 Potsdam, Germany, hanni@gfz-potsdam.de)

High-frequency acoustic interferometry is widely used to penetrate a medium and measure the reflection signature, which can reveal details about the inner structure of the medium. It is a very helpful and one of the most accurate techniques for determination elastic properties of different materials being capable to measure sound wave velocities in very small samples under high pressures. The ultrasonic interferometry system operating at 0.6-2.1 gigahertz $(\mathrm{GHz})$ frequencies was developed in the Bavarian Geoinstitute of the University of Bayreuth for in situ high pressure and temperature experiments. High pressures are reached by using diamond anvil cell, and a Pt-resistive heater allows reaching high temperatures. The experimental setup is equipped with a laser system, which allows measuring a shift of ruby fluorescence line at every given temperature.

3:40

5pPAb2. Estimation of thermophysical properties of fluids under high pressure from speed of sound measurements. Jean Luc Daridon (Université de Pau et des Pays de l'Adour, Laboratoire des Fluides Complexes, UMR 5150, BP 1155, 64000 Pau, France, jean-luc.daridon@univ-pau.fr)

The thermophysical properties of pure substances in fluid state as functions of temperature and pressure are of great interest not only for industrial applications (for example in the oil and gas industry), but also for fundamental aspects in view of developing models for an accurate representation of dense fluids. Now these measurements are difficult to perform under pressure, particularly for non singlephase systems, at atmospheric pressure. An interesting alternative consists in using the ultrasonic velocity which can be determined experimentally with a high degree of accuracy including at high pressures and high temperatures, and which presents the advantage of giving access to various derived properties. This potential, which is specific to ultrasonic velocity in fluids, has been the starting point for the investigation of a large number of pure liquids and gases as well as of several types of mixtures by ultrasonic measurements. In this work we will review the procedures used to evaluate thermophysical properties as a function of pressure from speed of sound measurements. The validity of the different approaches will be checked by comparison to several thermophysical properties measured in an extended pressure range. The accuracy reached for each property by the different procedures will be bring out.

\section{Contributed Papers}

\section{4:00}

5pPAb3. New results on the sound velocity measurements under extreme conditions using time-resolved picosecond acoustic technique. Frédéric Decremps (IMPMC, Université Paris VI, 140, rue de Lourmel, 75015 Paris, France, frederic.decremps@impmc.jussieu.fr), Laurent Belliard (INSP - UMR 7588 CNRS \& Université Pierre et Marie Curie, 140 Rue de Lourmel, 75015 Paris, France, lbelliar@ccr.jussieu.fr), Bernard Perrin (INSP - UMR 7588 CNRS \& Université Pierre et Marie Curie, 140 Rue de Lourmel, 75015 Paris, France, bernard.perrin@insp.jussieu .fr), Michel Gauthier (IMPMC, Université Paris VI, 140, rue de Lourmel, 75015 Paris, France, michel.gauthier@impmc.jussieu.fr)

In this presentation, recent works on the pressure and temperature dependence of the sound velocity will be discussed. We have used a newly developed method combining the time-resolved picosecond optical technique and a diamond anvil cell [1]. This setup makes possible accurate measurements of the attenuation and velocity of longitudinal waves in the Ghz range, and opens the elastic investigations of all materials (opaque, transparent, single- or polycrystal, liquids) up to several Mbar and thousands of $\mathrm{K}$. The experimental method will be first described, with a discussion of the factors limiting the possibilities and the technique accuracy. [1] F. Decremps, L. Belliard, B. Perrin, and M. Gauthier, Phys. Rev. Lett, to be pubished in January 2008.

\section{4:20-4:40 Break}

\section{4:40}

5pPAb4. Elastic moduli at high temperatures with two different ultrasonic methods. Ludivine Bourgeois (Commissariat à l'Energie Atomique (CEA), Centre de Valduc, 21120 Is sur Tille, France, ludivine .bourgeois@cea.fr)

"The elastic moduli and specially the shear modulus $\mathrm{G}$ is one of the parameters needed to implement the constitutive relations. These mechanical 
properties can be measured for a solid or a liquid with different ultrasonic methods. This paper deals with the determination of $G(T)$ with two different methods: a contact delay-line ultrasonic device which has been developed to measure the shear and compression waves velocities up to $1000 \mathrm{~K}$ on cylindrical specimens and a contactless (laser) ultrasonic device which can measure surface acoustic waves velocities in metals up to the melting point Results on metals such as Al without phase transition and for Co through the phase transition will be presented."

\section{$5: 00$}

5pPAb5. Measurements under high pressure of ultrasonic velocity in glycerol. Hassina Khelladi (Faculty of Physics, University of Science and Technology Houari Boumedienne, BP 32 El Allia, Bab-Ezzouar, 16000 Algiers, Algeria, hassinakhelladi@yahoo.fr), Frédéric Plantier (Université de Pau et des Pays de l'Adour, Laboratoire des Fluides Complexes, UMR 5150, BP 1155, 64000 Pau, France, frederic.plantier@univ-pau.fr), Jean Luc Daridon (Université de Pau et des Pays de l'Adour, Laboratoire des Fluides Complexes, UMR 5150, BP 1155, 64000 Pau, France, jean-luc.daridon@univ-pau.fr), Hakim Djelouah (Faculty of Physics, University of Science and Technology Houari Boumedienne, BP 32 El Allia, Bab-Ezzouar, 16000 Algiers, Algeria,djelouah_hakim@yahoo.fr)

Glycerol has been the subject of significant scientist interest. Indeed, glycerol is a polyalcohol and the presence of three hydroxyl groups per molecule makes glycerol a complex system to explore. The purpose of this investigation is to measure under high pressure the ultrasonic wave velocity in glycerol, from which a number of important thermodynamic properties could be derived and determined as a function of pressure and temperature. Pressure and temperature ranges exploited in this experimental investigation of various glycerol properties, are extended respectively from $0.1 \mathrm{MPa}$ to $100 \mathrm{MPa}$ and from $10{ }^{\circ} \mathrm{C}$ to $100{ }^{\circ} \mathrm{C}$. A high pressure measurement cell equipped with temperature and pressure monitoring and control instrumentation was used. A time of flight method was exploited to measure, under high pressure, the ultrasonic wave velocity at different temperatures. The resulting experimental data of ultrasonic wave velocity in glycerol combined with measurements at atmospheric pressure, of density, specific heat and thermal expansion coefficient were used to derive density at elevated pressures. As isentropic compressibility is linked to ultrasonic wave velocity and density by means of the Newton-Laplace equation, this intrinsic physical property is easily deduced. These results led to the behavior of each physical property as a function of temperature and pressure.

\section{$5: 20$}

5pPAb6. Laser optoacoustic study of near-critical states and phase transitions in metals. Alexander Y. Ivochkin (Moscow State University International Laser Centre, Leninskie gory, 1, 119992 Moscow, Russian Federation, ivochkin@yandex.ru), Alexander G. Kaptilniy (Joint Institute for High Temperatures, Russian Academy of Science, Izhorskaya str., 13/19, 125412 Moscow, Russian Federation, drc@pochta.ru), Alexander Karabutov (Moscow State University, MSU, 1, building 2, GSP-2, Leninskiye Gory, 119992 Moscow, Russian Federation, akarabutov@gmail.com)

Pulsed laser optoacoustic technique is used for generation and study of near-critical states and phase transitions in metals. Metal surface is confined by a layer of transparent dielectric. In this case the efficiency of pressure generation is much greater than in case of the free surface so it is possible to achieve states of metals with relatively high thermodynamic parameters: $\mathrm{P}$ $\sim 10^{4}$ atm and $\mathrm{T} \sim 10^{4} \mathrm{~K}$ with a table-top laser system. The experimental setup for simultaneous measurements of pressure, temperature, and reflectivity of metal surface with nanosecond temporal resolution was assembled. Q-switched Nd:YAG laser with pulse duration $\sim 10 \mathrm{~ns}$ and pulse energy $\sim 1 \mathrm{~J}$ was used. Pressure was measured using $\mathrm{LiNbO}_{3}$ piezotransducer. Temperature was obtained with optical pyrometer. Lead and mercury were chosen as test metals. Pressure pulses up to $1 \mathrm{kbar}$ in lead and up to $7 \mathrm{kbar}$ in mercury (with $\mathrm{T} \sim 2400 \mathrm{~K}$ - super-critical area of the phase diagram for $\mathrm{Hg}$ ) were obtained. The curve of laser heating process in P-T coordinates was plotted. The measurements of optical properties showed considerable decrease of surface reflectivity both for lead and mercury at high laser fluences due to increase of temperature and density decrease.

\section{$5: 40$}

5pPAb7. Effect of intense neutron dose radiation on piezoceramics. Franck P. Augereau (IES/Université Montpellier II, Université Montpellier II, Place Eugène Bataillon, 34095 Montpellier, France, Franck.Augereau@ies.univ-montp2.fr), Jean-Yves Ferrandis (Radio Application Division, NEC Corporation, Université Montpellier II, Place Eugène Bataillon, $34095 \quad$ Montpellier, France, ferrandi@lain.univ-montp2.fr), Jean-François Villard (CEA Saclay, 91191 Gif sur Yvette Cedex, France, jean-francois.villard@cea.fr), Damien Fourmentel (CEA Saclay, 91191 Gif sur Yvette Cedex, France, damien.fourmentel@cea.fr), Mark Dierckx (SCK-CEN, Boeretang 200, B-2400 Mol, Belgium, mdierckx@sckcen.be), Jan Wagemans (SCK-CEN, Boeretang 200, B-2400 Mol, Belgium, jwageman@SCKCEN.BE)

Four grades of commercial PZT materials have been exposed to nuclear radiation during five months in an irradiation channel of the BR1 research reactor at SCK ${ }^{\circ} \mathrm{CEN}$ (Belgium). This experimental study was performed in the framework of the Joint Instrumentation Laboratory with the CEA French Commission of Atomic Energy to validate these materials for future applications in severe conditions such as online measurements in irradiation experiments performed in research reactors. For this purpose, thin piezoelectric discs were irradiated while a remote network analyser continuously monitored the frequency response of their electrical impedance. The total neutron dose has reached a level of 1.5.1017 neutrons $/ \mathrm{cm}^{2}$. Positive and negative shifts of the peak resonance frequency have been recorded but in any case with a variation lower than $1 \%$. On the other hand, the amplitude of the electrical impedance at resonance frequency has largely decreased with even a reduction by factor two or three for some piezoelectric cells. Transitory effects have also been detected for these two parameters as function of the reactor activity. Additional thermal and gamma radiation effect have been investigated. Similarly, some piezoelectric cells glued on glass delay line have been tested with satisfactory results to these stresses.

\section{6:00}

5pPAb8. Ultrasonic exploration at extreme shallow underground in submerged soil. Kunihiko Seo (Toin University of Yokohama, 1614 Kurogane-cho, Aoba-ku, 225-8502 Yokohama, Japan, seo.ylk@gmail.com), Takashi Shirakawa (Toin University of Yokohama, 1614 Kurogane-cho, Aoba-ku, 225-8502 Yokohama, Japan, t_shira7@yahoo.co.jp), Tsuneyoshi Sugimoto (Toin University of Yokohama, 1614 Kurogane-cho, Aoba-ku, 225-8502 Yokohama, Japan, tsugimot @ cc.toin.ac.jp)

Now a lot of land mines remain buried in the world, so that the clearance of them is required. As a tool of removing land mines, equipments using electromagnetic radiation are often employed. However, there is a problem that the land mines in the flooded soil such as in Southeast Asia cannot be detected in the rainy season. Therefore, the new way using sound waves will be profitable to detect the land mines in the flooded soil. In this research, the acoustic exploration at very shallow area in submerged sand is examined at a water tank in the lab. First we measure the propagation property of ultrasound of $120 \mathrm{kHz}$ in the shallow submerged sand, and examine underground imaging. As a result, the acoustic velocity is measured at about $1500 \mathrm{~m} / \mathrm{s}$ and the attenuation is measured at about $-19 \mathrm{~dB} / \mathrm{m}$. And next, shallow underground exploring by using acoustic shielding boards is carried out. As a result, underground images in the water tank simulating the submerged soil are obtained. Then acoustic shielding boards can block wave which propagates specific route. This will make another exploration method possible. 


\title{
Session 5pPAc
}

\section{Physical Acoustics: General Topics in Physical Acoustics II}

\author{
Walter Lauriks, Cochair \\ Lab. ATF, Katholieke Universiteit Leuven, Celestijnenlaan 200D, Leuven, B-3001, Belgium \\ Andi Petsculescu, Cochair \\ University of Louisiana, Department of Physics, P.O. Box 44210, Lafayette, LA 70504, USA
}

\section{Contributed Papers}

\begin{abstract}
4:00
5pPAc1. An automated 3 dimensional scanning system for validation of acoustical simulation results. Simo-Pekka Simonaho (University of Kuopio, P.O.Box 1627, 70211 Kuopio, Finland, simo-pekka.simonaho@uku.fi)

To validate acoustical simulation results, a great number of measurement points are needed especially in 3 dimensional cases. These measurements can be extremely laborious when done manually. Also, the spatial information of the measurement points has to be accurate. In this work, an automated 3-D scanning system with high spatial resolution for validation of acoustical simulation results is introduced. The system consists of a multi channel data acquisition hardware, a microphone array and a 3-D scanning system. The movement of the microphone array is controlled by the data acquisition hardware. The performance of the automated 3-D scanning system is demonstrated and the experimentally measured pressure fields are compared to simulation results.
\end{abstract}

\section{4:20}

5pPAc2. Signal processing of impedance spectrum for speed of sound and pressure measurement in plane or radial resonators. Eric Rosenkrantz (Radio Application Division, NEC Corporation, Université Montpellier II, Place Eugène Bataillon, 34095 Montpellier, France, eric.rosenkrantz@ies.univ-montp2.fr), Jean-Yves Ferrandis (Radio Application Division, NEC Corporation, Université Montpellier II, Place Eugène Bataillon, 34095 Montpellier, France, ferrandi@lain.univ-montp2 .fr), Gerard Leveque (Institut d'Electronique du Sud UMR-CNRS 5214, Université Montpellier II, Place Eugène Bataillon, 34095 Montpellier, France, Gerard.leveque@ ies.univ-montp2.fr)

The impedance of gas contained between two plane walls is a periodic function of the frequency. The frequency interval between two resonances of the gas is equal to $\mathrm{c} / 2 \mathrm{D}$. Furthermore, the amplitude of the resonances is proportional to the pressure. We describe a signal processing to easily deduce the speed of sound and the pressure of the gas from the impedance spectrum. We show that the modulus of the Fourier transform of the modulus of impedance, called "Tempograph," contains all information about the gas. In some industrial cases the container is a cylinder or a sphere which can be used as a radial resonator exited by a radial wave $[1,2]$. The frequency response of such resonator at high frequencies is quasi-periodic and thus the same signal processing can be used. [1] M. F. Narbey, et al., "Determination of the composition of a gas mixture in a nuclear fuel rod by an acoustic method." INSIGHT, 42(9), 603-605 (2000). [2] A. Olson, "Helium bottle pressure measurement by portable ultrasonic technique." 1989, Rapport de Boeing nAD-A208 994, http://stinet.dtic.mil/str/index.html.

\section{4:40}

5pPAc3. Absorption and velocity of acoustical waves in binary solutions of poly (ethylene glycol) and water. Rajendra Kumar Singh (Department of Physics, Banaras Hindu University, 221005 Varanasi, India, rksingh_17@rediffmail.com), Manish Pratap Singh (Banaras Hindu
University, 221005 Varanasi, India, mps_bhu@yahoo.co.in),Rishi Pal Singh (Banaras Hindu University, 221005 Varanasi, India, rishisingh80 @ rediffmail.com)

A resonator technique has been devoleped to measure ultrasonic velocity and absorption for aqueous solutions of PEG of different molecular weights. The velocity has been measured at different frequencies and the concentrations (by weight) ranged from $1 \%$ to $10 \%$ of poly (ethylene glycol) in water. Adiabatic compressibility has been obtained at different temperatures, using experimental value of velocity and density. Viscocity has also been measured in wide temperature range . Ultrasonic absorption has been measured in the frequency range $400 \mathrm{KHz}-50 \mathrm{MHz}$, using resonator technique and pulse technique in temperature range $400-650{ }^{\circ} \mathrm{C}$. Observations showed that the ultrasonic absorption decreases with increasing temperature at a given concentration and also increases with concentration at a given temperature. The velocity increases with increasing temperature and concentration. Shear viscosity has been found to decrease with temperature but increases with concentration. Velocity studies show that as the polymer concentration increases a more rigid molecular structure is formed by bonding between the large polymer molecules.

\section{5:00}

5pPAc4. Acoustic field in a spherical resonator: effect of modal coupling due to small perturbations. Cécile Guianvarc'H (Institut National de Métrologie (LNE-INM/Cnam), 61 rue du Landy, 93210 La Plaine Saint Denis, France, cecile.guianvarch@cnam.fr), Laurent Pitre (Institut National de Métrologie (LNE-INM/Cnam), 61 rue du Landy, $93210 \mathrm{La}$ Plaine Saint Denis, France, pitre@cnam.fr), Arnaud Guillou (Institut National de Métrologie (LNE-INM/Cnam), 61 rue du Landy, 93210 La Plaine Saint Denis, France, guillou.arnaud@gmail.com), Michel Bruneau (Laboratoire d'Acoustique de l'Université du Maine, Avenue Olivier Messiaen, 72085 Le Mans, France, michel.bruneau@univlemans.fr), Anne-Marie Bruneau (Laboratoire d'Acoustique de l'Université du Maine (LAUM, UMR CNRS 6613), Avenue Olivier Messiaen, 72085 Le Mans, France, anne-marie.bruneau@univ-lemans.fr)

The international community recently recommended a re-definition of the kelvin referring to the value of the Boltzmann constant $k$, which must thus be known with a relative uncertainty of $10^{-6}$. The measurement of the acoustic resonance properties of a gas filled spherical or quasi-spherical resonator is an appropriate method to do this with these requirements. Actually, a detailed modeling of the acoustic field in the resonator is required for the determination of $k$. Several phenomena must be taken into account including heat conduction, shear and bulk viscosity of the gas, the real shape of the resonator, the acoustic input impedance of small acoustic elements flush-mounted on the wall (tubes, transducers)... . Significant theoretical studies have already been done in which these perturbations are accounted for separately, the coupling between them being neglected. The scope here is thus to provide a unified model for the acoustic field in the cavity including all these perturbations and the resulting modal coupling, and applying it on a simple practical configuration: a spherical resonator filled with argon, acoustic transducers being flush-mounted on the wall. 


\section{$5: 20$}

5pPAc5. Towards a theory for arbitrarily shaped sound field reproduction systems. Sascha Spors (Deutsche Telekom Laboratories, Ernst-Reuter-Platz 7, 10587 Berlin, Germany, sascha.spors@telekom.de), Jens Ahrens (Deutsche Telekom Laboratories, Ernst-Reuter-Platz 7, 10587 Berlin, Germany, jens.ahrens@telekom.de)

The simple source approach predicts that a distribution of appropriately driven loudspeakers (secondary sources) enclosing a given listening area is suitable for the physical recreation of any desired exterior virtual sound field within that listening area. A specific class of sound reproduction approaches (e.g., higher-order Ambisonics) is based upon the explicit solution of the simple source formulation with respect to the secondary source driving function. To the knowledge of the authors, such an explicit solution is currently only available for specific geometries of the sound reproduction system. This contribution presents a theoretical framework for the derivation of the explicit solution for the driving function of arbitrarily shaped secondary source arrangements. It is based upon the expansion of the reproduced wave field into a series of orthogonal basis functions. These basis functions emerge from the respective underlying geometry. It is shown that most of the sound reproduction systems that are based upon the simple source formulation can be seen as specialization of the presented approach to a particular geometry and basis function.

\section{$5: 40$}

5pPAc6. Numerical computation of reflected and transmitted waves at a fluid/solid interface. Laure Bossy (AREVA - CEZUS Research Center, Avenue Paul Girod, 73403 Ugine Cedex, France, laure.bossy@espci.fr), Marie-Françoise Cugnet (AREVA - CEZUS Research Center, Avenue Paul Girod, 73403 Ugine Cedex, France, mariefrancoise.cugnet@areva.com), Emmanuel Bossy (Laboratoire Photons et Matière, ESPCI/CNRS, 10 rue Vauquelin, 75231 Paris Cedex 05, France, emmanuel.bossy@espci.fr), Didier Cassereau (Laboratoire Ondes et Acoustique, 10, rue Vauquelin, 75231 Paris, France, didier.cassereau @ espci.fr)

In this paper, we propose a numerical computation of the different waves generated when a spherical incident pulse is reflected and transmitted by a fluid/solid interface. In addition to the standard reflected and transmitted waves that propagate inside the volume, various surface waves can also be found in both propagation media. In the fluid, we can observe the longitudinal and transverse head waves, and the so-called leaky Rayleigh wave that generalizes the Rayleigh wave in a semi-infinite free solid medium to the case of an immersed interface. Similar effects can also be observed on the transmitted displacement field inside the solid material. We compare different numerical approaches, including semianalytic methods (high-frequency approximation coupled to ray modeling approach) and implicit methods (finite elements and/or finite differences scheme), each method having its own advantages and inconvenients, and domains of validity. These different methods are used to evaluate the field reflected by the interface ; the transmitted displacement field is also analyzed from the same point of view. The plane and curved geometries will be analyzed and the influence of the curvature of the interface will be pointed out.

\section{6:00}

5pPAc7. V(z) oscillations in acoustic microscope at upward defocusing. Anton V. Kozlov (MSU, Leninskie Gory, Bld. 1-2, 119991 GSP-1 Moscow, Russian Federation, av_kozlov@inbox.ru), Vladimir G. Mozhaev (MSU, Leninskie Gory, Bld. 1-2, 119991 GSP-1 Moscow, Russian Federation, vgmozhaev@mail.ru)

Acoustic microscopy is widely used for imaging and study of elastic properties of transparent and opaque materials. As a rule, multiple periodic oscillations in the output signal $V$ of a reflection acoustic microscope can be observed in the case of negative defocusing of the wide-angle acoustic lens, i.e., when its focus is a distance $z$ below the solid sample surface. The main well-known mechanism for $V(z)$ oscillations is the interference of radiation reflected perpendicularly from the sample surface and re-radiation of leaky Rayleigh waves generated on the sample by the lens. This effect explains high contrast imaging in reflection scanning acoustic microscopy, and it is a popular method to study properties of solids by measuring Rayleigh wave speeds. As it is shown in this work, bulk acoustic waves in the sample can also give rise to $V(z)$ oscillations. A new mechanism of such oscillations is predicted in the case of positive defocusing (focusing above the sample surface) in acoustic microscopy of anisotropic plates exhibiting negative acoustic refraction. The ray model of this effect shows a possibility to find a relationship between extrema of $V(z)$ curve and separate points on the acoustic slowness surface of the sample.

FRIDAY AFTERNOON, 4 JULY 2008

ROOM 351, 5:00 TO 6:20 P.M.

\title{
Session 5pPAd
}

\section{Physical Acoustics: Scattering and Diffraction II}

\author{
Michael L. Oelze, Cochair \\ University of Illinois at Urbana-Champaign, Beckman Institute, 405 N Mathews, Urbana, IL 61801, USA \\ Jean-Marc Conoir, Cochair \\ Institut Jean Le Rond d'Alembert-UMR CNRS 7190, Université Paris 6, tour 55-65, 4 place Jussieu, Paris, 75005, France
}

\section{Contributed Papers}

\section{5:00}

5pPAd1. The physics of wedge diffraction: A model in terms of elementary diffracted waves. Mitsuhiro Ueda (Predio Meguro Science Laboratory, 4-20-13 Meguro, Meguro-ku, 153-0063 Tokyo, Japan, ueda-mt @ nifty.com)

A model for wedge diffraction is constructed using the virtual discontinuity principle of diffraction [1]. In the model diffracted waves are described by the sum of two elementary diffracted waves that are calculated by integrating the potential along the half line issued from the vertex of wedge. The wedge of aperture angle $\pi / \mathrm{n}(\mathrm{n}=1,2,3, \ldots)$ is nondiffractive since its potential can be expressed by the sum of direct waves from the point source and its mirror images. The nondiffractive wedges are useless in the conventional analysis of diffraction since there are no diffracted waves in the potential. But in this model diffracted waves of these wedges are cancelled out in the summing process. Thus the elementary diffracted waves are existed even in these wedges and far field solution for them is obtained for the nondiffractive wedge. Due to its simple structure it can be extended to the arbitrary wedge without any modification and the far field solution of diffracted waves is derived by summing the extended elementary diffracted waves in the model and it coincides with the rigorous one literally. Thus the model is verified firmly by this simple calculation. 1. M. Ueda, JASA, 95, p.2354 (1994). 


\section{$5: 20$}

5pPAd2. A global search tool for the equivalent source method and its applications to the scattering problem. Yves J.r. Gounot (UFRJ/COPPE, Universidade Federal do Rio de Janeiro, 21941-972 Rio de Janeiro, Brazil, ygounot@mecanica.ufrj.br), Ricardo E. Musafir (UFRJ/COPPE, Universidade Federal do Rio de Janeiro, 21941-972 Rio de Janeiro, Brazil, rem @ serv.com.ufrj.br)

Low computational-cost solutions to the acoustic scattering problem can be obtained with the equivalent source method (ESM), provided the sources are adequately positioned. Because this last point represents often a complicated task - mainly responsible for the not much widespread use of the method - a technique that hurdles this difficulty, called ESGA, has been previously proposed (Gounot and Musafir, Internoise 2004). Based on a combination of genetic algorithm with ESM, the ESGA is a global search tool that provides, given a set of monopoles, their 'optimal' positioning and complex amplitudes. The technique efficiency is here shown through a number of three-dimensional scattering problems. The algorithm is also used in order to identify, for each of the different cases considered, typical geometrical arrangements of monopoles which provide good solutions.

\section{$5: 40$}

5pPAd3. Convergence of correlations in multiply scattering media. Eric Larose (LGIT - CNRS - Université Joseph Fourier, Maison des Géosciences, 1381 rue de la Piscine, BP 53, 38041 Grenoble, France, Eric.Larose@obs.ujf-grenoble.fr), Arnaud Derode (Laboratoire Ondes et Acoustique, ESPCI, Université Paris 7, CNRS, 10 rue Vauquelin, 75005 Paris, France, arnaud.derode@ujf-grenoble.fr), Philippe Roux (LGIT CNRS - Université Joseph Fourier, Maison des Géosciences, 1381 rue de la Piscine, BP 53, 38041 Grenoble, France, philippe.roux@obs.ujf-grenoble.fr), Michel Campillo (LGIT - CNRS Université Joseph Fourier, Maison des Géosciences, 1381 rue de la Piscine, BP 53, 38041 Grenoble, France, Michel.Campillo@obs.ujf-grenoble.fr)

Correlations of ambient seismic or acoustic noise are now widely used to reconstruct the impulse response between two passive receivers as if a source was placed at on of them. Applications include terrestrial and solar seismology, underwater acoustics and structural health monitoring. Never- theless, for a given set of data, correlations do not only yield automatically the Green function between the sensors, but also contains residual fluctuations that might "blur" the images. [Gizon et al, Astrophys. J. 614 (2004); Weaver and Lobkis, J. Acoust. Soc. Am. 117 (2005); Sabra et al., J. Acoust. Soc. Am. 118 (2005)]. We propose a model to describe the "signal-tofluctuations" ratio in the correlations in the case of nonstationary wavefields, and more particularly in the case of scattering media. The work includes theoretical derivations and numerical simulations. The role of multiple scattering in the rate of convergence of the correlations toward the Green function is quantitatively evaluated [Larose et al, (submitted 2008)].

\section{6:00}

5pPAd4. Reflection and transmission coefficients of a fluid slablike region containing a depth-varying random distribution of cylinders. Jean-Marc Conoir (Institut Jean Le Rond d'Alembert-UMR CNRS 7190, Université Paris 6, tour 55-65, 4 place Jussieu, 75005 Paris, France, conoir@1mm.jussieu.fr), Sébastien Robert (LOA, UMR CNRS 7587, ESPCI, 10 rue Vauquelin, 75231 Paris, France, Sebastien.Robert@espci.fr), Abdelhak El Mouhtadi (LOMC- FRE CNRS 3102- Groupe Ondes Acoustiques, Université du Havre, place R. Schuman, 76610 Le Havre, France, abdelhak.ilel@gmail.com), Francine Luppé (LOMC- FRE CNRS 3102- Groupe Ondes Acoustiques, Université du Havre, place R. Schuman, 76610 Le Havre, France, francine.luppe @ univ-lehavre.fr)

This work deals with multiple scattering by a random distribution of parallel elastic cylinders immersed in a fluid slablike region. The concentration of scatterers inside the slab is supposed to vary slowly with depth, and the WKB method is used to calculate the reflection and transmission coefficients of the slab. In order to do so, the continuity conditions on the boundaries between the slab and the surrounding fluid are needed. They follow from the application of Twersky's theory to the case of a slab with a given constant concentration of scatterers, which shows that both pressure and normal displacement are continuous, provided an effective mass density of the slab is correctly defined. The results of the WKB are successfully compared to those obtained from the discretization of the slab into layers of constant concentrations of cylinders and the use of Twersky's theory.

FRIDAY AFTERNOON, 4 JULY 2008

ROOM 241, 2:00 TO 6:20 P.M.

\title{
Session 5pPPa
}

\section{Psychological and Physiological Acoustics and Speech Communication: Acoustic Features and Speech Perception II}

\author{
Jont B. Allen, Cochair \\ University of IL, 405 N. Mathews, Room 2061 Beckman Inst. (MC 251), Urbana, IL 61801, USA \\ Sarah Hawkins, Cochair \\ University of Cambridge, Department of Linguistics, Sidgwick Avenue, Cambridge, CB3 9DA, UK
}

\section{Invited Papers}

5pPPa1. Reception of phonetic features in fluctuating background noise maskers. Christian Lorenzi (Univ Paris Descartes, CNRS, Ecole Normale Superieure, DEC, 29 rue d'Ulm, 75005 Paris, France, lorenzi@ens.fr)

We will review recent studies investigating the reception of phonetic features (voicing, manner and place of articulation) in nonstationary background maskers. In each study, consonant identification was assessed in steady and amplitude-modulated speech-shaped noise at signal-to-noise ratios yielding about $50 \%$ correct in steady noise. The rate and depth of amplitude modulation applied to the noise masker were either fixed or systematically varied. Confusion matrices were compiled across listeners and the amount of release from masking (percent information received in nonsteady minus steady noise) was calculated for each phonetic feature and experimental condition. Speech and noise mixtures were (i) left intact (unfiltered), (ii) lowpass filtered $(<1.5 \mathrm{kHz}$ ), or iii) processed in order to 
degrade spectral (place of excitation in the cochlea) and/or temporal fine structure cues. Overall, the results indicate that release from masking typically reported in normal-hearing listeners (i.e., the substantial improvement in speech reception in fluctuating noise compared to steady noise) is not identical across phonetic features. This suggests that the ability to "glimpse" into background noise valleys involves multiple auditory processes constrained by both peripheral and central factors.

5pPPa2. Identification of intervocalic consonants in stationary and nonstationary noise. Martin Cooke (Sheffield University, Computer Science Department, Regent Court, 211 Portobello St., S1 4DP Sheffield, UK, m.cooke@dcs.shef.ac.uk), Odette Scharenborg (Centre for Language and Speech Technology, Radboud University Nijmegen, Erasmusplein 1, 6525 HT Nijmegen, Netherlands, O.Scharenborg@let.ru.nl)

The factors which underlie the perception of consonants in noise remain poorly understood. In this study, native listeners identified 24 English consonants spoken by eight talkers presented in nine intervocalic contexts with varying stress position. Listeners were tested in 5 noise conditions: tokens were masked by stationary speech-shaped noise, a competing talker, three and eight speaker babble and speech-modulated noise, all of which have the long-term spectrum of speech. The rank ordering of consonant identification scores in stationary noise was highly-correlated $(\mathrm{r}=0.9, \mathrm{p}<0.0001)$ with a similar condition reported by Phatak and Allen [JASA 121: 2312-2326, 2007], but less so in the four nonstationary noise backgrounds ( $\mathrm{r}=0.74, \mathrm{p}<0.001)$. In particular, /y/, /r/, /l/, /f/, /ch/, /sh/, /m/ and most of the plosives showed a wide variation in ranking. These findings suggest that, in addition to the long-term spectrum of the masker, consonant identification is noise is affected by other factors such as temporal fluctuations in the masker, misallocation of foreground/background components and attention.

\section{2:40}

5pPPa3. The interaction of glimpsing, pitch and vocal tract length in the recognition of concurrent syllables. Martin D. Vestergaard (Centre for the Neural Basis of Hearing, Department of Physiology, Development and Neuroscience, University of Cambridge, Downing Site, CB23EG Cambridge, UK, mdv23@cam.ac.uk), Nicolas R. Fyson (Centre for the Neural Basis of Hearing, Department of Physiology, Development and Neuroscience, University of Cambridge, Downing Site, CB23EG Cambridge, UK, nickfyson@gmail.com), Roy D. Patterson (Centre for the Neural Basis of Hearing, Department of Physiology, Development and Neuroscience, University of Cambridge, Downing Site, CB23EG Cambridge, UK, rdp1@cam.ac.uk)

In multispeaker environments, human listeners use the temporal misalignment of competing speech signals to improve recognitionan effect referred to as "glimpsing." When the temporal envelopes of concurrent syllables pairs are carefully matched to preclude glimpsing, listeners were observed to use vocal tract length (VTL) and glottal pulse rate (GPR) cues to improve recognition. This paper reports an investigation of the interaction between glimpsing and these vocal cues. Syllables were synthesized with a vocoder to simulate speakers with widely different combinations of GPR and VTL. Recognition of one syllable in the presence of a concurrent syllable was measured as the vocal cues and the temporal alignment of the syllables were varied. The effect of glimpsing was most pronounced when the vocal cue differences between the target and distracter syllables were small. Furthermore, there was a strong effect of consonant type (stops, fricatives, or sonorants) and an asymmetry between consonant-vowel (CV) and vowel-consonant (VC) syllables. The lowest recognition rate was observed, not at perfect temporal alignment but rather at a distracter lag of $-50 \mathrm{~ms}$ for CVs and $100 \mathrm{~ms}$ for VCs. The results are analyzed with confusion matrices. Research supported by the UK MRC [G0500221, G9900369].

\section{3:00}

5pPPa4. Phoneme confusions as a function of noise, spectral resolution and L2 experience. Robert Shannon (House Ear Institute, 2100 W. Third St., Los Angeles, CA 90057, USA, shannon@hei.org), Monica Padilla (House Ear Institute, 2100 W. Third St., Los Angeles, CA 90057, USA, mpadilla@hei.org)

Consonant and vowel confusion matrices were measured from normal hearing listeners with varying degrees of experience in English. There were five listeners each in the following categories: native English speakers, and Spanish speakers who were immersed in English at the ages of 0-5 years, 5-10 years, 10-18 years, and over 18 years. 12 vowels (hVd) or 18 consonants (vCv) were presented and responses were collected in a confusion matrix. Spectral resolution was varied by using a noise-band vocoder with 2, 4, 6, 8, and 16 channels, as well as unprocessed speech. All stimuli were presented in speech shaped noise at SNR levels of $-5 \mathrm{~dB}$ to $+15 \mathrm{~dB}$ in 5 $\mathrm{dB}$ steps as well as in quiet. Noise and spectral resolution had a similar effect on voicing, manner and place of articulation, and also had similar effects as a function of L2 experience. For native speakers of Spanish the duration of L2 experience had the largest effect on voicing cues, less effect on manner cues, and almost no effect on place cues.

5pPPa5. The perceptual flow of phonetic feature processing. Steven Greenberg (Technical University of Denmark, Center for Applied Hearing Research, Ørsteds Plads, Building 352, 2800 Lyngby, Denmark, steveng@ silicon-speech.com), Thomas Ulrich Christiansen (Technical University of Denmark, Center for Applied Hearing Research, Ørsteds Plads, Building 352, 2800 Lyngby, Denmark, tuc@oersted.dtu.dk)

How does the brain process spoken language? It is our thesis that word intelligibility and consonant identification are insufficient by themselves to model how the speech signal is decoded - a finer-grained approach is required. In this study, listeners identified 11 different Danish consonants spoken in a Consonant + Vowel $+[1]$ environment. Each syllable was processed so that only a portion of the original audio spectrum was present. Three-quarter-octave bands of speech, centered at 750, 1500, and $3000 \mathrm{~Hz}$, were presented individually and in combination with each other. The conditional, posterior probabilities associated with phonetic-feature decoding were computed from confusion matrices in order to deduce the temporal flow of phonetic processing. Decoding the feature, Manner-ofArticulation, depends on accurate decoding of the feature Voicing (but not vice-versa), and decoding Place-of-Articulation requires 
precise decoding of Manner (but not the converse). From these data, we conclude that Voicing is processed prior to Manner-ofArticulation, and that Manner is decoded prior to Place-of-Articulation. Voicing and Manner cues are often correctly decoded in conditions where Place is not. This asymmetric pattern of feature decoding may provide extra-segmental information of utility for speech processing, particularly in adverse listening conditions.

3:40

5pPPa6. Understanding the complex modulation spectrum for consonants and consonant features. Kenneth W. Grant (Walter Reed Army Medical Center, Army Audiology and Speech Center, 6900 Georgia Ave. NW, Washington, DC 20307-5001, USA, grant@tidalwave.net), Sandeep A. Phatak (Walter Reed Army Medical Center, Army Audiology and Speech Center, 6900 Georgia Ave. NW, Washington, DC 20307-5001, USA, s.a.phatak@gmail.com), Elena Grassi (Walter Reed Army Medical Center, Army Audiology and Speech Center, 6900 Georgia Ave. NW, Washington, DC 20307-5001, USA, elena.grassi@gmail.com)

Speech intelligibility is highly dependent on the magnitude and phase characteristics of the low-frequency modulation spectrum. However, unlike more traditional representations of speech, such as the spectrogram, associating details of the modulation spectrum to specific phonemes and subphonemic units of speech has not been readily forthcoming. In the present study we used local time reversals of the speech waveform between 20-160 ms to selectively distort portions of the complex modulation spectrum. Normal-hearing subjects were tested on a consonant recognition task and a detailed analysis of the perceptual confusions was performed. Consistent with earlier results using sentence-length materials, average consonant intelligibility declined as the length of the time reversal segment increased. Further analyses were conducted to determine the effect of time-reversal segment duration on the amount of information transmitted for individual consonants (including specific consonant productions) and acoustic features for voicing, manner of articulation, and place of articulation. An acoustic analysis using a biologically motivated auditory processing model was also performed to determine the effect of time reversals on cochlear and cortical representations of speech. The relations between changes to the complex modulation spectrum and the percent information transmission of selected speech segments and features are discussed.

\section{4:00-4:20 Break}

$4: 20$

5pPPa7. Can CV intelligibility predict speech intelligibility? Sarah Hawkins (University of Cambridge, Department of Linguistics, Sidgwick Avenue, CB3 9DA Cambridge, UK, sh110@cam.ac.uk)

This paper begins by reviewing the speech perception literature to predict cues that would and would not be expected to survive energetic masking of various types. The focus is especially (but not exclusively) on spectrotemporal cues to stops in the vicinity of the segment boundary in CV syllables. The second part of the paper discusses influences that can restrict the generality of research findings from isolated CV syllables. This includes ways in which CV syllables change in different phonetic contexts and styles of speech, contributions of the visual modality, and other uses of top-down information, such as phonotactic, lexical, semantic and syntactic probability. The paper concludes by asking whether-and how-simple measures such as CV intelligibility can be used to reflect intelligibility of speech in real-life communicative situations.

\section{4:40}

5pPPa8. The role of the cochlear processing in human speech recognition. Jont B. Allen (University of IL, 405 N. Mathews, Room 2061 Beckman Inst. (MC 251), Urbana, IL 61801, USA, jontallen@ieee.org), Marion Regnier (208 S. 3rd St. Apt 5A, Brooklyn, NY 11211, USA, marion.regnier@gmail.com), Sandeep Phatak (Walter Reed Hospital, Silver Springs, MD 20901, USA, s.a.phatak@gmail.com), Feipeng Li (University of IL, 405 N. Mathews, Room 2061 Beckman Inst. (MC 251), Urbana, IL 61801, USA, fli2@uiuc.edu)

Little is know about how the auditory system decodes speech. We may think of speech communication re Shannon's source-channel model, thus viewed, the most complex part of the speech communication channel is the auditory system (the receiver). In my speechperception research, I have attempted to limit the assumptions, and have thus fallen back on Shannon's basic source-channel model. The basic tool is the confusion matrix (CM) for isolated natural consonant and vowels $(\mathrm{CV})$, as a function of the speech to noise ratio (SNR), with several types of masking noise. We have used large numbers of talkers and listeners (i.e., 20). In a second experiment we selectively remove islands of speech in time-frequency, and then correlate the resulting modified speech against subject scores. Our most important conclusions are: (1) The across-frequency onset transient portion of the signal is typically the most important. (2) The spectral regions of these transient are used to code different consonants. (3) While the frequency regions for a given consonant are correlated to the following vowel, this may not be important for perception. (4) Compact spectral-temporal amplitude modulations components (e.g., a $10 \mathrm{~Hz}$ modulation) do not seem to play a significant role, at least above $1-2 \mathrm{kHz}$.

\section{Contributed Papers}

\section{5:00}

5pPPa9. Spectral and temporal modulations essential to spoken word, gender and timbre identification. Frédéric E. Theunissen (UC Berkeley, Dept. of Psychology, 3210 Tolmant Hall, Berkeley, CA 94720-1650, USA, theunissen@berkeley.edu), Taffeta Elliott (UC Berkeley, Dept. of Psychology, 3210 Tolmant Hall, Berkeley, CA 94720-1650, USA, taffeta@ berkeley .edu)

Human speech and musical sounds contain complex spectral and temporal modulations. Speech intelligibility, perception of melody, and identification of source characteristics (e.g., speaker gender or musical timbre) de- pend on spectrotemporal modulations but can be surprisingly robust to drastic spectral and temporal degradations. We systematically explored which restricted spectral and temporal modulations are essential to the perception of complex sounds. Degraded sentences and musical sounds were obtained by a novel modulation filtering procedure performed on the sound spectrogram. Temporal modulation filtering smeared the amplitude envelope by removing changes above particular Hz. Spectral modulation filtering smeared the spectral energy across frequency bands by removing changes above particular cyc/kHz. We further complemented this low-pass filtering with more specific notch-filtering. Speech intelligibility, gender recognition 
and musical instrument identification were assessed in psychophysical experiments. We determined that spectral modulations below $\sim 3.75 \mathrm{cy}$ $\mathrm{c} / \mathrm{kHz}$, and temporal modulations between 1 and $7 \mathrm{~Hz}$ are essential for speech comprehension. Gender identification however required the presence of higher spectral modulations. Similarly the timbre and pitch of instruments was affected differentially by notch filters in these two regions of the modulation spectrum. Our research could be used to guide the design of optimal signal processing in hearing aids and cochlear implants.

\section{$5: 20$}

5pPPa10. A model with compression for estimating speech intelligibility in quiet and in noise. Koenraad S. Rhebergen (AMC Dept. of Clinical and Experimental Audiology, AMC, Clinical and Experimental Audiology, 1105 Amsterdam, Netherlands, k.s.rhebergen @amc.uva.nl), Johannes Lyzenga (Vrije Universiteit Medical Center, Boelelaan 1117, 1081 HV Amsterdam, Netherlands, j.lyzenga@vumc.nl)

For speech reception thresholds (SRTs), measured in normally-hearing listeners using various types of stationary noise, the Speech Intelligibility Index (SII, ANSI S3.5-1997) model predicts a fairly constant speech proportion (of about 0.3) necessary for sentence intelligibility. For SRTs in quiet, the estimated speech proportions are often lower, and show a larger inter-subject variability, than found for speech in noise near normal speech levels. This might be related to the fact that cochlear compression is larger at normal speech levels than near the threshold for speech in quiet. The SII model does not take this into account. The present model attempts to alleviate this problem by including cochlear compression. It is based on a loudness model for normally-hearing and hearing-impaired listeners [ANSI S3 .4-2007]. It estimates internal excitation levels of the speech, accounts for the compressed effective dynamic range of the internal speech signal, and calculates the proportion of speech above threshold using similar spectral weighting as used in the standard SII. The present model and the standard SII were used to predict SRTs in quiet and noise for both normally-hearing and hearing-impaired listeners. The present model predicted speech intelligibility with less variability than the standard SII.

\section{5:40}

5pPPa11. Listeners' sensitivity to talker differences in voice-onset-time: Segments versus features. Rachel $M$. Theodore (Northeastern University, Dept. of Psych. - 125 NI, 360 Huntington Ave., Boston, MA 02115-5000, USA, r.theodore@ neu.edu), Joanne L.
Miller(Northeastern University, Dept. of Psych. - 125 NI, 360 Huntington Ave., Boston, MA 02115-5000, USA, j.miller@neu.edu)

Recent findings indicate that listeners are sensitive to talker differences in phonetic properties of speech, including voice-onset-time (VOT) in wordinitial voiceless stop consonants. Here we extend earlier findings from our laboratory [J. S. Allen and J. L. Miller, J. Acoust. Soc. Am. 115, 3171-3813 (2004)] by examining the level of representation underlying this sensitivity. In familiarization phases, listeners heard two talkers produce pain. Critically, word-initial VOTs were manipulated such that one talker produced short VOTs and the other talker produced long VOTs. In test phases, listeners were presented with a short-VOT and long-VOT variant of either pain or cane; in both cases, listeners were asked to select which of the two VOT variants was most representative of a given talker. Results to date indicate that which variant of pain is selected at test is in line with listeners' exposure during training (replicating earlier findings), and that this effect holds even when listeners are tested on cane, which begins with a different voiceless stop than heard during training. These results suggest that listeners are sensitive to talker differences in VOT at the level of a phonetic feature, rather than at the level of a particular phonetic segment.

\section{6:00}

5pPPa12. Amplitude modulation of noise cues voicing distinction in fricatives. Jonathan Pincas (University of Surrey, GU2 7XH Guildford, UK, jon@ pincas.co.uk), Philip J. Jackson (University of Surrey, Centre for Vision, Speech and Signal Processing, GU2 7XH Guildford, UK, p.jackson @ surrey.ac.uk)

The aperiodic noise source in fricatives is characteristically amplitude modulated by voicing. Previous psychoacoustic studies have established that observed levels of AM in voiced fricatives are detectable, and its inclusion in synthesis has improved speech quality. Phonological voicing in fricatives can be cued by a number of factors: the voicing fundamental, duration of any devoicing, duration of frication, and formant transitions. However, the possible contribution of AM has not been investigated. In a cue trading experiment, subjects distinguished between the nonsense words "ahser" and "ahzer." The voicing boundary was measured along a formant-transition duration continuum, as a function of AM depth, voicing amplitude and masking of the voicing component by low-frequency noise. The presence of AM increased voiced responses by approximately $30 \%$. The ability of AM to cue voicing was strongest at greater modulation depths and when voicing was unavailable as a cue, as might occur in telecommunication systems or noisy environments. Further work would examine other fricatives and phonetic contexts, as well as interaction with other cues. 


\title{
Session 5pPPb
}

\section{Psychological and Physiological Acoustics and Computational Acoustics: Computational Auralization II}

\author{
Durand R. Begault, Cochair \\ NASA Ames Research Center, Mail Stop 262-2, NASA ARC, Moffett Field, CA 94035, USA \\ Lauri Savioja, Cochair \\ Helsinki University of Technology, Department of Media Technology, PO Box 5400, TKK, 02015, Finland
}

\section{Contributed Papers}

\begin{abstract}
2:00
5pPPb1. Externalization in binaural synthesis: effects of recording environment and measurement procedure. Florian Völk (AG Technische Akustik, MMK, TU München, Arcisstr. 21, 80333 München, Germany, florian.voelk@mytum.de), Fabian Heinemann (AG Technische Akustik, MMK, TU München, Arcisstr. 21, 80333 München, Germany, hef@mmk.ei.tum.de), Hugo Fastl (AG Technische Akustik, MMK, TU München, Arcisstr. 21, 80333 München, Germany, fastl@mmk.ei.tum.de)

Databases of head related impulse responses (HRIRs) for binaural synthesis can be measured either in anechoic or reflective environments. If high synthesis quality is needed, miniature microphone measurements are performed in the ear canals of each individual user (individual measurement). Sometimes impulse responses measured in the ear canals of one individual are used for synthesis for other persons (nonindividual measurement). In most other cases artificial head measurements are used. This paper considers the dependence of the perceived distance of auditory images (externalization) on the measurement procedure (individual, nonindividual, artificial head) and on the recording environment (anechoic, reflective). For each measurement the same system and the same setup, especially the same geometric parameters, are used. Differences in the corresponding impulse response databases are determined and related to the subjective relative externalization differences in the front, in the back, and to both sides. For each direction a seven point rating scale was used. Statistical analysis suggests that the measurement parameters applied influence the externalization of auditory images.
\end{abstract}

\section{2:20}

5pPPb2. Smart sound environments: merging intentional soundscapes, nonspeech audio cues and ambient intelligence. Ralf Jung (Universität des Saarlandes, LS Wahlster, FB Informatik, Bldg. E 1 1, Room 1.18, 66123 Saarbrücken, Germany, rjung@cs.uni-sb.de)

We introduce an intelligent audio notification system for multiuser environments that provides users with information about events (e.g., important emails) in a more discreet and non-distracting way. The peripheral awareness of individual-related events is done by using nonspeech audio cues which can be seamlessly integrated into artificial background soundscapes. These ambient soundscapes are self-composed with respect to well-known perceptual constraints such as auditive Gestalt laws as well as music psychological findings. To follow a hierarchical approach for the notification sounds we use notification instruments, ambient noises and traditional alert signals that are grouped by their level of intrusiveness. Since the notification system also follows a human-centered approach it takes parameters like user preferences, his/her current position in the environment and the type of event into consideration to decide which notification is the appropriate at this time. In the paper, we will describe the architecture of the personalized ambient audio notification service, compositional constraints as well as some findings of a user study in which we tested successfully the efficiency of our system with 25 subjects.

\section{2:40}

5pPPb3. A virtual auditory environment for investigating the auditory signal processing of realistic sounds. Sylvain Favrot (CAHR, Department of Electrical Engineering, DTU, Ørsteds Plads, Bygning 352, 2800 Kgs. Lyngby, Denmark, sf@oersted.dtu.dk), Jörg M. Buchholz (CAHR, Department of Electrical Engineering, DTU, Ørsteds Plads, Bygning 352, 2800 Kgs. Lyngby, Denmark, jb@oersted.dtu.dk)

In the present study, a novel multichannel loudspeaker-based virtual auditory environment (VAE) is introduced. The VAE aims at providing a versatile research environment for investigating the auditory signal processing in real environments, i.e., considering multiple sound sources and room reverberation. The environment is based on the ODEON room acoustic simulation software to render the acoustical scene. ODEON outputs are processed using a combination of different order Ambisonic techniques to calculate multichannel room impulse responses (mRIR). Auralization is then obtained by the convolution of the mRIR with an acoustic signal. The derivation of the mRIRs takes into account that (i) auditory localization is most sensitive to the location of the direct sound and (ii) that auditory localization performance is rather poor for early reflections and even worse for late reverberation. Throughout the VAE development, special care was taken in order to achieve a realistic auditory percept and to avoid "artifacts" such as unnatural coloration. The performance of the VAE has been evaluated and optimized on a 29 loudspeaker setup using both objective and subjective measurement techniques.

\section{3:00}

5pPPb4. Real-time auralization system based on beam-tracing and mixed-order Ambisonics. Markus Noisternig (LIMSI-CNRS, B.P. 133, 91403 Orsay, France, markus.noisternig@limsi.fr), Lauri Savioja (Helsinki University of Technology, Department of Media Technology, PO Box 5400, 02015 TKK, Finland, Lauri.Savioja@tkk.fi), Brian F. Katz (LIMSI-CNRS, B.P. 133, 91403 Orsay, France, brian.katz@limsi.fr)

Auralization, the final step in computational room acoustic simulations, aims to make audible the acoustics of complex virtual architectural spaces in a realistic and accurate manner. This paper presents a novel real-time auralization system comprising a geometry engine, a beam-tracer, and an audio renderer. The computation of early reflection paths is based on an efficient beam-tracing algorithm capable of real-time detection of specular reflection paths in a static geometry with one or several moving listener(s). For simpler rooms, the real-time performance is maintained even with dynamic geometries and sources. Results of the beam-tracer, sent to the audio renderer, consist of visible reflection paths and their accumulated material attenuation. From this geometrical and acoustical data, listener position-related 3D room impulse responses are generated applying a higher-order virtual Ambisonics approach. Final rendering of the binaural room impulse response (BRIR) is made taking into account the listener's head-orientation. As higher order reflections are more diffuse in nature, they may be encoded using lower Ambisonic orders, thereby reducing computational load. The environment combines high quality audio with visual rendering realized using the open source platforms Pure Data and VirChor respectively. This auralization framework provides direct audio-visual feedback in real-time for VR environments. 


\section{Invited Papers}

3:20

5pPPb5. Real-time auralization of modifiable rooms. Dirk Schröder (Institute of Technical Acoustics, RWTH Aachen University, Neustr. 50, 52066 Aachen, Germany, dsc@akustik.rwth-aachen.de), Ingo Assenmacher (Virtual Reality Group, RWTH Aachen University, Seffenter Weg 23, 52074 Aachen, Germany, assenmacher@rz.rwth-aachen.de)

Immersive virtual environments are a powerful tool for acousticians and architects to design buildings if the virtual reality system provides an interactive imaging of virtual sound sources with respect to the rooms' physical aspects. Current implementations using hybrid room acoustic simulation methods (e.g., combining image sources and ray tracing) enable the user to walk freely in such virtual architectural spaces, whereby the position/orientation of sound sources are interactively manipulable to detect possible acoustic defects, e.g., flutter echoes. In the case of coupled rooms, sound transmission effects must be included into the real-time simulation in order to identify deficient airborne sound insulation, whereby current implementations only support a change of state (open/closed) of fixed room-connecting elements, e.g., doors and windows. However, in scenarios like an architectural planning stage, it is convenient to manipulate the room geometry more freely, e.g., via the interactive positioning of stage reflector panels, but common spatial data structures, e.g., BSP- or Octrees do not efficiently support these operations. For this purpose, the concept of Spatial Hashing, which originates from computer graphics for collision detection of deformable objects, is applied to the simulation process. This adaptation also features an efficient identification and update process of image sources.

\section{3:40}

5pPPb6. Comparison of auralisation results betwee measurements and simulations of line arrays with high resolution modeling data. Wolfgang Ahnert (Ahnert Feistel Media Group, Arkonastr. 45-49, 13189 Berlin, Germany, wahnert@ada-acousticdesign.de), Stefan Feistel (Ahnert Feistel Media Group, Arkonastr. 45-49, 13189 Berlin, Germany, sfeistel@afmg.eu), Ralph Bauer-Diefenbach (Ahnert Feistel Media Group, Arkonastr. 45-49, 13189 Berlin, Germany, rbauer@adaacousticdesign.de)

In a concert hall a direct comparison of several line arrays for a new sound system has been made. At different locations the binaural impulse response has been measured and used for auralisation. In a computer model of the hall the measured line array was implemented. The single array sources have been modeled in different modes like far-field cluster, simple module array or as a highresolution loudspeaker array. By means of the new developed SpeakerLab Module these source simulators have been created and afterwards used to calculate binaural impulse responses at the corresponding seats equal to the measurements. After that an auralisation routine has been used. The results are compared for the different degrees of source resolution and with the measurements. Beside acoustic measures the subjective sound quality of the different auralisation results is reported.

\section{4:00-4:20 Break}

\section{Contributed Paper}

\section{$4: 20$}

5pPPb7. Investigation on the restitution system influence over perceived Higher Order Ambisonics sound field: a subjective evaluation involving from first to fourth order systems. Stephanie Bertet (IRCAM, 1 Place Igor Stravinsky, 75004 Paris, France, Stephanie.Bertet@ircam.fr), Jérôme Daniel (France Telecom R\&D, 2 avenue Pierre Marzin, 22300 Lannion, France, jerome.daniel@orange-ftgroup.com), Etienne Parizet (Laboratoire Vibrations Acoustique, Insa Lyon, 25 bis, av. J. Capelle, 69621 Villeurbanne Cedex, France, etienne.parizet@insa-lyon.fr), Olivier Warusfel (IRCAM, 1 Place Igor Stravinsky, 75004 Paris, France, Olivier.Warusfel@ircam.fr)

Among the spatial audio reproduction techniques over loudspeakers, the Higher Order Ambisonics (HOA) approach is based on a sound field spherical harmonics decomposition. By truncating the decomposition to the Mth order, it remains a finite number of components that form the spatial HOA format. The more components are used to encode the sound field, the finer the spatial resolution is. Similarly, the size of the area where the sound field is accurately recreated is proportional to the order. For an Mth encoding order, $\mathrm{N}=2 \mathrm{M}+2$ equally distributed loudspeakers are recommended for a homogeneous reproduction in the horizontal plane. Adding loudspeakers does not change the spatial resolution. However, what is the influence of the restitution system on the perceived sound field? An experiment was designed in order to compare four systems (from first to fourth order) and a reference one, using similarity ratings obtained from pairwise comparisons. Two sound scenes were used, simulating an audio conference and a scene in a kitchen at home. 25 listeners participated to the experiment. The results were analysed using the Indscal method. The perceptual space appeared to be a two dimensional one, highlighting the influence of the order and the number of loudspeakers on the reproduced scenes.

\section{Invited Papers}

5pPPb8. Recording of anechoic symphony music. Tapio Lokki (Helsinki University of Technology, P.O. Box 5400, 02015 TKK, Finland, Tapio.Lokki@tkk.fi), Jukka Pätynen (Helsinki University of Technology, P.O. Box 5400, 02015 TKK, Finland, jpatynen@tml.hut.fi), Ville Pulkki (Helsinki University of Technology, P.O. Box 5400, 02015 TKK, Finland, Ville.Pulkki@tkk.fi)

When designing the acoustics of a concert hall, it would be beneficial to be able to use real recording of a symphony orchestra in auralization. The technical constraints for such recordings are high. First, the instruments have to be recorded separately, as in simultaneous recording the cross talk between microphones could not be avoided. Second, the recording room should be anechoic. Third, the instruments have different sound radiation patterns, thus they should be recorded with multiple microphones around them. Therefore, we end up recording each instrument individually in an anechoic chamber with multiple microphones. The remaining problem is to achieve a common timing as an ensemble between the individually recorded instruments. This was solved by first recording a video of a conductor conducting a pianist playing the whole score. The players in an anechoic chamber then followed the conductor in a monitor while 
listening the pianist on headphones. Four short passages, from two to four minutes, from different music styles were recorded. The recordings were made with 20 low-self-noise microphones, mounted on the shape of a dodecahedron. Finally, we discuss the musical and technical quality of recorded sound, and the response by the musicians, who were professional orchestra players.

\section{5:00}

5pPPb9. Uni-Verse Acoustic Simulation System: interactive real-time room acoustic simulation is dynamic 3D environments. Peter Lundén (Interactive Institute, Box 1197, SE-164 26 Kista, Sweden, plu@tii.se)

Uni-Verse Acoustic Simulation System (UVAS) is a newly developed interactive room acoustic simulation system that can handle dynamically changing 3D geometric models in real-time. The system can share such models with other application, such as visual renderers or 3D modeling tools, over a network using the Verse protocol. UVAS is implemented using the beam-tracing method. It is build as two separate but highly integrated parts. The first part is handling the geometry, it's responsibility is to find audible sound sources and relevant reflection paths in the simulated environment. The second part is handling the audio rendering, producing the audible result of the simulation based on information given by the first part. This paper will focusing on the first part

\section{$5: 20$}

5pPPb10. Case study of measurements and computer modeling auralization results for medium-sized multipurpose halls. Hari V. Savitala (Charles M Salter Associates, Inc., 130 Sutter St, Suite 500, San Francisco, CA 94104, USA, hari.savitala@cmsalter.com), Jason Duty (Charles M Salter Associates, Inc., 130 Sutter St, Suite 500, San Francisco, CA 94104, USA, jason.duty@cmsalter.com), Christopher Peltier (Charles M Salter Associates, Inc., 130 Sutter St, Suite 500, San Francisco, CA 94104, USA, christopher.peltier@cmsalter.com)

This case study focuses on auralizations and their accuracy in modeling medium-sized multipurpose halls (400-600 seats). Within each hall, impulse response measurements were taken with drapes deployed and retracted. The same configurations were modeled in the room acoustics program ODEON. Acoustical parameters, such as T20, T30, C50, C80, were used to check the agreement of the model to the measurements. A systematic approach was then used to adjust model parameters to match the real-world measurements. The modified and unmodified auralizations were then used to determine if any differences could be perceived in an informal listening evaluation. The auralizations and listening evaluation results are presented to better understand how to accurately auralize medium-sized multipurpose halls.

\section{Contributed Papers}

\section{$5: 40$}

5pPPb11. Real-time 3D audio for digital cinema. Pau Arumi (Universitat Pompeu Fabra - Fundació Barcelona Media, Ocata, 1, 08003 Barcelona, Spain, parumi@iua.upf.edu), David Garcia (Fundacio Barcelona Media, Carrer Ocata $\mathrm{n}^{\circ}$ 1, 08003 Barcelona, Spain, dgarcia@iua.upf.edu), Toni Mateos (Universitat Pompeu Fabra - Fundació Barcelona Media, Ocata, 1, 08003 Barcelona, Spain, toni.mateos@barcelonamedia.org), Adan Garriga (Universitat Pompeu Fabra - Fundació Barcelona Media, Ocata, 1, 08003 Barcelona, Spain, adan.garriga@barcelonamedia.org), Jaume Durany (Universitat Pompeu Fabra - Fundació Barcelona Media, Ocata, 1, 08003 Barcelona, Spain, jaume.durany@upf.edu)

We present a real-time 3D audio system with a number of nice features: it is suited for plausible reference with the visual environment, it is real-time capable, it can process multiple moving sound sources and listeners in a normal CPU. In our approach, a database of pressure and velocities impulseresponses (IRs) is computed offline for each (architectural) environment using physically based ray-tracing techniques. During playback, the real-time system retrieves IRs corresponding to the sources and target positions, performs a low-latency partitioned convolution and smoothes IR transitions with cross-fades. Finally, the system is flexible enough to decode to any surround exhibition setup. The software has been developed within the CLAM open-source audio framework. We present a real scenario where these techniques were successfully applied: an augmented-reality film with 3D audio within the context of the IP-RACINE project for digital cinema. The shooting was done with a high-end prototype camera with zoom and position tracking which enabled the real-time motion of a subjective listener within the scene. Our technology enabled the film director to both pre-hear surround audio of an augmented-reality scene shooting and fine-tune audio rendering in post-production.

\section{6:00}

5pPPb12. Near-field binaural synthesis, experimental progress report. Dylan Menzies-Gow (De Montfort University, Queens Building, LE1 9BH Leicester, UK, dylan@dmu.ac.uk)

A methodology was previously presented for displaying high quality binaural images of near-field complex sources, using wave reconstruction. Multipole representations of objects are transformed to Fourier-Bessel and plane wave expansions at the listener, before conversion to binaural signals. One advantage of this approach is that does not require special HRTF information other than the planewave HRTFs, and can fully render the complex field of a near object. As a first step towards a full working system, a realtime implementation is described here for displaying a monopole source using a six degrees-of-freedom infrared head tracking device. 


\title{
Session 5pSC
}

\section{Speech Communication: Multimodal Speech Technology}

\author{
Gerasimos Potamianos, Cochair \\ IBM T. J. Watson Research Center, RTE 134, Yorktown Heights, NY 10598, USA \\ Gerard Bailly, Cochair \\ GIPSA-lab. Dept Speech \& Cognition, INPG, 46, av. Félix Viallet, Grenoble, 38031, France
}

\section{Contributed Paper}

\begin{abstract}
2:00
5pSC1. An ultrasound-based silent speech interface. Thomas Hueber (ESPCI - Telecom Paris, 10 rue Vauquelin, 75005 Paris, France, hueber@ieee.org), Gerard Chollet (Telecom Paris Tech, 46 rue Barrault, 75013 Paris, France, chollet@enst.fr), Bruce Denby (Université Paris VI, ESPCI - Laboratoire d'Electronique, 10 rue Vauquelin, 75005 Paris, France, denby@ieee.org), Gerard Dreyfus (Université Paris VI, ESPCI - Laboratoire d'Electronique, 10 rue Vauquelin, 75005 Paris, France, gerard.dreyfus@espci.fr), Maureen Stone (Vocal Tract Visualization Lab, Depts of Biomedical Sciences and Orthodontics, University of Maryland Dental School, 650 W. Baltimore St., Baltimore, MD 21201, USA, mstone @umaryland.edu)
\end{abstract}

The paper proposes the use of ultrasound scans of tongue movement and video sequences of the lips to synthesize speech. A speech synthesizer driven only by video acquisitions may be qualified as a "silent speech inter- face," which could be used by laryngectomyzed patient as an alternative to tracheo-esophageal speech, for voice communication where silence must be maintained, or in very noisy environments. Our system is based on the building of a one-hour audiovisual corpus of phonetic units, which associates visual features extracted from video with acoustic observations. The ultrasound and optical images are interpreted as a linear combination of standard configurations obtained by principal components analysis (PCA) from a phonetically balanced subset of typical frames. HMM-based stochastic models trained on these visual features sequences are subsequently used to predict phonetic targets from video-only data. Finally, a Viterbi unit selection algorithm is used to find the optimal sequence of acoustic units given both this phonetic prediction and the sequence of visual features. The system is able to perform phonetic transcription from video-only speech data with over 55\% correct recognition, on continuous speech, using neither phonotactic nor linguistic constraints.

\section{Invited Papers}

5pSC2. Multimodal control of talking heads. Gerard Bailly (GIPSA-lab. Dept Speech \& Cognition, INPG, 46, av. Félix Viallet, 38031 Grenoble, France, gerard.bailly@gipsa-lab.inpg.fr), Oxana Govokhina (GIPSA-lab. Dept Speech \& Cognition, INPG, 46, av. Félix Viallet, 38031 Grenoble, France, oxana.govokhina@gipsa-lab.inpg.fr), Gaspard Breton (Orange R\&D, 4 rue du Clos Courtel, 35512 Cesson-Sévigné, France, gaspard.breton@ orange-ftgroup.com)

Multimodal speech synthesis has been devoted for years to the rendering of linguistic or paralinguistic content - i.e., parametrized but discrete information - by continuous audible and visible consequences of speech articulation, eventually complemented by facial expressions, gaze and other body gestures including head, hand, and arm movements. Articulatory synthesizers (producing sounds from gestures) intrinsically compute coherent audiovisual signals but do not presently compete with data-driven techniques: most talking heads are nowadays controlled by models built using human audiovisual data. These control models should replicate the laws governing the coherence of observed multimodal signals and the correct phasing relations between salient events of the multimodal stream. We will report on two comparative evaluations of various lip-sync models (dealing with post-synchronization between speech sounds and articulatory movements) and present a trainable control model that learns automatically phasing relations between acoustic and gestural events. This model can be further extended to capture the fine temporal structure of multimodal scores and a first application to the synchronization between speech and head, face and hand movements during cued speech production will be presented.

\section{2:40}

5pSC3. Statistical conversion of speech parameter trajectory for mapping between features of different modalities. Tomoki Toda (Nara Institute of Science and Technology, 8916-5 Takayama-cho, Ikoma, 630-0192 Nara, Japan, tomoki@ is.naist.jp)

A state-of-the-art speech parameter conversion technique and its application to a mapping between features of different modalities are reviewed. Many statistical approaches to the parameter conversion have been studied particularly for voice conversion in speech synthesis research. A typical method conducts the parameter conversion frame by frame based on the minimum mean square error using a Gaussian mixture model of the joint probability density of input and output parameters [Y. Stylianou et al., IEEE Trans. SAP, 6(2), 131-142 (1998)]. Although this method is reasonably effective, the deterioration of the conversion accuracy is caused by essential problems of the frame-based conversion process. Recently a conversion method based on the maximum likelihood estimation of a parameter trajectory has been proposed [T. Toda et al., IEEE Trans. ASLP, 15(8), 2222-2235 (2007)]. This method realizes the appropriate converted parameter sequence by (1) using not only static but also dynamic feature statistics and (2) considering a global variance feature 
of the converted parameters. It has been reported that this method is effective in several applications such as a spectral determination from articulatory movements, an acoustic-to-articulatory inversion mapping, and a conversion of body-transmitted speech into airtransmitted speech.

\section{Contributed Paper}

\section{3:00}

5pSC4. A comparison of visual features for audiovisual automatic speech recognition. Nasir Ahmad (Loughborough Univ, LE11 3TU Leicestershire, UK, n.ahmad@lboro.ac.uk), Sekharjit Datta (Loughborough Univ, LE11 3TU Leicestershire, UK, s.datta@lboro.ac.uk), David Mulvaney (Loughborough Univ, LE11 3TU Leicestershire, UK, d.j.mulvaney@lboro.ac.uk), Omar Farooq (Loughborough Univ, LE11 3TU Leicestershire, UK, o.farooq@lboro.ac.uk)

The use of visual information from speaker's mouth region have shown to improve the performance of automatic speech recognition (ASR) systems. This is particularly important in presence of noise which even in moderate form severely degrades the speech recognition performance of systems us- ing only audio information. Various sets of features extracted from speaker's mouth region have been used to improve upon the performance of an ASR system in such challenging conditions and have met many successes. To the best of authors knowledge, the effect of using these techniques on recognition performance on the basis of phonemes have not been investigated yet. This paper presents a comparison of phoneme recognition performance using visual features extracted from mouth region-of-interest using discrete cosine transform (DCT) and discrete wavelet transform (DWT). New DCT and DWT features have also been extracted and compared with the previously used one. These features were used along with audio features based on Mel frequency cepstral coefficients (MFCC). This work will help in selecting suitable features for different application and identify the limitations of these methods in recognition of individual phonemes.

\section{3:20-3:40 Break}

\section{Invited Paper}

\section{3:40}

5pSC5. Spatial rendering of audiovisual synthetic speech use for immersive environments. Markus Noisternig (LIMSI-CNRS, B.P. 133, 91403 Orsay, France, markus.noisternig@limsi.fr), Brian F. Katz (LIMSI-CNRS, B.P. 133, 91403 Orsay, France, brian.katz@limsi.fr), Christophe D’Alessandro (LIMSI-CNRS, B.P. 133, 91403 Orsay, France, cda@limsi.fr)

Synthetic speech is usually delivered as a mono audio signal. In this project, audiovisual speech synthesis is attributed to a virtual agent moving in a virtual three-dimensional scene. More realistic acoustic rendering is achieved by taking into account the position of the agent in the scene, the acoustics of the room depicted in the scene, and the orientation of the virtual character's head relative. 3D phoneme dependant radiation patterns have been measured for two speakers and a singer. These data are integrated into a Text-ToSpeech system using a phoneme to directivity pattern transcription module which also includes a phoneme to viseme model for the agent. In addition to the effects related to agent's head orientation for the direct sound, a room acoustics model allows for realistic rendering of the room effect as well as the apparent distance as depicted in the virtual scene. Real-time synthesis is implemented in a $3 \mathrm{D}$ audio rendering system.

\section{4:00}

5pSC6. Audiovisual automatic speech recognition: Progress and challenges. Gerasimos Potamianos (IBM T. J. Watson Research Center, RTE 134, Yorktown Heights, NY 10598, USA, gpotam@us.ibm.com)

The paper overviews recent progress and challenges in a number of audiovisual speech processing technologies with main emphasis on the problem of automatic speech recognition. It is well known that visual channel information can improve automatic speech processing for human-computer interaction. To automatically process and incorporate such information into automatic systems, a number of steps are required that are surprisingly similar accross speech technologies. Crucial above all is the issue of feature representation of visual speech and its robust extraction. In addition, appropriate integration of the audio and visual representations is required, in order to ensure improved performance of the bimodal systems over audio-only baselines. These topics are discussed in detail in the talk, with main emphasis on their application to the speech recognition problem in the challenging environments of automobiles and smart rooms.

\section{Contributed Paper}

\section{4:20}

5pSC7. Analysis and synthesis of nonverbal facial motion. Jonas Beskow (KTH Speech, Music and Hearing, Lindstedtsvägen 24, 10044 Stockholm, Sweden, beskow@kth.se), Björn Granström (KTH Speech, Music and Hearing, Lindstedtsvägen 24, 10044 Stockholm, Sweden, bjorn @ speech.kth.se)

Until recently, most efforts in audio-visual speech synthesis have been concerned with verbal content. However, in human-human communication it is obvious that nonverbal signals plays an important role, such as when expressing emotions and attitudes. Interaction is also often regulated using facial cues, for example gaze, head and eyebrow movements. Some of these cues have a direct coupling to the speech signal, while other occur during both while speaking and listening. When applying interactive talking agents in man-machine systems, nonverbal signals may be very important in easing the flow of communication. In a series of experiments we have been exploring the function of nonverbal facial motion. These studies include an experiment on the interaction between expressive speech and prominence, as well as an attempt to synthesize emotions and attitudes in a talking head, using 3D motion capture data. Further we will report on a real-time experiment with human-human avatar-mediated conversation, where the subjects' turntaking behavior is affected by facial motion in the avatars. 


\title{
Session 5pUWa
}

\section{Underwater Acoustics and ECUA: Sound Propagation in 3D Environments II}

\author{
David C. Calvo, Cochair \\ U.S. Naval Res. Lab., Acoust. Div., Code 7142, 4555 Overlook Ave. SW, Washington, DC 20375, USA \\ Michael Taroudakis, Cochair \\ University of Crete \& FORTH/IACM, Vassilika Vouton, P.O. Box 1385, Heraklion, 711 10, Greece
}

Invited Paper

$1: 40$

5pUWa1. Stability of wavefronts at sound propagation in highly structured three-dimensional environments. Oleg A. Godin (NOAA/ESRL, 325 Broadway, Mail Code R/PSD99, Boulder, CO 80305-3328, USA, Oleg.Godin@noaa.gov)

\begin{abstract}
Extensive numerical modeling of long-range propagation of sound and seismic waves as well as observations of underwater acoustic fields with line arrays reveal that wavefronts are often much more stable and predictable than the rays comprising these wavefronts. This paper considers multiple scattering of sound by environmental inhomogeneities with spatial scales small compared to the propagation range but large compared to the wavelength. These inhomogeneities include 3D variations in sound speed and current velocity that are small compared to the average sound speed, can be either random or deterministic, and are superimposed on an arbitrary slowly-varying background. A theoretical explanation of wavefront stability in highly-structured environments is achieved by demonstrating that end points of rays launched from a point source and having a given eikonal (phase) are scattered primarily along the wavefront corresponding to the same eikonal in the unperturbed environment. The ratio of displacements of the ray end points along and across the unperturbed wavefront is proportional to the number of uncorrelated scattering events. The results apply to conventional rays and to horizontal rays describing propagation of adiabatic normal modes in almost-layered media. The origin of relative stability of wavefronts compared to rays is traced back to Fermat's principle.
\end{abstract}

\section{Contributed Paper}

\section{2:00}

5pUWa2. Range and cross-range propagation effects in a liquid wedge overlaying an elastic bottom. Piotr Borejko (Vienna University of Technology, Karlsplatz 13/E206/3, A-1040 Vienna, Austria, pb@allmech.tuwien .ac.at)

The penetrable-wedge model, a constant density isospeed layer of fluid with a pressure-release horizontal surface and a sloping elastic bottom, provides an extensive insight into the role of the ocean bottom in acoustic propagation from an underwater source. In particular, it is a realistic model of a rock-bottom ocean near a shoreline that accounts for horizontal refraction and allows for a ground wave. This paper discusses some new results for small and large range propagation for two penetrable-wedge models: one where the shear wave speed in the bottom is lower than the sound speed in the fluid and the other where the shear wave speed is higher. The operational representation of the ray-integral solution for the acoustic field from a point source in a penetrable wedge of fluid is further developed to the stage at which one can compute the exact, other than the omission of diffraction at the wedge apex, pressure response curve, as recorded at a receiver, due to an arbitrary time variation of the pressure at the source in a $3^{\circ}$ wedge. The three-dimensional propagation effects are examined for range transmission when the receivers are located up-slope and down-slope of the source, and for cross-range transmission when the receivers are located cross-slope of the source.

\section{Invited Papers}

5pUWa3. Depth-dependent resonant target strength analysis of a dense Atlantic Herring school from wide-area OAWRS and localized 3D morphology sensing. Daniel Cocuzzo (Northeastern University, 302 Stearns Center, Rm 311, 360 Huntington Ave, Boston, MA 02115, USA, dcocuzzo@ece.neu.edu), Zheng Gong (Northeastern University, 302 Stearns Center, Rm 311, 360 Huntington Ave, Boston, MA 02115, USA, zgong@ece.neu.edu), Mark Andrews (Northeastern University, 302 Stearns Center, Rm 311, 360 Huntington Ave, Boston, MA 02115, USA, Andrews.mar@neu.edu), Ioannis Bertsatos (Massachusetts Institute of Technology, Room 5-435, 77 Massachusetts Avenue, Cambridge, MA 02139, USA, ibertsat@mit.edu), Tianrun Chen (Massachusetts Institute of Technology, Room 5-212, 77 Massachusetts Avenue, Cambridge, MA 02139, USA, trchen@mit.edu), Hector Pena (Institute of Marine Research, PO Box 1870, 5817 Bergen, Norway, hector.pena@imr.no), Thomas C. Weber (University of New Hampshire, Ctr. for Coastal and Ocean Mapping, 24 Colovos Road, Durham, NH 03824, USA, weber@ccom.unh.edu), Nicholas Makris (Massachusetts Institute of Technology, Room 5-212, 77 Massachusetts Avenue, Cambridge, MA 02139, USA, makris@mit.edu), Purnima Ratilal (Northeastern University, 302 Stearns Center, Rm 311, 360 Huntington Ave, Boston, MA 02115, USA, purnima@ece.neu.edu)

The depth-dependent target strength of Atlantic Herring is estimated at several distinct bandwidths close to their resonance frequency for a localized, highly dense school observed during the NOPP-sponsored Gulf of Maine Experiment on September 22, 2006. An ocean acoustics waveguide remote sensing (OAWRS) system was deployed near George's Bank to investigate the migration and spawning behavior of fish over wide areas. In conjunction with OAWRS, a Simrad EK60 conventional fish-finding echosounder (CFFS) 
and a Reson Seabat 7125 multibeam sonar system were deployed to provide local depth extent and 3D volume morphology of the dense herring school. The calibration of low-frequency target strength derived from OAWRS data using localized CFFS density and multibeam 3D volume estimates as inputs is discussed. The correlation between the mean depth of the vertically migrating herring school and its resonance frequency is investigated. The results are compared with a theoretical model for 3D resonance scattering from fish swimbladder modeled as a spheroidal bubble. This analysis may allow inference of fish depth and species classification based on the scattered frequency response of targets imaged by OAWRS. Implications for classifying general localized targets, biological or man-made, are discussed further.

\section{2:40}

5pUWa4. Multiple forward scattering through an ocean waveguide with 3D random inhomogeneities. Nicholas Makris (Massachusetts Institute of Technology, Room 5-212, 77 Massachusetts Avenue, Cambridge, MA 02139, USA, makris@mit.edu), Purnima Ratilal (Northeastern University, 302 Stearns Center, Rm 311, 360 Huntington Ave, Boston, MA 02115, USA, purnima@ece.neu.edu), Tianrun Chen (Massachusetts Institute of Technology, Room 5-212, 77 Massachusetts Avenue, Cambridge, MA 02139, USA, trchen@mit.edu)

Analytic expressions have been derived for the mean and spatial covariance of the acoustic field multiply forward scattered though a stratified ocean waveguide containing 3D random surface or volume inhomogeneities [Ratilal and Makris, J. Acoust. Soc. Am. 118, 3532-3559 (2005)]. These expressions are further used to derive the temporal coherence of an acoustic signal propagated through 3D random inhomogeneities. Field moments are given in terms of moments of the scatter function density of the 3D random inhomogeneities, which enables straightforward application to a broad range of 3D scatterers. Here we give examples of the attenuation, dispersion and loss of temporal coherence expected after multiple forward scattering through (1) random internal waves in both continental shelf and deep ocean environments, (2) fish schools, and (3) random wind-generated bubbles in continental shelf and surf-zone area. We show that 3D scattering effects become important when the Fresnel width exceeds the cross-range coherence scale of the inhomogeneities, and can lead to substantial power loss.

\section{Contributed Papers}

\section{3:00}

5pUWa5. Application of the matrix Rytov method to the calculation of the coherence function of a sound field in an oceanic waveguide. Alex G. Voronovich (NOAA/Earth System Research Laboratory, 325 Broadway, Boulder, CO 80305, USA, alexander.voronovich@noaa.gov), Vladimir E. Ostashev (NOAA/Earth System Research Laboratory, 325 Broadway, Boulder, CO 80305, USA, vladimir.ostashev@noaa.gov)

Closed equations for the coherence function of a monochromatic sound field propagating in a statistically inhomogeneous 3D oceanic waveguide have high dimensions and are difficult to solve even with the use of modern computers. Significant reduction of the dimension of the problem was achieved by assuming that sound speed fluctuations are statistically isotropic in a horizontal plane. However, even in this case calculation of the coherence function for a megameter range takes about a day. In this paper, we develop an approximate solution of the closed equations for the coherence function which is similar to a matrix version of the Rytov method. An explicit expression for the coherence function is obtained which contains exponent of an "interaction" matrix. This matrix is determined in terms of the acoustic and internal wave modes and spatial spectrum of the sound speed fluctuations. It is shown that the matrix Rytov method provides an accurate solution for the coherence function which coincides with the solution of the closed equations within a few percent. Calculation of the coherence function now takes only about an hour. This allows us to study in detail the dependence of the coherence function on parameters of the problem.

\section{3:20}

5pUWa6. Measurements of 3D propagation in the shelf environment Kevin D. Heaney (Oasis Inc., 11006 Clara Barton Dr., Fairfax Station, VA 22039, USA, oceansound04@yahoo.com)

In the Fall of 2007 measurements were made to calibrate the acoustic environment on the shallow water shelf off the coast of Florida. The continental shelf is quite flat (less than 1 degree slope) for a region approximately $10 \mathrm{~km}$ wide. Transmissions to a cross-shelf $900 \mathrm{~m}$ horizontal line array were made from a source transiting along the shelf. The signal transmissions in- clude broadband LFM $(20-420 \mathrm{~Hz})$ and a comb of narrow band frequencies spanning the same range. Narrowband beamforming results show the clearly identified source. The arrival angle for the source is as expected until a distance of approximately $30-40 \mathrm{~km}$ when there is an apparent bearing shift in-shore of up to 25 degrees. This behavior is expected in propagation on a shelf (of greater slope) but its behavior is surprising. The phenomenon was observed for several runs at various source and water depths. In order to explain the phenomenon, a hybrid adiabatic normal mode-Parabolic Equation method will be applied to the environment. This model computes the vertical modes and phase speeds at each location and then uses the PE to propagate each mode individually using it's spatially varying phase speed and attenuation. Comparisons of theory and data will be made.

3:40

5pUWa7. Observations of out of plane arrivals for long range low frequency transmission in shallow water. Harry Deferrari (Univ. of Miami, 4600 Rickenbacker Causeway, Miami, FL 33149, USA, hdeferrari @ rsmas.miami.edu)

Two recent experiments have used long horizontal arrays to receive broadband low frequency signals propagated over long ranges in shallow water. Both used $\mathrm{m}$-sequence signals that resolve pulse arrivals in time with each arrival associated with a single acoustic mode of propagation. At moderate propagation ranges, out to $20 \mathrm{~km}$, wave fronts for all modes are observed to be parallel, implying an orderly two-dimensional propagation. At a much longer range, $80 \mathrm{~km}$, a number of separable arrivals are observed but not necessarily with a one-to-one correspondence with modes. The paths appear to be stable and coherent in time implying that they are true Fermat paths, but their wavefront arrival angles differ suggesting the same mode is arriving from several directions, that is, by curved (out of plane paths). The paths could result from wedge effects from gentle slopes perpendicular to the propagation path or possibly from chaotic interaction with random facets of the bottom. In any case, the ultimate limitation for horizontal spatial coherence and array resolution may be the multipath interference of bundles of out of plane arrivals.

4:00-4:20 Break 


\section{Invited Papers}

$4: 20$

5pUWa8. Hydroacoustic blockage prediction and measurement at Diego Garcia using the Adiabatic Mode Parabolic Equation Model. Zachary Upton (BBN Technologies, 1300 N. 17th Street, Suite 400, Arlington, VA 22209, USA, zupton@bbn.com), Michael D. Collins (U.S. Naval Res. Lab., Acoust. Div., Code 7142, 4555 Overlook Ave. SW, Washington, DC 20375, USA, michael.collins@nrl.navy.mil), Jay Pulli (BBN Technologies, 1300 N. 17th Street, Suite 400, Arlington, VA 22209, USA, jpulli@bbn .com)

Underwater explosion monitoring with sparse sensors at long ranges relies on the efficient propagation of acoustic energy in the sound fixing and ranging (SOFAR) channel. When sound traveling in this channel encounters an island or seamount, it will either diffract, scatter, or be converted into seismic energy. Signals observed on the opposite side of these obstructions have been affected by some combination of these processes, and models of global detection and localization depend on knowing these effects. We present a study using the Adiabatic Mode Parabolic Equation (AMPE) model to predict these processes in three dimensions at the Chagos Archipelago. Predictions at 5,10, and $20 \mathrm{~Hz}$ are compared with measurements of approximately $300 \mathrm{~T}$-wave signals from six years of earthquakes on either side of the Chagos Archipelago. These have been recorded at the hydrophone arrays around Diego Garcia. The result of this 360-degree analysis, and the agreement with observed data, demonstrate the utility of the model in understanding the physical effects of these obstructions.

\section{$4: 40$}

5pUWa9. Investigation of 3D benchmark problems in underwater acoustics: a uniform approach. Frederic Sturm (Laboratoire de Mécanique des Fluides et d'Acoustique (UMR CNRS 5509), Ecole Centrale de Lyon, Centre acoustique, 36, avenue Guy de Collongue, 69134 Ecully Cedex, France, frederic.sturm@ec-lyon.fr)

In underwater acoustics, most of the three-dimensional effects on sound wave propagation are usually described by modelers considering one of the following shallow-water benchmark problems: a wedge-shaped waveguide, a canyon, a seamount, and a sinusoidal (corrugated) bottom. These test cases have been thoroughly analyzed individually considering both harmonic point sources (emitting at very low frequencies, for some obvious problems of CPU time and memory limitation) and broadband source pulses (with also very-low central frequencies). In the present work, we report numerical results corresponding to the propagation of broadband pulses in the four above-mentioned test cases. The numerical simulations are performed using a fully 3D parabolic equation based model coupled with a Fourier synthesis technique to handle the time dependence of the source signal. The objective is to propose a uniform representation of the numerical results so as to facilitate the comparison of the 3D effects present in each of the four benchmarks. Snapshots of the propagating pulses at very close successive times are compared with $2 \mathrm{D}$ results. In addition, movies of the propagating pulses are shown for each test case and compared to each other. Movies strongly facilitate the observation and thus the understanding of the 3D effects experienced by all the propagating waves.

\section{Contributed Paper}

\section{5:00}

5pUWa10. Using parallel programming and a three-dimensional visualization cave to map the acoustic energy distribution from a seismic array in the ocean. Natalia Sidorovskaia (Department of Physics, University of Louisiana, UL BOX 44210, Lafayette, LA 70504-4210, USA, nas @ louisiana.edu), Arslan Tashmukhambetov (Department of Physics, University of New Orleans, New Orleans, LA 70148, USA, atashmuk @uno.edu), George E. Ioup (Department of Physics, University of New Orleans, New Orleans, LA 70148, USA, geioup@uno.edu), Juliette W. Ioup (Department of Physics, University of New Orleans, New Orleans, LA 70148, USA, jioup@uno.edu)

Modeling and visualization of the dynamic acoustic field during a seismic exploration survey represent a computational challenge due to broadband, directional nature of the acoustic signal radiated by a seismic array. Standard acoustic propagation models (RAM and SWAMP) are upgraded for parallel processing and tested in the LONI (the Louisiana Optical Network Initiative) environment, using the Louisiana fiber optics grid computing network to model the three-dimensional time-varying acoustic field in the ocean during a seismic exploration survey. The generated volume of data is transferred and visualized in the advanced immersive visualization environment, supported by Louisiana Immersive Technology Enterprise (LITE) facilities. The proposed technology is one of the first steps in developing real-time monitoring of the acoustic energy distribution in a large oceanic volume. This can be beneficial for environmental impact assessment and regulation and for seismic survey design. [Research supported in part by the Joint Industry Project through OGP and by ITI of University of Louisiana at Lafayette.]

\section{Invited Paper}

5pUWa11. Effects of solitons on acoustic energy flow in three dimensions. Kevin B. Smith (Naval Postgraduate School/Naval Undersea Warfare Center, Code PH/Sk, Department of Physics, Monterey, CA 93943, USA, kbsmith@nps.edu), John A. Colosi (Naval Postgraduate School, Code OC/Cj, Department of Oceanography, Monterey, CA 93943, USA, jacolosi@nps.edu)

The impact of a train of nonlinear solitons on the propagation of acoustic energy in shallow water is examined. The soliton perturbations are based on an analytic formulation that produces a train of five soliton waves. Each wave front is parallel and has infinite extent in the horizontal direction. The acoustic field is modeled using a three-dimensional (3D) split-step Fourier parabolic equation (SSF/PE) approach defined in Cartesian coordinates. The standard PE approximation is employed in both depth and cross-range directions. Both pressure and particle velocity fields are computed in a self-consistent manner, allowing a full description of the 3D acoustic intensity field which describes the flow of energy in the presence of the solitons. Individual, low-order modes are extracted from the propagating field so that the impact on specific modes may be examined. The analysis is performed at various frequencies and 
for various source-receiver geometries relative to the soliton train. Emphasis is placed on the focusing and defocusing of acoustic energy between the various soliton waves. The impact of such soliton perturbations on signal variability and bearing resolution at the receiver will be quantified. [Work supported by ONR 321OA.]

\title{
Contributed Papers
}

$$
\text { 5:40 }
$$

5pUWa12. High-frequency underwater acoustic propagation in a port using the three-dimensional method of images. Pierre-Philippe J. Beaujean (Florida Atlantic University, SeaTech Campus, 101 North Beach Road, Dania Beach, FL 33004, USA, pbeaujea@seatech.fau.edu), Matthew D. Staska (International Transducer Corporation, 869 Ward Drive, Santa Barbara, CA 93111, USA, MStaska@channeltech.com)

A computer-efficient model for underwater acoustic propagation in a shallow, three-dimensional rectangular duct closed at one end has been developed using the method of images. The duct simulates a turning basin located in a port, surrounded with concrete walls and filled with sea water. The channel bottom is composed of silt. The modeled impulse response is compared with the impulse response measured between $15 \mathrm{kHz}$ and $33 \mathrm{kHz}$. Despite small sensor-position inaccuracies and an approximated duct geometry, the impulse response can be modeled with a relative echo magnitude error of $1.62 \mathrm{~dB}$ at worst, and a relative echo location error varying between $0 \%$ and $4 \%$ when averaged across multiple measurements and sensor locations. This is a sufficient level of accuracy for the simulation of an acoustic communication system operating in the same frequency band and in shallow waters, as time fluctuations in echo magnitude commonly reach $10 \mathrm{~dB}$ in this type of environment.

\section{6:00}

5pUWa13. Acoustic mode beam effects of nonlinear internal gravity waves in shallow water. Timothy Duda (Woods Hole Oceanographic Institution, 98 Water Street, Bigelow 107, MS-12, Woods Hole, MA 02543, USA, tduda@whoi.edu), Ying-Tsong Lin (Woods Hole Oceanographic Institution, 98 Water Street, Bigelow 107, MS-12, Woods Hole, MA 02543, USA, ytlin@whoi.edu), James F. Lynch (Woods Hole Oceanographic Institution, 98 Water Street, Bigelow 203A, MS-11, Woods Hole, MA 02543, USA, jlynch@whoi.edu)

Ducting of sound between short-wavelength nonlinear internal gravity waves in coastal environments has been demonstrated by substantial evidence. The ducting takes a unique form for each of the acoustic normal modes. Some consequences of this are examined here using threedimensional parabolic equation modeling and theory. For a pair of waves having a broadband $200-\mathrm{Hz}$ source placed between (i.e., in the duct), strong interference patterns within the duct are developed for each mode after a few kilometers. Some of the energy escapes at high angle with respect to the duct direction. Termination of the internal wave duct, an observed feature, results in beams of energy unique to each mode to radiate outward. Specific cases having water depths of order $80 \mathrm{~m}$ and propagation distances of 20 to $30 \mathrm{~km}$ are examined. Situations where one or more modes are completely absent at selected positions are compared with similar events observed in the field.

FRIDAY AFTERNOON, 4 JULY 2008

ROOM 342A, 1:40 TO 6:20 P.M.

\section{Session 5pUWb}

\section{Underwater Acoustics and ECUA: Scattering From Objects Near Boundaries}

\author{
Eric Thorsos, Cochair \\ Applied Physics Laboratory, University of Washington, 1013 NE 40th St, Seattle, WA 98105, USA \\ Mario Zampolli, Cochair \\ NATO Undersea Research Centre, Viale San Bartolomeo 400, La Spezia, 19126, Italy
}

\section{Invited Papers}

\section{$1: 40$}

5pUWb1. Measurement and modeling of targets deployed on and within sand sediments. Kevin L. Williams (Applied Physics Laboratory, University of Washington, 1013 NE 40th St, Seattle, WA 98105, USA, williams@apl.washington.edu), Eric Thorsos (Applied Physics Laboratory, University of Washington, 1013 NE 40th St, Seattle, WA 98105, USA, eit@apl.washington.edu), Steven Kargl (Applied Physics Laboratory, University of Washington, 1013 NE 40th Street, Seattle, WA 98105, USA, kargl@troutmask.apl.washington.edu), Joseph Lopes (Naval Surface Warfare Center - Panama City Division, 110 Vernon Ave, Panama City, FL 32407, USA, joseph.1.lopes@navy.mil), Raymond Lim (Naval Surface Warfare Center - Panama City Division, 110 Vernon Ave, Panama City, FL 32407, USA, raymond.lim@navy.mil), Carrie Dowdy (Naval Surface Warfare Center - Panama City Division, 110 Vernon Ave, Panama City, FL 32407, USA, carrie.dowdy@navy.mil)

Acoustic signatures of elastic targets located near sediment interfaces include effects due to energy interacting with the sediment. Therefore, modeling target response also requires models of scattering from, penetration into and propagation within ocean sediments. We first describe at-sea and test pond measurements carried out on "proud" (target resting on the sediment) and buried targets at frequencies in the range of 2 to $50 \mathrm{kHz}$. The results from some of these measurements are then compared to models incorporating various levels of sophistication relative to both the target and the sediment physics. The modeling hierarchy includes the following: (1) simple sonar equation estimates that treat the target physics via a frequency dependent target strength and use formally averaged results for sediment scattering, (2) realization level modeling that allows calculation of sediment and target scattering for individual pings with 
sufficient fidelity to carry out synthetic aperture processing (for a proud target only its geometrical scattering is considered while the elastic response can be included for a buried target), (3) T-matrix and finite element modeling in which the target elastic response is included but sediment scattering is treated using formal averages and/or flat surface approximations. [Work supported by the Office of Naval Research and the Strategic Environmental Research and Development Program, USA.]

\section{2:00}

5pUWb2. Synthetic aperture sonar imaging of simple finite targets near a sediment-water interface. Steven Kargl (Applied Physics Laboratory, University of Washington, 1013 NE 40th Street, Seattle, WA 98105, USA, kargl@ troutmask.apl.washington.edu) , Kevin L. Williams (Applied Physics Laboratory, University of Washington, 1013 NE 40th St, Seattle, WA 98105, USA, williams@apl.washington.edu), Eric Thorsos (Applied Physics Laboratory, University of Washington, 1013 NE 40th St, Seattle, WA 98105, USA, eit@apl.washington.edu), Darrell R. Jackson (Applied Physics Laboratory, University of Washington, 1013 NE 40th Street, Seattle, WA 98105, USA, drj@apl.washington.edu), Dajun Tang (Applied Physics Laboratory, University of Washington, 1013 NE 40th St, Seattle, WA 98105, USA, djtang@apl.washington.edu)

Synthetic aperture sonar (SAS) is used often to detect targets that are either proud or buried below a sandy sediment interface where the nominal grazing angle of incidence from the SAS to the point above a buried target is below the critical grazing angle. A numerical model for scattering from simple targets in a shallow water environment will be described, and can be used to generate pings suitable for SAS processing. For buried targets, the model includes reverberation from the rough seafloor, penetration through the interface, target scattering, and propagation back to the SAS. The reverberation and penetration components are derived from first order perturbation theory where small-scale roughness and superimposed ripple can be accommodated. For proud targets, the simulations include the scattering from the target where interaction with the seafloor is included through simple acoustic ray models. The interaction of the target with an incident field is based on a free field scattering model. Simulations will be compared to both benchmark problems and measurements over a frequency range of $10-30 \mathrm{kHz}$. These comparisons further support sediment ripple structure as the dominant mechanism for subcritical penetration in this frequency range. [Work supported by the US Office of Naval Research.]

$$
\text { 2:20 }
$$

5pUWb3. Experiments and numerical modeling of low to midfrequency scattering from elastic objects near the sea floor. Mario Zampolli (NATO Undersea Research Centre, Viale San Bartolomeo 400, 19126 La Spezia, Italy, zampolli@nurc.nato.int), Alessandra Tesei (NATO Undersea Research Centre, Viale San Bartolomeo 400, 19126 La Spezia, Italy, tesei@nurc.nato.int), Finn B. Jensen (NATO Undersea Research Centre, Viale San Bartolomeo 400, 19126 La Spezia, Italy, jensen@nurc.nato.int), Gaetano Canepa (NATO Undersea Research Centre, Viale San Bartolomeo 400, 19126 La Spezia, Italy, canepa@nurc.nato.int)

The scattering of low to mid-frequency sound (1-10's of $\mathrm{kHz}$ ) from submerged elastic structures of size $\mathrm{O}(1 \mathrm{~m})$ is a topic of interest to the underwater acoustics community. In the first part of the presentation, a brief description of the relevant components of the EVA experiment is given. The purpose of the sea trial was the acquisition of high-fidelity echoes from submerged spherical and cylindrical targets, made of composite materials with internal layered structure. The second part of the presentation is focused on the finite-element modeling technique developed at NURC for investigating the scattering from axially symmetric submerged elastic objects. Particular attention is dedicated to the computation of the far field at a distance from the target via the Helmholtz-Kirchhoff integral, using the near field sampled on the target surface, together with Green's functions capable of describing a two-layered water-sediment fluid medium. Those geometries, for which the overall axial symmetry is broken by the presence of the water-sediment boundary, can be treated approximately by taking into account the boundary-reflected incident field, as well as the first order interaction between the targetscattered echo and the sea floor. The numerical technique is validated by comparison with data collected during the EVA trial.

$$
\text { 2:40 }
$$

5pUWb4. Modeling bottom penetration for buried target detection. Raymond Lim (Naval Surface Warfare Center - Panama City Division, 110 Vernon Ave, Panama City, FL 32407, USA, raymond.lim@ navy.mil), Gary S. Sammelmann (Naval Surface Warfare Center - Panama City Division, 110 Vernon Ave, Panama City, FL 32407, USA, gary.sammelmann@navy.mil)

Sonar detection of targets buried in underwater sediments has been found to be complicated by surface roughness. In particular, current-induced ripples can diffract energy down into sandy sediments to enhance buried target detection at shallow sonar grazing angles. To validate these effects, models encompassing the dominant propagation mechanisms as well as faithfully representing the target in the environment have been used. This paper describes our efforts to adapt transition matrix and perturbation theory models to provide realistic predictions of buried target response for spherical and cylindrical shapes. Combining these models of scattering and penetration required adopting some approximations to reduce computation time while retaining accuracy. Steps taken to verify and exercise the resulting models reveal some sensitivities that accentuate the need for accurate environmental and setup ground truth for validation of detection mechanisms. [Work supported by the Office of Naval Research and the Strategic Environmental Research and Development Program, USA.]

\section{3:00}

5pUWb5. Scattering by a partially exposed nearly rigid cylinder: Experiments and analysis. Kyungmin Baik (Washington State University, Physics and Astronomy Department, Pullman, WA 99164-2814, USA, nupho27@dreamwiz.com), Philip L. Marston (Washington State University, Physics and Astronomy Department, Pullman, WA 99164-2814, USA, marston@wsu.edu)

The backscattering from a partially exposed circular cylinder was measured for broad side illumination under conditions where the contributions associated with the elastic response of the cylinder were expected to be weak. Grazing illumination was used. Since the objective was to investigate the transition in the number of reflected rays with increasing exposure, it was convenient to partially submerge the cylinder through the free surface of a tank of water. The magnitude of the scattering was measured for ka between 9.6 and 16 where $\mathrm{k}$ is the acoustic wave number and a is the radius of the cylinder. The scattering varied smoothly as a function of the cylinder's 
exposure in agreement with analytical results based on a Kirchhoff approximation [K. Baik and P. L. Marston, IEEE J. Oceanic Eng. (accepted)]. The analysis is easily modified for the case a cylinder breaking through a flat hard interface. For the different types of rays, path length calculations (as a function of the exposure) that are part of that analysis are also relevant to identifying echoes in SAS images of partially exposed cylinders and spheres. [Research supported by ONR.]

5pUWb6. Elastic and interfacial contributions to SAS images of tilted metal cylinders: Laboratory experiments. Jon La Follett (Washington State University, Physics and Astronomy Department, Pullman, WA 99164-2814, USA, LAFOLLEJ@mail.wsu.edu), Kyungmin Baik (Washington State University, Physics and Astronomy Department, Pullman, WA 99164-2814, USA, nupho27@dreamwiz.com), Philip L. Marston (Washington State University, Physics and Astronomy Department, Pullman, WA 99164-2814, USA, marston@wsu.edu)

Laboratory experiments were carried out to explore interfacial and elastic contributions to synthetic aperture sonar (SAS) images of a solid aluminum circular cylinder having flat ends. Some of the elastic responses for free field backscattering as a function of tilt angle could be interpreted using prior ray-based theory of generalized Rayleigh wave contributions [K. Gipson and P.L. Marston, J. Acoust. Soc. Am. 106, 1673-1680 (1999); 107, 112-117 (2000)]. Simplified acoustic holography was also used to interpret aspects of the free field bistatic response. To study the effects of proximity to a flat reflecting surface, the cylinder was hung through the free surface of a water tank and monostatic SAS images were acquired by scanning the transducer location along a horizontal line. This arrangement partially simulates SAS images of cylinders on the ocean bottom at grazing incidence. There were bright contributions to the SAS images of tilted cylinders associated with direct elastic rays as well as with indirect elastic rays due to acoustic reflections from the free surface of the water tank. [Research supported by ONR.]

\section{3:40}

5pUWb7. Elastic scattering by partially-solid-filled spherical shell on the seabed: Model-data comparison and physical understanding. Alessandra Tesei (NATO Undersea Research Centre, Viale San Bartolomeo 400, 19126 La Spezia, Italy, tesei@nurc.nato.int), Mario Zampolli (NATO Undersea Research Centre, Viale San Bartolomeo 400, 19126 La Spezia, Italy, zampolli@nurc.nato.int), Gaetano Canepa (NATO Undersea Research Centre, Viale San Bartolomeo 400, 19126 La Spezia, Italy, canepa@nurc.nato.int)

Low- to mid-frequency elastic scattering measurements were conducted in the range from 5 to $40 \mathrm{ka}$ on a spherical composite shell deployed proud on a sandy seabed. The object consists of a thin-walled shell made of layers of a random-fiber material, and then filled partially with an isotropic solidified epoxy resin and partially with sea water. A scaled version of the object was measured in a water tank under free field conditions. The target responses obtained with and without interaction with the seafloor boundary were compared to simulations achieved by the NURC modeling tool Axiscat. The temporal echoes of the objects were analyzed in terms of elastic waves supported by the structure, on the basis of a ray model. The strongest elastic components come from the interior solid filler. The experimental data of the sphere on the seabed were acquired in October 2006 during the EVA'06 trial off the Island of Elba. The free field data were collected in the NURC water tank.

\section{4:00-4:20 Break}

5pUWb8. Robust recognition and characterization of man-made objects in shallow water using time-frequency analysis. Shaun D. Anderson (Georgia Institute of Technology, Woodruff School of Mechanical Engineering, Graduate Box 1000, Atlanta, GA 30332, USA, sanderson49@gatech.edu), Karim G. Sabra (Georgia Institute of Technology, School of Mechanical Engineering, 771 Ferst Drive, NW, Atlanta, GA 30332-0405, USA, karim.sabra@me.gatech.edu), Manell E. Zakharia (French Naval Acadamey, BP 600, 29240 Brest-Armees, France, manell.zakharia@ecole-navale.fr), Mario Zampolli (NATO Undersea Research Centre, Viale San Bartolomeo 400, 19126 La Spezia, Italy, zampolli@nurc.nato.int), Henrik Schmidt (MIT, 77 Mass Ave, 5-204, Cambridge, MA 02139, USA, henrik@mit.edu), William A. Kuperman (MPL, Scripps Institution of Oceanography, University of California, San Diego, La Jolla, CA 92093-0238, USA, wkuperman@ucsd.edu)

For underwater sonar, time-frequency analysis, in particular Wigner-Ville analysis, has been shown to be a relevant tool for discriminating a man made target (shell) from a natural one of the same shape (solid) and even to estimate some target characteristics (shell thickness, shear velocity...). This processing tool takes advantage of the evolutional, time dependent aspect of the echo spectrum. The estimated time-frequency patterns can be used for detection and wideband classification of sonar echoes in order to reduce false alarms. In particular, the so-called "coincidence pattern" appearing for specific frequency range is a robust time-frequency signature of manmade shells. A time-frequency analysis will be presented to understand echo formation mechanisms using a standard spherical shell model target model. The influence of the medium parameters as well as the source-receiver configuration will be investigated in free space and then extended to the case of a shallow water waveguide. The proposed approach will be tested using target scattering data collected during Experiments for Validation of Acoustic modeling techniques (EVA) sea test on the north shore of Isola D'Elba, Italy. Application to mine-hunting sonar systems will be discussed. 
5pUWb9. Bi-static scattering from buried, elastic objects in shallow water waveguides. Henrik Schmidt (MIT, 77 Mass Ave, 5-204, Cambridge, MA 02139, USA, henrik@ mit.edu), Deep Ghosh (MIT, 77 Mass Ave, 5-204, Cambridge, MA 02139, USA, dghosh @ mit.edu)

The emerging autonomous network technology is enabling new operational paradigms for the concurrent detection, classification and localization of seabed objects by collaborating AUVs. Thus, such networks can exploit the bi-static enhancement of targets which are stealthy to conventional mono-static sonars, and the resonance properties of manmade targets. Under the GOATS and SWAMSI programs MIT in collaboration with NURC have addressed the fundamental issues associated with the development of such a new sonar concept. Through a series of joint experiements, various aspects of the interaction of elastic targets, completely or partially buried in the seabed have been investicated, including the evanescent coupling of low-frequency sound $(1-10 \mathrm{kHz})$ into the seabed, the coupling with structural waves in the targets, and the 3D scattering back into the water column. The analysis is performed using a spectral virtual source scattering model with an embedded spectral Green's function generator which incorporates all multiple scattering effects between the target and the seabed. The target response is represented uniquely by an impedance matrix which may be computed separately using analytical or numerical methods, depending on the target geometry. The scattering model has been combined with the OASES code to provide a comprehensive simulation environment including all the shallow water waveguide physics.[Work Supported by ONR].

\section{Contributed Paper}

\section{5:00}

5pUWb10. Full field modeling of multiaspect scattering from buried objects. Ilkka Karasalo (FOI, Gullfossgatan 6, SE 16490 Stockholm, Sweden, ilkka.karasalo@foi.se)

Results are presented from a computational study of imaging of objects buried in the seabed under shallow water, using a rail-mounted active sonar and synthetic aperture processing. The medium is modeled as range independent, composed of a shallow water layer above a seabed of a muddy sediment containing the objects, and deeper subbottom layers. The objects have simple shape and structure, with diameters in the range $15-195 \mathrm{~cm}$. From selected positions along the rail, the sonar insonifies the objects by
LFM pulses from a directive transmitter, and records the backscattered echoes with a horizontal uniform line array (ULA), or optionally a pair of vertically separated ULAs. The signals from all sonar positions are integrated coherently by synthetic aperture processing for enhanced azimuthal resolution in the images of the objects. The parameters of the models of the medium, the objects and the experimental geometry are chosen to approximate those of a sea trial conducted in the Stockholm archipelago in 2004. The model-predicted scattered field is computed using the XFEM-S code, based on a frequency-domain boundary integral equation (BIE) formulation of scattering from a smooth object in a layered fluid-solid medium. Comparisons of model predictions with experimental results are presented.

\section{Invited Papers}

5pUWb11. Broadband scattering from spherical shells in a waveguide: modeling and classification. John A. Fawcett (DRDC Atlantic, PO Box 1012, Dartmouth, NS B2Y 3Z7, Canada, john.fawcett@drdc-rddc.gc.ca)

In this presentation, the exact expression for scattering from a sphere in a Pekeris waveguide (e.g., Sammelmann and Hackman, J. Acoust. Soc. Am., 82, 1987) is discussed. The importance of the sphere/interface rescattering terms is considered. A computationally faster multipath expansion approach is derived and its accuracy compared with the exact approach. For sufficiently high frequencies and in the case where the rescattering terms can be ignored, the multipath approach yields accurate predictions. The broadband scattering from an elastic-shelled sphere in a Pekeris waveguide is considered as a function of the frequency (or time for a pulse) and the sphere's range and depth in the waveguide. The classification problem is also discussed. The echos (time series or spectra) from a large set of spheres with varying parameters are generated and grouped into six classes corresponding to the various shell thicknesses and materials. Simple classifiers based upon temporal or spectral representations are considered for the spheres in free space and in a waveguide

\section{$5: 40$}

5pUWb12. Backscattering from an elastic target near a water-sediment interface at oblique incidence: First results of tank experiments. Jean-Pierre Sessarego (Laboratory for Mechanics and Acoustics CNRS, 31 chemin Joseph Aiguier, 13009 Marseille, France, sessarego@lma.cnrs-mrs.fr), Anatoliy N. Ivakin (Applied Physics Laboratory, University of Washington, 1013 NE 40th Street, Seattle, WA 98105, USA, ivakin@apl.washington.edu), Régine Guillermin (Laboratory for Mechanics and Acoustics CNRS, 31 chemin Joseph Aiguier, 13009 Marseille, France, guillermin@1ma.cnrs-mrs.fr)

Sound scattering from a target situated near a water-sediment interface was studied in laboratory conditions in order to control separately all the parameters involved in the scattering process. Targets of different sizes were ensonified with wide band transducers covering the frequency range $200 \mathrm{kHz}$ to $1 \mathrm{MHz}$. First, the target scattering strength was measured in the free space conditions, and the scattering strength of the water-sediment interface was measured at oblique incidence. These characteristics were used to provide a rough estimate for the signal-to-noise ratio for the second set of experiments where the target was situated near the interface to study effects of target-boundary interactions. The intensity of the total scattered field was measured as a function of the beamwidth, transducer/object and object/interface distances, frequency, grazing angle, target size and the interface roughness parameters. The interface considered here is a flattened sand surface which was studied earlier [Ivakin and Sessarego, High frequency scattering from flattened sand sediments: effects of granular structure, J. Acoust. Soc. Am., 122, (5) 2007]. The targets were spherical glass beads of different size. Side scan sonar images are presented and possibilities of their qualitative interpretation are discussed. 


\title{
Contributed Paper
}

\section{6:00}

5pUWb13. Estimates of scattering strength for buried cylindrical targets ensonified by evanescent waves. David C. Calvo (U.S. Naval Res. Lab., Acoust. Div., Code 7142, 4555 Overlook Ave. SW, Washington, DC 20375, USA, david.calvo@nrl.navy.mil), Mario Zampolli (NATO Undersea Research Centre, Viale San Bartolomeo 400, 19126 La Spezia, Italy, zampolli@nurc.nato.int), Alessandra Tesei (NATO Undersea Research Centre, Viale San Bartolomeo 400, 19126 La Spezia, Italy, tesei@nurc.nato int)

It is known that low-frequency subcritical sound waves can significantly scatter from targets buried in a seabed due to the significant penetration depth of the incident evanescent wave. Past computational work on scattering by buried spherical shells has been done, for example, using a T-matrix
Method [R. Lim et al., J. Acoust. Soc. Am. 93, 1762-1783 (1993)], a Virtual Source Method [I. Lucifredi and H. Schmidt., J. Acoust. Soc. Am. 120, 3566-3583 (2006)], or finite-element methods [Zampolli et al., J. Acoust. Soc. Am., in press]. In addition to high-fidelity results which are expected from the preceding numerical methods, it desirable to have approximate analytical/asymptotic predictions of multistatic scattering strength for a variety of homogeneous or layered buried targets. Focusing on buried cylinders of infinite or finite-length, we first compute scattering using an approximate method that makes use of separation of variables and neglects multiple scattering between the interface and the target. Results are compared with those generated using the Axiscat/NURC/COMSOL finite-element method. Asymptotic estimates are then presented for scattering strength for objects completely buried in the seafloor for a flat interface (Work sponsored by ONR and NURC.)

FRIDAY AFTERNOON, 4 JULY 2008

ROOM 341, 2:00 TO 6:00 P.M.

\section{Session 5pUWc}

\section{Underwater Acoustics and ECUA: Automatic Target Recognition, Sensors and Algorithms}

\author{
Gerald Dobeck, Cochair \\ Naval Surface Warfare Ctr., Coastal Systems Station, Dahlgren Div., Panama City, FL 32407-7001, USA \\ Marc Pinto, Cochair \\ NATO Undersea Research Centre, Viale San Bartolomeo 400, La Spezia, 19126, Italy \\ Yvan Petillot, Cochair \\ School of Engineering and Physical Sciences, Heriot-Watt University, Edinburgh, EH14 4AS, UK
}

Invited Paper

2:00

\begin{abstract}
5pUWc1. A track-before-detect algorithm for active sonar based on a hidden Markov model. Nigel H. Parsons (Thales Underwater Systems Ltd., Dolphin House, Ashurst Drive, Bird Hall Lane, Cheadle Heath, SK3 0XB Stockport, UK, nigel.parsons@uk .thalesgroup.com)

An active sonar track-before-detect algorithm is described. It is based on a hidden Markov model which uses a Viterbi algorithm to estimate the log-likelihood ratio of the presence or absence of a target in tracks within a state space representing a set of ranges, bearings, range rates and bearing rates, assuming a set of transition probabilities of changes in range rate and bearing rate. A detection is declared if the log-likelihood ratio exceeds a certain threshold and subsequently an HMM tracker, operating on a much smaller state space, is then employed. The performance of this algorithm on simulated data is evaluated. It is shown that, for moving and manoeuvring targets, the detection performance is significantly better than that of a conventional algorithm.
\end{abstract}

\section{Contributed Paper}

2:20

5pUWc2. Forward looking techniques for environment modeling, obstacle detection and characterization. Isabelle Quidu (ENSIETA E3I2 Lab., 2 rue François Verny, 29806 Brest Cedex 9, France, isabelle.quidu@ensieta.fr), Yann Dupas (Groupe d'Etudes Sous-Marines de l'Atlantique (GESMA), BP 42, 29240 Brest Armées, France, yann.dupas @dga.defense.gouv.fr)

Military underwater robots are designed to perform complex underwater missions in both known and unknown environments. To achieve these tasks, an Autonomous Underwater Vehicle (AUV) must be supplied with appropriate sensors to deal with unpredictable events that can put it in danger, and with a high degree of decisional autonomy. In this paper, we have studied the architecture of forward looking sensors to allow the creation of a 3D model of the environment presenting the seabed and the obstacles that are on the path of the AUV. Our approach is based on experimental trials using different and complementary ways (sonars with several configurations) to gather an information as complete as possible. This information will be processed by the vehicle during a survey mission. Practically, we create a reference model of a static environment using a multibeam system which produces bathymetric images at different grazing angles. In the same environment we then use a Forward Looking Sonar intended for the recognition of detected echoes in comparison with the reference model. If an echo cannot be related to a known object on the reference map, it is considered as an obstacle, and the map is updated. 


\section{Invited Paper}

2:40

5pUWc3. Automated change detection with area matching. John Dubberley (Naval Research Laboratory, Bldg. 1005 Rm D-23, Stennis Space Center, MS 39529, USA, john.dubberley@nrlssc.navy.mil), Marlin Gendron (Naval Research Laboratory, Bldg. 1005 Rm D-23, Stennis Space Center, MS 39529, USA, marlin.gendron@nrlssc.navy.mil), Maura Lohrenz (Naval Research Laboratory, Bldg. 1005 Rm D-23, Stennis Space Center, MS 39529, USA, maura.lohrenz@nrlssc.navy.mil)

When resurveying a geographic area of the seafloor during sidescan change detection operations, an automated method to match bottom objects imaged previously with objects imaged in the resurvey can increase efficiency and accuracy. The geographic position of a new object relative to a historical object is a good indicator of a match. However, due to position error within either survey, there may be more than one spatially-close object in the new imagery. To complicate matters further, the reflected energy from the new object may be significantly different given a different incidence angle in the resurvey or the partial burial of the object. In addition, the resurveyed object image may be below the threshold set for automatic recognition and falsely eliminated. This presentation will address these problems and suggest possible methods for matching "constellations" of bottom objects by Dijkstra's minimum cost - maximum flow algorithm, control point matching, and the data-association procedure.

\section{Contributed Paper}

\section{3:00}

5pUWc4. An acoustic barrier based on amplitude variations of the ray paths and double beamforming. Barbara Nicolas (GIPSA-lab, dep. DIS, 961, rue de la Houille Blanche, 38402 St Martin d'Hères, France, barbara.nicolas@gipsa-lab.ingg.fr), Philippe Roux (LGIT - CNRS - Université Joseph Fourier, Maison des Géosciences, 1381 rue de la Piscine, BP 53, 38041 Grenoble, France, philippe.roux@obs.ujf-grenoble.fr), Ion Iturbe (GIPSA-lab, dep. DIS, 961, rue de la Houille Blanche, 38402 St Martin d'Hères, France, ion.iturbe@gipsa-lab.inpg.fr), Jérôme I. Mars (GIPSA-lab, dep. DIS, 961, rue de la Houille Blanche, 38402 St Martin d'Hères, France, jerome.mars@gipsa-lab.inpg.fr)

The objective of this work is to build an acoustic barrier to detect and localize a target between two vertical arrays of sensors. To perform this detection/localisation, we record the signal between each source (of the source array) and each reveiver (of the receiver array). Using these data, we extract the different ray paths between sources and receivers thanks to a new signal processing method: double beamforming. Then, we show that ray paths and their arrival times are not affected by a target in the medium but that ray amplitudes change. As a result, it is possible to use amplitude variation of the rays to find the target localisation. To validate these methods we perform ultrasonic experiments in a tank. These experiments are often used in underwater acoustics as they emulate shallow water waveguides: indeed, by multiplying the frequency by a factor $\mathrm{x}$, distances are divided by the same factor. As acoustic and elastic propagation properties are not affected by this scaling down, it is possible to achieve "oceanic experiments" in a simple tank. Results of double beamforming and target detection are shown.

\section{Invited Papers}

5pUWc5. Advanced ATR techniques based on High-Resolution SAS Sensors. Enrique Coiras (NATO Undersea Research Centre, Viale San Bartolomeo 400, 19126 La Spezia, Italy, coiras@nurc.nato.int), Johannes Groen (NATO Undersea Research Centre, Viale San Bartolomeo 400, 19126 La Spezia, Italy, groen@nurc.nato.int), Benjamin Evans (NATO Undersea Research Centre, Viale San Bartolomeo 400, 19126 La Spezia, Italy, evans@nurc.nato.int), Marc Pinto (NATO Undersea Research Centre, Viale San Bartolomeo 400, 19126 La Spezia, Italy, pinto@nurc.nato.int)

Automatic Target Recognition (ATR) is a key element of expeditionary Mine Countermeasures (MCM) and port protection operations. Most existing approaches to ATR are currently based on high resolution sonar sensors, which provide enough information to obtain satisfactory detection and classification performance for the large World War 2 mine types (e.g., 2 m long cylinder). False alarm rates, however, are still unacceptably high for modern mines, which constrains the way operations are undertaken and often requires either confirmation or re-evaluation by a human operator. The introduction of new AUV-mounted Synthetic Aperture Sonars (SAS) increases the resolution, quality and range of acquired sonar images, which broadens the set of machine vision and computer image analysis techniques that can be used for underwater ATR operations. In this paper we study the impact that the increased quality and resolution have on performance gains and false alarm reduction. A number of classification algorithms are selected to represent the pool of existing approaches to target detection and classification, and their performances are estimated using both simulated and real image data in order to quantify the benefits associated to the new SAS technology. Evolution and near-future plans are discussed, introducing emerging bio-sonar sensors, anomaly detectors and autonomous AUV systems.

\section{3:40}

5pUWc6. Rapid distinction of dumpsite objects using Multiple-Aspect Scattering - Results from scaled tank experiments. Philippe Blondel (University of Bath, Department of Physics, Claverton Down, BA2 7AY Bath, UK, pyspb@bath.ac.uk)

Toxic dumpsites on the seafloor are causing increasing environmental concern, but traditional sonar imaging strains to distinguish objects in unconsolidated sediments, in particular in cluttered terrains. Scaled tank experiments were conducted with four different cylinders (fluid-filled and solid aluminium, air-filled and solid stainless steel, respectively) and two seabed types (silt and gravel), using the facilities at the University of Bath. The setup was a 10:1 scaled version of the EC-SITAR sea trials site in the Stockholm Archipelago (Sweden). The main aim of these experiments was to design efficient surveying strategies, later used at sea. Our studies showed large variations depending on the aspect of these targets and their bistatic imaging configuration. These variations can be directly related to the shapes of the targets (e.g., dimensions, presence of ribs), their content (hollow or solid) and the material of the shells (e.g., 
stainless steel or aluminium). They are quantified using the combined L4 norm of the time-domain signals at each aspect. Using appropriate ranges of multistatic configurations and imaging each target at three distinct aspects $\left(45^{\circ}\right.$ apart), it is possible to successfully distinguish between similar targets with distinct contents and/or material, even in cluttered terrains.

\section{4:00-4:20 Break}

\section{Contributed Papers}

\section{4:20}

5pUWc7. Target detection of man made objects in side scan sonar images - segmentation based false alarm reduction -. Max Neumann (Freie Universität Berlin, Takustr. 9, 14195 Berlin, Germany, papperlapapp@gmail.com), Christian Knauer (Freie Universität Berlin, Takustr. 9, 14195 Berlin, Germany, christian.knauer@inf.fu-berlin .de), Bodo Nolte (Forschungsanstalt der Bundeswehr für Wasserschall und Geophysik, Klausdorfer Weg 2-24, 24148 Kiel, Germany, bodonolte@bwb.org), Dieter Brecht (Forschungsanstalt der Bundeswehr für Wasserschall und Geophysik, Klausdorfer Weg 2-24, 24148 Kiel, Germany, dieterbrecht@bwb.org), Wolfgang Jans (Forschungsanstalt der Bundeswehr für Wasserschall und Geophysik, Klausdorfer Weg 2-24, 24148 Kiel, Germany, wolfgangjans@bwb.org), Alfons Ebert (FGAN-FOM, Gutleuthausstraße 1, 76275 Ettlingen, Germany, a.ebert@fom.fgan.de)

This paper presents a fast and robust algorithm for significantly reducing the number of false detections caused by screening algorithms for side scan sonar (SSS) images. The presented algorithm consists of two processing steps. First, an iterative segmentation process is carried out for seperating the image into shadow and background. This segmentation is based on an energy function which combines the local neighborhood segment information and the amplitude of a pixel. By minimizing this function, a clear shadow, the most significant target characteristic, will be extracted. Second, based on the region of interest (ROI) and the shadow contour, a robust classification approach is applied, utilizing the area of the shadow, the first two statistical moments of the pixel amplitude and the existence of parallel lines (Hough transformation). This algorithm was tested using a data set with approx. 2400 ROIs containing about 200 targets and 270 targetlike stones or sandrippel. This data set was gathered during five different measurement campaigns in the Baltic Sea and the Mediterranean Sea using three different
SSS systems Benthos C3D, Klein2000 and Marine Sonics). These data were collected by FWG and WTD71 as well as by Atlas Electronik with the SeaOtter MK1 AUV.

\section{$4: 40$}

5pUWc8. Sonar target-phase measurement and effects of transducer-matching. Philip Atkins (University of Birmingham, Department of Electronic, Electrical and Computer Engineering, Edgbaston, B15 2TT Birmingham, UK, p.r.atkins@bham.ac.uk), Alan Islas (University of Birmingham, Department of Electronic, Electrical and Computer Engineering, Edgbaston, B15 2TT Birmingham, UK, AXI743@bham.ac .uk), Kenneth G. Foote (Woods Hole Oceanographic Institution, Woods Hole, MA 02543, USA, kfoote@whoi.edu)

Active sonar systems normally detect and classify a target based on the amplitude of the received echo or the induced Doppler shift. However, additional classification information may be available from the phase shift introduced by some targets as a result of the boundary conditions. For example, reverberation from the sea surface and scattering from fish swimbladders introduce an additional phase shift that may not be present in returns from an acoustically stiffer seabed or synthetic target. Algorithms based on the use of subband correlators are presented for measuring the phase shifts introduced by the boundary conditions on stationary and moving targets when insonified by broadband transmissions. These techniques are used to remove the phase shifts introduced by the unknown target. However, the unknown phase characteristics of the transducer, matching circuit, and electronic circuitry of a sonar system imply that target-phase measurements are very difficult to make in any practical system. The effects of adding a Butterworth-derived matching circuit to a Reson TC2130 transducer are presented for the case of sinusoidal frequency-modulated excitation of solid elastic and thin elastic-shelled hollow spheres. It is concluded that target-phase measurements can enhance the classification performance of a suitably calibrated sonar system.

\section{Invited Papers}

\section{5:00}

5pUWc9. Evaluation of portable high-frequency sonars for diver identification. Anna Crawford (Defence R\&D Canada Atlantic, P.O. Box 1012, 9 Grove St, Dartmouth, NS B2Y 3Z7, Canada, anna.crawford@drdc-rddc.gc.ca), Vance Crowe (Defence R\&D Canada Atlantic, P.O. Box 1012, 9 Grove St, Dartmouth, NS B2Y 3Z7, Canada, vance.crowe@drdc-rddc.gc.ca), Thomas Pastore (NATO Undersea Research Centre, Viale San Bartolomeo 400, 19126 La Spezia, Italy, Pastore@ nurc.nato.int), Ronald Kessel (NATO Undersea Research Centre, Viale San Bartolomeo 400, 19126 La Spezia, Italy, Kessel@nurc.nato.int)

Obtaining a positive identification is a critical step in most tiered harbour protection strategies for countering underwater intruders. It is generally recognised that sonar is one of the best tools for underwater imaging, however operating in a harbour environment presents challenges. As part of an on-going harbour protection research project, small easily portable high-frequency sonar systems are being investigated as a means to equip small response craft with intruder identification capability. Several systems are being considered, with the most comprehensive testing by DRDC to date being done on small Canadian-made sonars. Tests were conducted in local harbour waters in Halifax, Canada, and in La Spezia, Italy, through participation in the NATO Undersea Research Centre Response Against Diver Intrusions (RADI) joint trial, conducted in November 2007. A variety of small sonars and manned and unmanned response craft were used during the RADI trial. Evaluation of the performance of these devices for the task of diver identification in realistic conditions will be discussed.

\section{$5: 20$}

5pUWc10. Features for propagation-invariant classification of underwater targets. Patrick Loughlin (University of Pittsburgh, 348 Benedum Engineering Hall, Dept. of Electrical \& Computer Engineering, Pittsburgh, PA 15261, USA, loughlin@engr.pitt.edu), Greg Okopal (University of Pittsburgh, 348 Benedum Engineering Hall, Dept. of Electrical \& Computer Engineering, Pittsburgh, PA 15261, USA, gno1@pitt.edu)

As sound propagates in shallow water, it is subject to frequency-dependent spreading and attenuation (dispersion and damping). In active sonar, these propagation-induced changes can be detrimental to automatic classification because the observed backscatter depends on the propagation environment and how far the wave has traveled. One way to address this problem is to develop propagation-invariant 
features of the wave that can be used in automatic classification. In this talk, we present temporal, spectral, and cepstral moment-like features of a wave that are invariant per mode to dispersion and damping. Classification results on numerical simulations of the backscatter from different steel shells propagating in a Pekeris waveguide with damping and random variations will be presented. [Supported by ONR grants N00014-06-1-0009 and N00014-07-10355]

\title{
Contributed Paper
}

5:40

5pUWc11. MCM sensor requirements: performance measures. Samantha Dugelay (Dstl, Building A32, Winfrith Technology Centre, DT2 8WX Dorchester, UK, sdugelay@dstl.gov.uk)

This paper presents a comparison of two measures of performance suitable to characterise operational performance of mine hunting sonars in various environmental conditions. The first measure is a direct adaptation of information theory and bounds the capability of a sonar to distinguish between objects, i.e., a classification capability. The bounds take into account the amount of distinguishable pixels between objects and the statistical information content of each pixel. Successfully applied in Radar, this approach has also demonstrated the performance of synthetic aperture sonar at NURC using a Rayleigh distribution for statistical pixel distribution. The measure is now being further developed to include high resolution distributions such as K-law which readily appear in high resolution images. The second measure, Johnson's criteria aims to provide guidance on resolution required for operator detection, classification and identification. This measure originally derived from experiments on night vision images is now being modified to simultaneously incorporate target highlight and shadow information in varying environmental conditions. Finally, the predictions of these measures will in the future be compared to each other and to current system performances.

FRIDAY AFTERNOON, 4 JULY 2008

ROOM 342B, 2:20 TO 3:40 P.M.

\section{Session 5pUWd}

\section{Underwater Acoustics and ECUA: Acoustic Data Fusion}

\author{
Eric Maillard, Cochair \\ Reson Inc, Golet, CA, USA \\ Benoit Zerr, Cochair \\ DGA/GESMA, BP42, Brest Armées, 29240, France
}

\section{Contributed Papers}

2:20

5pUWd1. Multisegmentation of sonar images using belief function theory. Mounir Dhibi (ENSIETA E3I2, 2 rue François Verny, 29806 Brest Cedex 9, France, mounir.dhibi@ensieta.fr), Romain Courtis (I2ETA Romain Courtis, GESMA/SDP/GDM, BP 42, 29240 Brest Armées, France, Romain.Courtis@dga.defense.gouv.fr), Arnaud Martin (ENSIETA E3I2, 2 rue François Verny, 29806 Brest Cedex 9, France, arnaud.martin@ensieta .fr), Isabelle Quidu (ENSIETA - E3I2 Lab., 2 rue François Verny, 29806 Brest Cedex 9, France, isabelle.quidu@ensieta.fr)

Today side scan sonar is one of the most efficient sensors for Rapid Environment Assessment missions. Unfortunately, features extracted from a given area are strongly dependent on the relative position of the sensor (e.g., due to the shadow or the gain variation). That could conduct to a bad segmentation of the seabed. However, due to the fact that operational systems give very often multiple views of the same area we use the redundancy. In this work, we propose to fuse multiview segmentations in order to outperform the seabed classification. First we present a way to characterize the seabed using as a start point, a texture analysis in order to extract parameters on images. Then, a classification method allows allocating a class according to the type of sediment for the different standpoints. The proposed classifier fusion is based on the belief function theory. We present results from a set of experiments conducted to evaluate the proposed approach with real sonar images and we discuss them.
2:40

5pUWd2. Elimination of corner-turning in FFT-based sonar array beamforming. Jacob Barhen (Oak Ridge National Laboratory, 1 Bethel Valley Road, Oak Ridge, TN 37831-6015, USA, barhenj@ornl.gov), Travis Humble (Oak Ridge National Laboratory, 1 Bethel Valley Road, Oak Ridge, TN 37831-6015, USA, humblets@ornl.gov), Michael Traweek (Office of Naval Research, 875 North Randolph Street, Arlington, VA 22203, USA, Mike.Traweek@navy.mil)

The expected availability, in the near future, of an ultralow power version of the revolutionary IBM CELL multicore processor opens unprecedented opportunities for implementing sophisticated signal processing algorithms faster and within a much lower energy budget. The concept of "corner turning" has been, for many decades, at the heart of array beamforming via Fourier transforms. As widely reported in the open literature (both for sonars and radars), the computational sequence involving corner turning operations, i.e., the sequence: temporal Fourier transforms --> data cube corner turning --> spatial Fourier transforms, constitutes one of the primary obstacles to achieving high-performance and lower power dissipation (by reducing the number of times memory is accessed). Even with the emergence of novel multicore processors, leading providers (e.g., Mercury Computers) still include explicit corner turning stages in their computational flow architectures for multidimensional array processing. The primary innovation reported in this paper addresses the development of a computational scheme that avoids altogether the corner turning stage. We discuss its implementation on currently available CELL technology (65 nm SOI) and demonstrate close to an order of magnitude speed-up compared to the scheme with corner turning implemented on the same processor. 
5pUWd3. Multiple-sensor fusion approach to seabed classification. Benoit Zerr (DGA/GESMA, BP42, 29240 Brest Armées, France, benoit.zerr@dga.defense.gouv.fr), David Kerneis (ENST Bretagne Dept. ITI, Technopôle Brest-Iroise - CS 83818, 29238 Brest Cedex 3, France, david.kerneis@enst-bretagne.fr), Basel Solaiman (ENST Bretagne Dept. ITI, Technopôle Brest-Iroise - CS 83818, 29238 Brest Cedex 3, France, basel.solaiman@enst-bretagne.fr)

Seabed classification is key issue for civilian and military underwater applications, from offshore exploitation to mine counter measure. Most of the existing automated classification techniques relies on the analysis of the data provided by a single sensor, supposed to unambiguously separate the different classes of seabed. In this paper we present a different approach which considers that, even if a sensor cannot tell the differences between two classes, classification will improve by considering that the seabed belongs to one of these two classes, and, further, that the analysis of the data from another sensor can resolve the ambiguity. For each sensor, the classification is achieved in a conventional way by feature extraction and supervised classification. The fusion of the results implements the theory of evidence through Dempster-Shafer method. After a description of the method, the paper discusses the experimental results from the fusion of information delivered by three sensors: an imaging sidescan sonar, a vertical echo sounder and an interferometric bathymetric sonar. The major part of the experimental data has been acquired by towed or hull mounted sensors. As these sensors are a subset of the payloads operated simultaneously by the new DGA-SHOM DAURADE AUV, preliminary seabed classification results in covert REA missions will also be presented and discussed.
5pUWd4. Acoustic data fusion devoted to underwater vegetation mapping. Claire Noel (Semantic TS, 39 Ch Buge, 83110 Sanary, France, noel@semantic-ts.fr), Christophe Viala (Semantic TS, 39 Ch Buge, 83110 Sanary, France, viala@semantic-ts.fr), Michel Coquet (Semantic TS, 39 Ch Buge, 83110 Sanary, France, coquet@semantic-ts.fr), Benoit Zerr (DGA/GESMA, BP42, 29240 Brest Armées, France, benoit.zerr @ dga.defense.gouv.fr), Thierry Perrot (CEVA, Presqu'île de Pen Lan BP3, 22710 Pleubian, France, thierry.perrot@ceva.fr)

This paper presents research tasks conducted by SEMANTIC TS, in collaboration with GESMA, aimed to develop a mapping method for underwater vegetation lying on seabed. First stage is to develop a method for detecting and characterizing vegetation on the seabed using the acoustic response from a conventional single beam echo sounder. This new method is then operated simultaneously with multibeam sonar producing micro-relief information and side scan sonar providing gray scale levels associated with bottom reflectivity. Then fusion of these three data is processed. We show efficiency of these multisensor data fusion concept to get very precise seabed vegetation mapping in a way reducing truth control (video and diving investigations). Sensors and method accuracy allow obtaining, like in biomedical field, real 3D scan pictures of seabed vegetation. This study is first applied to posidonia and cymodocea, which play a key role in Mediterranean's echosystem. Then, extension of the method is investigated to address laminaria which may significantly affect the performance of acoustic and optical sensors used for sea-mines detection and this paper presents results of data fusion mapping on an Atlantic sea area covered by luminaria, studied and well known by the CEVA.

FRIDAY AFTERNOON, 4 JULY 2008

ROOM 342B, 4:00 TO 6:20 P.M.

\title{
Session 5pUWe
}

\section{Underwater Acoustics and ECUA: Noise Suppression, Robust Direction of Arrival, and Target Strength Estimation}

\author{
Georges Bienvenu, Cochair \\ Thales Naval Division, Thales Underwater Systems, France \\ Ronald A. Wagstaff, Cochair \\ National Center for Physical Acoustics, University of Mississippi, University, MS 38677, USA
}

\section{Contributed Papers}

\begin{abstract}
4:00
5pUWe1. "Venus's-noisetrap" high-gain adaptive processor. Ronald A. Wagstaff (National Center for Physical Acoustics, University of Mississippi, University, MS 38677, USA, rwagstaf@olemiss.edu), Heath E. Rice (National Center for Physical Acoustics, University of Mississippi, University, MS 38677, USA, herice@olemiss.edu)

The Venus's-noisetrap is a high gain adaptive signal processor inspired by Venus's-flytrap, an insect eating plant. The Venus's-noisetrap utilizes single sensor or beamformed spectral data. It "traps" the data samples by modifying the governing equations in a manner that each spectral sample is adaptively "trapped" before averaging. The trapping process is a fluctuationbased temporal coherence determination of whether the time history in each spatial or spectral bin is signal or noise. Signals are set "free", while the noise remains trapped, blocking it from participating in the averaging process. For ocean acoustic data, the processor automatically identifies signals from submerged sources. In addition, the Venus's-noisetrap achieves large signal-to-noise ratio (SNR), high spectral and spatial resolution, and auto-identification of signals. The techniques that cause this processor to
\end{abstract}

mimic the Venus's-flytrap will be discussed, and the method of continuously adapting the governing equations to the unpredictable signal and noise environment will be illustrated. Results will be presented to show the processor's large SNRs and corresponding enhancements in spectral and spatial resolution.

\section{$4: 20$}

5pUWe2. Suppression of oceanic reverberation by subspace methods. Xuan Li (Institute of Acoustics, Chinese Academy of Sciences, 100080 Beijing, China, happyxuanli@hotmail.com), Xiaochuan Ma (Institute of Acoustics, Chinese Academy of Sciences, 100080 Beijing, China, maxc@mail.ioa.ac.cn), Chaohuan Hou (Institute of Acoustics, Chinese Academy of Sciences, 100080 Beijing, China, hch@mail.ioa.ac.cn)

Acoustic signal processing in shallow water environments is a challenging problem because of the presence of reverberation. Based on some models of reverberation, data from sensors array is pretreated to suppress reverberation. Considering reverberation as a sum of echoes of transmitted 
signal, the principal component inverse (PCI) algorithm deletes the largest singulars of data matrix, which is constructed from array data. However, estimating a threshold which is needed in PCI is difficult in practice. In this paper, two new subspace methods, Deleting Big Eigenvalues and Subspace Projection are proposed. The two novel methods, substituting automatic signal-number estimation for threshold estimation, are operated via eigendecomposition. According to a simulation which takes broadband linear modulated frequency signal as transmitted signal, these two methods show a similar performance but smaller computing quantity compared with PCI.

\section{$4: 40$}

5pUWe3. Coherent effects of flow- and pressure-hull of a generic submarine on target scattering in an active sonar performance model. Pieter Schippers (TNO-D\&V-Underwater Technology, Oude Waalsdorperweg 63, Post Box 96864, 2509 JG The Hague, Netherlands, pieter.schippers @ tno.nl)

Since the late eighties the sonar performance model ALMOST for active and passive sonar is under development at TNO. For active detection performance, first a point target was used, with a single target strength value dependent on parameters like aspect angle, based on measurements or other sources. However by now there is a demand for TS of ships and wakes with realistic dimensions and characteristics. A generic sub was modeled with additional software routines, as a pixel file. A newly developed time domain model for hull reflection was implemented, also using scattering pixels, assuming multiple scattering with damping in the metal hull layer. This extension of the pixel modelling is evaluated versus literature data. Some modeling results of target strength computations are shown, for a generic submarine with pressure hull, with aspect angle, frequency and bandwidth as parameters.

\section{5:00}

5pUWe4. Robust capon beamformer for port/starboard discrimination of twin-line array. Zaixiao Gong (National Laboratory of Acoustics, Institute of Acoustics, Chinese Academy of Sciences, NO.21, Bei-Si-huan-Xi Road, 100080 Beijing, China, gzx @mail.ioa.ac.cn)

Compared with the single-line array, the twin-line array have the potential to solve the port/starboard discrimination problem. Conventional method of geometric phase shifting has been used to distinguish the port/starboard of the target. But it can only be used to solve the problem when the frequency bandwidth is limited. The method based on optimum beamforming for hydrophone triplets can hardly be applied with the twin-line arrays. Aimed at the twin-line arrays port/starboard discrimination problem, a method based on the robust capon beamformer is proposed in this paper, which has advantages of concise algorithm and robustness against the aberration of the array shape. And the method works better with wider frequency bandwidth. Simulation and on sea experiment data are analyzed to verify the method.

\section{$5: 20$}

5pUWe5. A numerical method for array sensor noise field calculation in detection performance optimization. Chao Sun (Institute of Acoustic Engineering, Northwestern Polytechnical University, 710072 Xi'an, China, csun@nwpu.edu.cn), Bo Yang (Institute of Acoustic Engineering, Northwestern Polytechnical University, 710072 Xi'an, China, yangbo@mail.nwpu.edu.cn), Yixin Yang (Institute of Acoustic Engineering, Northwestern Polytechnical University, 710072 Xi'an, China, yxyang @ nwpu.edu.cn)

Different from the generally adopted criteria of minimizing the sidelobe level and maximizing the array gain in the weighting design of a sonar array, an approach was proposed recently to optimize the shading weights with the aim of maximizing the deflection coefficient in the square-law detector, which in essence suppresses the self-noise by including the noise informa- tion at array sensors in the optimization procedure. When several noise sources are present and/or an analytical expression of the noise transfer function is not available, the sensor noise needs to be measured in forming the sensor noise response cross correlation matrix required in the optimization which is very demanding when an array with large number of sensors is considered. To avoid the tedious work of noise measurement, a numerical method is developed in this paper. In this method, the main self-noise sources are assigned with different positions and strengths and the noise field at the array sensors is calculated via either the finite element method or the boundary element method. Tank experiment validated the effectiveness of this method. By using this method, the detection performance of a sensor array can be predicted before it is physically built and tested in under practical conditions.

\section{$5: 40$}

5pUWe6. Improving spatial resolution of interferometric bathymetry in multibeam echosounders. Gerard Llort-Pujol (ENST-Bretagne, Dpt ITI, CS 83818, 29238 Brest Cedex 03, France, gerard.llort @enst-bretagne.fr), Christophe Sintes (ENST-Bretagne, Dpt ITI, CS 83818, 29238 Brest Cedex 03, France, christophe.sintes @enst-bretagne.fr), Xavier Lurton (Institut Français de Recherche pour l'Exploitation de la Mer, NSE/AS, BP 70, 29280 Plouzané, France, lurton @ifremer.fr)

Most multibeam echosounders used in seafloor mapping use the interferometry method for bathymetry measurement, based on the zero-crossing of the phase difference between two sub-arrays. In this approach, only one sounding is computed per formed beam, and the spatial resolution is linked to the beam footprint extent. Using the whole content of the phase difference signal vs time makes it possible ideally to get a bathymetry data sampled at the very resolution of the digitised signal. A trade-off is however to be found between this resolution improvement and the increase in the measurement noise. The performances of interferometric bathymetry are presented, together with the constraints of the resolution-accuracy trade-off, and a presentation of a high-resolution algorithm for interferometric bathymetry applicable to multibeam systems. This is illustrated by results obtained on a wreck, showing the remarkable improvement achievable in resolution over a real target. The potentialities for this method are promising, since it allows to process, in the same beam, targets on the seafloor and inside the water column; simplifications in multibeam sounders array structure and processing are also to consider.

\section{6:00}

5pUWe7. Adaptive removal of a known interference and its scattered energy. Yung P. Lee (SAIC, 4001 N. Fairfax Dr, Suite 175, mail stop 1-11-1, Arlington, VA 22203, USA, yung.p.lee@saic.com), William Lee (Duke University, Department of Electrical and Computer Engineering, Durham, NC 27708, USA,wwl3@duke.edu)

In underwater passive processing, the nearby fast moving loud surface ships produce nonstationary interference field and limit most sonar performance. After 9/11, AIS (automatic identify system) is required for ships larger than 60 's, providing ground truth of positions, speeds, and other ancillary information for the nearby surface ships. This study investigates adaptive removal of a known loud signal using the ground truth information and acoustic propagation modeling. In addition, scattered energy away from the loud signal is identified through a delay correlation processing and is also removed. In a segment of MAPEX2k experiment conducted by SACLANT Centre off the west coast of Italy on November 28, 2000, the research vessel ALLIANCE towed an array at $2 \mathrm{~m} / \mathrm{s}$ passing by a moored controlled source. The controlled source transmitted a loud 1-s $150-500 \mathrm{~Hz}$ LFM sequence every $15 \mathrm{~s}$. In this setting, the fixed controlled source generated a nonstationary interference field received on the towed array. With the knowledge of the array tow track and source position the performance of removing the known interference and its scattered energy in a real shallow water environment is examined. 


\title{
Session 5pUWf
}

\section{Underwater Acoustics and ECUA: Sound Generation and Attenuation}

\author{
Theodore G. Jones, Cochair \\ U.S. Naval Research Laboratory, Code 6795, 4555 Overlook Ave. SW, Washington, DC 20375, USA \\ Andrzej Zak, Cochair \\ Polish Naval Academy, Smidowicza 69, Gdynia, 81-103, Poland
}

\section{Contributed Papers}

\begin{abstract}
4:40
5pUWf1. Neural classification of ships hydroacoustic signatures Andrzej Zak (Polish Naval Academy, Smidowicza 69, 81-103 Gdynia, Poland, a.zak@amw.gdynia.pl)

The paper presents method of classification of hydroacoustic signatures generated by moving ship. Classification is a procedure in which individual items are placed into groups based on quantitative information on one or more characteristics inherent in the items. In this paper the hydroacoustics signals classification is understood as the process of automatically recognition what kind of object is generating acoustics signals on the basis of individual information included in generated sounds. Hydroacoustics signal classification is a difficult task and it is still an active research area. Automatic signal classification works based on the premise that sounds emitted by object to the environment are unique for that object. However this task has been challenged by the highly variant of input signals. The paper includes discussion about unique of sound generated by moving ships. To solve problem of hydroacoustic signatures classification the Kohonen networks which belongs to group of self organizing networks where chosen. Hydroacoustic signals were acquired on the Polish Navy Range during the complex ship measurement. At the end the results of classification of underwater noises made by ship were presented.
\end{abstract}

\section{5:00}

5pUWf2. Underwater acoustic generation with narrow and broadband lasers. Theodore G. Jones (U.S. Naval Research Laboratory, Code 6795, 4555 Overlook Ave. SW, Washington, DC 20375, USA, ted.jones@nrl.navy.mil), Melissa K. Hornstein (U.S. Naval Research Laboratory, Code 6795, 4555 Overlook Ave. SW, Washington, DC 20375, USA, melissa.hornstein@nrl.navy.mil), Antonio C. Ting (U.S. Naval Research Laboratory, Code 6795, 4555 Overlook Ave. SW, Washington, DC 20375, USA, antonio.ting@nrl.navy.mil), Zachary W. Wilkes (Research Support Instruments, Inc., 4325 Forbes Blvd, Ste B, Lanham, MD 20706, USA, zachary.wilkes@nrl.navy.mil), Dennis A. Lindwall (U.S. Naval Research Laboratory-Stennis, Marine Geosciences Division, Code 7432, Stennis Space Center, Stennis, MS 39529, USA, lindwall@nrlssc.navy.mil)

Underwater laser acoustic sources, generated by intense pulsed lasers on above-water and underwater platforms, are under investigation. In a novel configuration, a tailored intense broadband laser pulse can be designed to propagate many meters underwater and compress at a predetermined remote location. Controlled compression of these optical pulses is governed by a combination of optical group velocity dispersion (GVD) and nonlinear Kerr self-focusing, resulting in photoionization, localized heating, and shock generation. Recent and ongoing experiments include near-field acoustic source characterization using lens-focused pulses of a broadband $400 \mathrm{~nm}$ Ti: sapphire laser, as well as $1064 \mathrm{~nm}$ and $532 \mathrm{~nm}$ narrowband YAG laser pulses. Also, the nonlinear optical Kerr index of water at $400 \mathrm{~nm}$ was precisely measured. Acoustic source characterization includes measurements of photoacoustic energy conversion efficiency, acoustic power spectrum, and directivity. Experimental results will be presented, and laser sources and techniques for underwater acoustic generation will be compared. [This work is supported by the U.S. Office of Naval Research.]

\section{$5: 20$}

5pUWf3. Recent progress on the theoretical modeling of underwater acoustics induced by sonic booms. Johnson C. Wang (The Aerospace Corporation, System Planning and Engineering, El Segundo, CA 902454691, USA, Johnson.C.Wang@aero.org), Charles P. Griffice (The Aerospace Corporation, System Planning and Engineering, El Segundo, CA 90245-4691, USA, Charles.P.Griffice@aero.org), Adam M. Fincham (University of Southern, Aerospace \& Mechanical Engineering, 854 W Downey way, Los Angeles, CA 90089-1191, USA, afincham@usc .edu), John R. Edwards (U. S. Air Force, Air Force Space Command, 483 N. Aviation Blvd., El Segundo, CA 90245-2808, USA, John.Edwards @LOSANGELES.AF.MIL), Adel A. Hashad (U. S. Air Force, Air Force Space Command, 483 N. Aviation Blvd., El Segundo, CA 90245-2808, USA, Adel.Hashad@LOSANGELES.AF.MIL)

This paper, review and status in nature, consists of three parts: (1) The salient nature and results of the published papers from an ocean sonic boom (OSB) project will be reviewed including theoretical and experimental studies of the wavy surface effect on the underwater acoustics, a threedimensional theory of underwater acoustics and the underwater acoustics induced by a sonic boom traveling at hyper-velocity speeds. (2) New unpublished results will be reported including studies of sonic boom underwater overpressures affected by ocean finite depth and ocean stratification (variable sound speed). These studies are possible due to the derivation of a semi-similar transformation for the underwater acoustics governing equations and the application of high performance computers. (3) The preliminary results of the work-in-progress will also be discussed including threedimensional extensions of former professor H. K. Cheng's two-dimensional wavy surface theory and laboratory verification.

\section{$5: 40$}

5pUWf4. Influence of a resonance changer on the sound radiation of a submarine. Sascha Merz (University of New South Wales, School of Mechanical and Manufacturing Engineering, 2052 Kensington, Australia, sascha.merz@gmx.net), Roger Kinns (University of New South Wales, School of Mechanical and Manufacturing Engineering, 2052 Kensington, Australia, roger.kinns@aol.com), Nicole J. Kessissoglou (University of New South Wales, 2052 Sydney, Australia, n.kessissoglou@unsw.edu.au)

In order to reduce the excitation of the submarine hull through the shaft, a vibration attenuation system, called a resonance changer, can be implemented in the propeller/propulsion system. The effectiveness of such a system in reducing the low frequency sound radiation characteristics of a submarine is investigated. Only sound radiation due to fluctuating propeller forces, which are generated by the operation of the propeller in a nonuniform wake, is considered. These fluctuating forces are transmitted to the submarine hull through the fluid, as well as through the propeller shaft. Both 
types of excitation cause hull vibration and sound radiation. The accordion modes of the pressure hull, are particularly efficient sound radiators. Parameters for the resonance changer system have been optimised previously by considering only excitation of the hull through the shaft. It is shown that the effectiveness of the resonance changer at different frequencies is modified significantly in a typical full-scale implementation, due to the sound radiation from the propeller. The effect on performance is increased further when the vibration of the propeller itself is taken into account. Therefore overall optimisation of any resonance changer system requires a comprehensive model. Some of the principal effects are explored in this paper.

\section{6:00}

5pUWf5. The research on measuring the coefficient of sound absorption in turbid seawater. Qi Li (Harbin Engineering University, College of Underwater Acoustic Engineering, 150001 Harbin, China, chizhafengyun1979@126.com), Yongwei Liu (Harbin Engineering
University, College of Underwater Acoustic Engineering, 150001 Harbin, China, chizhafengyun1979@126.com)

When naval mine-hunting sonars and side-scan surveying sonars operating at $20 \sim 60 \mathrm{kHz}$ are working in shallow coastal waters, the viscothermal absorption of sound by suspended mud in the water may greatly decrease their detection properties. This kind of water is also characterized as turbid seawater. However, the research on measuring the coefficient of sound absorption in turbid seawater has little been done. The main difference between turbid seawater and clear seawater is that there are mud particles suspending in turbid seawater. It is also the main reason why sound absorption in turbid seawater is greater than that in clear seawater. In the paper, the coefficient of sound absorption at $20 \sigma 60 \mathrm{kHz}$ in turbid seawater has been measured by the reverberation technique. Results demonstrate that if the concentration of the mud is bellow $0.11 \mathrm{~kg} / \mathrm{m}^{3}$, the mud in turbid seawater doesn't cause additional absorption. When the concentration of the mud is between 0.14 and $0.49 \mathrm{~kg} / \mathrm{m}^{3}$, the coefficient of sound absorption in turbid seawater is as twice at least as that in clear seawater. 